\title{
Behavioral barriers to success in education
}

Citation for published version (APA):

de Gendre, A. M. C. (2021). Behavioral barriers to success in education. [Doctoral Thesis, Maastricht University]. ROA. https://doi.org/10.26481/dis.20210329ag

Document status and date:

Published: 01/01/2021

DOI:

10.26481/dis.20210329ag

Document Version:

Publisher's PDF, also known as Version of record

\section{Please check the document version of this publication:}

- A submitted manuscript is the version of the article upon submission and before peer-review. There can be important differences between the submitted version and the official published version of record.

People interested in the research are advised to contact the author for the final version of the publication, or visit the DOI to the publisher's website.

- The final author version and the galley proof are versions of the publication after peer review.

- The final published version features the final layout of the paper including the volume, issue and page numbers.

Link to publication

\footnotetext{
General rights rights.

- You may freely distribute the URL identifying the publication in the public portal. please follow below link for the End User Agreement:

www.umlib.nl/taverne-license

Take down policy

If you believe that this document breaches copyright please contact us at:

repository@maastrichtuniversity.nl

providing details and we will investigate your claim.
}

Copyright and moral rights for the publications made accessible in the public portal are retained by the authors and/or other copyright owners and it is a condition of accessing publications that users recognise and abide by the legal requirements associated with these

- Users may download and print one copy of any publication from the public portal for the purpose of private study or research.

- You may not further distribute the material or use it for any profit-making activity or commercial gain

If the publication is distributed under the terms of Article $25 \mathrm{fa}$ of the Dutch Copyright Act, indicated by the "Taverne" license above, 
Behavioral Barriers to Success in Education 
(C) 2021 Alexandra Marie Catherine de Gendre

All rights reserved. No part of this publication may be reproduced, stored in an automated data system, or transmitted in any form or by any means, electronic, mechanical, photocopying, recording or otherwise, without prior permission of the author.

The author acknowledges financial support from the Department of Economics and the Graduate School of Business and Economics (GSBE) at Maastricht University, the Research Centre for Education and the Labour Market (ROA), and the University of Sydney.

This book was typeset by the author using $\mathrm{LT}_{\mathrm{E}} \mathrm{X}$. The cover is art work by Geoffrey de Gendre.

Published by ROA

Postbus 616

6200 MD Maastricht

ISBN: 978-90-5321-594-4

Printed in the Netherlands by Canon 


\title{
Behavioral Barriers to Success in Education
}

\author{
DISSERTATION \\ to obtain the degree of Doctor at Maastricht University \\ on the authority of the Rector Magnificus, Prof. Dr. Rianne M. Letschert \\ in accordance with the decision of the Board of Deans, \\ to be defended in public on \\ Monday 29 March 2021 at 9:30 hours
}

by

Alexandra Marie Catherine de Gendre 


\section{Supervisors:}

Prof. dr. Olivier Marie

Prof. dr. Rolf van der Velden

\section{Assessment Committee:}

Prof. dr. Didier Fouarge (chair)

Prof. dr. Deborah Cobb-Clark, University of Sydney

Prof. dr. Hessel Oosterbeek, University of Amsterdam

Prof. dr. Dinand Webbink, Erasmus University of Rotterdam

Prof. dr. Inge de Wolf 


\section{Acknowledgments}

I did not need to write a dissertation about the value of peers, parents and teachers to realize their importance. I have been very lucky to meet such people throughout my education in general, and during my PhD journey in particular.

I am grateful to Olivier Marie, my advisor, for challenging me, trusting me and supporting me over the years - ever since I showed up at his office in 2016 with a bunch of half-backed ideas!

I also thank Rolf van der Velden, my second advisor, for welcoming me with open arms at ROA, and for trusting me every step of the way, especially when it came to working a distance from Melbourne and to funding my research using Dutch administrative data.

Thank you also to Didier Fouarge, Inge de Wolf, Deborah Cobb-Clark, Hessel Oosterbeek and Dinand Webbink for accepting to assess my dissertation, and for their most encouraging feedback on my work.

The Melbourne Institute, the University of Melbourne and the Lifecourse Centre felt like home from my very first research visit. Deborah Cobb-Clark, Dave Ribar, John de New took me in as part of the PhD cohort. Victoria Baranov, Leslie Martin, and Renaud Coulomb were always so generous with their time, feedback, mentorship and friendship. Gigi Foster and Stefanie Schurer gave me lots of advice on how to navigate life as a young female economist. Tom Wilkening, Maria Recalde, Siqi Pan, Nisvan Erkal, Boon Han Koh and Edwin Ip welcomed me in the Australasian experimental community.

I am grateful to Thomas Dohmen for taking me on board as his TA, and for being there for me as a mentor every time I needed professional or research advice. Being Steffen Künn's TA for course in personal economics has also been a great teaching experience as well as a source of inspiration for my own research. 
It's hard to express just how thankful I am to Ulf Zölitz and Jan Feld for all the chats, which have helped me grow in recent years. It is not just because you are great conference mates, or because your research inspired me, but also because you are amazing mentors and friends.

Stepping foot in the academic profession would not have been as exciting without a fantastic community of friends around the world: Ira, Caro, Diana, Melisa, Anna, Jan \& Trish, Ulf, Ingo, Nicko, Maria \& Cosma, Henrik \& Sarah, Shush, Jose, Luca, Marloes, Kim, Pieter, Maria Z., Marion \& Joost, Frauke \& Diego, Paul, Myrte, Bart, Olga, Julie \& Laurent, Nico \& Amélie, Cain, Renaud, Susan \& Kevin, JK \& Katy, Jordy \& Ellis, Leslie \& Jeremy, Victoria \& Paul, Edwin \& Tammy, Dave \& Cathy, Sarah \& Rami. Many of you were also mentors, and I am so grateful for your guidance.

Je remercie enfin ma famille, mon inspiration et mon soutien inconditionnel. Geo, Lili et Franck, Diane et Arnaud, Papa et Maman, un immense merci. Je dois aussi tant, enfin, à mon Nico, qui m'a soutenu de manière infaillible, m'a encouragé et m'a toujours poussé à progresser à chacune des étapes. 
Author:

Alexandra Marie Catherine de Gendre
Dissertation Advisors:

Prof. dr. Olivier Marie

Prof. dr. Rolf van der Velden

\section{Behavioral Barriers to Success in Education}

\section{Thesis Summary}

This dissertation investigates the role of aspirations and beliefs in explaining children's educational outcomes, and explores how they can exacerbate socioeconomic disadvantage in education. Chapter 1 proposes an easily-implementable methodology to estimate the potential size of aspirational poverty traps in a given population - an important quantity which indicates the scope for reducing achievement gaps by lifting aspirations. Chapter 2 shows that policy uncertainty about student finance can alone deepen achievement gaps as early as upon entering middle school. This finding highlights that reducing uncertainty in student finance could alleviate barriers in access to higher education for vulnerable populations. Chapter 3 indicates that higher-achieving peers not only improve test scores, but also trigger a host of behavioral changes in children and parents, yet these intermediate responses do not explain peer effects in test scores. This finding suggests that peer assignment policies such as tracking or school admission policies can be leveraged to increase students' aspirations and reduce achievement gaps. Chapter 4 shows that teachers rely on imperfect signals about children's ability, and form systematically biased expectations about their potential, intensifying disadvantage in the short and longer run. This study, however, also suggests that the consequences of teacher bias can be limited by admissions policies in which teacher assessments have little weight. This dissertation, overall, demonstrates that aspirations and beliefs of students, parents and teachers matter for children's educational success, and that educational policies may reduce socioeconomic disadvantage through their impact on aspirations and beliefs. 



\section{Contents}

Acknowledgments . . . . . . . . . . . . . . . i i

Thesis Summary . . . . . . . . . . . . . . . . iii

Introduction $\quad 1$

1 Quantifying Aspirational Poverty Traps 31

1.1 Introduction . . . . . . . . . . . . . . . . . . . . 31



1.2.1 Aspirations Failure in Dalton, Ghosal and Mani (2016) . . . 36

1.2.2 Theory Expansion . . . . . . . . . . . . . . . . . 38

1.2.3 A Macro-Economic Interpretation of the Model . . . . . . . 40

1.3 Empirical Methodology . . . . . . . . . . . . . . . . . . . . . . 42

1.4 Empirical Application . . . . . . . . . . . . . . . . . . 44

1.4.1 Data . . . . . . . . . . . . . . . . 44

1.4.2 Results . . . . . . . . . . . . . . . 47

1.5 Robustness of Findings . . . . . . . . . . . . . . . . . . . . . . . 53

1.5.1 Non-Cognitive Skills . . . . . . . . . . . . . . . . . 54

1.5.2 Credit Constraints . . . . . . . . . . . . . . 57

1.5.3 Networks in Job-Search . . . . . . . . . . . . . . . . . 58

1.6 Conclusions . . . . . . . . . . . . . . . . . . . 59

2 Policy Uncertainty and Early Discouragement $\quad 65$

2.1 Introduction . . . . . . . . . . . . . . . . . 65

2.2 Institutional Setting . . . . . . . . . . . . . . . . . . . . . 70

2.2.1 Secondary School in The Netherlands . . . . . . . . . . . 70

2.2.2 Post-Secondary Education and Student Finance . . . . . . . 70

2.2.3 Student Finance Reform Announcements and Implementation 74

2.3 Conceptual Framework . . . . . . . . . . . . . . . . 75

2.3.1 Setting . . . . . . . . . . . . . 75

2.3.2 Model Predictions: Reform Announcements and Implementation 77

2.4 Data . . . . . . . . . . . . . . . . . . . . 79

2.4.1 Sample Selection . . . . . . . . . . . . . . . 80

2.4.2 Students Tracking Outcomes over Time . . . . . . . . . . . . 81

2.5 Empirical Strategy . . . . . . . . . . . . . . . . . . . . . 82

2.6 Main Results . . . . . . . . . . . . . . . . . . . . . . 85

2.6.1 Effects on Tracking Outcomes ............ 85 
2.6.2 Effects on Track Dropout _. . . . . . . . . . . . . . 87

2.7 Additional Results and Supporting Evidence . . . . . . . . . . . . . 89

2.7.1 Heterogeneous Effects by Family Income . . . . . . . . . . . 90

2.7.2 Heterogeneous Effects by Student Gender . . . . . . . . . . . 90

2.7.3 STEM Specialization and Dropout . . . . . . . . . . . 90

2.7.4 Later Effects: Living Arrangements and Labor Supply . . . 91

2.8 Limitations and Sensitivity of Findings . . . . . . . . . . . . . . 93

2.8.1 Alternative Specifications of Time Trends . . . . . . . . . . . 93

2.8.2 A Systematic Exploration of Potential Confounding Factors 94

2.8.3 Measurement of Tracking Outcomes with Error . . . . . . . 94

2.9 Conclusions . . . . . . . . . . . . . . . . . . . 95

3 Mechanisms of Exposure to High-Achieving Peers 105

3.1 Introduction . . . . . . . . . . . . . . . . . . . . . 105

3.2 Peer Effects in Education . . . . . . . . . . . . . . . . . . . . 108

3.3 Institutional Setting and Data . . . . . . . . . . . . . . . . . . 113

3.3.1 Education in Taiwan . . . . . . . . . . . . . . . . 113

3.3.2 The Taiwan Education Panel Survey . . . . . . . . . . . . . 115

3.4 Empirical Strategy . . . . . . . . . . . . . . . . . . . . . 119

3.4.1 Testing for Random Assignment of Peer Groups . . . . . . . 119

3.4.2 Random Assignment to Classrooms in TEPS . . . . . . . . . 122

3.5 Main Results . . . . . . . . . . . . . . . . . . . . . . . . . . . 129

3.5.1 Academic Peer Effects on Test Scores . . . . . . . . . . . . . 129

3.5.2 Academic Peer Effects on Educational Inputs . . . . . . . . 131

3.5.3 Mediation of Academic Peer Effects . . . . . . . . . . . . . . 135

3.6 Sensitivity Analyses . . . . . . . . . . . . . . . . . . . 139

3.6.1 Robustness of Identification Strategy . . . . . . . . . . . . . 139

3.6.2 Robustness to Measurement Error and Classroom Sampling 143

3.6.3 Randomization Inference and Multiple Hypothesis Testing 146

3.6.4 Heterogeneous Peer and Mediated Effects . . . . . . . . . . . 148

3.7 Conclusions . . . . . . . . . . . . . . . . . . . . . . . 149

4 Siblings and Teachers' Biased Expectations $\quad 159$

4.1 Introduction . . . . . . . . . . . . . . . . . . . 159

4.2 Institutional Setting and Data . . . . . . . . . . . . 163

4.2 .1 Institutional Setting . . . . . . . . . . . . . 163

4.2 .2 Data . . . . . . . . . . . . . . . . 165

4.3 Empirical Strategy . . . . . . . . . . . . . . . . . . . . 169

4.3.1 Identifying Variation . . . . . . . . . . . . . . . 169

4.3.2 Balancing Tests of Older Sibling Rank . . . . . . . . . . . 170

4.3.3 Estimation Equation . . . . . . . . . . . . . . . 173

4.4 Main Results . . . . . . . . . . . . . . . . . . . . . . . . . 174

4.4.1 Sibling Rank Effects on Test Scores . . . . . . . . . . . . . . 174

4.4.2 Sibling Rank Effects on Teacher Assessment . . . . . . . . . 177

4.4.3 Long-Run Effects of Sibling Rank . . . . . . . . . . . . . . . 180

4.5 Robustness of Findings . . . . . . . . . . . . . . . . . . . . . . . 182 
4.6 Mechanisms . . . . . . . . . . . . . . . . . . . . . . . 184

4.6.1 School Admission Rules . . . . . . . . . . . . . . . . 184

4.6 .2 Salience . . . . . . . . . . . . . . . . 186

4.6.3 Parental Investments . . . . . . . . . . . . . . . . . . 187

4.6.4 Sibling Interactions . . . . . . . . . . . . . . . . . . . . . . 189

4.7 Policy Implications . . . . . . . . . . . . . . . . . . . . . . . 190

4.8 Conclusions . . . . . . . . . . . . . . . . . . . . . . . . 192

$\begin{array}{ll}\text { Conclusion } & 195\end{array}$

$\begin{array}{ll}\text { References } & 209\end{array}$

$\begin{array}{ll}\text { A Appendix to Chapter } 1 & 231\end{array}$

A.1 Proofs ............................. 231

A.2 Data Preparation . . . . . . . . . . . . . . . . . 232

A.3 Additional Tables and Figures . . . . . . . . . . . . . . . . . 234

B Appendix to Chapter 2 247

B.1 Institutional Details . . . . . . . . . . . . . . . . . . 247

B.2 Proofs . . . . . . . . . . . . . . . . . 256

B.3 Additional Analyses . . . . . . . . . . . . . . . . . . . . . 260

B.4 Sensitivity Analyses . . . . . . . . . . . . . . . . . . . . . . 280

$\begin{array}{ll}\text { C Appendix to Chapter } 3 & 297\end{array}$

C.1 Construction of Standardized Scales in TEPS . . . . . . . . . . . . 297

C.2 Additional Tables . . . . . . . . . . . . . . . . . . . . . . . . . 303

C.3 The Fishing Algorithm . . . . . . . . . . . . . . . . . . . . 318

C.3.1 Sorting of students into classrooms within schools in TEPS . 318

C.3.2 The Fishing Algorithm . . . . . . . . . . . . . . . . . . . . . 321

C.4 Fishing Algorithm in Simulated Data . . . . . . . . . . . . . . . . . 330

C.4.1 The Data Generating Process (DGP) for our Simulations . . 330

C.4.2 Performance of the Fishing Algorithm . . . . . . . . . . . . 333

C.4.3 A Practitioner's Guide for Researchers Wanting to Use our Fishing Algorithm . . . . . . . . . . . . . . . 341

D Appendix to Chapter $4 \quad 343$

D.1 Data Preparation . . . . . . . . . . . . . . . . . . 343

D.2 Empirical Specification . . . . . . . . . . . . . . . . . . 345

D.3 Additional Tables and Figures . . . . . . . . . . . . . . . . . . . 348

D.4 Impact of Rank in the Long-Run . . . . . . . . . . . . . . . . 357

$\begin{array}{ll}\text { Valorization Addedum } & 361\end{array}$

$\begin{array}{ll}\text { Curriculum Vitae } & 362\end{array}$

ROA Dissertation Series $\quad 363$ 



\section{List of Tables}

1.1 Summary Statistics of the NLSY79 . . . . . . . . . . . . . . . 45

1.2 Baseline Model of Intragenerational Social Mobility . . . . . . . . . 47

1.3 Two-Threshold Regression Models of Intragenerational Social Mobility 50

1.4 Three-Threshold Regression Models of Intragenerational Social Mobility 52

1.5 Intragenerational Social Mobility and Locus of Control . . . . . . . 56

2.1 Student Finance in the Netherlands Before and After 2015 . . . . . 73

2.2 Summary Statistics of Grade 10 Students in the Netherlands . . . . 80

2.3 The Effects of Policy Announcements on Track Enrollment . . . . . 86

2.4 The Effects of Policy Announcements on Track Dropout . . . . . . 89

3.1 Mechanisms of Peer Effects Explored in Previous Literature . . . . 111

3.1 Description of Academic Achievement and Input Measures in TEPS 118

3.2 Balancing and Sorting Tests on the TEPS Trimmed Sample . . . . 127

4.1 Summary Statistics on Sibling Pairs, Years 2003 to 2016 . . . . . . 168

4.2 Balancing Tests: The Effect of Older Sibling Rank on Pre-Determined Characteristics of Younger Siblings . . . . . . . . . . . . . . 172

4.3 The Effects of Older Sibling Rank on Younger Sibling Test Scores . 176

4.4 The Effects of Older Sibling Rank on Younger Sibling Teacher Assessment . . . . . . . . . . . . . . . . . . . 178

4.5 The Effects of Older Sibling Rank on Long-Term Educational Outcomes181

A.1 Baseline Intragenerational Mobility . . . . . . . . . . . . . . . 235

A.2 Intragenerational Social Mobility with Two Thresholds . . . . . . . 236

A.3 Intragenerational Social Mobility with Three Thresholds . . . . . . 237

A.4 Intragenerational Social Mobility and Locus of Control . . . . . . . 238

A.5 Intragenerational Social Mobility and Self-Esteem . . . . . . . . . . 240

A.6 Intragenerational Social Mobility and Self-Concept . . . . . . . . . 242

A.7 Intragenerational Social Mobility and Credit Constraints . . . . . . 244

A.8 Intragenerational Social Mobility and Network-based Job Search Strategies . . . . . . . . . . . . . . 246

B.1 Financial Aid Amounts and Take-Up Rates, 2002-2018 . . . . . . . 253

B.1 Financial Aid Amounts and Take-Up Rates, 2002-2018 . . . . . . . 254

B.2 Financial Aid Eligibility and Repayment Conditions, 2002-2018 . . 255

B.3 Effects on Track Enrollment by Family Income . . . . . . . . . . . . 263 
B.4 Effects on Track Dropout by Family Income . . . . . . . . . . . . . 264

B.5 Effects on Track Enrollment by Gender . . . . . . . . . . . . . . 265

B.6 Effects on Track Dropout by Gender . . . . . . . . . . . . . . . . 266

B.7 Effects on Enrollment in STEM . . . . . . . . . . . . . . . 269

B.8 Effects on STEM Dropout . . . . . . . . . . . . . . . . 272

B.9 Effects on Enrollment in STEM, by Family Income . . . . . . . . . 273

B.10 Effects on STEM Dropout, by Family Income . . . . . . . . . . . . 274

B.11 Effects on Enrollment in STEM, by Gender . . . . . . . . . . . . . 275

B.12 Alternative Specification - Main Effects on Track Enrollment . . . . 280

B.13 Alternative Specification - Main Effects on Track Dropout . . . . . 281

B.14 Alternative Specification - Effects on Enrollment in STEM . . . . . 281

B.15 Alternative Specification - Effects on STEM Dropout . . . . . . . . 282

B.16 Employment Prospect of Tracks, by Education Level . . . . . . . . 284

B.17 High School Graduation Rates and Time to Graduation . . . . . . . 286

B.18 Number of Schools, Pupils, and Education Expenditures in the Nether-

lands . . . . . . . . . . . . . . . . . . . . 294

C.1 Construction of Standardized Scales of Educational Inputs in TEPS 298

C.1 Construction of Standardized Scales of Educational Inputs in TEPS 299

C.1 Construction of Standardized Scales of Educational Inputs in TEPS 300

C.1 Construction of Standardized Scales of Educational Inputs in TEPS 301

C.1 Construction of Standardized Scales of Educational Inputs in TEPS 302

C.2 The Effect of Peer Test Scores on Students' Own Test Scores in Wave 2303

C.3 The Effect of Peer Test Scores on Educational Inputs in Wave 2 . . 304

C.4 Returns to Educational Inputs from Cumulative Value-Added Models 305

C.5 Academic Peer Effect Mediated by Educational Inputs . . . . . . . 306

C.6 Permutation-Based Classroom-Level Sorting Tests in Estimation Sample307

C.7 Non-Parametric Sorting Test in Estimation Sample . . . . . . . . . 308

C.8 Sensitivity of Estimates to Latent Class Threshold in Posterior Probability in the Fishing Algorithm . . . . . . . . . . . . . . . . . . 309

C.9 Oster (2019) Proportional Selection on Unobservable Characteristics in Initial Sample . . . . . . . . . . . . . . . . . . 310

C.10 The Effect of Peer Ability on Students' Own Ability, Using Alternative Measures of Ability . . . . . . . . . . . . . . . . 311

C.11 Using Instrumental Variable Estimators to Account for Measurement Error in Ability . . . . . . . . . . . . . . . . . . . . . 311

C.12 Using a Mixed Ability IV Approach . . . . . . . . . . . . . . . . . . 312

C.13 Using Sojourner (2013) Correction for Incomplete Classroom Sampling313

C.14 Corrected P-Values: Randomization Inference and Step-Down Familywise Error Rate Adjustment Procedures . . . . . . . . . . . . . . 314

C.15 Heterogeneous and Mediated Effects of Peer Ability . . . . . . . . . 315

C.16 Heterogeneous Value-Added . . . . . . . . . . . . . . . . 316

C.17 35 Education Systems in Comparative Perspective in TIMSS 1999 • 317

C.18 Balancing and Sorting Tests on the TEPS Initial Sample . . . . . . 320

C.19 The Fishing Algorithm: Finite-Mixture Model Estimates . . . . . . 327

C.20 Summary Statistics of Key Variables in TEPS Across Samples . . . 329 
C.21 Performance of the Fishing Algorithm in Simulated Data - Case 1 . 334

C.22 Performance of the Fishing Algorithm in Simulated Data - Case 2 . 338

C.23 Performance of the Fishing Algorithm in Simulated Data - Case 3 . 340

D.1 The Effects of Older Sibling Rank on Younger Sibling Test Scores Across Specifications . . . . . . . . . . . . . . . . 346

D.2 The Effects of Older Sibling Rank on Younger Sibling Test Scores Across Ability Functional Form . . . . . . . . . . . . . . . . . . 347

D.3 The Effects of Older Sibling Ranks on Younger Sibling Track Recommendation, Controlling for Older and Younger Sibling Overall Score . . . . . . . . . . . . . . . . . . . . . . . . 348

D.4 The Effect of Older Sibling Rank on Younger Sibling Track Recommendation, Controlling for Older Sibling Subject Scores and Younger Sibling Overall Score . . . . . . . . . . . . . . . . . . . . 349

D.5 Robustness of Sibling Rank Effects to Strategic Delay . . . . . . . . 350

D.6 Heterogeneous Effects of Sibling Rank: by Population Density of Residential Area . . . . . . . . . . . . . . . . . . 351

D.7 Heterogeneous Effects of Older Sibling Rank on Teacher Subjective Assessment of Younger Siblings . . . . . . . . . . . . . . . . . 352

D.8 Heterogeneous Effects of Older Sibling Rank: by Migration Background353

D.9 Heterogeneous Effects of Older Siblings' Rank: by Presence in the Household of Older Child Eligible to Means-Tested Higher Education Financial Aid . . . . . . . . . . . . . . . . . . . 354

D.10 Heterogeneous Effects of Older Siblings' Rank: by Sibling Gender-Match355

D.11 Sibling Rank When the Importance of Teacher Assessment Increases 356

D.12 Long-Term Effects of Own Rank on Later Educational Outcomes . 359 



\section{List of Figures}

1.1 Macroeconomic Testable Predictions after Dalton et al. (2016) . . . 40

1.2 Detected Thresholds and Model Fit Criteria . . . . . . . . . . . . . 49

1.3 Location and Size of Aspirational Poverty Traps in the United States 53

1.4 Locus of Control and Permanent Income . . . . . . . . . . . . . . 55

2.1 Tracking Trends in The Netherlands, Years 2005-2017 . . . . . . . . 81

2.2 Track Dropout in The Netherlands, Years 2006-2017 . . . . . . . . . 88

3.1 The Education System in Taiwan . . . . . . . . . . . . . . . . . . . 114

3.2 Schools Identified as Defying Random Assignment Using the Fishing Algorithm . . . . . . . . . . . . . . . . . . . 125

3.3 The Effect of Higher-Achieving Peers Test Scores on Students' own Test Scores in Wave 2 . . . . . . . . . . . . . . . . . . . 129

3.4 The Effect of Higher-Achieving Peer Test Scores on Educational



3.5 Returns to Educational Inputs from Cumulative Value-Added Models 137

3.6 Academic Peer Effects Mediated by Educational Inputs . . . . . . . 139

4.1 The Education System in the Netherlands . . . . . . . . . . . . . . 164

4.2 Remaining Variation in Older Sibling Rank in the Preferred Specification 170

A.1 Self-Esteem and Permanent Income . . . . . . . . . . . . . . . 239

A.2 Self-Concept and Permanent Income . . . . . . . . . . . . . . . . 241

A.3 Access to Loans and Aspirational Poverty Traps . . . . . . . . . . . . 243

A.4 Network-Based Job Search Strategies and Initial Family Net Worth 245

B.1 The Dutch Education System . . . . . . . . . . . . . . . . . 248

B.2 Google Trends Associated to Student Finance Reform Details . . . 256

B.3 Enrollment In Tracks, by Ability and Teacher Recommendation . . 261

B.4 Trends in STEM Specialization in High School . . . . . . . . . . . . 268

B.5 Share of Dropout from STEM and Medicine Specialization in High School . . . . . . . . . . . . . . . . . . 271

B.6 Living Arrangements and Commuting Distance . . . . . . . . . . . 276

B.7 Labor Supply of Young Adults in the Netherlands, Years 2006-2017 278

B.8 Youth Unemployment Rate, 15 to 19 Year-Old Population . . . . . 285

B.9 Parental Unemployment Rate in our Estimation Sample, Since 2004289

B.10 Teacher Track Recommendations over Time . . . . . . . . . . . . . 292 
B.11 CITO-Based Track Recommendations over Time . . . . . . . . . . . 292

B.12 European Trends Towards Vocational Education over Time . . . . . 295

B.13 European Trends Towards STEM Fields in Tertiary Education over

Time . . . . . . . . . . . . . . . . . . 296

C.1 The School-Level Concentration of Classrooms Based on Test Scores,

With $B=400$ Classroom Assignment Permutations . . . . . . . . . 324 


\title{
Introduction
}

\author{
"The main hope of a nation \\ is the proper education of its \\ youth".
}

Erasmus

\section{The Promise of Equality of Opportunity in Education}

One of the most successful ideas of the Enlightenment-perhaps the most successful idea - has been the universal right to education. Throughout the 19th and 20th century, education has progressively become one of the largest and consistently unchallenged public investments in most countries. This culminated in the adoption in 1948 of the Universal Declaration of Human Rights, which states that "everyone has the right to education. [...] Technical and professional education shall be made generally available and higher education shall be equally accessible to all on the basis of merit." (Article 26, Paragraph 1). Ever since, education has been generally perceived as a universal right, and providing access to education as a duty of governments.

The general idea that access to education is a moral objective to strive for has led to a global expansion of education throughout the 20th century. While the average years of schooling in the Netherlands was just below 3 years in 1870, it was above 12 years in 2017; in China over the same period, most people received no formal schooling in 1870 to almost 8 years in 2017 (Lee and Lee, 2016). Lee and Lee (2016) show that as much as in 1870 most of the world population received at most 3 years of formal education, compulsory schooling laws across the world have led to the world population receiving on average 7 years of schooling by 2010 . 
The expansion of education was achieved through large public spending programs: in 1940, the Netherlands spent $1.4 \%$ of GDP on education expenditures and $5.5 \%$ in 1993; in the United States, education expenditures represented 4\% of GDP in 1960 and $5.5 \%$ in 1993. Public investment took place through various educational policies, used across the world to provide greater access to education to ever larger number of children from all social classes: school building programs, compulsory schooling laws, government-backed student loans and subsidies tuition fees and scholarships, teaching schools. The expansion of education reached children of all sub-populations, thus achieving an immense decline in inequality in educational attainment (Crespo Cuaresma et al., 2013).

The expansion of education throughout the 20th century largely paid off with the fastest economic growth the world has ever seen. Roser and Ortiz-Ospina (2016), among many others, show that the expansion of education in the 20th century correlates with the progress of democracy and with large increases in GDP per capita across the world. In an unprecedented endeavor, Hendren and Sprung-Keyser (2020) estimate the return on investment of 133 historical policies in the United States since the 1950s, and find that education and early life health investments yield the largest returns, essentially because they target young populations whose gains in earnings translate in higher taxes paid back to the community over ever longer lifetimes.

Socioeconomic gaps remain, however, disappointingly pervasive at all levels of education. Family background remains the single most important determinant of educational achievement, in spite of decades of educational policies and interventions aiming at weakening the link between socioeconomic disadvantage and low educational attainment - ranging priority rules in school admissions, to school quality monitoring, education vouchers and charter schools. James Heckman has shown that socioeconomic gaps in child cognitive and non-cognitive development emerge in the first five years of a child's life, before even entering education, and never cease to increase during education. Susan Dynarski has shown throughout her career that poorer children and children of less educated parents are systematically less prepared to enter college, less likely to go to college, and less likely to graduate.

In many developed countries, the education expansion has stalled in the wake of the Great Recession, and recent years have even seen a resurgence of socioeconomic gaps in educational attainment between the rich and the poor, between young men and women, between migrant and native children. In the European Union, the rising number of youth that are not in employment, education or training-largely 
low-educated young men, with large disparities between countries - has revealed that education systems are increasingly failing to prepare youth to enter labor markets and contribute to society. In the United States, Raj Chetty questions the myth that America is a "land of opportunity": throughout several influential studies, he shows that the earnings of working adults are largely explained by early life experiences with teachers, neighborhoods, and family, yet good experiences are systematically associated with higher family socioeconomic status.

Recent studies reveal that disadvantaged children might face soft barriers in access to higher education, often arising from complex and inefficient design of education institutions. In 2015, Caroline Hoxby and Sarah Turner have revealed that information is a barrier to higher education: a large share of high-achieving students from low-income background in the United States don't even apply for college because they over-estimate the costs of higher education (Hoxby and Turner, 2015). In 2012, Eric Bettinger, Bridget Terry Long and Phillip Oreopoulos have shown that institutional complexity is also a barrier: many students fail to graduate from college because they struggle navigating the complex financial aid system (Bettinger et al., 2012). In 2018, Alberto Alesina, Michaela Carlana, Elena La Ferrara and Paolo Pinotti have shown that prejudice in the school system is another barrier: teachers themselves hold negative stereotypes against immigrant children, and play a role in their lower educational trajectories (Alesina et al., 2018). These studies all reveal that closing socioeconomic gaps in educational achievement will require to rethink the roots of disadvantage.

While the 20th century has been one of education expansion, the 21st century must be one of education revolution: gaining a deeper understanding of the origins of socioeconomic disadvantage, identifying the multi-faceted barriers hindering the success of disadvantaged children, and redesigning policies levers to close socioeconomic gaps.

\section{A New Paradigm of Persisting Achievement Gaps: Behavioral Barriers}

The idea that complexity leads to worse decisions is not new. Behavioral scientists such as Dan Ariely, Cass Sunstein and Richard Thaler have revealed over the past 20 years that people's decisions can be nudged by the tiniest details - a default option, the framing of a question. They have also shown that nudges are simple and cheap 
to implement, and yield powerful results, making them ideal tools for policymakers.

Yet beyond the policy implications of nudges, what we have learned is that internal constraints matter a great deal for people's decisions-perhaps as much as hard, external constraints like budgets or production constraints. In his best-selling psychology book "Thinking Fast and Slow", Daniel Kahneman shows that people make imperfect decisions that depend greatly on their immediate environment (Kahneman, 2011). In his seminal anthropological work in 2006, Arjun Appadurai argues that poor people don't just lack material resources, but also "the capacity to aspire" (Appadurai, 2004). Economists Anandi Mani, Anuj Shah, Sendhil Mullainathan and Eldar Shafir formulated an even bolder hypothesis: that beyond material deprivation, the state of poverty in itself creates mental scarcity, imposing psychological constraints to decision-making (Shah et al., 2012; Mani et al., 2013). In an influential study in 2012, they show that experimentally induced mental scarcity impedes cognitive functioning, which leads directly to worse decisions. Since then, recent studies in economics have found that poverty causes stress, loss of self-control, inattention, all of which in turn have a negative causal impact on decision-making (e.g. Haushofer and Fehr, 2014; Bernheim et al., 2015). Thus, the idea that people could make choices under internal constraints has brought new perspectives in economics, borrowing from other social and human sciences with a long tradition of studying the role of beliefs, values and identities in people's decisions - psychology, sociology, anthropology, ethnology and history.

This idea that there could exist internal constraints, creating behavioral barriers to economic success also brought deep change in the design of development interventions and anti-poverty programs, to focus not just on improving material conditions but also on the improving the psychological state of the poor. In a series of studies, Travis Lybbert and Bruce Wydick argue that hopelessness is an essential aspect of poverty, such that psychological coaching should be an core component of anti-poverty programs (Lybbert and Wydick, 2018). They implemented this program around the world, to find important and persistent positive impacts on indicators of poverty reduction. The World Health Organization, recognizing the large potential gains from implementing low-cost psychological coaching, has since launched "Thinking Healthy", a world-wide poverty-reduction program based on cognitive behavioral therapy.

The existence of internal constraints has immense implications for the economics of education, possibly leading to a major overhaul of many common policy tools to close socioeconomic gaps. In an influential article in 2016, Adam Lavecchia, 
Heidi Liu and Philipp Oreopoulos review an impressive number of interventions, and conclude that "education represents a relatively new avenue for behavioral economics, one that holds many opportunities. [...] The examples we've presented here suggest that interventions shaped by behavioral theory are likely cost-effective and easy to implement, while delivering significant results. They are exciting, testable and tenable" (Lavecchia et al., 2016). In her presidential address at the European Economic Association in 2018, Elena La Ferrara argued that economists should pay more attention to aspirations, how they form and how they drive life-changing decisions, such as going to university and what to study: "Aspirations are a potentially important determinant of individuals' educational investments and occupational choice. This opens the way for policy interventions aimed at changing people's aspirations as a way of lifting them out of poverty." (La Ferrara, 2019).

Very recent studies - most of them not even yet published in academic journals - have started to explore internal constraints in education. Mukherjee (2015) show that Indian students and parents' ethnic identity and caste can lead them to refrain from investing in higher education. Guyon and Huillery (Forthcoming) find that poor children and children of migrant backgrounds in France have lower educational aspirations than their peers, at all levels of ability.

This dissertation builds on this emerging body of work testing for new forms of behavioral barriers and constraints, and explores how they exacerbate socioeconomic disadvantage in education. This dissertation makes four key contributions to the discipline. Chapter 1 proposes an easily-implementable methodology to estimate the potential size of aspirational poverty traps in a given population. This is an important quantity which indicates the scope for reducing achievement gaps by lifting aspirations. Chapter 2 shows that policy uncertainty about student finance can alone create or exacerbate achievement gaps as early as upon entering middle school. This finding highlights that access to higher education could be improved by reducing uncertainty in student finance, and that uncertainty about future policies can harm vulnerable populations, worsening inequality of opportunity. Chapter 3 indicates that high-achieving peers not only improve test scores, but also make children exert less school effort, increase their aspiration to go to university, and increase their parents' time investments and strictness. More importantly perhaps, this study also shows that peer effects in test scores are not explained by intermediate effects on students' effort, aspirations and parental time investments and strictness. This finding suggests that peer assignment policies such as tracking or school admission policies can be leveraged to achieve lower achievement gaps and increase students' aspirations. Chapter 4 shows that i) teachers rely on imperfect signals about children's ability, 
and form systematically biased expectations about their potential, ii) teacher bias disadvantages vulnerable students, and iii) has long-lasting consequences on STEM major choice in high school. Suggestive findings also indicate that teacher bias can be limited through admissions policies in which teacher assessments have little weight.

Before presenting the problem statement of this dissertation and delving into each chapter in more detail, I argue in the next section why my research belongs to economics, and what the key challenges are conceptually, methodologically and empirically.

\section{An Economic Approach to Behavioral Barriers in Education}

Building on insights from several other disciplines, this dissertation belongs to economics for three key reasons: first, because it builds on classical economic theory and enriches economic theory to study internal constraints in education; second because it relies on modern econometric tools and quasi-experimental research designs that are common to economists, and third and perhaps more importantly because it provides answers that are directly useful to contemporary discussions in economics.

Compared to other social sciences, economics has traditionally studied 1) how isolated individuals make decisions in light of their environment, 2) how individual decisions aggregate into market-level outcomes such as prices, and 3) how markets interact to produce societal-level equilibria such as social norms. Within the social sciences, the most distinguishing feature of economics is its systematic use of the scientific method, much like the natural sciences. Since the early days of the discipline, economist have focused on developing parsimonious and falsifiable theoretical models of human behavior, and systematically designing experiments to test empirical predictions of models.

Within economics, the economics of education is a field in which researchers study the drivers of children's educational outcomes, and how to design education systems to give the same chances to all while efficiently allocating resources. Although a relatively young sub-field, the economics of education has become one of the densest ones, in which economists explore these two problems through two essential frameworks: the human capital theory and the education production function framework. 
Human capital theory expands the borders of classical microeconomic theory to the labor market and to education. Stemming from the pioneering work of Gary Becker and Jacob Mincer in the 1960s, human capital theory proposes that workers' skills are a special type of capital affecting the productivity of firms; wages are paid to workers as a return on their investment, and the level of wages depends on the contribution of their human capital to firm production (see e.g. Becker, 1964; Mincer, 1958). As a consequence, people's decision to acquire education is a decision to invest in human capital; educational choices should thus resemble investment choices, and depend on factors such as costs, returns, and risk. Since the 1960s, economists keep finding evidence consistent with this theory; Charles et al. (2018) recently showed, for example, that local housing booms and bursts change relative wages for high and low educated workers, which in turn affect the decisions of local students to go to college or not.

The education production framework expands the borders of classical microeconomic theory of firms, emphasizing the role of public resources in the educational outcomes of children. The education production framework links education "outputs" to "inputs", thus opening a new avenue for studying the optimal allocation of public resources between children, and therefore the design of education policy (see e.g. Hanushek, 1979). In this framework, human capital can be seen as the output of a production process involving inputs of all sorts, such as teacher wages, teacher training, class size or school quality. Thus, one can hope to explain heterogeneity in educational outcomes as the results of differences in abilities and choices, and differences in resources. Under some modelling assumptions, it thus allows the researcher the relate changes in outputs to changes in inputs.

Modern research in the economics of education has sought to expand both human capital theory and the education production framework. In an influential study published in 2003, Petra Todd and Kenneth Wolpin proposed an augmented education production function, incorporating that the human capital of children is the result of repeated investments of multiple agents (parents, teachers, schools) - an insight from developmental psychology (Todd and Wolpin, 2003). Since Todd and Wolpin's influential work, an emerging strand of literature has started systematically exploring how various agents and inputs interact in the education system, to achieve more credible estimates of the impact of educational policies. For example, Pop-Eleches and Urquiola (2013) show that parents of children who are admitted to elite secondary schools reduce their money and time investment in their children. This study thus indicates that estimating the impact of school quality on child test scores must account for how parents adjust their inputs. 
At the knowledge frontier in the economics of education, one of the key challenges is to understand what drives individual beliefs and how they affect educational outcomes in the form of internal constraints creating behavioral barriers to the success of some. Understanding how beliefs affect education outcomes is of crucial importance: if differences in outcomes between low and high socioeconomic status students are driven by individual preferences, then differences in outcomes are simply heterogeneity and don't necessarily reflect inequality of opportunity; if, however, differences in outcomes arise from differences in beliefs, then informing individuals might be required to ensure the same chances are given to all. In addition, while beliefs can be changed with information, altering preferences is at best barely possible, if not ethically questionable.

Yet studying beliefs in economics turns out to be challenging from both a theoretical and an empirical perspective. Theoretically, it requires relaxing some core assumptions in both human capital theory and the education production function framework; empirically, it requires careful research designs performed on well-chosen, highly detailed data, originating from settings with specific institutional features.

\section{The Conceptual Problem of Internal Constraints in Economics}

At the theoretical frontier, studying the role of beliefs in educational outcomes requires augmenting human capital theory and the education production function framework by relaxing the seemingly innocuous assumption that individuals have complete information. Yet, lifting this fundamental assumption is problematic in three ways.

First, conceptually, it is unclear how to operationalize beliefs in economic decision models, and consequently how to measure beliefs. In arguably some of the most important work on beliefs in economics, Charles Manski lays out a program for integrating beliefs in economic models: he first proposes to provide a structure to beliefs in the form of subjective probabilistic expectations, which allows beliefs to be compared between individuals and within individual, and then argues that with appropriate measures of subjective expectations, we can relate changes in outcomes to changes in subjective expectations induced by shocks in the decision environment of individuals (Manski, 1993a, 2000).

Second, even with measures of subjective expectations, once we lift the assumption that decision-makers are fully informed, we encounter a fundamental identification problem: with different information, agents are likely to express different preferences, 
such that separately assessing the role of beliefs from preferences on observed outcomes becomes strenuous. Some studies for example explore experimentally to what extent belief-changing information shocks affect preferences over outcomes. Thus, to study the role of beliefs on educational outcomes, it is best to choose a setting where individual preferences are less likely to play an important role.

Third, economists do not yet have the theoretical tools to aggregate the decisions of individuals with heterogeneous beliefs into markets, and further into societal-level equilibria. In a series of theoretical studies since 2007, Douglas Berheim and Antonio Rangel have been slowly developing the theoretical foundations for welfare economics incorporating findings from behavioral economics.

In a series of studies on health insurance markets since 2015, Benjamin Handel presents a striking illustration of how difficult it is to incorporate beliefs in modern economic models to conduct welfare analyses and policy. He shows that i) people systematically under invest in health insurance due to misinformation and behavioral biases; ii) wrong decisions are eventually costlier for disadvantaged people who suffer from generally worse health; lastly, iii) ignoring the presence of these biases, we would design insurance policies that are highly regressive.

Yet compared to the economics of health care insurance, the economics of education presents the additional layer of complexity that multiple agents and multiple inputs interact in producing child human capital. In such, even with augmented human capital theory and the education production framework to incorporate the beliefs of children, parents and teachers, drawing clear policy implications has never been harder.

\section{Empirical Challenges of Estimating Behavioral Barriers in Education}

At the empirical frontier, studying the role of beliefs in educational outcomes requires carefully designed experiments, and utmost caution when interpreting statistical estimates and deriving policy implications.

Over the past 30 years, the "credibility revolution" in applied microeconomics has shown that for empirical studies to infirm or support economic models of decisions, they must remain as close as possible to the ideal experiment. An ideal experiment is a theoretical experiment that allows the researcher to discern unarguably the empirical predictions of one model from any the predictions of another model, following the scientific method. Yet constructing such ideal experiments or even 
staying close to this standard is a challenge for three key reasons.

First, turning a broad problem statement into an ideal experiment is not as obvious as it may sound. It requires formulating a narrow research question focused on a causal relationship of interest, that is informative of a broader theoretical economic model. This approach thus consists in focusing on a specific parameter at a time in a theoretical model, and to conceptually determine the causal effect of this factor on a given outcome of interest. Thus, the first main challenge of modern empirical microeconomics of education is to achieve causal estimates of a particular factor on education outcomes.

Second, even once we have identified a target relationship of interest between a factor and an outcome of interest, constructing a research design that is close to the ideal experiment is also not trivial. To meet this goal, the researcher must find or construct an empirical setting in which a randomly selected group of people is assigned to a treatment - say a given value of the factor of interest, while another randomly selected group is assigned to a placebo - say a baseline value of the factor. This way, the mean difference in outcomes between the treatment and the placebo or control groups is an unbiased estimator of the causal effect of the treatment on the outcome. Thus, the second main challenge of modern empirical microeconomics of education is to argue to what extent statistical estimates do capture the true causal effect of a factor on an outcome.

Chapter 2 tests whether disadvantaged students are discouraged early on from university education, due to uncertain costs of studying, a mechanism hypothesized by some studies to contribute to achievement gaps in college enrolment (Lochner and Monge-Naranjo, 2016). To test this hypothesis, this chapter estimates the effect of exogenous, unexpected policy announcements which took place in the Netherlands in 2010 to study how students change their educational orientation in response to increasing uncertainty about future financial aid at university. The treatment is receiving information about the upcoming policy. Treated students are thus those who receive information, and control students are those who do not receive information. Since the policy announcements took place at the national level, the treatment affects all students from the release of the policy announcement, thus, to the extent that the policy announcement is as good as random, such that past cohorts are comparable to treated cohorts, we can achieve causal estimates of the effect of the policy announcement on changes in the education choices of treated students.

Chapter 3 estimates the impact of classroom peers on a myriad of educational inputs, 
and whether intermediate input responses explain student test scores. This chapter uses a natural experiment which takes place in the education system of Taiwan: a national mandate dictates that middle school children must be randomly assigned to classes within their school. Thus, conditional on being in a given school, we can compare the effect of being randomly exposed to classroom peers of various types on standardized test scores. In this configuration, the treatment that children are assigned to is not binary but is the classroom composition, such as the average test scores of classroom peers: the comparison thus becomes children in the same school assigned to either a classroom with lower or higher achieving peers. To the extent that children are indeed similar across classrooms within schools, this comparison yields causal estimates of the impact of classroom peer characteristics on educational inputs and test scores.

Chapter 4 estimates the impact of older siblings on teacher subjective assessment about the ability of children. In this chapter, I use the relative rank of older siblings in their primary school class in a national test of ability that Dutch pupils take upon exiting primary school. In this setting, we compare two similar younger siblings, whose older siblings had the same teacher and received the same score in the test, but one of them was top of his class, and the other was not. In this quasi-experiment, the treatment is having an older sibling with a higher relative rank. Thus, to the extent that younger siblings are similar, this comparison yields causal estimates of the impact of sibling rank on younger siblings' outcomes.

Naturally, each methodology has its flaws. The validity of each estimation methodology relies on assumptions which cannot always directly be tested. In applied microeconomics, since the "credibility revolution", researchers have been increasingly expected to discuss the limitations of their methodological choices, the assumptions under which estimates uncover causal relationships between treatments and outcomes, and how much we can interpret from them. This endeavor to achieve ever more methodological transparency, has come at the cost of lengthier yet more narrowly-defined research questions, but with the gain of more precise and robust findings.

\section{Data Challenges to Study Behavioral Barriers in Education}

Finding statistical data about students' experience in education used to be troublesome. Historically, researchers relied on large surveys such as labor force surveys, which only report outcomes of but never processes in education, and household surveys such as the DNB Household Survey in the Netherlands, which almost never 
collect student test scores or ability measures, nor student experience in education. There were also occasional cohort studies, such as the National Longitudinal Survey of Youth in the United States, which have the advantage of following respondents for a long time, yet information about schooling environments is almost never available, and natural attrition and small samples often make it laborious to explore nuanced mechanisms. Lastly, these types of data sources are rare, because of their high implementation and maintenance cost, not available in many countries and at any time. Even then, accessing existing data of this type is often easier said than done.

The past 10 years have, however, witnessed an explosion in the use of new data sources, starting with administrative individual-level data. Figlio et al. (2016) argue that the "promise of administrative data in education research" is three-fold: large sample, often population-level data, allowing researchers to i) achieve more precise estimates to bring new answers to older researcher questions; ii) focus on small, vulnerable populations which are under-represented in surveys; and iii) highlight new mechanisms which have small but important theoretical implications. Lastly, in some settings, administrative data can be linked to uncover or test new phenomena: in the United-States, the states of Texas, North-Carolina or Florida have notoriously allowed researchers to link education records to birth records, which allowed, for instance, David Figlio and co-authors to show the long-term negative impact of adverse health conditions at birth on educational outcomes of children (Figlio et al., 2014). Luckily, I was granted access to the Dutch administrative data infrastructure, CBS, thanks to which I was able to conduct research using linked education registries, birth, housing and labor market records for all Dutch pupils since the mid 2000s.

Administrative microdata sources do not only originate from government agencies, nor do they always represent the entire population. Some of the most innovative studies of the past 10 years in economics have been conducted using administrative data from isolated educational institutions. These sources give access to highly detailed, new information on the progress of students throughout the education system. This permits new research designs to emerge, and the testing of finer mechanisms on intermediate outcomes, e.g. retention, orientation and specialization in majors, test scores and teacher assessments. For example, Todd Stinebrickner and Ralph Stinebrickner collected a panel study at Berea College in United States in the 2000s, linked the data to administrative records to explore how students learn about their ability as they progress, and ultimately why students drop out (see e.g. Stinebrickner and Stinebrickner, 2001, 2006). Ulf Zölitz and Jan Feld have used the administrative records of the School of Business and Economics at Maastricht University and written some of the most convincing articles in recent years on peer 
effects (see e.g. Feld and Zölitz, 2017; Zölitz and Feld, Forthcoming).

Still, administrative microdata sources are not a panacea. First, accessing administrative data sources remains challenging and restricted for confidentiality reasons. Not many administrations give access to their administrative data, and often only to citizens or residents. Acquiring individual records from school districts, individual schools or firms is even more complicated because, as much as access can easily be granted, it can just as easily be revoked. Second, many high-quality administrative microdata cannot be matched to other sources. For example, in the United-States, administrative education microdata is only available at the state-level, but due to high geographic mobility, many students disappear from state-level administrative records because they moved to another state. Second, even when available, administrative data remains costly, sometimes prohibitively costly to acquire. Third, since administrative data were never meant for research purposes, they require a large investment in time and effort to understand institutional context, what exactly is being recorded and why. These data often don't come with detailed codebooks nor questionnaires, such that they also require experience with more standard data to diagnose issues.

Estimating behavioral barriers in education adds an additional set of complications. Conducting empirical research on behavioral barriers in education requires highquality measures of beliefs, which is not widely spread. Administrative data only records outcomes that are useful for administrative purposes, and individual beliefs, perceptions, as well as many social and demographic characteristics are typically not recorded. Even in survey data, occasional measures of beliefs are seldom good enough: they could be very noisy, or be formulated in a faulty manner such that there is no variation in answers. For example, the National Longitudinal Survey of Youth measures students' knowledge of occupations, but possible answers are sufficiently obvious that these data don't seem to contain a lot of useful information for researchers. The Taiwan Education Panel Study is one rare example of a panel study combining large-scale sampling, low attrition across waves of survey, and high-quality measures of beliefs and outcomes of students.

\section{Problem Statement and Dissertation Structure}

This dissertation investigates if, how and how much individual beliefs can become behavioral barriers in educational success. To address this problem statement, I broke it down into four research questions, addressed separately in four distinct 
studies:

1. What is the scope of internal constraints for explaining the persistence of socioeconomic inequality of opportunity in the long-run?

2. Are disadvantaged students discouraged early on from participating in universitypreparing education simply by uncertain access to financial aid?

3. Can we lift the aspirations of disadvantaged students by exposing them to high-achieving peers? How much do lifted aspirations translate into higher educational performance?

4. Can teacher beliefs become behavioral barriers to the success of disadvantaged children?

\section{Chapter I: Quantifying Aspirational Poverty Traps}

There is little evidence of the economic relevance of behavioral barriers in education as an explanation for the persistence of socioeconomic inequality. Therefore, an important and natural initial research question for any researcher entering this field of economics is: to what extent can we reasonably expect these types of barriers to matter in the long-run and at the macro-economic level?

As naïve as it may sound, this is a crucial question, both from an academic and from a policy perspective. Until we are able to answer this question, it remains unclear to what the extent we should care about behavioral barriers for the design of public policies. In Chapter $1^{1}$, I explore the scope of a specific type of behavioral barrier as an explanation for the persistence of inequality in long-term individual wealth: aspirational poverty traps, socioeconomic environments in which people systematically exert low effort and achieve low economic success. Yet if quantifying poverty traps is hard, quantifying aspirational poverty traps is yet harder. ${ }^{2}$

Conceptually, quantifying aspirational poverty traps requires to establish two separate cause-to-effect relationships linking individual circumstances and effort choices to outcomes: i) to establish at the micro-economic level how internal constraints makes people under-achieve relative to their potential; and ii) to establish to what extent internally-constrained v. unconstrained people compare in the long-run in the population. To this day, the evidence on the existence of and the impact of internal constraints on individual outcomes remains contextual and limited to small,

\footnotetext{
${ }^{2}$ Joint work with Nicolás Salamanca at the Melbourne Institute, The University of Melbourne.
} 
narrowly defined estimates. The evidence on the impact of internal constraints in a wider population and in the long-run is virtually nonexistent. Yet, even in the presence of short-term effects of behavioral barriers on educational outcomes of children, whether these effects would translate into long-term differences in outcomes remains unclear, let alone whether these differences would reflect macro-economic sub-optimal equilibria.

In this first project, I first propose a simple econometric methodology to estimate in any national context the location and size of aspirational poverty traps, under minimal data requirements. I start from Dalton et al. (2016)'s theoretical model describing how people form aspirations, and how these aspirations affect long-run investments, among which in education. This model is the first to show that theoretically, there could exist aspirational poverty traps. I extend the model to show that aspirational poverty traps imply systematically low intergenerational socioeconomic mobility, yet higher mobility than material poverty traps.

Quantifying empirically aspirational poverty traps using my methodology requires i) data on long-term trajectories of individual to assess the plausibility of such predictions - this kind of data turns out to be quite rare; and ii) nationally representative data. Individual outcomes in the long-run is key, because if small scale behavioral mechanisms have no impact in the long-run, then studying such mechanisms might not be our best use of research funding. On the other hand, if small scale behavioral mechanisms do matter in the long-run, then investing in channeling these mechanisms in policies could turn out to be the most efficient use of public funds. Nationally representative data is required because my methodology entails detecting changing regimes of social mobility in a given population. To be able to detect such changes, we need the maximum amount of data across the distribution of wealth.

I then provide some initial results on the empirical importance of aspirational failure as a factor explaining heterogeneous lifetime earnings of individuals. I apply this methodology to the United States, and find that roughly 5.5\% to $7.5 \%$ of the population could be in such a trap, and that these people would be located just below the median household in terms of wealth, around the 37 th and 42 nd percentile of the distribution of wealth.

This initial exercise is important because it suggests that there could be scope for improving the lives of an economically significant share of the population by raising their aspirations. This study calls for several avenues of follow-up research work. A first one could be to extend this analysis to many countries, in order to investigate the role of institutions in the existence, size and location of aspirational poverty 
traps. A second one, which I have chosen to pursue ever since, is to focus on how public policies can reduce or exacerbate inequality by triggering behavioral responses of individuals.

\section{Chapter II: Early Discouragement from Uncertainty About Financial Aid}

In this chapter, I investigate whether disadvantaged students could be discouraged early on in their educational career from participating in university-preparing education; this is a specific way that beliefs can become behavioral barriers to opportunities in education.

Over the past five years, several studies have shown that informing students about the cost of higher education can have a large impact on their decision whether to apply to higher education, at which institution and which major. One of the emerging ideas in this literature is that underprivileged students could be shying away from university-preparing education early in their educational career due to biased beliefs about the true cost of education (see Lochner and Monge-Naranjo, 2016). While a handful of studies have shown that disadvantaged students often over-estimate the cost of higher education and under-estimate their eligibility to financial aid, there is virtually no evidence that they shy away from universitypreparing education early on in their educational career. Yet understanding this early discouragement effect is crucial for designing optimal financial aid and higher education financing systems, balancing the right amount of incentive for students to exert study effort while attracting high-achieving students to higher education.

In Chapter $2^{3}$, I study the role of students and their families' beliefs in their early discouragement to pursue university-preparing education. To do this, I focus on the impact of policy uncertainty about financial aid on the tracking outcomes of secondary school students in the Netherlands.

There are two key reasons why policy uncertainty about financial aid is a good experiment to study whether the early discouragement effect exists. Firstly, while policy uncertainty is largely understudied in applied microeconomic studies, macroeconomic studies have shown that people respond to uncertainty in their decision environment: traders respond to central bank announcements even before any policy change takes

\footnotetext{
${ }^{3}$ Joint work with Jan Kabátek at the Melbourne Institute, The University of Melbourne.
} 
place, job seekers update their expectations about finding jobs when government reveal national unemployment statistics. Although many applied microeconomics studies show that people do learn from their experience, there is however hardly any evidence that students and families respond to uncertainty about future costs of higher education. Secondly, as much as administrative data does not collect individual beliefs, policy uncertainty prior to actual policy changes allows us under reasonable assumptions to interpret changes in educational trajectories as responses to changes in expectations, not responses to changes in actual prices.

To investigate how uncertainty about the future of financial aid can discourage some students from going to higher education, I exploit announcements of a controversial Dutch reform of financial aid, which turned universal, unconditional grants for higher education into income-contingent loans. Specifically, I use the staggered policy announcements of the "Sociaal Lensteelsel" and its later implementation, in the academic year 2015-2016: In June 2010, the Dutch government announced its general intention to move away from the subsidy-based system of student finance towards a loan-based system, without giving specific details. This announcement increased the expected price of higher education for all students by making the prospective receipt of financial aid uncertain. This initial announcement created a heated debate in the Netherlands, with news outlets, political parties and student trade unions heavily speculating on the government's potential bill while a general panic spread through the student population. In October 2012, after new elections, the government revealed in a second announcement the details of the new system, including its name "Sociaal Lensteelsel", its conditions and the first cohort of higher education students to be affected, while also revealing that vocational education students would be exempted.

I first propose a theoretical framework to describe in what way we can expect uncertainty about future financial aid conditions alone to affect educational decisions. I build on a simple model of human capital investments in which students make educational choices at every stage of their education anticipating on their complete trajectory. I then enhance this model by explicitly modelling uncertainty in parameters associated with student finance at higher education and studying the role of uncertainty in educational choices. The central prediction of my framework is that students' subjective probability of having to pay back financial aid can discourage low-income students from higher education, as early as when they can opt-out of tracks associated with higher probabilities of having to pay back.

Using administrative records for the universe of Dutch high school students, I 
find that the first policy announcement led to a sharp decrease in the share of students enrolling in college-preparing tracks, and to an immediate jump in the share of students dropping out of these tracks into the vocational track. The second announcement led to a further decrease in the share of students enrolling in collegepreparing tracks while dropout rates decreased. While we can expect students through the education system to respond to policy announcements concerning major university access reforms, this study makes a simple yet important contribution to this literature, by providing the first evidence that policy uncertainty alone about financial aid alone can lead a substantial share of secondary-school students to avoid college-preparing education.

Lastly, I perform a battery additional analyses to show that these results are unlikely to find better suiting alternative explanations. In particular, because all students were exposed to nation-wide announcement shocks at once, there is no obvious control group other than past cohorts, such that this study could only rely on a comparison of cohorts over time. The central concern with comparing cohorts before versus after policy announcements, is that there could be many confounding factors correlated with the timing of reform announcement shocks. To address this concern, I show that there are no trend breaks in 12 different potentially confounding factors correlated with the timing of reform announcements: 1) earnings of new graduates by education type, 2) employment rates of new graduates by education type, 3) minimum wage rates, 4) youth unemployment, in particular throughout the Great Recession, 5) high school graduation rates, 6) average time to graduation in postsecondary education, 7) costs of attending post-secondary education - tuition fees, other financial aid amounts, conditions, interest rates, 8) parental unemployment, 9) school capacity constraints, 10) other changes of the Dutch education system, 11) cross-country trends in track choice, and 12) cross-country trends in the take-up of STEM education.

Ultimately, this study tells us that policy uncertainty about the future can be devastating for people's educational plans. Children can shy away from university education as early as upon entering middle school, because of uncertainty about the future cost they will have to pay to go to university. This study suggests that policy announcements alone can exacerbate inequality of opportunities by triggering students' behavioral responses; students perceived uncertainty about financial aid affects their trajectories earlier than ever shown in previous research. 


\section{Chapter III: Lifting Aspirations for Better Educational Outcomes}

In this chapter, I explore whether exposing disadvantaged students to high-achieving peers can lift their aspirations, and whether lifting aspirations can improve educational achievement. Chapter $3^{4}$ follows up project on Chapter 1 . While Chapter 1 shows that there is scope for lifting people out of aspirational poverty traps, Chapter 3 provides some initial answers to the important question of how. Exposing children to high-achieving classroom peers appears as a naturally promising and relatively low-cost policy for lifting aspirations of students from disadvantaged backgrounds. Chapter 3 explores whether and to what extent classroom peers can improve educational outcomes by lifting aspirations.

Before even delving into how peers may or may not be able to lift aspirations, a major roadblock is that while an immense empirical literature has documented that peers in school do affect test scores, we still know very little about why and how peers matter. Several recent studies have elicited potential mechanisms of peer effects in isolation and across various settings, yet decades of research on education have shown that students performance is shaped by several simultaneous inputs from students, parents and teachers. The main issue with studying mechanisms in isolation is that it does not address the crucial question: what drives academic peer effects?

To address my research question, I need to investigate in one and the same setting 1) whether high-achieving peers matter for test scores, 2) whether high-achieving peers affect intermediate outcomes including aspirations, which are potential mechanisms through which peers affect test scores, and 3) whether high-achieving peers matter for test scores through these educational inputs. This study is the first to provide answers to all three steps jointly.

I use rich survey data from Taiwan on a representative sample of middle school pupils. Through a stratified nested sampling design, the survey collected detailed information about 20,000 students, their parents, teachers and school administrators, across multiple classrooms per school, firstly just after random assignment, and then two years later. Importantly, middle schools are mandated to randomly assign students to classrooms, such that students' exposure to higher- v. lower-achieving peers is as good as random within schools. I find that when exposed to classrooms

\footnotetext{
${ }^{4}$ Joint work with Nicolás Salamanca at the Melbourne Institute, The University of Melbourne.
} 
with 1 standard deviation higher-achieving peers, pupils experience a 5.4 percent of a standard deviation boost in standardized test score two years later. Next, I find that exposure to high-achieving peers also decrease student school effort such as homework, and increase university aspirations and expectations, as well as the time pupils spend with their parents. Perhaps most importantly, the positive effect of high-achieving peers on test scores is not explained by any of these intermediate effects.

While testing whether the data were indeed consistent with random assignment to classes, I designed the "Fishing Algorithm", a methodology I developed to exclude from the estimation sample specific schools where random assignment does not seem to hold. After first constructing an index of concentration of student types in classrooms within schools, the algorithm then uses randomization inference techniques and finite-mixture modelling to construct for each school a probability that pupils are assigned to classes not at random. Beyond the application of this algorithm in this paper, this algorithm is of independent interest and can be used, e.g. to detect clustered violations of randomization to treatment in RCTs.

I perform an extensive set of sensitivity analyses and find that my results are remarkably robust to various estimation decisions and identifying assumptions. Because these findings rely crucially on the assumption that students are indeed randomly assigned to their classroom within their school, I run multiple tests for conditional random assignment of children to classes in schools. I also propose alternative estimates with an exhaustive set of controls, and discuss to what extent correlated selection on unobservable characteristics could explain away the results. Because the mediation findings rely on the size of effects of high-achieving peers, I perform further sensitivity analyses: I further compute alternative estimates using bias corrections for measurement error in student ability and for incomplete sampling of classes, I use inference corrections using randomization inference and multiple hypotheses testing adjustments, and I extensive explore the extent of heterogeneity in peer effects and their mediation.

This is the first study showing causal evidence that randomly assigned high-achieving peers affect educational aspirations and expectations of students, and is also the first study to show that the effects of peers on intermediate educational inputs do not mediate the positive effects of peers on outcomes. This is an important finding, because if peers have a separate effect on beliefs and on outcomes, then they have the potential to create behavioral barriers that are unrelated to student performance by lowering aspirations. This study, however, also tells us that the potential of 
treatments increasing students' aspirations for improving outcomes remains limited: while exposing disadvantaged children to high-achieving peers might improve their aspirations, higher aspirations are unlikely to translate into higher performance, either because peers don't increase aspirations enough, or because aspirations don't translate much into better educational outcomes.

\section{Chapter IV: Teachers' Biased Beliefs and Inequality of Opportunity}

This last chapter address a key question: can teacher beliefs become behavioral barriers to the success of disadvantaged children? As exposure to teachers is hardly a margin that disadvantaged students can act upon, assessing whether teachers have biased beliefs about pupils, and the extent to which teachers' beliefs can exacerbate inequality of opportunity is crucial. Few studies have found evidence that some teachers hold biased views about their pupils, yet we know very little about why teacher bias arises, and what policies can reduce the consequences of bias. Recent randomized control trials have shown suggestive evidence that teacher bias may be reduce by training teachers to recognize their biases; these solutions, sadly, are neither scalable, nor are they immune to more pervasive types of biases. In Chapter 4, I focus on one of the simplest, most common ways that teachers might form biased expectations about the ability of a child: by knowing their older siblings.

I use administrative data from the Netherlands over 2003 to 2016, and exploit two specific features of the Dutch school system that allow me study teacher bias: first, pupils exit primary schools with a standardized test score which is comparable over time and throughout the Netherlands, and second teachers formulate a subjective assessment of their pupils' ability to pursue secondary education into one of three secondary school tracks. For every child, I construct his relative rank on the standardized test within his primary school cohort. As cohort composition varies between schools and within schools over time, for any specific test score, a child's relative rank goes from being at the top to being at the bottom of the cohort's ability distribution. For over 530,000 pairs of siblings, I then estimate the effect of older siblings' relative rank in their primary school cohort on their younger siblings' i) own primary school test score, and ii) teacher subjective assessment.

The relative rank of a child's older sibling is thus a good experiment to estimate teacher bias for two key reasons: firstly because, from the younger sibling's perspective, his older sibling's relative rank is as good as random conditional on the older sibling's absolute test score; secondly because, while the absolute performance of the older sibling contains information about cognitive ability that may legitimately also 
apply to the younger sibling (e.g. genetics, family resources and family environment), the relative rank of the older sibling is a direct function of the test scores of the older siblings' peers, and therefore contains no residual information about the younger child's endowments.

I find that older siblings' rank increases younger siblings' standardized test scores and, conditional on younger siblings' test scores, improves their teacher's subjective assessment. In a form of "shielding" effect, teachers are more lenient with younger siblings whose older siblings have already shown high academic potential relative to their cohort, even after controlling for the direct effect on younger siblings' own performance. I also find that older siblings' rank impacts the educational trajectory of their younger siblings in the long-run, by increasing their probability of graduating from high school with a STEM specialization. That older siblings' rank affects the test scores of younger siblings highlights that sibling spillovers effects may occur through motivation, even in the absence of additional human capital.

More importantly, my findings demonstrate that teacher biased expectations arising from older sibling rank effects also have the potential to exacerbate inequality of opportunity for disadvantaged students. When comparing native-born sibling pairs to first and second generation migrant sibling pairs, I find that older siblings with higher ranks provide a similar boosting effect on younger siblings' performance, but older siblings rank improve teachers' assessment only of native-born younger siblings. While teachers could have private information that families of migrant children tend to be more attracted towards vocational education, gender differences are more difficult to explain. Comparing sisters and brothers, I find that older sisters' rank has a larger positive effect on younger sisters' Math score compared to younger brothers', and a stronger positive effect on the probability that the teacher recommends the academic track for younger sisters compared to younger brothers. Older brothers' rank, on the other hand, has a larger negative effect on their younger brothers' Math score, but a large positive effect on their language score, and precisely estimated null effects on teacher recommendations. Overall, these findings indicate that children with different demographic characteristics are judged differently by teachers, possibly in a manner consistent with statistical discrimination. Because my study exploits sibling spillover effects to elicit teacher biased expectations, my results can be interpreted as a lower bound of the impact of teacher bias in general on children's educational trajectory.

Lastly, I explore to whether simple educational policies can reduce the adverse effects of disadvantage on teacher bias. To do this, I further exploit two educational 
policies that reduced the role of teacher subjective assessments on the admission of pupils at secondary schools and tracks. I find that when teacher assessment matter less for the admission chances of pupils in secondary school, sibling rank effects on teachers' subjective assessment seems to disappear, while the effect on younger siblings' performance remains.

This study highlights that teachers' beliefs about pupils' ability can be unrelated to their true ability, and have long-lasting impact of children's educational outcomes. At the same time, my findings also suggest that teachers' expectations can potentially be altered by educational policies.

Lastly, I conclude this dissertation with a summary of the contributions of each study, their limitations, and perspectives on future avenues for research. 

Chapter I:

Quantifying Aspirational Poverty Traps 

The first chapter of this dissertation investigates the empirical relevance of internal constraints as a potential explanation for the persistence of inequality in longrun labor market outcomes and wealth. In Chapter 1, we focus on a specific type of behavioral barrier as an explanation for the persistence of socioeconomic inequality: aspirational poverty traps, socioeconomic environments in which people systematically exert low effort and achieve low economic success.

We first develop a methodology to estimate the location and the size of aspirational poverty traps in any population using a modest amount of data. We start from the theoretical model of Dalton et al. (2016) which describes how individuals make decisions to invest either high or low effort to achieve future wealth, under budget constraints and with aspirational goals. In their model, there are two types of individual decision-makers: on the one hand, fully rational individuals who fully internalize that their aspirations affect their effort, such that they choose both their aspirations and their effort level; on the other hand, behavioral decision-makers have not internalized that aspirations affect effort, such that their effort choices are entirely dependent on their initial draws of aspirations. Two concrete examples will help illustrate this idea. Imagine two individuals: Peter, and Paul. Peter and Paul both have the same childhood dream of becoming firemen. Growing up, Peter updates his dream to teacher, which requires exerting more study effort in the form of going to university. Accordingly, Peter exerts this extra effort, and eventually becomes a teacher. Paul, on the other hand, takes his childhood dream unquestioningly as given, and accordingly does not go to college. Dalton et al. (2016)'s model is the first to show that, theoretically, there could exist aspirational poverty traps. The key lessons from their model is twofold: on the one hand, people who imperfectly update their aspirations have lower chances of receiving high wages or accumulate wealth compared to people who can update their aspirations over time to self-motivate to exert high effort; on the other hand, if poverty increases the chance that a person imperfectly updates his aspirations, then the sole existence of this imperfect aspiration updating implies that there will be aspirational poverty traps. 
To understand this second insight, consider again Peter and Paul. An aspirational poverty trap emerges if pupils like Peter tend to belong to wealthy families, while pupils like Paul tend to belong to poorer families.

In the theoretical part of Chapter 1, we extend the model of Dalton et al. (2016) to determine two key quantities: the size and the location of aspirational poverty traps in a given distribution of permanent income or lifetime wealth. We show that the existence of imperfect aspiration updating in the population implies systematically low intergenerational socioeconomic mobility at the bottom of the distribution of initial conditions driven by low aspirations, then discreetly increasing rates of within-lifetime income growth as initial conditions improve. Importantly, that intragenerational and intergenerational socioeconomic mobility is nonlinearly distributed in the population is a stylized fact, that now receives a deeply rooted economic interpretation.

A second important contribution of this study is to provide us with a simple methodology, with minimal data requirements, to estimate the size and location of aspirational poverty traps: with data on lifetime earnings, structural breaks in intragenerational mobility indicate the location of the population potentially trapped in low-aspiration low-effort equilibria.

In the empirical part of the study, we provide some initial results on the empirical importance of aspirational failure as a factor explaining heterogeneous lifetime earnings of individuals, using well-known survey data from the United-States to showcase our methodology in the case of the United States. Since 1979, the National Longitudinal Survey of Youth 1979 has been following annually or bi-annually a representative sample of over 12,000 people born between 1957 and 1964. Since our main theoretical finding is that the presence of behavioral people precisely leads to regime-switching points in intragenerational socioeconomic mobility, we estimate models of intragenerational socioeconomic mobility using threshold regressions. Although threshold regressions have not traditionally been used in this literature, threshold regression models present the key advantage that they allow the relationship between regressors such as initial conditions and outcome variables such as permanent income to switch discreetly, without necessarily knowing ex-ante the location of these switching points. Our methodology provides us with some of the first estimates of the location of regime-switching points, and then estimate models of intragenerational mobility with structural breaks.

Our theoretical analyses indicate that aspirational poverty traps are located in the middle region the distribution of intragenerational mobility, and our empirical 
analysis using the NLSY79 indicates that approximately $5.5 \%$ to $7.5 \%$ of the population could be in such traps in the United States, located just below the median household in terms of wealth, around the 37th and 42nd percentiles.

Our additional analyses suggest that our empirical findings are unlikely explained by other possible explanations. In particular, we show that the structural breaks we find in the relationship between initial conditions and lifetime earnings are not driven by systematic differences in locus of control, self-esteem nor job search strategies. 



\section{Quantifying Aspirational Poverty Traps ${ }^{1}$}

\subsection{Introduction}

Inequality of opportunity is one of the most pressing socioeconomic issues in the 21st century, and has been widely studied by social scientists since the beginning of the 19th century. A central research question in this field is to understand how cross-sectional inequality transmits from one generation from the next. A handful of studies show theoretically and empirical how local institutions and market failures can generate poverty traps, and economists have only recently begun to understand that poverty traps come in different types. Classical poverty traps caused external constraints creating material deprivation, are characterized by the poor being so constrained by their material well-being that they cannot improve it. ${ }^{2}$

Recently, a growing literature in economics has proposed a new approach to poverty, and has shown that material deprivation often generates a psychological burden, which can hinder decision-making and forward-looking behavior, such as human capital investments. Focusing on psychological or behavioral dimensions of poverty, the new economics of poverty shows that the psychological burden of poverty can be thought of as imposing internal constraints that impair decision-making (e.g. Bertrand et al., 2004; Mani et al., 2013). ${ }^{3}$

\footnotetext{
${ }^{1}$ This study is joint work with Nicolás Salamanca at the Melbourne Institute.

${ }^{2}$ Several types of material deprivation have been documented to generate classical poverty traps, for instance: poor health conditions and malnutrition (Dasgupta and Ray, 1986), limited access to credit or insurance (Loury, 1981; Banerjee and Newman, 1991, 1993; Galor and Zeira, 1993), the resources to develop productive skills (Kremer, 1993), or well-developed institutions (Bardhan, 1997), and the limiting effect of people's geographical and social environment (Hoff and Sen, 2005, 2006).

${ }^{3}$ Several types of psychological factors of poverty have already been documents: hopelessness and the lack of willpower (Duflo, 2012), the cognitive load imposed by poverty (Mani et al., 2013; Shah et al., 2012), the role of identity in retaining and acting upon information (Duflo et al., 2011b), low self-control (Bernheim et al., 2015) and systematically low aspirations (Bernard et al., 2014;
} 
Recent theoretical findings argue that there could be aspirational poverty traps, a specific type of behavioral poverty traps which hinders forward-looking behavior and investments. First documented in the seminal anthropological work of Appadurai (2004), aspirational poverty traps are a particular type of state in which loweffort equilibria are perpetuated not by people's lack of resources but by depressed aspirations to improve their own welfare - deprivation of the "capacity to aspire". Ray (2006) theorized how aspirations can work as incentives for people to invest in their future, and Genicot and Ray (2017) extends this theory to focus on how aspirations are socially-determined and what effects this can have on the distribution of income in the long-run. These models predict a U-shape relationship between individual initial conditions, aspirations and investments: when aspirations are too far or too close from initial conditions, they fail at providing the right incentives to invest. Dalton et al. (2016) are the first to show that theoretically aspirations failure can actually generate aspirational poverty traps that are empirically and conceptually distinct from classical poverty. ${ }^{4}$ Studying aspirational poverty traps is crucial, as stressed by La Ferrara (2019)'s presidential address at the Journal of the European Economic Association and Ray and Genicot (2019)'s recent review of the literature. ${ }^{5}$ Both studies indicate that the existence of such traps is a paradigm-shifter in the way we think about anti-poverty programs in developed and in developing economies. ${ }^{6}$

More generally, the existence of aspirational poverty traps also has important implications for the way we understand social mobility and inequality. Classical approaches to economic inequality and social mobility can be divided into two broad categories: the first approach focuses on measuring intergenerational mobility using

Beaman et al., 2012; Dupas et al., 2020).

${ }^{4} \mathrm{~A}$ notable other and earlier study outside of the development literature and in line with Dalton et al. (2016) is the study of Page et al. (2007), which offers an interpretation of aspirations as reference points in a prospect theory framework, and provides experimental evidence that aspirations drive the choices of students.

${ }^{5}$ Notable empirical studies on aspirations and associated concepts such as reference points in economics include: Beaman et al. (2012); Bernard et al. (2014); Macours and Vakis (2014); Mukherjee (2015); Lybbert and Wydick (2018); Ross (2019); Janzen et al. (2017); Guyon and Huillery (Forthcoming); Contreras Suarez and Cameron (2020).

${ }^{6}$ First, the existence of such traps implies that there are people who we can pull out of poverty by readjusting their expectations through interventions, which could be substantially less costly than their corresponding transfers from a traditional welfare program (e.g. Bernard et al., 2014; Goux et al., 2017). Second, the existence of such traps could also explain why for some people cash transfers are ineffective in helping them out of poverty (e.g. Dupas et al., 2020; Contreras Suarez and Cameron, 2020). Lastly, the existence of aspirational poverty traps could also explain why treatments combining cash transfers with non-cognitive skills training and psychological support have been so effective at improving people's outcomes (Heckman and Kautz, 2013). 
correlations between parents' and their children's' earnings at the same age in their life-cycle, and the second approach focuses on measuring the role of specific factors in the earnings of one generation -the children's generation - controlling for initial socioeconomic circumstances.

In the first approach to social mobility, the existence of aspirational poverty traps could imply distinct regimes of the transmission of socioeconomic conditions from one generation to the next, through parental decisions and external economic conditions that children are exposed to during their childhood. Aspirational poverty traps could contribute to explain some of the latest evidence on racial differences in intergenerational mobility, even after comparing children growing up in the same block and with similar fam(Chetty et al., 2020). Aspirational poverty traps could also contribute to explain that Durlauf et al. (2017) and Kourtellos et al. (2020) find evidence of non-linear intergenerational mobility in the United-States, with low mobility at the top and at the bottom of the wealth distribution, and high mobility around the mean. They show that a part of this non-linear pattern is related to non-cognitive skills of parents. Expanding on the seminal work of Becker and Tomes (1979), Becker et al. (2018) propose a unifying theoretical model of intergenerational mobility that incorporates the role of parental non-cognitive skills in the trajectory of their children. Thus, aspirational poverty traps could explain the finding that low-SES parents invest less in the human capital of their children than more affluent parents. A handful of studies has found some evidence for this mechanism. Janzen et al. (2017) show that parents in rural Nepal form aspirations about the schooling of their children based on their neighbors, and that these aspirations predict actual investments in human capital. Beaman et al. (2012) show that, in India, a policy allowing women to be elected in local councils increased the education and occupational aspirations of parents of girls.

In the second approach to social mobility, the existence of aspirational poverty traps could imply distinct regimes for the level of or the return to individual factors in lifetime earnings, thus contributing to cross-sectional heterogeneity in earnings level and trajectories. There is growing correlational evidence that disadvantaged people have lower aspirations (Bernard et al., 2014; Guyon and Huillery, Forthcoming), and there is also emerging evidence that people's aspirations have a causal impact on their own educational outcomes (Goux et al., 2017; Ross, 2019), yet more research is needed to understand how, why and when aspirations form and can be pivotal for educational outcomes of children. Above all, we know very little about the role of aspirations on long-run inequality, and in such, whether aspirational poverty traps exist. There is, in fact, little empirical evidence of the existence and economic 
relevance of aspirations, and of aspirational poverty traps in particular.

In this study, we propose a simple and tractable methodology to determine the size and location of aspirational poverty traps under minimal data requirements. We extend the model of aspirations failure of Dalton et al. (2016) to show theoretically that aspirational poverty traps would imply empirically sharp changes in income growth rates in the cross-sectional distribution of socioeconomic initial circumstances, that is, aspirational poverty traps would generate three distinct regimes in intragenerational social mobility: until some threshold in family earnings, children with low initial circumstances experience low social mobility and remain at the lowest percentiles in the distribution of earnings within their generation; from a higher point in the distribution of family earnings, children with highest initial circumstances experience low social mobility and remain at the highest percentiles in the distribution of earnings within their generation. In between these two thresholds, we find a zone of high intragenerational social mobility, which upper part corresponds to an aspirational poverty trap. Aspirational poverty traps thus correspond to a regime of intermediate intragenerational social mobility, where individual aspirations could be pivotal in making better forward-looking decisions.

We then propose an application to the United States using data from the National Longitudinal Survey of Youth 1979 (NLSY79). We estimate thresholds regression models of intragenerational mobility, and find robust evidence for non-linear intragenerational mobility, that is, we show that there are sharp changes in income growth experienced by individuals starting from different socioeconomic circumstances. Although we cannot directly pinpoint to the causal role of aspirations in determining individual income growth regimes, we argue that the sharp changes in the rate of income growth in the cross-section can be used as an indication of the location of aspirational poverty traps in the distribution of initial circumstances, and an upper bound of the size of these traps. We find that roughly $5.5 \%$ to $7.5 \%$ of the US population could be in an aspirational poverty trap, and concerns children with initial conditions around the 37 th and the 42nd percentile of the distribution of wealth.

Our empirical methodology brings us close to Durlauf et al. (2017), who use threshold regressions to document nonlinearities in intergenerational mobility in the United States, which they consider to be the first evidence for what they call "status traps" a type of socioeconomic disadvantage below which intergenerational mobility sharply drops. Our study contributes to the literature in three ways. First, our study complements the literature on intergenerational mobility which has recently started 
to document systematic heterogeneity in social mobility: Durlauf et al. (2017) and Kourtellos et al. (2020) emphasize that parental cognitive and non-cognitive traits are important for the intergenerational social mobility of their children, while Chetty and Hendren (2018) and Chetty et al. (2020) suggest that Black youth in the Unites States experience better outcomes when paternal figures are present. Our study complements this literature by providing new micro-economic foundations to the empirical regularity that social mobility is non-linear. Thus, our study complements theirs by showing that aspirational poverty traps would empirically materialize by the existence of non-linear individual income growth over the life-cycle, in other words, intragenerational mobility. Second, our study provides some of the first empirical grounds for the existence of aspirational poverty traps in a developed context. Third and perhaps most importantly, our study provides a simple and easily implementable methodology with minimal data requirement to determine whether there could be aspirational poverty traps, their location and their size. This methodology is useful for the design of policy interventions targeted at disadvantaged youth. With an idea of which part of the population could be in an aspirational poverty traps, policymakers could target research studies more easily, and design more efficient interventions aimed at raising aspirations.

This paper is organized as following. Section 1.2 reviews the aspiration failure model by Dalton et al. (2016), and presents our macro-economic implication for intragenerational social mobility. Section 1.3 presents our estimation methodology. Section 1.4 details our empirical application to the United States using the National Longitudinal Survey of Youth 1979. Section 1.5 discusses the robustness of our empirical findings, and Section 1.6 concludes. Technical details can be found in Appendix.

\subsection{A Theory of Aspirational Poverty Traps}

Aspirational poverty traps have been modelled theoretically in three main models. Ray (2006) develops a theoretical model in which individual aspirations work as incentives for investment behavior, and Genicot and Ray (2017) extends this model to focus on long-term dynamics of inequality under different regimes of aspirations formation. Dalton et al. (2016) is the only study, to the best of our knowledge, which explicitly shows how a lack of aspirations generates poverty traps that are conceptually different from classical poverty traps. In this sense, Dalton et al. (2016) provide another way of looking at the relationship between aspirations and effort provision. In their model, failing to aspire is a cognitive bias in which individuals 
fail at integrating that their aspirations and effort provision are endogenous. They show that even when this cognitive bias is randomly distributed in society, poverty worsens the consequences of this cognitive bias, thus generating a poverty trap that is conceptually different from a classical poverty trap. We build on their findings by deriving testable predictions from their theory that would allow us to empirically test the existence of aspirational traps and quantify their economic importance. We show that aspirational traps manifest themselves empirically through a highly non-linear relationship between people's initial and final wealth. The essential features of this non-linear relationship, which can inform us of the existence and significance of aspirational traps, can be estimated using threshold regressions with multiple thresholds.

\subsubsection{Aspirations Failure in Dalton, Ghosal and Mani (2016)}

In this subsection, we briefly explain the aspiration failure model of Dalton et al. (2016), which gives rise to the aspirational traps that we later analyze. We keep their notation throughout the paper. The model describes how people with exogenouslygiven heterogeneous levels of initial wealth $\left(\theta_{0} \in \mathbb{R}_{+}\right)$and potentially endogenous aspirations about their final wealth $\left(g \in \mathbb{R}_{+}\right)$make effort choices $(e \in\{0,1\})$, and how all of these elements together jointly determine their final wealth $\left(\theta \in \mathbb{R}_{+}\right)$. Formally, people's utility function is modeled as:

$$
u(e, g, \theta)=b(\theta)+\nu\left(\frac{\theta-g}{\theta}\right)-c(e)
$$

where $b$ describes classical consumption utility from final wealth, $\nu$ is a referencedependent value function capturing the additional utility gained or lost from comparing one's final wealth to a reference point - in this case the aspired level of final wealth $g-$, and $c$ is a cost of effort function. These functions are assumed to be well-behaved and to fulfill some additional conditions discussed in Dalton et al. (2016). The final wealth production function is assumed to be $\theta=(1+e) / \theta_{0}$, reflecting the assumption that, for a given level of effort exerted, wealthier people can obtain a higher level of final wealth. Finally, this model classifies individuals into two mutually exclusive types, rational or behavioral, as defined by the way their aspirations are determined ${ }^{7}$.

\footnotetext{
${ }^{7}$ Dalton et al. (2016) consider consistent effort-aspiration pairs as equilibria for their model, which effectively rules out situations where a person fails to meet their aspirations (as opposed to e.g.,
} 
In this model, aspirations $g$ are in principle endogenous and are affected by the level of effort exerted by people. Herein, however, lies the difference between rational and behavioral individuals. Since more effort is rewarded with higher final wealth, people's intention to exert higher effort should also result in them having higher aspirations, and this relation is fully internalized by rational individuals. Choices of rational individuals are thus described by them choosing effort levels $e$ to maximize Equation (1.1) subject to $g=\theta=(1+e) / \theta_{0}$. Behavioral individuals, on the other hand, are subject to a cognitive bias which makes them fail to fully internalize the fact that aspirations should respond to their exerted effort. Their choices are thus described by them choosing effort levels to maximize Equation (1.1) subject to $g=\bar{g}\left(\theta_{0}\right)$, where $\bar{g}$ is a fixed aspirations level akin to a steady state in an adaptive preference mechanism, and $g_{0}$ is the initial preference draw from a stable distribution which results in aspirations being set at $\bar{g} \cdot{ }^{8}$

The main results of Dalton et al. (2016) can be summarized as following:

1. There exists a threshold level of initial wealth, $\hat{\theta}$, which determines the behavior of rational individuals:

(a) If $\theta_{0}<\hat{\theta}$, rational agents will exert no effort $(e=0)$ and obtain $\theta=\theta_{0}$

(b) If $\theta_{0} \leq \hat{\theta}$, rational agents will exert effort $(e=1)$ and obtain $\theta=2 \theta_{0}$.

2. There exists two additional threshold levels of initial wealth, $\theta_{L}$ and $\theta_{H}$, which determine the behavior of behavioral individuals:

(a) If $\theta_{0}<\theta_{L}$, behavioral agents will exert no effort $(e=0)$ and obtain $\theta=\theta_{0}$

(b) If $\theta_{L} \leq \theta_{0} \leq \theta_{H}$, behavioral agents can end up in either a low-effort and low-wealth outcome $\left(e=0 ; \theta=\theta_{0}\right)$, or in a high-effort high-wealth outcome $\left(e=1 ; \theta=2 \theta_{0}\right)$.

(c) If $\theta_{0} \geq \theta_{H}$, behavioral agents will exert effort $(e=1)$ and obtain $\theta=2 \theta_{0}$.

3. Lastly, $\theta_{L} \leq \hat{\theta} \leq \theta_{H}$.

Ray (2006) and Genicot and Ray (2017) who also discuss inconsistent pairs. Our derivations also rely on this assumption. However, the authors do discuss in their appendix how their model can be extended to incorporate such aspiration gaps. The strength of their model is that aspirational poverty traps can emerge even when considering only consistent effort-aspiration pairs.

${ }^{8}$ For the sake of brevity, we introduce some notation at this point. See Dalton et al. (2016), p.174 for a more detailed explanation of this process, in particular their footnote 15. 
Among behavioral agents whose initial wealth lies between $\theta_{L}$ and $\theta_{H}$, some individuals will end up sub-optimally exerting low effort and achieve low wealth. This occurs for behavioral agents whose initial aspirations $g_{0}$ are too low and they failed to update them by noticing that if they exerted higher effort they could aspire to more.

Dalton et al. (2016) further characterize the range of initial aspirations $g_{0}$ for which agents are located in an aspirational poverty trap - traps from which they could in principle be pulled out. They define a stable cumulative distribution function $M$ from which $g_{0}$ is drawn, such that $M(x)=\mathbb{P}\left(g_{0}<x\right)$. They further show that there exists some level $\tilde{g}\left(\theta_{0}\right)$ which determines the effort regime that a behavioral agent will choose: draws of initial aspirations $g_{0}<\tilde{g}\left(\theta_{0}\right)$ lead to low-effort equilibria, while draws of initial aspirations $g_{0} \geq \tilde{g}\left(\theta_{0}\right)$ lead to high-effort equilibria. The authors lastly show that $\tilde{g}$ is a decreasing function of $\theta_{0}$ (Lemma 5), which implies some correlation between material poverty and low aspirations - a result which is consistent with the evidence found in developing countries.

Dalton et al. (2016)'s model makes several key contributions to the literature: they show that aspirational poverty traps can theoretically exist, and be conceptually and empirically different from classical poverty traps although correlate. There can be behavioral agents whose initial wealth is such that $\hat{\theta} \leq \theta_{0} \leq \theta_{H}$ and whose draw of initial aspirations land them in a low-effort equilibrium. These agents are in an aspirational trap, in the sense that they could be optimally exerting high effort and enjoying a high wealth had their initial aspiration not been set so low.

\subsubsection{The size and location of aspirational poverty traps}

In this section, we extend the model of Dalton et al. (2016) to derive the key quantities of interest to our study: the size and location of aspirational poverty traps. We first recast the model as follows:

1. The probability that a rational person lands in a low-effort, low-wealth equilibrium can be expressed as: $p= \begin{cases}1 & \text { if } \theta_{0}<\hat{\theta} \\ 0 & \text { if } \theta_{0} \geq \hat{\theta}\end{cases}$

2. The probability that a behavioral person lands in a low-effort, low-wealth equilibrium can be expressed as: $\tilde{p}= \begin{cases}1 & \text { if } \theta_{0}<\theta_{L} \\ M\left(\tilde{g}\left(\theta_{0}\right)\right) & \text { if } \theta_{L}<\theta_{0}<\theta_{H} \\ 0 & \text { if } \theta_{H}<\theta_{0}\end{cases}$ 
We can now determine the probability that any person lands in a low-effort, lowwealth equilibrium:

$$
\mathbb{P}= \begin{cases}1 & \text { if } \theta_{0}<\theta_{L} \\ \lambda+(1-\lambda) M\left(\tilde{g}\left(\theta_{0}\right)\right) & \text { if } \theta_{L}<\theta_{0}<\hat{\theta} \\ (1-\lambda) M\left(\tilde{g}\left(\theta_{0}\right)\right) & \text { if } \hat{\theta}<\theta_{0}<\theta_{H} \\ 0 & \text { if } \theta_{H}<\theta_{0}\end{cases}
$$

Where $\lambda \in\{0,1\}$ is the share of rational agents in the population, or the unconditional probability that an individual has the rational type.

Finally, we can now characterize final wealth outcomes $\theta$ as a function of $\mathbb{P}$, the thresholds $\theta_{L}, \theta_{H}$ and $\hat{\theta}$, and the wealth accumulation process determined by $\theta=(1+e) / \theta_{0}$ where effort is a binary choice:

$$
\theta= \begin{cases}\theta_{0} & \text { if } \theta_{0}<\theta_{L} \\ \lambda \theta_{0}+(1-\lambda)\left(2 \theta_{0}-\theta_{0} M\left(\tilde{g}\left(\theta_{0}\right)\right)\right) & \text { if } \theta_{L}<\theta_{0}<\hat{\theta} \\ 2 \lambda \theta_{0}+(1-\lambda)\left(2 \theta_{0}-\theta_{0} M\left(\tilde{g}\left(\theta_{0}\right)\right)\right) & \text { if } \hat{\theta}<\theta_{0}<\theta_{H} \\ 2 \theta_{0} & \text { if } \theta_{H}<\theta_{0}\end{cases}
$$

Equation (1.3) provides us with a first testable implication of this model in the form of a highly nonlinear relation between initial wealth and final wealth. To more easily characterize the form of this segmented relationship, we take the partial derivative of $\theta$ with respect to $\theta_{0}$, as follows:

$$
\frac{\partial \theta}{\partial \theta_{0}}= \begin{cases}1 & \text { if } \theta_{0}<\theta_{L} \\ \rho_{1} \equiv \lambda+(1-\lambda)\left(2-M\left(\tilde{g}\left(\theta_{0}\right)\right)-\theta_{0} \tilde{g}^{\prime}\left(\theta_{0}\right) M^{\prime}\left(\tilde{g}\left(\theta_{0}\right)\right)\right) & \text { if } \theta_{L}<\theta_{0}<\hat{\theta} \\ \rho_{2} \equiv 2 \lambda+(1-\lambda)\left(2-M\left(\tilde{g}\left(\theta_{0}\right)\right)-\theta_{0} \tilde{g}^{\prime}\left(\theta_{0}\right) M^{\prime}\left(\tilde{g}\left(\theta_{0}\right)\right)\right) & \text { if } \hat{\theta}<\theta_{0}<\theta_{H} \\ 2 & \text { if } \theta_{H}<\theta_{0},\end{cases}
$$

with $\rho_{1}=\lambda+(1-\lambda)\left[2-M\left(\tilde{g}\left(\theta_{0}\right)\right)-\theta_{0} \frac{\partial \tilde{g}\left(\theta_{0}\right)}{\partial \theta_{0}} \cdot \frac{\partial M\left(\tilde{g}\left(\theta_{0}\right)\right)}{\partial \theta_{0}}\right]$, and $\rho_{2}=\lambda+\rho_{1}$.

Proposition $11 \leq \rho_{1} \leq \rho_{2} \leq 2$.

Proof in Appendix A.1. 
This concludes on the testable predictions we derived from Dalton et al. (2016). We now turn to the empirical counterpart to these predictions.

\subsubsection{A Macro-Economic Interpretation of the Model}

In Figure 1.1 we present a stylized depiction of the relationship between initial conditions and final wealth. In this figure, $\theta_{L}, \theta_{H}$ and $\hat{\theta}$, correspond to the thresholds defined by Dalton et al. (2016). This figure helps us illustrate our predictions and provide a novel, macro-economic interpretation to their model.

Figure 1.1: Macroeconomic Testable Predictions after Dalton et al. (2016)

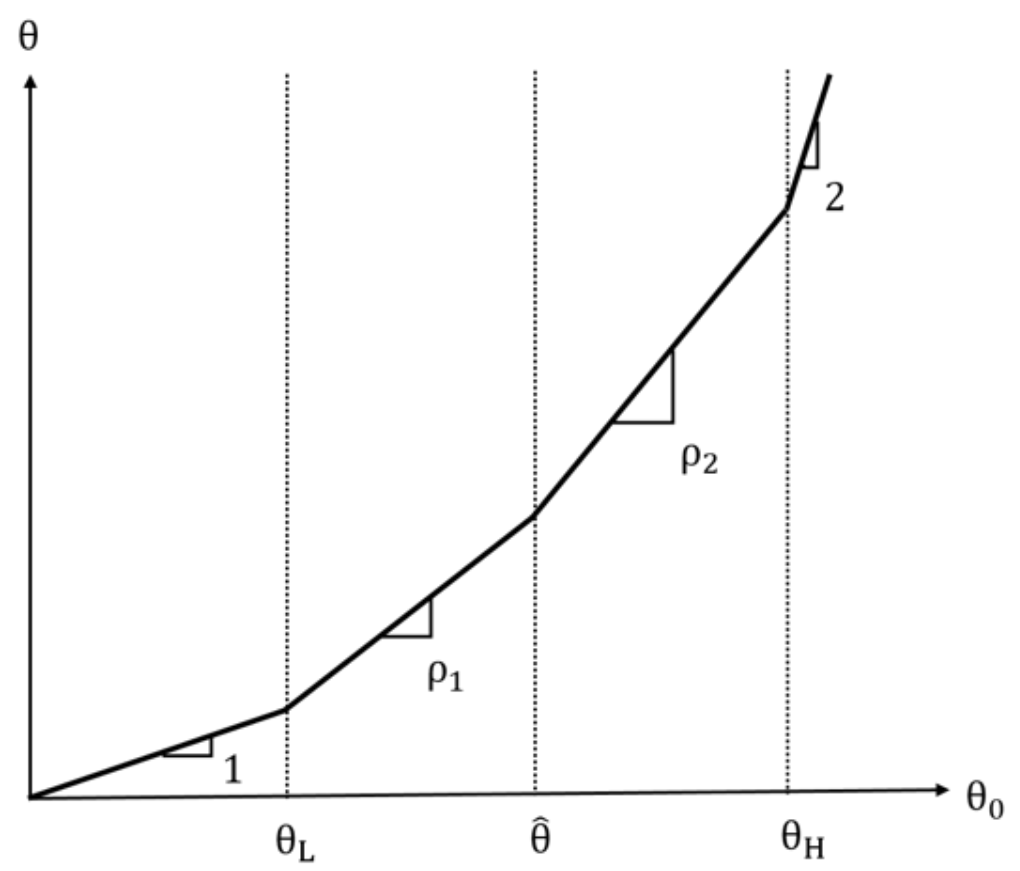

Note: This figure illustrates the cross-sectional relationship between initial wealth and final wealth based on our predictions from Dalton et al. (2016). Legend: $\theta_{L}, \theta_{H}$ and $\hat{\theta}$ are thresholds defined by Dalton et al. (2016), and and are slopes in wealth accumulation obtained in our calculations.

Individuals with initial wealth levels below $\theta_{L}$ will choose to exert low effort irrespective of whether they are the behavioral or rational type; as a consequence of this effort choice, they will not be able to increase their level of final wealth $\theta$ above their initial wealth. Thus, in this region of initial circumstances, individuals are unable to accumulate wealth at a faster pace than 1.

Individuals with initial wealth levels that are higher than $\theta_{L}$ but lower than $\hat{\theta}$ will exert low effort if they have the rational type and not increase their final wealth above the level of their initial wealth, while the choices of individuals with the behavioral 
type are subject to multiple equilibria depending on their initial aspiration draw. Those who had a draw of high aspiration will exert high effort and experience improved outcomes. Thus, in this region of initial circumstances, some behavioral individuals are able to accumulate more wealth than others, raising the average rate of wealth accumulation to $\rho_{1}$.

Individuals with initial wealth levels that are higher than $\hat{\theta}$ but lower than $\theta_{H}$ will exert high effort if they have the rational type and achieve higher final wealth compared to their initial wealth. Behavioral individuals will choose effort levels depending on their initial draw of aspirations, and a larger share of behavioral will now choose to exert high effort. Thus, in this region of initial circumstances, all rational individuals and some behavioral individuals are able to improve their final wealth, raising the average rate of wealth accumulation to $\rho_{2}$.

Lastly, individuals with initial wealth levels above $\theta_{H}$ will all choose to exert high effort irrespective of whether they are the behavioral or rational type. Therefore, in this final region of initial circumstances, individuals are all able to increase their initial wealth. Thus, the thresholds $\theta_{L}, \theta_{H}$ and $\hat{\theta}$ correspond to structural breaks in the process of wealth accumulation, and $\rho_{1}$ and $\rho_{2}$ correspond to wealth accumulation rates in the different regions of the income distribution. This gives us a testable implication for the model of Dalton et al. (2016): on cross-sectional data, we expect to find three structural breaks in the relationship between initial individual circumstances and final wealth, and increasing slopes in the relationship.

We therefore have a method to test for the existence, the size and the location of aspirational poverty traps. Behavioral individuals with initial wealth between $\hat{\theta}$ and $\theta_{H}$ who choose to exert low effort can theoretically be nudged into exerting higher effort and experience higher final wealth. These are the individuals in an aspirational poverty trap. The size of this trap is given by the number of individuals in this situation, that is, the number of behavioral individuals with initial wealth between $\hat{\theta}$ and $\theta_{H}$, who choose to exert low effort. With our parameters and distributional assumptions, this size corresponds to the integral of $(1-\lambda) M\left(\tilde{g}\left(\theta_{0}\right)\right)$ between $\hat{\theta}$ and $\theta_{H}$ (Equation (1.2)). Thus, an upper bound of the size of this trap is the population share of behavioral individuals between $\hat{\theta}$ and $\theta_{H}$. Note that this aspirational poverty trap, located between $\hat{\theta}$ and $\theta_{H}$, is distinct from a material poverty trap: individuals in this group are closer to the median of the income distribution than the left-tail of the distribution. Additional lessons can be drawn from this model; for example, note that $\rho_{1}$ and $\rho_{2}$ are also informative about the stochastic process determining aspirations $M\left(\tilde{g}\left(\theta_{0}\right)\right)$, the elasticity of aspirations to exogenous changes in income 
$\tilde{g}^{\prime}\left(\theta_{0}\right)$ and the unconditional probability $\lambda$ that an agent has the rational type.

\subsection{Empirical Methodology}

Our theoretical predictions indicate that the presence of aspirational poverty traps should manifest itself by nonlinearities in the relationship between initial wealth and final wealth. This implies that our empirical exercise will entail jointly estimating 1) point estimates of locations of breaks, and 2) point estimates of slope changes from one regime to the next.

We resort to threshold regression methods for this exercise. Threshold regression methods belong to the same family as statistical methods for the detection of structural breaks in data - similar methods are regime-switching models using Markov chains, splines, and segmented-regression analysis. Threshold regressions mostly gained popularity in macroeconomics and time series econometrics, because they provide simple and easily interpretable models for nonlinear relationships between variables. Hansen (2000) describes these methods as a natural way of exploring coefficient stability across subsamples in a population (e.g., poor versus rich) using linear regressions, when the subsamples to be explored are defined by a continuous variable, and one wishes to be agnostic as to the value of that variable that splits the sample. For example, a popular application in economics has been the idea that there exists a "tipping point" in neighborhood racial segregation in the United States (see e.g. Brock and Durlauf, 2001; Card et al., 2008).

The empirical counterpart of our theoretical problem is given by the following threshold regression equation: $Y_{i}=X_{i}^{\prime} \beta_{1} 1_{\left\{q_{1} \leq \gamma\right\}}+X_{i}^{\prime} \beta_{2} 1_{\left\{q_{1}>\gamma\right\}}+\epsilon_{i}$, where $\beta_{1}$ is a slope parameter on the subsample on which $q_{i}$ is below some threshold $\gamma$, and $\beta_{2}$ is a slope parameter on the complementary subsample where $q_{i}$ is above the threshold. This equation is usually rewritten into: $Y_{i}=X_{i}^{\prime} \beta_{1}+X_{i}^{\prime} \delta 1_{\left\{q_{1}>\gamma\right\}}+\epsilon_{i}$, where $\beta_{1}$ remains the regression coefficient parameter, $\delta=\beta_{2}-\beta_{1}$ is a discrepancy parameter and $\gamma$ is the threshold parameter.

Estimation and inference of threshold regression models when the all regressors and threshold variables are exogenous is discussed in detail in Hansen (2000). Caner and Hansen (2004) and Kourtellos et al. (2016) further discuss identification and inference when some of the regressors, including the threshold variable, are endogenous. Yu and Phillips (2018) propose a nonparametric estimator, the integrated difference kernel estimator (IDKE), which presents the advantage that the discrepancy coefficients 
can be statistically identified using only the local information around each threshold points. Thus, in contrast to Caner and Hansen (2004) and Kourtellos et al. (2016), the approach in $\mathrm{Yu}$ and Phillips (2018) does not require additional instruments for identifying either threshold points or discrepancy coefficients, although instruments can be used to increase efficiency — instruments remain, however, necessary for identifying slope parameter $\beta_{j}$.

We focus on the simplest case using the methodology proposed by Hansen (2000), which we adapt to detect multiple thresholds. Our setting requires us to estimate a regression with multiple threshold, which can be re-express from Equations 1.3 and 1.4, and Figure 1.1 as follows:

$$
\begin{aligned}
\theta_{i}=\alpha+\beta_{1} \theta_{0 i} & +\delta_{1} \theta_{0 i} 1\left\{\theta_{L} \leq \theta_{0 i}<\hat{\theta}\right\} \\
& +\delta_{2} \theta_{0 i} 1\left\{\hat{\theta} \leq \theta_{0 i}<\theta_{H}\right\} \\
& +\delta_{3} \theta_{0 i} 1\left\{\theta_{0 i} \geq \theta_{H}\right\}+\epsilon_{i}
\end{aligned}
$$

The parameters to be jointly estimated in this model are: i) the intercept $\alpha$; ii) the initial slope parameter $\beta_{1}$; iii) the three discrepancy coefficients $\delta_{j}$, with $j \in 1,2,3$; and iv) the three threshold location parameters $\theta_{L}, \hat{\theta}$ and $\theta_{H}$. In Equation (1.5), discrepancy parameters capture differences in wealth accumulation slopes from one regime to the next, and threshold location parameters correspond to regimes switching points in the distribution of initial conditions. We are only to a lesser degree interested in regression coefficients $\beta_{j}$. These are not directly informative for our problem and their estimates are not required for identifying the discrepancy coefficients $\delta_{j}$.

The estimation methodology proposed by Hansen (2000) uses a grid-search algorithm. For detecting the presence of one structural break in the relationship between two variables, the grid-search algorithm goes as follows:

1. for each possible value of $\gamma$ denoting the threshold location parameter in the $\mathrm{x}$-axis variable, estimate a spline model with a structural break at $x=\gamma$

2. Among all the spline models estimated - one per potential value of $\gamma$, find the value $\hat{\gamma}$ which minimizes the Concentrated Sum of Squares

3. Construct confidence intervals for the point estimate $\hat{\gamma}$ by inverting the likelihood-ratio.

4. Compare the fit of the model without structural break to the fit of the model 
with structural break at the estimated threshold location parameters, using a chosen information criterion (e.g. Bayesian, Akaike, Hannan-Quinn).

To continue searching for additional thresholds, take the model with $\hat{\gamma}$ as the baseline model, and repeat steps 1) to 4) separately for values of $\gamma$ above and below $\hat{\gamma}$.

At this stage, it is important to clarify that we are not treating the threshold points as exogenous realization of random variables, which may or may not have a causal effect on the wealth accumulation regime that individuals are subject to. That is to say, we are not claiming that we can fully isolate the causal effect of the thresholds on wealth accumulation of individuals and we are not aiming to conduct inference on any of the parameters. Rather, our aim is to test for the presence of such thresholds, and to estimate an economically relevant quantity using these thresholds.

\subsection{Empirical Application: Aspirational Poverty Traps in the United States}

To estimate the size of aspirational poverty traps using threshold regressions, we need data spanning the income distribution, to allow detection of structural breaks at various points in the relationship between initial conditions and later life earnings. Thus, a first and minimal data requirement for our study is a large, representative sample. Our testable predictions require, in addition, good measures for i) initial conditions, ii) final wealth, and iii) intermediate outcomes which can explain some of the relationship between initial conditions and final outcomes or changes in this

relationship across income. Such measures include proxies for effort, aspirations, or demographic characteristics which affect the wealth accumulation process.

\subsubsection{Data: The 1979 National Longitudinal Survey of Youth}

The National Longitudinal Survey of Youth 1979 meets our data requirements. It is a widely-used dataset from the United States, which has been following since 1979 a cohort of 12,686 individuals who were between 14 to 22 years old at the time of first interview. The survey was conducted yearly between 1979 and 1994, then biennially from 1994 onward. We decided to use the NLSY79 for several reasons: first it contains detailed information on respondents' participation to welfare programs of all sorts, as well as their spouse, which we need to determine the initial conditions of individuals. It contains rich background information on respondents and their 
family, which will be important for us to test whether rational or behavior decisionmakers can be identified ex ante and targeted with different types of interventions. Lastly, it also contains rich information on cognitive ability through the AFQT test, expectations about the future, hours worked and preferences which are all potential factors that affect the relationship between initial conditions and final outcomes.

Table 1.1: Summary Statistics of the NLSY79

\begin{tabular}{|c|c|c|c|}
\hline Characteristics: & $\begin{array}{l}\text { All } \\
(1)\end{array}$ & $\begin{array}{c}\text { Men } \\
(2)\end{array}$ & $\begin{array}{c}\text { Women } \\
(3)\end{array}$ \\
\hline Male & 0.50 & 1.00 & 0.00 \\
\hline Age & 35.16 & 35.14 & 35.18 \\
\hline AFQT score, perc. adj. & 50.48 & 50.30 & 50.67 \\
\hline Years of education & 12.67 & 12.55 & 12.79 \\
\hline \multicolumn{4}{|l|}{ Edu. attainment: } \\
\hline Less than high school & 0.17 & 0.19 & 0.15 \\
\hline High school diploma & 0.43 & 0.44 & 0.43 \\
\hline Some college & 0.21 & 0.19 & 0.24 \\
\hline 4-year college & 0.18 & 0.18 & 0.18 \\
\hline \multicolumn{4}{|l|}{ Race: } \\
\hline Hispanic & 0.16 & 0.16 & 0.16 \\
\hline Black & 0.25 & 0.25 & 0.25 \\
\hline Neither & 0.59 & 0.59 & 0.59 \\
\hline \multicolumn{4}{|l|}{ Household composition: } \\
\hline No children & 0.53 & 0.64 & 0.42 \\
\hline \multicolumn{4}{|l|}{ Marital status: } \\
\hline Never married & 0.38 & 0.43 & 0.33 \\
\hline Married / de facto & 0.46 & 0.43 & 0.48 \\
\hline Divorced, Separated, Widowed & 0.11 & 0.09 & 0.12 \\
\hline \multicolumn{4}{|l|}{ Parental education: } \\
\hline Mother years of education & 10.87 & 10.94 & 10.80 \\
\hline Father years of education & 10.95 & 11.00 & 10.89 \\
\hline N.Obs. & 12,686 & 6,403 & 6,283 \\
\hline
\end{tabular}

Our methodology involves estimating threshold regressions models of intragenerational mobility, that is, individual lifetime income growth. To do this, we regress a measure of permanent income on a measure of initial income. We construct two main measures of permanent income. Our first and main measure is the inverse hyperbolic sine of average family net worth between ages 40 and 48 . To construct this measure, we start by constructing yearly family net worth, by summing income 
from all sources net of all sources of debts. To avoid issues with temporary income loss in any one year which would generate noise in our measure, we take the average between ages 40 and 48. Finally, we take the inverse hyperbolic sine (IHS) of this average. The IHS presents the advantage that it behaves like the natural logarithm while allowing us to include observations with zero income, which is crucial for our purpose of maintaining the widest possible range of initial conditions to detect thresholds.

Our second measure is the residual in the regression of the first measure on an extensive set of control variables: age, AFQT adjusted score, the age of the youngest child in the household, sex, race, a dummy taking value 1 if the respondent grew up in the Southern states of the U.S., a dummy taking value 1 if the respondent was living in a rural area at age 14, the level of education of respondent and the year. ${ }^{9}$

We construct a measure of initial wealth conditions by taking the IHS of average family net worth between ages 22 and 25, which allows us to focus on the relationship between individual initial conditions and final outcomes after the effect of family background on human capital investments has already mostly been realized. Thus, this measure makes the comparison of this relationship between circumstances and outcomes more comparable across individuals with very different circumstances. We further construct the annual average number of working hours between ages 40 and 48 , and use it is a measure of individual effort in later life. We use this variable to explore one key prediction from Dalton et al. (2016), that "behavioral" individuals pay on average less effort compared to rational individuals, keeping income and observable characteristics constant.

In our regressions analyses, we include control variables that are likely to systematically affect either the initial conditions, final outcomes or the relationship between these two: AFQT score, highest educational attainment of respondents, their marital status and their fertility at age 22-25, parental educational attainment, sex, race, age, region of residence as defined by Census, a dummy taking value 1 if the respondent was living in a rural area at age 14, 0 otherwise. We exclude individuals which don't have at least three non-missing values in each of the key variables: marital status,

\footnotetext{
${ }^{9}$ We find our measures of initial and permanent income to be preferable to alternative measures commonly used in the literature on inter- and intragenerational mobility. Other studies use rankrank regression models, which capture a person's personal change in position within the distribution of wealth, or percentile-percentile regression models, which capture similarly individuals moving from a specific percentile to another, the result of which is a mobility table. Neither these methods are adequate for detecting structural breaks in initial conditions.
} 
number of children, educational level, initial income, final income, working hours in later life. Further technical details on data preparation can be found in Appendix A.2.

Table 1.1 presents summary statistics on our estimation sample, compared to the unrestricted initial sample. Overall, our estimation sample is very comparable to the raw extracted data: $50 \%$ of the sample is male, $15 \%$ have less than a high school degree by 1990 (when respondents are between 25 and 33 years of age), 45\% have a high school degree, $22 \%$ some college and $18 \%$ have completed a 4 -year college degree.

\subsubsection{Estimation Results}

\section{Baseline Intragenerational Socioeconomic Mobility}

Table 1.2 presents OLS estimation results for a baseline model of individual income growth over the life course. We regress an individual's permanent income (proxied by the log average household income between ages 40 and 48) on the individual's initial income (proxied by the log average individual income between ages 22 and 25). We find correlations between initial and permanent income ranging between 0.194 and 0.33 , which are stable to including more individual characteristics. This means that between 20 and $30 \%$ of individual permanent income is predicted by individual initial wealth after completing education.

Table 1.2: Baseline Model of Intragenerational Social Mobility

\begin{tabular}{|c|c|c|c|c|c|}
\hline \multirow[b]{3}{*}{$\begin{array}{l}\text { Initial Family Net Worth } \\
\text { (ages } 22-25, \text { IHS) }\end{array}$} & \multicolumn{5}{|c|}{ Final Family Net Worth (ages 40-48, IHS) } \\
\hline & (1) & $(2)$ & (3) & $(4)$ & $(5)$ \\
\hline & $\begin{array}{l}0.329 * * * \\
(0.018)\end{array}$ & $\begin{array}{l}0.256 * * * \\
(0.018)\end{array}$ & $\begin{array}{l}0.192 * * * \\
(0.018)\end{array}$ & $\begin{array}{l}0.197 * * * \\
(0.017)\end{array}$ & $\begin{array}{l}0.194 * * * \\
(0.017)\end{array}$ \\
\hline Dummies for zero wealth & & $\mathrm{Y}$ & $\mathrm{Y}$ & $\mathrm{Y}$ & $\mathrm{Y}$ \\
\hline Individual characteristics & & & $\mathrm{Y}$ & $\mathrm{Y}$ & $\mathrm{Y}$ \\
\hline Dummies for education level & & & & $\mathrm{Y}$ & $\mathrm{Y}$ \\
\hline AFQT & & & & & $\mathrm{Y}$ \\
\hline $\mathrm{R} 2$ & 0.07 & 0.16 & 0.26 & 0.28 & 0.29 \\
\hline N. Obs. & 5,131 & 5,131 & 5,131 & 5,131 & 5,131 \\
\hline
\end{tabular}

Note: This table presents the results of regressions of permanent net worth on initial net worth, as measured by the inverse hyperbolic sine of household net worth between ages 22 and 25. Each column represents a separate regression. Robust standard errors in parentheses. ${ }^{* * *} p<0.01$, ${ }^{* *}$ $p<0.05,{ }^{*} p<0.1$. Full table including all control variables in Appendix Table A.1. 
These correlation coefficients between initial conditions and final outcomes summarize complex processes in the intragenerational mobility, which combine stochastic and deterministic factors, as well as opportunities, endowments and effort. In our next step, we investigate nonlinearities in the relationship between initial conditions and final outcomes.

\section{Detection of Structural Breaks in Intragenerational Mobility}

Figure 1.2 shows that using the grid-search algorithm defined by Hansen (2000), we find two structural breaks in the relationship between initial conditions and final outcomes, which are consistent across the fit criterion we use.

In each panel of Figure 1.2, we plot the relationship between initial conditions on the x-axis, measured by log family net worth between ages 22 and 25 , and final outcomes on the y-axis, measured by log family net worth between ages 40 and 48 on the y-axis. Red lines indicate the estimated locations of structural breaks using the grid-search criterion until model fit could not be improved with additional estimated thresholds. Bigger markers indicate a larger number of individual observations. Importantly, we consistently find the same two structural breaks across the Bayesian (Figure 1.2a), Akaike (Figure 1.2b) and Hannan-Quinn information criteria (Figure 1.2c); these are the thresholds we plug into our threshold regression model in the next step of our analysis. 
Figure 1.2: Detected Thresholds and Model Fit Criteria

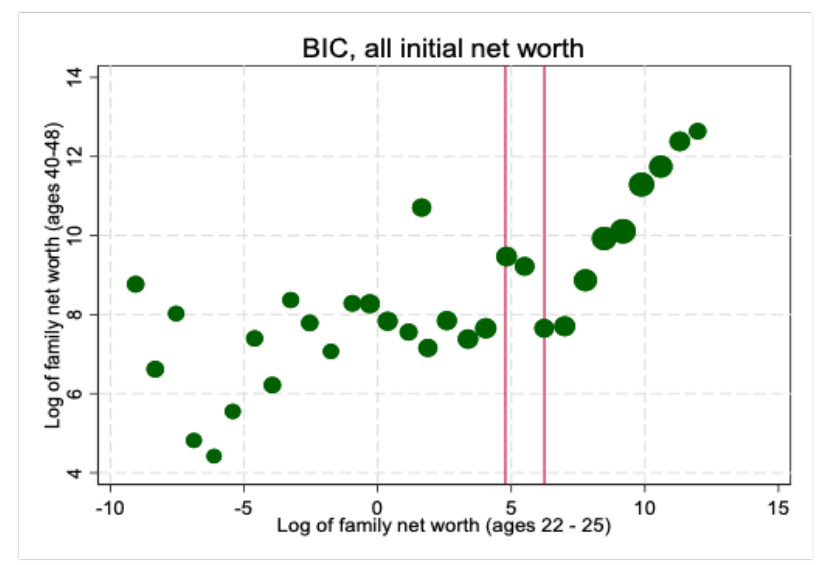

(a) Bayesian Information Criterion

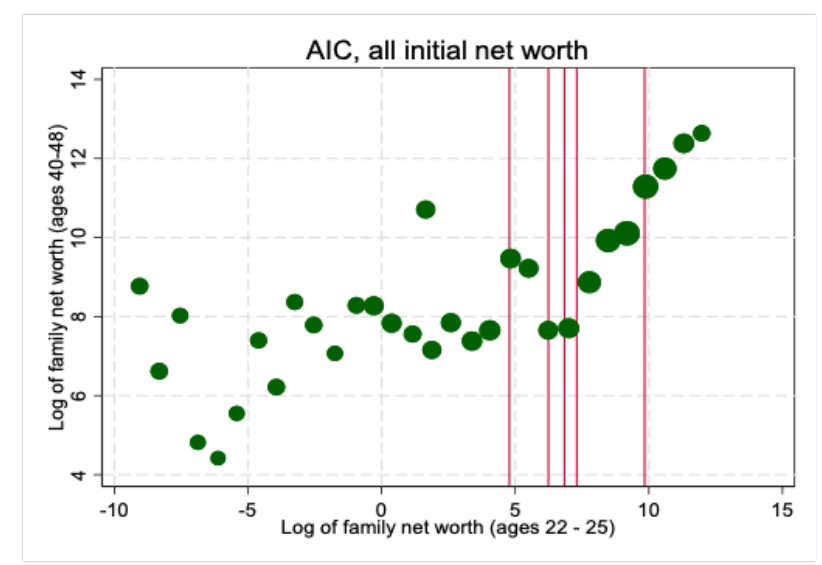

(b) Akaike Information Criterion



(c) Hannan-Quinn Information Criterion

Note: This figure represents the result of the sequential grid-search algorithm of Hansen (2000) used to detect thresholds in the relationship between log family net worth at ages 22 to 25 and log family net worth at ages 40 to 48. Red lines indicate the estimated locations of structural breaks using the grid-search criterion until model fit could not be improved with additional estimated thresholds. Bigger markers indicate a larger number of individual observations. 


\section{Models of Intragenerational Social Mobility with Structural Breaks}

Tables 1.3 and 1.4 support the hypothesis that there are important nonlinearities in the intragenerational social mobility, that are consistent with our main predictions that there could be a non-negligible part of the U.S. population locked in aspirational poverty traps. Our results consistently indicate at least 3 regimes of within-lifetime income mobility, with low mobility at left of the distribution of initial wealth, high mobility in the middle of the distribution, and again low mobility at the right tail of the distribution although higher than at the left tail of the distribution.

Table 1.3: Two-Threshold Regression Models of Intragenerational Social Mobility

\begin{tabular}{lcccc}
\hline & \multicolumn{3}{c}{ Final Family } & Net Worth $($ ages $40-48$, IHS $)$ \\
& $(1)$ & $(2)$ & $(3)$ & $(4)$ \\
\cline { 2 - 5 } & & & & \\
Initial Family Net Worth: & & & & \\
Below Threshold 1 & 0.038 & $0.073 *$ & $0.127 * * *$ & $0.113 * * *$ \\
& $(0.041)$ & $(0.039)$ & $(0.048)$ & $(0.039)$ \\
Between thresholds 1 and 2 & $-1.782 * * *$ & $-1.361 * *$ & $1.445 * * *$ & $-1.162 *$ \\
& $(0.683)$ & $(0.628)$ & $(0.324)$ & $(0.612)$ \\
Above Threshold 2 & $0.996 * * *$ & $0.658 * * *$ & $0.537 * * *$ & $0.533 * * *$ \\
& $(0.067)$ & $(0.068)$ & $(0.063)$ & $(0.067)$ \\
\hline Threshold 1 & 4.79 & 4.73 & 3.05 & 4.73 \\
Threshold 2 & 6.24 & 6.24 & 5.53 & 6.24 \\
Dummies for zero wealth & $\mathrm{Y}$ & $\mathrm{Y}$ & $\mathrm{Y}$ & $\mathrm{Y}$ \\
Individual characteristics & & $\mathrm{Y}$ & $\mathrm{Y}$ & $\mathrm{Y}$ \\
Dummies for education level & & & $\mathrm{Y}$ & $\mathrm{Y}$ \\
AFQT & & & & $\mathrm{Y}$ \\
\hline N. Obs. & 5,346 & 5,131 & 5,131 & 5,131 \\
\hline
\end{tabular}

Note: This table presents the results of regressions of permanent net worth on initial net worth, with 2 structural breaks. Initial net worth is measured by the inverse hyperbolic sine of household net worth between ages 22 and 25. Each column represents a separate regression. Robust standard errors in parentheses. ${ }^{* *} p<0.01$, ${ }^{* *} p<0.05,{ }^{*} p<0.1$. Full details in Appendix Table A.2.

Table 1.3 reports the results of threshold regression models of final family net worth on initial family net worth, without imposing any number of thresholds in the grid-search algorithm. The grid-search algorithm consistently detects two thresholds, which location is robust to including control variables. That threshold estimates are consistent across specifications suggests that the underlying process causing the structural breaks are orthogonal to the control variables. We find that the estimated correlation coefficient between initial conditions and final outcomes is consistent across specifications, but varies systematically around the thresholds: below the first 
threshold and above the second threshold, we find positive correlations, larger above the second threshold, while we find negative correlations between the thresholds.

Table 1.3 indicates low intragenerational mobility at left and right tails of the distribution of initial wealth, although higher mobility at the right tail, while the middle of the distribution is characterized by high mobility. This evidence is thus largely consistent with our predictions based on Dalton et al. (2016). Between the two thresholds, however, we find a negative correlation coefficient with large standard errors, which suggests a part of the distribution with important heterogeneity and potentially additional thresholds between the two thresholds we found. Lastly, we notice that the explanatory power of control variables varies along the spectrum of initial conditions: at the bottom of the distribution of initial conditions, the largest move in the correlation coefficient occurs when adding dummies for education level, while at the top of the distribution, the most important set of control variables is individual characteristics such as race and gender. This suggests that intragenerational mobility could be driven by systematically different processes at the different regions of initial conditions.

Table 1.4 reports the results of threshold regression models of final family net worth on initial family net worth, now imposing 3 thresholds in initial family net worth in the grid-search algorithm. Notice that by imposing to the grid-search algorithm the search of an additional threshold, we find a third threshold that is generally located on the right of the second threshold, but with a location that is not robust to the inclusion of control variables. In comparison, we find consistently 2 thresholds at the same locations as in Table 1.3. This is clear evidence that the model with 2 thresholds is preferable to a model with 3 thresholds, although Table 1.4 does provide suggestive evidence of a threshold located around log initial family net worth of 6 - this complements the notable difference in the correlation coefficients in regimes 2 and 3 .

\section{The Location and Size of Aspirational Poverty Traps}

Overall, our results indicate the presence of important nonlinearities in intragenerational social mobility in the United States, with low income mobility in the left tail of the distribution of initial conditions, high mobility in the middle, and lowest income mobility in the right tail of the income distribution. This evidence allows us to bound the size of a potential aspirational poverty trap and to determine its location in the population in Figure 1.3. 
Table 1.4: Three-Threshold Regression Models of Intragenerational Social Mobility

\begin{tabular}{lcccc}
\hline & \multicolumn{3}{c}{ Final Family Net Worth (ages 40-48, IHS) } \\
& $(1)$ & $(2)$ & $(3)$ & $(4)$ \\
\cline { 2 - 5 } & & & & \\
Initial Family Net Worth: & & & & \\
Below Threshold 1 & 0.037 & $0.073 *$ & $0.128 * * *$ & $0.113 * * *$ \\
& $(0.041)$ & $(0.039)$ & $(0.048)$ & $(0.039)$ \\
Between thresholds 1 and 2 & $-1.782 * * *$ & $-1.358 * *$ & $12.727 * * *$ & $-1.161 *$ \\
& $(0.683)$ & $(0.628)$ & $(4.796)$ & $(0.612)$ \\
Between thresholds 2 and 3 & $1.064 * * *$ & -5.339 & $1.427 * * *$ & -6.190 \\
& $(0.141)$ & $(4.795)$ & $(0.402)$ & $(4.872)$ \\
Above Threshold 3 & $0.384 * * *$ & $0.590 * * *$ & $0.536 * * *$ & $0.474 * * *$ \\
& $(0.137)$ & $(0.067)$ & $(0.063)$ & $(0.067)$ \\
\hline Threshold 1 & 4.79 & 4.73 & 3.05 & 4.73 \\
Threshold 2 & 6.24 & 6.24 & 3.53 & 6.24 \\
Threshold 3 & 9.84 & 6.84 & 5.53 & 6.84 \\
Dummies for zero wealth & $\mathrm{Y}$ & $\mathrm{Y}$ & $\mathrm{Y}$ & $\mathrm{Y}$ \\
Individual characteristics & & $\mathrm{Y}$ & $\mathrm{Y}$ & $\mathrm{Y}$ \\
Dummies for education level & & & $\mathrm{Y}$ & $\mathrm{Y}$ \\
AFQT & & & & $\mathrm{Y}$ \\
\hline N. Obs. & 5,346 & 5,131 & 5,131 & 5,131 \\
\hline
\end{tabular}

Note: This table presents the results of regressions of permanent net worth on initial net worth, with 3 structural breaks. Initial net worth is measured by the inverse hyperbolic sine of household net worth between ages 22 and 25. Each column represents a separate regression. Robust standard errors in parentheses. ${ }^{* *} p<0.01,{ }^{* *} p<0.05,{ }^{*} p<0.1$. Full details in Appendix Table A.3.

Figure 1.3 indicates the location and size of a potential aspirational poverty trap in the United States. The figure presents a kernel density plot of the inverse hyperbolic sign of initial net worth, and places the two thresholds that we have located using our threshold regressions. The first threshold corresponds to the 37 th percentile and the second threshold to the 42.5 th percentile of the distribution of initial family net worth. This implies that at most $5.5 \%$ of the U.S. population are potentially located in an aspirational poverty trap, and that this potential aspirational poverty trap would capture individuals in the low-middle range of the distribution of family wealth (around a log of initial family net worth of 4.8 to 6.3, corresponding to roughly $\$ 1,150$ to $\$ 1,650$ net worth at ages 22 to 25$)$. With three thresholds, this trap would roughly capture individuals between the 37 th and the 44.5 th percentile, for a total of roughly $7.5 \%$ of the population. 
Figure 1.3: Location and Size of Aspirational Poverty Traps in the United States

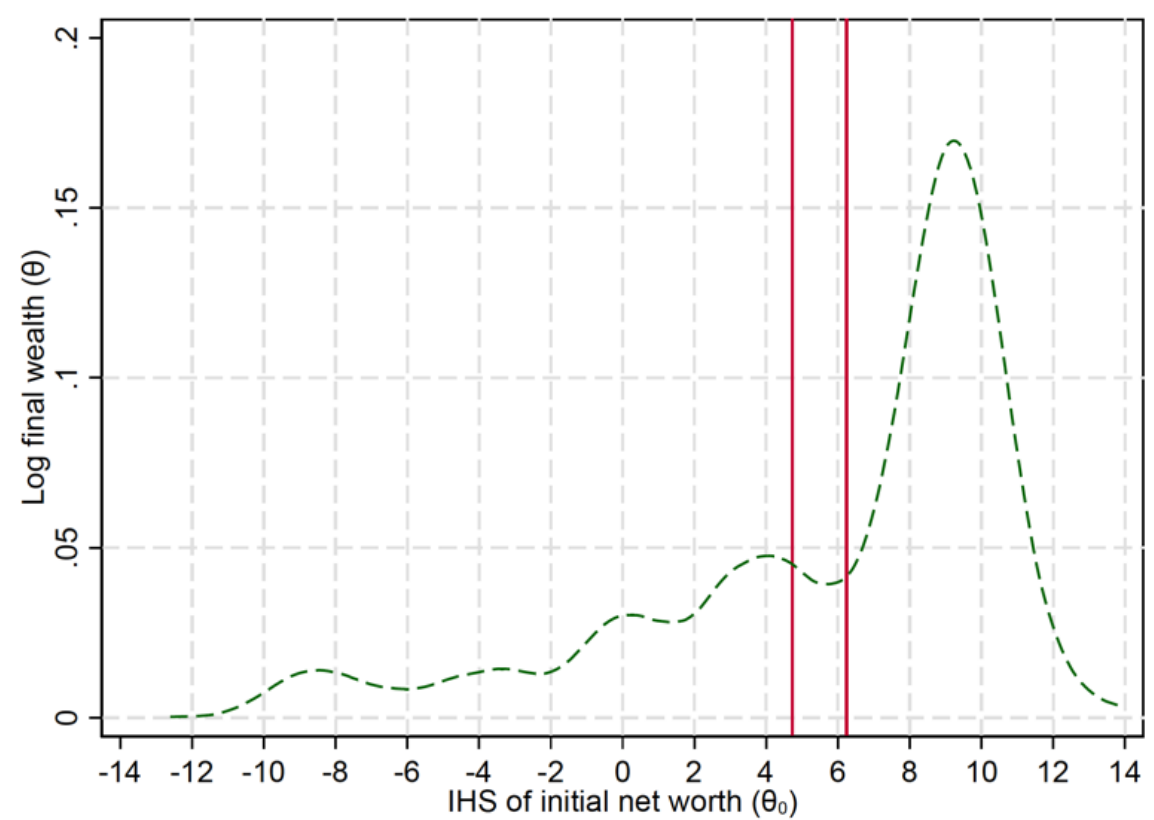

Note: This figure presents the results of a threshold-regression model of log family net worth at ages 40 to 48 on the inverse hyperbolic sine of family net worth at ages 22 to 25. The location of thresholds is obtained using the sequential grid-search algorithm of Hansen (2000), based on the Hannan-Quinn Information Criterion. Red vertical lines represent the location of thresholds detected using the grid-search algorithm. The green line represents a spline model of log permanent wealth on the inverse hyperbolic sine of initial wealth circumstances.

\subsection{Robustness of Findings}

Other models and mechanisms could produce such empirical regularities on the persistence of disadvantage, with different regimes of wealth accumulation over the life course.

We have already shown that our results are largely robust to specifications, and to searching for more thresholds. We now turn to discussing what other mechanisms than breaks in the formation of aspirations could explain our stylized fact. Our main concern is that there could be omitted factors correlated with initial conditions or final outcomes, which have structural breaks instead of the breaks we found in initial conditions. We call "confounding thresholds" these thresholds in confounding and omitted variables.

We consider three groups of factors that could in principle also produce regimeswitching points in the within lifetime accumulation of wealth: 1) non-cognitive 
skills; 2) credit constraints; 3) networks.

To test whether these factors could confound the low-social mobility traps we have identified as aspirational poverty traps, we construct a falsification test as following. For each factor, we start by taking our estimated thresholds in initial conditions as parameters in the model, and run the grid-search algorithm to detect the presence of additional thresholds. If we find no evidence of thresholds, we conclude that there are so significant nonlinearities in the factor considered that could generate regimes in wealth accumulation. If we do find evidence of thresholds in the factor, we then graphically investigate whether these structural breaks correspond roughly to the breaks we find in initial conditions - these are break that could be confounding our previous thresholds. We re-run our fully controlled threshold model, additionally controlling for the confounding variable, and observe whether the correlation coefficients in each regime of initial conditions is affected by this new control variable. Lastly, we re-run the fully interacted model allowing now for the confounding variable to vary across regimes of initial conditions. If we find that this variable has a significantly different explanatory power for final outcomes in each regime, then we can conclude that this variable is a confounding factor of the aspirational traps we have identified in our main analysis.

\subsubsection{Non-Cognitive Skills: Locus of Control, Self-Esteem, and Self- Concept}

Consider a model of human capital investments in which non-cognitive skills can be either high or low, and have positive labor market returns. If we additionally assume that a proportion of individuals in the population possess high non-cognitive skills, then non-cognitive skills could in principle generate different regimes in the accumulation of wealth, which could be observationally equivalent to the aspirational poverty trap that we have identified through our structural breaks. Non-cognitive skills would therefore confound our analyses if i) we detect breaks in the relationship between non-cognitive skills and final wealth, ii) non-cognitive skills correlate highly with initial conditions, and iii) the structural breaks we find in non-cognitive skills overlap with the breaks we find in initial conditions, or are close enough.

We use three measures of non-cognitive skills that have been widely used in the NLSY79 and that have been linked to a variety of outcomes and economic decisionmaking (see e.g. Heckman et al., 2006; Caliendo et al., 2015): locus of control measured in 1979 (Rotter, 1966), self-esteem measured in 1980 (Rosenberg, 1965), and self-concept measured in 1992 (Pearlin et al., 1981). We show results here for 
Figure 1.4: Locus of Control and Permanent Income

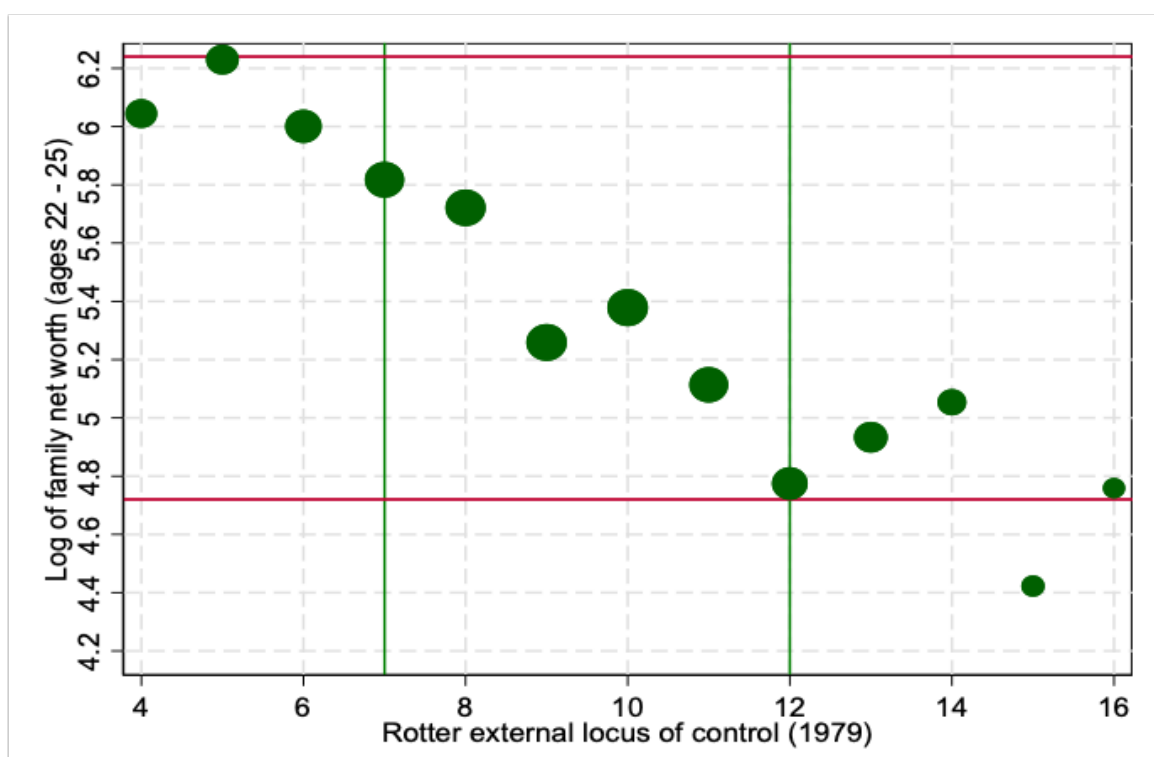

Note: This figure represents the results of the grid-search algorithm to detect the presence of thresholds in the relationship between Rotter's external local of control score and log family net worth at ages 22 to 25 (Rotter, 1966). Red horizontal lines represent the location of thresholds in log family net worth at ages 22 to 25 (initial conditions), and green vertical lines represent the location of potential thresholds in locus of control. Dots meeting at the crossing of a green and a red line indicates that the threshold detected in initial conditions could be driven by a correlated threshold in locus of control.

locus of control, and report the remaining results for self-esteem and self-concept in Appendix A.3. ${ }^{10}$

We first run our grid-search algorithm to detect the presence of structural breaks in the relationship between locus of control and final outcomes. Figure 1.4 plots the relationship between locus of control and final outcomes, green lines indicate the estimated thresholds in locus of control, and red lines recall the thresholds we find in initial wealth.

\footnotetext{
${ }^{10}$ For additional details on Rotter (1966); Rosenberg (1965); Pearlin et al. (1981) scales measured in the NLSY, see https://www.nlsinfo.org/content/cohorts/nlsy79/other-documentation/codebooksupplement/nlsy79-appendix-21-attitudinal-scales
} 
Table 1.5: Intragenerational Social Mobility and Locus of Control

Final Family Net Worth (at 40-48)

(2)

(3)

Initial Family Net Worth:

Below Threshold 1

$\begin{array}{ccc}0.189 * * * & 0.109 * * * & 0.109 * * * \\ (0.027) & (0.039) & (0.039) \\ 0.193 * * * & -1.305 * * & -1.292 * * \\ (0.022) & (0.595) & (0.594) \\ 0.169 * * & 0.534 * * * & 0.534 * * * \\ (0.068) & (0.067) & (0.067) \\ & -0.071 * * & \\ & (0.032) & \end{array}$

Rotter external locus of control (1979)

$(0.032)$

by region of initial wealth:

Below Threshold 1

Between thresholds 1 and 2

Above Threshold 2

Thresholds in locus of control:

Threshold 1

Threshold 2

Thresholds in initial wealth:

Threshold 1

$\begin{array}{ccc} & 4.73 & 4.73 \\ & 6.24 & 6.24 \\ \mathrm{Y} & \mathrm{Y} & \mathrm{Y} \\ \mathrm{Y} & \mathrm{Y} & \mathrm{Y} \\ \mathrm{Y} & \mathrm{Y} & \mathrm{Y} \\ \mathrm{Y} & \mathrm{Y} & \mathrm{Y} \\ 5,083 & 5,083 & 5,083\end{array}$

Threshold 2

Dummies for zero wealth

Individual characteristics

Dummies for education level

AFQT

5,083

Note: This table presents the results of threshold regressions of permanent net worth on initial net worth augmented by locus of control, using the sequential grid-search algorithm of Hansen (2000) for the detection of thresholds, and the Hannan-Quinn Information Criterion for model selection. Initial net worth is measured by the inverse hyperbolic sine of household net worth between ages 22 and 25. Each column represents a separate regression. Robust standard errors in parentheses. ${ }^{* * *} p<0.01,{ }^{* *} p<0.05,{ }^{*} p<0.1$. Full details in Appendix Table A.4. 
Table 1.5 shows that locus of control predicts final outcomes and presents potential structural breaks, but our intragenerational mobility correlation coefficients are robust to either controlling linearly for locus of control or flexibly across the regions of initial conditions. Column (1) reports the results of a threshold regression where we search for thresholds in locus of control. In this specification, we fully control for demographic characteristics, we plug in the estimates of thresholds in initial wealth as threshold parameters of the threshold regression model, and allow for the intragenerational mobility correlation coefficient to vary across regimes of initial conditions delimited by the estimated thresholds.

The specification in column (1) allows us to understand whether there are potential thresholds in locus of control that are distinct from the breaks in initial conditions, and whether potential regimes in locus of control change the relationship between initial conditions and final outcomes in a systematic way. We find evidence of two thresholds at respectively 7 and 12 in the Rotter scale of locus of control, suggesting three regimes in the relationship between locus of control and permanent income: individual with internal locus of control experience better final wealth outcomes, while individuals with external locus of control experience significantly lower wealth outcomes.

Comparing Column (2) of Table 1.5 to our preferred specification in Column (4) of Table 1.3, we find that controlling for locus of control barely affect our estimated correlation coefficients in each regime of initial conditions: this suggests that although locus of control is an important predictor of final wealth, it is does not highly correlate with the regimes in initial conditions. This finding is confirmed by Column (3) where we allow for locus of control to flexibly vary across regimes of initial conditions, and find that locus of control is not a strong predictor of outcomes within each regime.

We complement this analysis by considering self-esteem in Appendix Figure A.1 and Table A.5, and self-concept in Figure A.2 and Table A.6. We find similar results, and thus conclude that non-cognitive skills are unlikely to confound the aspirational poverty traps that we have identified using regimes in initial conditions.

\subsubsection{Credit Constraints: College Loans and Home Ownership}

Consider now a model of wealth accumulation in which there are constraints in access to credit. The presence of credit constraints has been the subject of much research in the United States, and it could entirely be possible that credit constraints generate different regimes in the accumulation of wealth, which could confound what 
we have identified as aspirational poverty traps. We consider two types of credits in the NLSY: educational loans, and mortgages.

Constraints in access to educational loans could generate structural breaks in the relationship between initial conditions and final outcomes by preventing poorer individuals from accessing higher education, and thus to higher paying occupations. Figure A.3a, however, provides graphical evidence summarizing our main threshold regression finding: we find no evidence of structural breaks in access to educational loans in the NLSY.

Discontinuities in access to mortgages could also generate regimes in wealth accumulation. Figure A.3b plots the share of individuals in our sample who are homeowners at age 25 by initial family net worth. This figure suggests important nonlinearities in the relationship between early home-ownership and initial conditions: a stable $20 \%$ of respondents are homeowners at age 25 for most of the sample, until roughly $\log$ family net worth at ages $22-25$ of 8 , from which home-ownership jumps to almost $90 \%$. We formally test for the presence of structural breaks in the data in threshold regressions in Table A.7 in Appendix. Note that home-ownership is a dummy so we are not searching for breaks in home-ownership status, but estimating the fully controlled threshold regression model first adding a control for home-ownership status, and then flexibly controlling for home-ownership status in each regime of initial condition. We find that home-ownership status does not linearly explain the relationship between initial conditions and final outcomes. When we flexibly control for home-ownership status across the three regimes of initial conditions, we find that home-ownership status has a systematically different contribution to the relationship between initial conditions and final outcomes, which suggests that our aspirational poverty traps could correlate with access to mortgages. However, the estimated correlation coefficients between initial conditions and final outcomes are stable to considering home-ownership as additional control variables, which suggests that the contribution of home-ownership to the relationship remains modest.

\subsubsection{Networks: Job Search and Current Job Found Through Personal Networks}

Consider now a model of human capital investments in which individuals rely on networks to access educational and labor market opportunities. In such a model, the presence of structural breaks in the intragenerational income mobility could reflect social clusters which are more or less organized or able to provide opportunities to their members. There is evidence of heterogeneity in human capital investments 
across immigrant communities in the United States, and there is also evidence of heterogeneity in returns to human capital investments across demographic groups. We explore this idea using questions about job search that asked in the 1982 wave of the NLSY. In 1982, respondents were asked how they found their current job, and they could answer a combination of job search strategies including friendship network, relatives, community organizations, school placement, teachers, personal contacts. Figure A.4 plots the share of respondents who indicated using a particular type of job search strategy against initial conditions. We could not detect the presence of any structural breaks in these relationships, except for community organizations as job search strategy, for which we find suggestive evidence that respondents around the median of initial wealth use this strategy. In Table A.8, we find that including a linear control for job search through community organizations has no impact on the correlation coefficients we find in each regime of initial conditions, but allowing for job search through community organizations to vary systematically across regimes, we find that the correlation coefficient in the left tail of the distribution of initial condition increases while it decreases substantially at the right tail of the distribution, and becomes insignificant in the middle of the distribution. This suggests that although the effect is modest, community-based job search strategies correlate with the regimes of wealth accumulation, and have distinct effects on the transmission of wealth.

\subsection{Conclusions}

In this paper, we propose a simple and tractable methodology for estimating the presence of aspirational poverty traps, their size and location in the distribution of wealth. To the best of our knowledge, this is the first study attempting such an exercise, in spite of the growing number of studies documenting the importance of aspirations for economic outcomes.

Our methodology provides insights on a key economic quantity for policy makers, since it would indicate how many individual lives could be dramatically improved by simple and low-cost interventions such as information campaigns about education, or role modelling interventions. We find that at most $7.5 \%$ of the US population could be located in such aspirational poverty traps.

Our estimates indicate that the population located in aspirational poverty traps is not quite poor: we find that aspirational poverty traps are located around the 37th to 42nd percentile of the distribution of wealth, that is, families with positive 
wealth not too far below the median. In addition, our findings complement an empirical regularity documented by several studies across several countries, that intergenerational social mobility is lowest at the tails of the income distribution. We complement this regularity by showing that it also applies to intragenerational mobility, that is, within person wealth accumulation within their lifetime. We find that wealth accumulation is slowest at the bottom of the income distribution, fastest at the top, with sharp changes in the slope of wealth accumulation within the life course.

Importantly, we started from a theory for the micro-foundations of aspirations poverty traps, we derive theoretical macro-economic predictions corresponding exactly to this empirical regularity. Thus, our study proposes for the first time a micro-founded explanation for the macro-economic regularity that social mobility is lowest at the tails of the distribution of income.

Further research could entail using alternative methods to detect structural breaks in data, to determine how robust is the location and size of the aspirational poverty trap that we found. Another possible route could be to find micro-economic exogenous shiftier of aspirations - such as income shocks, changes in one's social environment, policy announcements. With a valid treatment shifting aspirations, we could then estimate the causal mediating role of aspirations in the relationship between initial conditions and final outcomes. 
Chapter II:

Early Discouragement from Uncertainty

about Financial Aid 

The second chapter of this dissertation focuses on the consequences of internal constraints on the educational trajectories of disadvantaged children. In Chapter 2, we focus on uncertainty about financial aid as a natural experiment to study whether disadvantaged students could be discouraged at an early stage of their educational career from later participating in university-preparing education. As much as vast literature has shown that people do learn from their experience, there is little evidence that students and families respond to uncertainty about future costs of higher education, and how early they do so.

We evaluate the impact of policy uncertainty about financial aid on the tracking outcomes of secondary school students in the Netherlands, using administrative records for the entire population of Dutch pupils between 2005 and 2017. In the absence of direct measures of people's beliefs, a good experiment to study how beliefs respond to uncertainty is to exploit policy announcements prior to implementation. Policy announcements are information shocks that generate uncertainty about future states of the world. Because of this, policy announcements affect beliefs alone and not budget constraints. Therefore, we can then interpret changes in educational trajectories after policy announcements as responses to changes in beliefs, and not responses to changes in actual prices.

We study the introduction of the controversial Sociaal Lensteelsel in the Netherlands. This major reform turned universal and unconditional grants for higher education into income-contingent loans for students starting higher education in the academic year 2015-2016. Importantly, the policy was announced in stages, sparking a heated debate and triggering wild speculations about the future student finance system in news outlets, student unions and political parties. The first announcement in June 2010 took place as the Dutch government announced its intention to move away from the subsidy-based system of student finance towards a loan-based system, without specific details. The second announcement took place in October 2012, when the newly-elected government revealed the detailed reform plan, including the name of 
the new Sociaal Lensteelsel system, its conditions and its date of implementation, while also revealing that vocational education students would be exempted. Our analyses of Google Trends indicate that none of these details were known until October 2012.

The first contribution of this study is to conceptualize how these policy announcements are likely to affect beliefs for different types of students, and eventually their tracking outcomes. To do this, we propose a theoretical framework based on classical human capital theory to describe how uncertainty about financial aid affects educational choices of students. This framework predicts that when the chances of having to pay back for financial aid increase, low-income students are discouraged from pursuing educational tracks associated with higher probability of paying back, and they opt-out as early as they can from these tracks.

We find that the first policy announcement led to a sharp decrease in the share of students enrolling in tracks leading to higher education, and to an immediate jump in the share of students dropping out of these tracks into the vocational track. The second announcement led to a further decrease in the share of students enrolling in tracks leading to higher education, while dropout rates decreased.

We provide a battery of additional findings and sensitivity analyses, which indicate that alternative explanations for our findings are unlikely. In particular, we find that students also reorient towards higher-paying specializations such as STEM and Medicine field of study and are substantially less likely to drop out of these fields after receiving announcements. We find no evidence of trend breaks in 12 potential confounding factors - ranging from high school graduation rates and time to post-high school degree completion, to labor market opportunities for youth, costs of studying, school institutions, and broader macro-economic trends.

This study highlights that policy announcements can harm vulnerable students when they increase uncertainty about future costs of education. Announcements about future educational reforms can change the educational trajectories of vulnerable pupils, even if the reforms eventually implemented are milder than previously announced or don't eventually affect these particular students. Two specific features of the Dutch system make this lessons particularly relevant: on the one hand, early tracking forces children and families to specialize early in educational paths, and on the other hand, entry into post-secondary education requires track-specific diplomas, such that tracking mistakes are hard to correct even just a few years later in a child's life. 


\section{Policy Uncertainty and Early Discouragement ${ }^{1}$}

\subsection{Introduction}

In recent years, several studies have shown that informing students about the cost of higher education can have a large impact on their decision whether to apply to college, and it can also influence their choices of college location and study major (see e.g. Hoxby and Avery, 2013; Hoxby et al., 2013; Hoxby and Turner, 2015; Oreopoulos and Dunn, 2013; Pallais, 2015; Peter and Zambre, 2017). Lochner and Monge-Naranjo (2016) suggest that, in principle, low-SES students could be shying away from college-preparation because of uncertainty about their eligibility to financial aid, yet there is no evidence of this discouragement effect. This study is among the first to show that uncertainty about financial aid might affect students much earlier than in their final year of high school.

The effects of college financial aid on student outcomes have been analyzed in a multitude of national and institutional contexts. The consensus of this literature is that financial aid can increase college enrollment and graduation rates of financially constrained students. Yet, these effects vary considerably across countries. While studies based on the United States generally find large and positive effects (see Dynarski and Scott-Clayton, 2013), European studies find effects that are more ambiguous in terms of their magnitude and direction. ${ }^{2}$ Indeed, some European

\footnotetext{
${ }^{1}$ This study is joint work with Jan Kabátek at the Melbourne Institute. I am particularly thankful to my supervisor Olivier Marie for his advice and support throughout this project. I also thank participants at seminars and conferences for helpful comments, in particular Marianne Bitler and Esmee Zwier at the 21st IZA Summer School. I also thank Fatima El Meslaki and her team at Statistics Netherlands (CBS) for their support.

${ }^{2}$ see Canton and De Jong (2005); Belot et al. (2007); Kelchtermans and Verboven (2010) for studies in the Netherlands, Dearden et al. (2014); Azmat and Simion (2017); Murphy et al. (2019) in the United Kingdom, Hübner (2012) in Germany, Nielsen et al. (2010) in Denmark, Montalbán (2019) in Spain.
} 
studies even find null or small negative effects of financial aid on student performance and time to graduation (Belot et al., 2007; Murphy et al., 2019; Montalbán, 2019). This may be related to the fact that students in many European countries face little to no financial barriers to enter college (Chowdry et al., 2013); while non-selective admission procedures and low enrollment costs at European universities can attract students from many socioeconomic backgrounds, it can also attract low ability students and students exerting little study effort.

The policy discourse in several European countries has recently shifted towards replacing generous university subsidies by income-contingent loan programs (see e.g. Murphy et al., 2019). Proponents of this instrument argue that income-contingent loans are able to counter the negative selection inherent to non-targeted financial aid programs, while ensuring that financially-constrained students are provided with the necessary financial aid to pursue their degrees. The other major advantage of loanbased programs is that the cost of these programs is predicted to be substantially lower than the cost of current subsidy-based programs. ${ }^{3}$. Opponents to policy change from subsidy-based to loan-based programs argue that that the loss of the study subsidy and unwillingness to borrow may discourage some students from pursuing tertiary education. Although the magnitude of these discouragement effects is generally expected to be low, there is very little empirical evidence that these effects occur at all. ${ }^{4}$

Very little of the last argument is based on solid empirical footing, largely because national systems of student finance tend not to be subject to major structural changes, such that policymakers rely on lessons learned from changes of smaller targeted programs (see e.g. Belot et al., 2007; Dearden et al., 2014). Although these lessons may prove indicative of students' responses to major national reforms, they can also be distorted by a multitude of factors and intermediate mechanisms,

\footnotetext{
${ }^{3}$ While total government expenditures in education have steadily risen from $€ 20$ billion in 2000 to $€ 30$ billion in 2010, over the same period, student finance - subsidies and loans - have risen from $€ 2.4$ billion in 2000 to $€ 3.8$ billion in 2010, of which 1 billion alone in basic grant subsidies for living expenditures (for itemized details, see government yearly budgets (in Dutch) available https://www.rijksbegroting.nl) Student finance altogether has thus represented a stable 1/10th of total expenditures in education. Source: CBS Statline, accessed on August 28, 2020, series "Government; expenditure on education and student grants, loans since 1900".

${ }^{4}$ Following a national survey among the student population conducted in 2010, the National Student Union Lsvb suggested that a substantial share of students could drop out of education if student finance were ever to be replaced by a loan system (see e.g. news archives from 2010 https://www.nu.nl/binnenland/2217351/niet-studeren-zonder-studiefinanciering.html and opinion pieces such as https://www.volkskrant.nl/nieuws-achtergrond/studeren-wordt-iets-voor-derijken bb4bfd71/
} 
ranging from greater salience of national reforms to aggregate supply and demand effects. Another concern with using estimates from previous studies is that we do not know at what age do students start responding to the financial aid regime they expect to be exposed to during their studies. Some of the most prominent studies of financial aid programs are based on comparing the decisions made by two cohorts of secondary school students, with one cohort graduating right before some policy change and the other graduating right after (see e.g. Linsenmeier et al., 2006). However, if students start responding to financial aid ahead of the graduation year, we may expect reform effects to be more pronounced among following cohorts, who were exposed to information about the reform for a longer time. Other excellent studies have use descriptive approaches over the long run to characterize the impact of broad changes in student finance systems on the student population attracted to university education (see e.g. Dynarski and Bailey, 2011; Murphy et al., 2019).

In this paper, we use administrative records of all students residing in The Netherlands to study how secondary school students respond to an announcement and the later implementation of a major reform of student finance, which was eventually implemented in 2015. This reform turned a universal and unconditional subsidy for higher education students into a low-interest, income-contingent loan. By doing so, it increased the price of higher education for the median student by $50 \%$, from roughly $€ 2,300$ per year to roughly $€ 3,400$ per year. The reform announcement consisted of two stages. In June 2010, the Dutch government announced its intention to move away from the subsidy-based system of student finance to a loan-based system. This announcement increased the expected price of higher education for all students by making the prospective receipt of financial aid uncertain. The second stage followed in October 2012, when the government revealed the details of the new system, especially the first cohort of college students to be affected by the new regime. The government also revealed that vocational education students would be exempted from the reform, hence increasing the attractiveness of vocational studies relative to other post-secondary studies.

This announcement increased the expected price of higher education further by removing uncertainty regarding whether the new student finance regime would be implemented at all. The progressive information release makes this setting an ideal natural experiment for studying the effect of changes in beliefs about the price of higher education on early sorting in high school. We find that the first policy announcement led to a sharp decrease in the share of students enrolling in college-preparing tracks, and to an immediate jump in drop-out rates of these tracks. The second announcement led to a further decrease in the share of students enrolling 
in college-preparing tracks while dropout rates sharply decreased. Following the 2015 reform implementation, high school track choices resumed their pre-announcement trends, however the college-preparing track is yet to regain the student shares attained in year 2010 .

Our analyses yield additional results that are consistent with students responding to policy uncertainty and avoiding risky choices. First, students who respond most are those who would be most negatively impacted by policy uncertainty (i.e., low-income and low-ability students). Second, students who remain in college-preparing tracks become more likely to focus on STEM subjects, which allows them to choose college majors with higher earnings potential. Third, female students respond more than male students, which is in line with higher levels of risk aversion generally found, including in this setting (see e.g. Charness and Gneezy, 2012; Falk et al., 2018). Fourth, college students affected by the reform become more likely to cohabit with their parents, suggesting that students make living arrangements decisions to avoid taking larger student loans. Overall, this implies that students are attempting to minimize their indebtedness and maximize their repayment capacity.

To the best of our knowledge, only a handful of articles have assessed how do the different types of student finance affect students' decisions and outcomes, and there have been no studies focusing on the effects of uncertainty of financial aid receipt. ${ }^{5}$ Linsenmeier et al. (2006) and Rothstein and Rouse (2011) exploit a unique policy change in an elite college in the United-States, in which student loans were suddenly replaced by student grants for freshmen. Linsenmeier et al. (2006) show that admitted students eligible for grants were not more likely to enrol into the college compared to students eligible for loans. In contrast, Rothstein and Rouse (2011), show that students eligible for loans were more likely to choose majors that led to occupations with higher earnings potential. Field (2009) conducted a field experiment with law students at NYU, finding that the students were willing to

\footnotetext{
${ }^{5}$ There is a separate strand of literature showing that student under-utilize student loans. The three main explanations for this pattern are: i) there might be market failures in access to credit during college or later in the life cycle, such that it might be rational to avoid taking on early debt (see discussion in Rothstein and Rouse, 2011); ii) students lack information or are misinformed regarding the availability and conditions of student loans (e.g. Schmeiser et al., 2016); iii) students might be subject to debt aversion (see Rothstein and Rouse, 2011; Field, 2009). This may lead students to prefer working during their studies over borrowing (see e.g. Oosterbeek and van den Broek, 2009; Booij et al., 2012), at the expense of their study performance. A growing body of evidence has indicated that students tend to have wrong expectations about the costs and returns to college education, and that these expectations contribute to the SES gap in college enrollments (see e.g. Avery and Hoxby, 2004; Jacob and Linkow, 2011; Hoxby and Avery, 2013; Hoxby and Turner, 2015; Delavande and Zafar, 2019).
} 
trade away their preferred major and occupational choice so that they could avoid taking on debt. Both Rothstein and Rouse (2011) and Field (2009) argue that their findings are in line with students exhibiting substantial debt aversion. Dynarski et al. (2018) show that guaranteed financial aid over multiple years has positive effects on college enrollments of low-income students compared to non-guaranteed financial aid. ${ }^{6}$ Lastly, Bahrs and Siedler (2019) show that the introduction of tuition fees in German universities decreased the intentions of graduating high school students to pursue higher education degrees, and that low-income students were driving most of their estimated effect.

Our study makes a simple yet important contribution to this literature, by providing the first evidence that policy uncertainty alone about financial aid alone can lead a substantial share of secondary-school students to avoid college-preparing education. We also demonstrate that secondary-school students respond to the financial aid information well ahead of their graduation year, which has important consequences for empirical design of studies that focus on student responses to the incentives embedded in the system of higher education. Our findings suggest that financial aid can be used to increase the rates of college enrollment and reduce socioeconomic inequalities in higher education attainment.

This paper is organized as follows. Section 2.2 provides the necessary details on the institutional setting. Section 2.3 develops a conceptual framework to predict how does the uncertainty of financial aid receipt affect high school track choice and subject choice, and which students are more likely to respond to this uncertainty. Section 2.4 presents the data. Section 2.5 explains our identification strategy. Section 2.6 presents the main results. Section 2.7 presents additional results to support our framework, and Section 2.8 discusses in details robustness checks and threats to identification. Section 2.9 concludes.

\footnotetext{
${ }^{6}$ They conducted a randomized controlled trial among aid-eligible students at a four-years public college in the United States, in which the control group received a regular letter explaining the financial aid package for the first year of college, while the treatment group received additional guarantees their eligibility to financial aid during the entire four years of college.
} 


\subsection{Institutional Setting}

\subsubsection{Secondary School in The Netherlands}

Dutch children enter secondary school at age 12, following six years of primary schooling. The Dutch system of secondary schooling is distinct from other national systems in that it sorts children into ability-based tracks at a relatively early age. There are three available tracks: 1) vocational, 2) general, and 3) academic track. Students are assigned to tracks in secondary school based on their standardized test scores exiting primary school (CITO), on the subjective assessment of their head teacher exiting primary school, as well as based on their performance in the first years of secondary school. ${ }^{7}$ The timing of the track choice varies between secondary schools and students, however by the end of the third year, every secondary school student is assigned to a track. The initial track choice is crucial, because it shapes the entire educational path of the child. An example of this is that the university enrollment is only available to students who hold a diploma from the academic track. Students can change their initial track, but this is costly in terms of time and effort. In the fourth year, students in the general and academic tracks choose a 'subject profile'. This is a broad subject field that the students will focus on for the rest of their secondary schooling. The offered subject fields can be broadly divided into two categories: 1) STEM and Medicine, and 2) Social Sciences and Humanities. Similarly, to the initial track choice, the choice of the subject field is a high-stakes decision, because field-specific subjects often constitute pre-requisites for higher education courses in these fields. More details are available in Appendix B.1. ${ }^{8}$

\subsubsection{Post-Secondary Education and Student Finance}

In the last year of high school in April, students submit their applications to postsecondary education institutions. These institutions include: 1) vocational schools, 2) professional higher education institutions (also known as universities of applied

\footnotetext{
${ }^{8}$ As there is no catchment area policy in the Netherlands, parents can choose any secondary school for their child. Although technically secondary schools allocate students to tracks, parents can try to negotiate. If parents are unhappy with the tracking outcome of their child in a given school, their only option is to choose another school.
} 
sciences), and 3) universities. ${ }^{9}$ Post-secondary education in the Netherlands is almost exclusively public and non-selective. Tuition fees are set centrally, being indexed to consumer prices and fixed across programs and schools. In 2014, the nominal value of annual university tuition fees was $€ 1,903$, which is equivalent to roughly $9.5 \%$ of the full-time annual minimum wage. Tuition fees are low compared to tuition fees in Anglo-Saxon countries, but similar to tuition fees in neighboring countries such as France, Germany, Belgium, Luxemburg, Denmark, or Sweden (for a comparison of European systems see Eurydice, 2012).

In 1986, the Dutch government introduced a system of student finance for postsecondary students which offers 3 types of financial support: 1) a universal basic subsidy, 2) a means-tested subsidy, and 3) an opt-in loan with below-market interest rates. The universal basic subsidy was awarded at two rates depending on students' living arrangements, and it was accompanied by a free public transport card. The award rate of the means-tested subsidy depended on students' living arrangements, family composition and parental income. Eligibility for any financial support was conditional on: 1) being less than 30 years old, ${ }^{10} 2$ ) enrollment in a full-time or dual post-secondary program, and 3) being a Dutch national or a Dutch resident with same financing rights (for further details, see of Education of The Netherlands), 2018). Booij et al. (2012) show that Dutch students were well-informed about financial aid and its conditions. ${ }^{11}$

In 2015, the Dutch government implemented a major reform of the student finance system. This reform brought forth two main changes, which differed in coverage: 1) the universal basic subsidy was turned into a loan, and 2) the repayment period of student loans was extended from 15 to 35 years. The first applied only to students at Universities and Universities of Applied Sciences, while the second was universal. This meant that vocational school students were allowed to retain the universal basic subsidy and to enjoy the extended loan repayment period. The reform also simplified the means-tested subsidy, making it independent of living arrangements, which benefited students who would live with their parents in the absence of the policy change. The reform further changed the maximum amount of the means-tested subsidy, lowering it for students entering vocational schools, and increasing it for

\footnotetext{
${ }^{9}$ Universities and Universities of Applied Sciences constitute the Dutch higher education sector. Vocational schools are classified as post-secondary non-tertiary education institutions.

${ }^{11}$ Vocational school students younger than 18 years of age receive only the transport card. They become eligible for the basic subsidy from the first trimester after their 18th birthday. Students at Universities and Universities of Applied Sciences face no minimum age requirement.
} 
students entering higher education institutions. The latter was meant to compensate low-income students in higher education institutions for the loss of the basic subsidy. Table 2.1 summarizes key features of the old and the new system.

The first cohort of students affected by the reform entered post-secondary education institutions in the academic year 2015-2016. Students who started their studies prior to this academic year were allowed to finish their degrees under the old regime with the prospect that any financial aid for their follow-up degrees would be set according to the new financing rules. The eligibility criteria remained unaffected by the reform. The 2015 reform constituted a major change in the mode of student financing, leading to large changes in the price of higher education. For most higher education students, the change of subsidies increased the price of a 4-year degree by either $€ 5,000$ or $€ 14,000$ (depending on the students' living arrangements). ${ }^{12}$ This corresponds to two to six times the value of annual tuition fees in 2014 (€1903). However, not all students were made worse off. Disadvantaged students who were eligible for the full means-tested subsidy, and who were intending to live with their parents saw their total monthly subsidy increase by $€ 31$, amounting to a total reduction of $€ 1,488$ in the cost of a 3 -year degree. In addition, the expansion of the loan program and extension of the repayment period created a buffer for the overall price increase, benefiting particularly those students who would have taken up a student loan regardless of the policy change (approximately 10 to $15 \%$ of the students).

\footnotetext{
${ }^{12}$ For student living independently, the universal basic grant amounted to $€ 290$ monthly, so $€ 290 *$ $12 * 4=€ 13,920$ over the course of a 4-year bachelor degree; for student living with their parents, the grant amounted to $€ 100$ monthly, so $€ 100 * 12 * 4=€ 4,800$ for a 4-year bachelor degree. Assuming no interest, repaying these student debts over 30 years would correspond to monthly repayments of respectively $€ 40$ and $€ 15$.
} 
Table 2.1: Student Finance in the Netherlands Before and After 2015

\begin{tabular}{|c|c|c|}
\hline \multirow{2}{*}{\multicolumn{3}{|c|}{ Panel A. University and University of Applied Sciences }} \\
\hline & & \\
\hline \multicolumn{3}{|l|}{ Basic subsidy: } \\
\hline Living with parents & $€ 100.2$ & $€ 0$ \\
\hline Living independently & $€ 279.1$ & $€ 0$ \\
\hline \multicolumn{3}{|l|}{ Means-tested subsidy: } \\
\hline Maximum awarded & $€ 258.3$ & $€ 378.2$ \\
\hline Public transport card & Yes & Yes \\
\hline \multicolumn{3}{|l|}{ Opt-in components: } \\
\hline \multicolumn{3}{|l|}{ Tuition Fees credit } \\
\hline Amount & $€ 158.58$ & $€ 162.58$ \\
\hline \multicolumn{3}{|l|}{ Subsidized loan: } \\
\hline Maximum Amount & $€ 295.73$ & $€ 475.91$ \\
\hline Repayment period & 15 years & 35 years \\
\hline \multicolumn{3}{|c|}{ Panel B. Vocational education } \\
\hline \multicolumn{3}{|c|}{ Basic subsidy: } \\
\hline Living with parents & $€ 79$ & $€ 81$ \\
\hline Living independently & $€ 257.9$ & $€ 264.4$ \\
\hline \multicolumn{3}{|l|}{ Means-tested subsidy: } \\
\hline Maximum awarded & $€ 345$ & $€ 260.5$ \\
\hline Public transport card & Yes & Yes \\
\hline \multicolumn{3}{|l|}{ Opt-in components: } \\
\hline \multicolumn{3}{|l|}{ Tuition Fees credit } \\
\hline Amount & $€ 0$ & $€ 0$ \\
\hline \multicolumn{3}{|l|}{ Subsidized loan: } \\
\hline Maximum Amount & $€ 172.17$ & $€ 176.49$ \\
\hline Repayment period & 15 years & 35 years \\
\hline
\end{tabular}

Note: Information collected from yearly legal archives of Rijksoverheid. For detailed yearly information since 2002, see Tables B.1 and B.2 in Appendix B.1. 


\subsubsection{Student Finance Reform Announcements and Implementation}

The 2015 student finance reform was preceded by a long announcement period during which the initial reform proposal was being turned into an actionable bill. This period covered the years 2010-2014 and consisted of two main stages in which the information about the reform was progressively revealed to the public.

In the first stage, the government announced its intention to reform student finance. This announcement followed the June 2010 general elections, which took place in the context of austerity. Campaigns of political parties therefore emphasized budget cuts in non-priority areas. Education was one of the more controversial topics of the elections, with some parties advocating cuts to the education budget and other parties strongly opposing such measures. The general elections were unexpectedly won by the conservative-liberal party (VVD), which had campaigned in favor of removing the basic subsidy from the student finance package. At this stage, however, the winning party had no exact plan for implementing the reform of student finance. For prospective students, the election outcome introduced uncertainty about the future availability of financial aid, and thereby increased the expected cost of vocational and higher education.

In the second stage, the government revealed the prospective changes to the student finance system. This announcement took place in October 2012, following another round of general elections. Disagreements within the 2010 coalition led to its dissolution, and new elections were held in September 2012. Shortly after the elections, the new coalition (led again by VVD) announced the timing and exact contents of the student finance reform. The reform was to be introduced in the academic year 2014-2015, implementing the changes that we describe in Section 2.2.2. For prospective students, this resolved the uncertainty about prospective financial aid, further increasing the expected costs of higher education and lowering the expected cost of vocational education.

The eventual implementation of the reform was also facing challenges. In late May 2014, the coalition announced that the implementation of the reform would be postponed until September 2015 (academic year 2015-2016). This postponement was a result of political instability which led to delays in passing new legislation. The student finance bill was passed in May 2014, which was deemed too late for the new system to be implemented in the upcoming academic year. Note that, in late May, the next cohort of freshmen had already sent their applications to higher education programs and student finance at latest in April, under the expectation 
that they would enroll under the new regime of student finance.

The long announcement period allowed prospective students to respond to the reform well ahead of its implementation. The 2012 information shock in particular prompted a non-negligible number of high school graduates to expedite their post-secondary admissions (by skipping a gap year or by accepting a second-best non-selective program) in order to qualify for the old student finance package. ${ }^{13}$ We note that this strategic behavior is an identification concern for analyses of students' college-entry decisions, rendering the standard discontinuity-based methods of causal inference largely invalid. However, our analyses are unlikely to be affected by this behavior. This is because we focus on the decisions made by the secondary school students well ahead of their graduation. As such, the students who were making the studied decisions after the 2012 information shock were all under the impression that they will be subject to the new student finance regime, irrespective of their strategic choices. ${ }^{14}$

\subsection{Conceptual Framework}

In this section, we present a conceptual model of students' decision making. The model allows us to illustrate the responses of students to the reform and highlight the expected margins of heterogeneity in these responses. We build on the conceptual model of Charles et al. (2018), which we adapted and expanded to fit our setting. The key distinguishing feature is that our model allows us to study the effects of uncertainty regarding the prospective receipt of financial aid on students' decision making.

\subsubsection{Setting}

Similar to the actual setting we study, secondary school students in our model must choose among three mutually exclusive education tracks: vocational $(V)$, general $(G)$, and academic $(A)$. In contrast to the real setting, we treat the track choice as irreversible and deterministic of student's eventual education attainment. The tracks differ in terms of their length, with $V$ being shorter than $G$ and $G$ being shorter than $A$ (that is, $t_{V}<t_{G}<t_{A}$ ). For simplicity, we also assume that students

\footnotetext{
${ }^{14}$ Based on auxiliary analyses of our estimation sample, that are beyond the scope of this chapter.
} 
receive financial aid over the entire duration of their studies, and that the yearly financial aid rates are identical across tracks. To simplify notations, we also assume that students make decisions at the same time $t$.

Financial aid is defined as a government transfer $s$ that consists of a universal transfer $s^{0}$ and a means-tested transfer $s^{1}$. Receiving the means-tested transfer is conditional on student's eligibility status, such that $s=s^{0}+s^{1} 1\{$ eligible $\}$. The take-up of financial aid is assumed to be universal. Let $p_{0, j}$ and $p_{1, j}$ be the probabilities that $s^{0}$ and $s^{1}$ must be paid back to the government for a student in track $j$-i.e. the probabilities that the study subsidies are turned into loans. Students choose the track that maximizes their expected lifetime payoff. For a student $i$ with ability $\theta_{i} \sim \operatorname{unif}(0,1)$, the expected lifetime payoff of following track $j$ is $R_{i}^{j}\left(\theta_{i}\right)$.

Normalizing the expected lifetime payoff of the vocational track to $R_{i}^{V}\left(\theta_{i}\right)=0$, we can express the payoffs for academic and general tracks as:

$$
\begin{aligned}
R_{i}^{j}\left(\theta_{i}\right) & =\sum_{k=t_{j}+1}^{L-t_{j}} \mathbb{E}\left(\Pi_{t}^{j}\right) \\
& -\sum_{t=1}^{t_{V}}\left[(1+b)\left(F_{j}-F_{V}\right)+\left(\kappa_{j}-\kappa_{V}\right)\left(1-\theta_{i}\right)\right] \\
& -\sum_{t=t_{V}+1}^{t_{j}}\left[(1+b)\left(F_{j}-\left(1-p_{0}\right) s^{0}-\left(1-p_{1}\right) s^{1} 1 \text { eligible }\right)+\kappa_{j}\left(1-\theta_{i}\right)+\mathbb{E}\left(Y_{t}^{V}\right)\right]
\end{aligned}
$$

The first sum corresponds to the expected accumulated labor market premium of track $j$ over track $V$ from graduation $t_{j}$ until retirement $L . \Pi_{t}^{j}$ denotes the difference between stochastic wages $Y_{t}^{j}$ and $Y_{t}^{V}$ corresponding to the given time period. The next two sums represent the excess costs of pursuing education track $j$. The costs are computed over two periods, one covering the length of the vocational track $t_{V}$, and the other covering the excess time spent in track $j$. In the first period, the costs have two parts: 1) direct out-of-pocket costs $F_{j}$ in the form of living expenditures and tuition fees, and 2) psychic (or effort) costs of attending education $j, \kappa_{j}\left(1-\theta_{i}\right)$, where $\kappa_{j}$ captures the difficulty of track $j\left(\kappa_{V}<\kappa_{G}<\kappa_{A}\right)$. Psychic costs are decreasing with students' abilities $\theta_{i}$. The costs are compensated by financial aid ; however, this term is irrelevant in the first period as there are no differences in the receipt of financial aid across tracks. In the second period, the costs of track 
$j$ are compensated by the excess financial aid, and the psychic costs of vocational education are replaced by expected forgone earnings of graduates from the vocational track $\mathbb{E}\left(Y_{t}^{V}\right)$. We assume that these costs are covered by student loans procured at an interest rate $b$. We assume that students face no borrowing constraints, which is in line with the real setting we study, where students are allowed to borrow from the government regardless of their financial situation.

When choosing an education track, students take into account their own abilities. For each education track $j$, the expected lifetime payoff increases with ability $\left(\partial R_{i}^{j} / \partial \theta_{i}>0\right)$. To avoid corner solutions, we assume that students with lowest abilities reap the highest payoffs from the vocational track and the lowest payoffs from the academic track. This ordering is reversed for students with highest abilities.

$$
\begin{aligned}
& R_{i}^{V}(0)>R_{i}^{G}(0)>R_{i}^{A}(0) \\
& R_{i}^{V}(1)<R_{i}^{G}(1)<R_{i}^{A}(1)
\end{aligned}
$$

Under these assumptions, there exists an ability level $\theta_{G}^{*}$ at which the lifetime payoff functions $R_{i}^{V}(\theta)$ and $R_{i}^{G}(\theta)$ intersect, rendering the students at this ability level indifferent between tracks $V$ and $G$. Similarly, there exists an ability level $\theta_{A}^{*}$ at which students are indifferent between tracks $G$ and $A$. Accordingly, students with abilities lower than will choose track $V$, students with abilities in between $\theta_{G}^{*}$ and $\theta_{A}^{*}$ will choose track $G$, and students with abilities higher than $\theta_{A}^{*}$ will choose track $A$. The closed-form solutions for $\theta_{G}^{*}$ and $\theta_{A}^{*}$, and comparative statics of the effects of a policy shock are available in Appendix B.2.

\subsubsection{Model Predictions: Reform Announcements and Implementation}

Prediction 1 Overall reform effects We consider a simplified version of the 2015 reform. At baseline $B$, prior to the reform and its announcements, the probabilities of repayment $p_{0}$ and $p_{1}$ are zero for both the universal and the meanstested subsidy, such that we have: $p_{0 j}^{B}=p_{1 j}^{B}=0$. Under this policy regime, students sort themselves into education tracks according the baseline ability thresholds $\theta_{G}^{B *}$ and $\theta_{A}^{B *}$. Once implemented, the reform $B$ changes the student finance system by setting the probability of repaying the universal transfer to one for all students in tracks $A$ and $G$, that is, $p_{0 A}^{R}=p_{0 G}^{R}=1$. Students in track $V$ are exempted from this change, so $p_{0 V}^{R}=0$. The probability of repaying the means-tested transfer eventually remains zero under the new system, and all transfer rates remain the same, so we 
have $p_{1 A}^{R}=p_{1 G}^{R}=p_{1 V}^{R}=0$. This change shifts the ability thresholds to the right, with students above the first threshold moving from track $A$ to track $G$, and students above the second threshold moving from track $G$ to track $V$. Intuitively, we would expect the outflow from track $G$ to be stronger than the outflow from track $A$. This intuition is however not necessarily the case for all possible parameterizations of the model. In our case, this arises because we assume that the gap in duration of studies and difficulty is larger between general and academic tracks than it is between vocational and general tracks.

Prediction 2 Uncertainty and means-testing The reform implementation is preceded by a period of policy uncertainty $U$. Under this policy regime, students do not know whether they will receive any study subsidy. This means that the subjective probabilities of repayment increase for both the universal and the means-tested transfer $\left(p_{0 j}^{U}>0\right.$, and $\left.p_{1 j}^{U}>0\right)$. Similar to the reform itself, policy uncertainty alone shifts the ability thresholds rightwards from baseline, such that we have $\theta_{A}^{U *}>\theta_{A}^{B *}$ and $\theta_{G}^{U *}>\theta_{G}^{B *}$. Importantly, the magnitude of this shift is contingent on students' eligibility for the means-tested transfer.

On the one hand, students who are ineligible for the means-tested transfer respond to the policy uncertainty less than to the reform itself, because they still have a non-zero chance of receiving the universal subsidy.

Students who are eligible for the means-tested subsidy, on the other hand, respond more to policy uncertainty than the ineligible students, assuming the two groups have similar ability distributions around thresholds $\theta_{G}^{*}$ and $\theta_{A}^{*}$. This is because eligible students risk the repayment of both subsidy types. Although the model does not indicate whether the response of eligible students grows further under the reform regime $R$, under realistic parameterizations of the model, we can expect the eligible students to be less affected by the resolution of uncertainty than the ineligible students. This is because their loss of the universal subsidy is compensated by the newly-established certainty of receiving the means-tested subsidy. For sufficiently large values of $p_{1}$ and $s^{1}$, their response to $U$ may be actually stronger than their response to $R$.

Prediction 3 Uncertainty and gender Our model does not explicitly consider gender-specific effects. However, gendered responses may arise if male and female students differ from each other in characteristics that are relevant for their decision-making process. Here, we focus on the role of relative risk aversion. Women are generally found to be more risk averse than men (see e.g. Charness and Gneezy, 
2012; Falk et al., 2018) - including in controlled laboratory settings using university students - and this risk aversion may influence their behavior under policy uncertainty.

For the sake of simplicity, we assume that the higher risk aversion of female students translates into higher subjective probabilities of repayment under uncertainty. Within this setup, our model predicts female students to have stronger behavioral responses to policy uncertainty compared to male students. Once the reform is implemented and uncertainty is resolved, we expect gendered responses to become more aligned. We may, however, still see some residual gender differences if low-income students face uncertainty regarding the receipt of the means-tested subsidy. This would be more aligned with the real-world setting. Low-income secondary school students in the Netherlands are never completely sure that they will become eligible for the means-tested subsidy. This is because: 1) the eligibility is tested against future parental income, and 2) the income cut-offs are not disclosed to public.

\subsection{Data}

Our empirical analysis draws on data provided by the Dutch national statistics agency, Statistics Netherlands. The cornerstone of our dataset is the education register, which tracks the population of students at every level of the Dutch education system between years 2005 and 2017. ${ }^{15}$ Based on this registry information, we create a dataset of student-year education records, extracting the characteristics of the education program pursued by each student at the start of each academic year. The education register contains unique personal identifiers, which allows us to link it to other registry data and retrieve a rich set of students' background characteristics. These include gender, age, immigration background, employment and income histories (both own and parental), residential history, and standardized test scores taken in Grade 6 (age 11), accompanied by teacher's high school track recommendations.

\footnotetext{
${ }^{15}$ The education registry contains student enrollments at all levels as of December 1st of every year.
} 


\subsubsection{Sample Selection}

Our analyses use several subsets of the full education dataset. These smaller datasets contain students that are observed at the same stages of their education paths over the period of observation. Our primary analytical dataset contains students who are in their fourth year of secondary school (Grade 10). As outlined in Section 2.2.2, this is the first year in which we are able to determine the chosen education track for all secondary school students, and it is also the year in which academic and general track students choose their subject field. For other analyses, we use datasets of Grade 11 secondary school students, and first-year students in post-secondary education programs.

Table 2.2: Summary Statistics: Grade 10 High School Students in the Netherlands, years 2005-2017

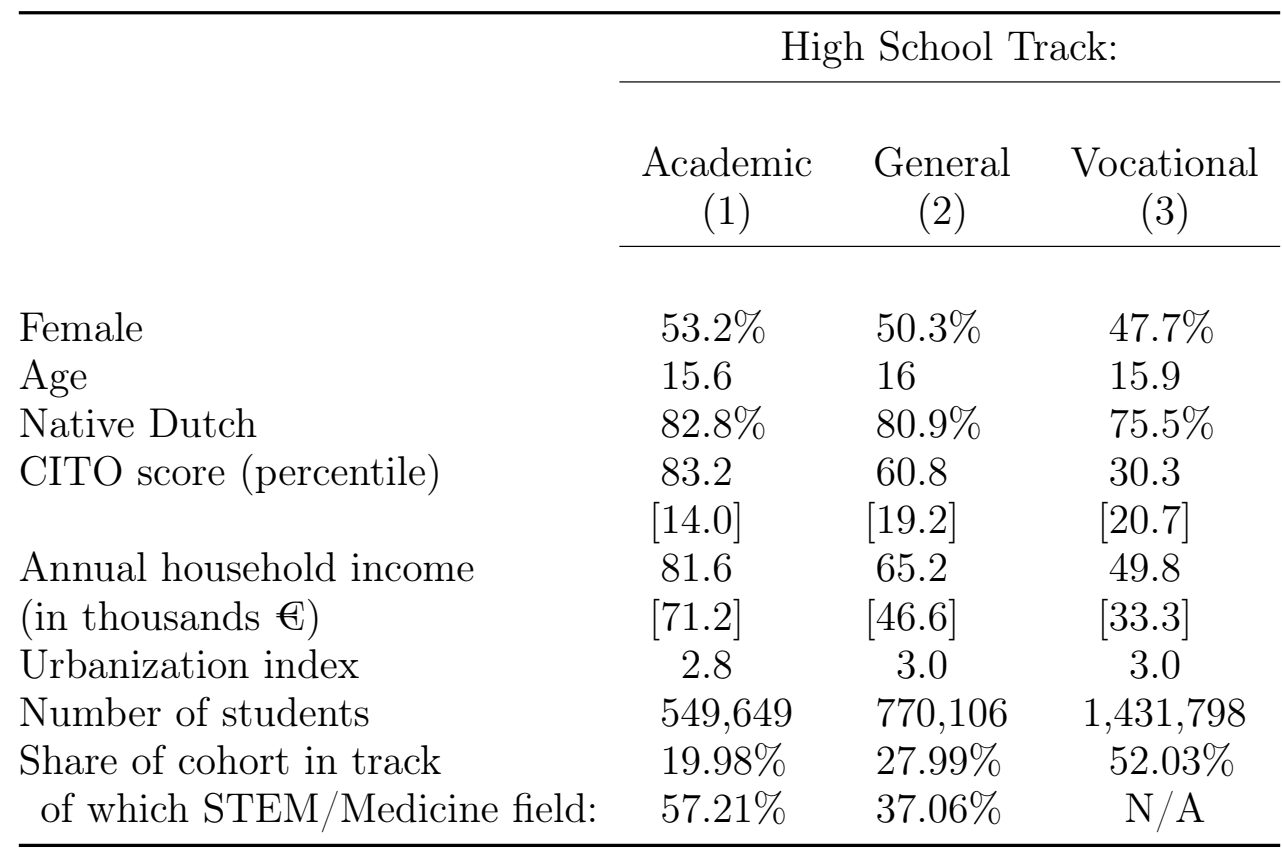

Note: This table presents summary statistics of the estimation sample. The sample covers the near-universe of Dutch students in Grade 10 between 2005 and 201\%. Numbers in brackets correspond to standard deviation from the mean. The Urbanization index ranges from 1: urban to 5: rural.

Our analytical datasets are not subject to any substantive selection criteria, as we are primarily interested in the reform effects on the entire cohort of secondary school students. We only exclude students who are enrolled in special education programs (which constitute a standalone secondary school track for children with learning disabilities), as they do not qualify for higher education.

In Table 2.2, we present descriptive statistics corresponding to the focal group of 
Figure 2.1: Tracking Trends in The Netherlands, Years 2005-2017

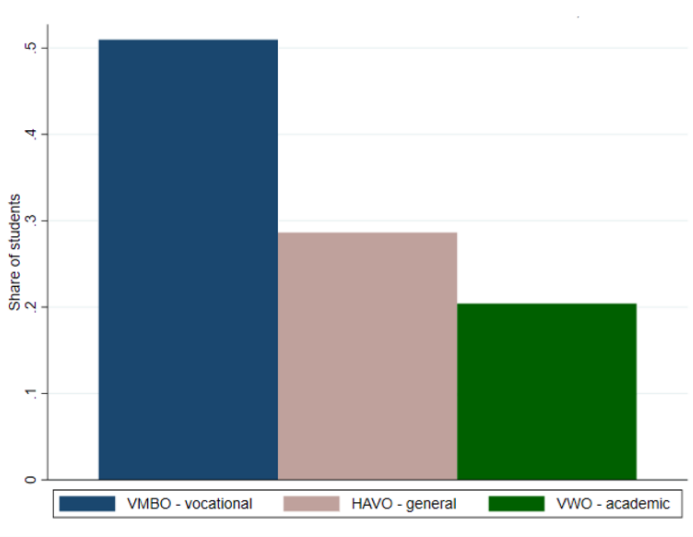

(a) Grade 10 track shares in 2005

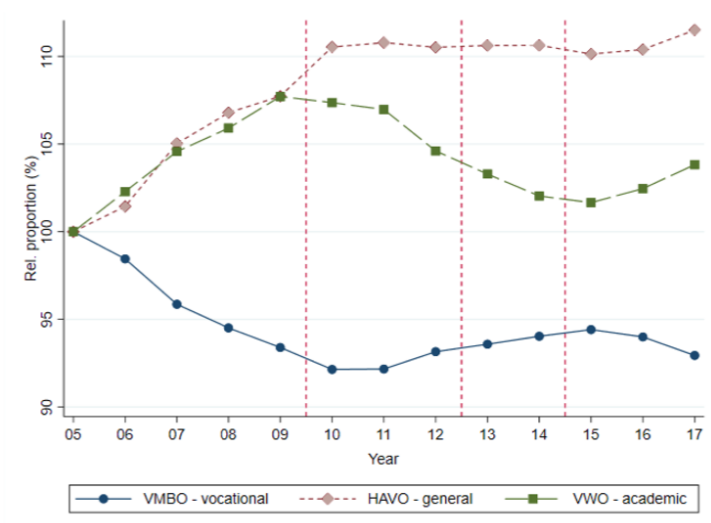

(b) Changes in the track shares relative to year 2005

Note: Authors' calculations of high school track shares over the period of observation. Figure 2.1a plots the Grade 10 high school track shares in year 2005, and Figure 2.1b tracks changes of the high school track shares over the next twelve years, taking the shares in year 2005 as the point of reference. Dutch education register data for Grade 10 high school students, excluding students in special education institutions, years 2005-2017. Red dashed lines indicate the timing of two reform announcements and the eventual reform implementation.

Grade 10 students. Over the period of our study, 20\% of students were following the academic track, $28 \%$ were following the general track, and $52 \%$ were following the vocational track. Among academic track students, $57 \%$ chose to study STEM and Medicine subjects. Among general track students this share was 28\%. Next, we note that students' characteristics differ systematically across tracks. Students in the academic track are more likely to be female, native Dutch, live in urbanized areas, and have higher standardized test scores and parental income. The characteristics of students in the vocational track are reversed.

\subsubsection{Students Tracking Outcomes over Time}

In Figure 2.1, we present the dynamics of Grade 10 track shares over the period of observation. The aim of this exercise is to highlight the trends in students' track choices at baseline, prior to any reform information shock, and to contrast them with the choice patterns observed during the period of reform announcement (2010-2014) and implementation (from 2015 onwards). The first panel of Figure 2.1 shows the Grade 10 track shares in the first year of observation (2005), and the second panel shows how these shares evolved over the following years.

In Figure 2.1, we present the magnitudes of track enrollment shares in relative terms, taking the 2005 track shares as reference points, to highlight dynamic changes in track 
enrollment trends over the period. The figure shows that over the pre-announcement period (2005-2009), the three high school track shares followed sustained trends. Academic and general tracks grew consistently between 2005 and 2009, to the point of being $5 \%$ larger in 2009 compared to 2005. This growth was at the expense of the vocational track, which saw a steady decline in student numbers during this period.

The first reform announcement took place in June 2010; although we do not see 2010 track shares already deviating from the pre-announcement trends as of the academic year 2010-2011, we do see a clear trend break starting with the academic year 2011-2012. That we do not yet see a trend break in 2010 is not surprising, given that the 2010 student cohort received the information only a few weeks prior to entering Grade $10{ }^{16}$

Starting with the 2011 cohort, however, we see a clear reversal of the pre-announcement trends. The share of students in the vocational track starts increasing, the share of students in the general track plateaus, and the share of students in the academic track starts declining. The trend reversal is sustained through both phases of the reform announcement and eventually the year 2015 when the reform was eventually implemented. From 2016 onward, our figure suggests that enrollments trends seem to revert to pre-reform trends, which suggests that the reform led to an overall drop in the demand for academic and general high school education, without however changing the underlying trends in demand for these education tracks.

\subsection{Empirical Strategy}

We are interested in modelling how students' trajectory in high school depends on their beliefs regarding the future receipt of financial aid. Because we cannot observe students' beliefs directly, we exploit the timing of the 2015 reform announcements as quasi-random shocks to those beliefs. As described above, the announcements generated exogenous variation in the probability of receiving the more generous prereform financial aid package, first by introducing uncertainty regarding its receipt, and later by setting the probability of receiving the universal subsidy to zero.

The primary outcome of interest is students' track, as observed in Grade 10. Since

\footnotetext{
${ }^{16}$ Pupils likely to respond to this first announcement shock in June 2010 are those leaving primary school with marginally passing Grade 6 test scores; for most pupils, test scores and teacher recommendations are already known by June.
} 
students can choose from three tracks, we model tracking using a multinomial logit model. Other outcomes of interest correspond to choices between two alternatives, leading to standard logistic model.

The dynamics of our outcomes of interest show substantive changes in students' behavior over the period of the reform announcement and implementation. In this section, we introduce the statistical models we use to quantify the effects of the reform on student's choices. We first outline the principal model specifications for the choice of high school track and the field of study, and then discuss the validity of assumptions for our models to identify causal effects of the policy announcements we study.

\section{A model of Tracking}

Our first set of analyses concerns how students choose their high school track (which we only observe with confidence in Grade 10). Since the choice is between three mutually exclusive high school tracks, we model students' tracking outcome in a multinomial logit framework as following:

$$
\mathbb{P}\left(Y_{i}=j^{*} \mid \mathbf{x}_{\mathbf{i}}, \beta\right)=\frac{\exp \left(\mathbf{x}_{\mathbf{i}} \beta_{j^{*}}\right)}{\sum_{j=1}^{3} \exp \left(\mathbf{x}_{\mathbf{i}} \beta_{j}\right)}
$$

Where $Y$ is a choice variable, $j^{*}$ is the selected high school track (Academic, General and Vocational), $\mathbf{x}_{\mathbf{i}}$ is a vector of covariates, and $\beta$ is a set of choice-specific parameter vectors, with

$$
\mathbf{x}_{\mathbf{i}} \beta_{j}=\beta_{1 j}+\beta_{2 j} f\left(t_{i}\right)+\sum_{y=2010}^{2017} \beta_{3 y j} 1\left\{t_{i}=y\right\}+\mathbf{z}_{\mathbf{i}} \beta_{4 j}
$$

where $i$ indexes students and $\mathrm{j}$ indexes high school tracks. The functional form consists of a time trend, a set of eight yearly dummies covering the period of the reform announcement and implementation, and students' observable characteristics including gender, immigration and ethnic background, parental income, and residential characteristics.

We consider two main specifications of the time trend: a linear time trend which 
stops in year 2009, and a linear time trend which runs throughout the entire period, corresponding respectively to assuming $f\left(t_{i}\right)=f_{1}\left(t_{i}\right)=t_{i} 1\left\{t_{i}<2010\right\}$, and $f\left(t_{i}\right)=f_{2}\left(t_{i}\right)=t_{i}$. The first specification controls for the pre-trend and assumes that the captured trend does not extend past the initial reform announcement, whereas the second specification assumes that the pre-trend continues beyond 2009.

The reform effects are captured by the set of eight yearly dummies covering the period of reform announcement and implementation. A specification with year dummies allows for cohort-specific responses to the decision environment they are in, while having one coefficient per period would allow us to identify the effects of the shocks on average across the cohorts treated by each shock, thus assuming that between two shocks cohorts respond similarly The set of yearly dummies is preferable to a single reform indicator due to the staggered announcement which made the reform and its details more salient to the later cohorts. Students who began their studies later had more time to adjust to the new student finance regime, which would imply stronger reform effects among the later cohorts. This model is estimated by maximum likelihood, and standard errors are clustered at the year level.

\section{A model of Track Dropout}

In our second set of analyses, we study how students decide to switch to a lower track. We model this decision using a logit model.

$$
\mathbb{P}\left(Y_{i}=j^{*} \mid \mathbf{x}_{\mathbf{i}}, \beta\right)=\frac{1}{1+\exp \left(-\mathbf{x}_{\mathbf{i}} \beta_{j}\right)}
$$

The model specification uses the same set of covariates as the model of track choice. We further use logit models to study other binary decisions, such as the choice of the field of study (STEM and Medicine or Social Science and Humanities), or to dropout from a field of study. These models are estimated by maximum likelihood, and standard errors are clustered at the year level.

Leveraging our linked, administrative data, we use an extensive set of control variables including gender, immigration and ethnic background, parental income, and residential characteristics. To account for the pre-announcement dynamics of track choices (see Figure 2.1), we use linear time trends, and include region fixed effects. 
We capture the effects of the reform announcements and later implementation using a set of yearly dummies that cover the period of reform announcements and implementation. The identifying assumptions are that the students are comparable across cohorts (conditional on their observed characteristics and the time trend), and that the only changing factor in their decision environments is their exposure to reform information shocks. Given that these are both strong assumptions, we conduct several supplementary analyses and discuss to what extent they are likely to hold (see Sections 2.7 and 2.8).

\subsection{Main Results}

\subsubsection{Effects on Tracking Outcomes}

The key results corresponding to the model of Grade 10 track choice are presented in Table 2.3. To simplify the exposition of our results, we aggregate the reform effect estimates into three coefficients, one for the first announcement period (2010-2012), then for the second announcement period (2013-2014), and the last one for the implementation period (2015-2017). These coefficients correspond to average reform effects over the given period of observation and they are derived by applying linear transformations to the original year-specific reform dummies. The estimates are presented as average marginal effects (AME), and they are expressed both in absolute (Panel A) and relative terms (Panel B).

In line with the trends in tracking outcomes presented in Figure 2.1, Table 2.3 indicates that policy uncertainty following the 2010 announcement lowered the share of academic track students by 1.07 percentage points, lowered the share of general track students by 0.54 percentage points, and increased the share of vocational track by 1.61 percentage points. These numbers capture the effects of the 2010 announcement, relative to the no-reform counterfactual in which the aggregate track shares are influenced only by students' observable characteristics and the general time trend.

The reform effects become more pronounced following the second announcement, which is in line with the corresponding resolution of uncertainty. During the postimplementation period, the effects reach their highest magnitudes. The academic and general track shares are respectively 3.57 and 3.22 percentage points lower, whereas the vocational track share is 6.8 percentage points higher than the no-reform 
Table 2.3: The Effects of Policy Announcements on Track Enrollment

\begin{tabular}{ccccc}
\hline & $\begin{array}{c}\text { Baseline } \\
\text { track } \\
\text { shares (\%) }\end{array}$ & Uncertainty & $\begin{array}{c}\text { Uncertainty } \\
\text { Resolution }\end{array}$ & $\begin{array}{c}\text { Post- } \\
\text { implementation }\end{array}$ \\
& $(2009)$ & $(2010-2012)$ & $(2013-2014)$ & $(2015-2017)$ \\
Track & $(1)$ & $(2)$ & $(3)$ & $(4)$ \\
\hline
\end{tabular}

Panel A: Absolute Effects (in percentage points)

\begin{tabular}{lcccc}
\hline Academic & 21.0 & $-1.07 * * *$ & $-2.29 * * *$ & $-3.57 * * *$ \\
& & $(0.06)$ & $(0.08)$ & $(0.12)$ \\
General & 29.0 & -0.54 & $-1.59 * * *$ & $-3.23 * * *$ \\
& & $(0.22)$ & $(0.30)$ & $(0.44)$ \\
Vocational & 50.2 & $1.61 * * *$ & $3.89 * * *$ & $6.80 * * *$ \\
& & $(0.26)$ & $(0.37)$ & $(0.53)$
\end{tabular}

Panel B: Relative Effects (in percent)

\begin{tabular}{lccc}
\hline Academic & $-5.37 * * *$ & $-11.57 * * *$ & $-18.09 * *$ \\
& $(0.30)$ & $(0.43)$ & $(0.62)$ \\
General & -1.94 & $-5.70 * * *$ & $-11.50 * * *$ \\
& $(0.77)$ & $(1.07)$ & $(1.54)$ \\
Vocational & $3.24 * * *$ & $7.83 * * *$ & $13.69 * * *$ \\
& $(0.53)$ & $(0.74)$ & $(1.06)$ \\
\hline
\end{tabular}

Note: Authors' estimates of three reform coefficients from a multinomial logit model of high school track enrollment as measured in Grade 10, with a linear time trend. The model uses 2,693,023 individual observations over years 2005201\%. Controls: region fixed effects, age, gender, migration status, ethnic background, parental income and employment status, and an urbanization index. ${ }^{* * *}=0.01$ significance level, ${ }^{* *}=0.05$ significance level, ${ }^{*}=0.1$ significance level.

counterfactual. Following the reform implementation in 2015, the growth of the yearly effects is sustained. This was to be expected, since all students entering Grade 10 from the academic year 2015-2016 were exposed to the second announcement in October 2012, ahead of starting their secondary schooling. Thus, they were able to fully internalize the information about the new student finance system when choosing their track.

These results strongly suggest that students anticipated the future costs of attending higher education when making their education track decisions. The relative magnitudes of the reform effects are well-aligned with our theoretical framework. First, we observe that the responses to policy uncertainty are smaller than the responses to the resolved uncertainty in the second announcement phase. Further, we see that under the initial policy uncertainty, the magnitude of student outflow from 
the academic track is similar to the magnitude of student inflow into the vocational track - the magnitudes of the two coefficients are not statistically different from each other. However, once students become aware that the vocational sector will be largely unaffected by the reform, the inflow into the vocational track intensifies, eventually becoming almost twice as large as the outflow from the academic track. We discuss alternative specification of time trends in Section 2.8.

\subsubsection{Effects on Track Dropout}

As described in Section 2.2, the education track and subject field choices that we observed when students are in Grade 10 are not necessarily final. Students may drop out of their tracks and fields in the following grades, and more than $5 \%$ of students do so at baseline. In Figure 2.2, we plot the yearly shares of students who switched to a lower track between Grades 10 and 11, combining together academic and general track students. We report drop-out rates only from 2006 because we start to observe the cohort of Grade 10 students in 2005. The drop-out rate in Grade 11 is stable during the pre-announcement period (2006-2009), amounting to approximately 2,800 students per year. Following the 2010 reform announcement, drop-out increases dramatically. It peaks in the academic year 2012-2013 with 4,000 Grade 11 students switching to a lower track.

After 2012, the rate of track drop-out declines, and even drops in the post-implementation period it below pre-announcement levels. This pattern is in line with our results regarding our Grade 10 track choice after 2015: since students observed in Grades 10 during the post-implementation period were fully informed about the reform when making their initial track decisions, they did not have to correct their choices in subsequent grades. In order to capture the effects of the student finance reform on the probability of switching to a lower track, we estimate a binomial model of switching choice. The set of covariates is almost the same as for the model of tracking in Grade 10. ${ }^{17}$

Table 2.4 shows the effects of the reform on the probability of switching to a lower track in Grade 11. Our model indicates that the first announcement in 2010 led to a 1.24 percentage point increase in switching from the academic and general tracks.

\footnotetext{
${ }^{17}$ We do not observe any substantive trends in track switching, so we don't include linear time trend among the set of covariates. We capture the small variation within the pre-announcement period by a linear pre-trend variable which is not extended into the reform period. We present results under alternative specifications in Appendix B.4.
} 
Figure 2.2: Track Dropout in The Netherlands, Years 2006-2017



Note: Authors' calculations of the incidence of switching to a lower high school track in Grade 11 over the period of observation. Track switching is recorded if a Grade 10 student enrolled in an academic (general) track in year 10, was recorded to enrol in a general (vocational) track in the subsequent academic year. Dutch education register data for Grade 11 high school students who were enrolled in either an academic or a general track in Grade 10, years 2006-201\%.

The reform effect following the second announcement in 2012 was slightly smaller, and there was no reform effect during the post-implementation period. That we find stronger effects on dropout rates following the first than the second announcement shock contrasts with our findings regarding initial track choices in Grade 10 track, yet our findings on dropout are consistent with our conceptual model: similar to the students observed in post-implementation period, many students observed during the second announcement period did not need to correct these choices, because they were already (partially) informed about the reform when making their initial track decisions.

Our results bind together students from the Academic and General tracks, which might actually have different outside options: while students leaving the Academic track overwhelmingly enter the General track, students leaving the General track overwhelmingly leave the public funded education system instead of pursuing the Vocational Track (additional analyses not shown here). It is likely that they go to privately funded schools, which specialize in preparing students for qualifying state examinations for entering higher education. If this is indeed the case, then this would further strengthen our argument that students and families respond to the policy announcements, as this type of school would offer a way of entering higher education 
Table 2.4: The Effects of Policy Announcements on Track Dropout

\begin{tabular}{ccccc}
\hline & $\begin{array}{c}\text { Baseline } \\
\text { track } \\
\text { dropout }(\%)\end{array}$ & Uncertainty & $\begin{array}{c}\text { Uncertainty } \\
\text { Resolution }\end{array}$ & $\begin{array}{c}\text { Post- } \\
\text { implementation }\end{array}$ \\
Outcome: & $(2009)$ & $(2010-2012)$ & $(2013-2014)$ & $(2015-2017)$ \\
& $(1)$ & $(2)$ & $(3)$ & $(4)$ \\
\hline
\end{tabular}

Panel A: Absolute Effects (in percentage points)

\begin{tabular}{|c|c|c|c|c|}
\hline $\begin{array}{l}\text { Track } \\
\text { Dropout }\end{array}$ & 4.1 & $\begin{array}{l}1.24 * * * \\
(0.17)\end{array}$ & $\begin{array}{l}1.10 * * * \\
(0.17)\end{array}$ & $\begin{array}{c}-0.08 \\
(0.17)\end{array}$ \\
\hline
\end{tabular}

Panel B: Relative Effects (in percent)

\begin{tabular}{llll}
\hline Track & $28.60 * * *$ & $25.47 * * *$ & -1.77 \\
Dropout & $(3.91)$ & $(3.90)$ & $(3.88)$ \\
\hline
\end{tabular}

Note: Authors' estimates of three reform coefficients from a logit model of track dropout measured in Grade 11 compared to high school track observed in Grade 10, assuming a linear pre-trend with does not extend past 2009. The model uses 958,512 individual observations over years 2006-2017. Controls: region fixed effects, age, gender, migration status, ethnic background, parental income and employment status, and an urbanization index. ${ }^{* *}=0.01$ significance level, ${ }^{* *}=0.05$ significance level, ${ }^{*}=0.1$ significance level.

before the implementation of the new student finance regime. Unfortunately, our data do not allow us to provide evidence for this behavior, since the education registry only records students enrolled in publicly funded schools.

\subsection{Additional Results and Supporting Evidence}

In this section, we discuss heterogeneity of the reform effects, and we analyze other decisions affected by the reform. First, we consider income heterogeneity. We use year-specific terciles of the parental income distribution to split the Grade 10 students into three groups (low-income, medium-income, and high-income). For each income group, we estimate a separate parameterization of the principal model of tracking and derive the group-specific reform effects. We present the key findings of these analyses in Table B.3 in Appendix B.3. 


\subsubsection{Heterogeneous Effects by Family Income}

Interpreting these effects is complicated by large baseline differences in track choice between the three income groups: low-income students are more likely to enroll in the vocational track, whereas high-income students are more likely to enroll in the general and academic tracks. In spite of these baseline differences, our estimated reform effects are generally aligned with our conceptual framework. Under policy uncertainty, we observe that a similar share of low-income and medium-income students started favoring the vocational track (2.17 and 2.29 percentage points, respectively). Upon the second announcement, low-income students have learned that they would be partially sheltered from the reform through increased meanstested subsidy for higher education. In line with this change of incentives, we see that their responses to the second announcement and eventual implementation are weaker than the responses of medium-income students. Similarly, low-income students were most likely to switch to a lower track under policy uncertainty, while medium-income students were most likely to switch to a lower track following the resolution of the uncertainty (see Table B.4).

\subsubsection{Heterogeneous Effects by Student Gender}

Second, we estimate the track choice model separately male and female students. Table B.5 presents our main results split by student gender. Female students respond to the initial policy uncertainty more than male students, which is in line with female students being more risk-averse than male students. Following the second announcement and extending to the post-implementation period, the responses of female and male students become more similar, although we still observe much higher dropout rates of female students from the academic track (Table B.6).

\subsubsection{STEM Specialization and Dropout}

Third, we analyze the effects of the reform on the Grade 10 choice of STEM + Medicine subject field (see Figure B.4 and Table B.7). Our model indicates that the first announcement in 2010 did not lead to an immediate change in the takeup of STEM + Medicine, consistent with our findings on initial track choice in Grade 10. However, we do see a 2.28 percentage points increase in the take-up of STEM + Medicine induced by the second announcement in 2012, which grows to 3.0 percentage points following the reform implementation in 2015. This lag in the effects of the reform suggests that choosing a different subject field may require more 
time and effort than choosing a different education track. ${ }^{18}$ Additional analyses show that reform announcements also lowered dropout rates out of subject field in Grade 11. Table B. 8 indicates a decline by $32 \%$ in the share of students dropping out of STEM + Medicine students into Social Sciences and Humanities (also see Figure B.5). Dropout from Social Sciences and Humanities toward STEM is extremely rare at baseline and remains so throughout.

These results suggest that, in response to the reform, students preferred specializations which are associated with better job prospects and higher earnings potential, which is in line with the findings of Field (2009) and Rothstein and Rouse (2011) in the United States. ${ }^{19}$

Another way of interpreting our results is that increased enrollments in STEM + Medicine field of study could be a result of a strong outflow of students interested in Social Sciences and Humanities to the vocational track. Our supplementary analyses suggest that this mechanism accounts for approximately a third of the observed response. The other two thirds are attributable to Dutch high school students acting upon the knowledge that they will have to take on student loans in the future, and choosing subject fields with better earnings prospects to secure the repayment of these loans. In addition, supplementary analyses show sizable heterogeneity with respect to gender and income: female students respond much more than male students and medium-income students respond the most, sheltered low-income students do not respond (full details and discussion in Appendix Tables B.9 to B.11).

\subsubsection{Later Effects: Living Arrangements and Labor Supply in Higher Education}

Fourth, we show that freshmen students who entered higher education under the new regime of student finance regime became much more likely to live with their parents during their first year of post-secondary education (see Figure B.6). University students were most affected; the share of students living at home increased by 18

\footnotetext{
${ }^{19}$ Although it is technically feasible, an immediate change of the subject field in response to the reform announcement can prove challenging. This is because the subject field choices encompass students' subject preferences and knowledge accumulated over the first three years of secondary school. Accordingly, reorienting to a different subject field may require development of new skills and knowledge. In contrast, the choice of an education track pertains largely to the desired level of complexity within the preferred subject field. This makes it easier to respond to reform announcements in terms track choices compared to subject field choices.
} 
percentage points (from 53\% to $71 \%$ ) between years 2014 and 2015 . While the average commuting time to school increased almost twofold, it seems like students' choice of place where to study were unaffected. For students starting post-secondary education in the academic year 2015-2016, their living arrangement decisions appear to be one of the primary and immediate margins of responses to the new financing regime. In contrast, we show in a fifth supplementary analysis that students in their first year of post-secondary education do not seem to respond to the new student financing regime through their employment choices: we find no sharp discontinuity around the implementation of the reform (see Figure B.7). The choice of the study major was likely affected as well, however the presence of substantive anticipation effects obscures the relative importance of this channel.

Lastly, respondents to the Studenten Monitor also show sharp changes over the period we study. The Studenten Monitor is a longitudinal survey of a representative sample of roughly 20,000 higher education students, which has been collected since 2001 by the Dutch Ministry of Education. Yearly reports describe how students experience and perceive their student life across a variety of topics, including work, financial aid, and budgeting. From 2010 onward, yearly reports have indicated a larger share of first-year post-secondary students state that they prefer minimizing student debt, at the expense of working while studying over taking on more debt and working less while studying. This suggests that student debt is a topic for higher education students, which became increasingly salient throughout the announcement period. From 2015 onward, fewer freshmen seek student finance and employment. Among respondents who do not take up a student loan, the three main reasons for doing so remain stable over the time: 1) parents do not agree with student borrowing (mostly college-educated parents), 2) students have fundamental objections with borrowing (mostly lower-educated parents), and 3) students lack knowledge about borrowing conditions. From 2015 onward, respondents become more likely to answer that they receive enough parental monetary support and do not feel the need to take up a loan. 2015 freshmen who do, however, take up student loans, are more likely to have been advised by their parents to take-up a loan, and less likely to declare that they are not willing to work to finance their studies. ${ }^{20}$

\footnotetext{
${ }^{20}$ see e.g. Van den Broek and Mens (2017); van den Broek et al. (2017); Van den Broek et al. (2006); Van den Broek and Van de Wiel (2005); Van den Broek et al. (2007)
} 


\subsection{Limitations and Sensitivity of Findings}

Our study uses a cohort design, which is imposed by the nation-wide announcement and implementation of the student finance reform. All students were exposed to the shocks at once, leaving us with no obvious control group other than past cohorts. This means that the identifying variation comes from year-on-year changes in choices made by subsequent cohorts of high school students, conditional on their observable characteristics and underlying time trends. The implicit assumption is that the aggregate conditional changes of students' behavior observed during the reform period are attributable to the reform and not to other unobserved processes. This identification strategy is subject to two main types of identification threats: misspecified time trends, and omitted variables correlated with the timing of reform shocks.

\subsubsection{Alternative Specifications of Time Trends}

For our principal model of track choice, we assume that the underlying time trends are linear. This is motivated by the apparent linearity of time trends observed over the pre-announcement period (as shown in Figure 2.1). It is also supported by the aggregate time trends observed in comparable European countries - which we have collected using yearly enrollments in university-preparing education tracks in neighboring countries with comparable education systems. The regional average time trend is linear over the entire observation period (2005-2015), exhibiting no signs of trend break between years 2010 and 2011 (see Appendix Figure B.12 and Figure B.13).

In order to assess sensitivity to this assumption, we have estimated models with alternative time trend specification. First, a model specification with quadratic time trends yielded similar estimates of the reform effects as our principal specification (not presented in this chapter). Second, we have also implemented a more radical treatment of the time trend, assuming that the linear time trend observed over the pre-announcement period does not extend beyond the first announcement. This means that in the absence of the reform, the conditional track shares would remain at their 2009 levels. Table B.12 presents our main results estimated using this model. While this adjustment necessarily affects the magnitudes of the reform effects, the results remain largely consistent with our theoretical framework: enrollment in the academic track declines throughout the reform announcement period, and enrollment in the vocational track rises after the second announcement. Enrollments in the 
general track remains slightly above the 2009 baseline, which implies that the number of students moving from the academic to the general track is greater than the number of students moving from the general to the vocational track. While this is theoretically possible, realistic parameterizations of our theoretical model would predict the opposite pattern. This, together with the linearity of international trends reinforces our conviction that our preferred model specification presented in Section 2.6 is appropriate for our analysis.

\subsubsection{A Systematic Exploration of Potential Confounding Factors}

We further explore the possibilities of confounding factors in Appendix B.4. We analyze the dynamics of various omitted variables and look for trend breaks that coincide with reform announcements and/or implementation. We consider the following variables: 1) earnings of new graduates by education type, 2) employment rates of new graduates by education type, 3) minimum wage rates, 4) youth unemployment, in particular throughout the Great Recession, 5) high school graduation rates, 6) average time to graduation in post-secondary education, 7) costs of attending post-secondary education - tuition fees, other financial aid amounts, conditions, interest rates, 8) parental unemployment, 9) school capacity constraints, 10) other changes of the Dutch education system, 11) cross-country trends in track choice, and 12) cross-country trends in the take-up of STEM education. Our exploration of these factors did not reveal any trend breaks which could confound our findings.

\subsubsection{Measurement of Tracking Outcomes with Error}

One concern could be that the trend break we find reflect earlier trend breaks in tracking outcomes of children, and not a trend break due to policy announcements. This concern arises because most children are assigned to a track before grade 10 , such that by studying tracking outcomes in grade 10, we could be identifying changes that occurred in grades 7 to 9 in years prior to 2010. In an earlier robustness check, we have found consistent evidence of trend breaks in announcement years for children in earlier grades, indicating that the trend breaks in tracking outcomes that we found in Grade 10 are not an artefact of trend breaks affecting Grade 9 students one year earlier, nor trend breaks affecting Grade 8 students two years earlier. This evidence also indicates that our results, if anything, would underestimate the true effect of policy announcements on tracking outcomes of pupils. 


\subsection{Conclusions}

In 2015, the Dutch government implemented a major reform of its post-secondary student finance system. This reform replaced the universal study subsidy scheme for higher education students by low-interest loans, thus raising the costs of a 4-year bachelor degree by roughly 5,000 to $€ 14,000$. The reform was revealed in two announcements. In 2010, the government announced its general intention to scale down the student finance system, thus creating uncertainty that students would receive any financial aid in the future. In 2012, the exact contents, timing and groups of students affected by the reform were revealed, which lifted the uncertainty and reduced the probability of receiving the universal subsidy to zero for affected students. In particular, it was only revealed in 2012 that vocational students would remain unaffected by the reform, and that means-tested financial aid would be maintained.

In this study, we analyse the effect of the reform on anticipatory decision making of secondary school students in the Netherlands. Our primary outcome of interest is the choice of secondary school track, which is instrumental for students' subsequent educational and professional pathways. We show that students' track choices are clearly contingent on the prospective regime of student financing. In line with our theoretical framework, students who are informed about the reform become less likely to choose academic and general tracks, which prepare them for higher education, and more likely to choose the vocational education track. We show that these effects emerge already under policy uncertainty following the initial announcement, and grow larger when this uncertainty is resolved through the second announcement. The reform announcements also led to a short-lived surge of older students switching to lower education tracks, correcting tracking decisions made with past information about financial aid. We show that each of these effects are stronger among the groups of students who were more affected by the reform.

We also explore several key additional outcomes. We show that the reform raised the number of academic and general track students specializing in STEM and Medicine subjects. This indicates that students who are resolved to pursue higher education do anticipate the higher costs of their degrees and refocus their attention to fields with higher earnings prospects. Similar changes were observed when analysing older students' decisions to abandon STEM and Medicine subjects and focus on Social Sciences and Humanities instead. Importantly, we note that these behavioural responses are driven almost exclusively by female students who are relatively under- 
represented in STEM fields. We further show that first-year students enrolled in higher education programs respond the actual implementation of the reform through different living arrangements: students at university and university of applied sciences are much more likely to remain living with their parents and commute to their schools on a daily basis. By avoiding the costs of rental housing, they lower their expenditures and reduce the need for additional financing through loans. This is also reflected in the take-up of student loans which increases only by 20 percentage points (from a stable roughly 30\% between 2001 and 2014 to almost 50\% in 2015). We do not see substantive changes of students' or parental employment.

Our findings highlight that changes to the mode of student financing can have far-reaching consequences for students' educational decisions. The 2015 reform led to a substantial resorting of secondary school students, reducing the demand for academic education and increasing the demand for vocational education. In the proximate future, this resorting is likely to translate into large changes in the demand for post-secondary degrees and it is also likely to alter the characteristics of new labor market entrants. Methodologically, our key contributions are twofold. The first is to show that the Dutch secondary school students are aware of the incentives embedded in the higher education system, and they respond to them well-ahead of high-school graduation. These incentives are shown to be shaping students' interests and effort levels throughout secondary school, which suggests that early salience of financial aid information is crucial for informed decision making. Our results imply that students and their families should be informed about college financial aid as early as in the first year of secondary school.

The second contribution is to show that uncertainty about the receipt of financial aid is sufficient to dissuade many students from pursing pathways that lead to higher education. This uncertainty alone can widen the achievement gaps between advantaged and disadvantaged students, with these effects being disproportionately larger among female students. We note that some of these effects were mitigated by the second announcement. By increasing the means-tested subsidy for higher education students, Dutch policy makers managed to limit the impact of the reform on low-income students. However, the low-income students were still observed to respond to the reform, likely because they were still facing considerable uncertainty with respect to the receipt and the applicable award rate of this subsidy. Better understanding how uncertainty about financial aid affects educational choices is a crucial avenue for future research.

We conclude that many effects of the 2015 reform are not yet available for researchers 
to study. We are so far unaware whether the 2015 reform affected the effort levels of students enrolled in higher education, for example through changing the average time to graduation or the likelihood of dropout. The effects are likely to have broader and longer-run effects on the labor market and in the Dutch economy. The decrease in demand for academic education may lead to shortages in some high-skill sectors of the economy, whereas the increase in take-up of STEM subjects may boost other high-skill sectors and improve national $R \& D$ indicators. In addition, the increase in demand for vocational education may suppress other white-collar sectors, but it may address some vocational shortages on the Dutch labor market. These are all extremely important avenues for future research. 



\section{Chapter III:}

Lifting Aspirations for Better Educational

Outcomes 

While the first chapter of this dissertation shows that there could be aspirational poverty traps, and the second chapter indicates that disadvantaged students could be discouraged from pursuing higher education, Chapter 3 now turns to exploring whether, conversely, we could lift the aspirations of disadvantaged students and ultimately improve their educational outcomes. In Chapter 3, we take the example of a low-cost and easily-implementable policy: exposure to high-achieving classroom peers.

In Chapter 3, we use data from Taiwan, where middle schools are mandated to randomly assign Grade 7 pupils to classrooms for a duration of two academic years. This natural experiment thus allows us to estimate the impact of randomized exposure to higher v. lower-achieving classroom peers on pupils' academic performance.

We find that when randomly exposed to classrooms composed of one standard deviation higher-achieving peers, pupils experience on average a 5.4 percent of a standard deviation boost in standardized test score two years later, a boosting effect on test scores that has been found in previous literature. Importantly, however, exposure to high-achieving peers could have adverse side effect on other outcomes of students, such that this results is not sufficient to conclude that we could simply expose disadvantaged students to high-achieving peers to improve their outcomes. For example, more challenging classrooms could lead to discouragement, and mental health issues.

We further study the impact of random exposure to higher-achieving classrooms peers on a myriad of intermediate outcomes including pupils' aspirations. Recent research has shown that to understand whether exposure to higher-achieving peers can improve educational performance through raised aspirations, we must include the complete set of other potential intermediate factors. We thus consider an extensive list of the most plausible factors explaining student test scores: student effort, initiative in class, cheating in exams and truant behavior, mental health, aspirations 
and expectations to go to university, and the child's perception of their school environment, as well as parental money and time investments, parental warmth, discipline and harshness, and teacher engagement.

We show that exposure to high-achieving peers also affects some intermediate outcomes of students: students pay significantly less time on homework, and are more likely to aspire to go to university. We also find that the parents of children exposed to higher-achieving classroom peers spend more time with their child. Perhaps most importantly, while we find positive effect of high-achieving peers on test scores and on several intermediate outcomes which likely affect educational performance, we find that the boosting effect of peers on test scores is not explained by any of these intermediate effects.

Our analyses allow us to exclude a certain number of possible explanations for this puzzle. We first show that our puzzling findings are not explained by a failure of our key identification assumptions to hold: we perform several alternative tests that confirm that children are as good as randomly assign to classrooms. We also discuss to what extent correlated selection on unobservable characteristics could explain away the results, and propose alternative estimates confirming that our results are not driven by selection on unobservable characteristics. Our sensitivity analyses also lead us to discuss measurement error that could stem from our sampling design, or from test scores that are imperfectly capturing true cognitive ability. We show that our findings are robust to using alternative test score measures, and are also robust to corrections for incomplete classroom sampling. We also propose alternative p-values for our statistics using randomization inference corrections and multiple hypotheses testing adjustments. Our findings are robust to both.

Lastly, we also explore at length the extent to which our puzzling inability to explain our academic peer effects with students' and parental intermediate responses could be explained by heterogeneity in peer effects and their mediation. We first show that exposure to higher-achieving peers has a similar boosting effect on test scores for various types of students - male v. female, richer v. poorer, ethnic minority v. majority, ... We then show that exposure to higher-achieving peers also has a similar effect on intermediate responses for these various types of students. Thus, our puzzling findings are not explained by heterogeneity in the underlying way that peers improve test scores.

The first lesson of this study is that exposing pupils to higher-achieving classroom peers is a valid and easily-implementable policy to improve their aspirations to go to university. In addition, the findings also show that, in this setting, there seem 
to be no behavioral perverse effects associated with exposure to higher-achieving classroom peers - we find indeed that pupils exposed to higher-achieving peers are not more likely to cheat on exams or adopt truant or externalizing behaviors such as smoking, substance abuse or physical violence, and these pupils are also not more likely to suffer from mental health problems such as depression or anxiety.

A second interpretation of this study is that, while lifting aspirations seems like a promising way to improve educational outcomes of students, it is unlikely to dramatically reduce achievement gaps. Based on this study and this particular setting, our findings suggest that lifting pupils' aspirations does not seem to significantly translate into higher test scores, either because exposure to higher-achieving peers does not increase aspirations enough, or because aspirations do not translate enough into better educational outcomes. 



\section{Mechanisms of Exposure to High-Achieving Peers ${ }^{1}$}

\subsection{Introduction}

Despite an immense literature in economics documenting the importance of peers for academic achievement, there is still much we do not know about their mechanisms. This remains an important limitation in our understanding of the theoretical underpinnings of peer effects, and limits our scope for using class assignment policies as a tool to improve student achievement and educational outcomes (Carrell et al., 2013; Sacerdote, 2014; Ushchev and Zenou, 2020).

One key reason why it is difficult to make headway in understanding the mechanisms behind peer effects is that this exercise requires large amounts of data from several sources. If we think of educational achievement as the output of an education production function which comprises several simultaneous inputs from students, parents and teachers (e.g. Cunha and Heckman, 2007), it is also natural to think that peers can affect educational achievement through any of these inputs. Datasets that collect information on all, or even many, of these sources are rare. Because of this limitation, the prevailing approach in the empirical literature on academic peer effects is to focus on the effect of higher-achieving peers on the few available outcomes and discuss why these isolated responses may be mechanisms through which academic peer effects operate. Formal analyses of the share of peer effects we can explain are often futile with so few mechanisms to look at, leaving the key unanswered question: what drives academic peer effects?

\footnotetext{
${ }^{1}$ This study is joint work with Nicolás Salamanca at the Melbourne Institute. I thank Jan Feld, Ulf Zölitz, Ingo Isphording, David Figlio and four anonymous referees at the Quarterly Journal of Economics for helpful comments. I also thank the Survey Research Data Archive (SRDA) in Taiwan for providing us with data access, and especially Wan-wen $\mathrm{Su}$ for facilitating our analyses of the Taiwan Education Panel Survey.
} 
To answer this question, we need three key elements in the same setting. First, we need to establish their existence; that there is a causal effect of being exposed to higher-achieving peers on students' own academic achievement. Second, we need to observe many potential factors that affect academic achievement. And third, we need to determine whether better peers improve academic achievement by shifting those factors. Many studies have gained access to one or two of these key elements, yet to date there is no study that provides empirical evidence all three of them jointly. We fill this gap.

In this paper, we first show the existence of academic peer effects, as others have done in different settings (for excellent reviews, see Sacerdote $(2011,2014)$ ). We exploit a mandate to randomly assign students to classrooms within schools in our setting as a lynch-pin in our identification strategy, and develop a method to identify and use only schools that adhere to this mandate in our analyses. We find that a one standard deviation (1 SD) increase in the average test scores of classroom peers at baseline increases own test scores by 5.2 percent of a standard deviation two years later.

Using rich data on students, parents, teachers and schools, we then estimate the causal effect of higher-achieving peers on a large battery of student, parent, teacher and school educational inputs, which are all potential mechanisms of academic peer effects. Together, our measures explain 71 percent of test scores two years later which suggest we have a very comprehensive set of measures in our data. Higher-achieving academic peers decrease students' school effort and increase students' university aspirations and their expected ability to go to university. Higher-achieving peers also increase parents' time investments and strict parenting style. We do not find effects of high-achieving peers on students' initiative in class, truancy, exam cheating, or academic self-efficacy. We also find no effects on parental investments in private tutoring, on emotional support or harsh parenting, or on parental aspirations for their child to go to university. Finally, we also find no effects on students' perceptions of their school environment, or on their teachers' engagement with students, reports of how hard to manage is the classroom, and how tired they feel about teaching. Some of the effects we do find complement existing evidence in the peer effects literature (Feld and Zölitz (2017) on perceived quality of peer interactions; Bursztyn and Jensen (2015) and Bursztyn et al. (2019) on social pressure and effort provision). Yet most of our estimates explore unstudied mechanisms behind academic peer effects; in fact, no study before has been able to test as many candidate mechanisms as we do. 
Combining our estimates of high-achieving peers on score and on educational inputs, we then answer the question: How much of the academic peer effect can be explained by our measured mechanisms? To do this, we begin by estimating the returns of all our educational inputs on academic achievement using high-quality cumulative valueadded models (Todd and Wolpin, 2007; Fiorini and Keane, 2014). Our estimates show large returns to many of our explored inputs. We then use these returns to map the effects of higher-achieving peers on educational inputs to academic achievement using mediation analyses (Gelbach, 2016). Our estimates show that our battery of educational inputs mediate a negative share of our academic peer effect - which means that the effects of high-achieving peers on educational inputs make it harder, not easier, to explain academic peer effects. This negative mediation is largely driven by the combined negative effect of high-achieving peers on student effort and its positive value-added returns. Our other inputs explored have a virtually null contribution to mediation. This is a surprising and important new insight for our understanding of academic peer effects and should help guide future research effort understanding their mechanisms.

Finally, we perform an extensive set of sensitivity analyses for our results including: additional tests for conditional random assignment, alternative estimates with an exhaustive set of controls, calculations of the degree of correlated unobserved heterogeneity needed to explain away our findings, corrections for measurement error in student ability and for incomplete sampling of classrooms, inference corrections using randomization inference and multiple hypotheses testing adjustments, and an extensive exploration of heterogeneity in peer effects, and their mediation.

Our paper makes several contributions to better understand the complex nature of academic peer effects. This is the first paper to provide a thorough test of the many possible mechanisms underlying academic peer effects, testing 19 of them covering all key agents in educational production. In one study, we cover the vast majority of achievement peer effect mechanisms - hypothesized or tested - in this vast literature (see Table 3.1). Most previous studies test only a few potential mechanisms for academic peer effects but never more than three or four at a time, and never in a formal mediation analysis (though two other studies, Gong et al. (2019) and Zölitz and Feld (Forthcoming), use mediation analyses to investigate mechanisms behind the effect of classroom peer gender). This is an important limitation since the many inputs in the education production function imply equally many mechanisms for peer effects to work through, and the only way to know how well we can explain peer effects is to jointly test all these potential mechanisms. The fact that after our efforts we still do not know how academic peer effects work is a testament to 
their complexity. Our findings rule out a host of mechanisms hypothesized in this extensive literature. When combined with previous findings, our results point to two additional mechanisms (direct peer-to-peer learning and endogenous teaching practices) and one empirical phenomenon (widespread heterogeneity in value-added functions across schools) as the most promising avenues to explain the surprisingly little mediation of academic peer effects.

We also make two methodological contributions to the empirical literature on peer effects. First, we develop an algorithmic approach to conducting balancing tests and identifying non-compliant schools in quasi-experimental peer effect designs. This is particularly useful in settings with partial compliance to random assignment of students to classrooms and no reliable way to know where compliance occurs and where it does not. In such settings, researchers often try and account for systematic violations of random assignment by controlling for additional characteristics beyond balancing characteristics, which complicates the interpretation of peer effect estimates and weakens identification strategies. Our approach is a transparent alternative to improve the validity of quasi-experimental research designs based on conditional random assignment without relying on conditioning pre-treatment covariates to account for failed randomization. Second, we provide a simple algorithm for randomization inference that observes the data structure of students within schools and within classrooms. Maintaining the data structure and, in particular, rigorously respecting assigned classroom sizes is crucial for correctly calculating permutationbased t-randomization p-values (Young, 2019) and for producing permutation-based tests of random assignment, which are commonly used in the empirical peer effects literature. We can provide Stata code for these two procedures upon request.

\subsection{Peer Effects in Education}

Economists have been interested in peer effects for a long time, and have published over 100 articles in economic journals since 2009 on peer effects in education alone, 28 of them in top 5 journals. ${ }^{2}$ One reason for the widespread interest in peer effects is that they could "be harnessed to cost-effectively improve public [...] services" (BenYishay and Mobarak, 2019). In other words, the existence of peer effects implies a social multiplier effect. Inspired by this promise in peer effects, an immense empirical literature rose to provide evidence on their existence and size - notable in education but in other fields as well. After two decades of studies, the existence of 
peer effects in education is a well-established fact. ${ }^{3}$

Peer effects are notoriously difficult to identify for two main reasons (Manski, 1993a): self-selection into peer groups (i.e., that similar people sort into the same groups) and the reflection problem (i.e., that estimates capture both my effect on my peers and the effect of my peers on me). Self-selection introduces bias in peer effects estimates arising from omitted variables. Reflection ties together the effects of (endogenous) peer interactions with the effect of (exogenous) peer characteristics, complicating the interpretation of peer effect estimates.

Empirical studies typically solve the reflection problem by estimating the reducedform effect of pre-assignment peer characteristics on student outcomes. Many studies have in addition convincingly solved the issue of self-selection by exploiting quasiexperimental assignment of students to peer groups. Two types of identification strategies have mainly been used to that end. The first strategy leverages (conditional) random assignment to peer groups within an institution. Examples include roommate assignment in college (Sacerdote, 2001; Stinebrickner and Stinebrickner, 2001, 2006; Zimmerman, 2003; Foster, 2006; Brunello et al., 2010; Griffith and Rask, 2014; Jain and Kapoor, 2015; Garlick, 2018), classroom/section/dorm assignment within institutions (Lyle, 2007; Kang et al., 2007; Graham, 2008; Carrell et al., 2009; De Paola and Scoppa, 2010; Burke and Sass, 2013; Carrell et al., 2013; Brady et al., 2017; Feng and Li, 2016; Feld and Zölitz, 2017; Huntington-Klein and Rose, 2018; Garlick, 2018), and study group assignment within classroom (Lu and Anderson, 2015; Hong and Lee, 2017). The second identification strategy uses natural variation in cohort composition. Examples include cross-cohort variation within an institution (Hoxby, 2000; Figlio, 2007); natural shocks or policy-driven changes affecting peer group composition (Angrist and Lang, 2004; Gould et al., 2004; Imberman et al., 2012; Figlio and Özek, 2019); admission cutoffs for schools or classrooms (Pop-Eleches and Urquiola, 2013); and experimental assignment to peer groups (Whitmore, 2005; Duflo et al., 2011a). ${ }^{4}$

\footnotetext{
${ }^{3}$ For brevity, we focus on studies of peer effects on academic achievement, but many other studies also document peer effects in e.g. college dropout (Stinebrickner and Stinebrickner, 2001), cheating in school (Carrell et al., 2008), job search (Marmaros and Sacerdote, 2002), substance abuse (Argys and Rees, 2008; Kremer and Levy, 2008; Card and Giuliano, 2013), crime (Deming, 2011), technology adoption (Oster and Thornton, 2012), consumption (Moretti, 2011), financial decisions (Ahern et al., 2014; Bursztyn et al., 2014) and beliefs (Boisjoly et al., 2006).

${ }^{4}$ It should be clear by now that there are very many studies of peer effects in education - see Sacerdote $(2011,2014)$ for two excellent reviews. For studies using cross-cohort variation within an institution see also: Hanushek et al. (2003); McEwan (2003); Arcidiacono and Nicholson (2005); Hanushek et al. (2009); Lavy and Schlosser (2011); Lavy et al. (2012a,b); Kiss (2013); Diette
} 
The main findings of this literature are that i) academic peer effects are positive but generally small; ii) the size of academic peer effects depends non-linearly on students' own academic ability; and iii) academic peer effects vary in large and seemingly unpredictable ways across settings.

Recent empirical studies have argued that academic peer effects could be largely driven by three types of mechanisms: i) student effort (e.g., Kang et al., 2007; Brunello et al., 2010), ii) group dynamics (e.g., Lavy and Schlosser, 2011; Lavy et al., 2012a; Bursztyn and Jensen, 2015; Brady et al., 2017; Feld and Zölitz, 2017), and iii) teacher effort or school resources (e.g., Duflo et al., 2011a; Chetty et al., 2011; Hoekstra et al., 2018; Todd and Wolpin, 2018). Table 3.1 lists several of these studies, classifying them by the type of mechanisms they explore.

A separate literature, yet directly relevant for our study, emphasizes the importance of parents as drivers of their children's academic achievement. This literature models academic achievement through an education production function framework - that is, as an output produced from students', parents' and teachers' inputs and governed by well-defined production technologies (such as dynamic or technical complementarities). Recent studies in this literature show, for example, that the benefits of class size reductions are driven by changes in student effort and classroom disruption (Lazear, 2001; Finn et al., 2003), as well as by changes in teacher behavior (Sapelli and Illanes, 2016) and parental investments (Bonesrønning, 2004; Jacob and Lefgren, 2007; Datar and Mason, 2008; Fredriksson et al., 2016). Recent studies estimate structural models of education production functions that include school peers, parents and neighborhoods as inputs (e.g. Agostinelli, 2018; Agostinelli et al., 2020).

and Uwaifo Oyelere (2014); Kramarz et al. (2015); Gibbons and Telhaj (2016). For studies using natural- or policy-driven shocks see also: Hoekstra (2009); Clark (2010); Vardardottir (2013); Jackson (2013); Abdulkadiroğlu et al. (2014); Dobbie and Fryer Jr (2014); Hoekstra et al. (2018). 


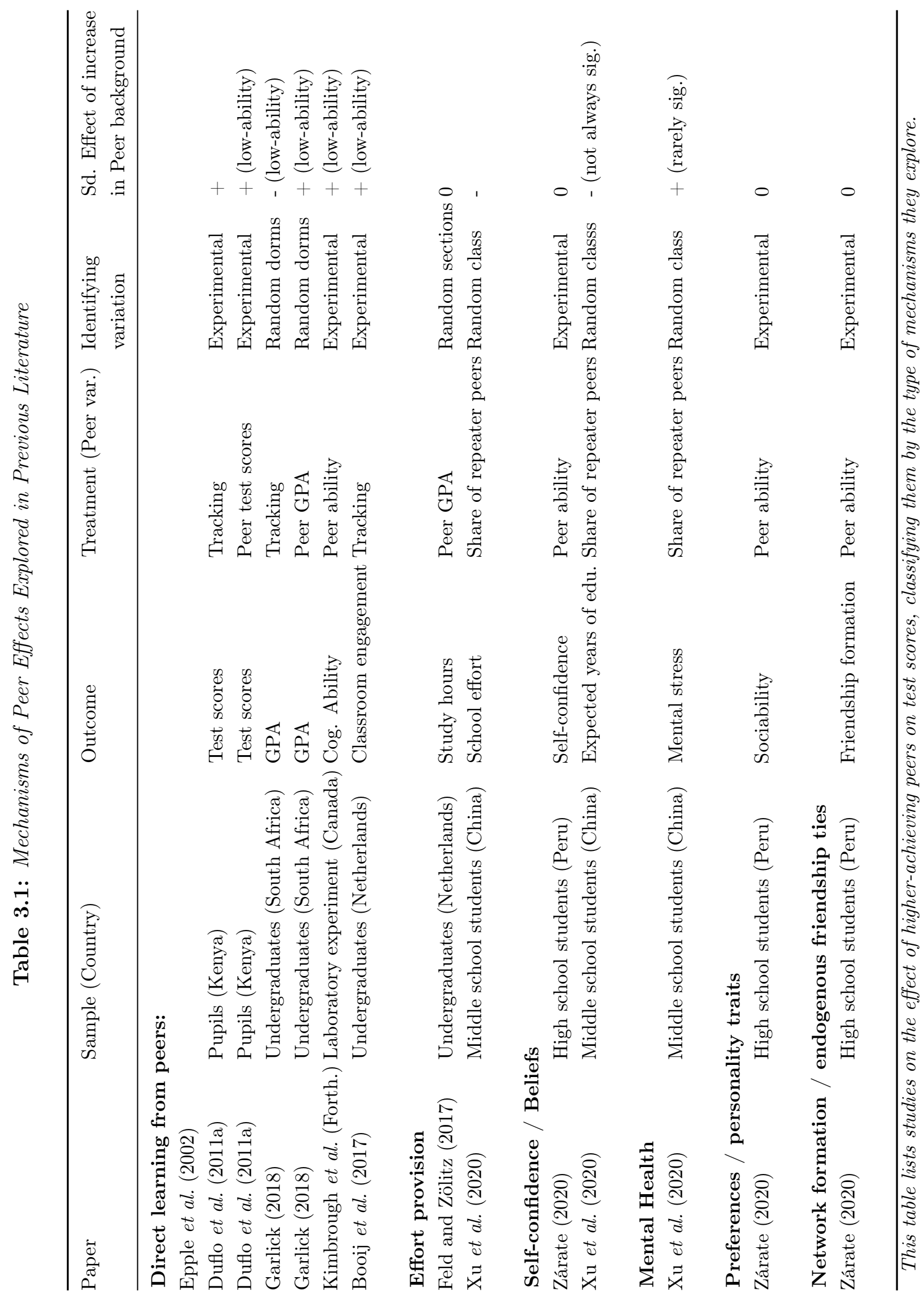




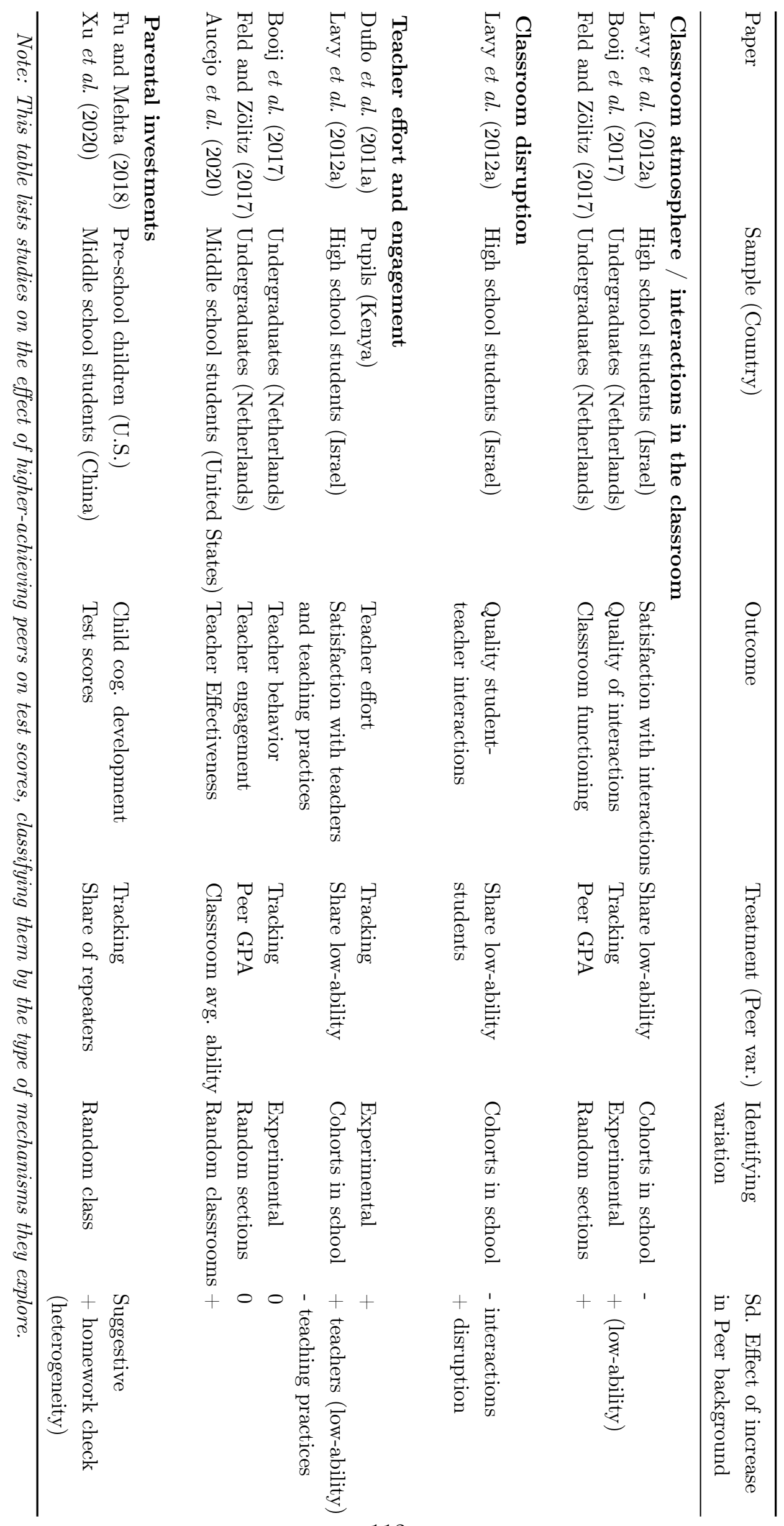


In this paper, we estimate the contribution of higher-achieving academic peers to students' test scores and to many educational inputs that may also contribute to improving test scores in their own right. Conceptually, our reduced-form models map the contribution of higher-achieving peers in a linearized version of education production functions. The downside of this approach is that we do not use economic structural information to improve identification. The upside is that our models are transparent in their identifying variation, econometrically tractable, and can easily be used to quantify the share of academic peer effects explained by educational inputs via standard mediation analyses. To take full benefit of this approach we exploit the pairing of Taiwan's policy of random classroom assignment within schools and the rich data in the Taiwan Educational Panel Survey, which we describe in detail in Section 3.3.

\subsection{Institutional Setting and Data}

\subsubsection{Education in Taiwan}

Figure 3.1 shows the basic organization of the Taiwanese educational system. Compulsory education in Taiwan starts at primary school, at 6 years old, and ends at the end of junior high school (middle school), around 15 years of age. In practice, however, 95 percent of students continue further onto either General or Vocational Senior High School or Junior College.

Since the democratization process in Taiwan started in the 1990s, junior high schools have been managed at the municipal level. Students can attend any school they chose but there is preferential school access based on catchment areas within each municipality. The educational curriculum is developed centrally by the Taiwanese Ministry of Education and has no subject specialization until only after junior high school. This unified curriculum is centered around sciences and mathematics and its adoption is often cited as the reason why Taiwanese pupils are consistently placed at the top on international educational rankings (e.g. 4th out of 72 countries in PISA 2015; Law (2004)).

Critical for our identification strategy, since the 1990s municipalities are also mandated by the government to ensure the random assignment of students and homeroom teachers to classrooms within schools. This requirement was formalized by the Implementation Guideline for Class Assignment of Junior High School Students, later 
Figure 3.1: The Education System in Taiwan

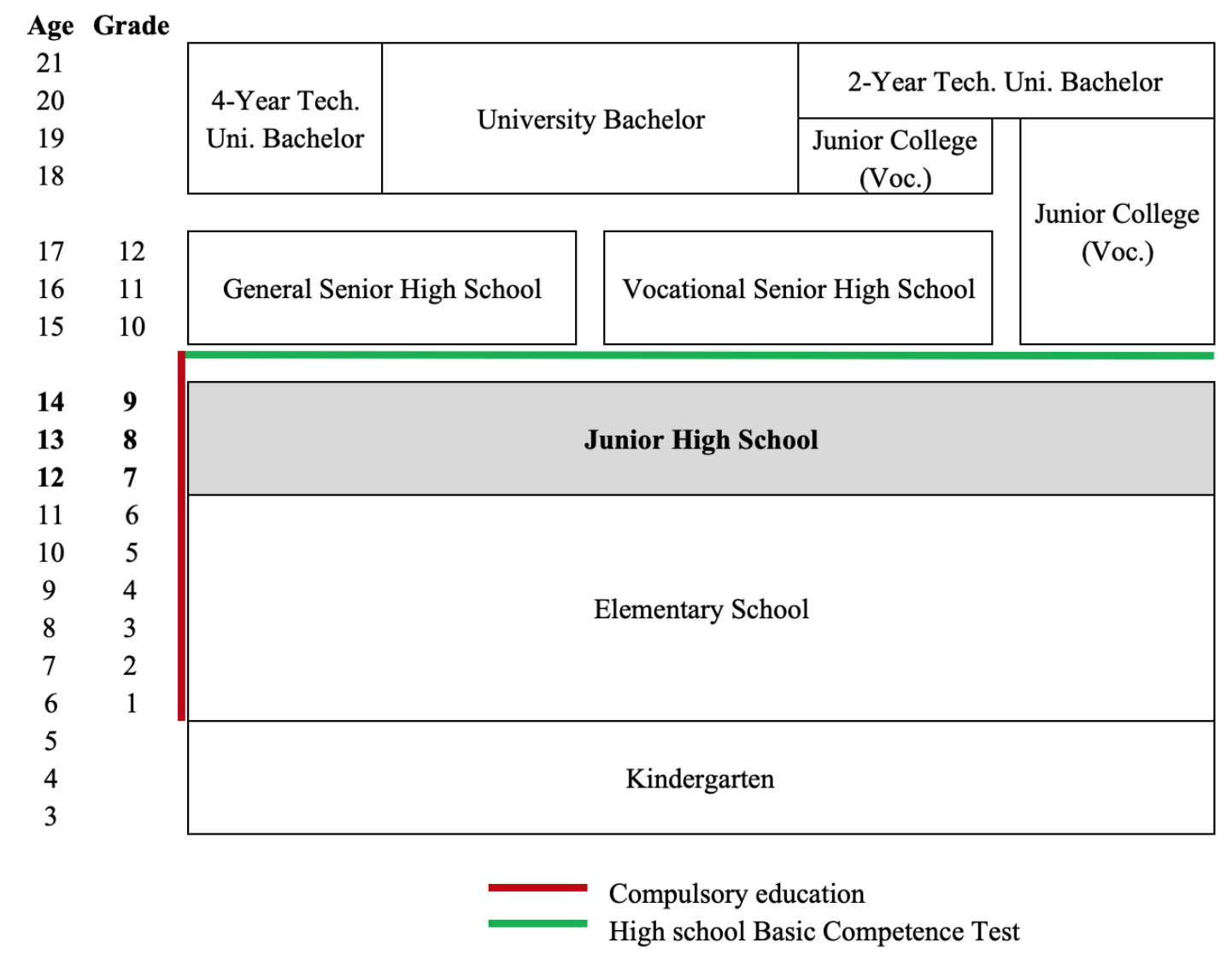

Authors' figure.

superseded by Article 12 of the Primary and Junior High School Act in $2004 .{ }^{5}$ Classroom assignment plays a persistent role in students' education since students typically remain with their assigned classroom and homeroom teacher (or Dao Shi) throughout all three years of junior high school.

Students take the National Basic Competence Test at the end of junior high school, which results play a key role for admissions to senior high schools and senior vocational schools. A good placement in these competitive schools, in turn, results in good placements in tertiary education programs, which have high returns in the labor market afterwards. Consequently, students spend time and effort preparing for these exams, and schools regularly organize practice exams and other forms of preparation. Parents are also engaged in their children's preparation, investing in extracurricular tutoring in mathematics, English and sciences largely through

\footnotetext{
${ }^{5}$ Additional details can be found at http://edu.law.moe.gov.tw/EngLawContent.aspx.
} 
cram schools - private extra-curricular institutions preparing for higher education entrance examinations - throughout junior high school or even earlier.

\subsubsection{The Taiwan Education Panel Survey}

We use data from the Taiwanese Education Panel Survey (TEPS), a project jointly funded by the Ministry of Education, the National Science Council, and the Academia Sinica. The TEPS is a nationally representative longitudinal survey of the education system in junior high school, senior high school, vocational senior high school, and junior college. The TEPS is a multiple respondent survey, collecting linked information on students, parents, teachers, and school administrators.

We focus on the junior high school sample of the TEPS because the timing of interviews allows us to use measures of student ability and educational inputs upon random assignment to classrooms in Grade 7. The TEPS junior high school sample includes information on more than 20,000 students, their parents, their teachers and their school administrators over two waves. The first wave was collected in early September 2001 at the beginning of students' first year of junior high school, right after their assignment to classrooms. The second wave was collected in 2003, at the beginning of the students' last year of junior high school. ${ }^{6}$

Paired with the mandate of random assignment to classrooms in schools, there are three other key features of TEPS that aid our study. First, its sampling framework allows us to observe a random sample of classmates in each junior high school classroom included in the survey. TEPS follows a stratified nested sampling procedure where first 338 randomly selected junior high schools were sampled (45 percent of all high schools in the country at the time), with different sampling strata for urban and rural areas, public and private schools, and senior high and vocational schools. In each of these schools an average of three classrooms of first-year students were then randomly sampled. In each of these classrooms, around 15 students were then randomly sampled. The mandated maximum class size at the time was 35 students per class, which implies that observed students in any classroom generally represent a random half of the classroom. ${ }^{7}$ This sampling framework is similar to

\footnotetext{
${ }^{6}$ In contrast, TEPS conducted the first round of interviews of students in general and vocational senior high school, and junior college in their second year, one year after assignment to classrooms, barring us from having baseline measures of ability and educational inputs for these samples.

${ }^{7}$ There are other minor sampling restrictions that are irrelevant for our empirical design, we refer the interested reader to TEPS technical reports.
} 
that of the National Longitudinal Study of Adolescent to Adult Health (Add Health), a panel study of a nationally representative sample of middle and high school pupils in the United States. Add Health is unique in collecting friendship ties and in observing multiple cohorts of students in each school, which makes it particularly appealing for peer effect and network research (e.g. Agostinelli, 2018; Elsner and Isphording, 2017; Card and Giuliano, 2013; Bifulco et al., 2011; Calvó-Armengol et al., 2009).

Second, and unlike Add Health, students in the TEPS take a standardized test in waves 1 and 2 called the Comprehensive Analytical Ability test. This test measures of students' cognitive ability and analytical reasoning, and it was specifically designed to capture gradual learning over time. There are 75 multiple-choice question in the test, covering general reasoning, mathematics, Chinese and English. These questions were taken from an extensive bank of questions which includes adapted questions from other international standardized tests, as well as questions provided by education and field experts in Taiwan. The Comprehensive Analytical Ability test scores, constructed as the sum of all correct answers, provide excellent measures of academic ability for students and their peers. ${ }^{8}$

Third, TEPS provides a wealth of questions measuring student behavior, attitudes and beliefs in and outside the school environment, parent-child interactions and parental investments, as well as detailed information on teachers and school administrators. Many of these measures have multiple raters, combining questions asked to students, parents, teachers and school administrators. We aggregate all these questions to construct an extensive battery of measures of student, teacher and parent inputs in students' educational production function. This large set of input measures allows us to comprehensively explore potential mechanisms behind academic peer effects.

Based on previous literature, we identified key inputs of students, parents and teachers in the education production function that are potential mediating factors of ability peer effects. For inputs with multiple potential measures, we first identify entire blocks of items in the questionnaires of all respondents - e.g. blocks of items related to study effort reported by students, parents and teachers. We then eliminate

\footnotetext{
${ }^{8}$ There is some evidence that female students may be disadvantaged compared to male students in multiple-choice measures of cognitive ability (see e.g. Willingham and Cole, 2013). This is not a concern for our study since cognitive ability is measured in a consistent manner throughout the TEPS, and our regression analyses always control for ability at baseline. Nevertheless, we discuss robustness of our results to measurement error in cognitive ability in Section 3.6.2
} 
very low correlates to maximize the informational content of each index and reduce noise. To do this, we compute Spearman correlations between all items under consideration, assess their Cronbach's alphas, and perform an exploratory factor analysis. Once we narrow down the list of items for a scale, we perform an additional confirmatory factor analysis to validate these items and ensure their item factor loadings have similar magnitudes. Finally, for each of these potential student, teacher and parent mediating factors and in each wave, we construct a summative scale that adds up the answers to each item in the scale. See Appendix C.1 for a detailed explanation and Appendix Table C.1 for summary statistics and factor loadings on all scale questions.

We measure student inputs through five scales of student school effort, initiative in class, mental health, truancy, and academic self-efficacy, and three additional dummies for whether students cheat in exams, aspire to go to university, and expect to be able to go to university. We measure parental inputs through four scales of money investments, time investments, parental strictness and parental support, and three additional dummies for whether parents have conflicts with their child, use harsh punishment, and aspire for their child to go to university. Lastly, we measure school and teacher inputs though two scales of student-perceived quality of the school environment and of teacher engagement, and two additional dummy variables for whether teachers reports that the classroom is hard to manage and whether they feel tired of teaching.

Table 3.1 shows a high-level summary of the academic ability and educational input measures we construct using the TEPS data. For each measure we list the number of items used and the number of unique values each measure takes. Wave 1 measures include many pre-assignment characteristics used in Section 3.4.2 to provide evidence of random assignment of students to classrooms within schools through a series of tests and methods we explain in the next section. 
Table 3.1: Description of Academic Achievement and Input Measures in TEPS

\begin{tabular}{|c|c|c|c|c|c|}
\hline \multirow[b]{2}{*}{ Measure } & \multirow[b]{2}{*}{ Description } & \multicolumn{2}{|c|}{ Wave 1} & \multicolumn{2}{|c|}{ Wave 2} \\
\hline & & $\begin{array}{l}\mathrm{Nb} \text { of } \\
\text { Items } \\
(1)\end{array}$ & $\begin{array}{c}\text { Distinct } \\
\text { Values } \\
(2)\end{array}$ & $\begin{array}{l}\text { Nb of } \\
\text { Items } \\
(3)\end{array}$ & $\begin{array}{c}\text { Distinct } \\
\text { Values } \\
(4)\end{array}$ \\
\hline \multicolumn{6}{|c|}{ Student inputs } \\
\hline Test scores & Comprehensive Analytical Ability std. test & 75 & 66 & 75 & 59 \\
\hline School effort & $\begin{array}{l}\text { Study effort, homework on time (in English, } \\
\text { Chinese \& math class) }\end{array}$ & 7 & 23 & 7 & 25 \\
\hline Initiative in class & $\begin{array}{l}\text { Initiative to ask and answer questions } \\
\text { (in English, Chinese \& math class) }\end{array}$ & 3 & 12 & 3 & 12 \\
\hline Cheating in exams & Student ever cheats in exams & 1 & 2 & 1 & 2 \\
\hline Mental health & $\begin{array}{l}\text { Feeling troubled, depressed, suicidal, nervous, } \\
\text { unfocused, pressured, irritated, isolated, guilty }\end{array}$ & 6 & 19 & 12 & 22 \\
\hline Truancy & $\begin{array}{l}\text { Skipping class, fighting, watching porn, drinking } \\
\text { alcohol, stealing, running away from home }\end{array}$ & 6 & 19 & 4 & 10 \\
\hline Academic self-efficacy & $\begin{array}{l}\text { Focus, diligence, conscientiousness, initiative, } \\
\text { eloquence, organization, cooperation, curiosity }\end{array}$ & 7 & 22 & 10 & 19 \\
\hline University aspirations & Student wants to go to university & 1 & 2 & 1 & 2 \\
\hline University expectations & Student expects to be able to go to university & 1 & 2 & 1 & 2 \\
\hline
\end{tabular}

\section{Parental investments}

Money investments

Time investments

Parent-child conflict

Parental strictness

Parental support

Harsh parenting University aspirations

$\begin{array}{lcccc}\text { Out-of-school tutoring for child: cost, intensity } & 2 & 10 & 3 & 10 \\ \text { Going to bookstores, cultural events with child } & 2 & 7 & 2 & 11 \\ \text { Student quarrels with father and mother } & 1 & 2 & 1 & 2 \\ \text { Father and mother use strict discipline with child } & 2 & 7 & 2 & 17 \\ \text { Father and mother discuss future, listen, } & 8 & 25 & 8 & 7 \\ \text { carefully, worry, give advice, accept as is } & & & & \\ \text { Parents use harsh punishment with student } & 1 & 2 & 1 & 2 \\ \text { Parents want student to go to university } & 1 & 2 & 1 & 2\end{array}$

School atmosphere and Teacher engagement

\begin{tabular}{|c|c|c|c|c|c|}
\hline School environment & $\begin{array}{l}\text { Student perception: school study ethos, campus } \\
\text { safety, school fairness, staff engagement }\end{array}$ & 5 & 16 & 5 & 16 \\
\hline Classroom management & Teacher assessment: classroom is hard to manage & 1 & 2 & 1 & 2 \\
\hline Teacher engagement & $\begin{array}{l}\text { Student perception: teacher knows names, } \\
\text { encourages students who work hard, uses several } \\
\text { different teaching materials, gives homework, } \\
\text { cares about students, reviews quest. after exams }\end{array}$ & 6 & 19 & 6 & 36 \\
\hline Teacher tired & Teacher is tired of teaching & 1 & 2 & 1 & 2 \\
\hline
\end{tabular}

Note: This table presents our summative scales, and their associated survey items in each wave of TEPS. Col. (1) and (3) report the number of items used in the construction of each summative scale resp. in Wave 1 and Wave 2, and Col. (2) and (4) indicates the number of distinct values summing each item. For example, the summative scale "School effort" in Wave combines answers to 7 items over 23 distinct values; thus, school effort ranges from 0 to 23. 


\subsection{Empirical Strategy}

\subsubsection{Testing for Random Assignment of Peer Groups}

Peer effect studies vary widely in their preferred test of random assignment to peers. In this section, we briefly review and discuss the type of tests used in the literature.

A growing number of peer effects studies have relied on experimental or quasiexperimental data in which students are randomly assigned to peer groups. This literature typically uses three types of test to show that data is consistent with (conditional) random assignment of students to groups. In the first method, researchers regress student $i$ 's pre-determined characteristics on the classroom leave-out mean - that is, the classroom mean after excluding student $i$ - of the key regressor of interest. This key regressor is usually a classroom leave-out mean of ability, gender or other pre-determined student behavior (see e.g. Carrell et al., 2013; Eble and $\mathrm{Hu}, 2019)$. A significant coefficient on the classroom leave-out mean indicates that students "treated" with peers differ in that pre-determined characteristic. Because this test mirrors balancing-of-covariates tests in the experimental literature, we refer to them as balancing tests.

In the second method, researchers regress student $i$ 's pre-determined characteristics on classroom leave-out mean of that same characteristic (e.g. Sacerdote, 2001). A positive coefficient on the characteristic classroom leave-out mean indicates that students are sorted into classrooms based on the characteristics tested; hence we call these sorting tests. Guryan et al. (2009) observe that empirically, even under random assignment, coefficients of sorting tests present a small negative bias; they show that this small, mechanical negative correlation between own and peer characteristics seems to disappear when controlling for school-level leave-out-mean of the characteristic. Jochmans (2020) shows that Guryan et al. (2009)'s empirical correction results in low power for detecting sorting. He further derives analytical expressions for this bias in within-school estimators and proposes a bias-corrected sorting test which solves the power issue of previous sorting tests.

In the third method, researchers run permutation-based (sorting or balancing) tests (e.g. Carrell et al., 2013; Lim and Meer, 2017). These tests go as follows. While keeping the core structure of the data (e.g., assignment to schools), researchers simulate what would happen under random assignment to treatment (e.g., to classrooms). Based on this new placebo assignment they then calculate key placebo 
statistics of interest - sometimes for sorting tests, sometimes for balancing tests, and sometimes for their main results. They repeat this process, say 10,000 times, and each time store their key placebo statistics. Finally, they calculate the proportion of times their placebo statistic has a more extreme value than their actual key statistic. They then calculate the proportion of times the coefficient of the placebo classroom leave-out mean is more extreme than their coefficient of the classroom leave-out mean as observed. This proportion of more extreme occurrences under placebo is a simulation-driven empirical p-value for a test of random assignment and can be judged by typical standards of statistical significance. These empirical p-values could be calculated for many statistics of interest, including for sorting and balancing tests but also for such tests at the school or even classroom level. When many of these empirical p-values are calculated, researchers can aggregate them into one overarching statistical test using goodness-of-fit tests for the distribution of p-values, which should be standard uniform under random assignment to treatment.

All three methods above are valid ways to produce evidence of quasi-random assignment, yet all methods also have their shortcomings. Neither method naturally corrects for multiple testing when researchers use many pre-determined characteristics in their tests. Using multiple hypotheses testing corrections (e.g. Benjamini and Hochberg, 1995; Romano and Wolf, 2005a,b) can, in turn, severely decrease test power. Another approach is to joint-test the significance of all pre-determined characteristics in predicting treatment but these joint tests have a tendency to over-reject, especially when using cluster-robust inference methods (Pei et al., 2019). Permutation tests have the additional problem of being relatively complex to program since researchers are required to keep most of the data structure identical (e.g., assignment to schools, number of classrooms in each school, class size) while still reassigning treatment at random, then correctly recalculate all treatment measures, and ensure that treatment variation is correctly accounted for in all estimates which is harder with discrete measures of pre-assignment characteristics like gender or race. In addition, goodness-of-fit tests used to aggregate many empirical p-values in permutation tests, such as the Kolmogorov-Smirnoff test, have known power issues.

Given the volume of peer effect studies out there, it is no surprise that in many of them there is evidence of some systematic assignment to peer groups (e.g. Krueger, 1999; Krueger and Whitmore, 2001; Whitmore, 2005; Dee, 2004; Ammermueller and Pischke, 2009; Balsa et al., 2018). When tests of random assignment reject the null that students are randomly assignment to peer groups, researchers have used three types of econometric strategies. 
A first approach is to adapt the econometric specification and adjust the interpretation of estimates accordingly (Krueger (1999), for example, estimates intent-to-treat effects rather than treatment effects), or to consider the size of the selection bias when interpreting results (e.g. Dee, 2004). This can be appropriate if the evidence on systematic assignment is weak, quantitatively small, and does not hint at further systematic assignment based on unobservable characteristics that affect student outcomes. The cost, however, is that estimates might be biased if any of these conditions fail.

A second approach is to remove treatment clusters where the data are consistent with some form of systematic assignment to treatment (e.g. Krueger, 1999; Whitmore, 2005; Chetty et al., 2011). This approach is valid if there are clear reasons to believe that random assignment applies to some known treatment clusters but not others, which usually requires intimate knowledge of the institutional background behind the data and the presence of markers of these known clusters. In complex institutional settings, removing data clusters suspected of systematic assignment to treatment quickly becomes unfeasible and can be very costly in terms of statistical power.

A third approach is to control for pre-assignment characteristics that reveal systematic assignment in the preferred specification, thus relying on mean independence of treatment conditional on these characteristics (e.g. Lavy and Schlosser, 2011; Gong et al., 2019). This approach is not costly in terms of power and does not require intimate knowledge of the institutional background, yet it assumes (often implicitly) that controlling for characteristics related to systematic assignment fully accounts for related unobserved characteristics that also determine assignment. Economist are often wary of this assumption. This third approach also comes with other shortcomings. In particular, it assumes that a single parameter function (e.g., linear) in the pre-assignment characteristics is sufficient to account for systematic assignment. This assumption is unlikely to hold if there are several such characteristics or several treatment clusters that differ in their drivers of systematic assignment. Parametrically relaxing this assumption can quickly become costly in terms of power. Perhaps more importantly, controlling for pre-assignment characteristics changes the interpretation of the peer effect estimates, often making them less immediately available for designing better peer group assignment policies. For example, unbiased peer effect estimates that control for parental education can only be used to predict outcomes of reassignment policies that hold parental education constant - a difficult exercise unless student reassignment to classrooms is done explicitly on parental education, which is unlikely to happen in practice. 
In sum, there are several ways to test for random assignment of students to peer groups and several ways to deal with an eventual rejection of random assignment. None of the tests are perfect, nor are the solutions. In the next section, we show our main test for random assignment in the TEPS and refer the interested reader to Section 3.6.1 for the additional tests we run. The case of the TEPS also presents an interesting challenge that combines i) a national mandate of random assignment of students to classrooms within schools, ii) incentives for parents and schools to violate this mandate if they believe that higher-achieving peers affect student outcomes, and iii) unusually rich pre-assignment data to test the outcome of these two clashing institutional features.

Perhaps more importantly, none of the existing tests are designed to identify specific treatment clusters where random assignment is unlikely to hold. In Section 3.4.2 we propose such procedure: a new data-driven method for finding subsamples where quasi-random assignment is credible, which is particularly useful in complex institutional settings such as ours.

\subsubsection{Random Assignment to Classrooms in TEPS}

Our identification strategy exploits random assignment of students to classrooms. If random assignment holds, we expect our treatment of interest, classroom leaveout-mean of peer ability, to be as good as randomly assigned to students. Random assignment to treatment is the main identification assumption under which our coefficient estimate yields a causal estimate of the effect of peer ability on subsequent outcomes. Therefore, we first show that our data are generally consistent with random assignment to classrooms, and then show that our treatment is as good as randomly assigned to students.

To show that the data are consistent with random assignment to classrooms within schools, we run sorting tests in the complete TEPS data on standardized test scores and 17 pre-assignment characteristics. We start from the complete sample, to prevent missing values to lead to over-rejecting sorting tests of random assignment. In this complete data, we find evidence of sorting by student ability and by several other student characteristics. We take this as evidence of non-perfect compliance with the mandate of random assignment of students to classrooms within schools across the entire TEPS data (see Appendix Table C.18).

There are many reasons why, in defiance of the national mandate of random assignment, we could find evidence of systematic assignment of students to classrooms. 
These can range from school principals occasionally catering to some parents' preferences for their child to be assigned to some classrooms, to institutionally allowed "talent" classrooms that pool high-ability student together, to a more concerning blatant disregard for the national mandate across schools. We develop a data-driven procedure that helps us determine the reason behind this seeming violation of random assignment in the data, and identify a sample where random assignment likely holds. We describe the key features of this procedure below, and refer the interested reader to a more complete description in C.3.

\section{The Fishing Algorithm}

Since the law in Taiwan has an explicit mandate of random assignment of students to classrooms, we suspect that rejecting the null of sorting tests is most likely driven by few schools that systematically sort students. Unfortunately, our data does not allow us to infer directly which are these schools to exclude them from our analysis.

We therefore designed a sample trimming method, which combines randomization inference, clearly pre-defined selection rules and latent-class modeling. Our Fishing Algorithm is a data-driven approach to identify and exclude the few schools that show evidence inconsistent with conditional random assignment. Since the norm should be random classroom assignment at the school level and since we are interested in ability peer effects, we focus on trimming schools that systematically sort students of similar academic ability into classrooms. This allows to exclude entire treatment clusters (schools) rather than within-cluster treatment cells (e.g., classrooms) which might leave non-random treatment in the remaining cells (i.e., because they are the complement of non-random treated cells). Our method, however, can be easily adapted to trim schools that sort on any observed characteristic in the data, and even on multiple characteristics at once. ${ }^{9}$

\footnotetext{
${ }^{9}$ Our Fishing Algorithm is also not restricted to finding sorter schools; it can be used to find sorter classes (i.e., classes where students are likely to be sorted based on ability). More generally, it can be adapted to find violations of balancing in any setting with cluster treatment assignment, such as treated villages within countries, or families within neighborhoods. Note also that, in general, our Fishing Algorithm is not equivalent to controlling for observable characteristics to achieve conditional balancing. Our approach combines knowledge of the intended level of treatment assignment (schools) and the nature of the treatment (peers) to non-parametrically identify treatment clusters that likely defy random assignment. Once non-random assignment is detected, we remove entire treatment clusters rather than trying to keep them and account for the non-randomness via controls. Only very stringent selection on observable procedures should be able to capture endogeneity as we do, and even then these would have to apply flexible control functions at the cost of many degrees of freedom and interpretability of estimates.
} 
The key five steps of the Fishing Algorithm are the following. First, we construct for each school a measure of strength of sorting, indicating how strongly the school sorts students of similar ability into the same classrooms. This measure is akin to a Herfindahl-Hirschman index of ability concentration in classrooms within each school, with larger values indicating stronger ability sorting in classrooms in the school. We call this measure $H_{s}$. Second, for each school we use several permutations of random assignment of students to classrooms within school without replacement and construct, for each simulated classroom assignment, its corresponding simulated index $H_{s}^{\text {random }}$. This procedure recovers the distribution of ability concentration in classrooms for each school under the null of random assignment. Third, for each school we compute the share of permutations for which the observed index $H_{s}$ in the data was larger than the simulated index $H_{s}^{\text {random }}$ under the null of random assignment, and call this share $S_{s}$. Under perfect compliance with random assignment, we expect the distribution of $S$ over schools to be uniform over the [0,1] interval; if random assignment violated in some schools, we expect more values of $S_{s}$ close to 1. Fourth, we estimate the latent probability that each school is a sorter (i.e., a school that sorts students into classrooms more strongly than chance would allow). We do this using latent class modelling - an atheoretical data-driven partitioning method that finds observations (e.g., school shares $S_{s}$ ) that are likely to be generated by the same stochastic process (e.g., ability-sorted classroom assignment). Using school-level data, we fit a finite mixture model where the outcome is $S_{s}$, the regressors are constants for each latent class, and we include school-level variables that could help identify sorter schools (such as the share of parents who report pushing to get their children assigned to a better classroom). One or more of the latent classes in this model correspond to schools with improbably high $S_{s}$ - the sorter schools - and the model itself produces school-level posterior probabilities of each school belonging to this latent sorter class. In the fifth and final step, we flag sorter schools based on whether their posterior probability of belonging to the latent sorter class is larger than the combined probabilities of belonging to all other classes. As mentioned above, a more complete description of this Fishing Algorithm can be found in C.3, and we provide validation of this procedure using simulated data in C.4. ${ }^{10}$

\footnotetext{
${ }^{10}$ The question on parental pushiness belongs to a block of questions regarding parental investments in their child's education, including whether parents have or plan on sending their child to study abroad, have changed jobs to be more available for their child, or have relocated to a better educational district to aid their child's education. We focus on pushy parents because it is the only one of these items that relates directly to classroom assignment and thus our treatment of interest, which could affect our within-school estimates.
} 
Most schools in the TEPS data show evidence consistent with random assignment, whereas some schools present obvious evidence of sorting (Appendix Figure C.1). As illustrated in Figure 3.2, our Fishing Algorithm identifies 106 out of the 333 schools in TEPS as sorter schools, which we exclude from our estimation sample. This leaves us with a trimmed sample of 13,685 students in these schools, allocated to 853 classrooms (68 percent of the TEPS data). Our trimmed sample is very similar to the overall TEPS data in terms of all key student and parent characteristics in wave 1, and is also similar to our final estimation sample of 11,029 observations with complete information on student and peer test scores and educational inputs (Appendix Table C.20).

Figure 3.2: Schools Identified as Defying Random Assignment Using the Fishing Algorithm



This figure shows the school-level distribution of our measure for whether schools sort students into classrooms more strongly than chance would allow, given the school size, number and classroom size and student composition. The probability of being a sorter school is the posterior probability of being in a latent class classified as sorters by us and calculated based on a finite mixture model of school sorting using several school averages of parental characteristics as class predictors. See C.3 for details.

An important concern in applying our Fishing Algorithm is over-trimming; that is, to remove schools that by chance look like sorters but are not. Our algorithm will unavoidably result in some schools being over-trimmed, and these schools would have contributed useful variation to identify peer effects. With severe over-trimming, peer effects could be less precisely estimated at best, and biased at worst (upwards 
if e.g., peer effects are strongly driven by positive effects of higher-achieving peers on high-achieving students). However, over-trimming is also easily diagnosed in our algorithm; it is revealed by negative and significant post-trimming sorting tstatistics. If negative post-trimming sorting t-statistics occurs, researchers should make efforts to improve the performance of the Fishing Algorithm (by e.g., finding better predictors of sorter schools or exploring different latent lass structures or models). If no improvement can be made, it is important to highlight the overtrimming brought on by the algorithm and cautiously interpret findings accordingly. Fortunately, in our application of the Fishing Algorithm to TEPS we find virtually no evidence of over-trimming.

\section{Sorting and Balancing Tests in our Trimmed Sample}

Table 3.2 presents the results of sorting and balancing tests on the trimmed sample, once we exclude the schools likely to be non-compliant with the mandate of random assignment. ${ }^{11}$ Columns (2) and (3) show sorting tests t-statistics, to be compared to standard normal critical values, whereas columns (4) and (5) show coefficients and standard errors of balancing regressions of pre-assignment characteristics on peer ability.

The main endogeneity concern in our estimates is ability sorting of students; that is, that high-ability students are assigned together in the same classroom. This type of sorting is concerning because, if ability is dynamically self-productive as in e.g., Cunha and Heckman (2007), it would bias peer effect estimates upwards. The first row of Table 3.2 shows that this sorting is not a concern in our trimmed sample.

Another common endogeneity concern is whether students are sorted in productive characteristics other than ability, say parental income. This kind of sorting is tested in the second and third columns, second row and below, of Table 3.2. Sorting on parental income can introduce bias in peer effects estimates if these characteristics are related to student achievement. Note, however, that if income sorting were related to students' achievement at baseline, this sorting would have already been reflected in the baseline achievement sorting. This still leaves the possibility that

\footnotetext{
${ }^{11}$ For this discussion, it is useful to keep in mind the omitted variable bias formula for our peer effect estimator $\beta$ : $\mathbb{E}(\beta \mid X)-\beta=\gamma \rho$, where $\gamma$ is the conditional effect of any omitted factor on student outcomes and $\rho$ is proportional to the correlation between the omitted factor and our peer achievement leave-out-mean. Evaluating all endogeneity concerns against this formula is an enlightening way to map econometric endogeneity concerns to economic principles.
} 
parental income has not been productive for student achievement at baseline but might become productive afterwards. If that is the case, income sorting at baseline can still bias peer effects upwards over and above achievement sorting.

Table 3.2: Balancing and Sorting Tests on the TEPS Trimmed Sample

\begin{tabular}{|c|c|c|c|c|c|}
\hline \multirow{3}{*}{ Treatment Variable: } & \multirow[b]{3}{*}{$\begin{array}{c}\text { Students } \\
\text { (1) }\end{array}$} & \multirow{2}{*}{\multicolumn{2}{|c|}{$\begin{array}{c}\text { Sorting tests (t-statistic) } \\
\text { Peer outcome } \\
\text { leave-out mean }\end{array}$}} & \multirow{2}{*}{\multicolumn{2}{|c|}{$\begin{array}{c}\text { Balancing tests } \\
\text { Peer ability } \\
\text { leave-out-mean [std] }\end{array}$}} \\
\hline & & & & & \\
\hline & & $\begin{array}{c}\text { Guryan et al. } \\
(2009) \\
(2)\end{array}$ & $\begin{array}{l}\text { Jochmans } \\
(2020) \\
(3)\end{array}$ & $\begin{array}{l}\text { Coef. } \\
(4)\end{array}$ & $\begin{array}{l}\text { Std. err. } \\
\text { (5) }\end{array}$ \\
\hline \multicolumn{6}{|l|}{ Pre-assignment characteristics: } \\
\hline Student test scores [std] & 13,685 & -0.2 & 0.1 & & \\
\hline Female student & 13,685 & 2.1 & -0.2 & 0.008 & $(0.011)$ \\
\hline Student born before 1989 & 13,611 & -0.8 & 0.6 & -0.005 & $(0.010)$ \\
\hline Household income $>$ NT $\$ 100 \mathrm{k} / \mathrm{mo}$. & 13,454 & -0.7 & -0.3 & $-0.019 * * *$ & $(0.007)$ \\
\hline College-educated parent(s) & 13,084 & -0.8 & 0.8 & 0.001 & $(0.009)$ \\
\hline Parent(s) work in government & 13,023 & 1.4 & 0.0 & 0.010 & $(0.007)$ \\
\hline Ethnic minority parent(s) & 13,081 & 2.2 & 1.4 & -0.004 & $(0.009)$ \\
\hline \multicolumn{6}{|l|}{ Since primary school: } \\
\hline Student always prioritized studies & 13,593 & -1.7 & 0.8 & -0.010 & $(0.009)$ \\
\hline Student always reviews lessons & 13,583 & -0.2 & 1.7 & 0.003 & $(0.008)$ \\
\hline Student likes new things & 13,554 & 1.5 & 2.4 & -0.001 & $(0.011)$ \\
\hline \multicolumn{6}{|l|}{ During primary school: } \\
\hline Student was truant & 13,489 & 1.6 & -0.7 & 0.000 & $(0.011)$ \\
\hline Student had mental health issues & 13,486 & -0.7 & 0.2 & -0.004 & $(0.010)$ \\
\hline Student quarreled with parents & 13,502 & -1.5 & -1.2 & -0.001 & $(0.009)$ \\
\hline \multicolumn{6}{|l|}{ Before junior high school: } \\
\hline Had private tutoring & 13,525 & 0.3 & 1.4 & 0.004 & $(0.012)$ \\
\hline Family help with homework & 13,013 & 1.2 & 0.8 & $-0.020 * *$ & $(0.008)$ \\
\hline Student in gifted academic class & 13,554 & -1.2 & 1.8 & 0.013 & $(0.008)$ \\
\hline Student in arts gifted class & 13,554 & 2.2 & 2.9 & -0.013 & $(0.015)$ \\
\hline $\begin{array}{l}\text { Parents made efforts to place child } \\
\text { in better class }\end{array}$ & 13,508 & 2.2 & 3.2 & $0.035 * * *$ & k $(0.010)$ \\
\hline
\end{tabular}

Note: This table presents the results of balancing and sorting tests in our trimmed sample of 232 schools and 850 classrooms. All estimators include school fixed effects. The reference distribution for the Guryan et al. (2009) and the Jochmans (2020) sorting statistics is the standard normal. The last column reports cluster-robust standard errors at the classroom level. ***, ** and * mark estimates statistically different from zero at the 90, 95 and 99 percent confidence level.

Table 3.2 shows that there is no evidence of sorting on other characteristics in our trimmed sample, especially when using the Jochmans (2020) state-of-the-art test. There is some evidence of sorting on intellectual curiosity and, perhaps more importantly, sorting for students enrolled in gifted arts classrooms and students 
whose parents report making efforts to get them assigned to particular classrooms. Several institutional settings, including TEPS, could allow for this type of sorting to occur over and above achievement sorting.

For student sorting on other characteristics to introduce bias in our peer effect estimates, however, a second necessary condition is for the student characteristic to be related to our peer achievement leave-out-mean measure. The last two columns of Table 3.2 show these tests. In our trimmed sample, the only potentially concerning characteristic which i) could affect student achievement over and above baseline achievement, ii) students are sorted on at baseline, and iii) is also related to peer achievement at baseline is whether parents made efforts to get their child assigned to a particular classroom. Of all the other characteristics that we test, only family income and family engagement with homework before baseline are related to peer achievement, and they are negatively related at that. This last finding rather suggests a potential slight over-trimming in our Fishing Algorithm (since pre-trimming these relationships were, if anything, positive (Appendix Table C.18). Regardless, in our main specifications we include the corresponding controls for household income, family engagement with homework, gifted art classroom assignment, and parents' pushiness to get child assigned to a particular classroom, which we jointly refer to as balancing controls. These balancing controls are not crucial for our empirical design, nor do they affect any of our main results. ${ }^{12}$

Overall, our algorithm is an effective way to identify schools that systematically assign student to classrooms in our data. In the schools identified by the algorithm as balanced we find no substantive evidence of systematic assignment, and we will keep this trimmed sample as our estimation sample throughout our main analyses. In Section 3.6 we also show the results of a battery of additional sorting tests, discuss in detail other ways to identify our estimates, explore the issues of sample selectivity, and compare our trimmed sample with the initial TEPS sample.

\footnotetext{
${ }^{12}$ Also, note that due to the power in our data, we detect small differences in balancing tests that would have likely gone unnoticed in other designs. Our ex-post Minimum Detectable Effects (MDEs) for our balancing tests are as small as 2.2 percentage points in the chance of being female, and less than 1 percentage point in the likelihood of having a migrant background. For comparison, the MDEs of balancing tests are 17 percent of a standard deviation in math test scores in the STAR data (Dee, 2004), and 25 percentage points for being female and 10 percentage points for migrant in the Add Health data (Bifulco et al., 2014).
} 


\subsection{Main Results}

\subsubsection{Academic Peer Effects on Test Scores}

Now that we have established a sample where conditional random assignment of students to classrooms holds, we go on to establish the existence of academic peer effects.

Figure 3.3: The Effect of Higher-Achieving Peers Test Scores on Students' own Test Scores in Wave 2

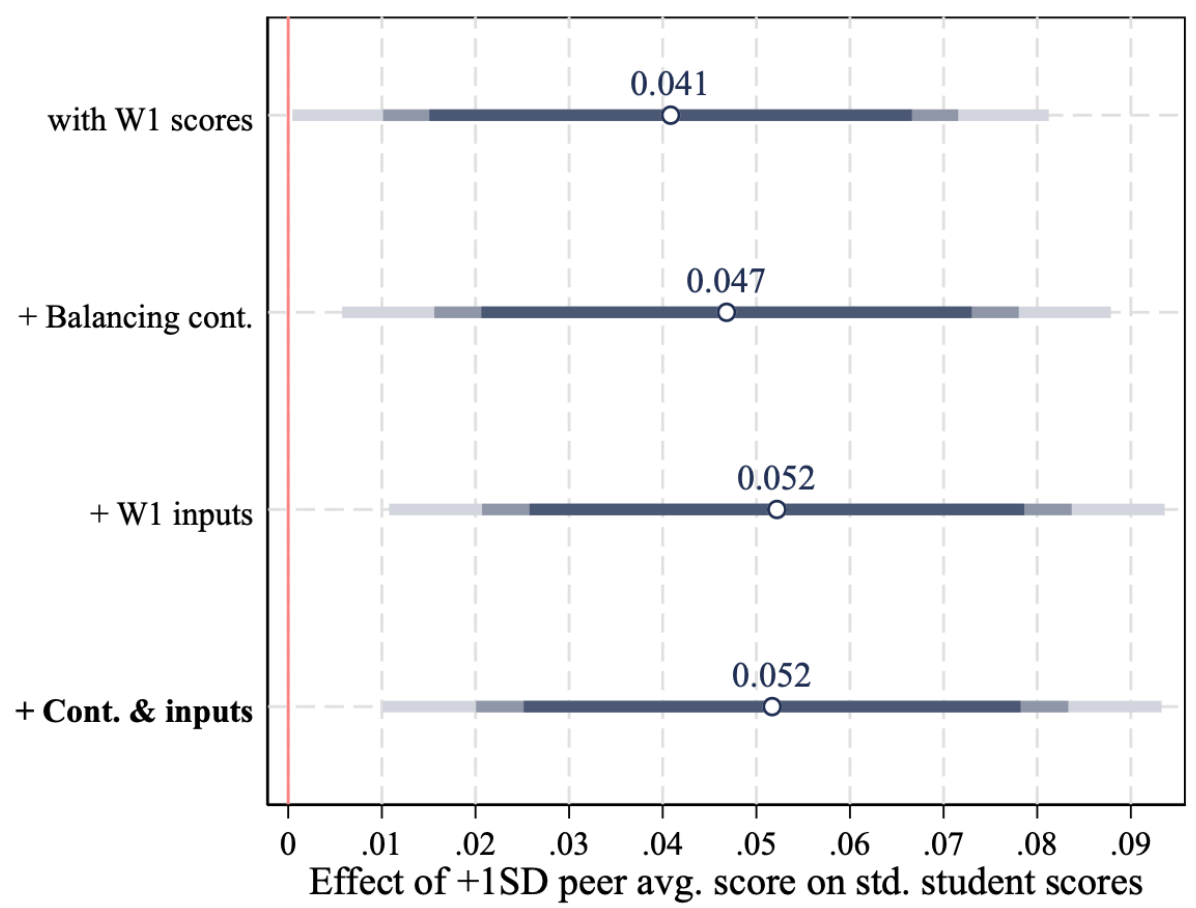

This figure reports estimates of regressing standardized student test scores in wave 2 on standardized average peer test scores in wave 1 in our sample containing 232 schools, 850 classes, and up to 11,029 students. Rows present results of models with different sets of control variables. The Baseline model includes wave 1 student test scores and school fixed effects. Balancing controls include household income, family engagement with homework, gifted art class assignment, and parents' efforts to get child assigned to a particular classroom. W1 inputs include standardized scales of student inputs (school effort, initiative in class, truancy, academic self-efficacy, and mental health), parent inputs (investment in private tutoring, time investments, parental strictness and parental support), school and teacher inputs (school environment and teacher engagement). Horizontal bars show the 99\%, 95\% and 90\% confidence intervals for each estimate, based on standard errors clustered at the classroom level. Estimates in this figure are also shown in Appendix Table C.2.

In its most basic form, we test for the existence of academic peer effects in our

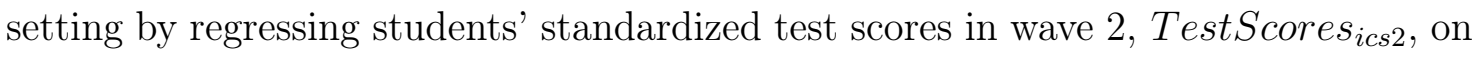


the standardized classroom leave-out mean of test scores in wave $1, \overline{\text { TestScores }}_{i c s 1}^{-i}$, our measure of average peer test scores. To this simplest specification we add school fixed effects and students' own test scores in wave 1 . We do not include homeroom teacher fixed effects since these teachers are also randomly assigned to classrooms so they cannot confound our peer effect estimates (see Section 3.3.1 and Chang et al. (2020)). Moreover we do not observe the same homeroom teacher across multiple classrooms so our estimates would not be econometrically identified in a teacher fixed effect model. We do consider specifications with and without the additional balancing controls (household income, family engagement with homework, gifted art classroom assignment, and parents' pushiness to get child assigned to a particular classroom) and standardized scales of student inputs (school effort, initiative in class, truancy, academic self-efficacy, and mental health), parent inputs (investment in private tutoring, time investments, parental strictness and parental support), school and teacher inputs (school environment and teacher engagement). We do this to assess the extent to which these covariates could capture omitted variable bias in our peer effect estimates. We cluster standard errors at the classroom level.

Figure 3.3 shows strong positive peer effects in our setting. It further shows that including balancing controls or wave 1 inputs does not qualitatively change our estimates, though it does slightly increase precision. This estimate stability is a reassuring result which provides strong evidence of no omitted variable bias in our estimates, especially given the wide range of controls included in our educational input measures.

Our preferred specification is on the last row of Figure 3.3, highlighted in bold. This specification controls for school fixed effects and student wave 1 test scores, as well as all wave 1 educational inputs and our four balancing covariates. It therefore identifies academic peer effects within the Todd and Wolpin (2003) cumulative value-added specifications; holding constant past outputs and educational inputs. This will prove important in the following sections. Our preferred estimates can be re-expressed as:

TestScores $_{i c s 2}=\underset{(0.017)}{0.054} \overline{\text { TestScores }}_{i c s 1}^{-i}+\underset{(0.009)}{0.562}$ TestScores $_{i c s 1}+\hat{\theta}^{\prime}$ Control $_{i c s 1}+\hat{\mu}_{s}$

where Controls $s_{i c s 1}$ includes balancing controls and wave 1 educational inputs.

These estimates imply that having one standard deviation higher average peer test 
scores in wave 1 increase own test scores by 5.2 percent of a standard deviation in wave 2. Comparing effect sizes in this literature is quite difficult; differences in standardized effect sizes across studies could capture true differences in responses to peer ability but could also reflect differences in standard deviations in peer achievement and student outcomes across settings. Assuming these standard deviations are comparable across studies, our peer effects are also similar (e.g. Imberman et al., 2012; Brunello et al., 2010; Booij et al., 2017). Compared to studies where students are randomly assigned to peer groups, our estimates are around the median of estimate. Yet our estimated effect measures the impact of two years' worth of exposure to classroom peers, which represents a strong dose compare to most comparable studies, thus our effect could also be seen as relatively small. ${ }^{13}$

To give this number more perspective, our estimated effect of a 1SD increase in average peer scores is about a tenth of the estimated effect of a 1SD increase in students' own lagged test scores. Our peer effect estimate is about half the marginal effect of having at least one college-educated parent, and about a sixth of the unconditional test score gap between children of two-parent households and single-parent households.

Another way of sizing the impact of higher-achieving peers is through the lens of socioeconomic inequality. Due largely to school sorting, the peers of poor students (with household monthly incomes under NT $\$ 20,000$, corresponding to the poorest 10 percent in the sample) have 68 percent of a standard deviation lower scores than the peers of rich students (with household monthly incomes over NT $\$ 100,000$, corresponding to the top 15 percent). The rich-poor test score gap in wave 2 test scores gap is 1.1 standard deviations. Putting these two numbers together, our linear peer effects imply that 3.5 percent of the rich-poor gap in standardized test scores can be explained by the richer students' access to higher-achieving peers.

\subsubsection{Academic Peer Effects on Educational Inputs}

In this section, we estimate the impact of higher-achieving academic peers on nineteen educational inputs in order to explain how academic peer effects work. We estimate variations of Equation (3.1) using our measures of educational inputs in wave 2 as

\footnotetext{
${ }^{13}$ The combination of partial classroom sampling and random assignment of students to classes in TEPS implies that these and all other peer effect estimates in our main results might be biased downwards (Sojourner, 2013). We discuss the source of this bias, and present and interpret corrected estimates, in Section 3.6.2.
} 
outcomes. Figure 3.4 shows the effect of a $1 \mathrm{SD}$ increase in average peer test scores on wave 2 educational inputs in our estimation sample. Each row shows the effect of peer test scores on a different educational input. We show the unconditional mean of each outcome in square brackets to give context to these estimates. Navy blue estimates show effects student inputs, maroon estimates show effects on parent inputs, and teal estimates show effects on school and teacher inputs.

A 1SD increase in average peer test scores decreases students' school effort in wave 2 by 5.2 percent of a standard deviation. While these effects are a priori surprising, they are difficult to benchmark against previous findings. Many studies have hypothesized study effort to be a key mechanism through which peer effects operate, yet a handful of them provide estimates of effort responses to high-achieving peers. The few studies that do find mixed evidence (Feld and Zölitz, 2017; Mehta et al., 2019; Fang and Wan, 2020).

The negative effect of higher-achieving peers on student effort might seem surprising. One possible explanation is that exposure to high achieving peers could constitute a form of relative performance feedback, which can affect effort decisions (Azmat and Iriberri, 2010). Intuitively, students start with a prior belief about their ability relative to their peers', and those exposed to high-achieving peers update their belief downwards. This has a flow-on effect on effort, which can be positive if students have competitive or rank-preferences (e.g. Azmat et al., 2019; van Lent and Souverijn, 2020; Clark et al., 2020) or negative if students become discouraged by the tournament-like stakes in the classroom (Bedard and Fischer, 2019). Our negative effects on effort in the TEPS data suggests the discouragement effect is the larger of the two.

A 1SD increase in average peer test scores also increases students' aspirations to go to university by 1.6 percentage points, and their expectations of actually going to university by 2 percentage points. These seem like small effects, corresponding to around 3-5 percent of their respective unconditional means, but become more sizeable when compared to the effect of other known shifters of aspirations. One could compare them, for example, to the 8.5 percent increase in parents' higher education aspirations for girls from opening access to male-dominated professions in India (Beaman et al., 2012), the 5.2 percent increase in educational aspirations of castpriming in high-casts in India as well (Mukherjee, 2015), or the precisely-estimated null effect of university information on educational aspirations of Colombian students (Bonilla-Mejía et al., 2019).

A 1SD increase in peer test scores also increases parents' time investment by 8.1 
Figure 3.4: The Effect of Higher-Achieving Peer Test Scores on Educational Inputs in Wave 2

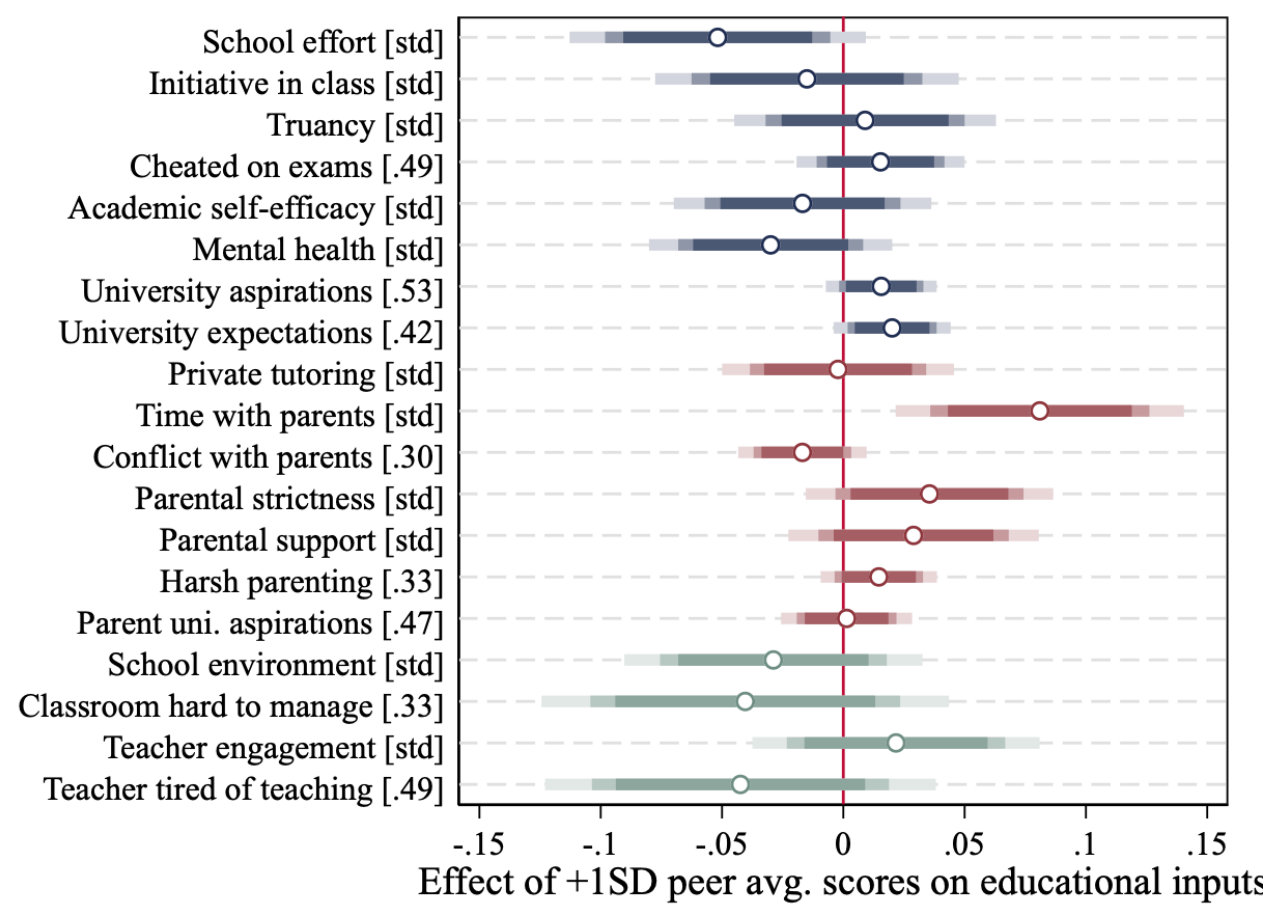

This figure reports estimates of regressing educational input measures in wave 2 on standardized average peer test scores in wave 1 in our sample containing 232 schools, 850 classes, and up to 11,029 students. Rows present results of models with different educational inputs as outcomes. Unconditional means of each outcome are shown in square brackets, and [std] marks outcomes that have been standardized to have a mean of zero and a standard deviation of one. All models control for school fixed effects, student test scores in wave 1, balancing controls, and educational inputs in wave 1. Student, parent, school and teacher inputs are shown in navy blue, maroon, and teal. Horizontal bars show the 99\%, 95\% and 90\% confidence intervals for each estimate, based on standard errors clustered at the classroom level. Estimates in this figure are also shown in Appendix Table C.3.

percent of a standard deviation. Our time investment measure in TEPS focuses in dinner time spent with parents, yet our estimated peer effect could be compared to half of the impact of having one student more in one's classroom on parents' likelihood of helping the child with homework in Fredriksson et al. (2016), or with a fifth of the effect of a child attending a marginally worse school in Pop-Eleches and Urquiola (2013). 1SD higher-achieving classroom peers also increases parental strictness by 3.6 percent of a standard deviation, a small effect that has no benchmark in the peer effects literature.

Finally, Figure 3.4 also shows that we cannot detect effects of higher-achieving peers on many educational inputs that have previously been considered as key potential mechanisms behind peer effects, such as student initiative in the classroom, 
classroom disruption and the quality of peer interactions in the classroom (e.g. Booij et al., 2017; Feld and Zölitz, 2017). We estimate null effects on all measures of parental investment or parenting behavior other than parental time investments. This finding is important because while we find no parental behavioral responses to classroom peer ability, previous studies have shown evidence of parental behavioral responses to other types of public investments such as school admissions, classroom size, and teacher qualifications. Lastly, we also find precisely estimated null effects on mechanisms that others have found potentially relevant including on students' perception about their school environment (e.g. Feld and Zölitz, 2017), and on teacher engagement, classroom management and tiredness (Lazear, 2001; Duflo et al., 2011a; Golsteyn et al., Forthcoming).

Importantly, our null effects on most of these mechanisms are precisely estimated. Between all our estimates, the largest standard error for a standardized educational input is 0.026. A standard ex-post Minimum Detectable Effect (MDEs) size calculation with 95 percent confidence and 80 percent power implies that we could have detected effects as small as 7.3 percent of a standard deviation for outcomes such as initiative in class or teacher engagement. A 7.3 percent of a standard deviation in an outcome is a relatively small detectable effect; close to 10 percent of the gender gap in effort (women pay more effort than men), 18 percent of the difference between private tutoring investments of top-income parents and the rest, or 9 percent of the difference between the time investments of two-person and single-parent households.

Overall, we show that higher-achieving peers decrease student effort, increase student aspirations and expectations to attend university, an increase in parental time investments and parental strictness. We can make sense of the first two, seemingly contradicting, results in the lens of existing theories of performance under uncertainty; they could be consistent with exposure to higher achieving peers as a form of relative performance feedback. The sign of these estimates is in line with the theoretical model and recent field evidence of Azmat et al. (2019). The latter result on time investments provide new insights on the relatively thin evidence base on parents' behavioral responses to school inputs. Our effects suggest that parents complement school inputs (i.e., better school peers) by increasing their own time investment. This collides with evidence that parents tend to treat school inputs and own time investments and parental strictness as substitutes (Pop-Eleches and Urquiola, 2013; Fredriksson et al., 2016) but is consistent with other evidence from Taiwan that showing that parents complement teacher qualifications with financial investments of their own (Chang et al., 2020). 
More relevant is that - depending on the productivity of these educational inputs for student achievement - these input responses could all be legitimate mechanisms for explaining our 5.2 percent of a standard deviation effect of higher-achieving peers on test scores. In the next section, we calculate how much of our estimated academic peer effect can be explained by these mechanisms.

\subsubsection{Mediation of Academic Peer Effects}

We are now able to formally ask how much of the 5.2 percent effect of higher-achieving peers on students' test scores can be explained by their intermediate impact on educational inputs. To do this we follow the decomposition in Gelbach (2016), which we adapt to our setting in order to use only within-school variation by modifying the b1x2 Stata package.

This decomposition calculates the total mediated effect $(M E)$ of educational inputs on peer effects:

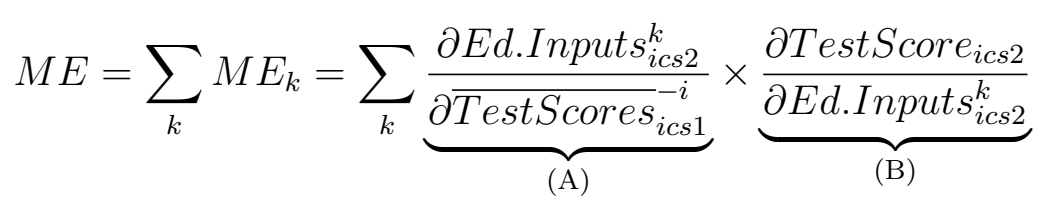

where Ed.Inputs ics $2^{k}$ stands for educational input in our set of inputs. The terms (A) are the causal effects of higher-achieving peers in wave 1 on educational inputs in wave 2 as shown in Figure 3.4. The only remaining pieces for the calculation of $M E$ are therefore the terms which are the partial returns (i.e., holding other inputs constant) to each of the educational inputs on student scores in wave 2.

There is no ideal experiment for estimating (B), not even by independently and randomly varying each educational input over a period of two years and then estimating their causal impact on student test scores. The reason, as expressed by Todd and Wolpin (2003), is that such experiments would identify "policy parameters" - effect identified out of variation not subject to choices of parents or schools but exogenously induced - rather than "production function" parameters. Policy parameters are identified by variation in inputs exogenously pressed onto people, rather than by naturally-occurring variation through people's investment decisions across the population (see e.g. Imai et al., 2013; Keele et al., 2015). Thus, policy parameters answer many important questions but they do not recover returns to inputs, so their use is limited in a mediation analysis as described by Equation (3.2). 
Todd and Wolpin (2003) argue for using (cumulative) value-added models to estimate the (B) term of Equation (3.2). Todd and Wolpin (2007) and Fiorini and Keane (2014), among others, discuss these models in detail and show that they can identify the returns to educational inputs under relatively weak conditions, and we find ourselves in an ideal scenario for estimating these models. This is because in our setting we i) always use within-school variation which accounts for unobserved school-level heterogeneity, ii) can control for standardized test scores in wave 1, iii) can control for a myriad of educational inputs in wave 1, and iv) only need to estimate returns over a two-year period. For all these reasons, we estimate the terms (B) as the $\hat{\beta}_{k}$ from the within-school cumulative value-added model:

$$
\text { TestScore }_{i c s 2}=\sum_{k=1}^{K} \beta_{k} \text { Ed.Inputs }_{i c s 2}^{k}+\delta \text { Covariates }_{i c s 1}+\gamma_{s}+\nu_{i c s 2}
$$

where Covariates ${ }_{i c s 1}$ includes student test scores, average peer test scores, and all other educational inputs in wave 1 . To the extent that our school fixed effects account for school-level unobserved heterogeneity $\gamma_{s}$ and extensive set of high-quality covariates account for endogeneity in observable educational inputs, Equation (3.3) will identify unbiased estimates of the average partial return to each of the $K$ educational input in our data. ${ }^{14}$

Figure 3.5 presents within-school cumulative value-added estimates of the total and partial average returns of educational input in wave 2. Total effects are return parameters estimated one input at the time. Partial effects are the return parameters estimates $\hat{\beta}_{k}$ obtained from Equation (3.3) with the complete set of $K$ inputs include as regressors together. In other words, they are the returns of each educational input $k$ holding constant all other $K-1$ inputs. We rescale test scores and all continuous inputs in wave 2 so that each value can easily be interpreted as the return of a one standard deviation increase in standard deviations of scores. The circles show the

\footnotetext{
${ }^{14}$ School fixed effects are theoretically not necessary for identifying our value-added parameters, yet we include them to easily map our academic peer effects on inputs onto a mediation analysis using shared modelling assumptions. Our value-added parameters do not change when we use classroom fixed effects, student fixed effects, or remove fixed effects altogether. Attenuation due to classical measurement error could also affect our value-added estimates, yet this would not meaningfully change our conclusions since i) most of our input measures combine several items, which reduces measurement error substantially, and ii) our measures tend to have excellent interitem consistency and high Cronbach alphas, which suggest little measurement error left in them. Back-of-the-envelope models with reasonable levels of attenuation assumed for our value-added models support these conclusions.
} 
Figure 3.5: Returns to Educational Inputs from Cumulative Value-Added Models

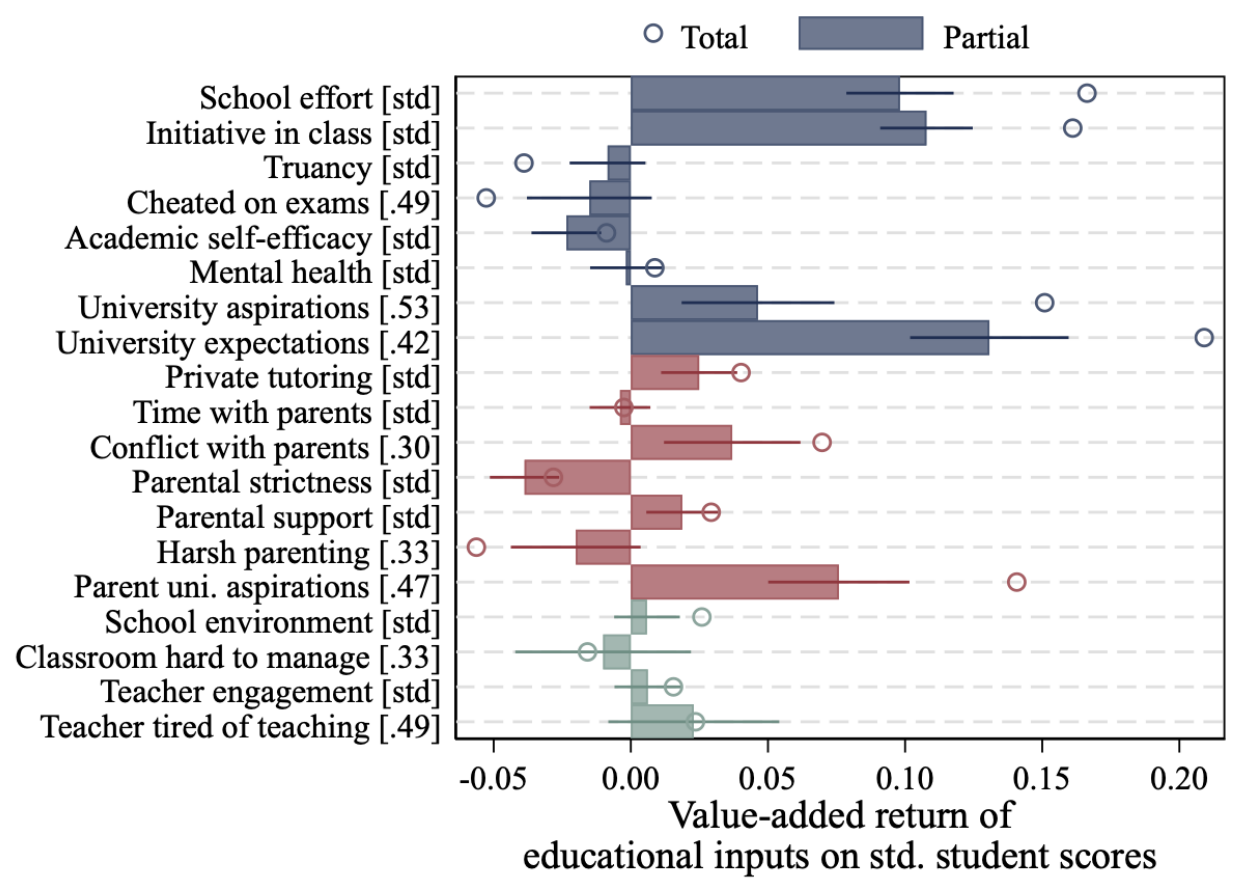

This figure reports coefficient estimates of regressing student test scores in wave 2 on educational inputs in wave 2 in our estimation sample containing 232 schools, 850 classes, and up to 11,029 students. Rows present coefficients of different regressors; Unconditional means of each input are shown in square brackets and [std] marks inputs that have been standardized to have a mean of zero and a standard deviation of one; circles show total effects (one input at the time) and bars represent partial effects (all inputs jointly). All models control for school fixed effects, student test scores, average peer test scores, and educational inputs in wave 1. Student, parent, school and teacher inputs are shown in navy blue, maroon, and teal. Spikes show $95 \%$ confidence intervals on partial effects based on standard errors clustered at the classroom level. These results are also available in Appendix Table C.4.

total returns of each input, and the bars show the partial effect of each input with their corresponding 95 percent confidence interval.

We obtain precise estimates of the average partial returns to all educational inputs. The first row in Figure 3.5, for example, shows that a 1SD increase in school effort between waves 1 and 2 carries an average return of 9.3 percent of a standard deviation in test scores in wave 2 . There are also positive returns to students' initiative in class, university aspirations and expectations, as well as parental money investment in the form of private tutoring, parental support and university aspirations for their child. There is evidence of negative returns to students' academic self-efficacy, and parental strictness and harshness. The differences between total and partial average 
returns reflect the fact that many of these inputs are correlated. ${ }^{15}$

Figure 3.6 puts together the results from Figures 3.3 to 3.5 to produce estimates of the mediated effect of peer effects by our measured educational inputs, as per Equation (3.2). This figure reports the mediated effects based on a Gelbach (2016) decomposition of our academic peer effect estimate using only within-school variation in our complete sample, and allowing errors to be correlated across the scores and input equations. The bar in green shows that our mechanisms explain a negative and statistically but not economically significant amount of our estimated peer effect - which means that the effect of higher-achieving peers on these inputs and their estimated return jointly make it harder, not easier, to explain the academic peer effects. Jointly, all our educational inputs explain only -0.8 percent of a standard deviation of the 5.2 percent of a standard deviation academic peer effect. This negative mediation is chiefly driven by the negative effects of higher-achieving peers on effort combined with the large and positive estimate of the returns to effort on academic achievement. None of the other inputs we consider has a statistically or economically significant mediating effect.

Overall, the results in this section show that, in spite of having precise estimates of i) academic peer effects and of ii) the effects of higher-achieving peers on educational inputs, which could potentially act as mechanisms for these peer effects, our potential mechanisms explain practically nothing of peer effects. These new results show the difficulties of learning about the mechanisms that drive social interaction effects and suggest that the prevailing microeconometric approach to exploring these mechanisms can be of limited use. Puzzling results such as these open a number of questions and can prove to be a knowledge base to build on, as long as its foundations are solid. Precisely because of this, in the next section we show that our main results and conclusions are robust to a myriad of specification checks and potential concerns. In particular, section 3.6.4 shows that these results on the absence of mediation are not hiding heterogeneity in the sense that we find little heterogeneity in the effect of high-achieving peers on scores and inputs across different subgroups of students.

\footnotetext{
${ }^{15}$ The $R^{2}$ in our cumulative value added model is 0.71 which suggests we explain a substantial amount of the variation in wave 2 test scores with our data. Our measured inputs contribute the vast majority of the explanatory power; the same model without school fixed effects has only a slightly lower $R^{2}$ of 0.69 . There is also not much scope for within-classroom variation to contribute additional explanatory power since adding classroom fixed effects only increases the $R^{2}$ to 0.74 .
} 
Figure 3.6: Academic Peer Effects Mediated by Educational Inputs

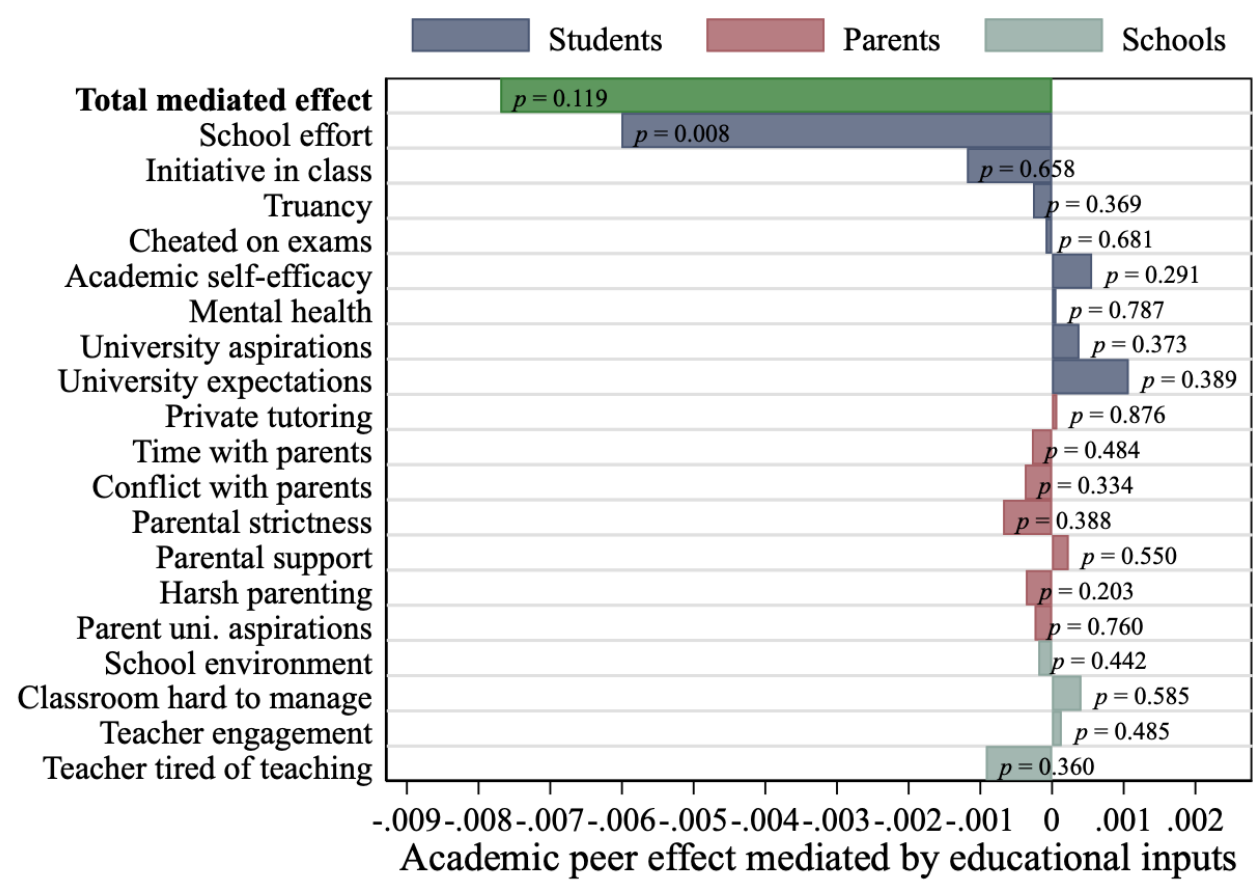

This figure reports the mediated effects based on a Gelbach (2016) decomposition of our academic peer effect estimate using only within-school variation in our estimation sample containing 232 schools, 850 classrooms, and up to 11,029 students. These estimates are produced using a modified version of the $61 \times 2$ Stata package. Rows present the mediated effect of different educational inputs in wave 2. All models control for school fixed effects, student test scores, average peer test scores, and educational inputs in wave 1. The total mediated effect is shown in green, and student, parent, school and teacher inputs are shown in navy blue, maroon, and teal. p-values shown are based on standard errors clustered at the classroom level. These results are also available in Appendix Table C.5.

\subsection{Sensitivity Analyses}

In this section, we discuss the sensitivity of our results along four dimensions: i) robustness to changes in our identification strategy; ii) robustness to the effects of measurement error in our data; iii) robustness of our inference to different constructions of standard errors; and iv) robustness of our conclusions on the mediation analyses to the presence of heterogeneous peer effects.

\subsubsection{Robustness of Identification Strategy}

Here, we first provide additional evidence of random assignment of students to classrooms within schools in our trimmed sample using permutation-based sorting 
tests, and using non-parametric sorting tests. Many of these tests have become standard in the empirical peer effects literature. We then exploit the richness of our data - in particular the fact that we observe many pre-assignment characteristics of students, parents and teachers - to show that proportional selection on unobservable characteristics is very unlikely to be driving our results.

\section{Permutation-Based Sorting Tests}

In the empirical peer effects literature, permutation-based tests of random assignment of students to peer groups have become very popular. These tests compared the actual student group composition in the data to counterfactual compositions simulated under the null of random assignment, as described in Section 3.3.2. As an additional check for random assignment in our data, we estimate permutation-based sorting tests akin to those in e.g., Carrell and West (2010); Lim and Meer (2017, 2020) in our trimmed sample.

For these tests, we simulate 10,000 classrooms under the null of random assignment of students to classrooms within schools. We do so by randomly drawing sampled students with replacement and keeping the core structure of the data-respecting students' assignment to schools, and number and size of classrooms within each school. We then calculate the mean of our key pre-treatment characteristics in each of the 10,000 synthetic classrooms. Finally, for each classroom, we count the times the synthetic classroom mean of each characteristic was more extreme than the actual classroom mean, relative to the schools mean. The share of times this happens corresponds to the classroom-level empirical p-value of a test of random assignment of students to classrooms within schools based on that characteristic. Appendix Table C.6 shows these permutation-based empirical p-values for each key pre-determined characteristic separately. Under random assignment, the shares in the second through fourth column should be close to the nominal rejection rates of 0.10, 0.05 and 0.01 in most or all rows. The evidence in this table strongly supports the idea of random assignment to classrooms within schools in our trimmed sample.

\section{Non-Parametric Sorting Test}

As implemented, balancing tests and sorting tests all have one important shortcoming: their linearity. Balancing tests, for example, assess whether female students are assigned to higher-achieving peers. Sorting tests try to capture whether female students end up in classrooms with other female students. But these tests do not truly test for what random assignment would imply: whether classrooms systematically 
differ in these pre-assignment characteristics in any way. In other words, these tests do not test non-parametrically for systematic assignment of students to classrooms. A few studies do use this non-parametric sorting test (Ammermueller and Pischke, 2009; Sojourner, 2013; Feld and Zölitz, 2017).

We implement this test in the following steps. First, we estimate school-by-school regressions of each pre-assignment characteristic on a set of classroom dummies. Second, we jointly test the statistical significance of these classroom dummies and collect the p-values of these tests. We end up with a set of 2,790 p-values; one for each of the 227 schools in our sample and each of our key 18 pre-assignment characteristics. We then note that, under the null of random assignment of classrooms to schools, these p-values should be uniformly distributed. Therefore, as a third step, we check whether more than ten, five and one percent of the school-level p-values fall under the nominal values $0.10,0.05$ and 0.01 for each characteristic.

Appendix Table C.7 shows empirical p-value distributions for each characteristic separately. Consistent with our tests in Section 3.4.2, these results also show some evidence of minor sorting based on intellectual curiosity, gifted arts classroom enrolment, and parents pushing for assignment to particular classrooms. Overall, however, these tests provide yet again evidence in strong support of random assignment to classrooms within schools in our trimmed sample.

\section{Different thresholds for our Fishing algorithm}

An important and somewhat arbitrary decision in implementing our Fishing Algorithm is deciding when to classify any particular school as not compliant with the mandate of random assignment. Recall from Section 3.4.2 that we do so based on how each school's probability to belong to the latent class of sorters - the latent sorter probability, for short. Our intuitive thumb rule is: a school is a sorter if its latent sorter probability is larger than all other latent class probabilities combined. However, this is not the only way to classify such schools. Another approach is to pick a fixed probability threshold and consider any school with a latent sorter probability above that threshold as a sorter.

In Appendix Table C.8 we show how all our main results on academic peer effects change had we implemented this fixed threshold approach at different levels, ranging from 0.5 (relatively strict, removing all schools that are "more likely than not" to be sorters) to 1 (very relaxed, effectively removing only schools for which $S_{s}$ is equal to 1 too). The bottom of the table shows that the sorting statistic of Jochmans 
(2020) grows monotonically with the threshold, as expected, and starts rejecting the null of no sorting for thresholds of 0.7 and above. For thresholds below 0.7, we find consistent academic peer effects on test scores of around 5 percent of a standard deviation, as well as consistent negative effects on student effort and positive effects on students' university expectations, and time with parents. We find weaker and less consistent evidence of positive effects on students' university aspirations, parental support, and teacher engagement, and negative effects on conflict with parents. In general for thresholds 0.7 and below all coefficients are very stable and compare well to our main effects. For thresholds above 0.7, where sorting tests fail, we tend to find larger academic peer effects on test scores, smaller effects in magnitude on student effort and time with parents, and larger effects in magnitude for parental support, parents' university aspirations, and class management. Most of these differences are consistent with stronger ability sorting into classrooms for these schools. Overall, Appendix Table C. 8 shows that our results are not overly sensitive to which threshold we use in our fishing algorithm as long as the resulting estimation sample passes Jochman's sorting test.

\section{Proportional Selection on Unobservable to Observable Characteristics}

Our trimmed sample is chosen in a data-driven way that ensures that key pre- assignment characteristics are unrelated to average peer test scores. This identification strategy relies on our ability to find data that reflects a clean quasi-experiment in classroom allocation, yet systematically excludes entire schools from our sample, which might lead to sample selection issues. Still, we ask ourselves whether the few observable characteristics that remain correlated to higher-ability peers could present reasonable concerns about unobserved heterogeneity. This calls for an analysis of proportional selection on observable characteristics, as discussed in Altonji et al. (2005) and Oster (2019). The two conditions for this analysis to make sense are i) that our observable characteristics for these analyses are a random sample of all determinants of student achievement, and ii) that the number of observed and unobserved determinants of student achievement are large and neither element is dominating. Along the argument lines of Altonji et al. (2005), we assume that the TEPS fulfils both conditions.

We implement this analysis by calculating the $\delta$ statistic from Oster (2019); the share of proportional selection needed to explain away the entire peer effect we estimate. Values of $\delta>1$ imply that the selection on unobservable characteristics would need to be at least as large as the selection on observable characteristics to explain away the entire peer effect estimate, which, given the data and data context, 
is an unreasonable assumption. A $\delta<1$ implies that the omitted variable bias from unobservable variables positively correlated with the observable variables included would bias the peer effects away from zero, not towards, and should therefore not be concerning as confounders. In this type of analysis, thus, finding values of $\delta$ between zero and one is worrisome, and could indicate a potential concern for unobserved selection affecting results. The observables we use for these analyses are extensive: they include our balancing controls (household income, family engagement with homework, gifted art classroom assignment, and parents' pushiness to get child assigned to a particular classroom) and our standardized measures of student, parent, school and teacher educational inputs in wave 1. Assuming that selection on unobservable characteristics occurs in proportion to the selection on this set of variables implies, by exclusion, that school fixed effects and students' own test scores in wave 1 - a priori essential for our identification strategy and standard in the literature - cannot inform the proportional selection analyses. We also use a hypothetical maximum R-Squared value of 1.3 times the R-Squared of the unrestricted model, which is the standard choice for these analyses.

Appendix Table C.9 shows Oster's $\delta$ for all our main estimates estimating using the psacalc Stata command. For nearly all our estimates, Oster's $\delta$ is negative which implies that proportionally selection on unobserved confounders are unlikely to explain our effects. The one exception is the $\delta$ of 0.10 for the effect of higherachieving peers on parental investments in tutoring, which is anyway insignificantly different from zero so none of our conclusions change following the results of this analysis. Overall, we conclude that proportional selection on unobservable variables cannot explain away any of our findings.

\subsubsection{Robustness to Measurement Error and Classroom Sampling}

We now turn our focus to the measurement error in our data We show that our main estimates i) are robust to using different measures of student and peer academic ability, ii) are not attenuated by measurement error in average peer test scores, and iii) are not biased by the fact that we do not observe whole classrooms.

\section{Main Results with Alternative Measures of Ability}

Our main results use the TEPS scores in the comprehensive ability test. As discussed in Section 3.3.2, this test was designed by TEPS team and uses 75 multiple-choice question to measure of students' cognitive ability and analytical reasoning. However, 
after a series of factor analyses and after estimating 3-parameter Item Response Theory (IRT) models, the TEPS team could also identify two highly correlated but distinct subcomponents measuring analytical ability and mathematical ability based on disjoint subsets of test questions. The IRT models were also used to produce the standardized Bayesian posterior means of the three components identifiable in the test - the general ability component and the analytical ability and mathematical ability subcomponents. ${ }^{16}$

Appendix Table C.10 shows that our main results are robust to using the analytical and mathematical subcomponents of the comprehensive ability test scores as measures of student and peer ability (columns (1) and (2)). Our main results are also robust to using the Bayesian posterior means of these components, arguably a more precise and efficient measure of ability (columns (3) through (5)).

\section{Correction for Classical Measurement Error in Peer Ability}

Even in excellent measures of student and peer ability, such as the well-designed standardized test scores in TEPS, there will still be some measurement error. Under random assignment and with classical measurement error (i.e., independent of all covariates and of true ability), this measurement error will attenuate our peer effect estimates (Sojourner, 2013; Feld and Zölitz, 2017; Angrist, 2014). We can address this attenuation bias in two similar ways. Noting that the analytical and mathematical subcomponents of test scores are measured with disjoint sets of questions, we can use average peer test scores using one subcomponent as an instrument for average peer test scores using the other in an instrumental variable (IV) estimator (see e.g. Salamanca et al. (Forthcoming) for a similar approach to account for measurement error in personality traits). This approach would eliminate attenuation bias from classical measurement error under two assumption: i) that both subcomponents have a strong common element of overall ability, and ii) that measurement error in test questions is uncorrelated across subcomponents. The first assumption is well supported by our data and by the TEPS team factor and IRT analyses. The second assumption is stronger; if it does not hold it would result in some attenuation bias left in the IV estimate.

Appendix Table C.11 shows that, although less precisely estimated due to the usual

\footnotetext{
${ }^{16}$ See http://www.teps.sinica.edu.tw/description/TestingReport2004-2-10.pdf (in Mandarin) for a description of these analyses.
} 
efficiency loss from instrumental variable models, the IV point estimates are nearidentical to our main results (columns (1) and (2)). We thus view this as evidence of little attenuation bias due to classical measurement error in our estimates.

One potential problem with the estimators above is that the IV estimates need to be interpreted as academic peer effects in analytical and mathematical ability, rather than in comprehensive ability. We address this problem by constructing a "mixed IV" estimator. In this estimator, we first construct an ability measure that, for each student, is randomly defined as either the analytical subcomponent score or the mathematical subcomponent score with equal probability. This ability measure is therefore an equal-weighted average of the analytical and mathematical subcomponents and can be interpreted as measuring general ability. We call this our 'mixed ability' measure. We also construct an ability instrument that is defined by the same random process to be the subcomponent that was not assigned as ability. For example, if for student ability is measured as the analytical subcomponent score, then the ability instrument is defined as the mathematical subcomponent score. We call this our 'mixed ability instrument'. Under the same assumptions above, an IV estimate that instruments our mixed ability with our mixed ability instrument also corrects for attenuation bias while identifying academic peer effects using general ability, rather than analytical or mathematical ability. We show that this new estimator produces very similar results to our main peer effect on test scores (Appendix Table C.11, column (3)). It also produces slightly larger estimated magnitudes of the effect of higher-achieving peers on study effort and students' university aspiration and expectations, and similar estimates for the effect on parental time investments (Appendix Table C.12). Back-of-the-envelope calculations show that these slightly larger estimates do not change our conclusions on the mediated effects of higher-achieving peers. We thus conclude that measurement error does not alter any of our main findings.

\section{Sojourner (2013) Correction for Incomplete Classroom Sampling}

Many empirical peer effect studies, including ours, has incomplete classroom data which results in incomplete sampling of students' peer group. Sojourner (2013) shows that this issue can result in bias in peer effect estimates that is similar to classical attenuation bias under random assignment, and much more difficult to sign and quantify under non-random assignment. He also proposes a correction for this bias that relies on i) weighting estimates by the share of peers sampled and ii) controlling for these shares at the school level. Often these last controls are multicollinear with the weighted peer measures, so he also suggests less restrictive 
estimators that control for the share of peers sampled within predetermined school clusters. We implement both methods in our data to evaluate the extent of this bias in our main results. The left-most column on the table implements Sojourner's preferred correction which can lead to substantial loss of power because it heavily restricts the identifying variation used by the estimator. The second through sixth columns implement specifications which trade off more power for less bias reduction, from left to right.

Appendix Table C.13 shows substantially larger effects of higher-achieving peers on student test scores, ranging from 8.9 to 13.3 percent of a standard deviation which nevertheless remain within the range of estimates found in previous studies, especially considering that peers here have had two years to work their effect on student achievement. These corrections also yield proportionally larger effects on students' university aspirations and expectations and parental time investments, which is all consistent with Sojourner's findings and with the data originating from conditional random assignment to classrooms within schools. The analyses do not reveal other effects of higher-ability peers. Moreover, since the attenuation in all our estimates is roughly proportional, our conclusions about mediated peer effects remains unchanged. This suggests that not observing complete classrooms in our data could lead to understating the importance of academic peer effects, but does not affect our (in)ability to explain their mechanisms. ${ }^{17}$

\subsubsection{Randomization Inference and Multiple Hypothesis Testing}

Having established the robustness of our point estimates of peer effects, in this subsection we show that our inference on these effects is robust to i) constructing standard errors based on recent randomization inference techniques and ii) to accounting for multiple hypotheses testing in our standard error calculations.

We first reassess inference on our main results using the randomization-t procedure from Young (2019). Our analyses benefit from this procedure because of the potential influence of a few high-leverage students, classrooms or schools, and we want to ensure that our inference is robust to this occurrence. We also want to use inference that does not make strong assumption on the structure of error terms given the

\footnotetext{
${ }^{17}$ There are other potential issues with incomplete classroom sampling, especially if our peer effects varied with classroom or school size, if the classroom sampling rate were correlated with our regressors, or if our Fishing Algorithm were selecting schools with different average sampling rates. Fortunately, none of these occur in our data.
} 
complexity of the TEPS sampling design and peer treatment. Other benefits of randomization inference, such as i) correcting for few treatment clusters or ii) issues of joint testing are less important for this study, because i) we observe several classrooms per school, and ii) each regression has one treatment effect of interest.

We construct randomization-t based empirical p-values via a very similar simulation procedure to the one used for permutation tests. The key difference is that, in each simulation, we capture the t-statistics of interest - the coefficient of the key variable of interest divided by its cluster-robust standard error - and construct empirical p-values based the share of occurrences where simulated t-statistics are more extreme than our actual t-statistic of interest. We use 10,000 simulations of random assignment to classroom within schools to produce randomization-t empirical p-values for our main results. Appendix Table C.14 shows that when using randomization-t inference p-values for conducting inference, our main conclusions on the effects of higher-achieving peers hold at the $5 \%$ significance level for student achievement and parental time investments, and at the $10 \%$ significance level for student university aspirations and expectations.

In a second analysis, we adjust our inference for multiple hypotheses testing: the problem that the chance of falsely rejecting a correct null hypothesis increases with the number of tests performed. We adjust for this by implementing the RomanoWolf multiple hypothesis correction (Romano and Wolf, 2005a,b) using the rwolf Stata command (Clarke et al., 2019). This procedure ensures that the familywise error rate - the probability of committing at least one Type I error across a set of hypotheses tested - does not exceed its predetermined significance. We consider all our main results to be part of the same family of tests. Appendix Table C.14 shows that our main conclusions on the effect of higher-achieving peers on student achievement and on parental time investment hold at the $10 \%$ significance level, but our evidence on students' university aspirations and expectations now appear not to be statistically significant.

Overall, with these different inference methods we still find strong evidence of academic peer effects in our data but somewhat weaker evidence of significant effects on educational inputs. This reinforces our conclusions of no mediated effects for academic peer effects. 


\subsubsection{Heterogeneous Peer and Mediated Effects}

Finally, we explore the sensitivity of our mediation analyses. Our chief concern here is the possibility that our lack of meaningful mediation can occur not because educational inputs cannot explain academic peer effects, but rather as the result of heterogeneity peer effects across subgroups. Heterogeneity can occur in two forms: firstly, academic peer effects could vary widely across subgroups - a result found in several studies across ability (Carrell et al., 2009), gender (Whitmore, 2005; Lavy and Schlosser, 2011), race (Hoxby, 2000; Hoxby and Weingarth), but secondly and perhaps most importantly, the drivers of peer effects for each subgroup could also widely differ, as suggested by (Brady et al., 2017). For example, higher-achieving peers could improve test scores of low-ability students because they reduce the amount of classroom disruption (see e.g. Lavy et al., 2012a) and improve test scores of high-ability students because they increase effort. Yet we might be unable to detect enough mediation via truancy and effort on the average academic peer effect. This form of heterogeneity would wrongly lead us to conclude that truancy and effort cannot explain at least part of academic peer effects. One way to assess whether this particular type of heterogeneity is a likely explanation for our findings is to estimate the heterogeneity of peer effects and their mediation via educational inputs across various subgroups.

There are countless dimensions to explore heterogeneity in academic peer effects in our data. Based on existing heterogeneous effects in the academic peer literature, and on a broader literature on the sociodemographic predictors of student test scores, we explore peer effect and mediation heterogeneity across: student ability, gender, household income, parental education, public vs private schooling, and teacher experience. Appendix Table C.15 shows that, by and large, there is little subgroup heterogeneity in our estimated academic peer effects and their mediation. Academic peer effects are slightly larger at the top and middle of the student ability distribution, with highly experienced teachers, and in private schools, yet are the same across student gender, household income, and parental education. More importantly, our inputs can still mediate either small or negative parts of these academic peer effect for any one of these subgroups. Altogether, we show strong evidence of little heterogeneity in academic peer effects and in mediated effects.

We also consider the possibility that our mediation is affected heterogeneity of the value-added parameters across the peer ability distribution. This could occur if higher-achieving peers change e.g., the productivity of some teaching practices (Aucejo et al., 2020). There is evidence of similar heterogeneity in our data: having 
higher-achieving peers increases returns to student effort and initiative in class, decreases returns to private tutoring and time spent with parents, and increases the penalty for being in a hard-to-manage classroom (see Appendix Table C.16). This heterogeneity is broadly consistent with higher-achieving peers being complements to some student and teacher investments in class and substitutes to some parental investments at home in the education production function. However, note that for the two inputs where we find both significant academic peer effects and significant heterogeneity (school effort and time with parents), higher-achieving peers would increase the return to effort and decrease the return to time with parents. Since academic peer effects are negative for effort and positive for time with parents, this together adds up to even less mediation when we allow for heterogeneity in our value-added estimates.

Based on these results we conclude that subgroup heterogeneity is not a likely explanation for the fact that our many educational inputs do not mediate academic peer effects.

\subsection{Conclusions}

We estimate the effect of being randomly assigned to classrooms with higherachieving peers on students' standardized test scores two years later, and on 19 other intermediate outcomes of students, their parents, and their teachers. We conduct a formal mediation analysis of academic peer effects to explore several potential mechanisms, one of the first ones of its kind in a field with over twenty years of research and hundreds of articles. Our study thus gives the most comprehensive view of how much academic peer effects are explained by changes in educational investments in a setting with a credible identification strategy.

For producing these results, we use data in a setting with a well-documented countrywide mandate of random assignment of students to classrooms within schools. The data, however, shows that this random assignment was likely not upheld everywhere, which is not entirely surprising: we can think of legal and illegal ways in which sorting can still occur - for example, via allowed "talent" classrooms in schools, or due to principals sorting students into classrooms in defiance of the mandate. Similar violations to national mandates are common in similar settings (e.g. Gong et al., 2019; Eble and $\mathrm{Hu}, 2019$ ). We develop a data-driven procedure to remove schools likely to be defying the mandate of random assignment from our estimation sample and show that data in this trimmed sample is strongly consistent with random 
assignment. This Fishing Algorithm can be used to improve quasi-experimental designs in settings where random assignment to peers is suspected to be violated in some, but not all, assignment groups. It can more generally be used in any setting where researchers suspect imperfect compliance of (quasi-)experimental treatment assignment in some clusters.

One might wonder if our results can really tell us something about academic peer effects in other settings. Yet data from the Trends in International Mathematics and Science Study (TIMSS) in 1999 shows that Taiwan's educational setting is altogether not that different from many others across the world (Appendix Table C.17). And while students in Taiwan do spend relatively more days per year in school, and have lower rates of absenteeism, none of these differences helps us explain why we still find positive academic peer effects of similar size to many other studies, yet no evidence of mediation. ${ }^{18}$ Moreover, Taiwan is comparable to other countries - especially in the Australasia and Pacific region - in most other key dimensions including class size, student-teacher ratios, daily study hours, dropout rates, or class disruption.

Since our academic peer effects remain largely unexplained, it could be tempting to conclude that academic peer effects are unexplainable by current methods. Instead, we see at least two avenues for future research.

The first avenue is to keep on striving to find data on potential mechanisms. It is true that most potential mechanisms for academic peer effects proposed in previous studies feature in one way or another in the TEPS (see Appendix Table 3.1), many of them more carefully measured than ever before. Two notable exceptions are: direct learning from peers and detailed teaching practices. Measuring direct peer learning (e.g., discussing tasks and coordinate among group mates) requires data on peer-to-peer interactions which is difficult to gather, yet could indeed be the missing explanation for academic peer effects (e.g., Garlick, 2018; Zárate, 2020; Kimbrough et al., Forth.). Detailed teaching practices (e.g., how teachers pair students for group work or the amount of material covered in each lesson ) are also hard to measure yet some of them are strongly related to student achievement gains (e.g., Kane et al., 2011) and one can easily think of ways in which teachers adapt their teaching style to classroom ability.

\footnotetext{
${ }^{18}$ What some of these differences could explain, however, is why we find negative effects of higherachieving peers on student effort. Taiwanese students might be exerting so much effort already that the demoralizing effect of these peers takes hold easier.
} 
The second avenue is to further explore heterogeneity in the value-added of educational inputs. Imagine a world in which academic peer effects exist in every school, but they occur via different channels. In one school higher-achieving peers increase effort while in another they decrease class disruption, and in yet another they increase parental engagement. Such dramatic differences in the mechanisms of peer effects become increasingly likely with school segregation and specialization. In such a world, we would find positive peer effects across schools and no mediation on average, just as we do. Such cancellation of mechanisms is not unheard of; it is documented in Bursztyn et al. (e.g., 2019) where they show that making effort observable can either increase or decrease student effort depending on the social norm in the school. One could explore this hypothesis in the academic peer effect context via school-specific academic peer effects and value-added functions. Unfortunately the data requirements for such exercise are enormous: we would need rich data on many schools with many classrooms, where random assignment holds and with a longitudinal dimension to it. We simply do not know of a dataset with these features, not even the TEPS.

Our results also get us closer to using peer effects to confidently inform and design classroom assignment policies. A pervasive concern with systematic assignment policies is that their benefits might come with unmeasured cost on, e.g. classroom disruption, increasing stress and deteriorating mental health for both students and teachers, and higher effort to keep up with one's higher-achieving peers. Our study shows that many of these concerns are unfounded. If anything, higher-achieving peers seem to improve classroom atmosphere, an effect which has been found in e.g. Feld and Zölitz (2017). In the absence of measurable costs, our results suggest that higher-achieving peers could be an effective way to increase student achievement, even if we do not quite know how they work yet. 

Chapter IV:

Teachers' Biased Beliefs and Inequality of Opportunity 

As I have shown through Chapter 3, lifting students' aspirations could prove to have only a limited impact on test scores. In the last chapter of this dissertation, I explore whether teachers' beliefs can become behavioral barriers to the success of disadvantaged children in education - perhaps contributing to explaining the puzzling findings of Chapter 3. The goal of this last chapter is to broaden our discussion of inequality of opportunity in education, to highlight that behavioral barriers to success for disadvantaged children are pervasive and multifaceted. Eradicating these barriers will likely require holistic educational policies based on a deep understanding of how pupils, parents and teachers interact in the education system.

In Chapter 4, I present new empirical evidence of teacher bias, and highlight new mechanisms through which teacher bias operates. I estimate the effect of older siblings' relative rank in their primary school cohort, on the test scores and teacher assessment of their younger sibling - an effect indicating that teachers clearly exploit information that does not directly apply to the younger sibling. This represents a natural experiment of teacher learning, which I use to reveal perhaps the simplest, and most common ways that teachers could form biased beliefs about a child's academic ability: by knowing their older siblings. Older siblings' test scores do contain information that may legitimately also apply to the younger sibling (e.g. genetic predisposition, family resources, learning environment), yet their relative rank depends directly on their cohort composition: when comparing older siblings with the same test scores but only different relative ranks, any effect of relative rank on teachers' beliefs about younger siblings is unrelated to younger siblings' true ability. To capture teacher biased beliefs, I therefore use the following natural experiment: I compare the subjective assessment of teachers for two children with the same observable characteristics, who only differ in the relative rank that their older sibling had in their primary school cohort. Essentially, I estimate the effect of growing up with an older sibling who is at the top v. at the bottom of his or her cohort in primary school, fixing everything else, in particular actual test scores. 
To study this natural experiment, I use education records of all children in the Netherlands leaving primary school between 2003 to 2016. The Dutch educational system presents two institutional features that make it ideal to study this experiment. Pupils leave primary school with two key pieces of information about their ability: i) a standardized test score, that is comparable over time and across the Netherlands; and ii) a subjective assessment of their primary school teacher, about the child's "best fitting" secondary school track. Importantly, these two pieces of information are available in the Dutch education records, making it a unique case of administrative data in which beliefs are recorded.

My findings indicate that teachers do form biased opinions about younger siblings in response to the relative rank of their older sibling. Younger siblings who grow up with a higher ranked older sibling, keeping constant the older sibling test score, enjoy a significant boost in their test scores, as well as a higher chance that their teacher advises the academic track, even after accounting for younger siblings' own test scores. That is, the higher the relative rank of older siblings in their cohort, the more lenient teachers become with younger siblings, above and beyond the younger siblings' actual academic performance.

Additional analyses allow me to exclude a certain number of possible alternative interpretations of our findings. In particular, my findings are neither explained by parents exerting pressure on teachers, nor by parents increasing investments in their younger child in response to the relative rank of their older child.

I further show that the effect of sibling rank on teacher leniency exacerbates inequality of opportunity. First, I show that older siblings' rank in primary school has a longlasting impact on the educational trajectories of their younger sibling, by increasing their probability of specializing into STEM fields of study in high school. Enrolling in STEM higher education majors in the Netherlands requires high school specialization in STEM, and STEM majors are associated with higher wages on the labor market and higher employment probabilities. Thus, the effect of older siblings' rank in primary school has the potential to exacerbate or generate inequality of opportunity between younger siblings who grow up with high ranked or low ranked older siblings. Second, I show that teachers' response to sibling rank puts vulnerable children at a disadvantage, thus exacerbating socioeconomic gaps in educational outcomes. In a first exercise, I compare the effect of older siblings' rank on younger siblings' test scores and teacher assessments between native and migrant children. I find that the boosting effect of older siblings' rank on younger siblings' test scores is similar between native and migrant children, and yet the effect on teacher leniency is only 
present for native children. This result suggests that teachers tend to be positively biased towards native children, but it could also be that teachers have private information e.g. that families of migrant children are less interested in academic v. vocational education, such that interpreting track advice as teacher belief could be hiding family preferences rather than true teacher beliefs about the child's ability. I address this concern with a second exercise, comparing the effect of older sisters and brothers' rank on younger sisters and brothers' test scores and teacher assessments. Older sisters' rank has a larger positive effect on younger sisters' Math score than on younger brothers', and a larger positive effect on the probability that the teacher recommends the academic track. Older brothers' rank, on the other hand, has a larger and negative effect on younger brothers' Math score v. younger sisters', but a larger positive effect on younger brothers' language score, and precisely estimated null effects on teacher recommendations. The findings of these two exercises indicate overall that teachers form different expectations about children depending on their demographic characteristics, thus exacerbating inequality of opportunity.

Lastly, I provide suggestive evidence that teacher bias could be limited by lowering the weight of teacher assessments in school admission policies in the Netherlands. First I show that the effect of older siblings' rank on teacher assessment disappears in zones where secondary school admissions are lottery-based due to over-subscription to schools, while the effect of older siblings' rank on younger siblings' test score is stable. Second, I show that the effect of older siblings' rank on teacher assessment disappears for younger siblings exiting primary school after a reform that reduced the weight of teachers in younger siblings' primary school report card relative to test scores, while the effect of older siblings' rank on younger siblings' test score remained stable after the reform.

This study indicates that teachers' beliefs can and do become behavioral barriers to the success for disadvantaged children: teachers' beliefs about children's ability are systematically biased by their past experience, and have a long-lasting impact on their educational outcomes. As children are imposed their teachers, teachers' biased beliefs seem like a source of unacceptable inequality of opportunity that educational institutions would best eradicate. Importantly, this study also provides suggestive evidence that school admission policies could be a solution to eliminate behavioral barriers arising from teacher bias. While recent randomized control trials have shown suggestive evidence that training teachers to recognize their obvious biases could help reduce these biases, we argue that a more efficient approach could be, instead, to design better incentive systems. 



\section{Siblings and Teachers' Biased Expectations ${ }^{1}$}

\subsection{Introduction}

Through social interactions, public investments in any member of a network or community may also impact its non-treated members. Spillover effects through social interactions are often called "social multiplier" effects, because the existence of such spillovers increases the returns of public investments. In the presence of social multiplier effects, investing in few members of a community - e.g. through positive discrimination policies - could impact many members of a community, making it faster and cheaper to achieve desirable equilibrium outcomes, such as the reduction of inequality in educational attainment. At the same time, adverse experiences of some could exacerbate the consequences of socioeconomic disadvantage in education, increasing achievement gaps between privileged and underprivileged groups.

In this paper, I estimate the causal effect of older siblings' rank in primary school on their younger siblings' standardized test scores and subjective assessment of their teacher. Sibling spillover effects represent a special case of peer effects, in which many aspects of how peers interact are fixed: sibling share genetic endowments, family and social environments. This feature of sibling interactions allows researchers to focus on other driving factors explaining social interaction effects in general. I exploit the experience of older siblings as a treatment on their younger sibling to highlight two new, understudied channels through which social interaction effects occur: how teacher form subjective expectations about the academic ability of children, and how peers have a motivational effect on each other. I use high-quality

\footnotetext{
${ }^{1}$ I thank Jan Feld, Ulf Zölitz, Ingo Isphording and Nicolás Salamanca for helpful discussions at earlier stages of this project. I am also grateful to my supervisors Olivier Marie and Rolf van der Velden for their feedback, for helping me gain access to the Dutch administrative data and for contributing to funding this work (Statistics Netherlands CBS - Project number 8308). I also thank Fatima El Meslaki and her team at Statistics Netherlands for their support.
} 
administrative records from the Netherlands covering the universe of children in school between 2003 and 2016, a setting in which children exit primary school with a national standardized test score and a teacher recommendation regarding which track to follow in secondary school. In this setting, parents learn the test score of their child, but not their child's rank in class; only teacher know both scores and ranks of all children in class.

Although sibling spillover effects are notoriously difficult to estimate, a recent body of work has produced convincing evidence on the existence of these effects. Studies in this literature treat siblings as a particular type of peer, and have thus proposed research methodologies aiming to address the same three identification concerns as peer effect studies: 1) the reflection problem (Manski, 1993b, 2000), i.e. the inability to discern endogenous, contemporaneous effects arising in social interactions from exogenous effects arising from exposure to exogenous peer characteristics; and 2) selection, i.e. the inability to separate contextual effects from peer background characteristics, typically caused by endogenous peer group formation; and 3) common shocks, i.e. the inability to discern contextual effects from endogenous effects. So far, this literature has put forward two mechanisms. The first and main mechanism is direct transmission of human capital from one sibling, exogenously exposed to more or different schooling, to another sibling. Karbownik and Özek (2019) exploit cutoffs at the start of schooling as an exogenous assignment of sibling schooling experience; Altmejd et al. (2020) use university admission eligibility cutoffs in Chile, Croatia, Sweden and the United-States; Joensen and Nielsen (2018) use the eligibility of older siblings to an educational program; Nicoletti and Rabe (2019) use the standardized score of an older sibling in a national standardized test. The second mechanism is parental allocation of resources between siblings. Black et al. (2017) use the difference in exposure between a first and a second-born sibling to a disabled thirdborn sibling as a measure of parental attention. Autor et al. (2019) show that sibling spillovers contribute to patterns of social disadvantage.

This study contributes to the literature on sibling spillover effects in important ways. I propose a new methodology for estimating sibling spillover effects, which allows me to shed light on new mechanisms that have not yet been studied in this literature: teacher expectations and sibling motivation. I apply this methodology to the Netherlands, a unique setting which provides both high-quality standardized test scores and a subjective assessment of children by primary school teachers, 
which is ideal for studying the formation of teachers' expectations. ${ }^{2}$ I exploit the percentile rank of older siblings in their school cohort in a national standardized test at the end of primary school as a quasi-experiment on younger siblings. This strategy to identify sibling spillover effects solves the issues of reflection, selection and common shocks: 1) it solves the reflection problem by using older siblings' rank as a treatment affecting younger siblings before they enter their test year; 2) it further solves the selection issue by estimating rank effects at fixed absolute ability of the older sibling, and absorbing school-level heterogeneity for both siblings; and 3) it also solves the common shocks issue by absorbing heterogeneity at the level of cohorts within school for both siblings. I find that the percentile rank of older siblings has a strong and positive effect on the performance of younger siblings in language, Math and an overall test of ability. I also find that, after controlling for younger siblings' performance, the percentile rank of older siblings has significant influence on the subjective assessment that teachers have of younger siblings, in the form of a recommendation to pursue a given track in secondary school. The rank of older siblings in primary education has long-lasting impact of their younger siblings' educational trajectories: younger siblings enter secondary schools with higher relative ranks in their cohort, and are more likely to graduate from high school with a STEM specialization.

Long-lasting, older sibling rank effects also have the potential to exacerbate inequality of opportunity in education for disadvantaged students. Comparing native-born siblings to first and second generation migrant siblings, I find that older siblings with higher ranks provide a similar boosting effect on younger siblings' performance, but older siblings rank only increase the probability that teachers recommend academic education to native-born younger siblings. Comparing sisters and brothers, I find that older sisters' rank has a large positive effect on younger sisters' Math score, and a positive effect on teachers' probability to recommend the academic track for the younger sibling. Older brothers' rank, on the other hand, has a large negative effect on their younger brothers' Math score, but a large positive effect on language score, and null effects on teacher recommendations. These findings suggest that children with different demographic characteristics might be judged differently in the education system, with teachers forming expectations about children in a manner consistent with statistical discrimination. Because my study focuses on sibling spillover effects, my results can be interpreted as a lower bound of the impact of

\footnotetext{
${ }^{2}$ In later sensitivity analyses, I will conduct additional analyses excluding older siblings who are in the top 3 of their class and whose parents might be aware of their exact ranking.
} 
teacher bias in general on children's educational trajectory.

I further explore how educational policies can use sibling spillover effects to reduce inequality in education, at least reduce the negative consequences of teacher bias. Understanding how policies moderate sibling spillover effects on teacher assessment is important because it could prevent sibling spillover effects from exacerbating the educational consequences of socioeconomic disadvantage. I study two educational policies which change the incentives of teacher to provide biased recommendations and the incentives of parents to pressure teachers into inflating their assessment of children. The first policy changed admission procedures in secondary schools in 2014, increasing the weight of teacher assessment relative to standardized test scores in determining the track that a child follows in secondary school. I compare sibling pairs in which the younger sibling takes her test before v. after the reform which took place in 2014, and find that teachers became more likely to recommend the academic and general track after the reform. The second policy feature I study is the co-existence of geographic areas using lottery-based secondary school admission procedures, where teacher assessment has no impact, and standard admission procedures, where teachers emit assessments that are important for children's admission chances in secondary school. My effects on teacher subjective assessment are entirely driven by zones where standard admission procedures are used.

Because the methodology exploits the rank of children in school, this paper also contributes to a burgeoning literature on the importance of students' class rank for educational achievements, in two important ways. Firstly, this study is the first to investigate the importance of class rank between siblings on academic performance. Second, this study is the first to provide any evidence that teachers might respond to children's rank. The mechanisms suggested by the literature on the importance of ordinal rank in education are: 1) perceived own ability and academic self-efficacy; 2) increased effort, motivation, mental health; 3) responsive environment (parents, teachers, friends); 4) tracking in the school system; and 5) subsequent admissions in the school system. The literature has so far provided evidence in favor of the first two of these mechanisms. Elsner and Isphording (2017) and Murphy and Weinhardt (Forthcoming) show that students' self-concept, effort in school, motivation and mental health are positively affected by own percentile rank. Elsner et al. (2018) show that in the short run, university students adjust their effort allocation between subjects and choose their university major in response to their rank in tutorials. Denning et al. (2020) show that rank can also has longer run effects. These papers are not able to test for parental responses, teachers, friends, school tracking and admissions in subsequent schools. I provide unique empirical evidence on teachers' 
track recommendations upon exiting primary school, tracking and graduation in secondary school, university enrolments, and major choice.

Finally, this study also contributes to an important and emerging literature on the effect of teachers' expectations on their pupils' outcomes. The consensus that has emerged from this literature is that teacher bias is prevalent, and matters for test scores of children (e.g. Lavy, 2008; Alan et al., 2018; Carlana, 2019; Papageorge et al., 2020). ${ }^{3} \mathrm{An}$ important recent finding of this literature is, however, that some of this bias can be corrected either by revealing to teachers the extent of their bias (Alesina et al., 2018), or by changing incentive structures (Dee et al., 2019). My results provide the first evidence that teachers form expectations about a child after observing their sibling, and learn unequally across types of children. This study thus demonstrates that the experience that teachers accumulate with families has the potential to exacerbate inequality and the perpetuation of socioeconomic disadvantage in education; it also suggests that the undesirable consequences of teachers' formation of biased expectations can be reduced through the design of better incentives in the school system. ${ }^{4}$

\subsection{Institutional Setting and Data}

\subsubsection{Institutional Setting}

Figure 4.1 provides a simplified description of the Dutch educational system. Education in the Netherlands is compulsory from age 5 until age 16. Most children enter kindergarten at age 4 and start attending primary school two years later, in 1st Grade, around the age of 6 . Primary school takes six years to complete and ends around age 12, in 6th Grade, with a national standardized test covering mathematics, language and general academic ability. Primary schools must, by law, provide pupils exiting primary school at the end of 6th Grade with two key indicators of their academic ability: 1) an objective measure of academic ability in the form of a standardized test score externally graded, and 2) a subjective assessment given by the primary school teacher in the form of a track recommendation. This

\footnotetext{
${ }^{4}$ Other important studies on teacher grading bias include: Lavy and Megalokonomou (2019); Gershenson et al. (2016); Hanna and Linden (2012); Feld et al. (2016); Lavy et al. (2018); Lavy and Sand (2018); Burgess and Greaves (2013); Botelho et al. (2015); Diamond and Persson (2016); Cornelisz et al. (2019).
} 
recommendation is based on teachers' own observation of pupils' in class, and is meant to help parents and children choose the most appropriate secondary school track given the child's ability. A reform in 2014 changed the relative weight of these two factors in determining the track for children, I exploit this change in a later section to study how teacher incentives impact the response of teachers to siblings' ranks when assessing younger siblings. ${ }^{5}$

Figure 4.1: The Education System in the Netherlands

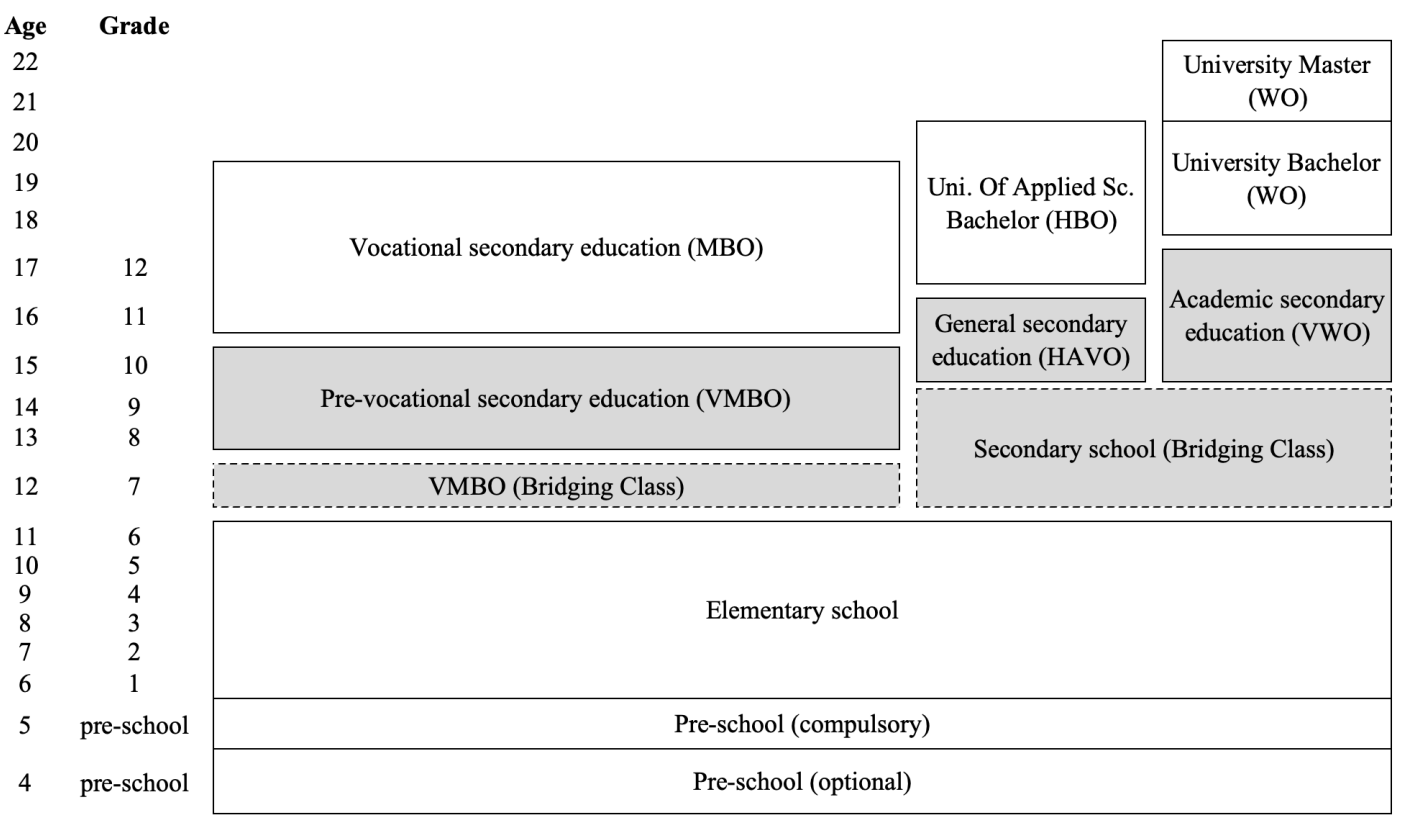

Note: Authors' figure. Simplified description of the Dutch educational system.

Students are then tracked in secondary school, and CITO test scores are used to help students and their parents in choosing the appropriate secondary school track for

\footnotetext{
${ }^{5}$ One test is overwhelmingly chosen by schools over the timeframe of my study. Since its creation in 2005, the CITO test has been given to all 6th Graders in over $80 \%$ of primary schools. Pupils take the CITO test between February and May during 6th Grade before exiting primary school, and receive their score in June when they are in the process of choosing their secondary school from 7th Grade onwards. The CITO test is designed every year by the CITOGroep, an independent private firm specialized in educational testing (akin to ETS in the US), which also grades the test independently from schools and the central government. The test is designed to be a test of cognitive ability with sections focusing on mathematics, language and general learning ability. Scores range between 501 and 550 points and are standardized such that the national annual average is always at 535 points. The CITOGroep recommends a mapping from pupils' scores onto high school types. The CITOGroep recommends the following mapping from CITO scores to tracks; by construction the shares of pupils in each bin are stable: 1) vocational track (from 501 to $532), 2$ ) either vocational or general track (from 533 to 536), 3) general track (from 537 to 539), 4) either general or academic track (from 540 to 544), and 5) academic track (from 545 to 550).
} 
each student's abilities and interests Students enter secondary school in 7th Grade and start with up to 3 years of common education to confirm students' academic ability. After these years of common education, students are tracked into one of three high school tracks: 1) academic, 2) general and 3) vocational. The vocational track ends in 10th Grade, and prepares students for a vocational secondary education program, which may or may not take the form of an apprenticeship. The general track ends one year later (11th Grade), and prepares students for professionallyoriented higher education. A general high school diploma constitutes the minimum requirement for enrolling into professional higher education programs, but does not make students eligible for university education. The academic track ends in 12th Grade; a diploma from academic high school constitutes the minimum requirement for enrolling into a research university. The academic track is generally considered to be more difficult than the general track, which is considered to be more difficult than the vocational track. After graduating from their high school track, students then pursue post-secondary education following their track. ${ }^{6}$

\subsubsection{Data}

\section{Education Registry Data from Statistics Netherlands}

I use data from the Dutch education registry linked to administrative records, allowing me to observe detailed demographic characteristics of pupils and families. I obtained the complete education history of children exiting primary school between 2003 and 2016, including detailed standardized test scores at the end of primary school, progression through the educational system and tracking, educational institutions attended, graduation outcome, post-secondary enrollment and major choice. Detailed characteristics about all household members include gender, date of birth, country of origin, migration background (including 1st- or 2nd-generation migration), household

\footnotetext{
${ }^{6}$ School choice is free in the Netherlands, in the sense that students can apply to any secondary school, irrespective of location, past grades and CITO score, or teacher track recommendation. School funding is fixed per student, which implies that secondary schools have no incentives to orient students towards one type of track over another, and therefore the admission process for secondary schools is largely non-selective. This system of free choice and unconditional funding has led to a concentration of secondary schools; the majority of schools is very large and offers all tracks. Roughly $3 \%$ of secondary schools are, however, selective in the sense that they do require CITO scores to be above some threshold, typically in order to offer a unique high-level academic track (Gymnasium). Although secondary schools decide eventually on the track that a pupil follows, parents and their child can influence this tracking outcome in two ways: 1) through discussion with the primary school teacher on the track recommendation the child receives, and 2) by choosing a school that only offers certain tracks.
} 
composition and residence at any point in time, as well as labor market and tax records.

These data are ideal for studying the effects of siblings' rank in primary school class on the expectations of teachers about younger siblings, since 1) I observe many cohorts in the same primary school over time; 2) standardized test scores allow me to compare cognitive ability within and across schools and cohorts; and 3) the data include the main teacher's track recommendations for secondary school, a direct measure of teachers' expectations that a child has the ability to graduate from a specific track.

I construct a dyadic dataset containing the universe of children who took the CITO test, with at least one older sibling who also took the CITO test in an earlier cohort. I describe in detail the data construction process in Appendix D.1. The full sample includes 975,256 unique children. I do not observe, however, teacher identifiers, which hinders me from constructing, e.g. teacher value-added or teacher bias measures using teacher fixed-effect estimates. Because of this limitation, I restrict the sample to younger sibling who are likely to have had the same teacher in primary school as their older sibling. I construct a dummy variable taking value 1 if three criteria are meet: 1 ) the younger sibling took the CITO test at least 1 year and at most 3 years after her older sibling, to avoid concerns of reverse causality and because the average turnover rate in primary school teachers is three years; 2) she took the test in the same primary school as her older sibling; and 3) in both siblings' cohorts, the primary school had fewer pupils taking the test than the yearly median school size, that is, the primary school can be considered small - close to one class per school. This set of restrictions leaves me with an estimation sample of $39 \%$ of dyads.

\section{Construction of Older Sibling Percentile Rank}

For every child $i$ in cohort $c$ of school $s$, I construct her absolute ordinal rank $R_{i c s}$ following Elsner and Isphording (2017) and Murphy and Weinhardt (Forthcoming), such that the child with the lowest score in his school cohort $c_{s}$ receives an absolute ordinal rank of 1 , and the child with the highest score receives a rank of $N_{c s}$, with $N_{c s}$ denoting the size of cohort $c_{s}$ in school $s$. To ensure that we capture the relative position of a child in the ability distribution of his school in a manner that is not sensitive to cohort and school size, I transform absolute ordinal ranks $R_{i c s}$ into percentile of ordinal ranks $\operatorname{rank}_{i c s}$, as follows: 


$$
\operatorname{rank}_{i c s}=\frac{R_{i c s}-1}{N_{c s}-1}
$$

With this transformation, the child with the highest score in his cohort now receives a percentile rank of 1 and the child with the lowest score a percentile rank of 0 . I construct percentile ranks for each subject and for the overall score.

\section{Summary Statistics}

Table 4.1 presents summary statistics of the initial sample, which contains 515,188 sibling dyads with complete information, of which $39 \%$ are likely to have had the same primary school teacher - the main selection criterion for remaining in my estimation sample. $78 \%$ of younger siblings take the CITO test between 1 and 3 years after their older sibling: $17 \%$ one year, $36 \%$ two years, and $25 \%$ three years after. $82 \%$ of younger siblings take the final primary school test in the same school as their older sibling. Children typically stay in primary school for 6 years, and seldom change primary schools. Importantly, I found no evidence that older sibling rank increases the probability of attending the same primary school; roughly half of siblings were registered in a small primary school. Overall, $39 \%$ of sibling dyads - roughly 200,000 dyads - meet all three criteria jointly, i.e. are likely to have the same teacher as their older sibling.

In the initial sample prior to imposing restrictions on the likelihood of having the same teacher, younger siblings are $50 \%$ likely to be female, and $83 \%$ live in households with both parents present. At the time when these younger siblings take their standardized test, $46 \%$ of them lived with one older sibling, $35 \%$ lived with two other siblings, $12 \%$ three other siblings, and $7 \%$ four other siblings or more; among these other siblings one at least is older. In my sample, families with more than two children are thus relatively rare. $19 \%$ of younger siblings are of migrant background. Household taxable income in the year prior to taking the test is 61,000 euros. $11 \%$ of pupils reside in one of the four largest cities in the Netherlands (Amsterdam, Rotterdam, The Hague and Utrecht). $73 \%$ of pupils with a test score have a recorded rack recommendation: $30 \%$ to pursue vocational education, $12 \%$ general education and $12 \%$ academic education; $9 \%$ a mixed recommendation towards general or vocational education, $9 \%$ academic or general education, and lastly $1 \%$ received an indistinct recommendation for all three tracks. ${ }^{7}$

${ }^{7}$ Note: Due to COVID-19, funding for this research project with Statistics Netherlands is tem- 
Table 4.1: Summary Statistics on Sibling Pairs, Years 2003 to 2016

\begin{tabular}{|c|c|c|}
\hline Variables: & Mean & Std. Dev. \\
\hline \multicolumn{3}{|l|}{ Characteristics of Younger siblings: } \\
\hline Female & 0.50 & 0.50 \\
\hline \multicolumn{3}{|l|}{ Household composition: } \\
\hline Two-parents household & 0.83 & 0.38 \\
\hline \multicolumn{3}{|l|}{ Number of siblings in same household (base: 2) } \\
\hline 3 & 0.35 & 0.48 \\
\hline 4 & 0.12 & 0.33 \\
\hline $5+$ & 0.07 & 0.25 \\
\hline \multicolumn{3}{|l|}{ Migration background (base: Native) } \\
\hline First-generation & 0.01 & 0.11 \\
\hline Second-generation & 0.18 & 0.38 \\
\hline \multicolumn{3}{|l|}{ Parental employment status: } \\
\hline Mother never in paid employment & 0.10 & 0.31 \\
\hline Father never in paid employment & 0.08 & 0.27 \\
\hline Household taxable income in year prior to CITO & 61,098 & 67,285 \\
\hline \multicolumn{3}{|l|}{ Number of years between siblings test-cohort (base: 1) } \\
\hline 2 & 0.36 & 0.48 \\
\hline 3 & 0.25 & 0.43 \\
\hline 4 & 0.12 & 0.32 \\
\hline 5 & 0.05 & 0.23 \\
\hline 6 & 0.03 & 0.16 \\
\hline 7 & 0.01 & 0.12 \\
\hline Same primary school as older sibling & 0.82 & 0.39 \\
\hline Small primary school & 0.50 & 0.50 \\
\hline Likely same teacher as older sibling & 0.39 & 0.49 \\
\hline Primary school in one of the four largest cities & 0.11 & 0.31 \\
\hline \multicolumn{3}{|l|}{ Younger siblings test scores: } \\
\hline Raw score in Dutch & 81.15 & 18.52 \\
\hline Raw score in Math & 47.99 & 14.61 \\
\hline Standardized CITO test score & 535.49 & 9.73 \\
\hline \multicolumn{3}{|l|}{ Teacher track recommendation: } \\
\hline Academic track & 0.12 & 0.33 \\
\hline Mixed-advice general or academic track & 0.09 & 0.29 \\
\hline General track & 0.12 & 0.33 \\
\hline Unspecific & 0.01 & 0.08 \\
\hline Mixed-advice vocational or general track & 0.09 & 0.28 \\
\hline Vocational track & 0.30 & 0.46 \\
\hline
\end{tabular}

Notes: This table presents summary statistics for the initial sample of 515,188 sibling dyads, based on education registry data from Statistics Netherlands over the years 2003 to 2016. 


\subsection{Empirical Strategy}

\subsubsection{Identifying Variation}

The goal is to identify the causal effect of the class rank of an older sibling on the academic performance, tracking outcomes and later human capital investments of their younger sibling. To do this, I exploit idiosyncratic changes in the cohort composition within primary schools over time, in a similar vein as Elsner and Isphording (2017); Denning et al. (2020) who estimate the effect of own class rank on own educational outcomes in a subsequent school.

Figure 4.2 displays the variation in older sibling rank that remains in my preferred specification, and that is used for regression analyses. For every older sibling's standardized score - from 501 to 550 -, older sibling percentile ordinal rank covers a wide range of values. With the average score of 535 , an older sibling's rank can range from lower $10 \%$ of the cohort to top $15 \%$ of the cohort. Naturally, this variation is smaller at the tails of the distribution of scores: with a score of 545 , the score from which unambiguously the older sibling is considered able to pursue the academic track, older sibling rank ranges from lower $45 \%$ of the cohort to top of the class.

To use this identifying variation, my preferred specification will always include school-by-cohort fixed effects of the younger sibling, two-way fixed effects for school and cohort of the older sibling, and dummies for each score in the older sibling test score distribution. I always cluster standard errors at the younger sibling school level. See Appendix D.2 for a detailed discussion on model specification and additional robustness checks on this issue.

Conditional on their primary school and test score, the cohort composition and therefore the rank of any child is as good as random since school entry is determined by date of birth and not amenable to manipulation. More importantly for my study, below I show that for any young child, their older sibling's rank from a previous cohort is conditionally random. ${ }^{8}$

porarily cut; whenever possible, this table will present summary statistics of the estimation sample. Also note that the 2014 reform introduced the possibility for teachers to revise their tracking recommendation; the results presented in this study reflect the final tracking recommendation.

${ }^{8}$ In tests not reported in this chapter, I found no evidence that older siblings' rank is systematically related to the probability that the younger sibling is in the same primary school. Table D.5 in Appendix D.3 shows that older sibling rank has a very small effect on the probability of being 12 at 
Figure 4.2: Remaining Variation in Older Sibling Rank in the Preferred Specification

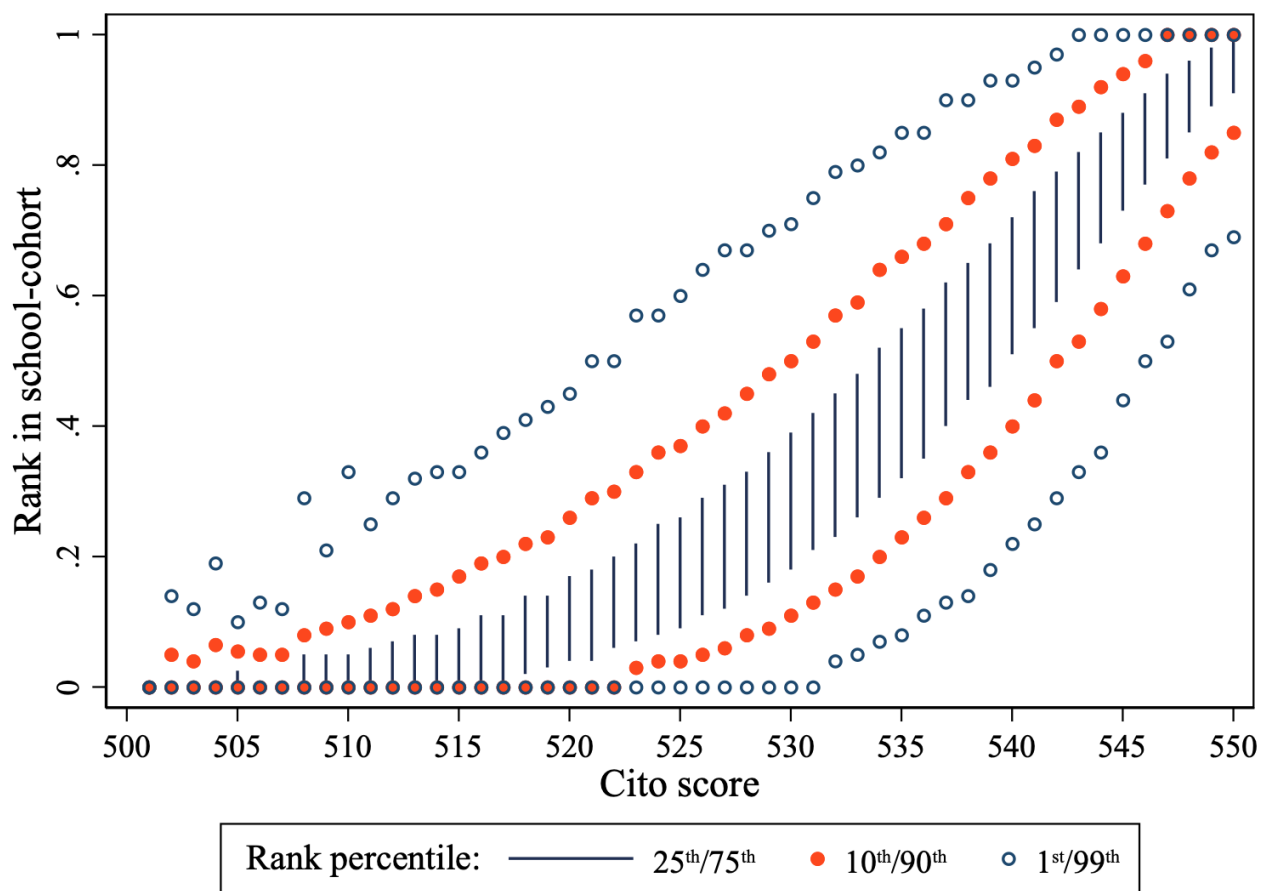

Note: This figure shows the remaining variation in older siblings' rank in the preferred specification. This specification regresses younger siblings' standardized test score on her older sibling's rank, including younger sibling cohort-by-school fixed effect, older sibling cohort fixed effect, point dummies controlling flexibly for the older sibling's standardized test score, dummies for the number of parents with a migrant background, birth year dummies of the younger sibling, and household taxable income in the year preceding the younger sibling's test. This figure is based on the sample of siblings who are likely to have had the same primary school teacher. For each standardized test score of the older sibling on the $x$-axis, a wide range of rank is observed in the data.

\subsubsection{Balancing Tests of Older Sibling Rank}

Table 4.2 shows that older sibling relative rank is as good as random for younger siblings. The table presents balancing tests on a series of baseline characteristics of the younger child. On the sample of children likely to have the same teacher, I perform these balancing checks by running my preferred specification with baseline

the time of the test, but I also show that my results on scores remain very similar when I restrict the sample to younger siblings who are 12 when taking the test, and who correspond to $80 \%$ of the sample of younger siblings. When I further restrict the sample to siblings in adjacent cohorts, and find that results on scores are identical whether younger siblings are or are not in their birth cohorts, while effects on teacher assessments become insignificant, due to low power. Table D.5 shows that sibling rank has no effect on the probability of having a teacher recommendation, and find a precisely estimated null effect. 
characteristics of younger siblings as outcome variable. Baseline characteristics are determined prior to the older sibling rank is given, such that any significant regression coefficient indicates some form of selection that could bias my main results.

Table 4.2 indicates that there is a small correlation between older sibling rank and household taxable income. This correlation occurs in the presence of some sort of school segregation. Given that this correlation remains after including school-bycohort fixed effects for the younger sibling and cohort fixed effects for the older sibling, this correlation likely indicates that some family-level heterogeneity remains as common shock.

There are two ways of accounting for this small correlation. The first way is to include balancing controls of family disadvantage in all regressions; I choose to include a variable for how many parents are of migrant background, family income in the year preceding the test, and the year of birth of the younger child. Including balancing controls implies a slight change in the interpretation of the results keeping constant the variables controlled for. An alternative method would be to use a family fixed-effects estimator, such as the one used in Black et al. (2017). The fixed effects estimator would come with two important complications for my analysis. First, the fixed effects estimator requires families with at least 3 CITO-test taking children per family; and in my setting, large families are a rare and selected sample; large families where children attend the same schools and are close in age is even rarer. Thus, the fixed effects estimator imposes further sample selection that may not be desirable for my analysis. Second, the fixed effects estimator identifies effects from variation within family between siblings scores and ranks. Thus, the fixed effects estimator would make older sibling rank effects even harder to interpret. For both these reasons, I prefer including balancing controls over the family fixed effects estimator. 


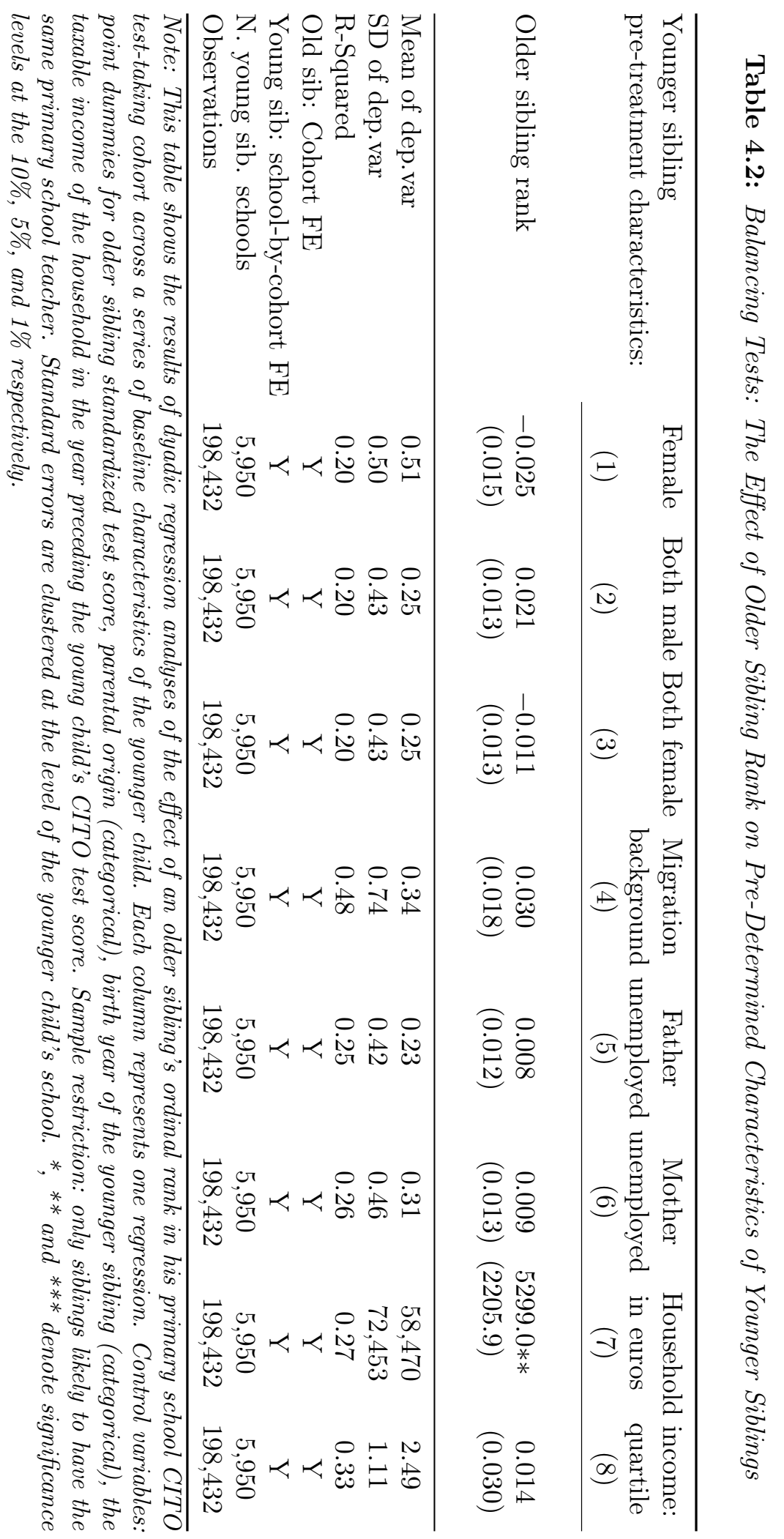




\subsubsection{Estimation Equation}

The goal is to estimate the effect of older sibling's rank on i) their younger siblings scores and ii) their younger siblings teacher assessment conditional on their performance.

I first estimate the effect of an older sibling's rank (child indexed 1 in a pair) on her younger sibling's test score (child indexed 2 in a pair), in school-cohort $s_{2}$, with the following reduced-form equation:

$$
\text { test } \text { score }_{2 s_{s}}=\alpha+\beta r_{1 s_{1}}+g\left(\text { test } \text { score }_{1 s_{1}}\right)+\gamma X_{2 s_{2}}+\lambda_{s_{2}}+\epsilon_{2 s_{2}}
$$

where $\beta$ is the coefficient of interest corresponding to the effect of the older sibling's rank $r_{1 s_{1}}$, within his school-cohort $s_{1}$.test score ${ }_{1 s_{1}}$ represents the standardized test score of the older sibling through the functional form $g(.) . X_{2 s_{2}}$ is a vector of individual characteristics of the younger sibling, in particular balancing controls (year of birth, migration background and family income). $\alpha$ is an intercept corresponding to average test scores in the younger sibling's cohort. $\lambda_{s_{2}}$ represents heterogeneity that is specific to the younger sibling's cohort and school, which is captured through a cohort-byschool fixed-effect - this includes for instance the mean and variance of ability of peers in the cohort, teacher quality, number of disruptive peers, etc. Finally, $\epsilon_{2 s_{2}}$ corresponds to an error term clustered at the school-cohort level. The thought experiment is thus to compare younger siblings who grow up with older siblings of a given level of ability, across all schools and cohorts, holding constant all school-cohort specific factors.

Next, I estimate the effect of an older sibling's rank on the teacher assessment of her younger sibling's ability, in school-cohort. To do this, I re-estimate the reduced-form Equation (4.2), where the outcome of interest is now the teacher track recommendation for the younger sibling, keeping constant the younger sibling's standardized test score, as follows:

track recommendation ${ }_{2 s_{s}}^{j}=\alpha+\beta r_{1 s_{1}}+g\left(\right.$ test score $\left._{1 s_{1}}\right)+\gamma_{1}$ test score $_{2 s_{s}}+\gamma_{2} X_{2 s_{2}}+\lambda_{s_{2}}+\epsilon_{2 s_{2}}$

Where track recommendation $2_{2 s}^{j}$ is a dummy variable taking value 1 if the teacher recommended track $j$ (Academic, General, Vocational). I estimate separate linear 
probability models using dummy variables as dependent variables, one per type of track recommendation, because rank effects are likely to affect non-linearly teacher assessments depending on the ability of the younger child. I estimate the effect of older sibling rank on the probability that a younger sibling is recommended a specific track $j$, given the younger sibling's overall performance in the standardized test. Thus, the rank coefficient estimate $\beta r_{1 s_{1}}$ can be interpreted as a bonus or a penalty at fixed performance of the younger sibling.

Equations (4.2) and (4.3) yield causal effects of older sibling's rank on younger sibling's outcomes to the extent that older siblings' rank is as good as random. The models represented by Equations (4.2) and (4.3) take care of potential concerns of endogeneity arising from unobservable characteristics driving both the rank of the older sibling and the score of the younger sibling. I take care of this endogeneity by 1) controlling for absolute ability of the older child, by including dummies for every point of standardized test score following Murphy and Weinhardt (Forthcoming), and 2) including a fixed effect for the school-cohort of the younger sibling, and 3) restricting our sample to siblings registered in the same primary school, and 4) including a fixed effect for the older sibling's cohort. In Appendix D.2, I discuss in details which fixed effects to include and which functional form to flexibly account for older sibling absolute ability.

\subsection{Main Results}

\subsubsection{The Effect of Older Sibling Relative Rank on the Performance of Younger Siblings}

Table 4.3 shows that older sibling rank has a significant and positive effect on younger sibling's performance. I show two sets of results. The first set of results in Panel A shows the effect of older sibling overall rank (controlling for overall points) on younger sibling overall performance (Column (1)) and subject-specific performance in Dutch (Column (2)) and Math (Column (3)). The second set of results in Panels $\mathrm{B}$ and $\mathrm{C}$ show the effect of older sibling subject-specific rank in Dutch (Panel B) and in Math (Panel C) (controlling for corresponding subject-specific points) on younger sibling overall performance and subject-specific performance in Dutch and Math.

In both sets of results, I find that older sibling rank has a significant and positive effect on both overall and subject-specific performance of younger siblings, which 
speaks to the robustness of the effect of older sibling rank on younger sibling performance. I also find consistently stronger effects of older sibling subject-specific rank on younger sibling overall score, compared to effects on the subject-specific performance of the younger sibling. That is, the effect of older sibling's rank in Math is larger on younger sibling's total score than on his Math score; the same goes for language rank and score. Nonetheless, I also find cross-subject effects of older sibling rank: growing up with a sibling who top of the class in Dutch also increases my test scores in Math. This suggests that siblings' influence is stronger within the same subject, but is not only subject-specific.

The effects I find are relatively large, and relatively similar to peer effects typically found in the literature using exogenously assigned classroom peers. Nicoletti and Rabe (2019) already note that sibling spillover effects in school are of comparable magnitude as peer effects. My effects are larger than those found in Denning et al. (2020) who study the effects of own class rank in primary school on later educational outcomes; they find that own rank in 3rd grade has a small effect on average on 8th grade scores, hiding large heterogeneity along the distribution of rank - that is, top ranks boosts later scores while bottom ranks depress later scores.

One reason why my results might be larger even though I consider rank effects between siblings is that I consider siblings who are between 1 and 3 years apart, while Denning et al. (2020) consider the same child 5 years after the rank is revealed. For comparison, I present estimates of the effects of own primary school rank on own later educational outcomes in Appendix D.4 - restricting the sample to older siblings in my estimation sample; my results are overall comparable to those found in the rank literature, but, perhaps unsurprisingly, these results are much more in line with those found by Karbownik and Özek (2019) who also study sibling spillover effects in education. They exploit cutoffs in the school starting age to estimate the effect of gaining an additional year of schooling on the standardized test scores of siblings between 3rd grade and 8th grade. They find a spillover effect of sibling additional human capital of up to 6 percent of a standard deviation, with a large SES gradient. They find that in low-income families, children who grow up with an older sibling born after the birth cutoff (who experience an additional year of schooling) have scores 14.3 percent of a standard deviation higher compared to children whose sibling was born right before the birth cutoff. They find however close to null effects for children in more affluent families. Although I cannot directly speak to this specific comparison on family income, my main effect ranges from 13.3 to 15 percent of a standard deviation. 
Table 4.3: The Effects of Older Sibling Rank on Younger Sibling Test Scores

\begin{tabular}{|c|c|c|c|}
\hline \multirow[t]{3}{*}{ Younger Sibling Outcome: } & \multicolumn{3}{|c|}{ Std. Test Scores: } \\
\hline & $\begin{array}{l}\text { Overall } \\
(1)\end{array}$ & $\begin{array}{l}\text { Dutch } \\
(2)\end{array}$ & $\begin{array}{l}\text { Math } \\
(3)\end{array}$ \\
\hline & Coef. Est. & Coef. Est. & Coef. Est. \\
\hline \multirow{2}{*}{$\begin{array}{l}\text { Older sibling rank: } \\
\text { Overall rank }\end{array}$} & & & \\
\hline & $\begin{array}{l}1.399 * * * \\
(0.245) \\
{[0.147]}\end{array}$ & $\begin{array}{l}1.595 * * * \\
(0.364) \\
{[0.092]}\end{array}$ & $\begin{array}{l}1.140 * * * \\
(0.323) \\
{[0.083]}\end{array}$ \\
\hline Dutch rank & $\begin{array}{l}1.426 * * * \\
(0.248) \\
{[0.15]}\end{array}$ & $\begin{array}{l}2.140 * * * \\
(0.374) \\
{[0.123]}\end{array}$ & \\
\hline Math rank & $\begin{array}{l}1.265 * * * \\
(0.251) \\
{[0.133]}\end{array}$ & & $\begin{array}{l}1.603 * * * \\
(0.324) \\
{[0.117]}\end{array}$ \\
\hline Mean of dep.var & 535.75 & 79.68 & 47.06 \\
\hline SD of dep.var & 9.51 & 17.34 & 13.72 \\
\hline Old sib: Cohort FE & $\mathrm{Y}$ & $\mathrm{Y}$ & $\mathrm{Y}$ \\
\hline Young sib: school-by-cohort FE & $\mathrm{Y}$ & $\mathrm{Y}$ & $\mathrm{Y}$ \\
\hline N. young siblings' schools & 5,950 & 5,950 & 5,950 \\
\hline Observations & 198,432 & 198,432 & 198,432 \\
\hline
\end{tabular}

Note: This table shows the results of dyadic regression analyses of the effect of older siblings' overall and subject-specific ranks on younger siblings' overall and subject-specific standardized test scores. Numbers in brackets correspond to effects size in SD. Each cell represents one regression. Control variables: point dummies for older sibling subject-specific test score (corresponding to rank - Panel A: total score; Panel B: language score; Panel C: Math score), the number of migrant parents (categorical), birth year of the younger sibling (categorical), the taxable income of the household in the year preceding the young child's test. Sample restriction: only siblings likely to have the same primary school teacher. Standard errors are clustered at the level of the younger child's school. ${ }^{*},{ }^{* *}$ and ${ }^{* * *}$ denote significance levels at the $10 \%, 5 \%$, and $1 \%$ respectively. 
I focus on the effect of older siblings' relative rank, on younger siblings' test scores, which rules out any direct effect of siblings' absolute ability. Thus, these results are important both for the literature on sibling spillover effects and for the literature on peer effects, since my results reveal new mechanisms through which sibling spillover effects occur (and peer effects in general, for that matter).

\subsubsection{The Effect of Older Sibling Relative Rank on Teachers' Subjective Assessment of Younger Siblings}

Table 4.4 shows that older siblings' rank matters for their younger siblings teacher assessment, above and beyond younger siblings own test scores. I present results for three different older sibling rank treatments — overall rank, Dutch rank and Math rank - on younger siblings' track recommendations, controlling for younger sibling's own corresponding test score (overall, Dutch or Math). Table 4.4 shows that older sibling rank increase the likelihood that teachers recommend younger siblings the Academic track, controlling for siblings' actual test score. The first row presents the effect of older siblings' overall rank on teacher recommendations for their younger siblings, controlling for younger sibling's own overall test score (and older siblings overall test score) (for the second and third row, replace overall by Dutch and Math respectively).

While the first row of Table 4.4 shows that older sibling overall rank has no effect on younger siblings' probability of receiving any particular track recommendation by their teacher, the second and third rows show that ranks in Dutch and Math have a positive and significant effect on younger siblings' probability of receiving a recommendation for the academic track (Column (1)). For a younger sibling with a given score in Math (Dutch), growing with a sibling who is top of the class as opposed to bottom of the class in Math (Dutch) increases the younger sibling's probability of receiving a top track recommendation by 2.4 (2.1) percentage point. As I only find this "teacher leniency" effect of siblings' relative rank for the academic track, my findings suggest that teachers rely more on older siblings' relative rank for younger siblings who would otherwise be marginal between the general and the academic track.

I further show in Table D.3 and Table D.4 in Appendix D.3 that the effects of older sibling rank on teacher track recommendations of younger siblings are robust across alternative ways of controlling for both older sibling ability and younger sibling ability. 
Table 4.4: The Effects of Older Sibling Rank on Younger Sibling Teacher Assessment

\begin{tabular}{|c|c|c|c|}
\hline \multirow[t]{2}{*}{ Younger sibling outcome: } & \multicolumn{3}{|c|}{ Track recommendation: } \\
\hline & $\begin{array}{c}\text { Academic } \\
\text { (1) }\end{array}$ & $\begin{array}{c}\text { General } \\
(2)\end{array}$ & $\begin{array}{c}\text { Vocational } \\
\text { (3) }\end{array}$ \\
\hline \multicolumn{4}{|l|}{ Older sibling rank: } \\
\hline Overall rank & $\begin{array}{c}0.010 \\
(0.008)\end{array}$ & $\begin{array}{c}0.008 \\
(0.009)\end{array}$ & $\begin{array}{c}-0.006 \\
(0.010)\end{array}$ \\
\hline Dutch rank & $\begin{array}{l}0.021 * * * \\
(0.008)\end{array}$ & $\begin{array}{c}0.008 \\
(0.009)\end{array}$ & $\begin{array}{c}-0.004 \\
(0.010)\end{array}$ \\
\hline Math rank & $\begin{array}{l}0.024 * * * \\
(0.008)\end{array}$ & $\begin{array}{c}0.007 \\
(0.009)\end{array}$ & $\begin{array}{r}-0.010 \\
(0.010)\end{array}$ \\
\hline Mean of dep.var & 0.12 & 0.12 & 0.30 \\
\hline SD of dep.var & 0.33 & 0.33 & 0.46 \\
\hline Old sib: Cohort FE & $\mathrm{Y}$ & $\mathrm{Y}$ & $\mathrm{Y}$ \\
\hline Young sib: school-by-cohort FE & $\mathrm{Y}$ & $\mathrm{Y}$ & $\mathrm{Y}$ \\
\hline N. young sib. schools & 5,950 & 5,950 & 5,950 \\
\hline Observations & 198,432 & 198,432 & 198,432 \\
\hline
\end{tabular}

Note: This table shows the results of dyadic regression analyses of the effect of an older sibling's rank in his primary school cohort on the track recommendation receives by the younger sibling. Each column-line represents one regression. Control variables: point dummies for older sibling score (first row: overall score; second row: Dutch language score; third row: Math score), younger sibling corresponding score, parental origin (categorical), birth year of the younger sibling (categorical), the taxable income of the household in the year preceding the young child's test. Sample restriction: only siblings likely to have the same primary school teacher. Standard errors are clustered at the level of the younger child's school. *, ** and *** denote significance levels at the 10\%, 5\%, and $1 \%$ respectively.

In Table D.3, I compare the effect of older siblings' rank overall, in Dutch and in Math, when controlling for the older siblings' overall test score and younger siblings' overall test score. These three treatments yield very comparable results to those shown in Table 4.4, where I instead control for older siblings' test scores matching the rank treatment (e.g. the effect of the overall rank, controlling for overall test score). That the effects don't vary when including a different set of controls for older sibling ability suggests that older siblings' ability is finely captured using point dummies, and does not vary importantly between subcomponents of the CITO overall test score.

In Table D.4, I compare the effect of one treatment - older sibling overall rank - when controlling for older siblings' test score overall, in Dutch only and in Math only, 
keeping constant the overall test score of the younger sibling as before. Table D.4 shows that the effect of older sibling overall rank on the probability that younger siblings receive a recommendation for the academic track is also robust, whether I control for older sibling' ability overall, in language or in Math. I find that for a younger sibling with a given score in Dutch (Math), growing with a sibling who is top of the class as opposed to bottom of the class in overall test score, at fixed older sibling score in Dutch (Math), increases the younger sibling's probability of receiving an academic track recommendation by 1.5 percentage point (1.8), and decreases the probability of receiving a vocational track recommendation by 1.9 percentage point (2.4). Overall, these results consistently suggest that teachers seem to be more lenient for younger siblings for whom they have received a signal that "academic ability runs in the family", an imperfect signal which relies on older siblings' relative rank in class.

These results suggest two ways that teachers update or form their beliefs about children's academic potential. First, I find a consistent "shielding" effect of siblings' rank on younger siblings: teachers seem to be more lenient with younger siblings whose older siblings have already shown high academic potential relative to their cohort, even after controlling for the direct effect of younger siblings' own performance. Second, I find consistently significant and "shielding" effects of older siblings' rank either when controlling for subject-specific ability of siblings or using subject-specific ranks of siblings as treatment variable, but not when considering siblings' overall test scores. These results suggest that when they formulate subjective assessment about younger siblings, teachers learn more from subject-specific rank information about older siblings than they learn from older siblings rank overall. This interpretation of the results is intuitive: teachers might be more able to form an idea about older siblings' true rank in class on a given subject, compared to older siblings' ability rank in the classroom across various skills. Teachers' perceived subject-ranks of older siblings are likely to correlate highly with their realized subject-ranks, because subject subcomponents in the standardized test are designed to measure subjectspecific ability. On the other hand, older siblings' overall standardized test score is a weighted sum across various subject tests. Teachers might not easily be able to compare pupils' ability across subject, and thus teachers perceived rank of older siblings overall might not correlate highly with realized rank in the overall test. 


\subsubsection{Long-Run Effects of Older Sibling Relative Rank}

Table 4.5 shows that older siblings' rank in primary school have a small, persistent impact on the educational trajectory of younger siblings. Controlling for the overall test score of younger siblings, I find that older siblings' primary school rank based on their overall test score has precisely estimated null effects on younger siblings' tracking outcomes and on attending the same secondary school as older siblings, yet I find that older siblings' rank has a significant effect on two longer-term outcomes of younger siblings. ${ }^{9}$

First, I find that older siblings' overall rank has a small positive effect (significant at the $10 \%$ level) on the relative rank of younger siblings in their cohort upon entering secondary school (Column (3)), which is a good measure of match quality between the child and the school. This effect could be interpreted in two ways. On the one hand, it could be that younger siblings enter the same secondary school as their older sibling - e.g. through priority listings or parental preferences. Since younger siblings enjoy higher scores as a result of their siblings' rank, they attend worse schools than they would in the absence of their older siblings. Thus, younger siblings enter their secondary school with slightly higher ranks. Column (1) shows, however, that siblings' rank has no significant effect on the probability of attending the same secondary school as the older sibling. On the other hand, that younger siblings enter secondary schools with a higher relative rank in their cohort could be a sign of common exposure to family environments with unobserved characteristics. Older siblings with top ranks could happen to be registered by their parents in school cohorts with lower level peers, and younger siblings similarly registered in secondary schools with lower level peers. Columns (4) and (5) show, however, that older siblings' rank does not affect the average quality of secondary schools in which younger siblings are registered, as measure by the average test score of younger siblings' cohort peers in secondary school, and by the share of top-scoring peers with CITO scores above 545 .

\footnotetext{
${ }^{9}$ In subsequent analyses, I plan to further explore whether older siblings' subject-specific ranks in Dutch and Math have a longer-term impact on younger siblings' outcomes. I suspect that older siblings' Dutch and Math ranks could have larger and significant effects on longer-term outcomes of younger siblings, because I found that compared to subject-specific rank, the overall rank of older siblings had smaller effects on younger siblings' test scores, and no discernible effect on teacher track recommendations for younger siblings.
} 


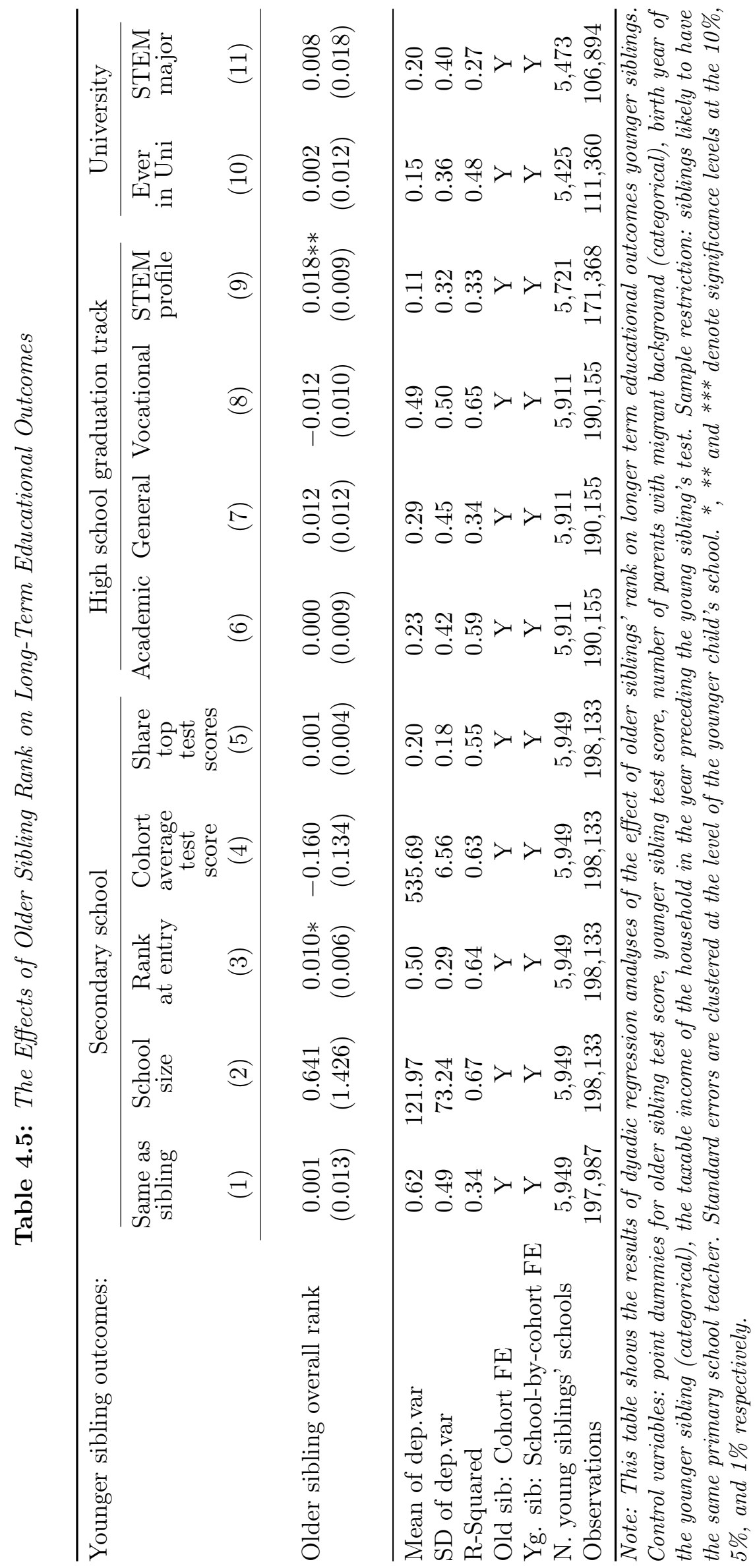


Second, older siblings' rank has a positive effect, significant at the $5 \%$ level, on the probability that younger siblings graduate high school with a STEM specialization: keeping younger sibling score constant, going from older sibling being the last to being the first in his primary school cohort increases the probability that the younger sibling graduates high school with a STEM specialization by 1.8 percentage point. This result is in line with Altmejd et al. (2020) who find that younger siblings are more likely to go to university, choose the same university and STEM majors when their older sibling passed university admission cutoffs and performs well at university.

Overall, my findings indicate that older siblings have persisting effects on their siblings, not only through their human capital, but also through their relative rank in their cohort.

\subsection{Robustness of Findings}

Recent research has shown that one's relative rank in a peer group is as good as random when researchers include absolute score fixed effects in regressions. Elsner et al. (2018) use data where students are randomly assigned to tutorials at university and find effects very close to those found by Murphy and Weinhardt (Forthcoming) in a setting where students are not quasi-experimentally assigned to cohorts. That relative rank effects are so close in settings with and without random assignment of students to peer groups suggests that relative rank effects (once controlling for absolute test score fixed effects) do not seem to suffer from endogeneity arising from selection into cohorts.

My results could nonetheless suffer from biases arising from two main sources of endogeneity: 1) testing with strategic delay; 2) selectively not taking the test or not having a recommendation. ${ }^{10}$

\footnotetext{
${ }^{10}$ Another form of sample selection could arise from endogenous fertility decisions. For my main results, younger siblings are already born when older siblings take their primary school tests, so endogeneity arising from younger siblings being present in the sample is not a concern. A remaining concern could still be that parents respond to the rank of the older sibling through fertility decisions related to a third child; one could imagine younger siblings receiving less parental investments if a third pregnancy is on the way or third child is born before the younger sibling takes his primary school exit test. In a next series of robustness checks, I plan on checking whether endogenous fertility decisions could affect my results.
} 
I first consider the possibility that parents could be strategically delaying their younger child from taking the standardized test after observing the rank of their older child. The motivation for strategic delay would be to increase the preparedness of younger children for the test. I look at strategic delay in two ways: 1) I directly estimate the effect of older sibling rank on the probability of being in one's normal age-cohort based on birth date; 2) I re-estimate my main results on scores restricting my sample to children who are in their normal age-based cohort. Table D.5 in Appendix D.3 shows that older siblings' rank increases slightly the probability of that younger siblings are in their school-age cohort, that is, turn 12 in the academic year of the test; this concerns $80 \%$ of younger siblings (Column (1)). This could indicate that younger siblings are somewhat slightly differently selected in response to their sibling's rank; this explanation, however is unlikely because as much as parents only learn the absolute score of their older child, but not the rank of their child in class. Only teachers know this rank because they know the scores of every child in class. The more likely explanation is that older siblings with higher ranks generate a small performance boost which might lead to slightly fewer grade retention. This performance boost, however, is small enough that my main results on scores are robust and stable when restricting the sample to younger siblings who are 12 when taking the test (see Columns (2) through (4)). I therefore conclude that strategy delay is not a major concern for my results.

I then consider sample selection. Older siblings' rank could affect the probability that a younger sibling takes the standardized test at all, or conditional on taking the test, that the younger sibling receives any teacher assessment. I further show in Column (5) that older siblings' rank also has no effect on the probability to be in the same primary school, or on the probability of having a teacher subjective assessment, these are precisely estimated null effect. I therefore conclude that selective attrition does not seem to be a concern for my results. ${ }^{11}$

\footnotetext{
${ }^{11}$ In a next iteration, I will perform permutation-based placebo tests. In each permutation, older sibling score will be kept constant but an artificial rank between 0 and 1 will be randomly assign. After running 1000 permutations, I will collect the average sibling rank effect across permutations, which represents the effect under the null hypothesis that sibling rank has no impact on younger sibling outcomes.
} 


\subsection{Mechanisms: Teachers, Parents and Siblings}

In the previous section, I show that older sibling rank has a significant and positive effect on younger siblings' performance and after controlling for their performance, on their teachers' subjective assessment through recommendations towards the academic track. For older sibling's rank to be as good as random, I control flexibly for older sibling's absolute ability, which excludes the possibility that my effects are driven directly by siblings' ability. This allows me to focus on three other channels through which older siblings' rank could matter for their younger siblings' test scores and their teachers' subjective assessment: 1) motivational effects between siblings, 2) parental investments, 3) teacher expectations.

Growing up with an older sibling who was best in his cohort, younger siblings could have higher motivation to work hard to be high-achievers themselves, which could explain not only the higher performance but also the improved teacher assessment of these younger siblings.

Although parents do not know exactly the rank of their child in class, they might receive some noisy signals about their child's relative rank in their cohort. These parents could therefore invest more in their younger child in response to their older child's rank, or be more engaged with the school or the teacher of their younger child. Parental responses could thus contribute to explain both the effect of older siblings' rank on the younger child's performance and on their teacher's assessment.

Lastly, it could also truly be that teachers simply form higher expectations for younger siblings whose older sibling was top of their class.

In this section, I conduct four heterogeneity analyses to test to what extent these three distinct theoretical mechanisms could be driving the main results. For each analysis, I explain the test, how the test highlights one specific channel, and how it shuts down other mechanisms.

\subsubsection{Lottery v. Standard Admission Rules and the Weight of Teacher Recommendations}

I first consider separately the effect of older siblings' rank on younger siblings' outcomes in the four largest Dutch cities - Amsterdam, Rotterdam, The Hague and Utrecht - against the remainder of the sample, mostly small towns and rural areas. 
The four largest cities are characterized by a very high population density. High population density has two consequences on incentives in the school system, both for parents and teachers. Firstly, secondary schools in these cities are often oversubscribed, such that lottery-based admissions are common practice (see De Haan et al., 2015; Oosterbeek et al., 2019; Ruijs and Oosterbeek, 2019). Under lottery-based admissions, track recommendations have little impact on the admission probability of a child, therefore there are no incentives to give any particular recommendation. Secondly, in highly density populated areas, any one secondary school receives many applications from several different primary schools, such that even in the absence of lottery-based admissions, the influence of any individual teacher's recommendation on the probability of admission of any given child is small.

Comparing older siblings' rank effects in the four largest cities against the rest of the Netherlands allows us to tell apart the motivational effect of older siblings' rank on younger siblings from the parental engagement and teacher expectation effects. If sibling spillover effects were driven by sibling role modeling effects alone, we should expect no heterogeneous effects of older sibling rank on teacher track recommendations since admission rules are unlikely to have any impact of the interactions between siblings. If sibling spillover effects are driven by teacher expectations and parental investments responses, on the other hand, we should expect that older sibling rank has no effects on teacher recommendations of younger sibling in the four largest cities, but has positive effects on teacher recommendations in small towns and rural areas.

Table D.6 shows that older sibling rank on test scores and teacher track recommendations are entirely driven by children residing in smaller urban areas and rural areas - for these children, older sibling rank increase the probability of receiving a recommendation for the academic track. In contract, I find precisely estimated null effects on track recommendations for sibling pairs residing in the four largest cities in the Netherlands. ${ }^{12}$

These heterogeneous effects between rural and urban schools suggest that teacher expectations and parental investments are important drivers of the effect of older siblings' ranks on teacher suggestive assessment of younger siblings.

\footnotetext{
${ }^{12}$ It could still be that schools in the largest cities experience larger cohorts and lower turnover than schools in the remainder of the country do. In a next step, I will try to narrow down schools that use lotteries within the largest cities v. schools that do not, and re-run analyses focusing on neighborhoods in which both types of schools co-exist.
} 


\subsubsection{Are Higher Ranking Older Siblings More Salient for Teachers?}

In classes in which older siblings are more noticeable, one could expect teachers to notice and remember them more. In these cases, one could expect teachers to respond more to older siblings' rank when assessing younger siblings. In comparison, it seems less likely that more noticeable siblings from teachers' perspective would make siblings more motivating for younger siblings, or induce more parental investments in response to their rank in class. These latter two channels would, thus, no explain the presence of heterogeneous effects of older sibling rank on teacher track recommendations of younger siblings. Thus, if older siblings' rank affects teacher subjective assessment through teachers learning, we should expect that older siblings ranks has a larger impact on teacher track recommendations when older siblings are noticeable.

I start by defining measures of how noticeable an older sibling is, that is, siblings who are more likely to stand out in their cohort. In Table D.7, I consider two ways in which older siblings might be noticeable in their cohort: 1) their relative rank, and 2) how many years apart are the two siblings. Firstly, older siblings might be more noticeable if they are at the top or bottom ranks of their cohort, compared to around the mean of their cohort's ability distribution. This would imply that the effect of older siblings' rank is non-linear. One simple way of testing this hypothesis is to split the sample of siblings and estimate piece-wise linear regression of my main specification. When I split the sample of siblings who are likely to share the same teacher into quartiles of older sibling rank, I don't have enough power to detect effects on teacher recommendations. A better way to run this analysis would have been to interact older sibling rank with my measure of older sibling salience.

Secondly, older siblings that are closer in age to younger siblings might be easier to remember for teachers. I now split the sample of siblings likely to have had the same primary school teacher by age difference, and estimate piece-wise linear regressions of older siblings' overall rank on teacher assessments of younger siblings. I only find a marginally significant positive effect on the probability of receiving a recommendation towards the vocational track for siblings who are two years apart. For the large part, however, I still don't have enough power to detect effects. Similarly, a better test would have been to estimate a model interacting the treatment variable with each dummy of distance in years.

Thirdly, in Table D.8, I consider an alternative way of studying teachers' expectations after their experience with older siblings and parents, which follows more closely the 
literature on teacher biases. I split the sample of siblings who are likely to have had the same primary school teacher into native- and foreign-born older siblings, and estimate separate regressions of the effect of older sibling overall rank on teachers' subjective assessments of younger siblings. I find that for native-born children, older siblings' rank has a small, yet significant and positive effect on the probability that younger siblings' teachers recommend the academic track; I find precisely estimated null effects on recommendations towards other tracks, which indicates that sibling ranks matter mostly for high ability younger siblings. For foreign-born children, however, I find no noisily estimated null effects on all recommendations, indicating that my regressions are low powered. This third test, however, is not sufficient to conclude that teachers alone adjust their expectations. Indeed, it could also be that native-born parents are more engaged and proactive in the school system compared to foreign-born parents who are likely to have less experience with the school system. In the next section, I look for evidence of parental investment changes in response to older siblings' ranks in school.

Thus, I find some suggestive evidence that teachers' expectations contribute to sibling spillover effects, yet further analyses are required to achieve clearer conclusions on the extent to which teachers import from their experience with a first sibling when judging a second sibling.

\subsubsection{Do Parents Adjust Investments Within the Family?}

Although parents do not know the exact rank of their older child in their primary school cohort, they might still get some noisy signals about this rank, and adjust their parental investments in subsequent children in response to this signal. Prior literature has found that parents respond to public investments through the schooling system, but the evidence is mixed regarding whether parental investments such as time and money complement or substitute public investments such as class size, teacher qualifications, and other indicators of school quality (e.g. Pop-Eleches and Urquiola, 2013; Fredriksson et al., 2016; Chang et al., 2020). Recent studies focused on sibling spillover effects suggest that parents do seem to adjust investments within the family (Landersø et al., 2019; Qureshi, 2018b; Black et al., 2017).

In my setting, parental responses to older siblings' rank could involve increasing time engaging with the primary school or pressuring the younger child's teacher into providing a recommendation for a desired track. A more direct and simple test of parental responses could be to compare younger siblings test scores when their family income increased. 
In the spirit of Fredriksson et al. (2016), I hypothesize that wealthier parents are better able to invest in their younger child in response to their older child's rank in class, compared to less affluent parents. If the effects on scores were mainly driven by siblings' interactions or teachers' expectations, we should expect no heterogeneous effects across family income. If parental investments are an important mechanism, we should expect large positive effect for younger siblings in rich families, and smaller effects for children in poorer families.

I exploit exogenous shocks to household income in the year when the younger sibling takes the test, arising from the presence of older siblings who are eligible v. ineligible to means-tested financial aid for higher education. For younger children growing up with eligible older children, parental resources are expected to be less taxed and thus parental investments in the younger child can be expected to increase, leading to higher test scores than in the absence of eligible older children.

In the Netherlands, means-tested financial aid for post-secondary education (aanvullende beurs) is a government payment of roughly 3,000 euros annually. I exploit discontinuities in the formula which determines eligibility to means-tested financial aid for post-secondary education over time. There are three reasons why I find it interesting to split the sample into families who did versus did not receive additional governmental transfers in the year preceding the younger sibling taking the test: 1) means-tested financial aid is a clear marker of socioeconomic disadvantage, since the income eligibility threshold is located roughly at the 30th percentile of the national distribution of household income; 2) eligibility starts at age 18 for all students enrolled in education, and applications to student finance are sent via the centralized applications platform of the educational system, thus most eligible students apply, and consequently selection into applying to financial aid and receiving it is minimal; and 3) means-tested financial aid of 3,000 euros annually relieves income constraints and thus might allow parents to shift resources from older siblings to younger siblings.

Table D.9 shows results splitting the sample into families where no other child was eligible to means-tested financial aid at the time when the older sibling was taking his test, and families where at least one child was already eligible; this allows us to compare the elasticity of younger siblings scores to relieving family income constraints. I find no detectable effects of older siblings' rank on younger siblings' scores, due to low power.

Thus, so far I don't have clear evidence of parental responses to older sibling rank affecting on younger siblings' scores. in a next step, I will run this analysis again in interaction models. In addition, I will also run a simple model of interaction with 
household income to estimate the income gradient of the effect of older siblings' rank on both test scores and teacher assessments. Lastly, I might consider other types of income shocks, such as eligibility to other welfare programs.

\subsubsection{Are Older Siblings Acting As Role Models?}

Older siblings' rank might matter through role model effects on younger siblings. I test this hypothesis by re-estimating my main effects on younger siblings' scores by gender of both siblings. Comparing the effect of older sibling rank on younger siblings' scores depending on the gender of both siblings tells us whether sibling interactions matter for sibling spillovers to occur.

For siblings who are around 12 years old, being of the same gender might be an important factor for spending time playing together, rather than helping each other out on homework. Another possibility is that older siblings exercise a role model influence on their younger sibling. Brenøe (2018) shows, for instance, that girls growing up with a brother are more likely to choose more female stereotypical studies compared to girls who grow up with a sister. Qureshi (2018a) uses data from Pakistan to show that additional human capital for older sisters increases the educational performance of younger brothers. Dahl et al. (2020) and Altmejd et al. (2020) find that the field of study of older siblings affects their younger sibling. Exploiting discontinuities in access to over-subscribed majors, Dahl et al. (2020) reveals that sibling spillovers in major choice depend on the gender-match of siblings. These findings echoes a recent body of work showing that exposure to female peers - e.g. in the classroom, in schools - contributes to gender differences in educational outcomes such as scores in Math and language and the likelihood of choosing a STEM major (Brenøe and Zölitz, 2020).

Table D.10 shows that the effect of older siblings' rank varies substantially depending on the gender composition of sibling pairs. Panel A shows effects when the older sibling is a brother, and Panel B when the older sister is a sister. In the first three columns of each panel, I show how the effects of older siblings' overall rank on younger siblings' scores interacted with a dummy taking value 1 if the younger sibling has the same gender. In Panel A, I find that when the older sibling is a brother, older siblings' rank has a 1.8 CITO-point stronger effect on performance in Dutch for boys compared to girls, and a 1.1 Cito-point weaker effect on performance in Math compared to girls.

In Panel B, however, I see that compared to male younger siblings, the rank of older 
sisters in the standardized test has a 1.6 CITO-point stronger effect on performance in Math female younger siblings, and a smaller effect on overall performance. That older sisters' overall rank affect younger sisters' scores through their performance in Math is suggestive of a role modelling effect of older sisters. That older brothers' overall rank, on the other hand, affects negatively their younger brothers' test score in Math and positively their test score in Dutch compare to younger sisters is suggestive of preferences for specialization between brothers. ${ }^{13}$

Perhaps more importantly, I also find that for girls compared to boys, their older sisters' rank has a stronger effect on their probability of being recommended the academic track by their primary school teacher. Column (4) of Panel B shows that younger sisters are 1.6 percentage point more likely than younger brothers to receive a recommendation for the academic track in response to the relative rank of their older sister. That this teacher leniency effect is only present for the academic track suggests, again, that teachers seem to use older sibling ranks as a signal for the younger sibling's ability only when younger siblings are of high ability. At the same time, I find that the relative rank of older brothers has no differential effect on younger sisters compared to younger brothers.

Thus, my set of results is consistent with both teachers updating expectations about girls' academic ability and older sisters acting as role models. The effect of older siblings' rank on scores seem to be driven largely by siblings affecting gender norms, which might in turn affect teachers' gender stereotypical norms.

\subsection{Policy Implications: Sibling Spillovers and Incentives in the School System}

In this section, I present suggestive evidence that sibling rank spillover effects could be related to incentives in the school system, which implies that educational policies could in principle be used to reduce the gap between advantaged and disadvantaged students in teacher assessments induced by sibling rank effects. I exploit a policy change in secondary school admission procedures which took place in 2014, to study how sibling spillover effects vary with incentives in the school system. My findings suggest that educational policies can improve incentives in order to reduce the

\footnotetext{
${ }^{13}$ I plan on exploring further this discussion in future work in this setting.
} 
potentially adverse ways that teachers form expectations about children based on their past experiences, in particular with their older siblings.

I exploit an educational reform, which changed the weight of teacher subjective assessment and standardized test scores for determining the tracks that pupils eventually pursue in secondary school. This reform of secondary school admission procedures was introduced in 2014 and decreased the importance of the standardized test score relatively to the primary school teacher's track recommendation. Before the reform, children would exit primary school with a report card containing their standardized test score and their primary school teacher's track recommendation. Whenever a child had a lower score than expected and corresponding to a lower track compared to their teacher's recommended track, parents could indicate to secondary schools that the test was not representative of the child's ability. From the academic year 2014-2015 onward, the track recommendation became the more important criterion in the report card of children exiting primary school. This reform therefore affects incentives in two ways: 1) it increased incentives for teachers to inflate subjective assessments of children, since assessments became more important for children's admissions to secondary schools; and 2) it also increased incentives for parents to put pressure on teachers to inflate teacher assessments. If the reform did indeed increase teachers and parents' incentives to inflate recommendations, then we should expect effects of siblings' rank to increase after $2014 .^{14}$

Table D.11 shows that after the policy change in 2014, older siblings' rank had a stronger positive effect on teachers' subjective assessment of younger siblings, compared to before the reform. This result suggests that teachers and parents do respond to older siblings' rank, when incentives are strong to do so. This implies that, conversely, policies in the school system can be designed to exploit sibling spillover effects in order to reduce socioeconomic inequality.

\footnotetext{
${ }^{14}$ For more details, see European Commission country note on the Netherlands, in particular: "As of school year 2014-2015 the advice on secondary education of the primary school is leading for admission to secondary education. The score on the attainment test is complementary and acts as an independent second item on admission.". Accessed on 16 July 2020 at https://eacea.ec.europa.eu/national-policies/eurydice/content/assessment-primary-education-32
} 


\subsection{Conclusions}

In this study, I propose new estimates of sibling spillover effects running through new, understudied channels of social interactions: teacher expectations about younger siblings, and a motivational effect of having successful older siblings. My findings reflect subtle changes in perceptions net of the direct effect of siblings' human capital.

I use Dutch administrative records covering the universe of pupils from primary school until university from 2003 until 2016, a unique setting in which children take a national standardized test upon exiting primary school, and in which primary school teachers emit a subjective assessment of each pupils' ability, which takes the form of a tracking recommendation for secondary schools. Because secondary school admissions are based on both the standardized test score and teacher assessments, I exploit quasi-experimental variation in the percentile rank of older siblings within their cohort, controlling for older siblings' exact test score. An advantage of this treatment is that it excludes parental responses as a key driver of sibling spillover effects: in this setting, teachers alone know the exact ranks of children, while parents only know the test score of their child.

I estimate the causal effect of older siblings' rank on the scores of their younger sibling in their primary school cohort and on the subjective assessment of their teacher. I find that older siblings' rank increases younger siblings' test scores, and that, after controlling for younger siblings' overall performance in the test, older siblings' rank in language and in Math improve teachers' subjective assessment of younger siblings. In addition, the rank of older siblings in primary has long-lasting impact of their younger siblings' educational trajectories: younger siblings enter secondary schools with higher relative ranks in their cohort, and are more likely to graduate from high school with a STEM specialization.

My results constitute the first evidence that sibling spillovers effects occur net of the direct effect of siblings' ability: through the expectations that teachers form, through the motivational effect of growing up with a successful sibling. An immediate broader implication of this finding is that qualitative aspects of family environments and how they interact with the school system matter a great deal for child development.

Yet my findings also indicate that sibling spillover effects have the potential to exacerbate the disadvantage of some social groups, or to maintain gaps in educational attainment between privileged and underprivileged groups. I find that although older siblings' rank equally increases test scores of younger siblings, teachers are 
more likely to recommend the prestigious academic track to native-born children, not children of migrant backgrounds. This finding suggests that teachers' learning is asymmetric. I also find that the gender composition of sibling pairs matters both for test scores and for teacher assessments: while older sisters' rank in Dutch and in Math increase the test scores of their younger sisters, older brothers' rank in Math increases the test scores in Dutch of their siblings and decreases their score in Math. In addition, older sisters' rank in Math increase the probability that younger sisters receive a teacher recommendation to pursue the academic track over younger brothers, this effect is absent for brother sibling pairs. These findings suggest that the motivational effect of siblings relates to sibling gender composition.

I further exploit two educational policies to investigate the extent to which teacher biased assessments can be neutralized through better institutions. I first exploit a policy change in 2014, which increased the weight of teacher assessments over test scores on the report card submitted as secondary school applications. Comparing younger siblings who took the test before v. after the academic year 2014-2015, I find that teacher became more likely to recommend the academic and general track in response to older siblings' rank. Second, I exploit the co-existence of geographic areas using lottery-based secondary school admission procedures, where teacher assessment has no impact, and standard admission procedures, where teacher assessments are crucial for children's admission chances in secondary school. My effects on teacher subjective assessment are entirely driven by zones where standard admission procedures are used. Both findings suggest that migrant younger siblings, for whom teachers are less lenient, could be made better off by reducing the impact of teacher assessments in secondary school admission procedures.

That older siblings' rank affect teachers' subjective assessments of younger siblings imply that for some disadvantaged groups, there might be returns to being top of the class in a worse school than bottom of the class in a top school. Yet, being of the class at fixed ability implies being surrounded by lower ability peers. Thus, the positive effects of higher ranks must be balanced against the loss in high-achieving peers.

Lastly, this study shows that such returns to being a big fish in a small pond are even larger than shown previously in the literature on rank effects in education. A direct policy implication of this result is that sibling spillover effects might truly constitute a social multiplier. As sibling spillover effects interact with incentives in the school system, sibling spillover effects amplify the impact of policies and their returns. 



\section{Conclusion}

Building on decades of economic research evaluating the impact of various educational policies on many outcomes of students, this $\mathrm{PhD}$ dissertation highlights the role of internal constraints in the persistence of socioeconomic disadvantage in education. The general ambition of this thesis is not to bring definitive answers regarding the existence and the extent of internal constraints in education-beliefs that students, parents and teachers might hold, which exacerbate socioeconomic gaps in educational outcomes. Rather, this dissertation sheds light on these emerging explanations and potentially new mechanisms through which socioeconomic disadvantage might persist in education, in spite of over a century of educational policies to improve access to opportunity.

I first present the key findings of this dissertation, and then discuss the limitations of this work, and suggest avenues for future research.

\section{New Approaches and New Answers to Old Questions}

\section{Chapter I: Quantifying Aspirational Poverty Traps}

The first chapter of this thesis indicates that there could exist aspirational poverty traps. A consequence of internal constraints preventing people from making optimal decisions, aspirational poverty traps are socioeconomic environments in which people systematically exert low effort and achieve low economic success. Since there is still little evidence on the economic relevance of internal constraints in education for the persistence of socioeconomic inequality, this first chapter addresses the crucial initial issue of knowing to what extent behavioral barriers are likely to matter in the long-run and at the macro-economic level. Chapter 1 indicates that theoretically, aspirational poverty traps are systematically related to intragenerational income mobility, and finds empirical evidence suggesting that a substantial part of the 
United States population could be in aspirational poverty traps.

Chapter 1 presents a new methodology to estimate the potential size of aspirational poverty traps in a given population, a decisive quantity suggesting the scope for achieving more equality of opportunity by lifting people's aspirations. Starting from a theoretical model describing how people's effort decision endogenously relate to their aspirations, I show that aspirational poverty traps at the micro-economic level generate threshold-like effects in intragenerational socioeconomic mobility at the macro-economic level. Thus, the existence of aspirational poverty traps explains an important and unexplained stylized fact: people in the middle of the income distribution enjoy high rates of income growth within their lifetime, while others remain stuck with little prospects of providing a better lifetime to their children. This study shows that the existence and location in the income distribution of these threshold-like structural breaks in intragenerational socioeconomic mobility indicate the size of aspirational poverty traps.

Conceptually, this study makes two key contributions. First, it expands previous literature regarding the existence of classical poverty traps to a radically new type of poverty trap, in which people exert low-effort and achieve low-outcomes, not because they lack material resources, but rather because they lack the "capacity to aspire" (Appadurai, 2004). This study indicates that theoretically, aspirational poverty traps at the micro-economic level translate into regimes of low v. high rates of income growth within people's lifetime, in other words, intragenerational income mobility. Thus, these findings suggest that low aspirations at the individual level can have far reaching consequences in the long-run, through low income growth within a person's lifetime which transmit to the next generation, and at the aggregate level, through large disparities between individuals achieving similar outcomes at radically different effort decisions. The existence of such types - those who realize that aspirations are endogenously formed, and those who don't - implies that we cannot hope to distinguish the deserving from the undeserving poor from simply observing either effort or outcomes. As aspirational poverty traps emerge from internal constraints, their existence is a paradigm-shifter: they imply a conceptual failure of the trade-off between equity and efficiency on which almost all social safety nets and anti-poverty programs rely.

The second way in which this study is innovative is its methodology to emphasize how aspirational poverty traps could be a key mechanism for the persistence of socioeconomic disadvantage. While studies revealing the presence of classical poverty traps rely on detailed measures of the institutional features preventing people from 
improving their material conditions, detecting the existence of aspirational poverty traps requires detailed experimental measures of aspirations and effort decisions, data that can reasonably be collected at the small scale of randomized controlled trials, but not at the national level in order to estimate the size of such traps and later design large-scale policies. Thus, the innovation of this study is to show how to estimate the size of aspirational poverty traps in the absence of detailed measures of aspirations linked to effort decisions.

This study also make an important empirical contribution. I apply this methodology to the United States using a widely-used panel survey, the National Longitudinal Survey of Youth, and I find that 5.5\% to $7.5 \%$ of the United States population could be stuck in an aspirational poverty trap, located just below the median household in terms of wealth, around the 37 th and 42 nd percentile of the distribution of wealth.

This study, lastly, also stresses that new theoretical tools are crucially needed to understand how internal constraints operate, and to renew traditional welfare policies. These tools are key to identifying specific policies and natural experiments which can potentially lift aspirations and relax internal constraints. Only then can we hope to estimate the extent to which behavioral constraints contribute to socioeconomic gaps in educational attainment.

\section{Chapter II: Early Discouragement from Uncertainty About Financial Aid}

The second chapter of this thesis investigates whether disadvantaged students could be discouraged early on in their educational career from participating in universitypreparing education, because of uncertainty regarding the future availability of financial aid. When a consequence of wrong beliefs, early discouragement is a specific way that beliefs can become behavioral barriers to opportunities in education. Chapter 2 shows that theoretically, uncertainty about student finance can in itself can create an early discouragement effect, and shows that, in practice, the Dutch recent reform of student finance could have discouraged a substantial part of the student population from enrolling in university-preparing tracks, which exacerbates achievement gaps.

Hypothesized yet largely understudied, uncertainty about financial aid could in principle discourage disadvantaged students from enrolling in university-preparing education. The first contribution of this study is to provide a theoretical framework that pinpoints how exactly uncertainty about future eligibility to higher education 
financial aid can create early discouragement effects, and could thus be a central mechanism through which socioeconomic gaps persist. The framework shows that the simple perspective of not receiving financial aid in higher education can make some students prefer educational paths with lower earnings prospects but no uncertainty about future costs of studying. This framework is crucial for understanding which students are most negatively impacted by uncertainty regarding financial aid, and why uncertainty alone is costly even for students who are eventually not impact by actual changes. In this sense, one could also consider that this study deals with background risk, a distinct literature which focuses on how people make decisions when their decision environment entails more or less risk, even when that risk does not apply directly to them.

A second contribution of this study is to combine the use of administrative data with policy announcements of a controversial reform of student finance. As much as administrative data does not collect direct measures of individual beliefs, in particular the state of students' knowledge about upcoming policy changes, exploiting exogenous, unexpected policy announcements prior to actual policy changes allows the researcher under reasonable assumptions to interpret changes in educational trajectories as responses to changes in expectations, not responses to changes in actual prices. While policy evaluation techniques typically assume that the timing of policy implementations is as good as random for individuals, this study shows that policy announcements can actually reveal substantial behavioral responses in anticipation of changes to come.

Beyond highlighting that the individual beliefs - students' and teachers' - matter for children's educational outcomes, this study also bears direct lessons for designing better, fairer and arguably cheaper student finance systems. As pointed by Lochner and Monge-Naranjo (2016) and more recently by Findeisen and Sachs (2016, Forthcoming), designing higher education financial aid that optimally trade-off equity and efficiency, relies crucially on achieving the perfect balance of incentives to study hard and attraction of talented students, and insurance of access for disadvantaged students. Chapter 2 indicates that access to higher education could be improved by reducing uncertainty in student finance; for example, by reducing early announcements before policies are actually ready to be revealed to the public, or by announcing early that disadvantaged students will be shielded. Recent work by Dynarski et al. (2018) also suggests that guaranteeing financial aid over multiple years could substantially improve access to college for low income students, compared to the standard year-to-year financial aid. 
Lastly, this study suggests that there could be an interesting tension between the political cost of imposing reforms without announcements, and the economic cost of policy uncertainty which arises from receiving too much and too early information about potential policy changes.

\section{Chapter III: Lifting Aspirations for Better Educational Outcomes}

The third chapter of this thesis investigates whether classroom assignment policies can improve aspirations, and improve test scores as a result. Chapter 3 indicates that high-achieving peers not only improve test scores, but also make children exert less school effort, while increasing their aspiration to go to university, and increasing the time they spend with their parents. Most importantly perhaps, this study shows overall that peer effects in test scores are not explained by intermediate effects on students' effort, aspirations and parental time investments.

This study makes several key contributions to the literature. Conceptually, this study builds on the education production function framework. In particular, we learn from Heckman and Pinto (2015) that in order to truly understand the mechanisms through which peer effects operate, we need to combine experimentally assigned peers with a wide range of potential educational inputs, and perform a formal mediation analysis. While recent studies in the literature on peer effects have only been able to either exploit data from settings with quasi-experimental assignments to peers but few inputs, or survey data with many inputs but no exogenous assignment to peers, the first contribution of this study is to show in the same setting: i) a causal effect of being exposed to higher-achieving peers on students' own academic achievement; ii) many potential factors that affect academic achievement; iii) causal effects of being exposed to high-achieving peers on these intermediate factors.

Methodologically, this study also presents a new algorithm combining randomization inference techniques, a well-known economic indicator of market concentration and finite mixture-modelling to detect schools which randomly assign students to classes from schools that do not. Indeed, the empirical findings of this study rely on data from Taiwan, where a national mandate dictates that middle school children must be randomly assigned to classes within their school. Because we found evidence of violation of random assignment in our data, we designed an algorithm to exclude clusters of schools using systematic assignment rules, such as ability sorting or art classes. This algorithm thus, opens the door to using many more datasets which fail to pass classical test of random assignment of students to classes, as well as improving estimate precision in datasets where random assignment has been found 
using under-powered tests.

Empirically, we estimate the impact of classroom peers on a myriad of educational inputs, some previously identified or hypothesized as potential mechanisms of academic peer effects, and several others which have not yet been studied in the peer effects literature - parenting styles and parental investments in particular. We find that although high-achieving peers significantly decrease student effort, increase aspirations and increase parental time investments, a mediation analysis reveals that these intermediate input responses don't explain why high-achieving peers increase student test scores.

Chapter 3 also indicates crucial avenues for future education policies. While the consensus in the peer effects literature is that reassignment policies have unforeseen consequences, this study shows several reasons why this might be the case -conceptually and methodologically. Peer assignment policies such as tracking or school admission policies can increase test scores while having several simultaneous effects on educational inputs which may or may not affect test scores in the short-run. This implies that in principle, school or classroom assignment policies enforcing exposure of disadvantaged students to high-achieving could be a low-cost way to reduce socioeconomic gaps in educational achievement, while nurturing the aspirations of disadvantaged students.

\section{Chapter IV: Teachers' Biased Beliefs and Inequality of Opportunity}

The fourth chapter of this thesis shows that behavioral barriers can also emerge from interactions between families and the school system. Chapter 4 reveals that growing up with a higher-ranked older sibling in primary school test scores, younger siblings achieve higher test scores, and receive better assessments by teachers above and beyond their performance.

The first empirical contribution of this study is to show that children's relative rank in school also impacts their younger siblings. This finding extends those of an emerging literature which shows that beyond actual performance, the relative rank of a child in school impacts his long trajectory in the short and in the long run. This literature is still in its early days, yet a consistent pattern has emerged in a variety of settings and at various levels of education: higher ranks in class increases test scores, boosts academic self-efficacy and attracts students to specialize in courses where they have highest ranks. This study is the first to show that students' rank in class also impacts their younger siblings, and the teachers of their younger siblings: 
younger siblings who grow up with higher ranked older siblings enjoy higher test scores, and controlling for their test scores, they receive more lenient subjective assessments from their teachers, and are more likely to graduate high school with STEM, a specialization with better labor market prospects.

The second contribution of this study is to shed light on two new mechanisms through which socioeconomic disadvantage can persist: teacher biased expectations about pupils' ability, and motivational effects from the relative performance of older siblings in the schooling system. Exploiting the relative rank of older siblings in primary school is a simple, common and good experiment to estimate teacher bias because older sibling's relative rank is as good as random for the younger sibling, conditional on the older sibling's absolute test score. Importantly, while previous studies have discussed the potential role of teachers in explaining why rank effects occur, this study is uniquely able to show that teachers do respond to children's rank. Yet, disadvantaged pupils are less likely to have high test scores, and thus less likely to have high ranks in class, such that their younger siblings are less likely to enjoy the positive spillovers of growing up with successful siblings.

This study delves deeper even into mechanisms and shows that teachers learn from older siblings' rank in a manner consistent with statistical discrimination: while native-born children enjoy the positive spillovers of older siblings' ranks on test scores and teacher subjective assessments, children with a migrant background only enjoy the effect on test scores, but not in teacher assessments; and as much as private information could explain that teachers are less lenient for migrant children than for native-born children, such explanations fail at explaining gendered effects.

This study calls for building institutional mechanisms to reduce the potentially negative impact of teacher bias on children's educational trajectories. Indeed, while the actual test score of the older sibling can reveal information about the ability of the younger sibling, the relative rank of the older sibling contains no information about the younger child's ability, since it entirely depends on the cohort composition of the older sibling. One possibility is to define school admission procedures that rely on test scores and less on teacher recommendations; some suggestive findings of this study indicate that teacher bias in subjective assessments declines or disappear when their weight in the secondary school admission procedures declines. 


\section{Limitations and New Perspectives}

\section{Improving our Understanding of Aspirational Poverty Traps}

Although Chapter 1 proposes an original methodology to estimate the potential size of aspirational poverty traps - a quantity that is directly usable for policy makers - , the exact mechanisms through which aspirational traps appear remain unclear. While mechanisms may not seem to be of primary importance for the design of policies, failing to understand the mechanisms driving aspirational poverty traps can ultimately lead to costly policy failure. Additional work is crucial to identify the mechanisms driving aspirational poverty traps.

A first avenue for research is to ensure that aspirational poverty traps are truly driven by low aspirations - low effort choices. If the structural breaks we find are systematically related to other factors than aspirations, then designing interventions to lift aspirations could prove costly and useless. We conduct robustness analyses to show that our breaks are not systematically associated to cognitive traits such as selfesteem and locus of control, yet our methodology also does not allow us to exclude definitely the presence of confounding factors affecting individual decisions. To isolate the specific role of aspirations in individual income growth, future work must rely on research designs focused on more local shocks to aspirations, as recommended by Dalton et al. (2016) themselves: comparing the aspirations and later life outcomes of individuals just above v. below eligibility to social security payments, or comparing the aspirations of students assigned to better v. worse classroom peers. I pursued the latter project in Chapter 3 using data from Taiwan, and I plan on pursuing the former project using social security data from Australia in my post-doctoral research.

A next avenue for research is to understand to what extent structural breaks are driven by individual behavioral responses to institutional features. Conceptually, it is indeed difficult to distinguish institutional features themselves such as tax breaks, or college admission cutoffs from endogenous changes in aspirations induced by the presence of such features. Further research could entail geographical and time comparisons. For example, comparing the size and location of structural breaks in the income distribution across multiple locations or countries could help identify the role of specific institutional features driving aspirational poverty traps, exploiting exogenous changes affecting their opportunities. For example, Beaman et al. (2012) study how the aspirations of parents for their daughters changed following a policy 
in India allowing women to sit on local councils. An advantage of comparing several geographical contexts - counties, regions or countries - is that it could also indicate whether our results are specific to the setting we study.

These limitations largely stem from the methodological choices we made for this study, in response to data limitations. While the study is appealing for its simplicity and its seemingly small data requirements, the main reason why our methodology does not allow us to exclude potential confounding factors or identify mechanisms is that it hinges on an indirect test for the presence of aspirational poverty traps: we identify the presence of aspirational poverty traps from their consequences on intragenerational mobility, that is, the presence of structural breaks in the relationship between initial wealth conditions and later life wealth. A direct test for the presence of aspirational poverty traps could entail, for example, finding a treatment lifting aspirations, and comparing the long-run outcomes of individuals treated v. untreated. A first example of such more direct test is Chapter 3, using classrooms peers as a potential treatment lifting aspirations. Although a promising avenue for future research, such studies will need to rely on treatment which have been shown to improve aspirations, and such treatments are yet to be found.

Another important limitation of this study is the data it uses. We use the National Longitudinal Survey of Youth 1979, a widely used and well-known cohort study from the United States. The NLSY is appealing because it contains detailed information about individuals throughout their lifetime: family composition and parental earnings during youth, personality traits measured early in life, personal earned and unearned income from first labor market entry almost until retirement. In spite of this wealth of data, the NLSY only contains at best 12,000 individuals, which limits the scope for analyses exploiting detailed geographical variation or time variation. Further work will almost certainly use additional data sources, for example data from the Opportunity Atlas data showcased in Chetty et al. (2020).

\section{Investigating How and Why Financial Aid Uncertainty can Discourage Students}

While Chapter 2 presents the reader with evidence that uncertainty of financial aid can discourage some students from enrolling in university-preparing education, our estimates are based on cohort comparisons only - past cohorts are used as counterfactual group for the cohorts treated with policy announcements. This chapter estimates the effect of exogenous, unexpected policy announcements to study how students change their educational orientation in response to increasing 
uncertainty about future higher education financial aid. In principle, the treatment consists in being exposed to information about the pending reform, such that the ideal research design would have compared in the same cohort, students who by chance received the information to those who, by chance, did not. Yet, policy announcements took place simultaneously at the national level, such that all students in a given cohort are either treated or not treated when starting secondary school tracks. To argue that our estimates identify a causal treatment effect of exposure to information, our best strategy is thus to discuss extent to which policy announcements were as good as random for students, and to what extent past cohorts are comparable to treated cohorts.

Additional analyses are, however, necessary to ensure that our results are not confounded by factors such as cohort-specific dynamics, or macro-economic conditions. A first, simple approach, could be to exploit the large number of observations in administrative data, and compare students at a much finer level of aggregation: for example, comparing students within schools by estimating models with school fixed effects, could improve the comparison of cohorts over time; similarly, comparing students with the exact same test score. An issue with this approach is that with each additional control variables, results loose interpretability while endogeneity arising from omitted variables remains largely unaccounted for. Another approach is to offer multiple comparisons to control groups within each cohort, although imperfect: ineligible students to means-tested aid, students ineligible to higher education, foreign students ineligible to student finance altogether, older students or very younger students. To isolate the pure effect of uncertainty in financial aid on student outcomes, further research should therefore focus on identifying settings amenable to more robust research designs.

An additional difficulty with Chapter 2 is that identifying the mechanisms through which students respond to uncertainty about financial aid remain unclear, largely due to limitations inherent to administrative data. Indeed, while we build intuition using our theoretical framework, we cannot exclude that our findings are driven by genuine changes in preferences induced by policy announcements, rather than income constraints made salient by policy announcements. In a section dedicated to robustness, we discuss the possibility of broader changes across cohorts, including preferences for labor supply over borrowing. Identifying with precision why the policy announcements we study created a discouragement effect will require, however, not only additional data such as preference measurement, borrowing behavior and labor supply, but also a longer time horizon. Alternatively, one could rely on structural modelling approaches, in which one can calibrate models using past 
data and assigning values to parameters such as risk preferences, and see whether the calibrated model predicts accurately educational outcomes following policy announcements.

Lastly, this study is based on data from the Netherlands, a specific setting in which student finance has been in place since 1986 and is perceived as a right. Thus, our empirical findings could be context-specific to a large extent. Additional work in other settings could help establish the robustness of the discouragement effect induced by uncertainty about student financial aid.

\section{Exploring How Classroom Peers Affect Student Outcomes}

Chapter 3 follows up on some of the issues identified from Chapter 1. In particular, Chapter 3 explores the impact of randomly assigned classrooms peers on aspirations of students, and the extent to which classroom peers affect test scores through their impact on aspirations. While this study solves some of the core limitations of Chapter 1, this study is not exempt of other issues.

One of the key limitations of this study is the linear-in-means model of peer effects that it relies on, and the conceptual assumptions it implies. Modeling peer effects is not obvious: there is no consensus in the literature as to how theoretically peer effects operate, and there is consequently no consensus either regarding the econometric modelling assumptions required to capture peer effects. In the linear-in-means model, the researcher implicitly assumes that the benefit of high-achieving peers is spread equally across all students - an assumption that has already been disproved on numerous occasions. Yet, the linear-in-means model remains the most common and parsimonious model of peer effects (see Sacerdote, 2014). We further discuss the possibility that our absence of mediation is explained by heterogeneity of peer effects - either in the sense that exposure to high achieving peers benefit children of various abilities differently, or in the sense that exposure triggers heterogeneous behavioral responses. While our evidence indicates that our findings are not driven by such heterogeneity, further analyses are required to fully explore this possibility.

Our analyses could also benefit from additional data. Recent advances in econometric theory have been instrumental for our empirical analyses to not suffer from a lack of precision that would have otherwise been a likely concern: Jochmans (2020) proposed a new sorting test solving an important power issue in the state-of-the-art test of Guryan et al. (2009), Young (2019) explained in detail how to improve permutation techniques and report randomization inference statistics, and Clarke 
et al. (2019) recently proposed a Stata package to conduct inference correcting for multiple hypothesis testing. While our main results don't suffer from a major lack of precision, additional observations could be helpful for our discussion of heterogeneous mediation analyses.

Our analyses could also benefit from observing more inputs. We could be unable to mediate our peer effects with any of the inputs considered because we are missing on key drivers of academic peer effects. Even though this seems unlikely, we cannot exclude this possibility. For example, it could be that our inputs fail at capturing social pressure in the classroom, a factor which recent research has shown to be an input driver of effort responses of students Bursztyn et al. (see e.g. 2019).

A natural concern for our findings is that they could reflect norms and institutions that are specific to Taiwan. For example, that parents invest a lot of money in tutoring or that the pressure is so high for students to perform in schools could be highly context specific. While the literature on peer effects in education is at a mature stage, and the broader literature on interdependent decision-making is a very active one, some of the crucial advances in the broader empirical literature on peer effects has come from new datasets in which peers are experimentally or quasi-experimentally assigned (see e.g. Bursztyn et al., 2014; Oster and Thornton, 2012). In education, however, data originating from such settings remain limited and not easily accessible. Thus, although our study exploit one such setting, it is not clear to what extent our findings teach us about peer effects in general, beyond Taiwan. Using data from the 1999 Trends In International Mathematics And Science Study (TIMSS), we show that Taiwan seems comparable to other countries on many commonly used indicators of schools systems, suggesting that our results in Taiwan may hold in many other settings.

Lastly, as we have already pointed at in our discussion on statistical power, many econometric advances are still to invent to enable researchers to use more data. Although the Fishing Algorithm we develop in this study is essential to eliminate schools where random assignment does not hold, we have also discovered that some findings can vary importantly depending on few outlier schools. Thus, our next work seeks to gain further understanding of the statistical properties of the standard econometric tools used in empirical peer effect research. 


\section{How and Why Teachers' Expectations Affect Student Outcomes}

Chapter 4 indicates that teachers' expectations about children's potential is systematically biased - in particular, teachers respond to older siblings' relative rank in primary school test scores. As much as this finding can seem intuitive, additional analyses are crucially needed to fully grasp why children's relative rank in primary school test scores also affects their younger siblings. The mechanisms behind these effects are complex. In future work, I expect to be exploring in further details two specific directions of mechanisms.

On the one hand, building on the literature on rank effects in education, it seems that top ranked children can be expected to build more self-confidence, which may or may not affect their siblings. The exact way this spillover could occur remains unclear: growing up as the younger sibling of a top student could discourage younger siblings, it could also foster a healthy competition. The magnitude and sign of such mechanisms could also depend non-linearly on the ability of younger siblings. A particular avenue for future research, which I have only begun to brush over, is the gender and ability composition of sibling pairs. On the other hand, building on the emerging and burgeoning literature on sibling spillover effects, it seems likely that parents also respond to information about the rank of their child. Previous literature has shown that parents often optimize parental investments - for example, Black et al. (2017) show that children who grow up with a disabled sibling benefit from less parental time. Future work will further explore parental responses to sibling rank and optimization strategies between siblings.

One of the important limitations of this study is also its strength: the administrative data it uses. On the one hand, exploiting administrative education records linked to birth records and family records has provided this study with a large sample of 530,000 sibling pairs, allowing us to detect small effects with a high degree of precision. On the other hand, the data remain limited in two ways. First, detailed information regarding any single individual is scarce and bound to information collected for administrative purposes, leaving little hope to identify finer mechanisms such as perceptions, aspirations, parental beliefs and child non-cognitive skills.

The second, and perhaps more important data limitation of Chapter 4, is that its findings depend crucially on observing primary school test scores. Since primary school test scores are only reported from 2003, the findings of this study remain relatively short term, and yet, the empirical relevance of the sibling rank effect for inequality of opportunity depends on whether and how much sibling rank effects 
affect longer term outcomes, such as earnings and employment.

Lastly, one could also offer alternative interpretations of this study's findings. I interpret the effect of siblings' rank on teacher subjective assessment as teacher bias, essentially because after controlling for the absolute test score of both siblings, that teachers remain more likely to recommend the academic track in response to higher sibling ranks is akin to a boosting effect of siblings rank in the absence of any additional human capital. Yet, it could also be that teachers are not biased but rather observe non-cognitive skills of younger siblings, who benefit from growing up with higher ranking older siblings: if older siblings with higher ranks are more confident and enjoy a better experience in secondary school, younger siblings could themselves develop non-cognitive skills which teacher think make them better suited for the academic track. I perform additional analyses comparing migrant children to native children on the one hand, and girls and boys on the other and find evidence suggesting that the results are not solely driven by a motivational effect of sibling rank, but further analyses are necessary making creative use the limited data available.

A second alternative explanation is that parents of higher ranks children push teachers to better assess their younger children. This could explain why the sibling rank effect on teacher assessment disappears once admissions rely less on teacher advice - when comparing the rank effect before and after a reform secondary school admissions, and comparing geographical zones using admissions with lotteries v. not. However, if parents were indeed pushing teachers to give academic recommendations to their younger children in response to their older child's rank, we would expect this effect to be particularly strong around the cutoff for the academic track. Yet I show in additional analyses that this is not the case. While we cannot exclude that some parents do know their child's rank, and could drive some of the results, further analyses excluding parents who could know the rank of their child (e.g. excluding the top and bottom 3 ranks in class) - should help further understand whether and to what extent parental behavioral responses are likely to contribute to the effects. 


\section{References}

AbdulkadiroĞlu, A., Angrist, J. and Pathak, P. (2014). The elite illusion: Achievement effects at boston and new york exam schools. Econometrica, 82 (1), $137-196$.

Agostinelli, F. (2018). Investing in children's skills: An equilibrium analysis of social interactions and parental investments. Unpublished Manuscript, Arizona State University.

-, Doepke, M., Sorrenti, G. and Zilibotti, F. (2020). It takes a village: the economics of parenting with neighborhood and peer effects. Tech. rep., National Bureau of Economic Research.

Ahern, K. R., Duchin, R. and Shumway, T. (2014). Peer effects in risk aversion and trust. The Review of Financial Studies, 27 (11), 3213-3240.

Alan, S., Ertac, S. and Mumcu, I. (2018). Gender stereotypes in the classroom and effects on achievement. Review of Economics and Statistics, 100 (5), 876-890.

Alesina, A., Carlana, M., Ferrara, E. L. and Pinotti, P. (2018). Revealing stereotypes: Evidence from immigrants in schools. Tech. rep., National Bureau of Economic Research.

Altmejd, A., Barrios-Fernández, A., Drlue, M., Goodman, J., Hurwitz, M., Kovac, D., Mulhern, C., Neilson, C. and Smith, J. (2020). O brother, where start thou? sibling spillovers on college and major choice in four countries. cep discussion paper no. 1691. Centre for Economic Performance.

Altonji, J. G., Eldder, T. E. and Taber, C. R. (2005). Selection on observed and unobserved variables: Assessing the effectiveness of catholic schools. Journal of political economy, 113 (1), 151-184.

Ammermueller, A. and Pischke, J.-S. (2009). Peer effects in european primary schools: Evidence from the progress in international reading literacy study. Journal of Labor Economics, 27 (3), 315-348.

Angrist, J. D. (2014). The perils of peer effects. Labour Economics, 30, 98-108.

- and LANG, K. (2004). Does school integration generate peer effects? evidence from boston's metco program. American Economic Review, 94 (5), 1613-1634. 
Appadurai, A. (2004). The capacity to aspire. Culture and public action, pp. 59-84.

Arcidiacono, P. and Nicholson, S. (2005). Peer effects in medical school. Journal of public Economics, 89 (2-3), 327-350.

Argys, L. M. and ReEs, D. I. (2008). Searching for peer group effects: A test of the contagion hypothesis. The Review of Economics and Statistics, 90 (3), $442-458$.

Artmann, E., Ketel, N., Oosterbeek, H. and Klaauw, B. (2018). Field of study and family outcomes. Tech. rep., CEPR Discussion Paper No. DP13033.

Aucejo, E. M., Coate, P., Fruehwirth, J., Kelly, S. and Mozenter, Z. (2020). Using global observation protocols to inform research on teaching effectiveness and school improvement: Strengths and emerging limitations. Education Policy Analysis Archives, 28, 62.

Autor, D., Figlio, D., Karbownik, K., Roth, J. and Wasserman, M. (2019). Family disadvantage and the gender gap in behavioral and educational outcomes. American Economic Journal: Applied Economics, 11 (3), 338-81.

Avery, C. and HoxBy, C. M. (2004). Do and should financial aid packages affect students' college choices? In College choices: The economics of where to go, when to go, and how to pay for it, University of Chicago Press, pp. 239-302.

Azmat, G., Bagues, M., Cabrales, A. and Iriberri, N. (2019). What you don't know... can't hurt you? a natural field experiment on relative performance feedback in higher education. Management Science, 65 (8), 3714-3736.

- and IRIBERRI, N. (2010). The importance of relative performance feedback information: Evidence from a natural experiment using high school students. Journal of Public Economics, 94 (7-8), 435-452.

— and Simion, S. (2017). Higher Education Funding Reforms: A Comprehensive Analysis of Educational and Labor Market Outcomes in England. IZA Discussion Papers 11083. Tech. rep., Institute for the Study of Labor (IZA).

BAhrs, M. and Siedler, T. (2019). University tuition fees and high school students' educational intentions. Fiscal Studies, 40 (2), 117-147.

Balsa, A., Gandelman, N. and Roldán, F. (2018). Peer and parental influence in academic performance and alcohol use. Labour Economics, 55, 41-55.

Banerjee, A. V. and Newman, A. F. (1991). Risk-bearing and the theory of income distribution. The Review of Economic Studies, 58 (2), 211-235.

- and - (1993). Occupational choice and the process of development. Journal of political economy, 101 (2), 274-298.

Bardhan, P. (1997). Corruption and development: a review of issues. Journal of economic literature, 35 (3), 1320-1346. 
Beaman, L., Duflo, E., Pande, R. and Topalova, P. (2012). Female leadership raises aspirations and educational attainment for girls: A policy experiment in india. science, 335 (6068), 582-586.

BECKER, G. (1964). S. human capital: A theoretical and empirical analysis, with special reference to education. Chicago: University of Press, 19 (6), 4.

Becker, G. S., Kominers, S. D., Murphy, K. M. and Spenkuch, J. L. (2018). A theory of intergenerational mobility. Journal of Political Economy, 126 (S1), S7-S25.

- and Tomes, N. (1979). An equilibrium theory of the distribution of income and intergenerational mobility. Journal of political Economy, 87 (6), 1153-1189.

BedARD, K. and Fischer, S. (2019). Does the response to competition depend on perceived ability? evidence from a classroom experiment. Journal of Economic Behavior \& Organization, 159, 146-166.

Belot, M., Canton, E. and Webbink, D. (2007). Does reducing student support affect scholastic performance? evidence from a dutch reform. Empirical Economics, $32(2-3), 261-275$.

Benjamini, Y. and HochBerg, Y. (1995). Controlling the false discovery rate: a practical and powerful approach to multiple testing. Journal of the Royal statistical society: series B (Methodological), 57 (1), 289-300.

BenYishay, A. and Mobarak, A. M. (2019). Social learning and incentives for experimentation and communication. The Review of Economic Studies, 86 (3), 976-1009.

Bernard, T., Dercon, S., Orkin, K., Taffesse, A. et al. (2014). The future in mind: Aspirations and forward-looking behaviour in rural Ethiopia. Centre for Economic Policy Research London.

Bernheim, B. D., RAY, D. and Yeltekin, Ş. (2015). Poverty and self-control. Econometrica, 83 (5), 1877-1911.

Bertrand, M., Mullainathan, S. and Shafir, E. (2004). A behavioraleconomics view of poverty. American Economic Review, 94 (2), 419-423.

Bettinger, E. P., Long, B. T., Oreopoulos, P. and Sanbonmatsu, L. (2012). The role of application assistance and information in college decisions: Results from the h\&r block fafsa experiment. The Quarterly Journal of Economics, 127 (3), 1205-1242.

Bifulco, R., Fletcher, J. M., Oh, S. J. and Ross, S. L. (2014). Do high school peers have persistent effects on college attainment and other life outcomes? Labour Economics, 29, 83-90. 
- - - and Ross, S. L. (2011). The effect of classmate characteristics on postsecondary outcomes: Evidence from the add health. American Economic Journal: Economic Policy, 3 (1), 25-53.

Black, S. E., Breining, S., Figlio, D. N., Guryan, J., Karbownik, K., Nielsen, H. S., Roth, J. and Simonsen, M. (2017). Sibling spillovers. Tech. rep., National Bureau of Economic Research.

Boisjoly, J., Duncan, G. J., Kremer, M., Levy, D. M. and Eccles, J. (2006). Empathy or antipathy? the impact of diversity. American Economic Review, 96 (5), 1890-1905.

BonesRønning, H. (2004). The determinants of parental effort in education production: do parents respond to changes in class size? Economics of Education Review, 23 (1), 1-9.

Bonilla-Mejía, L., Bottan, N. L. and Ham, A. (2019). Information policies and higher education choices experimental evidence from colombia. Journal of Behavioral and Experimental Economics, 83, 101468.

Booij, A. S., Leuven, E. and Oosterbeek, H. (2012). The role of information in the take-up of student loans. Economics of Education Review, 31 (1), 33-44.

- , - and - (2017). Ability peer effects in university: Evidence from a randomized experiment. The review of economic studies, 84 (2), 547-578.

Botelho, F., Madeira, R. A. and Rangel, M. A. (2015). Racial discrimination in grading: Evidence from brazil. American Economic Journal: Applied Economics, 7 (4), 37-52.

Brady, R. R., Insler, M. A. and Rahman, A. S. (2017). Bad company: Understanding negative peer effects in college achievement. European Economic Review, 98, 144-168.

Brenøe, A. A. (2018). Brothers Increase Women's Gender Conformity. Tech. rep., University of Zurich Working Paper.

Brenøe, A. A. and ZÖlitz, U. (2020). Exposure to more female peers widens the gender gap in stem participation. Journal of Labor Economics, 38 (4), 1009-1054.

Brock, W. A. and Durlauf, S. N. (2001). Discrete choice with social interactions. The Review of Economic Studies, 68 (2), 235-260.

Brunello, G., De Paola, M. and Scoppa, V. (2010). Peer effects in higher education: Does the field of study matter? Economic Inquiry, 48 (3), 621-634.

Burgess, S. and Greaves, E. (2013). Test scores, subjective assessment, and stereotyping of ethnic minorities. Journal of Labor Economics, 31 (3), 535-576.

Burke, M. A. and SAss, T. R. (2013). Classroom peer effects and student achievement. Journal of Labor Economics, 31 (1), 51-82. 
Bursztyn, L., Ederer, F., Ferman, B. and Yuchtman, N. (2014). Understanding mechanisms underlying peer effects: Evidence from a field experiment on financial decisions. Econometrica, 82 (4), 1273-1301.

-, Egorov, G. and Jensen, R. (2019). Cool to be smart or smart to be cool? understanding peer pressure in education. The Review of Economic Studies, 86 (4), $1487-1526$.

— and Jensen, R. (2015). How does peer pressure affect educational investments? The quarterly journal of economics, 130 (3), 1329-1367.

Caliendo, M., Cobb-Clark, D. A. and Uhlendorff, A. (2015). Locus of control and job search strategies. Review of Economics and Statistics, 97 (1), 88-103.

Calvó-Armengol, A., Patacchini, E. and Zenou, Y. (2009). Peer effects and social networks in education. The Review of Economic Studies, 76 (4), 1239-1267.

CAner, M. and Hansen, B. E. (2004). Instrumental variable estimation of a threshold model. Econometric Theory, pp. 813-843.

Canton, E. and De Jong, F. (2005). The demand for higher education in the netherlands, 1950-1999. Economics of Education Review, 24 (6), 651-663.

Card, D. and Giuliano, L. (2013). Peer effects and multiple equilibria in the risky behavior of friends. Review of Economics and Statistics, 95 (4), 1130-1149.

-, Mas, A. and Rothstein, J. (2008). Tipping and the dynamics of segregation. The Quarterly Journal of Economics, 123 (1), 177-218.

Carlana, M. (2019). Implicit stereotypes: Evidence from teachers' gender bias. The Quarterly Journal of Economics, 134 (3), 1163-1224.

Carrell, S. E., Fullerton, R. L. and West, J. E. (2009). Does your cohort matter? measuring peer effects in college achievement. Journal of Labor Economics, 27 (3), 439-464.

-, Malmstrom, F. V. and West, J. E. (2008). Peer effects in academic cheating. Journal of human resources, 43 (1), 173-207.

-, Sacerdote, B. I. and West, J. E. (2013). From natural variation to optimal policy? the importance of endogenous peer group formation. Econometrica, 81 (3), $855-882$.

- and WeSt, J. E. (2010). Does professor quality matter? evidence from random assignment of students to professors. Journal of Political Economy, 118 (3), 409-432.

Chang, S., Cobb-Clark, D. A. and Salamanca, N. (2020). Parents' Responses to Teacher Qualifications. IZA Discussion Paper Series, No. 13065. Tech. rep., Institute for the Study of Labor (IZA). 
Charles, K. K., Hurst, E., Notowidigdo, M. J. et al. (2018). Housing booms and busts, labor market opportunities, and college attendance. American Economic Review, 108 (10), 2947-2994.

Charness, G. and Gneezy, U. (2012). Strong evidence for gender differences in risk taking. Journal of Economic Behavior $\&$ Organization, 83 (1), 50-58.

Chetty, R., Friedman, J. N., Hilger, N., Saez, E., Schanzenbach, D. W. and YAGAN, D. (2011). How does your kindergarten classroom affect your earnings? evidence from project star. The Quarterly journal of economics, 126 (4), 15931660 .

- and Hendren, N. (2018). The impacts of neighborhoods on intergenerational mobility ii: County-level estimates. The Quarterly Journal of Economics, 133 (3), $1163-1228$.

-, - Jones, M. R. and Porter, S. R. (2020). Race and economic opportunity in the united states: An intergenerational perspective. The Quarterly Journal of Economics, 135 (2), 711-783.

Chowdry, H., Crawford, C., Dearden, L., Goodman, A. and Vignoles, A. (2013). Widening participation in higher education: analysis using linked administrative data. Journal of the Royal Statistical Society: Series A (Statistics in Society), 176 (2), 431-457.

Clark, D. (2010). Selective schools and academic achievement. The BE Journal of Economic Analysis \& Policy, 10 (1).

-, Gill, D., Prowse, V. and Rush, M. (2020). Using goals to motivate college students: Theory and evidence from field experiments. Review of Economics and Statistics, 102 (4), 648-663.

Clarke, D., Romano, J. P. and Wolf, M. (2019). The Romano-Wolf Multiple Hypothesis Correction in Stata. IZA Discussion Paper Series, No. 12845. Tech. rep., Institute for the Study of Labor (IZA).

Contreras Suarez, D. and Cameron, L. (2020). Conditional cash transfers: Do they result in more patient choices and increased educational aspirations? Economic Development and Cultural Change, 68 (3), 729-761.

Cornelisz, I., Meeter, M. and van Klaveren, C. (2019). Educational equity and teacher discretion effects in high stake exams. Economics of Education Review, 73, 101908.

Correia, S. (2018). Reghdfe: Stata module to perform linear or instrumentalvariable regression absorbing any number of high-dimensional fixed effects. stat software components. published online first: September 17, 2018. Statistical Software Components s457874, Boston College Department of Economics. 
Crespo Cuaresma, J., KC, S. and Sauer, P. (2013). Age-specific education inequality, education mobility and income growth. Tech. rep., WWWforEurope Working Paper.

Cunha, F. and Heckman, J. (2007). The technology of skill formation. American Economic Review, 97 (2), 31-47.

Dahl, G. B., Rooth, D.-O. and Stenberg, A. (2020). Family Spillovers in Field of Study. Tech. rep., National Bureau of Economic Research.

Dalton, P. S., Ghosal, S. and Mani, A. (2016). Poverty and aspirations failure. The Economic Journal, 126 (590), 165-188.

DAsGupta, P. and RAY, D. (1986). Inequality as a determinant of malnutrition and unemployment: Theory. The Economic Journal, 96 (384), 1011-1034.

Datar, A. and MAson, B. (2008). Do reductions in class size "crowd out" parental investment in education? Economics of Education Review, 27 (6), 712-723.

De Haan, M., Gautier, P. A., Oosterbeek, H. and Van Der Klaauw, B. (2015). The performance of school assignment mechanisms in practice. Tech. rep., CEPR Discussion Paper No. DP10656.

De Paola, M. and Scoppa, V. (2010). Peer group effects on the academic performance of italian students. Applied Economics, 42 (17), 2203-2215.

Dearden, L., Fitzsimons, E. and Wyness, G. (2014). Money for nothing: Estimating the impact of student aid on participation in higher education. Economics of Education Review, 43, 66-78.

DeE, T. S. (2004). Teachers, race, and student achievement in a randomized experiment. Review of economics and statistics, 86 (1), 195-210.

-, Dobbie, W., Jacob, B. A. and Rockoff, J. (2019). The causes and consequences of test score manipulation: Evidence from the new york regents examinations. American Economic Journal: Applied Economics, 11 (3), 382-423.

Delavande, A. and Zafar, B. (2019). University choice: The role of expected earnings, nonpecuniary outcomes, and financial constraints. Journal of Political Economy, 127 (5), 2343-2393.

Deming, D. J. (2011). Better schools, less crime? The Quarterly Journal of Economics, 126 (4), 2063-2115.

Denning, J. T., Murphy, R. and Weinhardt, F. (2020). Class rank and long-run outcomes. Tech. rep., National Bureau of Economic Research.

Diamond, R. and Persson, P. (2016). The long-term consequences of teacher discretion in grading of high-stakes tests. Tech. rep., National Bureau of Economic Research. 
Diette, T. M. and Uwaifo Oyelere, R. (2014). Gender and race heterogeneity: The impact of students with limited english on native students' performance. American Economic Review, 104 (5), 412-17.

Dobbie, W. and Fryer JR, R. G. (2014). The impact of attending a school with high-achieving peers: Evidence from the new york city exam schools. American Economic Journal: Applied Economics, 6 (3), 58-75.

Duflo, E. (2012). Women empowerment and economic development. Journal of Economic literature, 50 (4), 1051-79.

-, Dupas, P. and Kremer, M. (2011a). Peer effects, teacher incentives, and the impact of tracking: Evidence from a randomized evaluation in kenya. American Economic Review, 101 (5), 1739-74.

-, Kremer, M. and Robinson, J. (2011b). Nudging farmers to use fertilizer: Theory and experimental evidence from kenya. American economic review, 101 (6), 2350-90.

Dupas, P., Robinson, J. and SaAvedra, S. (2020). The daily grind: Cash needs and labor supply. Journal of Economic Behavior \&f Organization, 177, 399-414.

Durlauf, S. N., Kourtellos, A. and Tan, C. M. (2017). Status traps. Journal of Business \& Economic Statistics, 35 (2), 265-287.

DYNARSKI, S. and BAILEY, M. (2011). Inequality in post-secondary education. Whither Opportunity.

- Libassi, C., Michelmore, K. and Owen, S. (2018). Closing the gap: The effect of a targeted, tuition-free promise on college choices of high-achieving, lowincome students. Tech. rep., National Bureau of Economic Research.

- and Scott-Clayton, J. (2013). Financial aid policy: Lessons from research. Future of Children, 23 (1), 67-91.

EBle, A. and Hu, F. (2019). How important are beliefs about gender differences in math ability? transmission across generations and impacts on child outcomes. CDEP-CGEG Working Paper, 53.

Elsner, B. and Isphording, I. E. (2017). A big fish in a small pond: Ability rank and human capital investment. Journal of Labor Economics, 35 (3), 787-828.

—, - and ZÖLITZ, U. (2018). Achievement rank affects performance and major choices in college. University of Zurich, Department of Economics, Working Paper No. 300.

Epple, D., Newlon, E. and Romano, R. (2002). Ability tracking, school competition, and the distribution of educational benefits. Journal of Public Economics, 83 (1), 1-48. 
EURYDICE (2012). National student fee and support systems 2011/2012. Tech. rep., Eurydice Brussels, Belgium.

Falk, A., Becker, A., Dohmen, T., Enke, B., Huffman, D. and Sunde, U. (2018). Global evidence on economic preferences. The Quarterly Journal of Economics, 133 (4), 1645-1692.

FAnG, G. and WAn, S. (2020). Peer effects among graduate students: Evidence from china. China Economic Review, 60, 101406.

Feld, J., Salamanca, N. and Hamermesh, D. S. (2016). Endophilia or exophobia: beyond discrimination. The Economic Journal, 126 (594), 1503-1527.

- and ZÖLITZ, U. (2017). Understanding peer effects: On the nature, estimation, and channels of peer effects. Journal of Labor Economics, 35 (2), 387-428.

FEnG, H. and Li, J. (2016). Head teachers, peer effects, and student achievement. China Economic Review, 41, 268-283.

FiELD, E. (2009). Educational debt burden and career choice: Evidence from a financial aid experiment at nyu law school. American Economic Journal: Applied Economics, 1 (1), 1-21.

Figlio, D., Guryan, J., Karbownik, K. and Roth, J. (2014). The effects of poor neonatal health on children's cognitive development. American Economic Review, 104 (12), 3921-55.

-, Karbownik, K. and Salvanes, K. G. (2016). Education research and administrative data. In Handbook of the Economics of Education, vol. 5, Elsevier, pp. $75-138$.

— and ÖzEK, U. (2019). Unwelcome guests? the effects of refugees on the educational outcomes of incumbent students. Journal of Labor Economics, 37 (4), 1061-1096.

Figlio, D. N. (2007). Boys named sue: Disruptive children and their peers. Education finance and policy, 2 (4), 376-394.

Findeisen, S. and SACHS, D. (2016). Education and optimal dynamic taxation: The role of income-contingent student loans. Journal of Public Economics, 138, $1-21$.

— and - (Forthcoming). Optimal need-based financial aid. Journal of Political Economy.

Finn, J. D., Pannozzo, G. M. and Achilles, C. M. (2003). The "why's" of class size: Student behavior in small classes. Review of Educational Research, 73 (3), 321-368. 
Fiorini, M. and KeAne, M. P. (2014). How the allocation of children's time affects cognitive and noncognitive development. Journal of Labor Economics, 32 (4), $787-836$.

Foster, G. (2006). It's not your peers, and it's not your friends: Some progress toward understanding the educational peer effect mechanism. Journal of public Economics, 90 (8-9), 1455-1475.

Fredriksson, P., ÖCkert, B. and Oosterbeek, H. (2016). Parental responses to public investments in children: Evidence from a maximum class size rule. Journal of Human Resources, 51 (4), 832-868.

Fu, C. and Menta, N. (2018). Ability tracking, school and parental effort, and student achievement: A structural model and estimation. Journal of Labor Economics, 36 (4), 923-979.

GALOR, O. and ZeIRA, J. (1993). Income distribution and macroeconomics. The review of economic studies, 60 (1), 35-52.

GARLICK, R. (2018). Academic peer effects with different group assignment policies: Residential tracking versus random assignment. American Economic Journal: Applied Economics, 10 (3), 345-69.

GelbaCH, J. B. (2016). When do covariates matter? and which ones, and how much? Journal of Labor Economics, 34 (2), 509-543.

Genicot, G. and Ray, D. (2017). Aspirations and inequality. Econometrica, 85 (2), 489-519.

Gershenson, S., Holt, S. B. and Papageorge, N. W. (2016). Who believes in me? the effect of student-teacher demographic match on teacher expectations. Economics of education review, 52, 209-224.

Gibbons, S. and Telhaj, S. (2016). Peer effects: Evidence from secondary school transition in england. Oxford Bulletin of Economics and Statistics, 78 (4), 548-575.

Golsteyn, B., Non, A. and ZÖlitz, U. (Forthcoming). The impact of peer personality on academic achievement. Journal of Political Economy.

Gong, J., Lu, Y. and Song, H. (2019). Gender peer effects on students' academic and noncognitive outcomes: Evidence and mechanisms. Journal of Human Resources, pp. 0918-9736R2.

Gould, E. D., Lavy, V. and Paserman, M. D. (2004). Immigrating to opportunity: Estimating the effect of school quality using a natural experiment on ethiopians in israel. The Quarterly Journal of Economics, 119 (2), 489-526.

Goux, D., Gurgand, M. and Maurin, E. (2017). Adjusting your dreams? high school plans and dropout behaviour. The Economic Journal, 127 (602), 1025-1046. 
Graham, B. S. (2008). Identifying social interactions through conditional variance restrictions. Econometrica, 76 (3), 643-660.

Griffith, A. L. and RAsk, K. N. (2014). Peer effects in higher education: A look at heterogeneous impacts. Economics of Education Review, 39, 65-77.

Guryan, J., Kroft, K. and Notowidigdo, M. J. (2009). Peer effects in the workplace: Evidence from random groupings in professional golf tournaments. American Economic Journal: Applied Economics, 1 (4), 34-68.

Guyon, N. and Huillery, E. (Forthcoming). Biased aspirations and social inequality at school: Evidence from french teenagers. The Economic Journal.

Hanna, R. N. and Linden, L. L. (2012). Discrimination in grading. American Economic Journal: Economic Policy, 4 (4), 146-68.

Hansen, B. E. (2000). Sample splitting and threshold estimation. Econometrica, $68(3), 575-603$.

HanusheK, E. A. (1979). Conceptual and empirical issues in the estimation of educational production functions. Journal of human Resources, pp. 351-388.

-, Kain, J. F., Markman, J. M. and Rivkin, S. G. (2003). Does peer ability affect student achievement? Journal of applied econometrics, 18 (5), 527-544.

- - - and Rivkin, S. G. (2009). New evidence about brown v. board of education: The complex effects of school racial composition on achievement. Journal of labor economics, 27 (3), 349-383.

Haushofer, J. and Fehr, E. (2014). On the psychology of poverty. Science, 344 (6186), 862-867.

HeCKMAN, J. J. and KAutz, T. (2013). Fostering and measuring skills: Interventions that improve character and cognition. Tech. rep., National Bureau of Economic Research.

— and Pinto, R. (2015). Econometric mediation analyses: Identifying the sources of treatment effects from experimentally estimated production technologies with unmeasured and mismeasured inputs. Econometric reviews, 34 (1-2), 6-31.

-, Stixrud, J. and URzuA, S. (2006). The effects of cognitive and noncognitive abilities on labor market outcomes and social behavior. Journal of Labor economics, 24 (3), 411-482.

Hendren, N. and Sprung-Keyser, B. (2020). A unified welfare analysis of government policies. The Quarterly Journal of Economics, 135 (3), 1209-1318.

Hoekstra, M. (2009). The effect of attending the flagship state university on earnings: A discontinuity-based approach. The Review of Economics and Statistics, 91 (4), 717-724. 
-, Mouganie, P. and Wang, Y. (2018). Peer quality and the academic benefits to attending better schools. Journal of Labor Economics, 36 (4), 841-884.

Hoff, K. and Sen, A. (2005). Homeownership, community interactions, and segregation. American Economic Review, 95 (4), 1167-1189.

— and - (2006). The kin system as a poverty trap? In S. Bowles, S. N. Durlauf and K. Hoff (eds.), Poverty Traps, The Persistent Inequality Project, Princeton University Press, p. 95.

HonG, S. C. and LeE, J. (2017). Who is sitting next to you? peer effects inside the classroom. Quantitative Economics, 8 (1), 239-275.

HoxBy, C. (2000). Peer effects in the classroom: Learning from gender and race variation. Tech. rep., National Bureau of Economic Research.

— and Avery, C. (2013). The missing" one-offs": The hidden supply of highachieving, low-income students. Brookings Papers on Economic Activity.

-, Turner, S. et al. (2013). Expanding college opportunities for high-achieving, low income students. Stanford Institute for Economic Policy Research Discussion Paper, 12, 014.

Hoxby, C. M. and Turner, S. (2015). What high-achieving low-income students know about college. American Economic Review, 105 (5), 514-17.

- and Weingarth, G. (). Taking race out of the equation: School reassignment and the structure of peer effects. Tech. rep., Citeseer.

HÜBNER, M. (2012). Do tuition fees affect enrollment behavior? evidence from a 'natural experiment'in germany. Economics of Education Review, 31 (6), 949-960.

Huntington-Klein, N. and Rose, E. (2018). Gender peer effects in a predominantly male environment: Evidence from west point. In AEA Papers and Proceedings, vol. 108, pp. 392-95.

Imai, K., Tingley, D. and Yamamoto, T. (2013). Experimental designs for identifying causal mechanisms. Journal of the Royal Statistical Society: Series A (Statistics in Society), 176 (1), 5-51.

Imberman, S. A., Kugler, A. D. and Sacerdote, B. I. (2012). Katrina's children: Evidence on the structure of peer effects from hurricane evacuees. American Economic Review, 102 (5), 2048-82.

JACKSON, C. K. (2013). Can higher-achieving peers explain the benefits to attending selective schools? evidence from trinidad and tobago. Journal of Public Economics, 108, 63-77.

JACOB, B. A. and Lefgren, L. (2007). What do parents value in education? an empirical investigation of parents' revealed preferences for teachers. The Quarterly Journal of Economics, 122 (4), 1603-1637. 
and Linkow, T. W. (2011). Educational expectations and attainment. In G. J. Duncan and R. J. Murnane (eds.), Whither opportunity?: Rising inequality, schools, and children's life chances, Russell Sage Foundation, p. 133.

JAIN, T. and KAPOOR, M. (2015). The impact of study groups and roommates on academic performance. Review of Economics and Statistics, 97 (1), 44-54.

Janzen, S. A., Magnan, N., Sharma, S. and Thompson, W. M. (2017). Aspirations failure and formation in rural nepal. Journal of Economic Behavior \& Organization, 139, 1-25.

Jochmans, K. (2020). Peer effects and endogenous social interactions. arXiv preprint arXiv:2008.07886.

Joensen, J. S. and Nielsen, H. S. (2018). Spillovers in education choice. Journal of Public Economics, 157, 158-183.

Kahneman, D. (2011). Thinking, fast and slow. Macmillan.

Kalenkoski, C. M. and Pabilonia, S. W. (2010). Parental transfers, student achievement, and the labor supply of college students. Journal of Population Economics, 23 (2), 469-496.

Kane, T. J., Taylor, E. S., Tyler, J. H. and Wooten, A. L. (2011). Identifying effective classroom practices using student achievement data. Journal of human Resources, 46 (3), 587-613.

KANG, C. et al. (2007). Classroom peer effects and academic achievement: Quasirandomization evidence from south korea. Journal of Urban Economics, 61 (3), $458-495$.

Karbownik, K. and ÖzeK, U. (2019). Setting a Good Example? Examining Sibling Spillovers in Educational Achievement Using a Regression Discontinuity Design. Tech. rep., National Bureau of Economic Research.

Keele, L., Tingley, D. and Yamamoto, T. (2015). Identifying mechanisms behind policy interventions via causal mediation analysis. Journal of Policy Analysis and Management, 34 (4), 937-963.

Kelchtermans, S. and Verboven, F. (2010). Participation and study decisions in a public system of higher education. Journal of Applied Econometrics, 25 (3), $355-391$.

Ketel, N., Leuven, E., Oosterbeek, H. and van der Klaauw, B. (2016). The returns to medical school: Evidence from admission lotteries. American Economic Journal: Applied Economics, 8 (2), 225-54.

- , - - - and Van Der Klaauw, B. (2019). Do dutch dentists extract monopoly rents? Journal of Health Economics, 63, 145-158. 
Kimbrough, E. O., McGee, A. and ShigeokA, H. (Forth.). How do peers impact learning? an experimental investigation of peer-to-peer teaching and ability tracking. Journal of Human Resources, Forthcoming.

KISS, D. (2013). The impact of peer achievement and peer heterogeneity on own achievement growth: Evidence from school transitions. Economics of Education Review, 37, 58-65.

Korthals, R. A. (2015). Tracking students in secondary education: Consequences for student performance and inequality. Ph.D. thesis, Graduate School of Business and Economics, Maastricht University.

Kourtellos, A., Marr, C. and Tan, C. M. (2020). Local intergenerational mobility. European Economic Review, p. 103460.

—, Stengos, T. and Tan, C. M. (2016). Structural threshold regression. Econometric Theory.

Kramarz, F., Machin, S. and Ouazad, A. (2015). Using compulsory mobility to identify school quality and peer effects. Oxford Bulletin of Economics and Statistics, 77 (4), 566-587.

KREMER, M. (1993). The o-ring theory of economic development. The Quarterly Journal of Economics, 108 (3), 551-575.

— and Levy, D. (2008). Peer effects and alcohol use among college students. Journal of Economic perspectives, 22 (3), 189-206.

Krueger, A. B. (1999). Experimental estimates of education production functions. The quarterly journal of economics, 114 (2), 497-532.

— and Whitmore, D. M. (2001). The effect of attending a small class in the early grades on college-test taking and middle school test results: Evidence from project star. The Economic Journal, 111 (468), 1-28.

La Ferrara, E. (2019). Presidential address: Aspirations, social norms, and development. Journal of the European Economic Association, 17 (6), 1687-1722.

Landers $\varnothing$, R. K., Nielsen, H. S. and Simonsen, M. (2019). Effects of school starting age on the family. Journal of Human Resources, pp. 1117-9174R1.

Lavecchia, A. M., Liu, H. and Oreopoulos, P. (2016). Behavioral economics of education: Progress and possibilities. In Handbook of the Economics of Education, vol. 5, Elsevier, pp. 1-74.

LAVY, V. (2008). Do gender stereotypes reduce girls' or boys' human capital outcomes? evidence from a natural experiment. Journal of public Economics, 92 (10-11), 2083-2105. 
- and Megalokonomou, R. (2019). Persistency in teachers' grading bias and effects on longer-term outcomes: University admissions exams and choice of field of study. Tech. rep., National Bureau of Economic Research.

-, Paserman, M. D. and Schlosser, A. (2012a). Inside the black box of ability peer effects: Evidence from variation in the proportion of low achievers in the classroom. The Economic Journal, 122 (559), 208-237.

- and SAND, E. (2018). On the origins of gender gaps in human capital: Short-and long-term consequences of teachers' biases. Journal of Public Economics, 167, 263-279.

-, - and Shayo, M. (2018). Charity begins at home (and at school): Effects of religion-based discrimination in education. Tech. rep., National Bureau of Economic Research.

- and Schlosser, A. (2011). Mechanisms and impacts of gender peer effects at school. American Economic Journal: Applied Economics, 3 (2), 1-33.

- Silva, O. and Weinhardt, F. (2012b). The good, the bad, and the average: Evidence on ability peer effects in schools. Journal of Labor Economics, 30 (2), $367-414$.

LAW, W.-W. (2004). Translating globalization and democratization into local policy: Educational reform in hong kong and taiwan. International Review of Education, 50, 497-524.

LAZEAR, E. P. (2001). Educational production. The Quarterly Journal of Economics, 116 (3), 777-803.

LEE, J.-W. and LEe, H. (2016). Human capital in the long run. Journal of Development Economics, 122, 147-169.

Lim, J. and MeER, J. (2017). The impact of teacher-student gender matches random assignment evidence from south korea. Journal of Human Resources, 52 (4), 979-997.

- and - (2020). Persistent effects of teacher-student gender matches. Journal of Human Resources, 55 (3), 809-835.

Linsenmeier, D. M., Rosen, H. S. and Rouse, C. E. (2006). Financial aid packages and college enrollment decisions: An econometric case study. Review of Economics and Statistics, 88 (1), 126-145.

Lochner, L. and Monge-Naranjo, A. (2016). Student loans and repayment: Theory, evidence, and policy. In Handbook of the Economics of Education, vol. 5, Elsevier, pp. 397-478.

LOURY, G. C. (1981). Intergenerational transfers and the distribution of earnings. Econometrica: Journal of the Econometric Society, pp. 843-867. 
Lu, F. and Anderson, M. L. (2015). Peer effects in microenvironments: The benefits of homogeneous classroom groups. Journal of Labor Economics, 33 (1), 91-122.

LybBert, T. J. and Wydick, B. (2018). Hope as aspirations, agency, and pathways. In C. Barrett, M. Carter, J. Chavas and M. Carter (eds.), The Economics of Poverty Traps, National Bureau of Economic Research Conference Report, University of Chicago Press.

LYLE, D. S. (2007). Estimating and interpreting peer and role model effects from randomly assigned social groups at west point. The Review of Economics and Statistics, 89 (2), 289-299.

Macours, K. and VAKIS, R. (2014). Changing households' investment behaviour through social interactions with local leaders: Evidence from a randomised transfer programme. The Economic Journal, 124 (576), 607-633.

Mani, A., Mullainathan, S., Shafir, E. and Zhao, J. (2013). Poverty impedes cognitive function. science, 341 (6149), 976-980.

MAnski, C. F. (1993a). Identification of endogenous social effects: The reflection problem. The review of economic studies, 60 (3), 531-542.

- (1993b). Identification problems in the social sciences. Sociological Methodology, pp. $1-56$.

- (2000). Economic analysis of social interactions. Journal of economic perspectives, $14(3), 115-136$.

Marmaros, D. and Sacerdote, B. (2002). Peer and social networks in job search. European economic review, 46 (4-5), 870-879.

MCEwan, P. J. (2003). Peer effects on student achievement: Evidence from chile. Economics of education review, 22 (2), 131-141.

Mehta, N., Stinebrickner, R. and Stinebrickner, T. (2019). Time-use and academic peer effects in college. Economic Inquiry, 57 (1), 162-171.

MincER, J. (1958). Investment in human capital and personal income distribution. Journal of political economy, 66 (4), 281-302.

Montalbán, J. (2019). Countering moral hazard in higher education: The role of performance incentives in need-based grants. Tech. rep., Paris School of Economics Working Paper No. 2019-30.

Moretti, E. (2011). Social learning and peer effects in consumption: Evidence from movie sales. The Review of Economic Studies, 78 (1), 356-393.

MukherJee, P. (2015). The effects of social identity on aspirations and learning outcomes: a field experiment in rural india. Unpublished working paper, College of William and Mary. 
Murphy, R., Scott-Clayton, J. and Wyness, G. (2019). The end of free college in england: Implications for enrolments, equity, and quality. Economics of Education Review, 71, 7-22.

- and Weinhardt, F. (Forthcoming). Top of the class: The importance of ordinal rank. The Review of Economic Studies.

Nicoletti, C. and RABE, B. (2019). Sibling spillover effects in school achievement. Journal of Applied Econometrics, 34 (4), 482-501.

Nielsen, H. S., Sørensen, T. and Taber, C. (2010). Estimating the effect of student aid on college enrollment: Evidence from a government grant policy reform. American Economic Journal: Economic Policy, 2 (2), 185-215.

of Education of The Netherlands), D. D. U. O. M. (2018). Elgibility conditions to student finance in the netherlands.

Oosterbeek, H., Sóvágó, S. and KlaAuw, B. (2019). Why are schools segregated? Evidence from the secondary-school match in Amsterdam. Tech. rep., CEPR Discussion Paper No. DP13462.

- and van Den Broek, A. (2009). An empirical analysis of borrowing behaviour of higher education students in the netherlands. Economics of education review, 28 (2), 170-177.

Oreopoulos, P. and Dunn, R. (2013). Information and college access: Evidence from a randomized field experiment. The Scandinavian Journal of Economics, 115 (1), 3-26.

Oster, E. (2019). Unobservable selection and coefficient stability: Theory and evidence. Journal of Business \&6 Economic Statistics, 37 (2), 187-204.

— and Thornton, R. (2012). Determinants of technology adoption: Peer effects in menstrual cup take-up. Journal of the European Economic Association, 10 (6), $1263-1293$.

Page, L., Garboua, L. L. and Montmarquette, C. (2007). Aspiration levels and educational choices: An experimental study. Economics of Education Review, 26 (6), 747-757.

PAllais, A. (2015). Small differences that matter: Mistakes in applying to college. Journal of Labor Economics, 33 (2), 493-520.

Papageorge, N. W., Gershenson, S. and Kang, K. M. (2020). Teacher expectations matter. Review of Economics and Statistics, 102 (2), 234-251.

Pearlin, L. I., Menaghan, E. G., Lieberman, M. A. and Mullan, J. T. (1981). The stress process. Journal of Health and Social behavior, pp. 337-356. 
Pei, Z., Pischke, J.-S. and Schwandt, H. (2019). Poorly measured confounders are more useful on the left than on the right. Journal of Business \& Economic Statistics, 37 (2), 205-216.

Peter, F. H. and Zambre, V. (2017). Intended college enrollment and educational inequality: Do students lack information? Economics of Education Review, 60, $125-141$.

Pop-Eleches, C. and Urquiola, M. (2013). Going to a better school: Effects and behavioral responses. American Economic Review, 103 (4), 1289-1324.

QuRESHI, J. A. (2018a). Additional returns to investing in girls' education: Impact on younger sibling human capital. The Economic Journal, 128 (616), 3285-3319.

- (2018b). Siblings, teachers, and spillovers on academic achievement. Journal of Human Resources, 53 (1), 272-297.

RAY, D. (2006). Aspirations, poverty, and economic change. In A. Banerjee, R. Benabou and D. Mookherjee (eds.), Understanding Poverty, Oxford University Press, pp. 409-421.

- and Genicot, G. (2019). Aspirations: A review. Economic Development and Institutions Working Paper Series.

Romano, J. P. and Wolf, M. (2005a). Exact and approximate stepdown methods for multiple hypothesis testing. Journal of the American Statistical Association, 100 (469), 94-108.

- and - (2005b). Stepwise multiple testing as formalized data snooping. Econometrica, 73 (4), 1237-1282.

Rosenberg, M. (1965). Society and the adolescent self-image. Princeton university press.

Roser, M. and Ortiz-Ospina, E. (2016). Global education. Our World in Data.

Ross, P. H. (2019). Occupation aspirations, education investment, and cognitive outcomes: Evidence from indian adolescents. World Development, 123, 104613.

Rothstein, J. and Rouse, C. E. (2011). Constrained after college: Student loans and early-career occupational choices. Journal of Public Economics, 95 (1-2), 149-163.

Rotter, J. B. (1966). Generalized expectancies for internal versus external control of reinforcement. Psychological monographs: General and applied, 80 (1), 1.

Ruijs, N. and Oosterbeek, H. (2019). School choice in amsterdam: Which schools are chosen when school choice is free? Education Finance and Policy, 14 (1), 1-30. 
SACERdote, B. (2001). Peer effects with random assignment: Results for dartmouth roommates. The Quarterly journal of economics, 116 (2), 681-704.

- (2011). Peer effects in education: How might they work, how big are they and how much do we know thus far? In Handbook of the Economics of Education, vol. 3, Elsevier, pp. 249-277.

- (2014). Experimental and quasi-experimental analysis of peer effects: Two steps forward? Annual Review of Economics, 6 (1), 253-272.

Salamanca, N., De Grip, A., Fouarge, D. and Montizaan, R. (Forthcoming). Locus of control and investment in risky assets. Journal of Economic Behavior \& Organization, 177, 548-568.

Sapelli, C. and Illanes, G. (2016). Class size and teacher effects in higher education. Economics of Education Review, 52, 19-28.

Schmeiser, M., Stoddard, C. and Urban, C. (2016). Student loan information provision and academic choices. American Economic Review, 106 (5), 324-28.

Shah, A. K., Mullainathan, S. and Shafir, E. (2012). Some consequences of having too little. Science, 338 (6107), 682-685.

Sojourner, A. (2013). Identification of peer effects with missing peer data: Evidence from project star. The Economic Journal, 123 (569), 574-605.

Stinebrickner, R. and Stinebrickner, T. R. (2006). What can be learned about peer effects using college roommates? evidence from new survey data and students from disadvantaged backgrounds. Journal of public Economics, 90 (8-9), $1435-1454$.

Stinebrickner, T. and Stinebrickner, R. (2001). Peer effects among students from disadvantaged backgrounds. Tech. rep., University of Western Ontario, Centre for Human Capital and Productivity (CHCP).

Todd, P. and Wolpin, K. I. (2018). Accounting for mathematics performance of high school students in mexico: Estimating a coordination game in the classroom. Journal of Political Economy, 126 (6), 2608-2650.

TodD, P. E. and Wolpin, K. I. (2003). On the specification and estimation of the production function for cognitive achievement. The Economic Journal, 113 (485), F3-F33.

- and - (2007). The production of cognitive achievement in children: Home, school, and racial test score gaps. Journal of Human capital, 1 (1), 91-136.

Ushchev, P. and Zenou, Y. (2020). Social norms in networks. Journal of Economic Theory, 185, 104969. 
VAN DEN BERGE, W. (2018). Bad start, bad match? the early career effects of graduating in a recession for vocational and academic graduates. Labour Economics, 53, 75-96.

Van den Broek, A. and Mens, A. (2017). Onderzoeksverantwoording Studenten Monitor 2001-2015. Beschrijving databestand voor DANS. Tech. rep., ResearchNed Technical Report.

— and VAn DE Wiel, E. (2005). Leengedrag van studenten. Tech. rep., Nijmegen: ITS.

—, - , Pronk, T. and Sijbers, R. (2006). Studentenmonitor 2005. Tech. rep., Technical report, Nijmegen: ITS.

van den Broek, A., Wartenbergh, F., Bendig-Jacobs, J., Tholen, R., DuYsak, S. and NoOIJ, J. (2017). Monitor Beleidsmaatregelen 2016-201\%. Studiekeuze, studiegedrag en leengedrag in relatie tot beleidsmaatregelen in het hoger onderwijs, 2006-2016. Tech. rep., ResearchNed Technical Report.

Van den Broek, A., Wartenbergh, F., Wermink, I., Sijbers, R., Thomassen, M. and van Klingeren, M. (2007). Studentenmonitor 2006. Tech. rep., Technical report, Nijmegen: ITS.

VAn Lent, M. and Souveriun, M. (2020). Goal setting and raising the bar: A field experiment. Journal of Behavioral and Experimental Economics, p. 101570.

VArdardottir, A. (2013). Peer effects and academic achievement: a regression discontinuity approach. Economics of Education review, 36, 108-121.

Whitmore, D. (2005). Resource and peer impacts on girls' academic achievement: Evidence from a randomized experiment. American Economic Review, 95 (2), 199-203.

Willingham, W. W. and Cole, N. S. (2013). Gender and fair assessment. Routledge.

Xu, D., Zhang, Q. and Zhou, X. (2020). The impact of low-ability peers on cognitive and non-cognitive outcomes: Random assignment evidence on the effects and operating channels. Journal of Human Resources.

Young, A. (2019). Channeling fisher: Randomization tests and the statistical insignificance of seemingly significant experimental results. The Quarterly Journal of Economics, 134 (2), 557-598.

Yu, P. and Phillips, P. C. (2018). Threshold regression with endogeneity. Journal of Econometrics, 203 (1), 50-68.

ZÁrAte, R. A. (2020). More than friends: Beliefs and peer effects in the formation of social and academic skills. Available at SSRN 3595812. 
Zimmerman, D. J. (2003). Peer effects in academic outcomes: Evidence from a natural experiment. Review of Economics and statistics, 85 (1), 9-23.

ZÖLITZ, U. and FELD, J. (Forthcoming). The effect of peer gender on major choice in business school. Management Science. 



\section{A $\quad$ Appendix to Chapter 1}

\section{A.1 Proofs}

Proof of Proposition 1.

For this proof, we simplify notations to: $M$ for $M\left(\tilde{g}\left(\theta_{0}\right)\right), \tilde{g}^{\prime}$ for $\tilde{g}^{\prime}\left(\theta_{0}\right)$ and $M^{\prime}$ for $M^{\prime}\left(\tilde{g}\left(\theta_{0}\right)\right)$.

We first show that $1 \leq \rho_{1}$.

$$
\begin{aligned}
1 \leq \rho_{1} & \Leftrightarrow \lambda+(1-\lambda)\left(2-M-\theta_{0} \tilde{g}^{\prime} M^{\prime}\right) \geq 1 \\
& \Leftrightarrow(1-\lambda)\left(2-M-\theta_{0} \tilde{g}^{\prime} M^{\prime}\right) \geq 1-\lambda \\
& \Leftrightarrow 2-M-\theta_{0} \tilde{g}^{\prime} M^{\prime} \geq 1
\end{aligned}
$$

We know that $\forall \theta_{0}, 0 \leq M() \leq$.1 , so $1 \leq 2-M \leq 2$. This leaves us only to show that $-\theta_{0} \tilde{g}^{\prime} M^{\prime} \geq 0$. Since $M(\tilde{g}()$.$) is a decreasing function of \theta_{0}$, its partial derivative against $\theta_{0}$ is $\tilde{g}^{\prime} M^{\prime}$ which takes only negative values. Since $\theta_{0} \geq 0$, by pre-multiplying $\tilde{g}^{\prime} M^{\prime}$ by $-\theta_{0}$, we find that $-\theta_{0} \tilde{g}^{\prime} M^{\prime} \geq 0$ always holds, which implies that $1 \leq \rho_{1}$.

Next, we show that $\rho_{1} \leq \rho_{2}$.

$$
\begin{aligned}
r h o_{1} \leq \rho_{2} & \Leftrightarrow \rho_{1} \leq \lambda+\rho_{1} \\
& \Leftrightarrow 0 \leq \lambda
\end{aligned}
$$

which is always true by definition since $\lambda$ is a probability.

Lastly, we show that $r \mathrm{O}_{2} \leq 2$. 


$$
\begin{aligned}
r h o_{2} \leq 2 & \Leftrightarrow 2 \lambda+(1-\lambda)\left(2-M-\theta_{0} \tilde{g}^{\prime} M^{\prime}\right) \leq 2 \\
& \Leftrightarrow(1-\lambda)\left(2-M-\theta_{0} \tilde{g}^{\prime} M^{\prime}\right) \geq 2(1-\lambda) \\
& \Leftrightarrow 2-M-\theta_{0} \tilde{g}^{\prime} M^{\prime} \leq 2 \\
& \Leftrightarrow M+\theta_{0} \tilde{g}^{\prime} M^{\prime} \geq 0
\end{aligned}
$$

We know that $M+\theta_{0} \tilde{g}^{\prime} M^{\prime}$ is a decreasing function of $\theta_{0}$, so a sufficient condition for this inequality to hold is that it holds where $M+\theta_{0} \tilde{g}^{\prime} M^{\prime}$ reaches its minimum. On this portion of the income distribution, $M+\theta_{0} \tilde{g}^{\prime} M^{\prime}$ reaches its local minimum at $\theta_{H}$. In addition, since $M$ takes values in [0;1], we only need to show that $\theta_{H} \tilde{g}^{\prime}\left(\theta_{H}\right) M^{\prime}\left(\tilde{g}\left(\theta_{H}\right)\right) \geq 0$ to show that $M+\theta_{0} \tilde{g}^{\prime} M^{\prime} \geq 0$.

$$
\lim _{\theta \rightarrow \theta_{H}} \theta_{0} \tilde{g}^{\prime} M^{\prime}=\theta_{H} \tilde{g}^{\prime}\left(\theta_{H}\right) \lim _{\theta \rightarrow \theta_{H}} M^{\prime}(\tilde{g}(\theta))=0
$$

Therefore we have that $M+\theta_{0} \tilde{g}^{\prime} M^{\prime} \geq 0$ always holds, and thus $r h o_{2} \leq 2$ always holds. This concludes our demonstration of Proposition 1.

\section{A.2 Data Preparation}

Income and program participation-related variables Respondents were asked each survey year how much income they received in the past calendar year from a specific source: wages and salary, military service, business and farm, alimony, parental support. Respondents were also asked about the welfare benefits they received each month and in total in the past calendar: unemployment benefits, child support, AFDC (Aid to Families with Dependent Children), Foodstamps, SSI (Supplementary Security Income), veteran benefits, disability payments, social security payments and any other source of public assistance. We focus on yearly income and welfare variables. Whenever available, we also extract income and welfare variables related to the spouse of respondents. We do this because these variables are important for tax simulation, and thus reflect best the true wealth of households (see e.g. Dahl and Lochner (2012) who simulate EITC payments using the Taxsim9 tax simulation program from the NBER. ${ }^{1}$ ) We use each of the different sources of income reported by respondents, and group them in the following seven 
categories: (i) Income from wages, salary and tips (including income from military service), (ii) income from farm or business, (iii) unemployment compensation, (iv) income from other sources (savings, net rental income, social security income), (v) veteran benefits, disability payments and worker compensation, (vi) welfare benefits (AFDC, food stamps, SSI, and any other public assistance), (vii) child support. Lastly, we further aggregate these sources of income into three main categories which are used in the analysis: a) earned income (source (i)), b) unearned income (sources (ii) and (iii)), and c) non-taxable income (sources (iv) to (vii)). ${ }^{2}$

Cognitive ability We use the Armed Forces Qualifying Test score (AFQT) as a proxy for cognitive ability. The AFQT is a standardized test composed of a section for mathematics, vocabulary, science and automotive knowledge. The NLSY79 collects AFQT percentile score, as an indication of the relative performance of respondents compared to other respondents. The AFQT was administered to all respondents in 1980 independently of any observable characteristic, in particular schooling level or age. Thus, AFQT scores in the NLSY79 are likely to reflect true cognitive ability, as well as cohort, age as well as schooling effects. Following the literature, we build an adjusted AFQT score for each individual by first regressing the original AFQT percentile score on quarter of birth and age dummies, then categorizing the residuals of the regression into 100 percentiles, and finally using the residuals in the analysis.

Demographic characteristics In all regressions of individual wealth, we control for the education attainment of respondents, their marital status and their fertility. Educational attainment is reported in the NLSY79 as the highest number of completed years of education as of May 1st of the year of interview. Since most respondents make their decisions regarding higher education before 1983, we can construct a time invariant measure of highest educational level completed from 1983 onwards. Our variable is an ordered categorical variable taking values 0 for respondents with less a high school degree (strictly less than 12 years of education), 1 for respondents with a high school degree (12 years of education), 2 for respondents with some college education (between 12 and 16 years of education), and 3 for respondents with a 4 -college degree $(16+$ years of education).

Marital status and the number of children of respondents are also included in every

\footnotetext{
${ }^{2}$ Taxsim9 is maintained by Daniel Feenberg, NBER and the NBER. For more information, see this link.
} 
survey wave. The initial variable for marital status included in the NLSY79 takes values 0 if the respondent was never married, 1 if he is married, 2 if he is separated, 3 if he is divorced, 5 if he is remarried and 6 if he is widowed. We use this variable for our analysis of demographic characteristics of respondents. These variables are important controls in our regression analyses and in our description of rational and behavioral agents. We construct the number of dependent children as the number of children younger than 17 years old in the household, and the number of dependent as the number of children independently of their age.

Imputation procedure We follow Dahl and Lochner (2012)'s imputation methodology and impute missing values for each income source separately, marital status, education, and the number of children of respondents using simple interpolation from adjacent survey years. For these variables, most missing observations are generated by the biennial nature of the panel, and imputation should therefore not assign erroneous values.

\section{A.3 Additional Tables and Figures}


Table A.1: Baseline Intragenerational Mobility (full model)

\begin{tabular}{|c|c|c|c|c|c|}
\hline & \multicolumn{5}{|c|}{ Final Family Net Worth (ages 40-48, IHS) } \\
\hline & $(1)$ & $(2)$ & (3) & $(4)$ & $(5)$ \\
\hline Initial family net worth & $\begin{array}{l}0.329 * * * \\
(0.018)\end{array}$ & $\begin{array}{l}0.256 * * * \\
(0.018)\end{array}$ & $\begin{array}{l}0.192 * * * \\
(0.018)\end{array}$ & $\begin{array}{l}0.197 * * * \\
(0.017)\end{array}$ & $\begin{array}{l}0.194 * * * \\
(0.017)\end{array}$ \\
\hline Zero initial family net worth & & $\begin{array}{c}-2.880 * * * \\
(0.385)\end{array}$ & $\begin{array}{c}-1.657 * * * \\
(0.377)\end{array}$ & $\begin{array}{c}-1.202 * * * \\
(0.378)\end{array}$ & $\begin{array}{c}-1.158 * * * \\
(0.379)\end{array}$ \\
\hline Zero final family net worth & & $\begin{array}{c}-7.759 * * * \\
(0.189)\end{array}$ & $\begin{array}{c}-6.293 * * * \\
(0.214)\end{array}$ & $\begin{array}{c}-5.750 * * * \\
(0.221)\end{array}$ & $\begin{array}{c}-5.653 * * * \\
(0.220)\end{array}$ \\
\hline Female & & & $\begin{array}{c}0.063 \\
(0.152)\end{array}$ & $\begin{array}{c}-0.188 \\
(0.151)\end{array}$ & $\begin{array}{c}-0.188 \\
(0.150)\end{array}$ \\
\hline Black & & & $\begin{array}{c}-0.206 \\
(0.239)\end{array}$ & $\begin{array}{r}-0.429 * \\
(0.237)\end{array}$ & $\begin{array}{c}-0.294 \\
(0.241)\end{array}$ \\
\hline Non-Black, non-Hispanic & & & $\begin{array}{l}1.245 * * * \\
(0.215)\end{array}$ & $\begin{array}{l}0.837 * * * \\
(0.215)\end{array}$ & $\begin{array}{l}0.621 * * * \\
(0.226)\end{array}$ \\
\hline Never married & & & $\begin{array}{l}2.863 * * * \\
(0.397)\end{array}$ & $\begin{array}{l}2.078 * * * \\
(0.400)\end{array}$ & $\begin{array}{l}2.065 * * * \\
(0.400)\end{array}$ \\
\hline Married & & & $\begin{array}{l}7.575 * * * \\
(0.408)\end{array}$ & $\begin{array}{l}6.481 * * * \\
(0.412)\end{array}$ & $\begin{array}{l}6.403 * * * \\
(0.412)\end{array}$ \\
\hline No children & & & $\begin{array}{l}1.861 * * * \\
(0.446)\end{array}$ & $\begin{array}{l}1.066 * * \\
(0.438)\end{array}$ & $\begin{array}{l}1.042 * * \\
(0.437)\end{array}$ \\
\hline 1 child & & & $\begin{array}{l}1.320 * * * \\
(0.486)\end{array}$ & $\begin{array}{l}1.233 * * * \\
(0.477)\end{array}$ & $\begin{array}{l}1.225 * * \\
(0.476)\end{array}$ \\
\hline 2 children & & & $\begin{array}{l}1.051 * * \\
(0.474)\end{array}$ & $\begin{array}{c}0.676 \\
(0.464)\end{array}$ & $\begin{array}{c}0.694 \\
(0.464)\end{array}$ \\
\hline Lived alone before 1982 & & & $\begin{array}{r}-0.505 * \\
(0.273)\end{array}$ & $\begin{array}{r}-0.477 * \\
(0.270)\end{array}$ & $\begin{array}{r}-0.504 * \\
(0.269)\end{array}$ \\
\hline High school diploma & & & & $\begin{array}{l}1.006 * * * \\
(0.245)\end{array}$ & $\begin{array}{l}0.761 * * * \\
(0.251)\end{array}$ \\
\hline Some college & & & & $\begin{array}{l}1.959 * * * \\
(0.273)\end{array}$ & $\begin{array}{l}1.516 * * * \\
(0.291)\end{array}$ \\
\hline 4-College degree & & & & $\begin{array}{l}3.411 * * * \\
(0.280)\end{array}$ & $\begin{array}{l}2.746 * * * \\
(0.326)\end{array}$ \\
\hline AFQT (percentiles, adjusted) & & & & & $\begin{array}{l}0.015 * * * \\
(0.004)\end{array}$ \\
\hline $\mathrm{R} 2$ & 0.078 & 0.17 & 0.26 & 0.29 & 0.29 \\
\hline $\mathrm{N}$ & 5,131 & 5,131 & 5,131 & 5,131 & 5,131 \\
\hline
\end{tabular}

Note: This table presents the results of regressions of permanent net worth on initial net worth. Each column represents a separate regression. Robust standard errors in parentheses. ${ }^{* * *} p<0.01$, $^{* *}$ $p<0.05,{ }^{*} p<0.1$. Table 1.2 presents a summary version of this model. 
Table A.2: Intragenerational Social Mobility with Two Thresholds (full model)

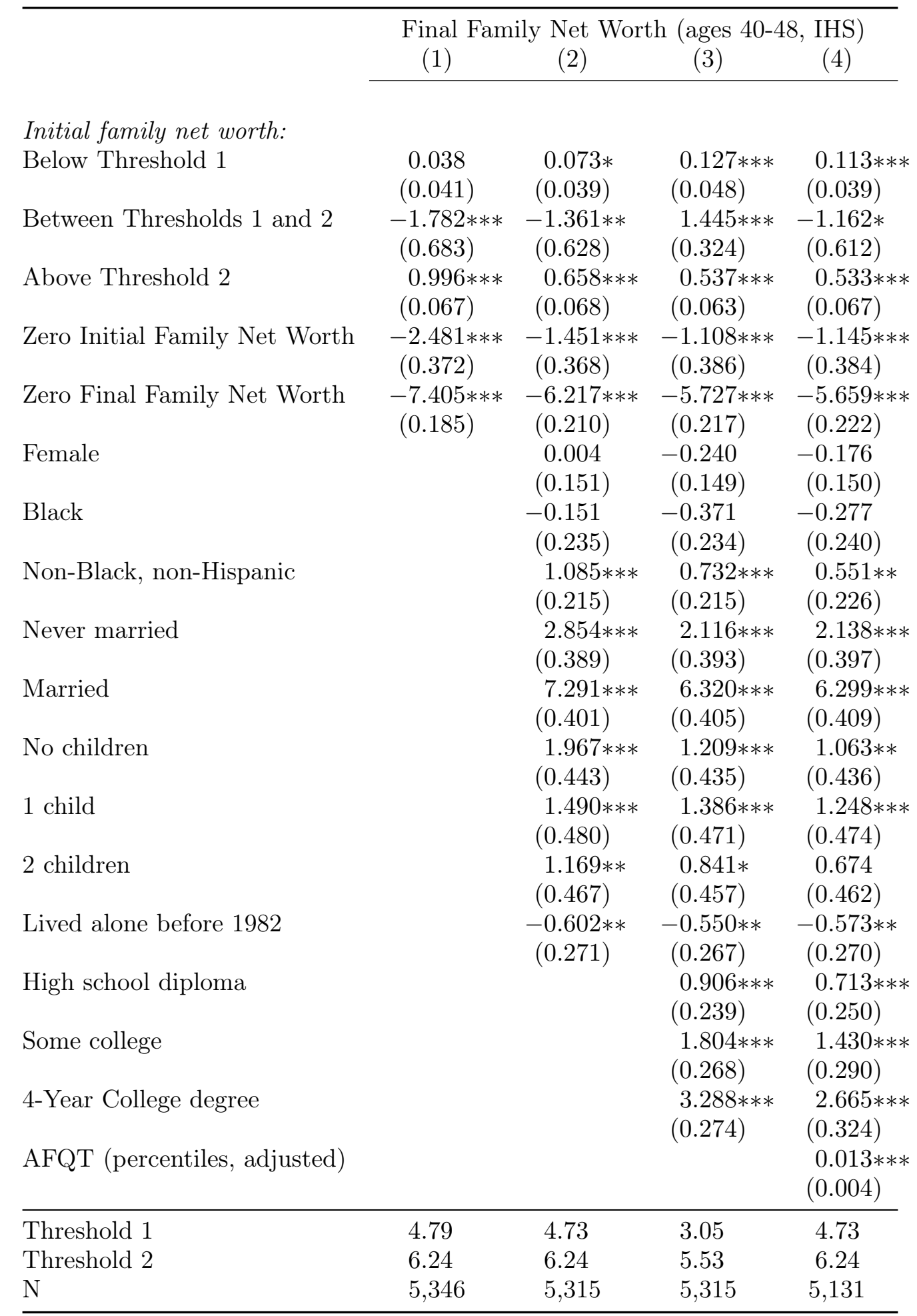

Note: This table presents the results of threshold regressions of permanent net worth on initial net worth, using a grid-search algorithm for the detection of thresholds and the HQIC criterion for model selection. Each column represents a separate regression. Robust standard errors in parentheses. ${ }^{* * *} p<0.01,{ }^{* *} p<0.05,{ }^{*} p<0.1$. Table 1.3 presents a summary version of this model. 
Table A.3: Intragenerational Social Mobility with Three Thresholds (full model)

\begin{tabular}{|c|c|c|c|c|}
\hline \multirow{3}{*}{$\begin{array}{l}\text { Initial family net worth: } \\
\text { Below Threshold } 1\end{array}$} & \multicolumn{4}{|c|}{ 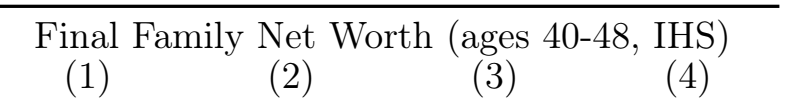 } \\
\hline & & & & \\
\hline & $\begin{array}{c}0.037 \\
(0.041)\end{array}$ & $\begin{array}{r}0.073 * \\
(0.039)\end{array}$ & $\begin{array}{l}0.128 * * * \\
(0.048)\end{array}$ & $\begin{array}{l}0.113 * * * \\
(0.039)\end{array}$ \\
\hline Between thresholds 1 and 2 & $\begin{array}{l}-1.782 * * * \\
(0.683)\end{array}$ & $\begin{array}{l}-1.358 * * \\
(0.628)\end{array}$ & $\begin{array}{l}12.727 * * * \\
(4.796)\end{array}$ & $\begin{array}{c}-1.161 * \\
(0.612)\end{array}$ \\
\hline Between thresholds 2 and 3 & $\begin{array}{l}1.064 * * * \\
(0.141)\end{array}$ & $\begin{array}{c}-5.339 \\
(4.795)\end{array}$ & $\begin{array}{l}1.427 * * * \\
(0.402)\end{array}$ & $\begin{array}{c}-6.190 \\
(4.872)\end{array}$ \\
\hline Above Threshold 3 & $\begin{array}{l}0.384 * * * \\
(0.137)\end{array}$ & $\begin{array}{l}0.590 * * * \\
(0.067)\end{array}$ & $\begin{array}{l}0.536 * * * \\
(0.063)\end{array}$ & $\begin{array}{l}0.474 * * * \\
(0.067)\end{array}$ \\
\hline Zero Initial Family Net Worth & $\begin{array}{l}-2.483 * * * \\
(0.372)\end{array}$ & $\begin{array}{l}-1.444 * * * \\
(0.368)\end{array}$ & $\begin{array}{l}-1.102 * * * \\
(0.386)\end{array}$ & $\begin{array}{l}-1.140 * * * \\
(0.384)\end{array}$ \\
\hline Zero Final Family Net Worth & $\begin{array}{l}-7.393 * * * \\
(0.185)\end{array}$ & $\begin{array}{l}-6.221 * * * \\
(0.210)\end{array}$ & $\begin{array}{l}-5.728 * * * \\
(0.217)\end{array}$ & $\begin{array}{l}-5.662 * * * \\
(0.222)\end{array}$ \\
\hline Female & & $\begin{array}{c}0.012 \\
(0.151)\end{array}$ & $\begin{array}{l}-0.236 \\
(0.149)\end{array}$ & $\begin{array}{r}-0.169 \\
(0.149)\end{array}$ \\
\hline Black & & $\begin{array}{l}-0.152 \\
(0.235)\end{array}$ & $\begin{array}{l}-0.366 \\
(0.234)\end{array}$ & $\begin{array}{c}-0.282 \\
(0.239)\end{array}$ \\
\hline Non-Black, non-Hispanic & & $\begin{array}{l}1.085 * * * \\
(0.214)\end{array}$ & $\begin{array}{l}0.735 * * * \\
(0.215)\end{array}$ & $\begin{array}{l}0.548 * * \\
(0.225)\end{array}$ \\
\hline Never married & & $\begin{array}{l}2.825 * * * \\
(0.389)\end{array}$ & $\begin{array}{l}2.104 * * * \\
(0.393)\end{array}$ & $\begin{array}{l}2.109 * * * \\
(0.398)\end{array}$ \\
\hline Married & & $\begin{array}{l}7.289 * * * \\
(0.401)\end{array}$ & $\begin{array}{l}6.323 * * * \\
(0.405)\end{array}$ & $\begin{array}{l}6.293 * * * \\
(0.409)\end{array}$ \\
\hline No children & & $\begin{array}{l}1.979 * * * \\
(0.442)\end{array}$ & $\begin{array}{l}1.225 * * * \\
(0.435)\end{array}$ & $\begin{array}{l}1.079 * * \\
(0.435)\end{array}$ \\
\hline 1 child & & $\begin{array}{l}1.503 * * * \\
(0.480)\end{array}$ & $\begin{array}{l}1.391 * * * \\
(0.471)\end{array}$ & $\begin{array}{l}1.270 * * * \\
(0.474)\end{array}$ \\
\hline 2 children & & $\begin{array}{l}1.174 * * \\
(0.466)\end{array}$ & $\begin{array}{r}0.834 * \\
(0.458)\end{array}$ & $\begin{array}{c}0.688 \\
(0.461)\end{array}$ \\
\hline Lived alone before 1982 & & $\begin{array}{c}-0.616 * * \\
(0.271)\end{array}$ & $\begin{array}{c}-0.538 * * \\
(0.267)\end{array}$ & $\begin{array}{c}-0.586 * * \\
(0.269)\end{array}$ \\
\hline High school diploma & & & $\begin{array}{l}0.931 * * * \\
(0.239)\end{array}$ & $\begin{array}{l}0.716 * * * \\
(0.250)\end{array}$ \\
\hline Some college & & & $\begin{array}{l}1.827 * * * \\
(0.268)\end{array}$ & $\begin{array}{l}1.430 * * * \\
(0.290)\end{array}$ \\
\hline 4-Year College degree & & & $\begin{array}{l}3.316 * * * \\
(0.274)\end{array}$ & $\begin{array}{l}2.665 * * * \\
(0.324)\end{array}$ \\
\hline AFQT (percentiles, adjusted) & & & & $\begin{array}{l}0.013 * * * \\
(0.004)\end{array}$ \\
\hline & & & 3.05 & 4.73 \\
\hline Threshold 2 & 6.24 & 6.24 & 3.53 & 6.24 \\
\hline Threshold 3 & 9.84 & 6.84 & 5.53 & 6.84 \\
\hline $\mathrm{N}$ & 5,346 & 5,315 & 5,315 & 5,131 \\
\hline
\end{tabular}

Note: This table presents the results of threshold regressions of permanent net worth on initial net worth, using a grid-search algorithm for the detection of thresholds and the HQIC criterion for model selection. Each column represents a separate regression. Robust standard errors in parentheses. ${ }^{* * *} p<0.01,{ }^{* *} p<0.05,{ }^{*} p<0.1$. Table 1.4 presents a summary version of this model. 
Table A.4: Intragenerational Social Mobility and Locus of Control (full model)

\section{(1)}

Final Family Net Worth

Initial family net worth:

Below Threshold 1

Between thresholds 1 and 2

Above Threshold 2

Rotter external locus of control (1979)

Rotter score by region of initial wealth:

Below Threshold 1

Between thresholds 1 and 2

Above Threshold 2

Zero Initial Family Net Worth

Zero Final Family Net Worth

Female

Black

Non-Black, non-Hispanic

Never married

Married

No children

1 child

2 children

Lived alone before 1982

High school diploma

Some college

4-Year College degree

AFQT (percentiles, adjusted)

$\begin{array}{ccc}0.189 * * * & 0.109 * * * & 0.109 * * * \\ (0.027) & (0.039) & (0.039) \\ 0.193 * * * & -1.305 * * & -1.292 * * \\ (0.022) & (0.595) & (0.594) \\ 0.169 * * & 0.534 * * * & 0.534 * * * \\ (0.068) & (0.067) & (0.067) \\ & -0.071 * * & \\ & (0.032) & \end{array}$

$-0.063$

(0.062)

$-0.132$

$(0.127)$

$-0.071 *$

(0.037)

$\begin{array}{ccc}-1.199 * * * & -1.188 * * * & -1.188 * * * \\ (0.382) & (0.388) & (0.387) \\ -5.662 * * * & -5.676 * * * & -5.678 * * * \\ (0.222) & (0.224) & (0.224) \\ -0.171 & -0.159 & -0.157 \\ (0.151) & (0.150) & (0.150) \\ -0.295 & -0.270 & -0.270 \\ (0.242) & (0.241) & (0.241) \\ 0.659 * * * & 0.587 * * * & 0.585 * * * \\ (0.226) & (0.226) & (0.226) \\ 2.120 * * * & 2.191 * * * & 2.188 * * * \\ (0.400) & (0.399) & (0.399) \\ 6.423 * * * & 6.321 * * * & 6.319 * * * \\ (0.412) & (0.410) & (0.410) \\ 0.958 * * & 1.002 * * & 1.004 * * \\ (0.436) & (0.435) & (0.435) \\ 1.236 * * * & 1.269 * * * & 1.269 * * * \\ (0.474) & (0.473) & (0.473) \\ 0.669 & 0.672 & 0.673 \\ (0.461) & (0.461) & (0.461) \\ -0.562 * * & -0.616 * * & -0.617 * * \\ (0.271) & (0.271) & (0.271) \\ 0.745 * * * & 0.683 * * * & 0.685 * * * \\ (0.252) & (0.251) & (0.252) \\ 1.491 * * * & 1.393 * * * & 1.394 * * * \\ (0.292) & (0.292) & (0.292) \\ 2.684 * * * & 2.594 * * * & 2.593 * * * \\ (0.327) & (0.325) & (0.326) \\ 0.013 * * * & 0.012 * * * & 0.012 * * * \\ (0.004) & (0.004) & (0.004) \\ & & \end{array}$

Thresholds in locus of control:

Threshold 1

Threshold 2 7

12

Thresholds in initial wealth:

Threshold 1

Threshold 2

$\mathrm{N}$

$\begin{array}{llr} & 4.73 & 4.73 \\ 5,083 & 6.24 & 6.24 \\ & 5,083 & 5,083\end{array}$

Note: This table presents the results of threshold regressions of permanent net worth on initial net worth augmented by locus of control, using a grid-search algorithm for the detection of thresholds and the HQIC criterion for model selection. Each column represents a separate regression. Robust standard errors in parentheses. ${ }^{* * *} p<0.01$, ** $p<0.05,{ }^{*} p<0.1$. Table 1.5 presents a summary version of this model. 
Figure A.1: Self-Esteem and Permanent Income



Note: This figure represents the results of the grid-search algorithm to detect the presence of thresholds in the relationship between Rosenberg's self-esteem score and log family net worth at ages 22 to 25. Red horizontal lines represent the location of thresholds in log family net worth at ages 22 to 25 (initial conditions), and green vertical lines represent the location of potential thresholds in self-esteem. Dots meeting at the crossing of a green and a red line indicates that the threshold detected in initial conditions could be driven by a correlated threshold in self-esteem. 
Table A.5: Intragenerational Social Mobility and Self-Esteem (full model)

(1)

Final Family Net Worth

(2)

(3)

Initial family net worth:

Below Threshold 1

Between thresholds 1 and 2

Above Threshold 2

Rosenberg self-esteem (1980)

$\begin{array}{lll}0.246 * * * & 0.132 * * * & 0.135 * * * \\ (0.050) & (0.048) & (0.048) \\ 0.213 * * * & 1.660 * * * & 1.665 * * * \\ (0.031) & (0.332) & (0.333) \\ 0.169 * * * & 0.508 * * * & 0.516 * * * \\ (0.021) & (0.064) & (0.064) \\ & 0.036 * & \\ & (0.020) & \end{array}$

Rosenberg score by region of initial wealth:

Below Threshold 1

$0.074 *$

$(0.043)$

0.073

$(0.057)$

0.011

Above Threshold 2

$(0.022)$
Zero Initial Family Net Worth

Zero Final Family Net Worth

Female

Black

Non-Black, non-Hispanic

Never married

Married

No children

1 child

2 children

Lived alone before 1982

High school diploma

Some college

4-Year College degree

AFQT (percentiles, adjusted)

$-1.081 * * * \quad-1.234 * * * \quad-1.220 * * *$

$\begin{array}{lll}(0.386) & (0.407) \quad(0.409)\end{array}$

$-5.551 * * * \quad-5.607 * * * \quad-5.581 * * *$

$\begin{array}{lll}(0.228) & (0.228) & (0.230)\end{array}$

$\begin{array}{lll}-0.189 & -0.189 & -0.189\end{array}$

$\begin{array}{lll}(0.152) & (0.151) & (0.152)\end{array}$

$\begin{array}{lll}-0.295 & -0.280 & -0.285\end{array}$

$\begin{array}{lll}(0.246) & (0.246) \quad(0.246)\end{array}$

$0.677 * * * \quad 0.616 * * * \quad 0.616 * * *$

$\begin{array}{lll}(0.229) & (0.230) & (0.229)\end{array}$

$2.041 * * * \quad 2.124 * * * \quad 2.125 * * *$

$\begin{array}{lll}(0.405) & (0.403) & (0.403)\end{array}$

$6.412 * * * \quad 6.293 * * * \quad 6.287 * * *$

$\begin{array}{lll}(0.416) & (0.413) & (0.413)\end{array}$

$\begin{array}{lll}1.024 * * & 1.075 * * & 1.060 * *\end{array}$

$\begin{array}{lll}(0.440) & (0.439) & (0.439)\end{array}$

$1.160 * * \quad 1.199 * * \quad 1.180 * *$

$\begin{array}{lll}(0.478) & (0.478) & (0.477)\end{array}$

$\begin{array}{lll}0.617 & 0.621 & 0.616\end{array}$

$\begin{array}{lll}(0.470) & (0.469) & (0.469)\end{array}$

$-0.568 * * \quad-0.636 * * \quad-0.627 * *$

$\begin{array}{lll}(0.275) & (0.276) \quad(0.276)\end{array}$

$0.760 * * * \quad 0.739 * * * \quad 0.717 * * *$

$\begin{array}{lll}(0.260) & (0.258) & (0.259)\end{array}$

$1.509 * * * \quad 1.428 * * * \quad 1.405 * * *$

$\begin{array}{lll}(0.301) & (0.300) & (0.301)\end{array}$

$2.717 * * * \quad 2.650 * * * \quad 2.621 * * *$

$\begin{array}{lll}(0.336) & (0.333) \quad(0.334)\end{array}$

$0.014 * * * \quad 0.012 * * * \quad 0.012 * * *$

$(0.004) \quad(0.004) \quad(0.004)$

Thresholds in self-esteem:

Threshold 1 - 17

$\begin{array}{ll}\text { Threshold } 2 & 17\end{array}$

Thresholds in initial wealth:

Threshold 1

Threshold 2

$\begin{array}{rlr} & 3.05 & 3.05 \\ 5,020 & 5.53 & 5.53 \\ & 5,020 & 5,020\end{array}$

Note: This table presents the results of threshold regressions of permanent net worth on initial net worth augmented by a measure of self-esteem, using a grid-search algorithm for the detection of thresholds and the HQIC criterion for model selection. Each column represents a separate regression. Robust standard errors in parentheses. ${ }^{* * *} p<0.01$, ** $p<0.05,{ }^{*} p<0.1$. 
Figure A.2: Self-Concept and Permanent Income

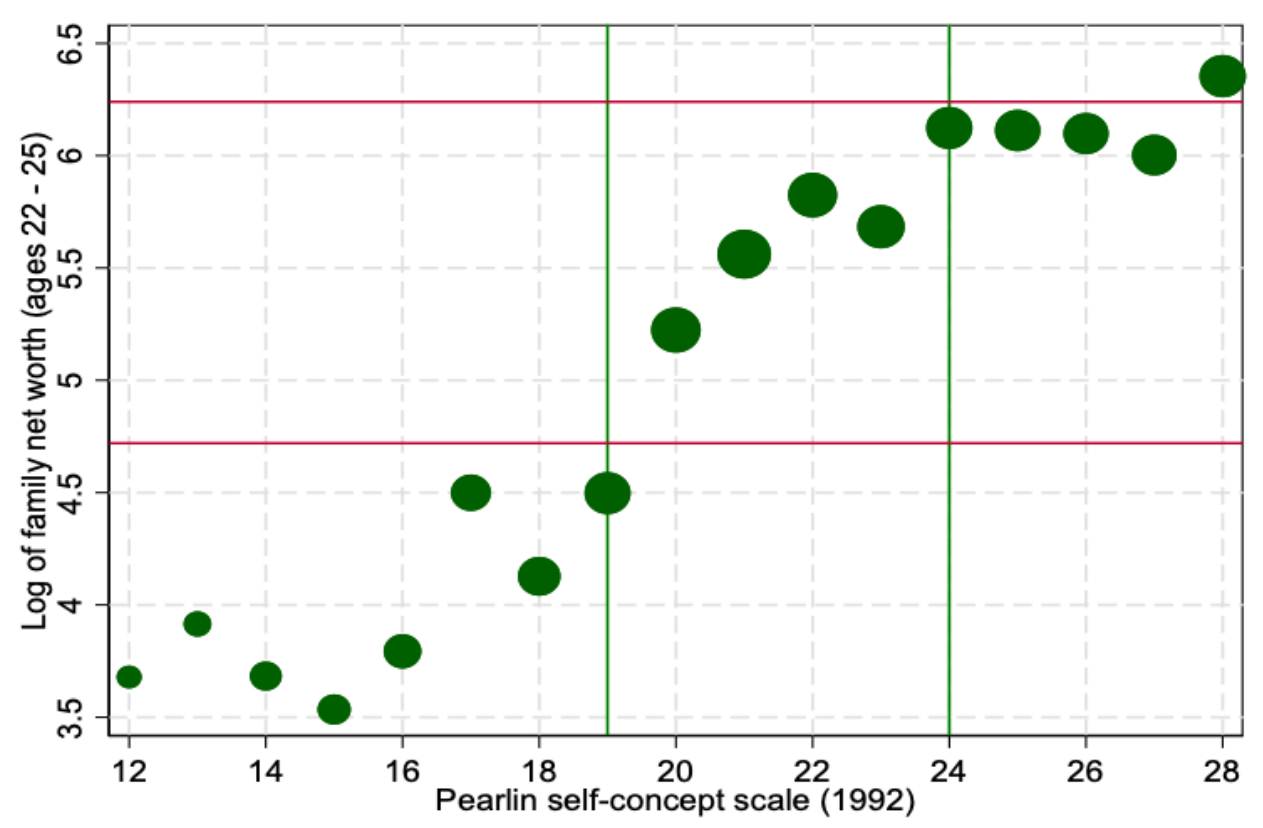

Note: This figure represents the results of the grid-search algorithm to detect the presence of thresholds in the relationship between Pearlin (1992)'s self-concept score and log family net worth at ages 22 to 25. Red horizontal lines represent the location of thresholds in log family net worth at ages 22 to 25 (initial conditions), and green vertical lines represent the location of potential thresholds in self-concept. Dots meeting at the crossing of a green and a red line indicates that the threshold detected in initial conditions could be driven by a correlated threshold in self-concept. 
Table A.6: Intragenerational Social Mobility and Self-Concept (full model)

(1)

Final Family Net Worth

(2)

(3)

Initial family net worth:

Below Threshold 1

Between thresholds 1 and 2

Above Threshold 2

Pearlin self-concept (1992)

Pearlin score by region of initial wealth:

$\begin{array}{ccc}0.130 * * * & 0.115 * * * & 0.128 * * \\ (0.039) & (0.039) & (0.054) \\ 0.217 * * * & -1.478 * * & 0.500 * * * \\ (0.024) & (0.646) & (0.177) \\ 0.185 * * * & 0.539 * * * & 0.540 * * * \\ (0.029) & (0.067) & (0.067) \\ & 0.099 * * * & \\ & (0.024) & \end{array}$

Below Threshold 1

Between thresholds 1 and 2

Above Threshold 2

Zero Initial Family Net Worth

Zero Final Family Net Worth

Female

Black

Non-Black, non-Hispanic

Never married

Married

No children

1 child

2 children

Lived alone before 1982

High school diploma

Some college

4-Year College degree

AFQT (percentiles, adjusted)

\begin{tabular}{|c|c|c|}
\hline & & $\begin{array}{c}0.026 \\
(0.049) \\
0.254 * * * \\
(0.059) \\
0.093 * * * \\
(0.028)\end{array}$ \\
\hline $\begin{array}{c}-1.154 * * * \\
(0.388)\end{array}$ & $-1.114 * * *$ & $-1.262 * * *$ \\
\hline$-5.574 * * *$ & $-5.514 * * *$ & $-5.453 * * *$ \\
\hline $\begin{array}{c}(0.224) \\
-0.195\end{array}$ & $\begin{array}{c}(0.226) \\
-0.187\end{array}$ & $\begin{array}{c}(0.231) \\
-0.199\end{array}$ \\
\hline$(0.152)$ & $(0.151)$ & $(0.151)$ \\
\hline-0.363 & -0.328 & -0.342 \\
\hline$(0.243)$ & $(0.242)$ & $(0.242)$ \\
\hline $0.640 * * *$ & $0.588 * * *$ & $0.574 * *$ \\
\hline$(0.225)$ & $(0.226)$ & $(0.226)$ \\
\hline $2.027 * * *$ & $2.112 * * *$ & $2.086 * * *$ \\
\hline$(0.404)$ & $(0.402)$ & $(0.402)$ \\
\hline $6.324 * * *$ & $6.226 * * *$ & $6.226 * * *$ \\
\hline$(0.415)$ & $(0.412)$ & $(0.413)$ \\
\hline $1.022 * *$ & $1.018 * *$ & $0.996 * *$ \\
\hline$(0.440)$ & $(0.439)$ & $(0.439)$ \\
\hline $1.200 * *$ & $1.209 * *$ & $1.196 * *$ \\
\hline$(0.479)$ & $(0.478)$ & $(0.478)$ \\
\hline 0.599 & 0.562 & 0.540 \\
\hline$(0.468)$ & $(0.466)$ & $(0.466)$ \\
\hline$-0.463 *$ & $-0.520 *$ & $-0.532 * *$ \\
\hline$(0.270)$ & $(0.270)$ & $(0.270)$ \\
\hline $0.765 * * *$ & $0.691 * * *$ & $0.687 * * *$ \\
\hline$(0.253)$ & $(0.252)$ & $(0.253)$ \\
\hline $1.496 * * *$ & $1.390 * * *$ & $1.398 * * *$ \\
\hline$(0.292)$ & $(0.292)$ & $(0.293)$ \\
\hline $2.640 * * *$ & $2.540 * * *$ & $2.549 * * *$ \\
\hline (0.328) & $(0.328)$ & $(0.328)$ \\
\hline $0.012 * * *$ & $0.010 * * *$ & $0.010 * * *$ \\
\hline$(0.004)$ & $(0.004)$ & $(0.004)$ \\
\hline
\end{tabular}

19

24

Threshold 1

Threshold 2

Thresholds in initial wealth:

Threshold 1

Threshold 2

$\begin{array}{rlr} & 4.83 & 2.36 \\ 4,984 & 6.24 & 6.24 \\ & 4,984 & 4,984\end{array}$

Note: This table presents the results of threshold regressions of permanent net worth on initial net worth augmented by a measure of self-concept, using a grid-search algorithm for the detection of thresholds and the HQIC criterion for model selection. Each column represents a separate regression. Robust standard errors in parentheses. ${ }^{* * *} p<0.01,{ }^{* *}$ $p<0.05,{ }^{*} p<0.1$. 
Figure A.3: Access to Loans and Aspirational Poverty Traps

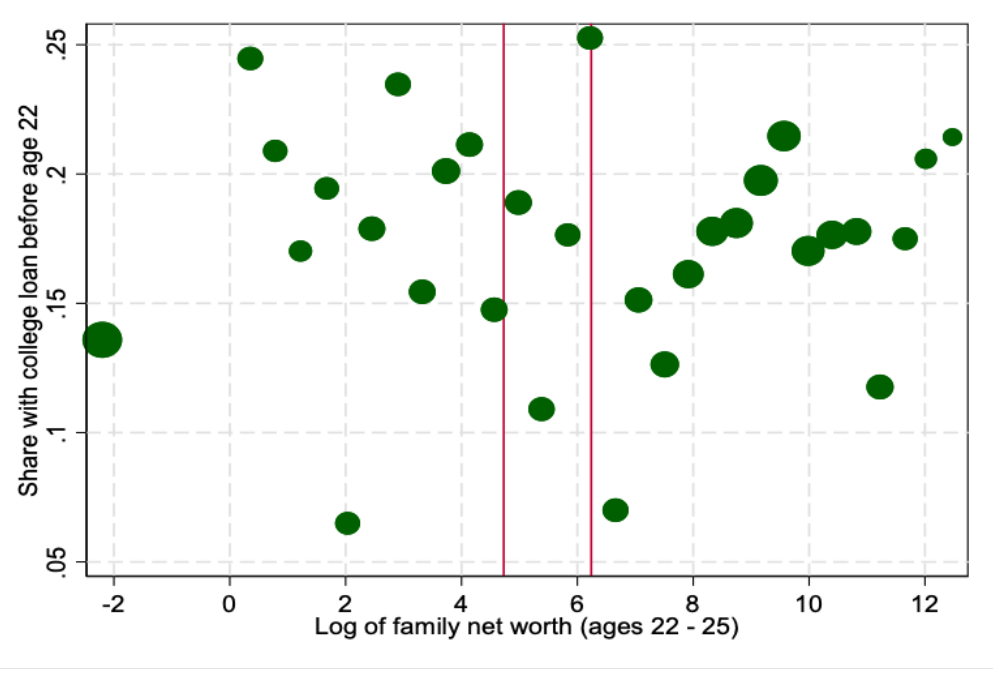

(a) Access to Educational Loans

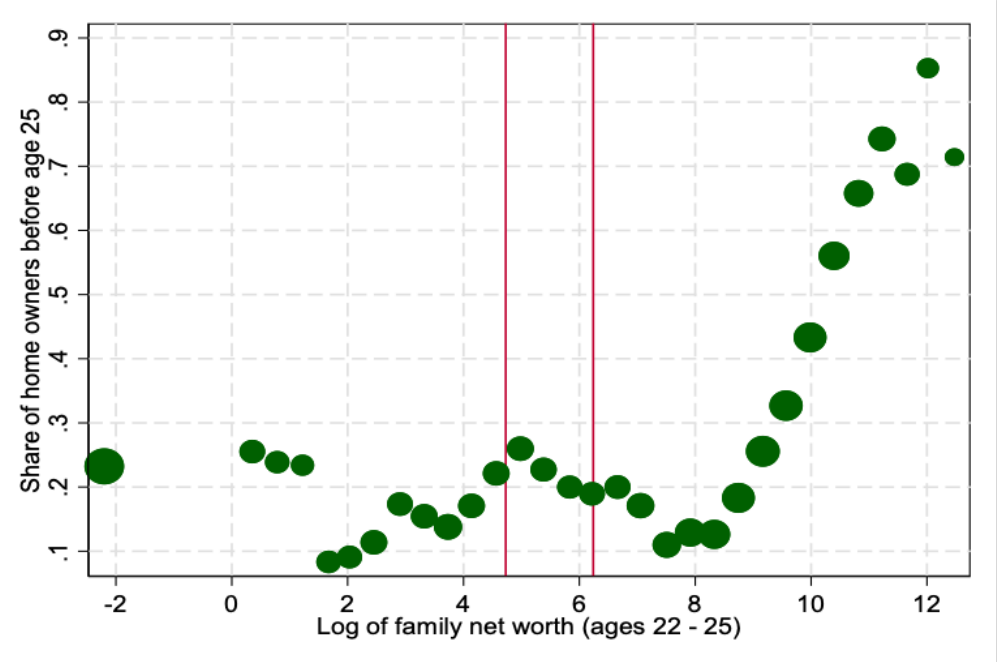

(b) Access to Home-Ownership at Age 25

Note: This figure represents the results of the grid-search algorithm to detect the presence of thresholds in the relationship between access to college loans (Figure A.3a) and mortgages ((Figure A.3b)) and log family net worth at ages 22 to 25. Red horizontal lines represent the location of thresholds in log family net worth at ages 22 to 25 (initial conditions), and green vertical lines represent the location of potential thresholds in the share of respondents with college loans before age 22 (Figure A.3a), and the share of homeowner respondents before age 25 (Figure A.3b). Dots meeting at the crossing of a green and a red line indicates that the threshold detected in initial conditions could be driven by a correlated threshold in access to college loans or mortgages, which are our measures of liquidity constraints. 
Table A.7: Intragenerational Social Mobility and Credit Constraints (full model)

Final Family Net Worth

(1) (2)

Initial family net worth:

Below Threshold 1

$\begin{array}{cc}0.113 * * * & 0.111 * * * \\ (0.039) & (0.039) \\ -1.162 * & -1.053 * * \\ (0.612) & (0.526) \\ 0.531 * * * & 0.612 * * * \\ (0.071) & (0.074) \\ 0.016 & \\ (0.181) & \end{array}$

Home-ownership by region of initial wealth:

Below Threshold 1

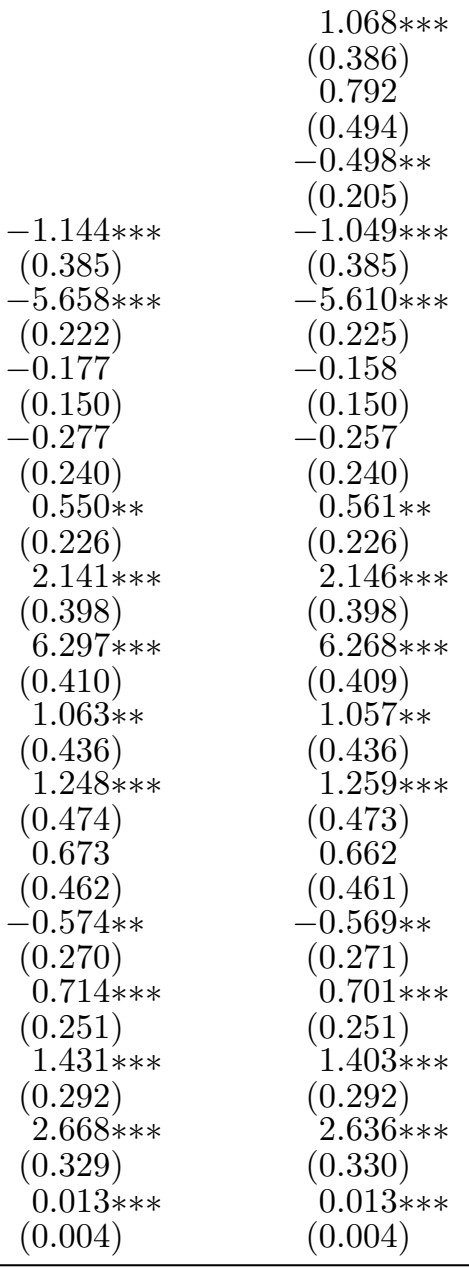

Thresholds in initial wealth:

Threshold 1

4.73

6.24

4.73

Threshold 2

5,131

6.46

$5,131 \quad 5,131$

Note: This table presents the results of threshold regressions of permanent net worth on initial net worth augmented by home-ownership status, using a grid-search algorithm for the detection of thresholds and the HQIC criterion for model selection. Each column represents a separate regression. Robust standard errors in parentheses. ${ }^{* * *} p<0.01$, ** $p<0.05,{ }^{*} p<0.1$. 
Figure A.4: Network-Based Job Search Strategies and Initial Family Net Worth

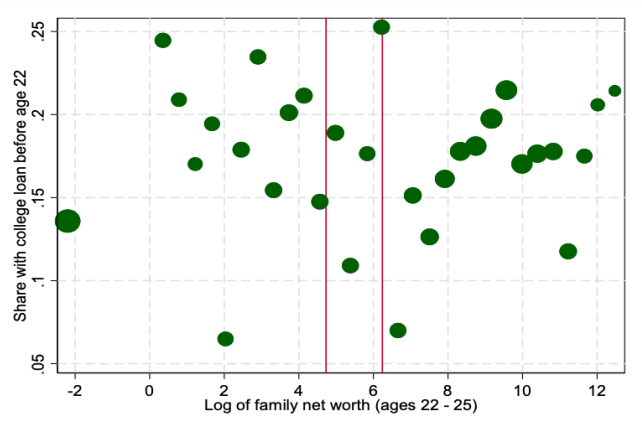

(a) Personal Network

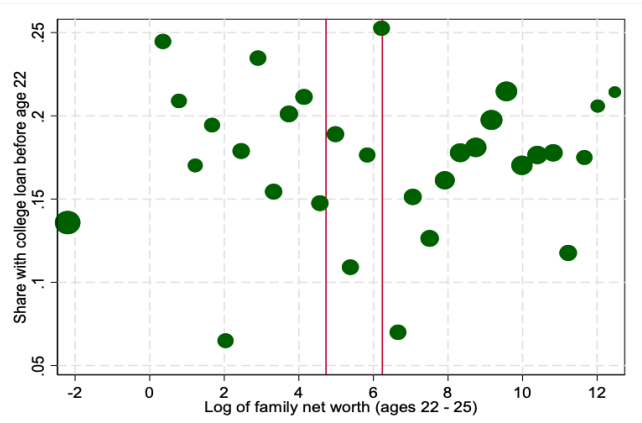

(c) Community Organization

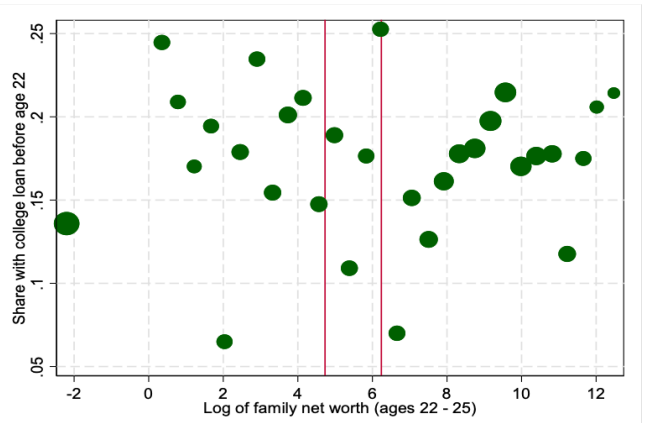

(e) Former Teachers

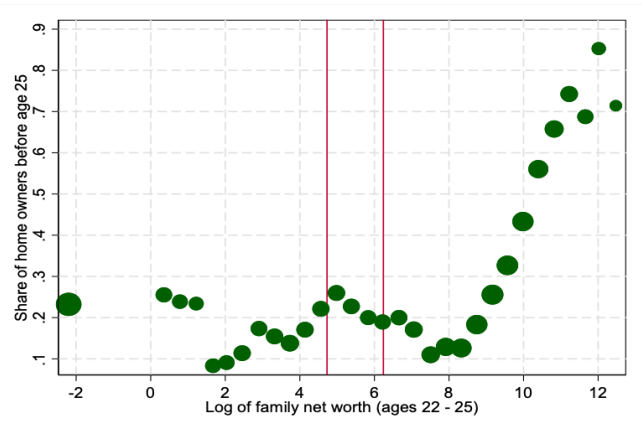

(b) Relatives

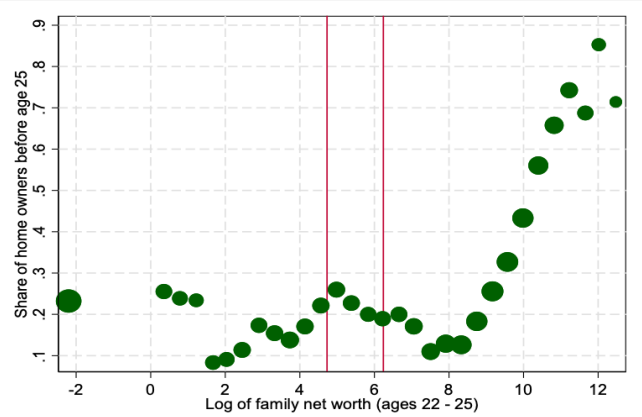

(d) School Placement Office

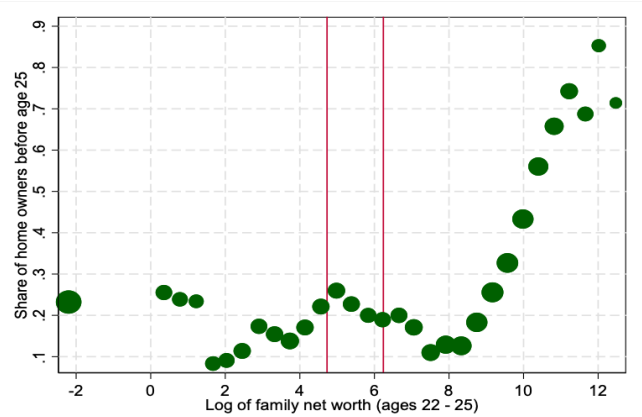

(f) Personal Contacts

Note: This figure represents the results of the grid-search algorithm to detect the presence of thresholds in the relationship between six types of job search strategies used by respondents to find their current job, and log family net worth at ages 22 to 25. Red horizontal lines represent the location of thresholds in log family net worth at ages 22 to 25 (initial conditions), and green vertical lines represent the location of potential thresholds in the share of respondents using any of the six job search strategies. Dots meeting at the crossing of a green and a red line indicates that the threshold detected in initial conditions could be driven by a correlated threshold in the use of network-based job search strategies, our measures of network strength. 
Table A.8: Intragenerational Social Mobility and Network-based Job Search Strategies (full model)

Final Family Net Worth

(1)

$$
\begin{gathered}
0.113 * * * \\
(0.039) \\
-1.164 * \\
(0.613) \\
0.533 * * * \\
(0.067) \\
-0.222 \\
(0.841)
\end{gathered}
$$

Job search through community org. (1982)

Job search by region of initial wealth:

Below Threshold 1

Between thresholds 1 and 2

Above Threshold 2

Zero Initial Family Net Worth

Zero Final Family Net Worth

Female

Black

Non-Black, non-Hispanic

Never married

Married

No children

\begin{tabular}{|c|c|}
\hline & 0.479 \\
\hline & $(0.953)$ \\
\hline & 0.000 \\
\hline & $(0.000)$ \\
\hline & -1.308 \\
\hline & $(1.557)$ \\
\hline$-1.146 * * *$ & $-1.271 * * *$ \\
\hline$(0.384)$ & $(0.381)$ \\
\hline$-5.659 * * *$ & $-5.691 * * *$ \\
\hline$(0.222)$ & $(0.219)$ \\
\hline-0.176 & -0.185 \\
\hline$(0.150)$ & $(0.150)$ \\
\hline-0.277 & -0.293 \\
\hline$(0.240)$ & $(0.239)$ \\
\hline $0.549 * *$ & $0.551 * *$ \\
\hline$(0.226)$ & $(0.225)$ \\
\hline $2.137 * * *$ & $2.080 * * *$ \\
\hline$(0.397)$ & $(0.398)$ \\
\hline $6.298 * * *$ & $6.297 * * *$ \\
\hline$(0.409)$ & $(0.408)$ \\
\hline $1.064 * *$ & $1.067 * *$ \\
\hline$(0.436)$ & $(0.435)$ \\
\hline $1.250 * * *$ & $1.277 * * *$ \\
\hline$(0.474)$ & $(0.475)$ \\
\hline 0.675 & 0.666 \\
\hline$(0.462)$ & $(0.460)$ \\
\hline$-0.573 * *$ & $-0.580 * *$ \\
\hline$(0.270)$ & $(0.270)$ \\
\hline $0.714 * * *$ & $0.743 * * *$ \\
\hline$(0.250)$ & $(0.250)$ \\
\hline $1.431 * * *$ & $1.465 * * *$ \\
\hline$(0.290)$ & $(0.291)$ \\
\hline $2.665 * * *$ & $2.705 * * *$ \\
\hline$(0.324)$ & $(0.325)$ \\
\hline $0.013 * * *$ & $0.013 * * *$ \\
\hline$(0.004)$ & $(0.004)$ \\
\hline
\end{tabular}

1 child

2 children

Lived alone before 1982

High school diploma

Some college

4-Year College degree

AFQT (percentiles, adjusted)
Thresholds in initial wealth:

Threshold 1

Threshold 2

4.73
6.24
5,131

6.54 6.84 5,131

Note: This table presents the results of threshold regressions of permanent net worth on initial net worth augmented by job-search strategies based on networks, using a grid-search algorithm for the detection of thresholds and the HQIC criterion for model selection. Each column represents a separate regression. Robust standard errors in parentheses. *** $p<0.01,{ }^{* *} p<0.05,{ }^{*} p<0.1$. 


\section{B $\mid$ Appendix to Chapter 2}

\section{B.1 Institutional Details}

\section{General details about the Dutch education system}

Education in the Netherlands is compulsory from age 5 until age 18. Most children enter kindergarten at age 4 and start attending primary school two years later, in Grade 1. Primary school takes six years to complete and ends with a standardized test (CITO) which covers mathematics, language and general learning ability. CITO test scores are used to assign students to the most appropriate high school track given a child's abilities and interests. The admission process for high schools is largely non-selective, meaning that the students can enroll in any high school they choose, irrespective of their grades and their CITO test scores - although a small share of selective secondary schools does apply selection criteria often based on prior test scores; these are almost always specialized in Gymnasium VWO tracks. Figure B.1 gives a schematic descriptive of the Dutch education system.

Primary schools must, by law, provide Grade 6 students exiting primary school with an objective measure of academic ability in the form of a standardized test score, and the school's recommendation of which high school track the child is best suited for. This implies that pupils and their parents decide on a high school at the end of Grade 6 based on three elements: 1) a standardized test score, 2) the primary school's advice regarding a high school track that best suits the child's abilities, 3) the child and her parents' preference for education between academic, general and vocational education.

Most children in our sample are in schools in which the standardized test is the CITO test. Since its creation in 2005, CITO has been given annually in more than $80 \%$ of Dutch schools to all Grade 6 pupils (12-year-olds). Dutch pupils take this test between February and May during Grade 6 before exiting primary school and receive 
Figure B.1: The Dutch Education System

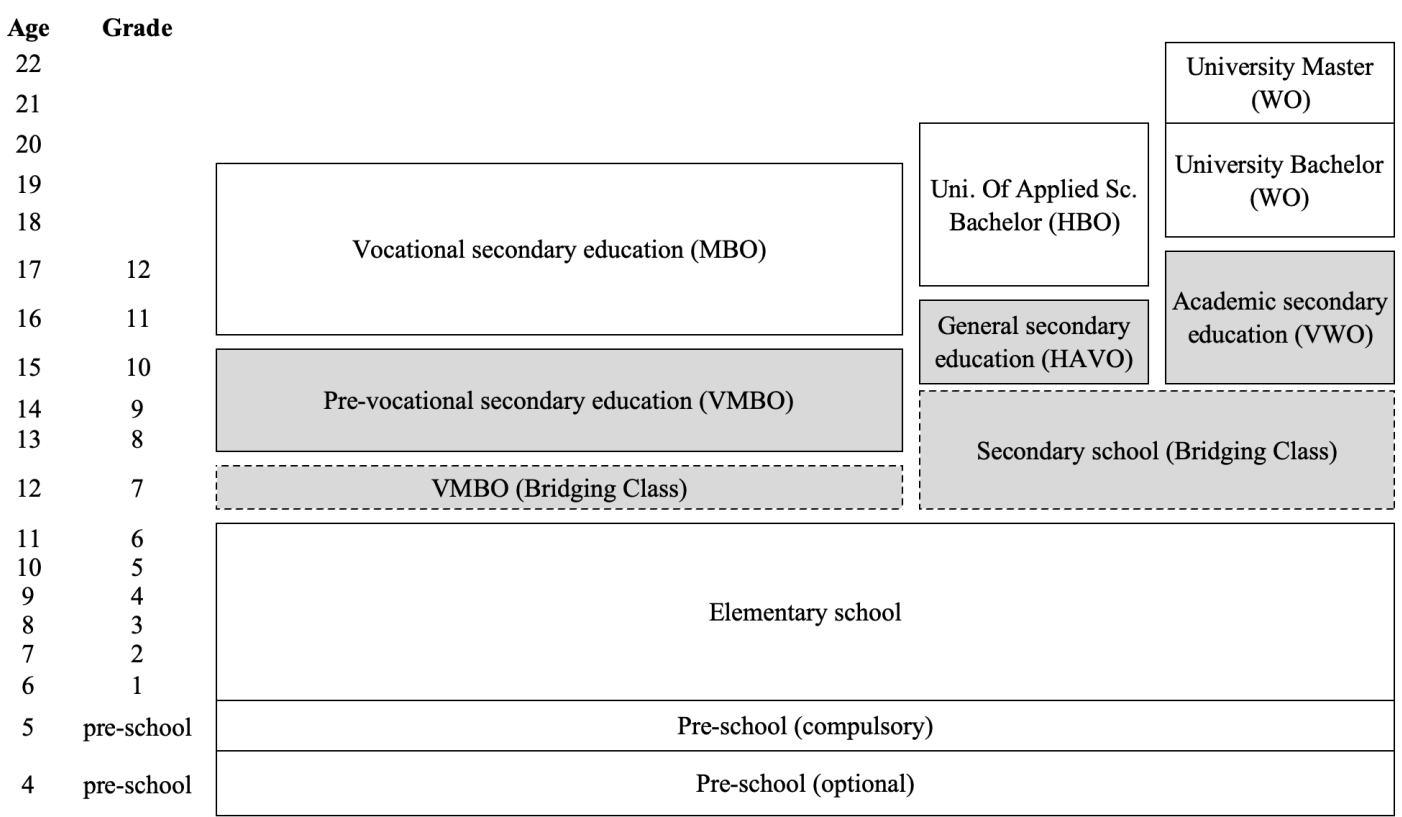

Note: Authors' own figure. This is a simplified description of the Dutch education system.

their score in June when they are in the process of choosing their secondary school from Grade 7 onward. The CITO test is designed every year by the CITOGroep, an independent private firm specialized in educational testing (akin to ETS in the US), which also grades the test independently from schools and the central government. The test is designed to be a test of cognitive ability with sections focusing on mathematics, language and general learning ability. Scores range between 501 and 550 points and are normalized such that the national annual average is 535 points. The CITOGroep recommends a mapping from pupils' scores onto high school types. The thresholds recommended by CITOGroep are fixed over the time frame of this study, and the shares of pupils in each bin are stable by construction. These are the publicly available mapping from CITO score onto high school tracks proposed by the CITOGroep:

1. 501-532: Vocational high school track

2. 533-536: Pre-Vocational or General high school track

3. 537-539: General high school track

4. 540-544: General or Academic high school track

5. 545-550: Academic high school track

Primary school teachers use their own observation of pupils' in class, as well as the CITO outcome of pupils to make their recommendation of high school type to pupils 
and parents. Teachers' advice is meant to help parents and children to choose the most appropriate high school for the child's ability. Over time, teachers gave fewer mixed recommendations (e.g. vocational/general high school, or general/academic high school) and slightly higher recommendations. The correlation between the recommendation that pupils receive, and their choice of high school type remains, however, stable over our time frame. The Dutch Inspectorate for education reports annually how children's chosen high school type relates to their CITO score and teacher's recommendation. Over the period we study, approximately 45 to $53 \%$ of pupils choose a high school type matching their recommendation, 23 to $26 \%$ choose a high school of a lower type than their recommendation, and 21 to $23 \%$ choose a type higher than their recommendation.

Students enter secondary school in Grade 7 and start with up to 3 years of common education, usually referred to as "bridging years". Following the bridging years, students are tracked into one of three high school tracks: 1) academic (VWO), 2) general (HAVO) and 3) vocational (VMBO). The academic track ends in Grade 12 and a diploma from academic high school constitutes the minimum requirement for enrolling into a university (WO). The general track ends one year earlier (Grade 11) and prepares students for professionally-oriented higher education. A general high school diploma constitutes the minimum requirement for enrolling in Universities of Applied Sciences (HBO) but does not make students eligible for university education. The vocational track ends in Grade 10 and prepares students for a vocational secondary education program (MBO), which may or may not take the form of an apprenticeship. The academic track is usually considered to be more difficult than the general track, which is usually considered to be more difficult than the vocational track.

Although secondary schools decide eventually on the track a pupil follows, parents and their child have a strong influence on this tracking outcome in 2 ways: 1) through discussion with the primary school on the track recommendation the child receives, and 2) through the choice of school. According to the principle of "free choice" defined in the so-called Mammoth Law of 1964, students cannot be strictly denied entry at any public school in the Netherlands, and schools received governmental funding according to the number of students annually registered. School funding is fixed per student irrespective of their high school type, which means that once a child is enrolled, secondary schools have no incentives to orient students towards one type of curriculum over another. This system of free choice and unconditional funding has led to a concentration of secondary schools, with the majority of schools being very large and offering all types of curriculum. 
A majority of secondary schools offers all tracks, yet the timing of tracking is part of a school structure, with some schools tracking students as early as grade 7 (first year of secondary school), thus not offering a bridge year, while other schools track students as late as grade 10 (fourth year of secondary school, thus offering three bridging years. Over our timeframe, Grade 7 pupils are about $80 \%$ in a bridge year, while this share reduces to $60 \%$ in Grade 8 and 2.5\% in Grade 9 (Korthals, 2015).

Once tracked, academic and general education students choose a field of study, and vocational students choose a professional trade. Fields are chosen by the end of Grade 9, and the field-specific curriculum spans the remaining years of high school, two in the case of the general track, and three in the case of the academic track. Students can choose among the following options: 1) Nature and Technology (Natuur en Techniek), 2) Nature and health (Natuur en Gezondheid), 3) Economy and society (Economie en Maatschappij) and 4) Culture and society (Cultuur en Maatschappij). Students can choose either a single field or a combination of two fields. Double specializations or mixed profiles are possible, yet students almost exclusively combine either the two STEM fields (1 and 2), or the two non-STEM fields (3 and 4 ). In this study, we consider a simplified choice of specialization, in which students choose either a curriculum in STEM and Medicine (field 1, field 2, or their combination), or a curriculum in the Humanities and Social sciences (field 3 , field 4 , or any remaining combinations).

In the last year of high school, students from the general and academic track apply to post-secondary institutions, and students from the vocational track apply to secondary vocational institutions. The admission process is largely non-selective, allowing students to enroll into any school and program available for their high school track diploma and their field of study. Applications are submitted through a unified online platform, where students also apply for student finance. ${ }^{1}$

\footnotetext{
${ }^{1}$ Selective admissions do exist for a small subset of over-demanded programs, such as medicine and medical sciences. For these programs, the selective admission process is based on a fixed number of spots (Numerus Fixus), and applications go through a stratified lottery system; applications for these specific programs are due in January, earlier in the academic year than the standard applications which are due in April. For a detailed description of the stratified lotteries for Numerus Fixus programs, see Ketel et al. (2016, 2019); Artmann et al. (2018).
} 


\section{General details on financial aid in the Netherlands}

The Dutch student finance system (Studiefinanciering) has been in place since 1986, and has since been organized into three parts. The first part is a basic, unconditional grant paid monthly for up to four years of higher education. The subsidy was intended to cover students' living expenses, and it was awarded at two rates, depending on students' living arrangements. The rates were set by a decree every calendar year, mirroring the gradual growth of tuition fees. In 2014, the monthly rate for university students living on their own was $€ 279.14$, which is equivalent to roughly $5.5 \%$ of the full-time minimum wage ( $€ 1,486$ monthly in 2014). The 2014 monthly rate for students living with parents was $€ 100.25$. The subsidy was also available for adult students attending secondary vocational education (MBO), and the two subsidy rates were $257.93 €$ and $79.04 €$, respectively.

The second type of aid was a means-tested subsidy awarded to students from disadvantaged backgrounds. The awarded amount was calculated by the Dutch authorities based on students' living arrangements, the taxable income of both parents two years prior to application, and household composition. In 2014, the maximum amount of means-tested subsidy a student could receive was $€ 258.35$ per month. The third type of available aid was an optional low-interest loan. The loan was provided by a government agency which guaranteed loan financing at below-market rates. There were three types of loans: 1) A basic loan depending on individual needs, 2) a loan to smooth payment of tuition fees expenses ("college tuition credit"), and 3) a loan to increase the duration of coverage of living expenses. The loan repayment amount was automatically adjusted by the tax office, so that the amount never exceeded $12 \%$ of taxable income. Students were expected to repay their loans over the period of 15 years following graduation, with monthly repayments calculated such that the total debt was repaid in exactly 15 years. If a student's post-graduation income was below full-time minimum wage, then the repayment was put on hold, and the residual amount due after 15 years was forgiven.

The third and last type if an opt-in loan with interest rates guaranteed below market rates. Table B.1 shows all financial aid amounts and conditions granted depending on a student's educational level, living situation and parental income. This table is organized as follows: Panel A shows information relative to higher education freshmen, and Panel B displays information relative to vocational education freshmen. In every panel, we decompose financial aid into its main components: i) basic, unconditional subsidy; ii) means-tested aid; iii) public transport card, iv) maximum monthly opt-in loan amount; v) tuition fees; and vi) the maximum monthly opt-in 
loan amount beyond the nominal duration of financial aid. In each category, we first indicate the different amounts granted depending on the living situation of the student, if applicable, then we show the year-to-change in the granted amount, and lastly the take-up rate of this aspect of financial aid if applicable. Overall, this table shows that financial aid conditions and amounts evolve in a smooth way, following the evolution of the consumer price index.

\section{Google Trends analysis of perceptions in the population}

Figure B.2 shows Google trends over the period 2004 to 2017, and reveals which parts of the student finance reform were known by the general public at which points in time. The query "basisbeurs" (basic grant) is relatively stable, with three main peaks in January and June 2010, and October 2012, secondary peaks in May 2014 and January 2015. In January 2010, large national demonstrations were organized by student trade unions against budget cuts in education mentioned during the political campaign. In May 2014, the government announced that the reform would be postponed by one year, until September 2015. In January 2015, the first cohort of students under the new regime of student finance started making their applications via the national platform, Studielink. In comparison, the query for "Sociaal leenstelsel" only appears in October 2012, precisely the week that the name of the new "social lending system" is revealed along with the details of its implementation. We also constructed an aggregate search query by summing queries on terms related to the abolition of student finance ("afschaffing studiefianciering", "afschaffing studiebeurs", etc. as well as variation on spelling of these queries), and find that this index peaks exactly in January 2010 and June 2010 as uncertainty, and disappears progressively after the announcement in October 2012. 


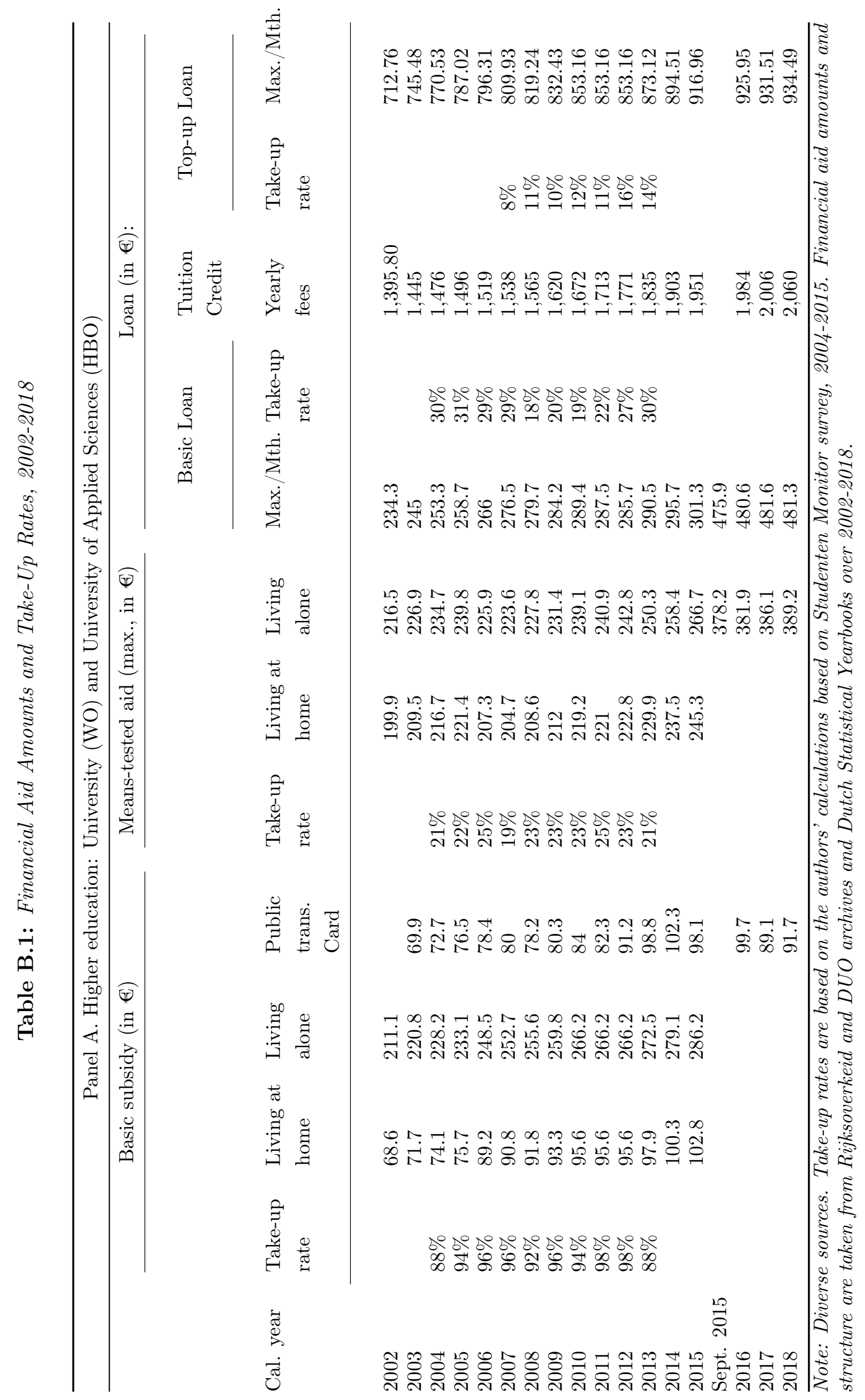




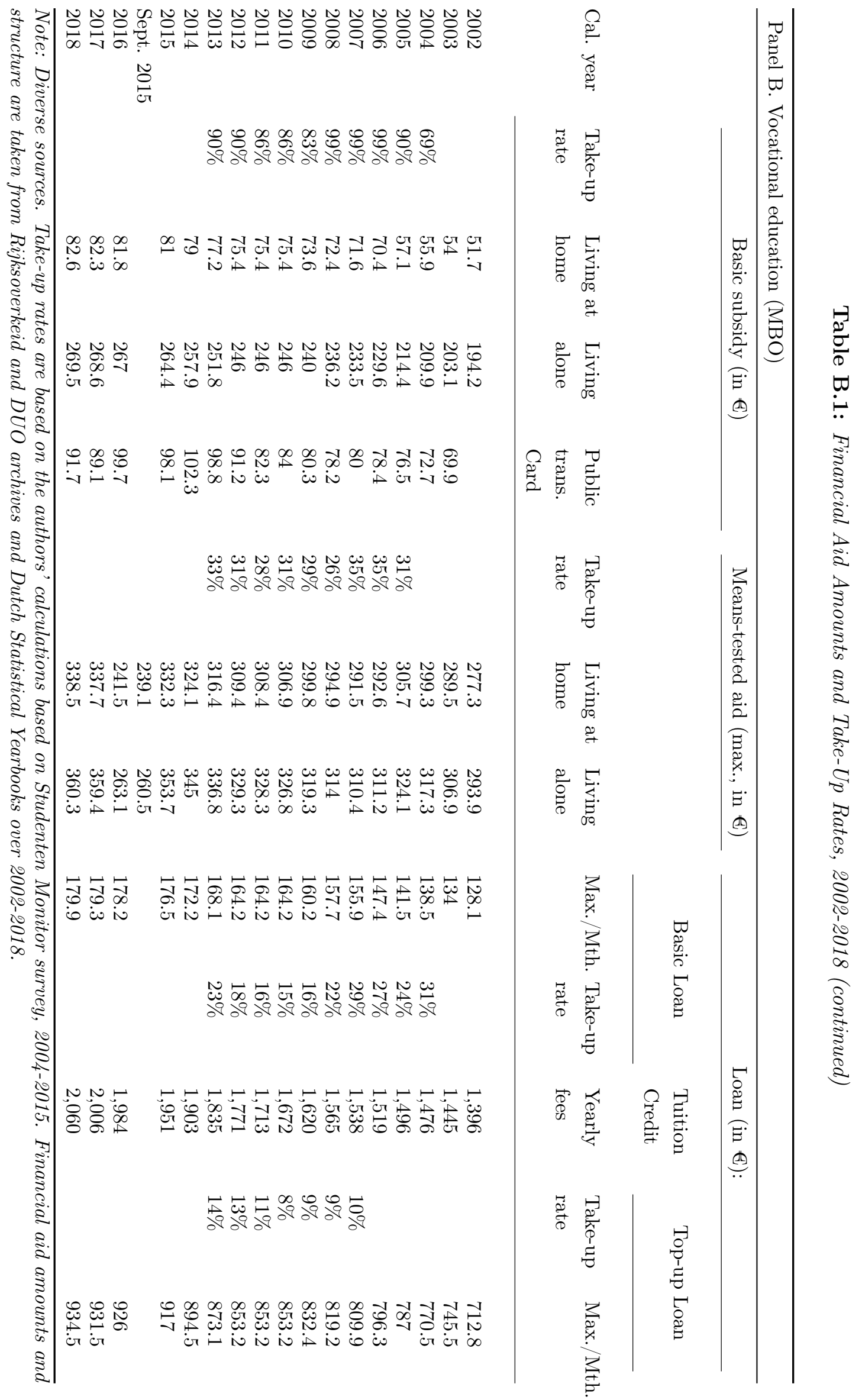


Table B.2: Financial Aid Eligibility and Repayment Conditions, 2002-2018

\begin{tabular}{|c|c|c|c|c|c|}
\hline \multirow{4}{*}{ Cal. year } & \multicolumn{4}{|c|}{ Means-tested aid: cutoffs in family taxable income } & \multirow{4}{*}{$\begin{array}{c}\text { Loan } \\
\text { Interest rate } \\
\text { (5) }\end{array}$} \\
\hline & \multicolumn{2}{|c|}{ Higher education (WO, HBO) } & \multicolumn{2}{|c|}{ Vocational education (MBO) } & \\
\hline & 2-Parent & Single parent & 2-Parent & Single parent & \\
\hline & $\begin{array}{l}\text { household } \\
\text { (1) }\end{array}$ & $\begin{array}{l}\text { household } \\
(2)\end{array}$ & $\begin{array}{l}\text { household } \\
(3)\end{array}$ & $\begin{array}{l}\text { household } \\
\text { (4) }\end{array}$ & \\
\hline 2002 & $26,703.50$ & $34,333.10$ & $13,351.80$ & $17,166.60$ & \\
\hline 2003 & $27,966.60$ & $35,957.10$ & $13,983.30$ & $17,978.50$ & \\
\hline 2004 & $29,021.00$ & $37,312.70$ & $14,510.50$ & $18,656.30$ & \\
\hline 2005 & $29,711.70$ & $38,200.70$ & $14,855.80$ & $19,100.40$ & \\
\hline 2006 & $30,551.30$ & $39,093.90$ & $15,275.70$ & $19,546.90$ & $2.74 \%$ \\
\hline 2007 & $31,008.50$ & $39,497.50$ & $15,504.20$ & $19,748.80$ & $3.70 \%$ \\
\hline 2008 & $31,856.30$ & $40,398.80$ & $15,928.20$ & $20,199.40$ & $4.17 \%$ \\
\hline 2009 & $33,638.90$ & $42,659.50$ & $16,819.50$ & $21,329.70$ & $3.58 \%$ \\
\hline 2010 & $33,775.30$ & $42,832.50$ & $16,887.70$ & $21,416.20$ & $2.39 \%$ \\
\hline 2011 & $34,515.00$ & $43,770.50$ & $17,257.50$ & $21,885.30$ & $1.50 \%$ \\
\hline 2012 & $34,898.10$ & $44,256.40$ & $17,449.10$ & $22,128.20$ & $1.39 \%$ \\
\hline 2013 & $35,337.90$ & $44,814.00$ & $17,668.90$ & $22,407.00$ & $0.60 \%$ \\
\hline 2014 & $35,857.30$ & $45,472.80$ & $17,928.70$ & $22,736.40$ & $0.81 \%$ \\
\hline 2015 & $33,781.20$ & $42,799.00$ & $16,890.60$ & $21,399.50$ & $0.12 \%$ \\
\hline Sept. 2015 & & & & & \\
\hline 2016 & $34,105.50$ & $43,209.90$ & $17,052.80$ & $21,605.00$ & $0.01 \%$ \\
\hline 2017 & $34,658.00$ & $43,909.90$ & $17,329.00$ & $21,955.00$ & $0.00 \%$ \\
\hline 2018 & $35,243.80$ & $44,652.00$ & $17,621.90$ & $22,326.00$ & $0.00 \%$ \\
\hline
\end{tabular}

Note: Financial aid conditions are taken from Rijksoverkeid and DUO archives and Dutch Statistical Yearbooks over 2002-2018. The exact amount of means-tested financial aid that students receive is calculated based on the amounts presented in Columns (1) to (4), which are yearly cutoffs in household taxable income depending on household composition. Other criteria also apply which are not publicly available. 
Figure B.2: Google Trends Associated to Student Finance Reform Details

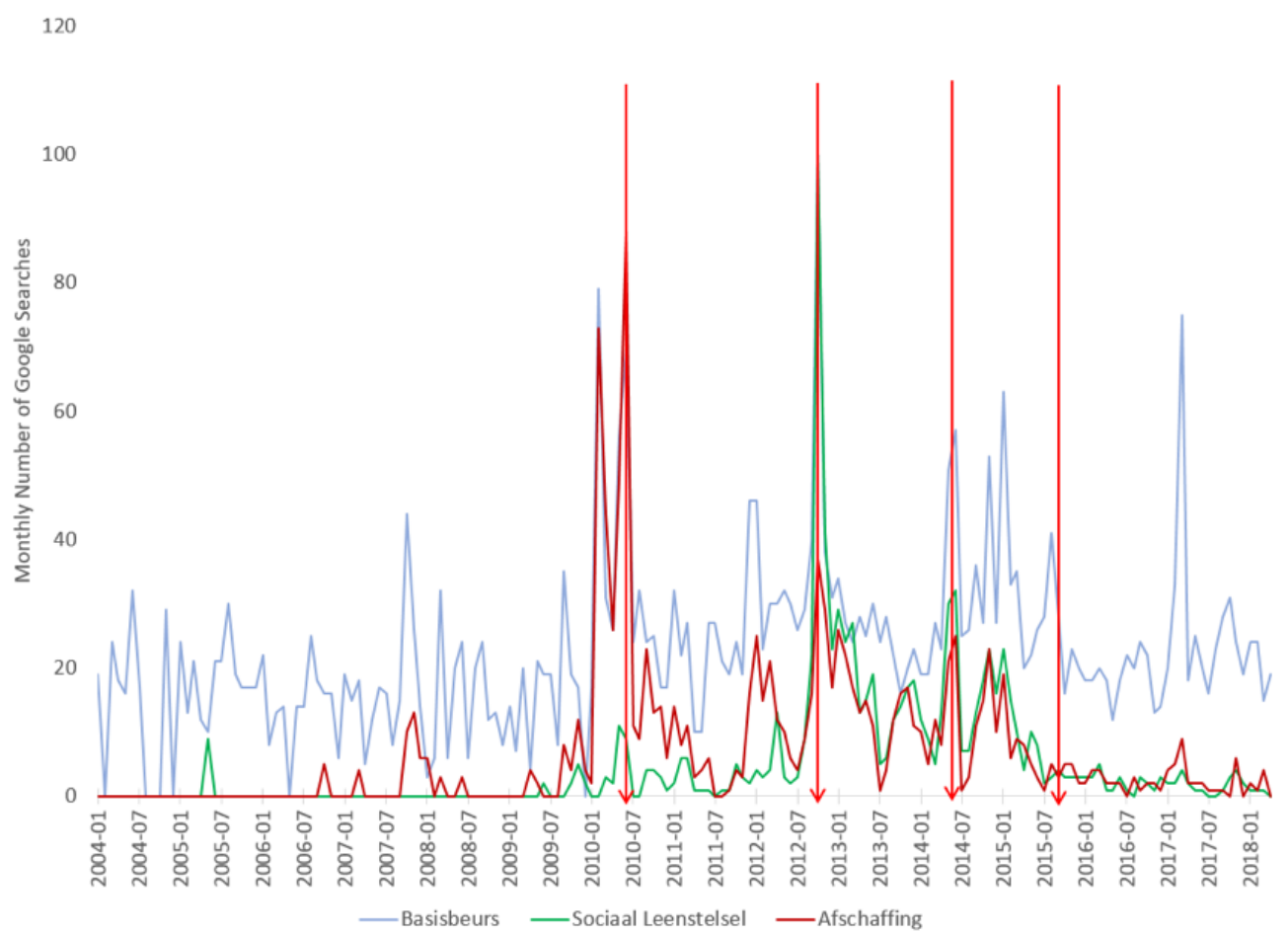

Note: This figure plots Google trends related to details of the old and new student finance system. Trends correspond to national monthly Google searches, normalized based on searches for "Basisbeurs" (In Dutch: basic subsidy of student finance), the blue line. The red line corresponds to "Afschaffing stufi" (in Dutch: removal of student finance), a synthetic trend we constructed by aggregating searches terms associated to the removal of Student finance, collected from news clippings. The green line corresponds to "Sociaal Leenstelsel" (in Dutch: social lending student finance system), the exact name of the new student finance regime, officially revealed on October 9, 2012.

\section{B.2 Proofs}

In this section, we derive comparative statics from our theoretical framework.

The first condition expresses that low-ability students have incentives to attend vocational education: they would reap negative benefits from enrolling into anything but the vocational track, because their effort cost would exceed their returns to any other type of schooling. The second condition expresses that high ability students have strong incentives to study at the academic track. The last condition describes the marginal student's case between general and academic education (with ability level $\left.\theta_{A}^{*}\right)$.

We can express $\theta_{G}^{*}$ and $\theta_{A}^{*}$ as a function of the lifetime payoff functions, using the 
equilibrium conditions above. $\theta_{G}^{*}$ solves $R_{i}^{G}\left(\theta_{G}^{*}\right)=R_{i}^{V}\left(\theta_{G}^{*}\right)$, and since $R_{i}^{V}\left(\theta_{G}^{*}\right)$ is normalized to 0 , we can express analytically that:

$$
\begin{aligned}
\theta_{G}^{*} & =1-\frac{1}{\kappa_{G}\left(t_{G}-t_{V}-1\right)-\left(\kappa_{G}-\kappa_{V}\right)\left(t_{V}-1\right)} \\
& \times\left[\sum_{k=t_{G}+1}^{L-t} \mathbb{E}\left(\Pi_{t+k}^{G}\right)-(1+b)\left[\left(F_{G}-\left(1-p_{0}\right) s^{0}-\left(1-p_{1}\right) s^{1}\right)\left(t_{G}-t_{V}-1\right)\right.\right. \\
& \left.\left.+\left(F_{G}-F_{V}\right)\left(t_{V}-1\right)\right]-\sum_{t=t_{V}+1}^{t_{G}} Y_{t}^{V}\right]
\end{aligned}
$$

$\theta_{G}^{*}$ is increasing in $\kappa_{G}$, illustrating that the harder general education relative to vocational education, the higher the ability level of the marginal student between these two options. $\theta_{G}^{*}$ is also increasing in the net additional cost of attending general education over vocational education, as well as in the cost of borrowing $b$, and in the opportunity cost given by the counterfactual wage a student would receive after graduating from vocational education instead of attending further years of general education. Lastly, $\theta_{G}^{*}$ is decreasing in the expected premium from general education: increasing premium from general education over vocational education drives a larger margin of students into general education.

Similarly, $\theta_{A}^{*}$ solves $R_{i}^{A}\left(\theta_{A}^{*}\right)=R_{i}^{G}\left(\theta_{A}^{*}\right)$, which eventually leads to:

$$
\begin{aligned}
\theta_{A}^{*} & =1-\frac{1}{\kappa_{A}\left(t_{A}-t_{G}-1\right)-\left(\kappa_{A}-\kappa_{G}\right)\left(t_{G}-1\right)} \\
& \times\left[\sum_{k=t_{A}+1}^{L-t} \mathbb{E}\left(\Pi_{t+k}^{A}\right)-(1+b)\left[\left(F_{A}-\left(1-p_{0}\right) s^{0}-\left(1-p_{1}\right) s^{1}\right)\left(t_{A}-t_{G}-1\right)\right.\right. \\
& \left.\left.+\left(F_{A}-F_{G}\right)\left(t_{G}-1\right)\right]-\sum_{t=t_{G}+1}^{t_{A}} Y_{t}^{G}\right]
\end{aligned}
$$

$\theta_{A}^{*}$ has a similar expression to that of $\theta_{G}^{*}$, except that for the student who is indifferent between general and academic education, the relevant counterfactual is general education, such that i) the counterfactual net cost of attending academic education is attending general education, and ii) the opportunity cost from attending academic education is now the premium from general education over vocational education after graduation from general education until graduation from academic 
education.

Comparative Statics Following Charles et al. (2018), we allow some shock $S$ to affect sorting through any parameter of the model. Thus, studying the effects of any policy shock on ability sorting implies taking the derivative of $\theta_{A}^{*}$ and $\theta_{G}^{*}$ against this policy shock $S$. Let us spell out how a shock $S$ can affect equilibrium sorting in our setting, and through which mechanisms.

$$
\begin{aligned}
\frac{\partial \theta_{G}^{*}}{\partial S} & =-\frac{1}{\kappa_{G}\left(t_{G}-t_{V}-1\right)-\left(\kappa_{G}-\kappa_{V}\right)\left(t_{V}-1\right)} \times\left[\frac{\partial}{\partial S} \sum_{k=t_{G}+1}^{L-t} \mathbb{E}\left(\Pi_{t+k}^{G}\right)\right. \\
& -\frac{\partial b}{\partial S}\left[\left(F_{G}-\left(1-p_{0}\right) s^{0}-\left(1-p_{1}\right) s^{1}\right)\left(t_{G}-t_{V}-1\right)+\left(F_{G}-F_{V}\right)\left(t_{V}-1\right)\right] \\
& -(1+b)\left(t_{G}-t_{V}-1\right)\left[\frac{\partial F_{G}}{\partial S}+s^{0} \frac{\partial p_{0}}{\partial S}+s^{1} \frac{\partial p_{1}}{\partial S}-\left(1-p_{0}\right) \frac{\partial s^{0}}{\partial S}-\left(1-p_{1}\right) \frac{\partial s^{1}}{\partial S}\right] \\
& \left.-(1+b)\left(t_{v}-1\right)\left[\frac{\partial F_{G}}{\partial S}-\frac{\partial F_{V}}{\partial S}\right]+\frac{\partial}{\partial S} \sum_{t=t_{V}+1}^{t_{G}} Y_{t}^{V}\right]
\end{aligned}
$$

Although this expression seems complicated, we can decompose it into terms which help us structure our thoughts on the possible mechanisms through which any shock $S$ can affect selection into tracks. The effect of any shock $S$ to the ability threshold $\theta_{G}^{*}$, which characterizes the decision problem of a person who is indifferent between vocational and general education, goes through four possible channels.

The first term represents the effect of $S$ on $\theta_{G}^{*}$ through increasing students' expectations about the wage premium of general over vocational education; as such, this channel attracts to general education a new margin of lower ability students who would have otherwise chosen to attend vocational education. This effect is weighted by the relative difficulty and duration of attending general education compared to vocational education; the harder and longer it is to attend general education compared to vocational education, the less one should expect $S$ to affect sorting.

The second term represents the intermediate effect of $S$ on $\theta_{G}^{*}$ through a change in the cost of borrowing to cover the additional costs of attending general education instead of vocational education; through this channel, $S$ has a positive effect on $\theta_{G}^{*}$, such that the marginal student choosing general education has higher ability than prior to $S$.

The third term represents the change induced by $S$ in the net cost of attending 
general versus vocational education, which can happen through any elements of this net cost - tuition fees, unconditional financial aid, means-tested aid, or the probabilities of having to repay financial aid. We interpret the part of this cost associated to financial aid as the change in the cost of having to save just in case $s^{0}$ and $s^{1}$ must eventually be paid back; this quantity is akin to a liability in accounting terms.

Lastly, the fourth term corresponds to the change induced by $S$ in the opportunity cost of attending general over vocational education, i.e. an increase in the outside wage level students would receive if they were to attend vocational education instead of general education. Importantly, at fixed level of financial aid $s^{0}$, a marginal increase in the probability $p_{0}$ of having to repay $s^{0}$ induced by the shock $S$ is likely to have a positive effect on selection into general education; the same applies for means-tested aid. This means that shocks to the expected probabilities of repayment increase resorting mostly among the weakest students who are marginal between vocational and general education.

Similarly, the effect of $S$ on $\theta_{A}^{*}$ also goes through the same four distinct channels. Similar to the effect of $S$ on $\theta_{G}^{*}$, the effect of $S$ through the expected wage premium from academic versus general education is weighted by the gap in difficulty and duration between general and academic education. Importantly, the effect of $S$ on $\theta_{A}^{*}$ are specific to the margin of students indifferent between general and academic education; for these students, what matters is the relative additional costs from attending academic over general - not vocational - education. In such, the level of costs incurred in academic or general education are not relevant, nor is the level of wages for vocational graduates, but rather the gap in cost between academic and general education, and outside wage for general education graduates.

Thus, a shock $S$ is expected to affect sorting through potentially four different types of channels running through changes in the lifetime expected payoff of each education type, the overall sign and magnitude of the effect being the sum of these distinct types of effect. In the absence of any other channels, a shock affecting only repayment uncertainty by increasing $p_{0}$ and $p_{1}$ would lower $\theta_{G}^{*}$ and $\theta_{A}^{*}$, and would lower $\theta_{G}^{*}$ to a larger extent than $\theta_{A}^{*}$. 


\section{B.3 Additional Analyses}

This section provides detailed additional evidence to support our interpretation that Dutch students chose alternative tracks in response to reform announcements in anticipation of future changes to financial aid.

\section{Main effects of reform announcements across subgroups}

We investigate differences in responses to reform announcements across groups of students for which our model predicts larger responses: across family income, ability and gender. The responses to announcements for these key subgroups that are more likely to be affected by the policy change in a specific way gives credit to our interpretation of the policy announcements as shocks to students' beliefs about the future of financial aid.

\section{Low v. high ability students' responses to reform announcements}

To compare the responses to announcements of low and high ability students within their relevant ability-peer group, we estimate the probability to be in a given track in Grade 10, conditional on the recommendation received by the primary school teacher around the time of the CITO test. One issue is that, given our data requirement to observe students in Grade 10, the first cohort for which we can use recommendations is the 2009 cohort of 10 th graders. A second issue is that there seem to be changes in trends of teachers' recommendations (see Figure B.10), such that changes in the probability of being in a given track in Grade 10 must account for changes in the probability of receiving any recommendation. 
Figure B.3: Enrollment In Tracks, by Ability and Teacher Recommendation
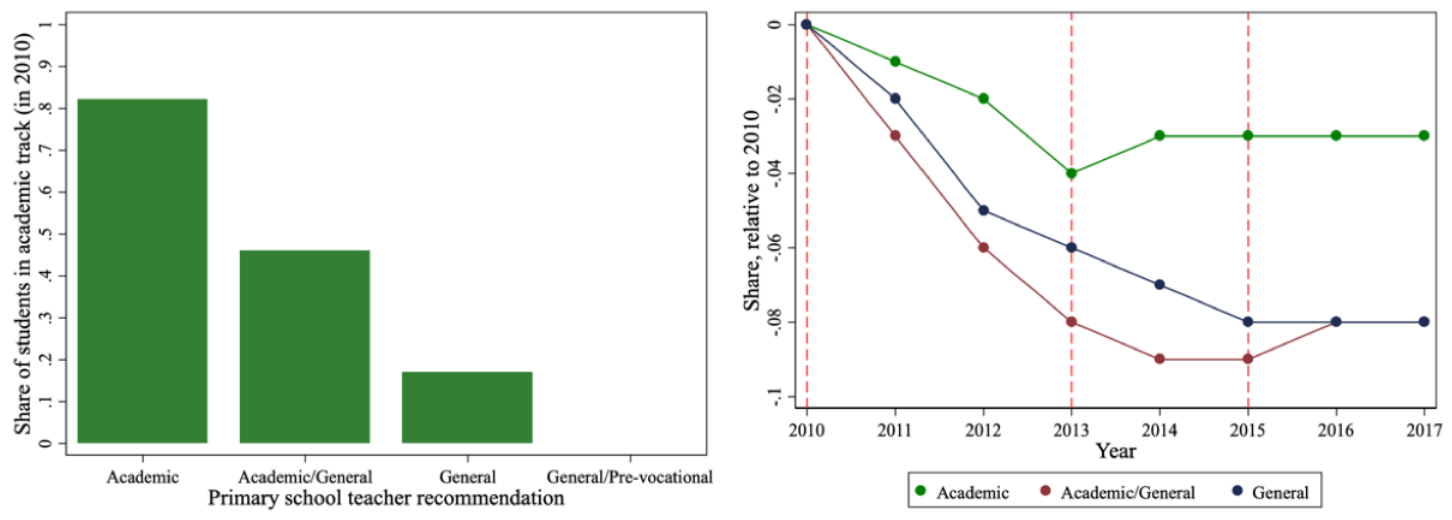

(a) Enrollment in the Academic track, by teacher track advice upon exiting primary school
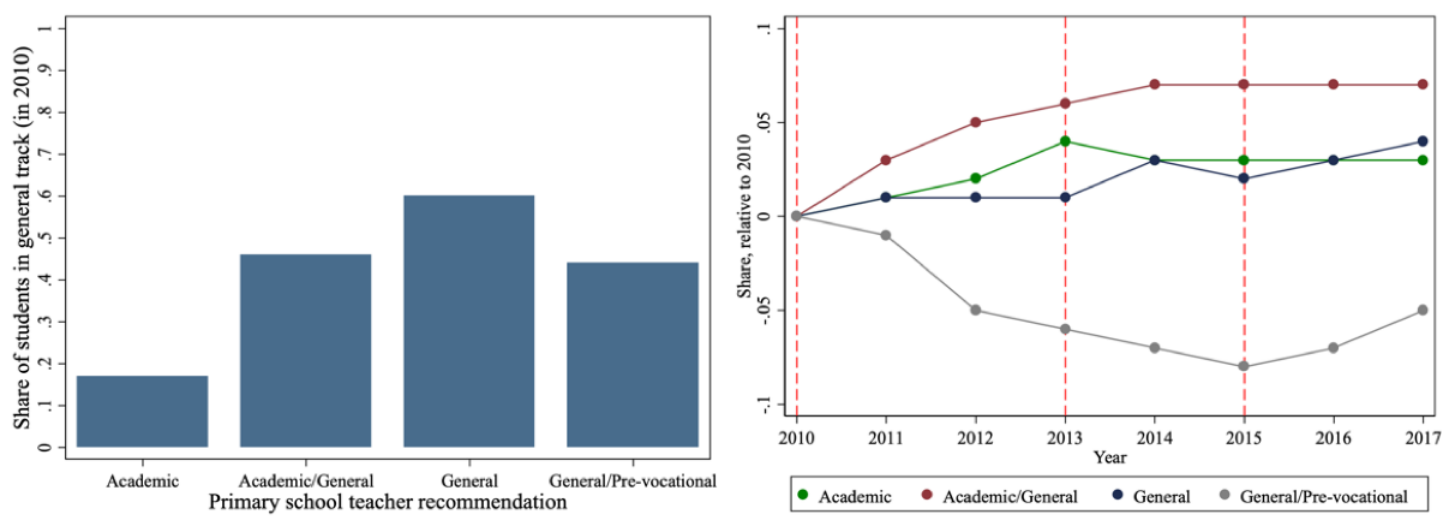

(b) Enrollment in the General track, by teacher track advice upon exiting primary school
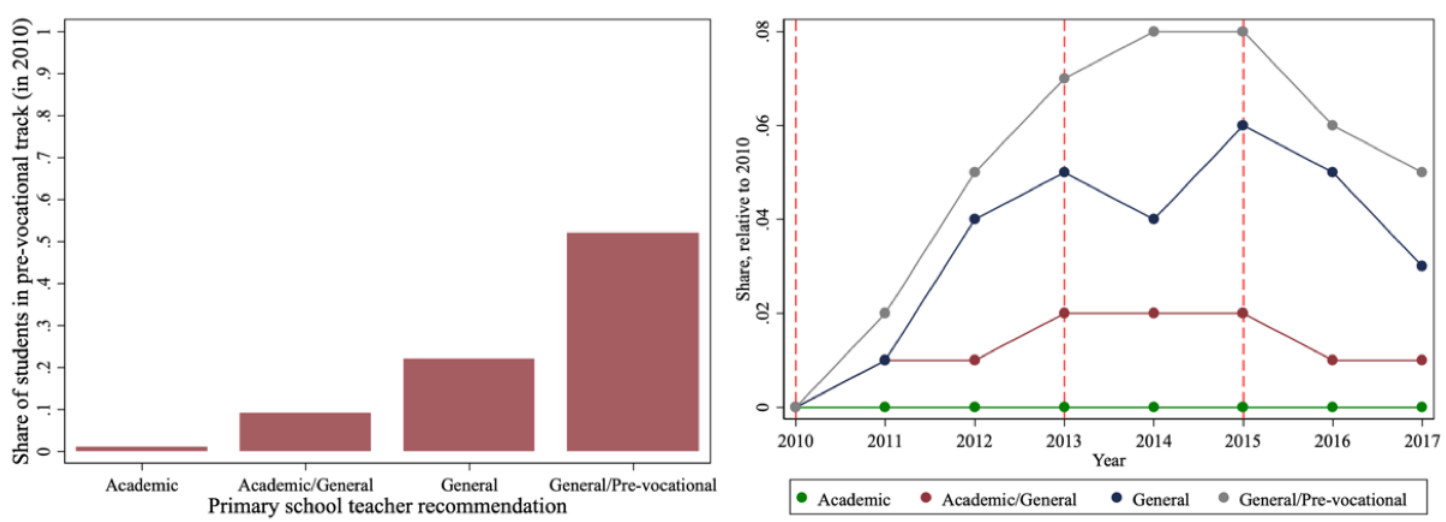

(c) Enrollment in the Vocational track, by teacher track advice upon exiting primary school

Note: This figure presents enrollment shares in the Academic, General and Vocational tracks, split by the type of track advice children received upon exiting primary school, as a measure of child cognitive ability. Figures on the left-hand side present these shares in 2010, and figures on the right-hand side present changes in shares relative to 2010 levels. Red dotted vertical lines mark the first cohorts of children affected after reform announcements in 2010, 2012 and implementation in 2015. 
Figure B.3 shows the change in the probability of being in a given track in Grade 10 conditional on a given recommendation, adjusting for the underlying trends in the probability of receiving any recommendation. Within each bin of teacher recommendation, the share of students going to a lower track than recommended declines, while the shares of students going to the same level or higher track than recommended increases. This is suggestive that after 2010, students within each track in Grade 10 have received relatively better recommendations than previous cohorts, which is in line with our main prediction on ability sorting.

\section{Income: Responses to reform announcements of low and high income students}

Our findings regarding heterogeneity by family income are also in line with the predictions we draw from our framework. For this analysis, we split students into terciles of the distribution of family income, as the lowest income tercile corresponds roughly to eligibility to means-tested financial aid. Our preferred measure of family income is the average total annual parental income from labor earnings and business revenues in the last five years. The means-tested part of student finance is tested against pooled income of both parents, regardless of whether the parents live together, thus we also pool maternal and paternal income. We average over five years to smooth out transitory income shocks. Our first observation is the presence of a strong income gradient in track choice in Grade 10, in the baseline year 2009. While $68 \%$ of low-income students enroll in the vocational track and $10 \%$ in the academic track, only $33 \%$ of high-income students enroll in the vocational track and $33.5 \%$ in the academic track. 
Table B.3: Effects on Track Enrollment by Family Income

\begin{tabular}{|c|c|c|c|c|c|}
\hline Track & Income: & $\begin{array}{c}\text { Baseline } \\
\text { track } \\
\text { shares (\%) } \\
(2009) \\
(1)\end{array}$ & $\begin{array}{c}(2010-2012) \\
(2)\end{array}$ & $\begin{array}{l}\text { Uncertainty } \\
\text { Resolution } \\
(2013-2014) \\
(3)\end{array}$ & $\begin{array}{c}\text { Post- } \\
\text { implementation } \\
(2015-2017) \\
(4)\end{array}$ \\
\hline
\end{tabular}

Panel A: Absolute Effects (in percentage points)

\begin{tabular}{llllll}
\hline Academic & Low & 10.0 & $-0.61 * * *$ & $-1.48 * * *$ & $-2.20 * * *$ \\
& Medium & 18.0 & $-1.18 * * *$ & $-2.54 * * *$ & $-4.09 * * *$ \\
& High & 33.5 & $-1.73 * * *$ & $-3.35 * * *$ & $-5.11 * * *$ \\
\hline General & Low & 22.0 & $-1.56 * * *$ & $-3.13 * * *$ & $-5.37 * * *$ \\
& Medium & 28.0 & -1.11 & $-2.64 *$ & $-4.97 * * *$ \\
& High & 33.5 & $0.83 * * *$ & $0.64 *$ & 0.15 \\
\hline Vocational & Low & 68.0 & $2.17 * * *$ & $4.61 * * *$ & $7.56 * * *$ \\
& Medium & 54.0 & $2.29 * * *$ & $5.18 * * *$ & $9.06 * * *$ \\
& High & 33.0 & $0.90 * * *$ & $2.71 * * *$ & $4.96 * * *$
\end{tabular}

Panel B: Relative Effects (in percent)

\begin{tabular}{llccc}
\hline Academic & Low & $-5.68 * * *$ & $-13.74 * * *$ & $-20.45 * * *$ \\
& Medium & $-7.03 * * *$ & $-15.14 * * *$ & $-24.39 * * *$ \\
& High & $-5.26 * * *$ & $-10.19 * * *$ & $-15.57 * * *$ \\
\hline General & Low & $-6.89 * * *$ & $-13.84 * * *$ & $-23.72 * * *$ \\
& Medium & -3.83 & $-9.07 *$ & $-17.09 * * *$ \\
& High & $2.46 * * *$ & $1.90 *$ & 0.44 \\
\hline Vocational & Low & $3.33 * * *$ & $7.07 * * *$ & 11.60 \\
& Medium & $4.30 * * *$ & $9.69 * * *$ & $16.97 * * *$ \\
& High & $2.82 * * *$ & $8.42 * * *$ & $15.41 * * *$ \\
\hline
\end{tabular}

Note: Authors' estimates of three reform coefficients from a multinomial logit model of high school track enrollment as measured in Grade 10, assuming a continued linear pre-trend extending past 2009. The models are split by parental income terciles, and use respectively 877,855; 889,294; and 898,283 individual observations over years 2005-2017. Controls: region fixed effects, age, gender, migration status, ethnic background, parental income and employment status, and an urbanization index. ${ }^{* * *}=0.01$ significance level, ${ }^{* *}=0.05$ significance level, ${ }^{*}=0.1$ significance level. 
Table B.3 reports the effects of policy announcements by family income, assuming a continued linear pre-trend extending beyond 2009. We find that the 2010 announcement had a stronger effect on low-income students, although it led students from all three income groups to avoid academic and general education. Importantly, the share of low-income students moving from academic to general education is smaller than the share moving from general to vocational education. In comparison, the share of higher income students between academic and general education is larger than that between general and vocational education, which is in line with our framework's predictions. After the 2012 announcement, the magnitude of effects increases for low-income students, and the most important margin for low-income students is general to vocational education. For high-income students, resorting mostly happens at the margin between academic and general education at first, but more resorting at the margin between general and vocational education starts to occur after 2012 to peak in the later period. Table B.4 further indicates that low-income students are substantially more likely than their peers from more privileged backgrounds to move to a lower track in Grade 11 after the announcement shocks in 2010 and 2012.

Table B.4: Effects on Track Dropout by Family Income

\begin{tabular}{|c|c|c|c|c|}
\hline Outcome: & Income: & $\begin{array}{c}(2010-2012) \\
(1)\end{array}$ & $\begin{array}{l}\text { Uncertainty } \\
\text { Resolution } \\
(2013-2014) \\
(2)\end{array}$ & $\begin{array}{c}\text { Post- } \\
\text { implementation } \\
(2015-2017) \\
(3)\end{array}$ \\
\hline \multicolumn{5}{|c|}{ Panel A: Absolute Effects (in percentage points) } \\
\hline Track Dropout & $\begin{array}{l}\text { Low } \\
\text { Medium } \\
\text { High }\end{array}$ & $\begin{array}{l}2.00 * * * \\
1.44 * * * \\
1.29 * * *\end{array}$ & $\begin{array}{l}2.00 * * \\
1.80 * * * \\
1.31 * * *\end{array}$ & $\begin{array}{l}1.01 \\
0.69 \\
0.75\end{array}$ \\
\hline \multicolumn{5}{|c|}{ Panel B: Relative Effects (in percent) } \\
\hline Track Dropout & $\begin{array}{l}\text { Low } \\
\text { Medium } \\
\text { High }\end{array}$ & $\begin{array}{l}38.98 * * * \\
29.54 * * * \\
35.55 * * *\end{array}$ & $\begin{array}{l}38.99 * * \\
36.78 * * * \\
35.99 * * *\end{array}$ & $\begin{array}{l}19.69 \\
14.19 \\
20.56\end{array}$ \\
\hline $\begin{array}{l}\text { Note: Authors' es } \\
\text { high school track } \\
\text { The dataset is spli } \\
\text { and } 458,403 \text { indivi } \\
\text { age, gender, migr } \\
\text { status, and an urb } \\
\text { level, } *=0.1 \text { sign }\end{array}$ & $\begin{array}{l}\text { mates of } t \\
\text { opout, ass? } \\
\text { by parental } \\
\text { ual observa } \\
\text { ion status, }\end{array}$ & $\begin{array}{l}\text { ee reform coeffi } \\
\text { ing a continued } \\
\text { ncome terciles a } \\
\text { ns over years } 20 \\
\text { thnic backgrou } \\
*^{* *}=0.01 \text { si }\end{array}$ & $\begin{array}{l}\text { ients from a log } \\
\text { linear pre-trend } \\
\text { nd uses respectiv } \\
\text { 06-2017. Contro } \\
\text { d, parental inco } \\
\text { gnificance level, }\end{array}$ & $\begin{array}{l}\text { it model of Grade } 11 \\
\text { extending past } 2009 \\
\text { ely 198,914; } 296,780 \\
\text { s: region fixed effects } \\
\text { me and employment } \\
* *=0.05 \text { significance }\end{array}$ \\
\hline
\end{tabular}




\section{Gender: Responses to reform announcements of female and male stu- dents}

Female students are more responsive than male students throughout the entire period, which partially consistent with the predictions we were able to draft from the framework. We assumed female students to be more risk averse than male students, and under this assumption, we predicted that mostly the 2010 announcement shock would lead girls to avoid general and academic tracks more than boys. We further predicted gender heterogeneous responses to announcements to at least reduce after the second announcement which resolving policy uncertainty.

Table B.5: Effects on Track Enrollment by Gender

\begin{tabular}{|c|c|c|c|c|c|}
\hline Track & Gender: & $\begin{array}{c}\text { Baseline } \\
\text { track } \\
\text { shares (\%) } \\
(2009) \\
(1)\end{array}$ & $\begin{array}{c}(2010-2012) \\
(2)\end{array}$ & $\begin{array}{c}\text { Uncertainty } \\
\text { Resolution } \\
(2013-2014) \\
(3)\end{array}$ & $\begin{array}{c}\text { Post- } \\
\text { implementation } \\
(2015-2017) \\
(4)\end{array}$ \\
\hline
\end{tabular}

Panel A: Absolute Effects (in percentage points)

\begin{tabular}{llllll}
\hline Academic & Male & 21.5 & $-0.37 * * *$ & $-1.41 * * *$ & $-2.60 * * *$ \\
& Female & 18.5 & $-1.77 * * *$ & $-3.19 * * *$ & $-4.54 * * *$ \\
\hline General & Male & 28.5 & -0.52 & $-1.67 * *$ & $-3.44 * * *$ \\
& Female & 27.5 & -0.57 & $-1.53 * * *$ & $-3.02 * * *$ \\
\hline \multirow{2}{*}{ Vocational } & Male & 50.0 & $0.89 * * *$ & $3.08 * * *$ & $6.04 * * *$ \\
& Female & 54.0 & $2.34 * * *$ & $4.72 * * *$ & $7.57 * * *$
\end{tabular}

Panel B: Relative Effects (in percent)

\begin{tabular}{llccc}
\hline Academic & Male & $-2.02 * * *$ & $-7.69 * * *$ & $-14.18 * * *$ \\
& Female & $-8.32 * * *$ & $-15.03 * * *$ & $-21.46 * * *$ \\
\hline General & Male & -1.87 & $-6.01 * *$ & $-12.38 * * *$ \\
& Female & $-2.03 *$ & $-5.41 * * *$ & $-10.67 * * *$ \\
\hline Vocational & Male & $1.73 * * *$ & $5.99 * * *$ & $11.73 * * *$ \\
& Female & $4.89 * * *$ & $9.85 * * *$ & $15.79 * * *$ \\
\hline
\end{tabular}

Note: Authors' estimates of three reform coefficients from a multinomial logit model of high school track enrollment as measured in Grade 10, assuming a continued linear pre-trend extending past 2009. The models are split by gender and use respectively 1,354,293 and 1,338,730 individual observations over years 2005-2017. Controls: region fixed effects, age, gender, migration status, ethnic background, parental income and employment status, and an urbanization index. ${ }^{* * *}=$ 0.01 significance level, ${ }^{* *}=0.05$ significance level, ${ }^{*}=0.1$ significance level.

Our main results on track enrollments in Grade 10 are reported in Table B.5, which shows results for the specification using a linear pre-trend continuing beyond 2009. 
We find that as uncertainty rises in 2010, mostly female students shy away from academic and general education towards vocational education. Yet female enrollment keeps on dropping relative to baseline in academic and general tracks in favor of enrollments in the vocational track. This is rather consistent with a progressive change in the stereotype that vocational education is more for boys than for girls. These results are also consistent for alternative specifications of time pre-trends.

Consistently, we also find in Table B.6 that the 2010 shock had heterogeneous effects across gender on the probability to drop out into a lower track. These results are similar to those found with our alternative specification. Overall, girls dropped out into a lower track less often than boys, and this gap increased over time, suggesting that the margin on which girls respond to reform announcements is initial track choice rather than re-optimizing throughout their track, while the opposite seems true for boys.

Table B.6: Effects on Track Dropout by Gender

\begin{tabular}{|c|c|c|c|c|}
\hline & & Uncertainty & $\begin{array}{l}\text { Uncertainty } \\
\text { Resolution }\end{array}$ & $\begin{array}{c}\text { Post- } \\
\text { implementation }\end{array}$ \\
\hline Outcome: & Gender: & $\begin{array}{c}(2010-2012) \\
(1)\end{array}$ & $\begin{array}{c}(2013-2014) \\
(2)\end{array}$ & $\begin{array}{c}(2015-2017) \\
(3)\end{array}$ \\
\hline
\end{tabular}

Panel A: Absolute Effects (in percentage points)

\begin{tabular}{lllll}
\hline Track Dropout & Male & $1.81 * * *$ & $2.27 * * *$ & $2.08 * * *$ \\
& Female & $1.16 * * *$ & $0.94 *$ & -0.47
\end{tabular}

Panel B: Relative Effects (in percent)

\begin{tabular}{lllll}
\hline Track Dropout & Male & $44.98 * * *$ & $56.37 * * *$ & $51.56 * * *$ \\
& Female & $25.35 * * *$ & $20.47 *$ & -10.25
\end{tabular}

Note: Authors' estimates of three reform coefficients from a logit model of Grade 11 high school track dropout, assuming a continued linear pre-trend extending past 2009. The models are split by gender and use respectively 440,807 and 517,705 individual observations over years 2006-2017. Controls: region fixed effects, age, gender, migration status, ethnic background, parental income and employment status, and an urbanization index. ${ }^{* * *}=0.01$ significance level, ${ }^{* *}=0.05$ significance level, ${ }^{*}=0.1$ significance level. 
Additional effects of reform announcements on field of study enrollments and dropout

We show that students choice of high school major and dropout decisions from majors is also consistent with students seeking less risky majors in response to policy uncertainty.

After students choose a track, learning about policy changes can change their preferred high school major. Under financial aid with higher chances of repayment, students have an incentive to choose a field of study which higher returns. This effect has already been documented in Rothstein and Rouse (2011) and Field (2009). They show that college student in the United-States became more likely to choose majors yielding lower returns after student loans were turned into grants; both papers conclude that this behavior is evidence of debt aversion. van den Berge (2018) shows that Dutch college students who graduate from STEM and Medicine programs have higher average earnings and better job opportunities than other Dutch college students, thus we consider specialization in STEM and Medicine fields of study as specialization in the higher earning majors in our setting.

\section{Enrollments in STEM and Medicine fields of study}

Purely descriptively at first, we find similar effects in the Dutch context among high school students affected by the policy announcements. Figure B.4 shows the raw share of students in the academic and general tracks enrolling into STEM and Medicine high school majors. Students' choice of field of study also seems to be affected by the reform.

Following the 2010 reform announcement, the share of students choosing STEM and Medicine has grown substantially from $44 \%$ to $50 \%$ in the span of three years. This growth in enrollment in STEM and Medicine levels off in the academic year 2014-2015 (students starting Grade 10 in this year are the first students who finished primary school following the first reform announcement) and declines somewhat in the last year of observation. Although STEM and Medicine majors are more popular in the academic track, trends in enrollments in STEM and Medicine are similar across general and academic track students after the 2010 announcement. $^{2}$

\footnotetext{
${ }^{2}$ Note that the pre-announcement trend is potentially distorted by a change in the classification of fields of study, which took place in the academic year 2007-08. Nonetheless, the figure shows a
} 
Figure B.4: Trends in STEM Specialization in High School

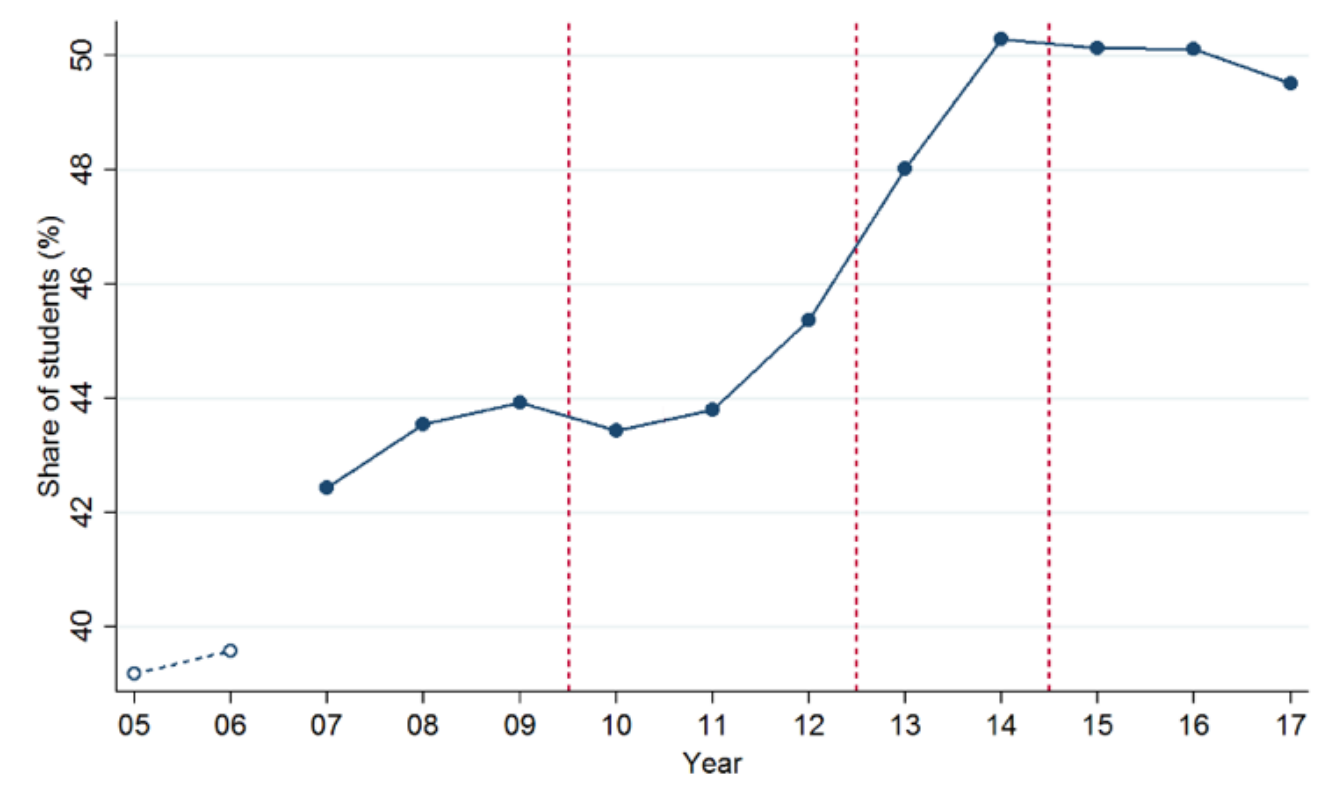

Note: Authors' calculations of the choice of STEM specialization in Grade 10 over the period of observation. STEM specialization corresponds to the following curricula: Nature and Technology, Nature and Health, and the combination thereof. Other curricula include Economy and Society, Culture and Society, and all other possible combinations. The line between years 2005-06 is dashed to highlight different curriculum classification rules which were in place during this period. Dutch education register data for Grade 10 high school students who were enrolled in either an academic or a general track in Grade 10, years 2005 to $201 \%$.

Table B.7 reports the effects of the reform announcements on the probability of choosing STEM or Medicine majors in Grade 10, conditional on being in a given track. The 2010 policy announcement led students to choose STEM and Medicine fields of study more significantly than before. We estimate the magnitude of these changes using a logit model of the choice of field of study.

We add to the baseline model specification a dummy variable for years 2005 and 2006. This is to account for the change of field classification which occurred between 2006 and 2007 and which caused a discontinuity in the pre-2010 trend. We prefer the specification with a continued linear time trend because the share of students in STEM and Medicine specialization was increasing throughout the baseline period 2005-2009, and because similar trends have been observed in other countries (see Figure B.13). The results in column (1) show that the share of STEM and Medicine students remained stable after the 2010 announcement which increased policy

mild pre-trend. 
Table B.7: Effects on Enrollment in STEM

\begin{tabular}{lcccc}
\hline & Baseline & Uncertainty & Uncertainty & Post- \\
STEM & & Resolution & implementation \\
share (\%) & & & \\
& $(2009)$ & $(2010-2012)$ & $(2013-2014)$ & $(2015-2017)$ \\
Outcome: & $(1)$ & $(2)$ & $(3)$ & $(4)$ \\
\hline
\end{tabular}

Panel A: Absolute Effects (in percentage points)

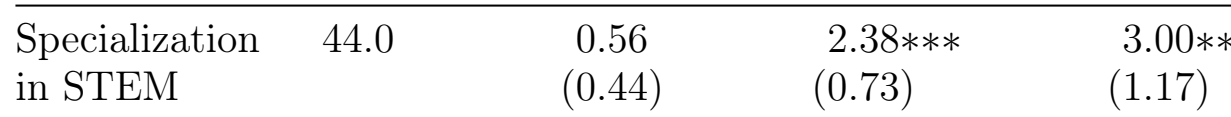

Panel B: Relative Effects (in percent)

\begin{tabular}{lccc}
\hline Specialization & -0.99 & $4.02 * * *$ & $5.27 * *$ \\
in STEM & $(0.77)$ & $(1.28)$ & $(2.06)$ \\
\hline
\end{tabular}

Note: Authors' estimates of three reform coefficients from a logit model of Grade 10 choice of STEM and Medicine specialization in academic and general high school tracks, with a linear pre-trend extending beyond 2009. The model uses 549,640 individual observations over years 2005-2017. Controls: region fixed effects, age, gender, migration status, ethnic background, parental income and employment status, an urbanization index and a dummy for the differently-classified period 2005-06. ${ }^{* * *}=0.01$ significance level, ${ }^{* *}=0.05$ significance level, ${ }^{*}=0.1$ significance level.

uncertainty, and rose after the 2012 announcement which resolved policy uncertainty, to stabilize and peak after implementation in 2015.

Table B.14 in Appendix B.4 shows the results for the alternative specification in which we assume the linear pre-trend was discontinued in 2009. This second specification of the model yields larger effects, with the share of STEM and Medicine students reaching 5.8 percentage points in the implementation period. Importantly, both model specifications indicate that enrollment in STEM and Medicine did not respond immediately to the rise in uncertainty in 2010, which is in contrast with enrollments in tracks. Our interpretation of this 'delay' of the reform announcement effect is that the decision to switch fields requires more effort than the decision to switch tracks. This is likely to be the case, if students start profiling themselves for a specific field well-ahead of their actual field choice in Grade 10. Students dropping out of their tracks into a less academic track are able to resume studying similar focal subjects, which minimizes the amount of effort and planning involved in the procedure. The field of study response of cohorts 2010-2012 is therefore diminished by the fact that these students spent up to three years of high school preparing for another specialization. 
The results of our analysis of fields of study could be influenced by the compositional changes among the students enrolling into the academic and general high school tracks. In principle, the increase of the share of STEM and Medicine could be a result of a strong outflow of students interested in humanities to the lower tracks of high school. We conduct supplementary analyses to show that such compositional changes are unlikely to explain the magnitude of this increase in the take-up of STEM and Medicine. Specifically, we analyze the field of study choices of students who switched from the academic track to the general track, and we find no evidence of a systematic outflow of students interested in social science and humanities. In fact, the share of STEM and Medicine specializations among the switching students is increasing, following a pattern similar to the one plotted in Figure 2.2. The share of STEM and Medicine specializations among switching students is, however, lower than the share found among students who decided to stay in the academic track. This can have a direct effect on the composition of fields of study: Assuming that the $2.5 \%$ of Grade 10 students who decided not to pursue the academic track in response to the reform would have chosen the same field of study as the students who switched tracks, we calculate that their outflow would account for a 1 percentage point change of the STEM and Medicine share. The results suggest that in response to the reform high school students started moving towards specializations which are associated with better job prospects and higher earnings potential. These results echo those of Rothstein and Rouse (2011) and Field (2009). Here we argue that Dutch high school students act upon the knowledge that they will have to take more student loans in the future and choose fields of study with better labor market prospects in order to secure the repayment of their student loans.

\section{Dropout of STEM and Medicine field of study}

We now focus on the decision to leave the STEM and Medicine curriculum and to take up the humanities and social sciences curriculum instead. Our focus is one-sided because switches from humanities to STEM are very rare. Figure B.5 shows the shares of students dropping out of STEM and Medicine majors in Grade 11 from 2008 onward. Students dropping out of majors represent a relatively small part of the student pool engaged in the STEM and Medicine specialization. In years 2008 to 2010 , they accounted for only $4.5 \%$ of students, and this share declined to $3.3 \%$ in 2013 at which point it stabilized.

Table B.8 reports the effects of policy announcements on dropping out of STEM or Medicine in Grade 11, conditional on being in STEM or Medicine in Grade 10. The share of students who leave the STEM and Medicine curriculum decreased by 
Figure B.5: Share of Dropout from STEM and Medicine Specialization in High School

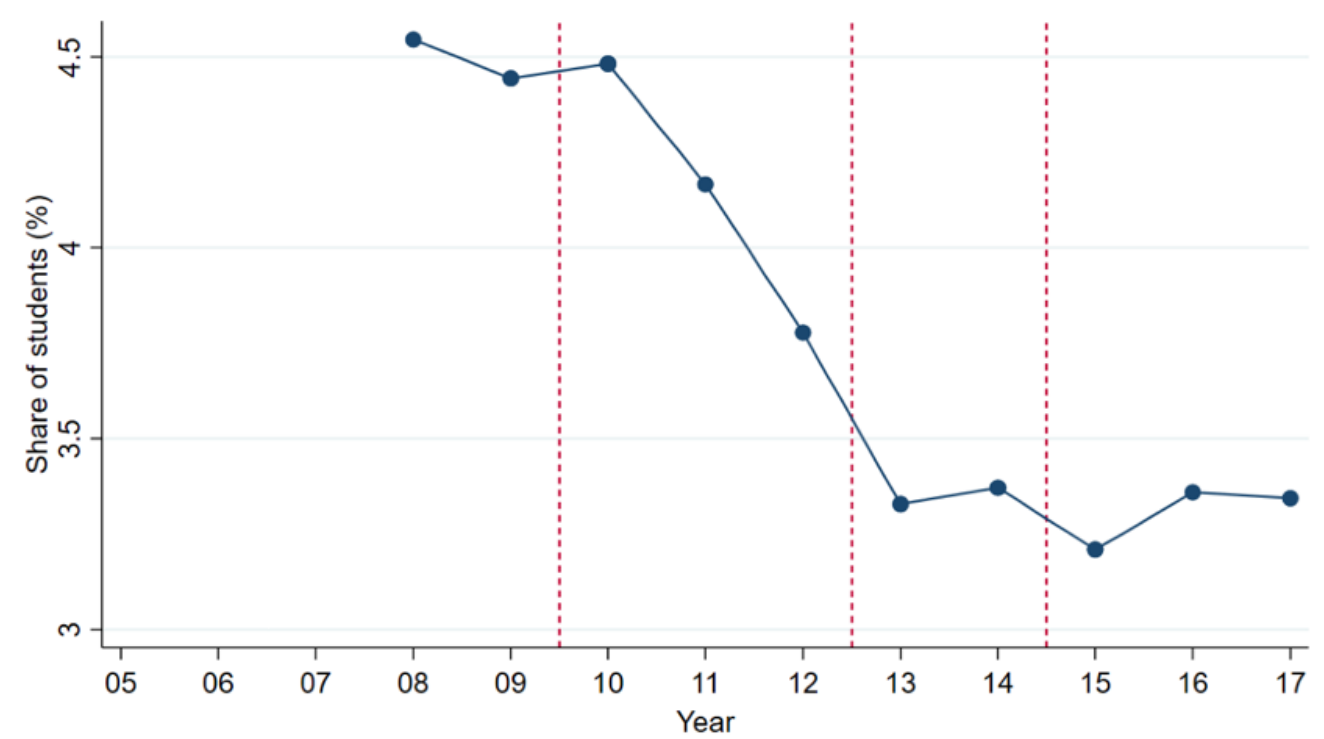

Note: Authors' calculations of the choice to switch from STEM specialization to a nonSTEM specialization in Grade 11 over the period of observation. STEM specialization corresponds to the following curricula: Nature and Technology, Nature and Health, and the combination thereof. Other curricula include Economy and Society, Culture and Society, and all other possible combinations. Dutch education register data for Grade 11 academic or general track high school students who chose the STEM specialization in Grade 10, years 2008 to $201 \%$.

0.30 percentage points after the 2010 announcement, further decreasing by 1.12 percentage points after the uncertainty is resolved in the 2012 announcement, and stabilizing at -1.21 percentage points after reform implementation in 2015 .

Table B.15 in Appendix B.4 reports the results for the alternative specification using a continued linear pre-trend, which yields similar results. The first year of analysis is 2008. We prefer the model specification which does not extrapolate the pre-trend, as for the model of track dropout in the main results section, because the time interval used to identify the pre-trend is too short, and because the share of switching students in the implementation period is sustained. Overall, these results indicate that students were also re-optimizing their high school orientation in terms of field of study following the changes in their decision environment induced by the reform announcements. 
Table B.8: Effects on STEM Dropout

\begin{tabular}{|c|c|c|c|c|}
\hline Outcome: & $\begin{array}{l}\text { Baseline } \\
\text { STEM } \\
\text { Dropout }(\%) \\
\quad(2009) \\
\quad(1)\end{array}$ & $\begin{array}{c}(2010-2012) \\
(2)\end{array}$ & $\begin{array}{l}\text { Uncertainty } \\
\text { Resolution }\end{array}$ & $\begin{array}{l}\text { Post- } \\
\text { implementation }\end{array}$ \\
\hline \multicolumn{5}{|c|}{ Panel A: Absolute Effects (in percentage points) } \\
\hline STEM Dropout & 4.5 & $\begin{array}{c}-0.30 * * * \\
(0.00)\end{array}$ & $\begin{array}{l}-1.12 * * * \\
(0.00)\end{array}$ & $\begin{array}{l}-1.21 * * * \\
(0.00)\end{array}$ \\
\hline \multicolumn{5}{|c|}{ Panel B: Relative Effects (in percent) } \\
\hline STEM Dropout & & $\begin{array}{c}-7.93 * * * \\
(0.04)\end{array}$ & $\begin{array}{c}-29.95 * * * \\
(0.08)\end{array}$ & $\begin{array}{c}-32.21 * * \\
(0.11)\end{array}$ \\
\hline \multicolumn{5}{|c|}{$\begin{array}{l}\text { Note: Authors' estimates of three reform coefficients from a logit model of Grade } 11 \text { choice } \\
\text { to dropout of STEM and Medicine into another subject specialization, assuming a break in } \\
\text { the pre-trend in 2009. The model uses } 451,512 \text { individual observations over years } 2006 \text { - } 201 \% \text {. } \\
\text { Controls: region fixed effects, age, gender, migration status, ethnic background, parental income } \\
\text { and employment status, an urbanization index and a dummy for the differently-classified period } \\
\text { 2005-06. }{ }^{* * *}=0.01 \text { significance level, }{ }^{* *}=0.05 \text { significance level, }{ }^{*}=0.1 \text { significance level. }\end{array}$} \\
\hline
\end{tabular}

\section{STEM and Medicine enrollment and dropout by family income}

Table B.9 indicates that students are not significantly more likely to enroll in STEM fields after the initial shock in 2010, irrespective of their family background. Following uncertainty resolution following the second announcement, however, middle and high income students became more likely to enroll in STEM fields. These results with the continued linear pre-trend specification, are similar to those obtained with the alternative specification (not presented in this chapter), which shows that low and medium income students were already responsive to the first announcement (by 0.43 percentage points and 0.81 percentage points respectively).

Table B.10 presents suggestive evidence regarding dropout and reorientation after one year, immediately following exposure to announcement shocks. Low income students who choose a STEM field are less likely to switch fields after uncertainty increased in 2010, and persistently after uncertainty is resolved from 2013 onward. For middle and higher income students, however, dropout rates in STEM majors peak after the second announcement, in 2013.

These results provide further evidence that high school students respond with anticipation to the incentives generated by student finance in higher education. As 
Table B.9: Effects on Enrollment in STEM, by Family Income

\begin{tabular}{|c|c|c|c|c|}
\hline Outcome: & Income: & $\begin{array}{c}(2010-2012) \\
(1)\end{array}$ & $\begin{array}{l}\text { Uncertainty } \\
\text { Resolution } \\
(2013-2014) \\
(2)\end{array}$ & $\begin{array}{c}\text { Post- } \\
\text { implementation } \\
(2015-2017) \\
(3)\end{array}$ \\
\hline \multicolumn{5}{|c|}{ Panel A: Absolute Effects (in percentage points) } \\
\hline $\begin{array}{l}\text { Specialization } \\
\text { in STEM }\end{array}$ & $\begin{array}{l}\text { Low } \\
\text { Medium } \\
\text { High }\end{array}$ & $\begin{array}{r}-0.8 \\
0.54 \\
-1.17\end{array}$ & $\begin{array}{l}1.52 * * \\
3.02 * * * \\
2.00 * *\end{array}$ & $\begin{array}{l}1.16 \\
5.43 * * * \\
2.09\end{array}$ \\
\hline \multicolumn{5}{|c|}{ Panel B: Relative Effects (in percent) } \\
\hline $\begin{array}{l}\text { Specialization } \\
\text { in STEM }\end{array}$ & $\begin{array}{l}\text { Low } \\
\text { Medium } \\
\text { High }\end{array}$ & $\begin{array}{r}-1.45 \\
0.97 \\
-2.01\end{array}$ & $\begin{array}{l}2.78 * * \\
5.44 * * * \\
3.45 * *\end{array}$ & $\begin{array}{l}2.12 \\
9.79 * * * \\
3.6\end{array}$ \\
\hline
\end{tabular}

Note: Authors' estimates of three reform coefficients from a logit model of Grade 10 choice of STEM and Medicine specialization in academic and general high school tracks, assuming a continued linear pre-trend beyond 2009. The dataset is split by parental income terciles and uses respectively 96,694; 150,621; and 299,946 individual observations over years 2006-2017. Controls: region fixed effects, age, gender, migration status, ethnic background, parental income and employment status, and an urbanization index. ${ }^{* * *}=0.01$ significance level, ${ }^{* *}=0.05$ significance level, ${ }^{*}=0.1$ significance level.

uncertainty peaked, vulnerable students were the ones responding; as uncertainty resolved in favor of vulnerable students, those responding most are middle and high income students who would not be eligible to receive means-tested aid. These students, therefore, faced the steepest increase of costs, while low-income students were eventually sheltered from the cost increase.

One issue is that it is difficult to conclude which students are made better or worse off by policy uncertainty, which led students to choose alternative tracks and high school majors. The proportion of middle-income students preparing for college dropped substantially, however among those who remained in the academic track there was a strong shift towards high-earning subjects. Students in the third income tercile also lowered their enrollments into the academic-track, yet unlike middle-income students, they did not change their subject specialization and chose more often to remain in the general track, rather than spilling over into the vocational track. Students from the relatively poorer families adjusted their track choices similarly to middle-income students, however their adjustment was less pronounced, and they did not adjust their specialization towards fields yielding higher returns. 
Table B.10: Effetcs on STEM Dropout, by Family Income

\begin{tabular}{|c|c|c|c|c|}
\hline Outcome: & Income: & $\begin{array}{c}(2010-2012) \\
(1)\end{array}$ & $\begin{array}{l}\text { Uncertainty } \\
\text { Resolution } \\
(2013-2014) \\
(2)\end{array}$ & $\begin{array}{c}\text { Post- } \\
\text { implementation } \\
(2015-2017) \\
(3)\end{array}$ \\
\hline \multicolumn{5}{|c|}{ Panel A: Absolute Effects (in percentage points) } \\
\hline $\begin{array}{l}\text { STEM } \\
\text { dropout }\end{array}$ & $\begin{array}{l}\text { Low } \\
\text { Medium } \\
\text { High }\end{array}$ & $\begin{array}{r}-2.07 * * * \\
0.46 * * * \\
0.33 * * *\end{array}$ & $\begin{array}{r}-3.43 * * * \\
0.38 * * * \\
-0.40 * * *\end{array}$ & $\begin{array}{r}-5.38 * * * \\
0.79 * * * \\
0.57 * * *\end{array}$ \\
\hline \multicolumn{5}{|c|}{ Panel B: Relative Effects (in percent) } \\
\hline $\begin{array}{l}\text { STEM } \\
\text { dropout }\end{array}$ & $\begin{array}{l}\text { Low } \\
\text { Medium } \\
\text { High }\end{array}$ & $\begin{array}{r}-48.08 * * * \\
13.14 * * * \\
9.01 * * *\end{array}$ & $\begin{array}{r}-79.70 * * * \\
11.03 * * * \\
-10.74 * * *\end{array}$ & $\begin{array}{r}-125.01 * * * \\
22.69 * * * \\
15.58 * * *\end{array}$ \\
\hline
\end{tabular}

Note: Authors' estimates of three reform coefficients from a logit model of Grade 11 choice to switch from STEM and Medicine to another subject specialization, assuming a continued linear pre-trend beyond 2009. The dataset is split by parental income tercile and uses respectively 96,693; 138,489; and 218,330 individual observations over years 2006-2017. Controls: region fixed effects, age, gender, migration status, ethnic background, parental income and employment status, and an urbanization index. ${ }^{* * *}=0.01$ significance level, ${ }^{* *}=0.05$ significance level, $*=0.1$ significance level.

\section{Gender and STEM and Medicine field of study enrollments}

We find strong gender differences in how students respond to reform announcements. Girls were responsible for almost the entire reform effect on STEM specialization and re-orientation. Table B.11 shows that girls were more proactive and more flexible in their choices than boys as early as uncertainty increased in 2010. These results also hold using our alternative specification (not presented in this chapter).

Once the reform is implemented in 2015, the take-up of STEM and Medicine among girls had increased by $10 \%$ compared to baseline, while the reform announcements and implementation coefficients for boys are close to zero and failing to yield statistical significance. At baseline, the take-up of STEM and Medicine among girls was considerably lower than among boys (38\% compared to 50\%). The student finance reform narrowed this gap considerably, although a small disparity remains even in the last year of observation (48\% compared to $51 \%$ ).

These results could reflect differences in forward-looking behavior of girls and boys. 
Table B.11: Effects on Enrollment in STEM, by Gender

\begin{tabular}{|c|c|c|c|c|c|}
\hline Outcome: & Gender: & $\begin{array}{c}\text { Baseline } \\
\text { STEM } \\
\text { share (\%) } \\
(2009) \\
(1)\end{array}$ & $\begin{array}{c}(2010-2012) \\
(2)\end{array}$ & $\begin{array}{c}\text { Uncertainty } \\
\text { Resolution } \\
(2013-2014) \\
(3)\end{array}$ & $\begin{array}{c}\text { Post- } \\
\text { implementation } \\
(2015-2017) \\
(4)\end{array}$ \\
\hline \multicolumn{6}{|c|}{ Panel A: Absolute Effects (in percentage points) } \\
\hline $\begin{array}{l}\text { Specialization } \\
\text { in STEM }\end{array}$ & $\begin{array}{l}\text { Male } \\
\text { Female }\end{array}$ & $\begin{array}{l}50.0 \\
38.0\end{array}$ & $\begin{array}{l}-1.18 \\
-0.35\end{array}$ & $\begin{array}{l}1.31 \\
2.77 * * *\end{array}$ & $\begin{array}{l}-0.13 \\
5.22 * * *\end{array}$ \\
\hline \multicolumn{6}{|c|}{ Panel B: Relative Effects (in percent) } \\
\hline $\begin{array}{l}\text { Specialization } \\
\text { in STEM }\end{array}$ & $\begin{array}{l}\text { Male } \\
\text { Female }\end{array}$ & & $\begin{array}{l}-1.91 \\
-0.67\end{array}$ & $\begin{array}{l}2.12 \\
5.3 * * *\end{array}$ & $\begin{array}{l}-0.21 \\
9.98 * * *\end{array}$ \\
\hline
\end{tabular}

Note: Authors' estimates of three reform coefficients from a logit model of Grade 10 choice of STEM and Medicine specialization in academic and general high school tracks, assuming a continuing linear pre-trend extending beyond 2009. The models are split by gender and use respectively 257,338 and 292,302 individual observations over years 2005-2017. Controls: region fixed effects, age, gender, migration status, ethnic background, parental income and employment status, and an urbanization index. ${ }^{* * *}=0.01$ significance level, ${ }^{* *}=0.05$ significance level, ${ }^{*}=0.1$ significance level.

They could also indicate gender differences in debt aversion. Lastly, gender differences in responses to reform announcements could also relate to parents' willingness to pay for education of their children, and how much this varies depending on the child's gender.

\section{Additional effects of policy announcements on living arrangements and commuting distance in post-secondary education}

We show further evidence in line with students exhibiting debt aversion. To avoid taking debt, students can prefer reducing expenditures, and living at home after high school instead of moving closer to university is one way of lowering expenditures. This decision puts students in front of a new trade-off between choosing a university closer to home or to commute more. For this exercise, we construct commuting distance from home to school as the Euclidian distance from the residential address of a student to his post-secondary institution. Addresses of post-secondary vocational institutions are not available in our data, so we focus on Universities of Applied Sciences and University students. 
Figure B.6: Living Arrangements and Commuting Distance

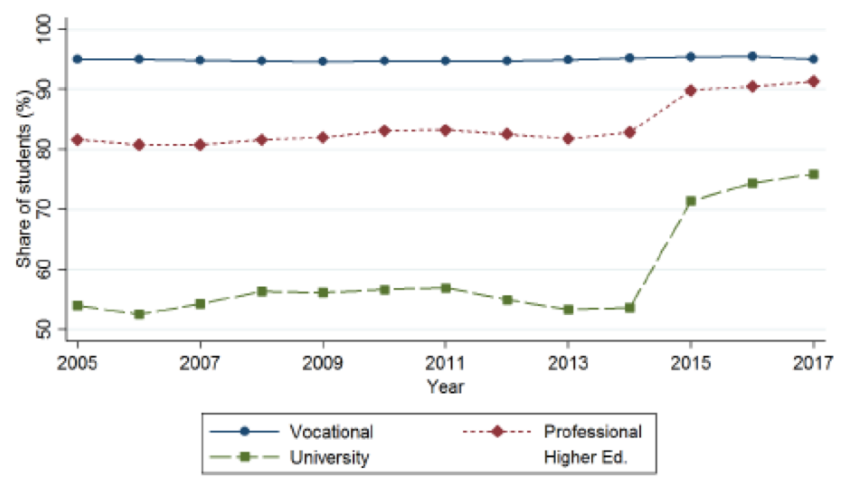

(a) Living arrangements of first-year higher education students

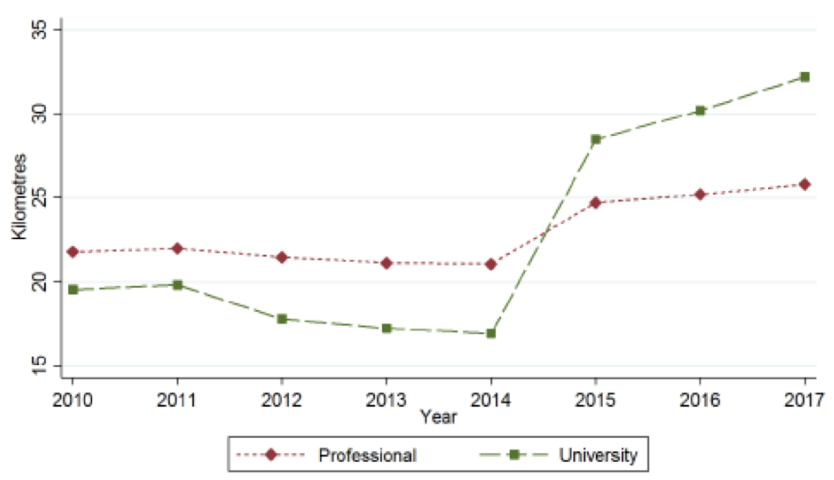

(b) Geographical distance from parental home to school

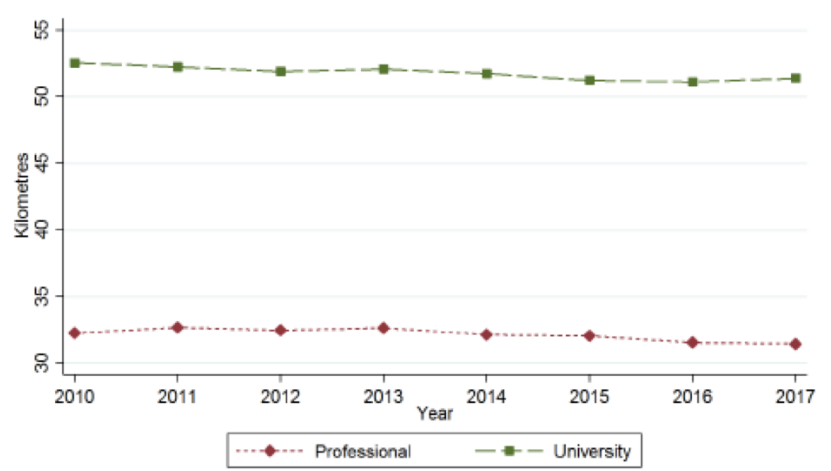

(c) Commuting distance form parental home to school

Note: Authors' calculations of the living arrangement choices among of first year students enrolled in vocational and higher education institutions. Living arrangements are retrieved at the end of the first semester; students are classified as living with their parents if parents are registered at the same residential address. The distance measures correspond to Euclidean distance between the student's residence and the institution attended. Dutch education register and municipal register data for first year students enrolled in vocational and higher education institutions, years 2010 to $201 \%$. 
Figure B.6 plots changes in living arrangements and commuting distance of students in their first year after graduating from high school, over our time frame. Figure B.6a show the probability that students live at home over time, depending on the type of post-secondary education they are enrolled in. The figure reveals two important patterns: 1) $95 \%$ of all vocational students, who eventually were not affected in 2015 , consistently live at home in their first year after high school, while 2) professional and academic higher education students are less likely to still live at home, but this probability increases dramatically in 2015, the first cohort of students eventually affected by the reform. This suggests that these students, who had no choice but to take-up loans, adjust their expenditures by living at home, and not having to pay rent for an independent apartment.

Figure B.6b shows that the distance from students' home to their university or institution remained stable throughout, suggesting that students are not choosing different universities than they did prior to 2015. Figure B.6c completes the picture by showing that these new living arrangements were made at the cost of commuting distance, and not the choice of institution. Students who entered higher education in 2015 under the new student finance regime preferred commuting slightly more to their institution rather than choosing institutions closer to home.

These new living arrangements are further evidence that students avoided accumulating debt in response to the new policy environment.

\section{Additional effects of policy announcements on labor supply and take-up of financial aid in post-secondary education}

We study students' labor supply, which is another margin on which students are likely to adjust to the new system of student finance. Previous literature (e.g. Kalenkoski and Pabilonia, 2010) has shown that parental transfer, financial aid and students' labor supply are substitutes, such that when financial aid decreases, students receiving low parental transfers are likely to increase labor supply.

Under the new regime of student finance, students faced higher costs of attending higher education (but not vocational education). Thus, we can expect to find an increase in the labor supply of higher education freshmen from 2015 onward, compared to baseline. Figure B.7 shows the employment rate of students in their first year after high school, relative to the employment rate of students in the same education level in 2006. We find that labor supply dynamics are similar across levels of education, suggesting that labor supply was not a margin that students heavily 
Figure B.7: Labor Supply of Young Adults in the Netherlands, Years 2006-2017

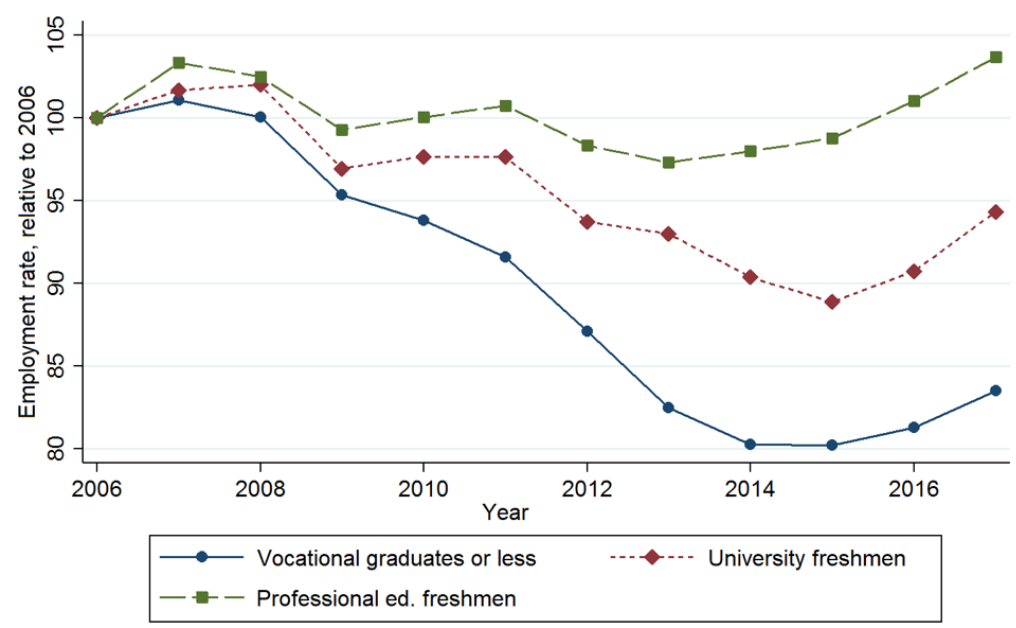

Note: Authors' calculations of the employment rates among vocationalschool graduates and freshmen enrolled in higher education institutions over the period of observation. Employment is recorded if the young adult was recorded to work within the span of the academic year starting in the respective calendar year. Dutch education register and tax register data for young adults, years 2006-2017.

used in our setting.

Other sources suggest that one reason why we are not finding that students labor supply changed in response to the new student finance environment, is that freshmen in 2015 re-optimized through other margins, in particular living arrangements.

To complement our main results from administrative records, we refer the reader to annual reports of the Studenten Monitor, a longitudinal survey of a representative sample of approximately 20,000 students in higher education, which has been collected since 2001 at the initiative of the Dutch Ministry of Education, Culture and Science. The Studenten Monitor contains a battery of questions on students' beliefs and perceptions about student finance, labor supply, as well as sources of income and budgeting choices during higher education.

The Studenten Monitor reveals three changes which suggest that students reoptimized their budget to avoid accumulating debt. The first change is a decline in the take up of student loans. After 2015, roughly 3\% of all Dutch freshmen had not applied to any financial aid. The take-up of student loans among freshmen respondents only increased by 20 percentage points in 2015, from a stable $27 \%$ from 2001 to 2014 to $47 \%$ after 2015. This is suggestive that there is a substantial share of students who preferred not claiming financial aid under the new system of student finance, with smaller differences in take-up behavior across family income 
after 2015. The share of students who claimed $90 \%$ of more of the maximum amount they are entitled to has declined over time, down to $31 \%$ in 2015 . This result is in line with the general pattern of under-utilization of student loans found in many settings, including the Dutch context. The average monthly amounts borrowed among students with a student loan has increased after the introduction of the new student finance regime, from $€ 394$ to $€ 439$ per month (an increase of $€ 44$ per month), with variation across type of higher education. ${ }^{3}$

The second change is a decline in the labor supply of students, compensated by an increase in parental investments. Freshmen in 2015 and onward choose more often than previous cohorts to neither take-up a student loan nor work - this is in line with the absence of significant change in labor supply which we found in the administrative records in Figure B.7. These students are supported by their parents both directly through increased parental transfers, and indirectly parents covering expenditures, including in-kind transfers through living arrangements at home.

The third change is in increase in the preferences of freshmen in 2010 for minimizing debt compared to previous cohorts. Among respondents who did not take-up a student loan, the main reasons for not borrowing remain stable over the time frame, and relate to either parents not agreeing with student borrowing (mostly highly educated parents), or having fundamental objections with borrowing (mostly lower educated parents), or students lacking knowledge about borrowing conditions. From 2015 onward, however, respondents were more likely to answer that they receive enough parental monetary support and don't feel the need to take up a loan, and substantially less likely to respond that they perform paid work to avoid taking-up a loan. 2015 freshmen who do, however, take up loans, are more likely to have been advised by their parents to take-up a loan, and less likely to declare that they are not willing to work to finance their studies. This suggests that even among borrowers, the trade-off over labor supply and loan take-up is less important than we expected, largely because families re-optimized their budget through living arrangements.

Overall, these changes in students' preferences and beliefs about debt and labor supply are suggestive of debt aversion. That students re-optimized their budget and avoided taking up loans from 2015 onward is suggestive that these preferences were already latent when the 2015 cohort was entering high school 5 years earlier, around the time of the 2010 announcement which increased policy uncertainty about the

\footnotetext{
${ }^{3}$ see also van den Broek et al. (2017)
} 
future of financial aid.

\section{B.4 Sensitivity Analyses}

Results under Alternative Specification

Table B.12: Alternative Specification - Main Effects on Track Enrollment

\begin{tabular}{lcccc}
\hline & $\begin{array}{c}\text { Baseline } \\
\text { track } \\
\text { shares (\%) } \\
(2009)\end{array}$ & Uncertainty & $\begin{array}{c}\text { Uncertainty } \\
\text { Resolution }\end{array}$ & $\begin{array}{c}\text { Post- } \\
\text { implementation }\end{array}$ \\
Outcome: & $(1)$ & $(2010-2012)$ & $(2013-2014)$ & $(2015-2017)$ \\
\cline { 2 - 4 } & & & $(3)$ & $(4)$
\end{tabular}

Panel A: Absolute Effects (in percentage points)

\begin{tabular}{lcccc}
\hline Academic & 21.0 & $-0.39 * * *$ & $-0.94 * * *$ & $-1.21 * * *$ \\
& & $(0.03)$ & $(0.03)$ & $(0.03)$ \\
General & 29.0 & $0.57 * * *$ & $0.62 * * *$ & $0.65 * * *$ \\
& & $(0.14)$ & $(0.14)$ & $(0.14)$ \\
Vocational & 50.2 & -0.18 & $0.32 *$ & $0.56 * * *$ \\
& & $(0.17)$ & $(0.17)$ & $(0.17)$
\end{tabular}

Panel B: Relative Effects (in percent)

\begin{tabular}{lccc}
\hline Academic & $-1.92 * * *$ & $-4.67 * * *$ & $-6.00 * * *$ \\
& $(0.18)$ & $(0.17)$ & $(0.17)$ \\
General & $1.98 * * *$ & $2.16 * * *$ & $2.24 * * *$ \\
& $(0.49)$ & $(0.49)$ & $(0.50)$ \\
Vocational & -0.34 & $0.66 *$ & $1.14 * * *$ \\
& $(0.33)$ & $(0.34)$ & $(0.34)$
\end{tabular}

Note: Authors' estimates of three reform coefficients from a multinomial logit model of high school track enrollment as measured in Grade 10, using a linear pre-trend that does not extend beyond 2009. The model uses 2,692,887 individual observations over years 2005-2017. Controls: region fixed effects, age, gender, migration status, ethnic background, parental income and employment status, and an urbanization index. *** $=0.01$ significance level, ${ }^{* *}=0.05$ significance level, ${ }^{*}=0.1$ significance level. This is an alternative specification compared to results shown in Table 2.3. 
Table B.13: Alternative Specification - Main Effects on Track Dropout

\begin{tabular}{lcccc}
\hline & $\begin{array}{c}\text { Baseline } \\
\text { track } \\
\text { dropout }(\%)\end{array}$ & Uncertainty & $\begin{array}{c}\text { Uncertainty } \\
\text { Resolution }\end{array}$ & $\begin{array}{c}\text { Post- } \\
\text { implementation }\end{array}$ \\
Outcome: & $(2009)$ & $(2010-2012)$ & $(2013-2014)$ & $(2015-2017)$ \\
& $(1)$ & $(2)$ & $(3)$ & $(4)$ \\
\hline
\end{tabular}

Panel A: Absolute Effects (in percentage points)

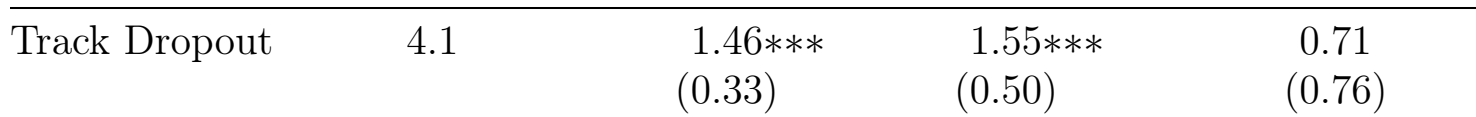

Panel B: Relative Effects (in percent)

\begin{tabular}{llll}
\hline Track Dropout & $33.78 * * *$ & $35.83 * * *$ & 16.37 \\
& $(7.55)$ & $(11.47)$ & $(17.50)$ \\
\hline
\end{tabular}

Note: Authors' estimates of three reform coefficients from a logit model of track dropout measured in Grade 11 compared to high school track observed in Grade 10, using a linear pre-trend extending beyond 2009. The model uses 958,512 individual observations over years 2006-2017. Controls: region fixed effects, age, gender, migration status, ethnic background, parental income and employment status, and an urbanization index. ${ }^{* * *}=0.01$ significance level, ${ }^{* *}=0.05$ significance level, ${ }^{*}=$ 0.1 significance level. This is an alternative specification compared to results shown in Table 2.4.

Table B.14: Alternative Specification - Effects on Enrollment in STEM

\begin{tabular}{lcccc}
\hline & Baseline & Uncertainty & Uncertainty & Post- \\
STEM & & Resolution & implementation \\
share $(\%)$ & & $(2013-2014)$ & $(2015-2017)$ \\
Outcome: & $(2009)$ & $(2010-2012)$ & $(3)$ & $(4)$ \\
\hline
\end{tabular}

Panel A: Absolute Effects (in percentage points)

\begin{tabular}{|c|c|}
\hline $\begin{array}{l}\text { Specialization } \\
\text { in STEM }\end{array}$ & 44. \\
\hline
\end{tabular}

Panel B: Relative Effects (in percent)

\begin{tabular}{lccc}
\hline Specialization & 0.44 & $6.87 * * *$ & $10.26 * * *$ \\
in STEM & $(0.38)$ & $(0.38)$ & $(0.39)$ \\
\hline
\end{tabular}

Note: Authors' estimates of three reform coefficients from a logit model of Grade 10 choice of STEM and Medicine specialization in academic and general high school tracks, assuming a break in the pre-trend in 2009. The model uses 549,640 individual observations over years 2005-2017. Controls: region fixed effects, age, gender, migration status, ethnic background, parental income and employment status, an urbanization index and a dummy for the differently-classified period 2005-06. ${ }^{* * *}=0.01$ significance level, ${ }^{* *}=0.05$ significance level, ${ }^{*}=0.1$ significance level. This is an alternative specification compared to results shown in Table B.\%. 
Table B.15: Alternative Specification - Effects on STEM Dropout

\begin{tabular}{|c|c|c|c|c|}
\hline Outcome: & $\begin{array}{c}\text { Baseline } \\
\text { STEM } \\
\text { Dropout }(\%) \\
(2009) \\
(1)\end{array}$ & $\begin{array}{c}(2010-2012) \\
(2)\end{array}$ & $\begin{array}{l}\text { Uncertainty } \\
\text { Resolution }\end{array}$ & $\begin{array}{l}\text { Post- } \\
\text { implementation }\end{array}$ \\
\hline \multicolumn{5}{|c|}{ Panel A: Absolute Effects (in percentage points) } \\
\hline STEM Dropout & 4.5 & $\begin{array}{c}-0.12 * * * \\
(0.01)\end{array}$ & $\begin{array}{c}-0.77 * * * \\
(0.01)\end{array}$ & $\begin{array}{c}-0.60 * * * \\
(0.02)\end{array}$ \\
\hline \multicolumn{5}{|c|}{ Panel B: Relative Effects (in percent) } \\
\hline STEM Dropout & & $\begin{array}{c}-3.25 * * * \\
(0.15)\end{array}$ & $\begin{array}{c}-20.60 * * * \\
(0.28)\end{array}$ & $\begin{array}{c}-15.94 * * * \\
(0.42)\end{array}$ \\
\hline \multicolumn{5}{|c|}{$\begin{array}{l}\text { Note: Authors' estimates of three reform coefficients from a logit model of Grade } 11 \text { choice } \\
\text { to dropout of STEM and Medicine into another subject specialization, assuming a break in } \\
\text { the pre-trend in } 2009 \text {. The model uses 451,512 individual observations over years } 2006 \text {-201\%. } \\
\text { Controls: region fixed effects, age, gender, migration status, ethnic background, parental income } \\
\text { and employment status, an urbanization index and a dummy for the differently-classified period } \\
2005-06 . * * *=0.01 \text { significance level, }{ }^{* *}=0.05 \text { significance level, }{ }^{*}=0.1 \text { significance level. } \\
\text { This is an alternative specification compared to results shown in Table B.8. }\end{array}$} \\
\hline
\end{tabular}




\section{Potential Confounding Factors}

In this subsection of Appendix B.4, we systematically explore potential confounding factors which could bias our results. We explore these factors by order of relevance, based on our conceptual framework.

\section{Expected Wage Premia and Opportunity Cost}

A natural concern is that the changes in track and study choice are a result of changes in track specific changes in returns to education and opportunity cost of studying.

Expected Wages Premia Using Dutch administrative data, van den Berge (2018) shows that earnings profiles of graduates from academic Universities (WO) and Universities of Applied Sciences (HBO) are very comparable since 1999, such that the wage premium from academic over general education is almost constant over time. As the framework indicates, the marginal student who is indifferent between general and academic education will consider not his expected level of earnings in academic or in general education, but rather his expected wage premium from graduating with an academic degree over a general degree. Similarly, the share of full-time students employed directly after education is very high, comparable and more importantly stable over our entire time period between graduates of vocational, general and academic education (see Table B.16).

We cannot directly observe students' wage premium expectations nor their expected employment probabilities. A growing body of work on the formation of beliefs (see e.g. Delavande and Zafar, 2019) shows, however, that students' expectations are realistic. Consequently, under the assumption that students have realistic expectations, it is unlikely that the effect of policy announcements on track choice and dropout would be driving solely by diverging expectations about wage premiums of general education over vocational education, and of academic over general education.

Opportunity Cost of Studying An important aspect of students' decision making is the opportunity cost of studying. For a student who is indifferent between academic and general education, the opportunity cost of studying is the premium the student would receive from graduating earlier from general education and receiving a general education wage premium. For a student who is indifferent between general and vocational education, however, this opportunity cost is the vocational graduate 
Table B.16: Employment Prospect of Tracks, by Education Level

Academic year:

$\begin{array}{lllllllll}2007 & 2008 & 2009 & 2010 & 2011 & 2012 & 2013 & 2014 & 2015\end{array}$

Panel A. Share of cohort in paid employment 6 months after leaving education

\begin{tabular}{llllllllll}
\hline Vocational education (MBO): & & & & & & & & & \\
Dropout & & 67.0 & 58.6 & 57.4 & 57.3 & 54.1 & 51.0 & 51.7 & 56.2 \\
Graduate & & 86.9 & 83.0 & 82.2 & 81.9 & 79.8 & 78.0 & 77.5 & 79.7 \\
Uni. Applied Sciences (HBO): & & & & & & & & & \\
Dropout & 80.1 & 79.7 & 75.3 & 73.9 & 75.3 & 73.3 & 70.0 & 71.6 & 74.0 \\
Graduate & 88.7 & 88.4 & 86.0 & 86.0 & 86.6 & 85.0 & 84.1 & 84.9 & 86.2 \\
University (WO): & & & & & & & & & \\
Dropout & 72.1 & 70.8 & 65.1 & 64.7 & 64.7 & 62.8 & 56.4 & 56.7 & 59.8 \\
Graduate & 81.8 & 81.1 & 79.3 & 77.4 & 77.9 & 75.9 & 74.0 & 75.7 & 76.7 \\
& & & & & & & & & \\
Panel B. Year-to-year \% changes & & & & & & & & \\
\hline Vocational education (MBO): & & & & & & & & & \\
Dropout & & & -13 & -2 & 0 & -6 & -6 & 1 & 9 \\
Graduate & & -4 & -1 & 0 & -3 & -2 & -1 & 3 \\
Uni. Applied Sciences (HBO): & & & & & & & & \\
Dropout & 0 & -6 & -2 & 2 & -3 & -5 & 2 & 3 \\
Graduate & 0 & -3 & 0 & 1 & -2 & -1 & 1 & 2 \\
University (WO): & & & & & & & & \\
$\begin{array}{l}\text { Dropout } \\
\text { Graduate }\end{array}$ & -2 & -8 & -1 & 0 & -3 & -10 & 1 & 5 \\
\hline
\end{tabular}

Note: CBS Statline - aggregate administrative data publicly available.

wage a student would receive for graduating earlier from vocational education over general education.

Our main findings are not in line with an alternative explanation relying on the Great Recession. Indeed, we would expect that the Great Recession increases incentives to choose longer educational tracks which promise higher labor market returns, compared to shorter tracks with lower labor market returns.

In addition, we can show that i) minimum wage is stable over the entire period and mandated by law, and ii) youth unemployment rate shows no sign of trend breaks between 2000 and 2017, in spite of the Great Recession. Figure B.8 shows the national youth unemployment rate for the 15-19 year old across countries surrounding the Netherlands, over the period 2000 to 2018. The Dutch unemployment rate is around 10 to $14 \%$ for the 15-19-year-old population, making it one of the lowest in the European Union and most stable throughout the crisis. Thus, it seems also unlikely that students' opportunity cost of studying suddenly increased in light of the policy announcement in 2010. Similar rates and dynamics also apply for other age ranges. Thus, the opportunity cost of studying is stable throughout our time-frame, and changes in the opportunity cost of studying would not explain away 
our results on either sorting into tracks nor dropout rates.

Figure B.8: Youth Unemployment Rate, 15 to 19 Year-Old Population

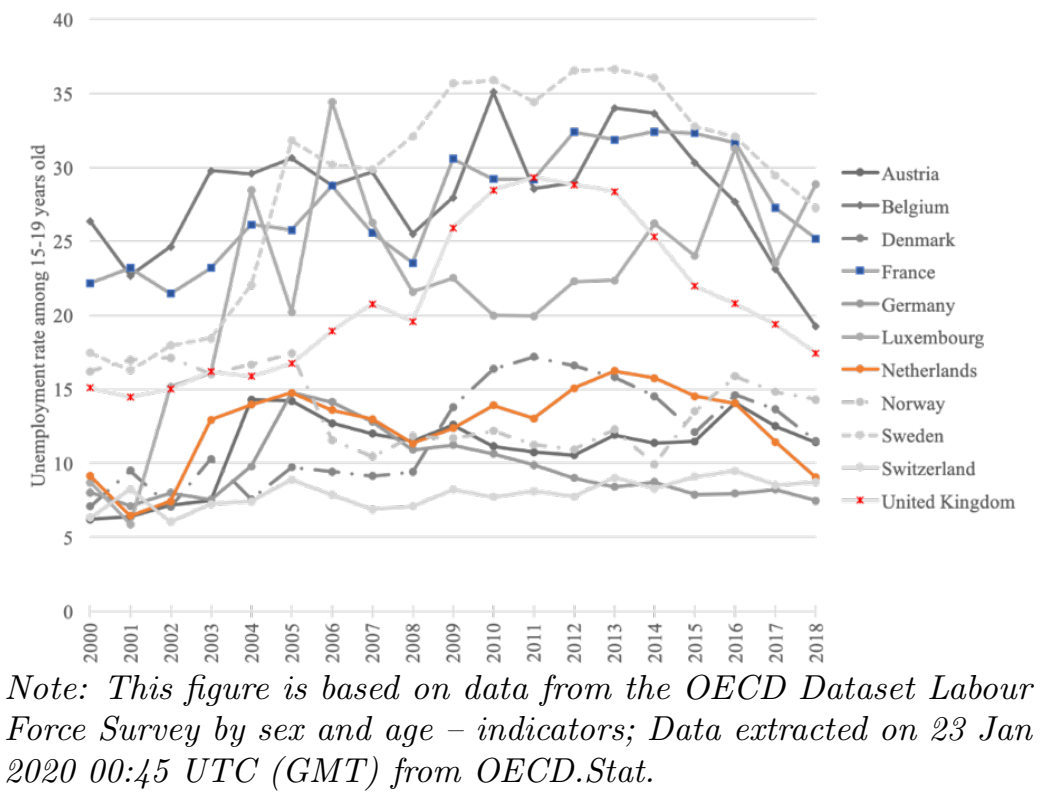

\section{Psychological Cost of Studying: Education Difficulty and Duration of Studies}

One might be concerned that our results are driven by changes in students' underlying cost of studying, either because high school track curricula or duration of studies coincidentally changed in 2010 .

High School Graduation Rates Panel A of Table B.17 shows that high school graduation rates are stable over time. Using aggregate data from the Dutch Ministry of Education (DUO Statline), we first note that the probability of graduating is highest for vocational students around $96 \%$, and lowest for academic students around $88 \%$. Second, we find a decline in graduation rates at academic and general high school in 2008 which is likely linked to curriculum reform which took place in 2007. The gap in graduation probabilities is stable between general and academic tracks, and remains small between general and vocational tracks before reverting to baseline from 2012 onward. Importantly, splitting these graduation probabilities by high school major within tracks, we see no differences between majors, such that diverging trends in the difficulty of tracks and majors within tracks cannot explain our results on selection into tracks, resorting after one year, major choice within tracks and major resorting after one year. 


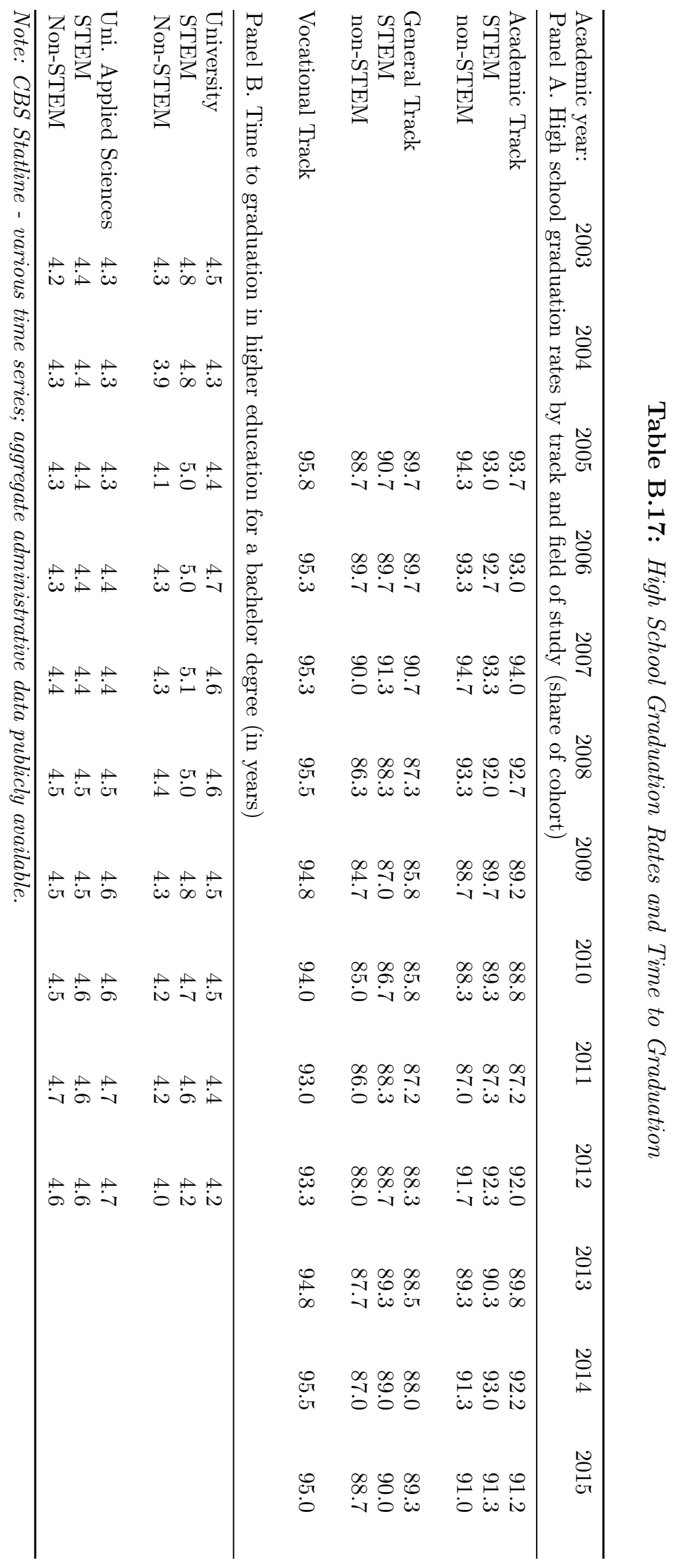


Average Time to Graduation in Higher Education Another way to think about the psychic cost of studying in a given educational track is to consider the average time to graduation in post-secondary education, as shown in Panel B of Table B.17. From 2003 until 2012, we find that the average time to graduation for students enrolled full-time in a first bachelor degree - the average scenario on which we expect prospective students to form expectations - is stable over time, ranging from between 4.25 up to 5 years across majors and type of institution. Students enrolled in Universities of Applied Sciences take on average 4.5 years to graduate from their first bachelor degree irrespective of whether they study in STEM or non-STEM majors, while students enrolled in research universities take on average 4.25 years if enrolled in non-STEM majors and 5 years if enrolled in STEM majors. Thus, diverging expectations about the psychic costs of studying across tracks and the natural following type of studies are unlikely to be main channel driving our results.

\section{Net Direct Out-of-Pocket Cost of Studying}

A concern could be that other direct costs of studying changed coincidentally at the time of reform announcements. Table B.1 shows that any costs associated to attending post-secondary were stable, as well as financial aid amounts. Table B.1 provides amounts of every aspect of financial aid since 2002, and Table B.2 shows that of loan conditions are also stable since 1996.

Interest Rate of Student Loans Loan reimbursement schemes are fixed over the period. The interest rate applied for students reimbursing their student loans varies from year to year, but varies very little (over the entire period, from $0 \%$ to $4 \%$ ), and is consistently below market rates for commercial loans. Over the period, interest rates follow a decreasing trend, from around $4 \%$ before 2008 to less than $1 \%$ from 2013 onward.

Tuition Fees Tuition fees are virtually non-existent in the Dutch education system until only post-secondary education. Parents may give a voluntary contribution to their child's school, e.g. for extra-curricular activities and school outings, but these contributions are negligible. No compulsory tuition can be charge to parents in either primary or secondary school. Tuition fees do, however, exist in post-secondary education and in secondary vocational education, yet these are low, and indexed to inflation, such that they increase at a low and stable rate over time of a few dozens of euros yearly. 
Financial Aid Amounts Student finance is stable until the 2015 reform. Amounts of grants are indexed to inflation and increase at a slow and steady pace. Income thresholds determining eligibility to the means-tested grant are also indexed and increase at the same rate. Eligibility conditions for the various part of student finance are fixed over the period. Overall, the policy announcements are unlikely to have affected students' sorting through changes in students' net cost of studying, as interest rates on student loans, tuition fees, and all other aspects of financial aid but repayment probabilities changed over the time-frame of our study.

Thus, we conclude that the 2010 and 2012 announcements affected students' sorting chiefly through a shock to students' subjective probabilities of repayment of financial aid.

\section{Parental Unemployment and the Great Recession}

A concern could be that we observe students shying away from academic and general education from 2010 onward not because of policy announcements about financial aid, but rather because their parents push them to enroll in vocational education which provides learning paths through paid apprenticeships. This could be the case if parental employment suddenly declined in 2010 and continued throughout. Yet, parental unemployment is mostly stable over the entire course of this study: Figure B.9 shows that fathers of students in our sample are employed in roughly $74 \%$ of cases, and mothers in roughly $67 \%$ of cases. Thus, changes in parental unemployment around the time of the policy announcements we study are unlikely to confound the effects we find. To reduce any remaining concern, we control for parental employment status and earnings in all our specifications. 
Figure B.9: Parental Unemployment Rate in our Estimation Sample, Since 2004

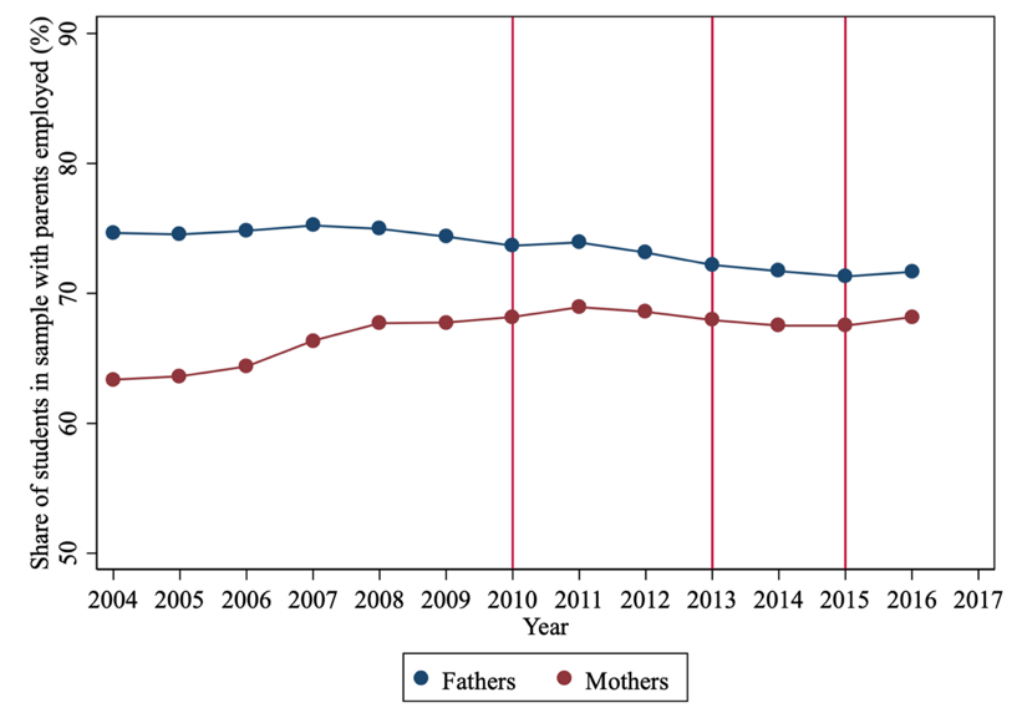

Note: Authors' calculations of the employment rates among parents of students in our main estimation sample. Employment is recorded if the parent was recorded to work within the span of the academic year starting in the respective calendar year. Dutch education register and tax register data, years 2004-2017.

\section{Education Supply and School Capacity Constraints}

Another concern could be that the changes in students' selection into tracks occurs because of underlying changes to the number of available spots in each track in 2010 and onward. School capacity constraints are not an issue in our setting for institutional reasons; Table B.18 provides yearly number of schools at all levels of education.

Over the period, the number of primary schools declined at a steady pace, from 7,500 institutions in 2007 down to 2,750 in 2013. This could suggest a strain on educational resources, if student to teacher ratios increased, but instead the average number of students in each school remains remarkably stable, at about 220 pupils per school over the same period. Instead, cohorts are getting smaller over the period, which would eventually result in smaller cohorts of secondary school students in subsequent years - thus freeing spots in every track and making it unlikely for potential capacity constraints to start binding for later cohorts. Smaller cohorts over time can become a concern if 1) later cohorts become smaller at the time of policy announcements, and 2) if the "missing students" were those who would have otherwise chosen to study in the academic and general tracks. We see a steady decline in cohort size, with no trend break in 2010, suggesting that this is not concern a likely potential 
confound for our effects.

The supply of education in secondary school provide further answers. The number of secondary schools is almost fixed over 2007 to 2013. The distribution of these schools by type of track offer is also fixed, with a stable $50 \%$ of these offering all three tracks to students, and $75 \%$ offering at least two tracks out of three. Of the remaining $25 \%$ of institutions, about $4 \%$ offers the general track only, $6 \%$ the academic track only, and $15 \%$ offers the vocational track only. One could worry that the distribution of students across these school changes as a result of the changing size of cohorts, yet this distribution is also remarkably stable, with almost $75 \%$ of students attending an institution offering all tracks. The average number of pupils per institution is, however, increasing, from approximately 1,400 students in 2007 to 1,460 in 2013 - this increase represents approximately an increase of 2 classes over the time-frame. These facts together reinforce our finding that capacity constraints are not concerning in the Dutch education system, as the cohort size variation seems to be distributed equally across the various types of institutions, as shown by the stable shares of students across the different types of schools and the stable number of schools of each type.

Lastly, the number of Universities (15) and Universities of Applied Sciences (40) is stable over the period of observation, as well as the number of vocational institutions (60). This stability indicates no openings or closings of post-secondary education institutions, thus leaving unchanged students' proximity to post-secondary education institutions over the time-frame of this study. Consequently, one should not be concerned that students' expectations regarding the possibility of attending postsecondary education should change over the period, lest for the reform information shocks that we study in this paper.

\section{Changes to School Funding Rules, Selection Rules, Education Policies}

Apart from the reform of student finance, the Netherlands was not subject to any other major reforms of either primary, secondary or tertiary education over the period of observation. The funding mechanisms for secondary and tertiary schools have not changed since the 1963 Mammoth Law of Secondary Education. The Mammoth Law develops the principle of "Free School Choice" ensures that students (and their parents) can choose any school of their liking. For all schools, the central government allocates budgets to schools according to the number of students enrolled as of October 1st. Schools that specialize in one type of track can, however, deny enrollment to students whose recommendation and CITO score 
does not match the school's track - yet this concerns only a small minority of school (around $5 \%$ of institutions, for about 2 to $3 \%$ of the student population see Table B.18). Thus, students' high school choice should not, in principle, be affected by capacity constraints. Similarly, application processes to secondary and post-secondary institutions are fixed over our time frame, as well as requirements for tertiary majors in the vast majority of programs. In a similar vein, the number of selective majors and their admission criteria are also stable over the period.

We found little evidence of other policies that could explain away our results. Two policy discussions required careful analysis. The first concern is a debate regarding the relative importance of CITO and of teacher recommendations in guiding students ending primary school towards their preferred track in secondary school. This discussion could be concerning if it changed trends in 2010 towards academic and general tracks. This debate, however, is not too concerning as it was a longstanding debate in the Dutch education system, which eventually led to a reform in 2014, with no specific highlights in 2010 that could explain trend breaks in enrollment in tracks, and even more strikingly dropout rates in 2010.

The second policy discussion that could have been concerning is a discussion fostered by the European Commission since 2004 regarding STEM fields, which the objective to improve regional economic integration. This policy discussion could be concerning if it created an underlying trend toward STEM fields. It is, however, not so concerning for two main reasons. First, although the discussion at the European level led to the creation of national agencies which role was to inform and incentivize students to study STEM topics, the Dutch agency Platform Beta-Techniek mostly used small-scale information interventions until 2012. Only in 2018 has this agency merged with industry lobbies to increase its impact. An exploration of Google Searches of terms associated to this discussion shows no sign that this was a wide concern for public opinion (not presented in this chapter).

\section{Broader Trends}

\section{Trends in Scores and Teacher Recommendations Exiting Primary School}

A concern could be changing underlying patterns of unobserved heterogeneity driving results on enrollments in tracks in Grade 10. Cohorts of students could change over time in their academic ability. The concern is that, the school system being comparable over time, students' ability is declining over time, such that fewer students enrolling in academic and general education reflects not a response to policy announcements but rather unobserved changes in the ability distribution in the 
student population.

Figure B.10: Teacher Track Recommendations over Time
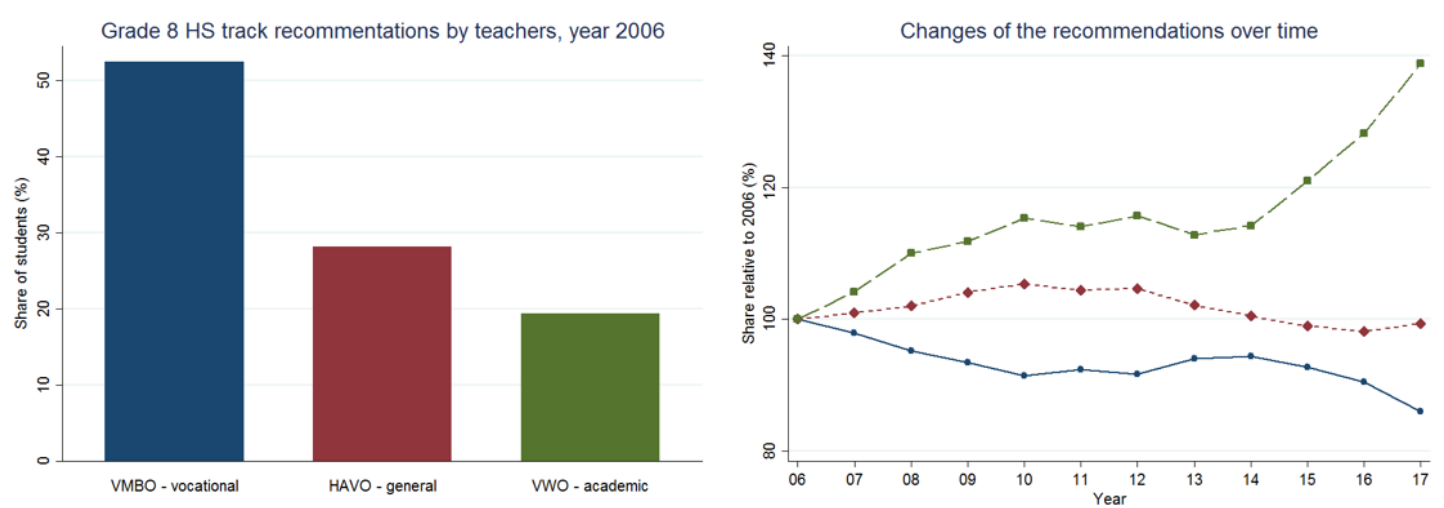

$\Longrightarrow$ VMBO - vocational $\cdots$ HAVO - general $\longrightarrow$ - WWO - academic

Note: Authors' calculations of teacher track recommendation trends for students in our main estimation sample. Track recommendations are recorded with CITO test score information each calendar year over our time-frame. Dutch education register, years 2006-201\%.

Figure B.11: CITO-Based Track Recommendations over Time
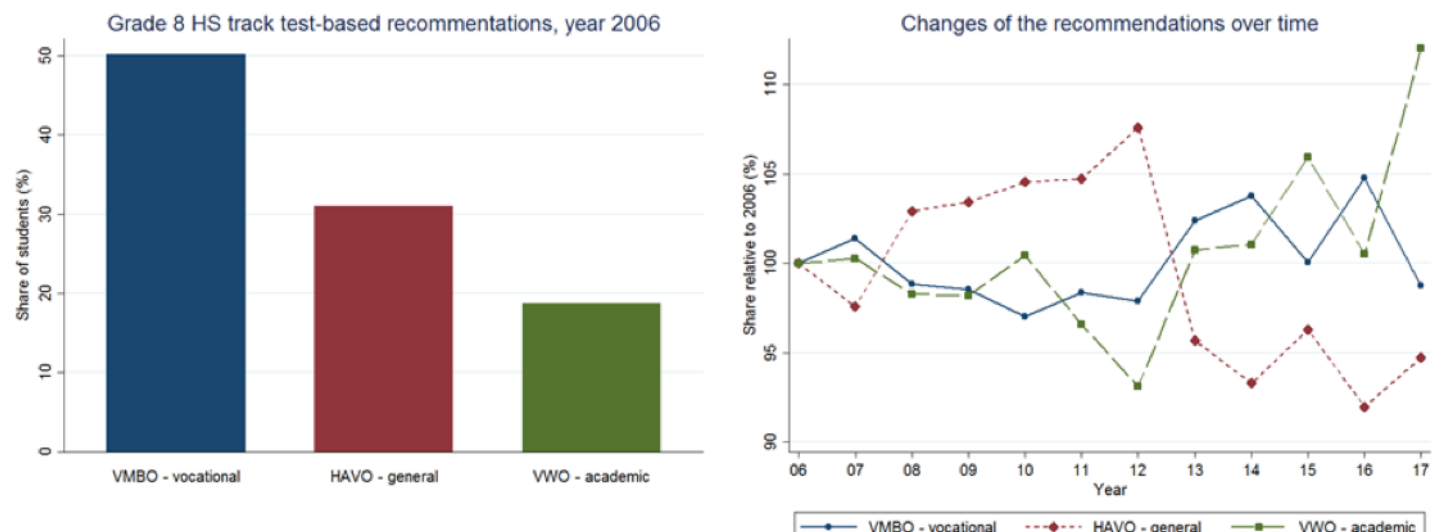

Note: Authors' calculations of CITO-based track recommendation trends for students in our main estimation sample. CITO test score results are recorded each calendar year over our time-frame. For each child with a CITO score, we construct a CITO-based track recommendation using the publicly available threshold in CITO scores recommended by the CITOGroep (see Appendix B.1 for exact thresholds). Dutch education register, years 2006-201\%.

We perform two types of checks: i) we check whether trends changed in the track recommendations that students receive at the end of primary school (Figure B.10), and ii) we check whether there are changes in the track recommendations that students would receive only based on their CITO scores, that is, whether students get worse CITO scores over time (Figure B.11). Track recommendations are given in the last year of primary school (Grade 6), and are available from the academic year 2005-2006 which implies that we do not observe the recommendations and test 
scores for the first four cohorts of students in our main dataset of Grade 10 students (these are the students who started Grade 6 in years 2001 to 2004).

Figure B.10 shows the initial distribution of teacher recommendations in year 2006 and the changes of the distribution over the period of observation, taking the 2006 levels as the starting point. The initial distribution of teacher's recommendations shown in the first panel is remarkably close to the distribution of actual Grade 10 track shares in 2005. The second panel shows that teachers' recommendations have been changing over time, yet in the opposite direction as would be consistent with a general decline in ability. Over our period, cohorts exiting primary school in 2007 to 2011 - whose final track assignment takes place between 2010 and 2014, during our policy announcements - received on average better track recommendations than earlier cohorts, yet these students end up choosing tracks that are lower than earlier cohorts - a pattern that goes against the hypothesis that teacher recommendations explaining resorting to lower tracks.

Figure B.11 shows high school recommendations over time as based on the CITO test scores directly, using the recommendations cut-offs recommended by the CITOGroep. If the test was not designed to be comparable across years, one could consider testbased recommendations to be noisier as scores could in principle be influenced by year-to-year variation in the difficulty of the CITO test. The design of the test insures against this concern. Recommendations based on CITO scores are consistent over time, thus changing students' abilities over time do not appear as a serious potential explanation for the trend reversal in high school track choice that we observe in 2010. However, the trend in test-based recommendations shows a break in 2013: fewer students achieve scores in the middle of the distribution. The cohort of pupils exiting primary school in 2013 differed from the 2012 cohort in that they were informed about the specific contents and the timeline of the financial aid reform. In the absence of any other change in 2012 regarding the test structure, we interpret this break as revealing a change in effort provision of students after the 2012 announcement. 
Table B.18: Number of Schools, Pupils, and Education Expenditures in the Netherlands

\begin{tabular}{lccccccc}
\hline $\begin{array}{l}\text { Academic year: } \\
\text { Panel A. Number of schools and institutions }\end{array}$ & & & & 2007 & 2013 \\
Elementary & 7,537 & 7,528 & 7,515 & 7,480 & 7,434 & 7,360 & 7,261 \\
Secondary & 645 & 647 & 644 & 646 & 646 & 645 & 645 \\
Vocational (MBO) & 61 & 60 & 59 & 58 & 57 & 57 & 57 \\
Uni. Applied Sciences (HBO) & 37 & 36 & 36 & 35 & 35 & 35 & 34 \\
University (WO) & 12 & 12 & 12 & 12 & 12 & 12 & 12
\end{tabular}

Panel B. Average number of students per institution, by level of education

\begin{tabular}{lccccccc}
\hline Elementary & 220 & 221 & 221 & 220 & 219 & 219 & 218 \\
Secondary & 1,404 & 1,391 & 1,401 & 1,406 & 1,421 & 1,440 & 1,458 \\
Vocational (MBO) & 7,821 & 7,994 & 8,239 & 8,438 & 8,396 & 8,269 & 8,195 \\
Uni. Applied Sciences (HBO) & 9,888 & 10,413 & 10,942 & 11,629 & 11,835 & 11,769 & 12,639 \\
University (WO) & 17,222 & 17,833 & 18,842 & 19,528 & 19,736 & 19,369 & 20,010
\end{tabular}

Panel C. Cumulative distribution of secondary school types by track offered (in \%)

\begin{tabular}{lccccccc}
\hline All tracks & 47 & 46 & 45 & 46 & 45 & 45 & 45 \\
Academic/General & 68 & 67 & 66 & 67 & 66 & 66 & 66 \\
General/pre-voc. & 73 & 73 & 72 & 73 & 72 & 72 & 72 \\
Academic & 79 & 79 & 78 & 79 & 78 & 78 & 79 \\
General & 81 & 81 & 80 & 81 & 81 & 81 & 82 \\
Pre-vocational & 100 & 100 & 100 & 100 & 100 & 100 & 100
\end{tabular}

Panel D. Cumulative distribution of secondary school students by school type (in \%)

\begin{tabular}{lccccccc}
\hline All tracks & 71 & 71 & 72 & 71 & 71 & 71 & 71 \\
Academic/General & 90 & 90 & 91 & 90 & 90 & 90 & 90 \\
General/pre-voc. & 93 & 93 & 94 & 93 & 93 & 93 & 93 \\
Academic & 96 & 96 & 97 & 96 & 96 & 96 & 97 \\
General & 97 & 97 & 98 & 97 & 97 & 97 & 98 \\
Pre-vocational & 100 & 100 & 100 & 100 & 100 & 100 & 100
\end{tabular}

Panel E. Cumulative distribution of education expenditures by education level (in \%)

\begin{tabular}{lccccccc}
\hline Elementary & 32.3 & 33.3 & 34.3 & 35.3 & 36.3 & 37.3 & 38.3 \\
Secondary & 55.0 & 54.8 & 56.0 & 55.6 & 55.0 & 56.0 & 56.2 \\
Voc./adult education & 67.1 & 66.6 & 68.0 & 67.5 & 66.6 & 67.6 & 67.4 \\
Uni. Applied Sciences (HBO) & 74.8 & 74.3 & 75.9 & 75.9 & 75.0 & 76.0 & 75.7 \\
University (WO) & 88.1 & 87.3 & 88.9 & 88.9 & 88.1 & 89.3 & 88.7 \\
Student Finance & 100 & 100 & 100 & 100 & 100 & 100 & 100 \\
\hline
\end{tabular}

Note: Source Rijksoverheid and Ministry of Education, Education Statistical yearbooks (2007-2013). 


\section{Macro-Trends in Europe Regarding University-Preparing Education and}

STEM Fields We show that the dynamics of educational choices in the Netherlands are unlikely to be driven by changes of macroeconomic conditions and other international trends. Figure B.12 shows academic high school enrollments in the Netherlands compared to neighboring European countries (Belgium (Wallonia and Flanders), Denmark, France, Germany, and Norway). These countries 1) are in the proximity of the Netherlands, 2) use comparable systems of secondary education, and 3) publish yearly statistics of high school track enrollments since 2005 at least, which we used to construct this figure. The figure plots the trend in the Netherlands against the country-specific trends indicated by grey lines, and their arithmetic average indicated by the dashed line. From the figure, we see that the dynamics of education choices in the Netherlands clearly deviate from the trends in comparison countries. In most countries, enrollments into academic high schools gradually rose over time, following sustained linear trajectories. None of the countries recorded a trend reversal similar to the one we observe in the Netherlands in 2010-2015. This gives us confidence that the results presented in our paper are not an artifact of broader changes of socio-economic and geo-political conditions in western Europe.

Figure B.12: European Trends Towards Vocational Education over Time

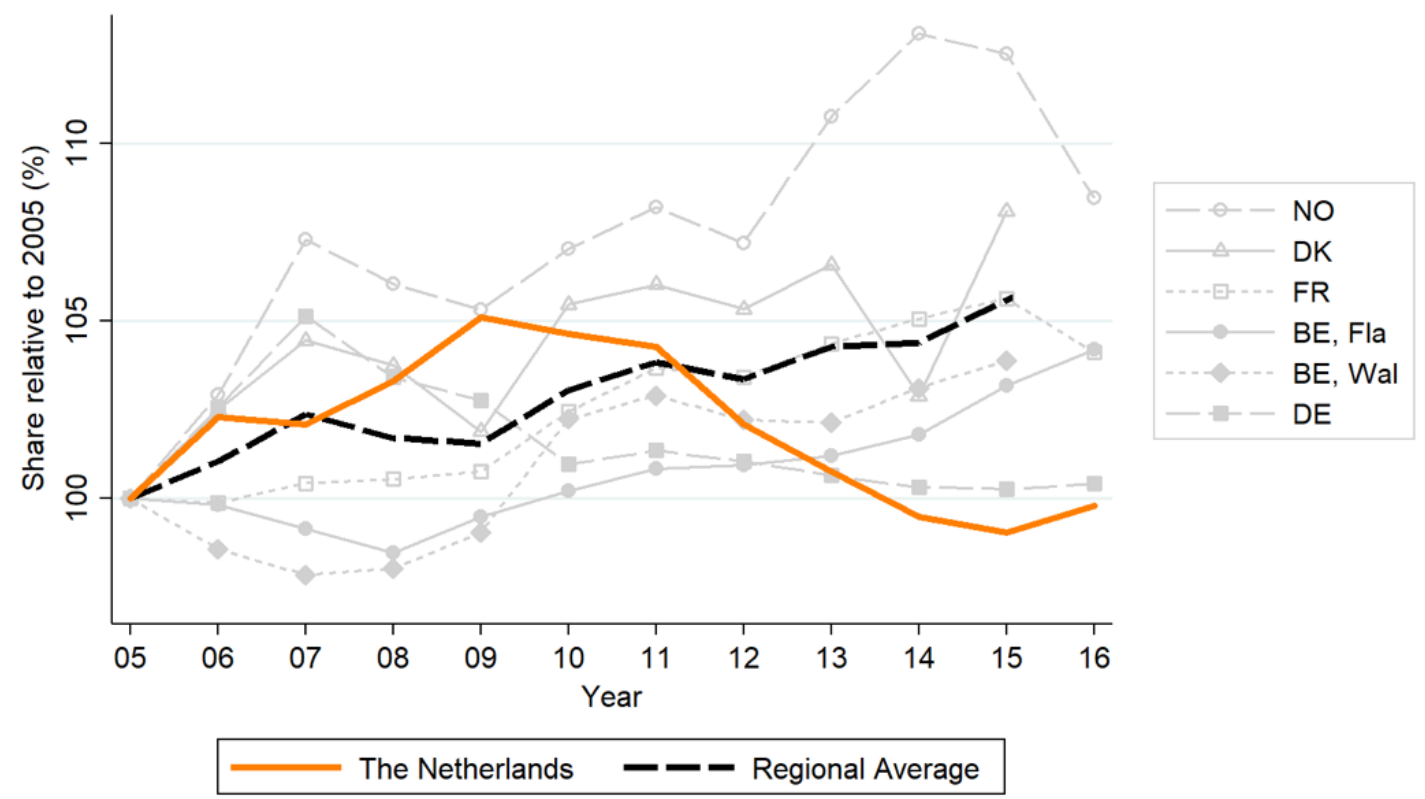

Note: This figure is based on statistical yearbooks and online publicly available aggregate administrative data of Norway, Denmark, France, Belgium (Flanders and Wallonia), Germany. 
Figure B.13: European Trends Towards STEM Fields in Tertiary Education over Time

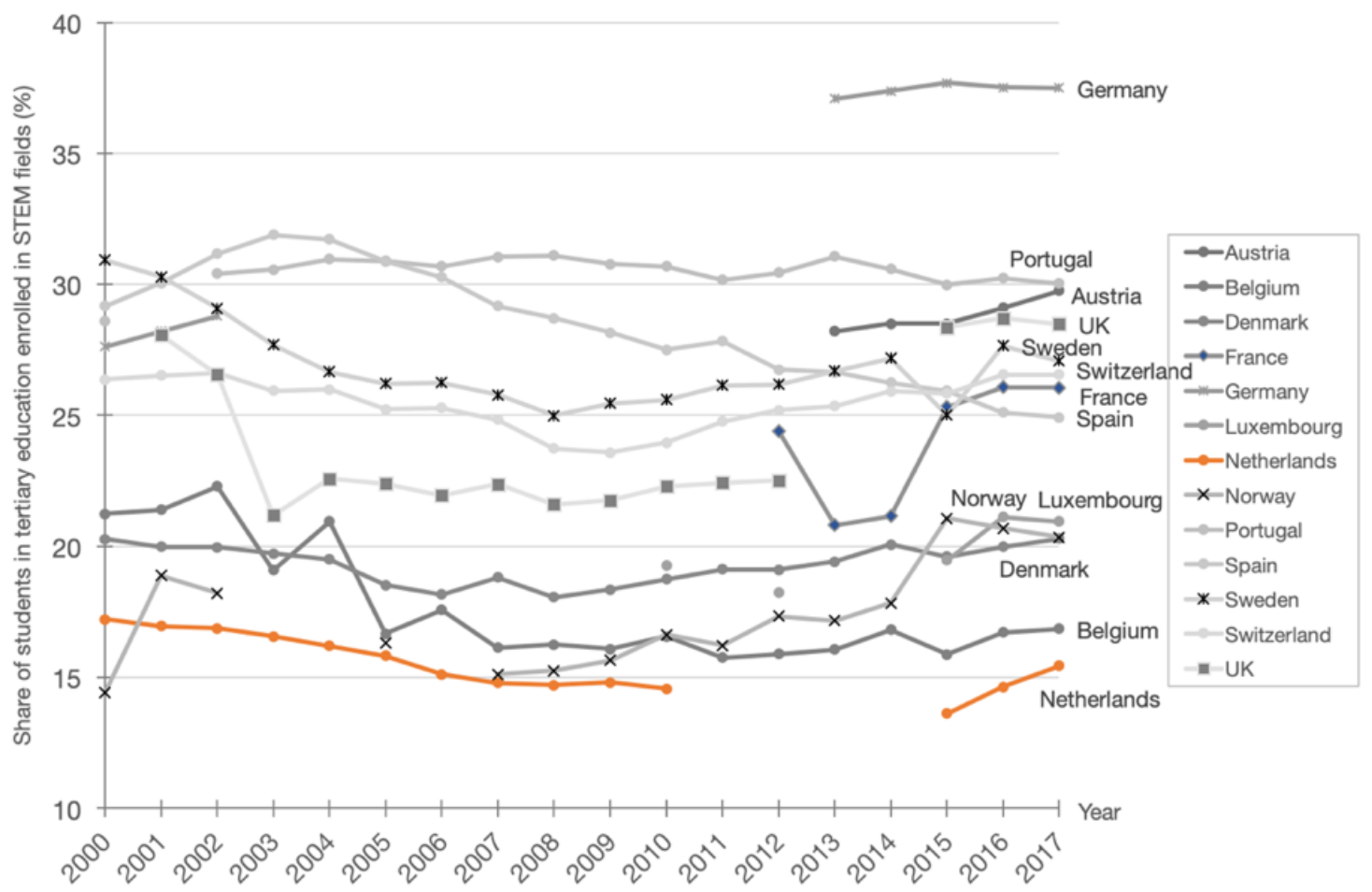

Note: This figure is based on Unesco UIS online data. Own calculations aggregating percentage of students in tertiary education enrolled in three fields of study: 1) Engineering, Manufacturing and Construction programs, 2) Information and Communication Technologies programs, and 3) Natural Sciences, Mathematics and Statistics programs. Both sexes. Data extracted on 22 Oct 2019 03:57 UTC (GMT) from UIS.Stat. 


\section{C $\quad$ Appendix to Chapter 3}

\section{C.1 Construction of Standardized Scales in TEPS}

We summarize the wealth of data available in TEPS into standardized summary indices using commonly used data reduction methods. We proceed as following:

1. Compute Spearman correlation of all potential variables in the factor to construct: eliminate very low correlates; Run preliminary PCA on remaining variables

2. Count number of missing values by individual across variables

3. Standardize each variable, construct preliminary index as row-mean across standardized variables

4. Cut preliminary index into deciles: construct bins of similar input

5. For each variable, construct median within index decile among people used for imputation. If missing item and less than $1 / 3$ missing, replace missing value by median within index decile.

6. Re-run PCA now using variables with imputed values, to check visually that factor with and without imputed values have same distribution

In the long table below, we report for each index we use:

1. the items used and their respondent (Teacher, Student or Parents),

2. the initial number of observations for each of these items separately,

3. PCA factor loadings before and after imputation,

4. the number of observations for the factor before and after imputation,

5. the eigenvalue of the first and second factors before and after imputation,

Factor for which no imputation has been performed are indicated by blanks for factor loadings after imputation, observations after imputation and eigenvalue of first factor after imputation. 
Table C.1: Construction of Standardized Scales of Educational Inputs in TEPS

Scale and Survey items used in scale

Factor loadings

Obs. Resp. Original Imputed

$(1)$

$(2)$

(3)

(4)

Effort wave 1

Chinese teacher's assessment of student effort in class

$\begin{array}{cccc}18,508 & \mathrm{~T} & 0.75 & 0.76 \\ 17,961 & \mathrm{~T} & 0.73 & 0.75 \\ 18,126 & \mathrm{~T} & 0.71 & 0.72 \\ 18,571 & \mathrm{~T} & 0.62 & 0.63 \\ 18,627 & \mathrm{~T} & 0.70 & 0.71 \\ 18,233 & \mathrm{~T} & 0.67 & 0.68 \\ 18,394 & \mathrm{~T} & 0.65 & 0.66 \\ & & 16,004 & 19,231 \\ & & 3.35 & 3.46 \\ & & 0.16 & 0.14\end{array}$

English teacher's assessment of student effort in class

Math teacher's assessment of student effort in class

\begin{tabular}{lcccc} 
Chinese teacher's report always completes homework on time 18,627 & $\mathrm{~T}$ & 0.70 & 0.71 \\
English teacher's report always completes homework on time 18,233 & $\mathrm{~T}$ & 0.67 & 0.68 \\
Math teacher's report always completes homework on time & 18,394 & $\mathrm{~T}$ & 0.65 & 0.66 \\
Factor observations & & & 16,004 & 19,231 \\
First factor eigenvalue & & & 3.35 & 3.46 \\
Second factor eigenvalue & & 0.16 & 0.14 \\
\hline
\end{tabular}

Effort wave 2

Chinese teacher's assessment of student effort in class

English teacher's assessment of student effort in class

$17,120 \quad \mathrm{~T}$

0.78

0.79

Math teacher's assessment of student effort in class

$16,509 \mathrm{~T}$

0.76

0.77

$16,612 \mathrm{~T}$

0.74

0.76

Dao Shi report student always completes homework on time $17,161 \mathrm{~T}$

Chinese teacher's report always completes homework on time 17,107 T

0.71

0.72

English teacher's report always completes homework on time 16,657

Math teacher's report always completes homework on time

16,698

0.68

0.69

Factor observations

First factor eigenvalue

$0.63 \quad 0.65$

$\begin{array}{lll}\mathrm{T} & 0.63 & 0.65 \\ \mathrm{~T} & 0.62 & 0.64\end{array}$

$14,251 \quad 17,950$

Second factor eigenvalue

$3.48 \quad 3.62$

$0.13 \quad 0.11$

Mental health wave 1

How often feeling down or frustrated

How often feeling troubled, worried

How often want to scream or smash something

How often feeling body shaking, unable to focus

How often feeling lonely

How often hopeless

Factor observations

First factor eigenvalue

Second factor eigenvalue

$\begin{array}{cccc}19,781 & \mathrm{~S} & 0.74 & 0.74 \\ 19,877 & \mathrm{~S} & 0.74 & 0.73 \\ 19,854 & \mathrm{~S} & 0.64 & 0.64 \\ 19,839 & \mathrm{~S} & 0.68 & 0.68 \\ 19,793 & \mathrm{~S} & 0.76 & 0.76 \\ 19,856 & \mathrm{~S} & 0.75 & 0.75 \\ & & 19,493 & 19,934 \\ & & 3.09 & 3.09 \\ & & 0.33 & 0.32\end{array}$

Mental health wave 2

How often feeling down or frustrated

How often want to scream or smash something

How often feeling body shaking, unable to focus

How often feeling lonely

How often feeling that you have bad fortune

How often feeling easily irritated by others

How often guilty, regret over some things

Factor observations

First factor eigenvalue

Second factor eigenvalue

$\begin{array}{llcc}18,716 & \mathrm{~S} & 0.71 & 0.71 \\ 18,712 & \mathrm{~S} & 0.67 & 0.67 \\ 18,695 & \mathrm{~S} & 0.62 & 0.62 \\ 18,676 & \mathrm{~S} & 0.64 & 0.64 \\ 18,658 & \mathrm{~S} & 0.59 & 0.59 \\ 18,682 & \mathrm{~S} & 0.62 & 0.62 \\ 18,654 & \mathrm{~S} & 0.58 & 0.58 \\ & & 18,355 & 18,782 \\ & & 2.82 & 2.83 \\ & & 0.24 & 0.24\end{array}$

Note: This table presents detailed factor loadings and number of observations used in the construction of our summative scales as imputation procedure. Col. (1) reports the initial number of complete observations available, Col. (2) indicates whether teachers (T), parents (P) or students (S) respond to each item, Cols. (3) and (4) report factor loadings on the first factor respectively before and after imputation. See Appendix C.1 for details about our imputation procedure. 
Table C.1: Construction of Standardized Scales of Educational Inputs in TEPS (continued)

Scale and Survey items used in scale

Factor loadings

Obs. Resp. Original Imputed

(1) (2) (3) (4)

Truancy wave 1

How often cutting or skipping classes

How often physical fights or quarrels with teachers

How often watching porn

How often substance abuse (tobacco, alcohol, drugs)

How often running away from home

How often stealing or destroying others' property

Factor observations

First factor eigenvalue

Second factor eigenvalue

\begin{tabular}{cccc}
19,846 & $\mathrm{~S}$ & 0.70 & 0.70 \\
19,790 & $\mathrm{~S}$ & 0.61 & 0.6 \\
19,867 & $\mathrm{~S}$ & 0.65 & 0.64 \\
19,865 & $\mathrm{~S}$ & 0.74 & 0.73 \\
19,880 & $\mathrm{~S}$ & 0.73 & 0.73 \\
19,862 & $\mathrm{~S}$ & 0.68 & 0.67 \\
& & 19,614 & 19,929 \\
& & 2.83 & 2.77 \\
& & -0.002 & -0.01 \\
\hline
\end{tabular}

\begin{tabular}{lcccc}
\hline Truancy wave 2 & & & & \\
\hline How often cutting or skipping classes & 18,718 & $\mathrm{~S}$ & 0.51 & 0.52 \\
How often physical fights or quarrels with teachers & 18,737 & $\mathrm{~S}$ & 0.59 & 0.59 \\
How often watching porn & 18,729 & $\mathrm{~S}$ & 0.42 & 0.42 \\
Factor observations & & & 18,611 & 18,799 \\
First factor eigenvalue & & & 0.78 & 0.79 \\
Second factor eigenvalue & & -0.07 & -0.07 \\
\hline
\end{tabular}

Self-efficacy wave 1

I am good at presentations or expressing my points of view

$\begin{array}{cccc}19,749 & \mathrm{~S} & 0.65 & 0.65 \\ 19,800 & \mathrm{~S} & 0.68 & 0.68 \\ 19,810 & \mathrm{~S} & 0.74 & 0.73 \\ 19,798 & \mathrm{~S} & 0.62 & 0.62 \\ 19,758 & \mathrm{~S} & 0.57 & 0.57 \\ 19,847 & \mathrm{~S} & 0.59 & 0.59 \\ 19,808 & \mathrm{~S} & 0.59 & 0.59 \\ & & 19,346 & 19,909 \\ & & 2.83 & 2.83 \\ & & 0.17 & 0.16\end{array}$

I am good at coordinating with other people in a group

I can plan things well no matter how trivial they are

I cooperate with everyone very well

I always come up with solutions to problems

I have always reviewed what I learn since elementary school

I always try to figure out answers whenever have questions

Factor observations

First factor eigenvalue

Second factor eigenvalue

Self-efficacy wave 2

I am good at presentations or expressing my points of view

I am good at coordinating with other people in a group

I can plan things well no matter how trivial they are

I cooperate with everyone very well

$\begin{array}{cccc}18,686 & \mathrm{~S} & 0.54 & 0.53 \\ 18,744 & \mathrm{~S} & 0.58 & 0.58 \\ 18,731 & \mathrm{~S} & 0.65 & 0.64 \\ 18,709 & \mathrm{~S} & 0.55 & 0.54 \\ 18,708 & \mathrm{~S} & 0.62 & 0.62 \\ 18,606 & \mathrm{~S} & 0.54 & 0.54 \\ & & 18,384 & 18,795 \\ & & 2.02 & 2.01 \\ & & 0.05 & 0.05\end{array}$

$\begin{array}{lllll}\text { I always come up with solutions to problems } & 18,708 & \mathrm{~S} & 0.62 & 0.62\end{array}$

My friends think of me as a person who always has lots of ideas $18,606 \quad \mathrm{~S} \quad 0.54 \quad 0.54$

Factor observations

First factor eigenvalue

$0.05 \quad 0.05$

\begin{tabular}{lcc} 
Second factor eigenvalue & 0.05 & 0.05 \\
\hline Note: This table presents detailed factor loadings and number of observations used in the construction
\end{tabular} of our summative scales as imputation procedure. Col. (1) reports the initial number of complete observations available, Col. (2) indicates whether teachers (T), parents (P) or students (S) respond to each item, Cols. (3) and (4) report factor loadings on the first factor respectively before and after imputation. See Appendix C.1 for details about our imputation procedure. 
Table C.1: Construction of Standardized Scales of Educational Inputs in TEPS (continued)

Scale and Survey items used in scale

Factor loadings

Obs. Resp. Original Imputed
(1)
(2)
(3)
(4)

Initiative in class wave 1

Chinese teacher report student initiative to participate in class 18,635

English teacher report student initiative to participate in class $18,307 \mathrm{~T}$

Math teacher report student initiative to participate in class $\quad 18,367 \quad \mathrm{~T}$

Factor observations

First factor eigenvalue

Second factor eigenvalue

$\begin{array}{cc}0.52 & 0.53 \\ 0.52 & 0.54 \\ 0.52 & 0.54 \\ 17112 & 19219 \\ 0.81 & 0.86 \\ -0.16 & -0.16\end{array}$

Initiative in class wave 2

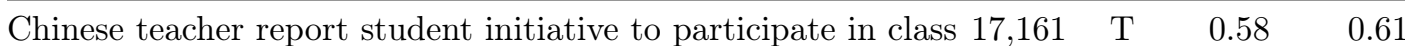

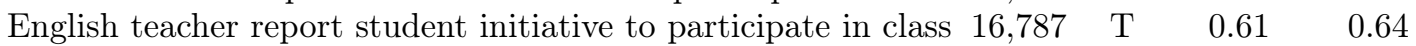

Math teacher report student initiative to participate in class $\quad \begin{array}{llll}16,698 & \mathrm{~T} & 0.59 & 0.62\end{array}$

Factor observations

$15,426 \quad 17,791$

First factor eigenvalue

$1.06 \quad 1.16$

Second factor eigenvalue

$-0.16-0.16$

\begin{tabular}{|c|c|c|c|c|}
\hline \multicolumn{5}{|l|}{ Money wave 1} \\
\hline Hours per week spent on tutoring in/outside school & 19,851 & $\mathrm{P}$ & 0.60 & 0.60 \\
\hline Amount paid for this child's tutoring classes & 19,710 & $\mathrm{P}$ & 0.60 & 0.60 \\
\hline Factor observations & & & 19,573 & 19,988 \\
\hline First factor eigenvalue & & & 0.71 & 0.73 \\
\hline Second factor eigenvalue & & & -0.25 & -0.25 \\
\hline \multicolumn{5}{|l|}{ Money wave 2} \\
\hline Hours per week spent on tutoring outside school & 18,747 & $\mathrm{P}$ & 0.78 & 0.78 \\
\hline Monthly expenditures this semester for this child's tutoring & 18,755 & $\mathrm{P}$ & 0.78 & 0.78 \\
\hline Factor observations & & & 18,586 & 18,916 \\
\hline First factor eigenvalue & & & 1.21 & 1.22 \\
\hline Second factor eigenvalue & & & -0.21 & -0.20 \\
\hline \multicolumn{5}{|l|}{ Time wave 1} \\
\hline How often parents go to bookstores or expos with child & 19,750 & $\mathrm{P}$ & 0.53 & 0.53 \\
\hline How often parents go to concerts or performances with child & 19,750 & $\mathrm{P}$ & 0.53 & 0.53 \\
\hline Factor observations & & & 19,743 & 19,757 \\
\hline First factor eigenvalue & & & 0.55 & 0.55 \\
\hline Second factor eigenvalue & & & -0.24 & -0.24 \\
\hline \multicolumn{5}{|l|}{ Time wave 2} \\
\hline Weekly number of dinners with the child & 18,783 & $\mathrm{P}$ & 0.44 & 0.45 \\
\hline Spouse: Weekly number of dinners with the child & 18,493 & $\mathrm{P}$ & 0.44 & 0.45 \\
\hline Factor observations & & & 18,457 & 18,819 \\
\hline First factor eigenvalue & & & 0.39 & 0.41 \\
\hline Second factor eigenvalue & & & -0.21 & -0.21 \\
\hline
\end{tabular}

Note: This table presents detailed factor loadings and number of observations used in the construction of our summative scales as imputation procedure. Col. (1) reports the initial number of complete observations available, Col. (2) indicates whether teachers (T), parents (P) or students (S) respond to each item, Cols. (3) and (4) report factor loadings on the first factor respectively before and after imputation. See Appendix C.1 for details about our imputation procedure. 
Table C.1: Construction of Standardized Scales of Educational Inputs in TEPS (continued)

Scale and Survey items used in scale

Factor loadings

Obs. Resp. Original Imputed

\begin{tabular}{lll}
$(1) \quad(2) \quad(3) \quad(4)$ \\
\hline
\end{tabular}

\begin{tabular}{lcccc}
\hline Parent strictness wave 1 & & & \\
\hline My father is strict & 19,851 & $\mathrm{~S}$ & 0.51 & 0.51 \\
My mother is strict & 19,842 & $\mathrm{~S}$ & 0.51 & 0.51 \\
Factor observations & & 19,739 & 19,928 \\
First factor eigenvalue & & 0.52 & 0.53 \\
Second factor eigenvalue & & -0.23 & -0.24 \\
\hline
\end{tabular}

Parent strictness wave 2

\begin{tabular}{llllll}
\hline How many of your parents set strict rules for your daily routine? & 18,828 & $\mathrm{~S}$ & 0.61 & 0.61
\end{tabular}

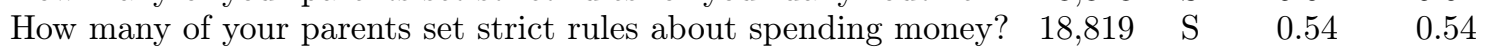

$\begin{array}{llllll}\text { How many of your parents set strict rules about demeanor? } & 18,806 & \text { S } & 0.63 & 0.63\end{array}$

$\begin{array}{llllll}\text { How many of your parents set strict rules about health habits? } & 18,731 & \mathrm{~S} & 0.60 & 0.60\end{array}$

$\begin{array}{llllll}\text { How many of your parents set strict rules about making friends? } & 18,821 & \mathrm{~S} & 0.57 & 0.57\end{array}$

How many of your parents uses guilt and emotional blackmail? $\quad \begin{array}{llllll}18,821 & \mathrm{~S} & 0.51 & 0.51\end{array}$

How many of your parents does not allow you to argue with them? 18,816 S $\quad \begin{array}{llll}0.50 & 0.50\end{array}$

$\begin{array}{lllll}\text { How many of your parents discipline you very strictly? } & 18,809 & \mathrm{~S} & 0.53 & 0.53\end{array}$

Factor observations $\quad 18,648 \quad 18,831$

$\begin{array}{lll}\text { First factor eigenvalue } & 2.54 & 2.55\end{array}$

Second factor eigenvalue $\quad 0.15 \quad 0.15$

Parent emotional support wave 1

My father discusses student's future study and career

My father discusses my feelings and thoughts

My mother discusses student's future study and career

My mother discusses my feelings and thoughts

My father accepts me as I am

My mother accepts me as I am

My family provides strong emotional support

In my family, we discuss together important decisions

Factor observations

First factor eigenvalue

Second factor eigenvalue

$\begin{array}{cccc}19,854 & \mathrm{~S} & 0.46 & 0.46 \\ 19,764 & \mathrm{~S} & 0.59 & 0.58 \\ 19,822 & \mathrm{~S} & 0.49 & 0.50 \\ 19,816 & \mathrm{~S} & 0.64 & 0.64 \\ 18,993 & \mathrm{~S} & 0.49 & 0.51 \\ 19,370 & \mathrm{~S} & 0.49 & 0.49 \\ 19,652 & \mathrm{~S} & 0.53 & 0.54 \\ 19,528 & \mathrm{~S} & 0.56 & 0.57 \\ & & 17,729 & 19,973 \\ & & 2.28 & 2.33 \\ & & 0.56 & 0.52\end{array}$

Parent emotional support wave 2

\begin{tabular}{|c|c|c|c|c|}
\hline My parents pay attention to my ideas and thoughts & 18,816 & $\mathrm{~S}$ & 0.66 & 0.66 \\
\hline I seek my parents' help when I encounter difficulties & 18,811 & $\mathrm{~S}$ & 0.67 & 0.67 \\
\hline My parents accept me as I am & 18,799 & $\mathrm{~S}$ & 0.62 & 0.62 \\
\hline Factor observations & & & 18,769 & 18,827 \\
\hline First factor eigenvalue & & & 1.27 & 1.27 \\
\hline Second factor eigenvalue & & & -0.15 & -0.15 \\
\hline
\end{tabular}

Note: This table presents detailed factor loadings and number of observations used in the construction of our summative scales as imputation procedure. Col. (1) reports the initial number of complete observations available, Col. (2) indicates whether teachers (T), parents (P) or students (S) respond to each item, Cols. (3) and (4) report factor loadings on the first factor respectively before and after imputation. See Appendix C.1 for details about our imputation procedure. 
Table C.1: Construction of Standardized Scales of Educational Inputs in TEPS (continued)

Scale and Survey items used in scale

Factor loadings

Obs. Resp. Original Imputed

(1) (2)

(3)

(4)

School environment wave 1

My school is an interesting place

My school is fair in terms of rewards and grading

The campus of my school is safe

My school cares about their students

My school has a great atmosphere for learning

Factor observations

First factor eigenvalue

Second factor eigenvalue

$\begin{array}{cccc}19,513 & \mathrm{~S} & 0.47 & 0.48 \\ 19,557 & \mathrm{~S} & 0.54 & 0.55 \\ 19,567 & \mathrm{~S} & 0.63 & 0.63 \\ 19,481 & \mathrm{~S} & 0.71 & 0.71 \\ 19,456 & \mathrm{~S} & 0.64 & 0.65 \\ & & 18,701 & 19,903 \\ & & 1.83 & 1.86 \\ & & -0.006 & -0.013\end{array}$

School environment wave 2

My school's requirements on students are quite reasonable

$\begin{array}{cccc}18,614 & \mathrm{~S} & 0.39 & 0.39 \\ 18,741 & \mathrm{~S} & 0.46 & 0.46 \\ 18,709 & \mathrm{~S} & 0.56 & 0.56 \\ 18,340 & \mathrm{~S} & 0.62 & 0.62 \\ 18,690 & \mathrm{~S} & 0.52 & 0.52 \\ & & 18,053 & 18,814 \\ & & 1.33 & 1.34 \\ & & 0.015 & 0.013\end{array}$

My school is fair in terms of rewards and grading

The campus of my school is safe

My school cares about their students

My school has a great atmosphere for learning

Factor observations

First factor eigenvalue

Second factor eigenvalue

$0.015 \quad 0.013$

Teacher engagement in class wave 1

How many of my teachers know the name of every student

How many teachers encourage student when they study hard

How many teachers use different teaching methods/materials

How many teachers give homework to increase students'

chance to practice

How many teachers ask reasons when students fail homework

How many teachers give a review after every exam

Factor observations

First factor eigenvalue

$\begin{array}{cccc}19,865 & \mathrm{~S} & 0.38 & 0.39 \\ 19,780 & \mathrm{~S} & 0.48 & 0.48 \\ 19,846 & \mathrm{~S} & 0.55 & 0.55 \\ 19,836 & \mathrm{~S} & 0.48 & 0.49 \\ & & & \\ 19,812 & \mathrm{~S} & 0.46 & 0.48 \\ 19,604 & \mathrm{~S} & 0.48 & 0.49 \\ & & 19,210 & 19,953 \\ & & 1.35 & 1.4 \\ & & 0.11 & 0.11\end{array}$

Second factor eigenvalue

$\begin{array}{cccc}18,795 & \mathrm{~S} & 0.70 & 0.70 \\ 18,784 & \mathrm{~S} & 0.73 & 0.73 \\ 18,783 & \mathrm{~S} & 0.62 & 0.62 \\ 18,772 & \mathrm{~S} & 0.62 & 0.62 \\ 18,795 & \mathrm{~S} & 0.53 & 0.53 \\ & & & \\ 18,784 & \mathrm{~S} & 0.45 & 0.45 \\ 18,744 & \mathrm{~S} & 0.53 & 0.53 \\ & & 18,590 & 18,820 \\ & & 2.56 & 2.56 \\ & & 0.17 & 0.17\end{array}$

Teacher engagement in class wave 2

class

How many teachers often discuss life goals, do career advice

How many teachers often recommend books, encourage reading

How many teachers often use real life and practical examples

How many teachers take free time to help students with personal issues

How many teachers often use guilt or emotional blackmail

How many teachers praise me when I study hard

Factor observations

First factor eigenvalue

0.17

0.17

Note: This table presents detailed factor loadings and number of observations used in the construction of our summative scales as imputation procedure. Col. (1) reports the initial number of complete observations available, Col. (2) indicates whether teachers (T), parents (P) or students (S) respond to each item, Cols. (3) and (4) report factor loadings on the first factor respectively before and after imputation. See Appendix C.1 for details about our imputation procedure. 


\section{C.2 Additional Tables}

Table C.2: The Effect of Peer Test Scores on Students' Own Test Scores in Wave 2

\begin{tabular}{lcccc}
\hline Outcome: & \multicolumn{4}{c}{ Student test scores in wave 2 [std] } \\
& $(1)$ & $(2)$ & $(3)$ & $(4)$ \\
\cline { 2 - 5 } & & & & \\
Peer test scores [std] & $0.041 * * *$ & $0.047 * * *$ & $0.052 * * *$ & $0.052 * * *$ \\
& $(0.016)$ & $(0.016)$ & $(0.016)$ & $(0.016)$ \\
Own test scores [std] & $0.737 * * *$ & $0.733 * * *$ & $0.589 * * *$ & $0.585 * * *$ \\
& $(0.007)$ & $(0.007)$ & $(0.009)$ & $(0.009)$ \\
& & & & \\
\hline R2 & 0.65 & 0.64 & 0.68 & 0.68 \\
School FE & $\mathrm{Y}$ & $\mathrm{Y}$ & $\mathrm{Y}$ & $\mathrm{Y}$ \\
Balancing controls & & $\mathrm{Y}$ & & $\mathrm{Y}$ \\
W1 inputs & & & $\mathrm{Y}$ & $\mathrm{Y}$ \\
Schools & 232 & 232 & 232 & 232 \\
Classes & 872 & 872 & 850 & 850 \\
Students & 13,086 & 12,173 & 11,702 & 11,029 \\
\hline
\end{tabular}

Note: This table reports estimates of regressing standardized student test scores in wave 2 on standardized average peer test scores in wave 1 in our sample containing 232 schools, 850 classrooms, and up to 11,029 students. Balancing controls include household income, family engagement with homework, gifted art classroom assignment, and parents' efforts to get child assigned to a particular classroom. W1 inputs include standardized scales of student inputs (school effort, initiative in class, truancy, academic self-efficacy, and mental health), parent inputs (investment in private tutoring, time investments, parental strictness and parental support), school and teacher inputs (school environment and teacher engagement). Standard errors clustered at the classroom level in parentheses. Estimates in this figure are also shown in Figure 3.3. 
Table C.3: The Effect of Peer Test Scores on Educational Inputs in Wave 2

\begin{tabular}{lccccc}
\hline Treatment variable: & \multicolumn{2}{c}{ Peer test scores [std] } & & \\
\cline { 2 - 3 } & Coef. & Std. err. & R2 & Classrooms & Students \\
& $(1)$ & $(2)$ & $(3)$ & $(4)$ & $(5)$ \\
\cline { 2 - 4 } & & & & & \\
Outcomes: educational inputs & & & & & \\
School effort [std] & $-0.052 * *$ & $(0.024)$ & 0.56 & 849 & 10659 \\
Initiative in class [std] & -0.015 & $(0.024)$ & 0.46 & 849 & 10558 \\
Truancy [std] & 0.009 & $(0.021)$ & 0.18 & 850 & 11113 \\
Cheated on exams [.48] & 0.015 & $(0.013)$ & 0.12 & 850 & 11078 \\
Academic self-efficacy [std] & -0.017 & $(0.021)$ & 0.15 & 850 & 11113 \\
Mental health [std] & -0.030 & $(0.019)$ & 0.16 & 850 & 11103 \\
University aspirations [.57] & $0.016 *$ & $(0.009)$ & 0.28 & 850 & 11115 \\
University expectations [.44] & $0.020 * *$ & $(0.009)$ & 0.29 & 850 & 11105 \\
Private tutoring [std] & -0.002 & $(0.019)$ & 0.37 & 850 & 11164 \\
Time with parents [std] & $0.081 * * *$ & $(0.023)$ & 0.08 & 850 & 11111 \\
Conflict with parents [.31] & -0.017 & $(0.010)$ & 0.06 & 850 & 11086 \\
Parental strictness [std] & $0.036 *$ & $(0.020)$ & 0.16 & 850 & 11133 \\
Parental support [std] & 0.029 & $(0.020)$ & 0.2 & 850 & 11133 \\
Harsh parenting [.33] & 0.015 & $(0.009)$ & 0.08 & 850 & 11133 \\
Parent uni. aspirations [.51] & 0.001 & $(0.010)$ & 0.33 & 850 & 11022 \\
School environment [std] & -0.029 & $(0.024)$ & 0.17 & 850 & 11126 \\
Classroom hard to manage [.33] & -0.040 & $(0.033)$ & 0.35 & 836 & 10534 \\
Teacher engagement [std] & 0.022 & $(0.023)$ & 0.11 & 850 & 11129 \\
Teacher tired of teaching [.49] & -0.042 & $(0.031)$ & 0.31 & 836 & 10527 \\
\hline
\end{tabular}

Note: This table reports estimates of regressing educational input measures in wave 2 on standardized average peer test scores in wave 1 in our sample containing 232 schools, 850 classrooms, and up to 11,164 students. Rows present results of models with different educational inputs as outcomes. Unconditional means of each outcome are shown in square brackets, and [std] marks outcomes that have been standardized to have a mean of zero and a standard deviation of one. All models control for school fixed effects, student test scores in wave 1, balancing controls, and educational inputs in wave 1. Standard errors clustered at the classroom level in parentheses. Estimates in this table are also shown in Figure 3.4. 
Table C.4: Returns to Educational Inputs from Cumulative Value-Added Models

Outcome: Student test scores in wave 2 [std]

\begin{tabular}{ccccc}
\hline \multicolumn{2}{c}{ Total effect } & & \multicolumn{2}{c}{ Partial effect } \\
\cline { 5 - 6 } \cline { 5 - 6 } Coef. & Std. err. & & Coef. & Std. err. \\
$(1)$ & $(2)$ & & $(3)$ & $(4)$ \\
\hline
\end{tabular}

Treatments:

School effort [std]

Initiative in class [std]

Truancy [std]

Cheated on exams [.48]

$\begin{array}{llll}0.166 * * * & (0.009) \quad 0.098 * * * \quad(0.010)\end{array}$

$0.161 * * * \quad(0.008) \quad 0.109 * * * \quad(0.009)$

$\begin{array}{llll}-0.039 * * * & (0.007) & -0.008 & (0.007)\end{array}$

Academic self-efficacy [std]

$-0.053 * * * \quad(0.011)$

$-0.015$

$(0.012)$

Mental health [std]

$-0.009$

(0.006)

$-0.024 * * *$

$(0.006)$

University aspirations [.57]

0.009

$(0.007)$

$-0.002$

$0.151 * * *$

(0.013)

$0.046 * * *$

$(0.007)$

University expectations [.44]

$0.209 * * *$

$(0.014)$

$(0.014)$

Private tutoring [std]

$0.040 * * *$

$(0.007)$

$0.131 * * *$

$(0.015)$

Time with parents [std]

Conflict with parents [.31]

$-0.003$

$(0.006)$

$0.025 * * *$

$(0.007)$

$0.070 * * *$

$(0.012)$

$-0.004$

$(0.006)$

Parental strictness [std]

Parental support [std]

$-0.028 * * *$

$0.037 * * *$

$(0.013)$

$0.029 * * *$

(0.006)

$-0.039 * * *$

$(0.006)$

Harsh parenting [.33]

Parent uni. aspirations [.51]

School environment [std]

Class hard to manage [.33]

Teacher engagement [std]

$-0.056 * * *$

$(0.006)$

$0.019 * * *$

$(0.007)$

$0.141 * * *$

$(0.011)$

$(0.012)$

$0.026 * * *$

$(0.013)$

$-0.020 *$

$-0.016$

$(0.006)$

$0.076 * * *$

(0.006)

$0.016 * *$

$(0.017)$

0.006

Teacher tired of teaching [.49]

0.024

$-0.01$

(0.006)

R2

(0.016)

0.006

Schools

0.023 0.71

232

850

Students

10,490

\begin{abstract}
Note: This table reports coefficient estimates of regressing student test scores in wave 2 on educational inputs in wave 2 in our estimation sample containing 232 schools, up to 850 classrooms, and up to 11,029 students. Rows present coefficients of different regressors. Unconditional means of each input are shown in square brackets and [std] marks inputs that have been standardized to have a mean of zero and a standard deviation of one. Total effects are estimated one input at the time, whereas partial effects are estimates of all inputs jointly. All models control for school fixed effects, student test scores, average peer test scores, and educational inputs in wave 1. Standard errors clustered at the classroom level in parentheses. These results are also available in Figure 3.5.
\end{abstract}


Table C.5: Academic Peer Effect Mediated by Educational Inputs

\begin{tabular}{|c|c|c|}
\hline \multirow[b]{3}{*}{ Total mediated effect } & \multicolumn{2}{|c|}{ Student test scores in wave } \\
\hline & $\begin{array}{c}\text { Coef. Est. } \\
\text { (1) }\end{array}$ & $\begin{array}{c}\text { Std. err } \\
\text { (2) }\end{array}$ \\
\hline & -0.008 & $(0.005)$ \\
\hline \multicolumn{3}{|l|}{ Mediated effect by: } \\
\hline School effort & $-0.006 * * *$ & $(0.002)$ \\
\hline Initiative in class & -0.001 & $(0.003)$ \\
\hline Truancy & -0.000 & $(0.000)$ \\
\hline Cheated on exams & -0.000 & $(0.000)$ \\
\hline Academic self-efficacy & 0.001 & $(0.001)$ \\
\hline Mental health & 0.000 & $(0.000)$ \\
\hline University aspirations & 0.000 & $(0.000)$ \\
\hline University expectations & 0.001 & $(0.001)$ \\
\hline Private tutoring & 0.000 & $(0.000)$ \\
\hline Time with parents & -0.000 & $(0.000)$ \\
\hline Conflict with parents & -0.000 & $(0.000)$ \\
\hline Parental strictness & -0.001 & $(0.001)$ \\
\hline Parental support & 0.000 & $(0.000)$ \\
\hline Harsh parenting & -0.000 & $(0.000)$ \\
\hline Parent uni. Aspirations & -0.000 & $(0.001)$ \\
\hline School environment & -0.000 & $(0.000)$ \\
\hline Classroom hard to manage & 0.000 & $(0.001)$ \\
\hline Teacher engagement & 0.000 & $(0.000)$ \\
\hline Teacher tired of teaching & -0.001 & $(0.001)$ \\
\hline
\end{tabular}

Note: This table reports the mediated effects based on Gelbach's (2016) decomposition of our academic peer effect estimate using only withinschool variation in our estimation sample containing 232 schools, up to 850 classrooms, and up to 11,029 students. These estimates are produced using a modified version of the $\mathrm{b} 1 \times 2$ Stata package. Rows present the mediated effect of different educational inputs in wave 2. All models control for school fixed effects, student test scores, average peer test scores, and educational inputs in wave 1. Standard errors clustered at the classroom level in parentheses. These results are also available in Figure 3.6. 
Table C.6: Permutation-Based Classroom-Level Sorting Tests in Estimation Sample

\begin{tabular}{|c|c|c|c|c|c|}
\hline & \multirow[b]{2}{*}{$\begin{array}{c}\text { Classrooms } \\
\text { (1) }\end{array}$} & \multicolumn{3}{|c|}{$\begin{array}{c}\text { Share of classes } \\
\text { with empirical } \\
\text { p-val. under }\end{array}$} & \multirow{2}{*}{$\begin{array}{c}\text { Avg. } \\
\text { p-value } \\
(5)\end{array}$} \\
\hline & & $\begin{array}{l}0.10 \\
(2)\end{array}$ & $\begin{array}{l}0.05 \\
(3)\end{array}$ & $\begin{array}{l}0.01 \\
(4)\end{array}$ & \\
\hline \multicolumn{6}{|l|}{ Pre-assignment characteristics: } \\
\hline Student test scores & 853 & 0.10 & 0.06 & 0.02 & 0.486 \\
\hline Female student & 853 & 0.06 & 0.04 & 0.02 & 0.562 \\
\hline Student born before 1989 & 853 & 0.10 & 0.05 & 0.01 & 0.490 \\
\hline Monthly household income $>\mathrm{NT} \$ 100,000$ & 853 & 0.09 & 0.04 & 0.01 & 0.491 \\
\hline College-educated parent(s) & 853 & 0.09 & 0.06 & 0.02 & 0.485 \\
\hline Parent(s) work in government & 853 & 0.08 & 0.04 & 0.01 & 0.487 \\
\hline Ethnic minority parent(s) & 853 & 0.08 & 0.04 & 0.01 & 0.494 \\
\hline \multicolumn{6}{|l|}{ Since primary school: } \\
\hline Student always prioritized studies & 853 & 0.12 & 0.06 & 0.01 & 0.491 \\
\hline Student always reviews lessons & 853 & 0.12 & 0.06 & 0.01 & 0.478 \\
\hline Student likes new things & 853 & 0.13 & 0.07 & 0.02 & 0.465 \\
\hline \multicolumn{6}{|l|}{ During primary school: } \\
\hline Student was truant & 853 & 0.08 & 0.04 & 0.01 & 0.498 \\
\hline Student had mental health issues & 853 & 0.10 & 0.06 & 0.02 & 0.495 \\
\hline Student quarreled with parents & 853 & 0.10 & 0.05 & 0.01 & 0.503 \\
\hline \multicolumn{6}{|l|}{ Before junior high school: } \\
\hline Student had private tutoring & 853 & 0.11 & 0.06 & 0.01 & 0.479 \\
\hline Family help with homework & 853 & 0.09 & 0.05 & 0.01 & 0.496 \\
\hline Student enrolled in gifted academic class & 853 & 0.09 & 0.05 & 0.02 & 0.466 \\
\hline Student enrolled in arts gifted class & 853 & 0.12 & 0.08 & 0.03 & 0.447 \\
\hline $\begin{array}{l}\text { Parents made efforts to place student } \\
\text { in better class }\end{array}$ & 853 & 0.13 & 0.07 & 0.02 & 0.465 \\
\hline
\end{tabular}

Note: This table shows the results of permutation-based class-level sorting tests, in our estimation sample containing 227 schools, 853 classrooms, and 12,816 students. For these tests, we simulate 10,000 classrooms under the null of random assignment of students to classrooms within schools, calculate the mean of pre-treatment characteristics in synthetic classroom, and construct class-level empirical p-values as the share of times synthetic classroom means were more extreme than actual classroom means relative to the schools mean. Each row presents class-level empirical p-values for a different pre-assignment characteristic. The last column shows the average p-value for all classrooms. 
Table C.7: Non-Parametric Sorting Test in Estimation Sample

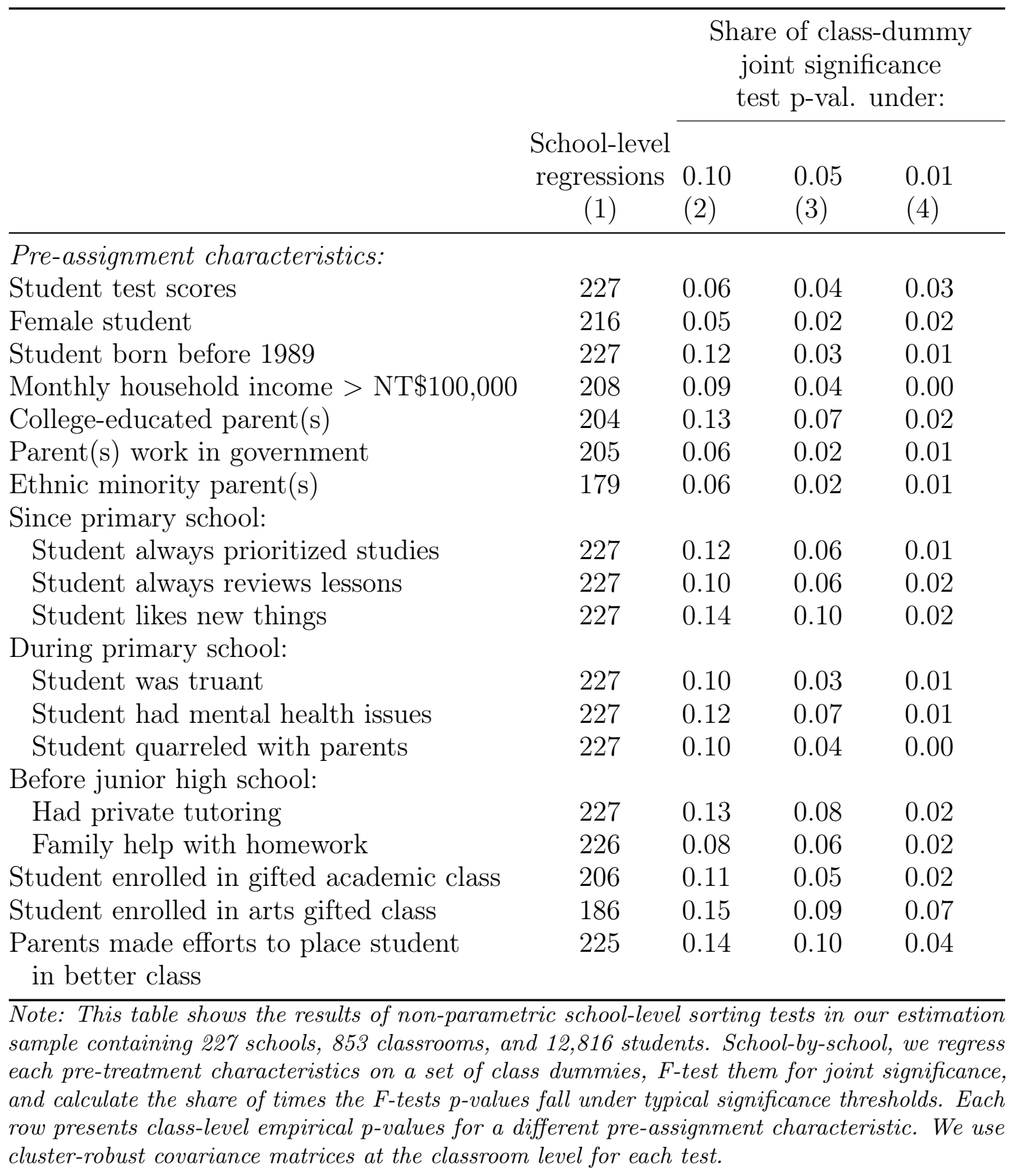


Table C.8: Sensitivity of Estimates to Latent Class Threshold in Posterior Probability in the Fishing Algorithm

\begin{tabular}{|c|c|c|c|c|c|c|}
\hline Outcome: & $\begin{array}{c}\text { Effect of p } \\
\text { (1) }\end{array}$ & $\begin{array}{l}\text { eer test scol } \\
\text { thresholds } \\
(2)\end{array}$ & $\begin{array}{l}\text { res [std] wit } \\
\text { for classific } \\
\quad(3)\end{array}$ & $\begin{array}{l}\text { ith different } \\
\text { cation as de } \\
\text { (4) }\end{array}$ & $\begin{array}{l}\text { t posterior } p \\
\text { efier school } \\
(5)\end{array}$ & $\begin{array}{c}\text { probability } \\
\text { (6) }\end{array}$ \\
\hline Test scores [std] & $\begin{array}{l}0.052 * * * \\
(0.016)\end{array}$ & $\begin{array}{l}0.047 * * * \\
(0.016)\end{array}$ & $\begin{array}{l}0.056 * * * \\
(0.015)\end{array}$ & $\begin{array}{l}0.066 * * * * \\
(0.014)\end{array}$ & $\begin{array}{l}0.080 * * * \\
(0.012)\end{array}$ & $\begin{array}{l}0.098 * * * \\
(0.011)\end{array}$ \\
\hline School effort [std] & $\begin{array}{c}-0.052 * * \\
(0.024)\end{array}$ & $\begin{array}{c}-0.050 * * \\
(0.022)\end{array}$ & $\begin{array}{r}-0.039 * \\
(0.021)\end{array}$ & $\begin{array}{c}-0.025 \\
(0.018)\end{array}$ & $\begin{array}{c}-0.026 * \\
(0.015)\end{array}$ & $\begin{array}{c}-0.018 \\
(0.014)\end{array}$ \\
\hline Initiative in class [std] & $\begin{array}{c}-0.015 \\
(0.024)\end{array}$ & $\begin{array}{c}-0.001 \\
(0.023)\end{array}$ & $\begin{array}{c}-0.016 \\
(0.022)\end{array}$ & $\begin{array}{c}-0.008 \\
(0.018)\end{array}$ & $\begin{array}{c}-0.015 \\
(0.017)\end{array}$ & $\begin{array}{c}-0.008 \\
(0.015)\end{array}$ \\
\hline Truancy [std] & $\begin{array}{c}0.009 \\
(0.021)\end{array}$ & $\begin{array}{c}0.007 \\
(0.021)\end{array}$ & $\begin{array}{c}-0.007 \\
(0.020)\end{array}$ & $\begin{array}{c}-0.015 \\
(0.016)\end{array}$ & $\begin{array}{c}-0.011 \\
(0.014)\end{array}$ & $\begin{array}{c}-0.017 \\
(0.013)\end{array}$ \\
\hline Cheated on exams [.48] & $\begin{array}{c}0.015 \\
(0.013)\end{array}$ & $\begin{array}{l}0.027 * * \\
(0.013)\end{array}$ & $\begin{array}{c}0.012 \\
(0.013)\end{array}$ & $\begin{array}{c}0.013 \\
(0.010)\end{array}$ & $\begin{array}{l}0.015 * \\
(0.009)\end{array}$ & $\begin{array}{c}0.007 \\
(0.008)\end{array}$ \\
\hline Academic self-efficacy [std] & $\begin{array}{c}-0.017 \\
(0.021)\end{array}$ & $\begin{array}{c}-0.004 \\
(0.019)\end{array}$ & $\begin{array}{c}-0.005 \\
(0.018)\end{array}$ & $\begin{array}{c}0.005 \\
(0.016)\end{array}$ & $\begin{array}{c}0.002 \\
(0.014)\end{array}$ & $\begin{array}{c}0.008 \\
(0.013)\end{array}$ \\
\hline Mental health [std] & $\begin{array}{r}-0.030 \\
(0.019)\end{array}$ & $\begin{array}{r}-0.020 \\
(0.018)\end{array}$ & $\begin{array}{c}-0.016 \\
(0.017)\end{array}$ & $\begin{array}{c}-0.013 \\
(0.015)\end{array}$ & $\begin{array}{c}-0.014 \\
(0.013)\end{array}$ & $\begin{array}{c}-0.009 \\
(0.012)\end{array}$ \\
\hline University aspirations [.57] & $\begin{array}{c}0.016 * \\
(0.009)\end{array}$ & $\begin{array}{c}0.013 \\
(0.008)\end{array}$ & $\begin{array}{c}0.012 \\
(0.008)\end{array}$ & $\begin{array}{l}0.008 \\
(0.007)\end{array}$ & $\begin{array}{c}0.011 * \\
(0.006)\end{array}$ & $\begin{array}{l}0.014 * * * \\
(0.005)\end{array}$ \\
\hline University expectations [.44] & $\begin{array}{l}0.020 * * \\
(0.009)\end{array}$ & $\begin{array}{l}0.015 * \\
(0.009)\end{array}$ & $\begin{array}{l}0.017 * * \\
(0.008)\end{array}$ & $\begin{array}{l}0.015 * \\
(0.008)\end{array}$ & $\begin{array}{c}0.012 * \\
(0.007)\end{array}$ & $\begin{array}{l}0.016 * * * \\
(0.006)\end{array}$ \\
\hline Private tutoring [std] & $\begin{array}{c}-0.002 \\
(0.019)\end{array}$ & $\begin{array}{c}0.024 \\
(0.018)\end{array}$ & $\begin{array}{c}0.020 \\
(0.017)\end{array}$ & $\begin{array}{c}0.015 \\
(0.014)\end{array}$ & $\begin{array}{c}0.012 \\
(0.012)\end{array}$ & $\begin{array}{c}0.001 \\
(0.011)\end{array}$ \\
\hline Time with parents [std] & $\begin{array}{l}0.081 * * * \\
(0.023)\end{array}$ & $\begin{array}{l}0.063 * * * \\
(0.022)\end{array}$ & $\begin{array}{l}0.061 * * * \\
(0.022)\end{array}$ & $\begin{array}{l}0.048 * * * \\
(0.017)\end{array}$ & $\begin{array}{l}0.043 * * * \\
(0.017)\end{array}$ & $\begin{array}{l}0.046 * * * \\
(0.014)\end{array}$ \\
\hline Conflict with parents [.31] & $\begin{array}{c}-0.017 \\
(0.010)\end{array}$ & $\begin{array}{r}-0.017 * \\
(0.009)\end{array}$ & $\begin{array}{c}-0.019 * * \\
(0.008)\end{array}$ & $\begin{array}{c}-0.014 * * \\
(0.007)\end{array}$ & $\begin{array}{c}-0.008 \\
(0.006)\end{array}$ & $\begin{array}{c}-0.003 \\
(0.006)\end{array}$ \\
\hline Parental strictness [std] & $\begin{array}{l}0.036 * \\
(0.020)\end{array}$ & $\begin{array}{c}0.029 \\
(0.018)\end{array}$ & $\begin{array}{c}0.024 \\
(0.017)\end{array}$ & $\begin{array}{c}0.011 \\
(0.015)\end{array}$ & $\begin{array}{c}0.011 \\
(0.014)\end{array}$ & $\begin{array}{c}-0.000 \\
(0.012)\end{array}$ \\
\hline Parental support [std] & $\begin{array}{c}0.029 \\
(0.020)\end{array}$ & $\begin{array}{c}0.031 * \\
(0.018)\end{array}$ & $\begin{array}{c}0.031 * \\
(0.017)\end{array}$ & $\begin{array}{l}0.031 * * \\
(0.014)\end{array}$ & $\begin{array}{l}0.034 * * * \\
(0.012)\end{array}$ & $\begin{array}{l}0.038 * * * \\
(0.011)\end{array}$ \\
\hline Harsh parenting [.33] & $\begin{array}{c}0.015 \\
(0.009)\end{array}$ & $\begin{array}{l}0.010 \\
(0.009)\end{array}$ & $\begin{array}{l}0.010 \\
(0.009)\end{array}$ & $\begin{array}{c}0.004 \\
(0.007)\end{array}$ & $\begin{array}{c}0.004 \\
(0.006)\end{array}$ & $\begin{array}{c}-0.004 \\
(0.006)\end{array}$ \\
\hline Parent uni. aspirations [.51] & $\begin{array}{c}0.001 \\
(0.010)\end{array}$ & $\begin{array}{c}0.006 \\
(0.009)\end{array}$ & $\begin{array}{c}0.005 \\
(0.009)\end{array}$ & $\begin{array}{c}0.007 \\
(0.007)\end{array}$ & $\begin{array}{c}0.009 \\
(0.006)\end{array}$ & $\begin{array}{l}0.018 * * * \\
(0.006)\end{array}$ \\
\hline School environment [std] & $\begin{array}{c}-0.029 \\
(0.024)\end{array}$ & $\begin{array}{c}-0.029 \\
(0.023)\end{array}$ & $\begin{array}{c}-0.022 \\
(0.022)\end{array}$ & $\begin{array}{c}-0.006 \\
(0.019)\end{array}$ & $\begin{array}{c}0.005 \\
(0.016)\end{array}$ & $\begin{array}{c}0.018 \\
(0.014)\end{array}$ \\
\hline Classroom hard to manage [.33] & $\begin{array}{c}-0.040 \\
(0.033)\end{array}$ & $\begin{array}{c}-0.037 \\
(0.030)\end{array}$ & $\begin{array}{c}-0.052 * \\
(0.028)\end{array}$ & $\begin{array}{c}-0.051 * * \\
(0.024)\end{array}$ & $\begin{array}{c}-0.052 * * * \\
(0.020)\end{array}$ & $\begin{array}{c}-0.058 * * * \\
(0.017)\end{array}$ \\
\hline Teacher engagement [std] & $\begin{array}{c}0.022 \\
(0.023)\end{array}$ & $\begin{array}{l}0.052 * * \\
(0.022)\end{array}$ & $\begin{array}{c}0.041 * \\
(0.021)\end{array}$ & $\begin{array}{c}0.021 \\
(0.019)\end{array}$ & $\begin{array}{c}0.020 \\
(0.016)\end{array}$ & $\begin{array}{c}0.022 \\
(0.014)\end{array}$ \\
\hline Teacher tired of teaching [.49] & $\begin{array}{c}-0.042 \\
(0.031)\end{array}$ & $\begin{array}{r}-0.016 \\
(0.029)\end{array}$ & $\begin{array}{c}-0.027 \\
(0.028)\end{array}$ & $\begin{array}{c}-0.026 \\
(0.024)\end{array}$ & $\begin{array}{r}-0.012 \\
(0.022)\end{array}$ & $\begin{array}{c}0.009 \\
(0.020)\end{array}$ \\
\hline $\begin{array}{l}\text { Excluded school if prob. of defier }< \\
\text { Jochmans (2020) sorting t-statistic } \\
\text { Schools } \\
\text { Classes } \\
\text { Students }\end{array}$ & $\begin{array}{c}0.5 \\
-0.5 \\
232 \\
850 \\
11,029\end{array}$ & $\begin{array}{c}0.6 \\
0.9 \\
247 \\
911 \\
11,800\end{array}$ & $\begin{array}{c}0.7 \\
1.9 \\
257 \\
951 \\
12,302\end{array}$ & $\begin{array}{c}0.8 \\
4.2 \\
283 \\
1,046 \\
13,526\end{array}$ & $\begin{array}{c}0.9 \\
\mathbf{5 . 3} \\
306 \\
1,129 \\
14,665\end{array}$ & $\begin{array}{c}1.0 \\
\mathbf{6 . 6} \\
328 \\
1,206 \\
15,687\end{array}$ \\
\hline \multicolumn{7}{|c|}{$\begin{array}{l}\text { This table reports coefficient estimates of regressing student outcomes in wave } 2 \text { on standardized average } \\
\text { peer ability in wave } 1 \text { in samples defined by taking different thresholds in the school-level posterior } \\
\text { probability of being a defier school, as defined by our fishing algorithm. All models include school fixed } \\
\text { effects, and students' own ability and educational inputs in wave } 1 \text {. At the bottom we report Jochmans } \\
\text { (2020)'s sorting t-statistic, noting that its reference distribution is the standard normal. T-statistics } \\
\text { larger than critical values for a two-sided test are shown in italics for } 95 \% \text { confidence and in bold for } \\
99 \% \text { confidence. Standard errors are clustered at the classroom level. *, ** and *** denote significance } \\
\text { levels at the } 10 \%, 5 \% \text { and } 1 \% \text {. }\end{array}$} \\
\hline
\end{tabular}


Table C.9: Oster (2019) Proportional Selection on Unobservable Characteristics in Initial Sample

Degree of selection required to explain effect of peer test scores on outcomes

(1)

(2)

Outcomes:

Test scores

School effort

$0.0-0.2$

Initiative in class

$0.0-0.1$

Truancy

Cheated on exams

$0.0 \quad-3.0$

Academic self-efficacy

$$
-0.1
$$$$
2.4
$$

0.0

$-0.5$

Mental health

0.0

$-5.7$

University aspirations

0.0

$-0.7$

University expectations

0.0

$-1.0$

Private tutoring

0.0

$-1.1$

Time with parents

$-0.1$

0.1

Conflict with parents

0.1

$-0.4$

Parental strictness

$-0.1$

$-3.4$

Parental support

$-0.1$

$-1.6$

Harsh parenting

$-0.1$

$-0.5$

Parent uni. Aspirations
School environment

0.0

$-4.8$

$-0.1$

0.0

Classroom hard to manage

0.0

$-0.7$

Teacher engagement

0.1

$-0.1$

Teacher tired of teaching

0.0

$-0.9$

$0.0 \quad 0.0$

Selection proportional to:

Balancing controls

W1 inputs

$\begin{array}{ll}\mathrm{Y} & \mathrm{Y} \\ & \mathrm{Y}\end{array}$

$\overline{\text { This table reports Oster's (2019) } \delta \text {, the share of proportional se- }}$ lection needed to explain away each estimate in our initial sample 332 schools, 1,241 classrooms and 14,383 students. Values of $\delta$ between zero and one imply that, under reasonable assumption, the effect can be explained by correlated unobservable characteristics. Each cell is an estimate from a separate analysis. All estimates are calculated using Oster (2019)'s psacalc Stata package, and assume a theoretical maximum $R$-square of one. All models control for school fixed effects and student test scores in wave 1. Pre-assignment characteristics are listed in Section 3.4.2. Educational inputs in wave 1 are listed in Section 3.5.1. 
Table C.10: The Effect of Peer Ability on Students' Own Ability, Using Alternative Measures of Ability

\begin{tabular}{|c|c|c|c|c|c|}
\hline \multirow[t]{4}{*}{ Outcome: } & \multicolumn{5}{|c|}{ Student ability in wave 2 [std] } \\
\hline & \multicolumn{5}{|c|}{ Measure of Ability used: } \\
\hline & \multirow[b]{2}{*}{$\begin{array}{c}\text { Analytical } \\
\text { (1) }\end{array}$} & \multirow[b]{2}{*}{$\begin{array}{l}\text { Math } \\
(2)\end{array}$} & \multicolumn{3}{|c|}{ IRT Bayesian posterior mean of: } \\
\hline & & & $\begin{array}{c}\text { General } \\
(3)\end{array}$ & $\begin{array}{c}\text { Analytical } \\
(4)\end{array}$ & $\begin{array}{l}\text { Math } \\
(5)\end{array}$ \\
\hline Peer ability [std] & $\begin{array}{l}0.042 * * \\
(0.018)\end{array}$ & $\begin{array}{l}0.046 * * * \\
(0.016)\end{array}$ & $\begin{array}{l}0.048 * * * \\
(0.017)\end{array}$ & $\begin{array}{l}0.043 * * \\
(0.019)\end{array}$ & $\begin{array}{l}0.047 * * * \\
(0.017)\end{array}$ \\
\hline Own ability [std] & $\begin{array}{l}0.389 * * * \\
(0.010)\end{array}$ & $\begin{array}{l}0.542 * * * \\
(0.009)\end{array}$ & $\begin{array}{l}0.606 * * * \\
(0.009)\end{array}$ & $\begin{array}{l}0.396 * * * \\
(0.010)\end{array}$ & $\begin{array}{l}0.558 * * * \\
(0.009)\end{array}$ \\
\hline$R^{2}$ & 0.46 & 0.61 & 0.70 & 0.49 & 0.64 \\
\hline
\end{tabular}

Note: This table reports coefficient estimates of regressing student's own ability in wave 2 on standardized average peer ability and own ability in wave 1 in our estimation sample containing 232 schools, 850 classrooms, and 11,029 students. The columns vary the measure of ability used for the analysis. The identification of analytical and mathematical subcomponents of ability and the Bayesian posterior mean calculation based on Item Response Theory (IRT) models, the TEPS team could also identify two highly correlated but distinct subcomponents measuring analytical ability and mathematical ability based on disjoint subsets of test questions. The IRT models were also used to produce the standardized Bayesian posterior means of the three components identifiable in the test-the general ability component and the analytical ability and mathematical ability subcomponents. All models include school fixed effects and educational inputs in wave 1. Standard errors are clustered at the classroom level. *, ** and *** denote significance levels at the 10\%, 5\% and $1 \%$.

Table C.11: The Effect of Better Peer Ability on Students' Own Ability, Using Instrumental Variable Estimators to Account for Measurement Error in Ability

\begin{tabular}{lccc}
\hline Outcome: & \multicolumn{3}{c}{ Student ability in wave 2 [std] } \\
\hline & \multicolumn{3}{c}{ Measure of Ability used: } \\
\cline { 2 - 4 } & Analytical & Mathematical & Mixed \\
& $(1)$ & $(2)$ & $(3)$ \\
\hline Peer ability [std] & $0.054 *$ & $0.042 *$ & $0.068 *$ \\
& $(0.029)$ & $(0.025)$ & $(0.029)$ \\
Instrument & & & \\
t-statistic of first-stage coefficient & 30.53 & Analytical & Alt. mixed \\
\hline
\end{tabular}

Note: This table reports coefficient estimates of instrumental variable regressions of student's test scores in wave 2 on standardized average peer ability in wave 1 in our estimation sample containing 232 schools, 850 classrooms, and 11,744 students. The measures of ability and the instrument vary across columns, as described in Section 3.6.2. All models include school fixed effects, and students' own test scores and educational inputs in wave 1. Standard errors are clustered at the classroom level. *, ** and ${ }^{* * *}$ denote significance levels at the $10 \%, 5 \%$ and $1 \%$. 
Table C.12: The Effect of Peer Ability on Educational Inputs, Using a Mixed Ability IV Approach

\begin{tabular}{|c|c|c|}
\hline & \multicolumn{2}{|c|}{ Mixed IV effect of peer ability [std] } \\
\hline & $\begin{array}{c}\text { Coef. Est. } \\
\text { (1) }\end{array}$ & $\begin{array}{c}\text { Std. err. } \\
\text { (2) }\end{array}$ \\
\hline \multicolumn{3}{|l|}{ Outcome: Educational inputs } \\
\hline School effort [std] & $-0.072 * *$ & $(0.034)$ \\
\hline Initiative in class [std] & 0.002 & $(0.038)$ \\
\hline Truancy [std] & 0.019 & $(0.034)$ \\
\hline Cheated on exams [.48] & 0.013 & $(0.022)$ \\
\hline Academic self-efficacy [std] & -0.014 & $(0.034)$ \\
\hline Mental health [std] & -0.052 & $(0.034)$ \\
\hline University aspirations [.57] & 0.022 & $(0.014)$ \\
\hline University expectations [.44] & 0.021 & $(0.016)$ \\
\hline Private tutoring [std] & -0.006 & $(0.031)$ \\
\hline Time with parents [std] & $0.113 * * *$ & $(0.038)$ \\
\hline Conflict with parents [.31] & -0.016 & $(0.017)$ \\
\hline Parental strictness [std] & $0.057 *$ & $(0.031)$ \\
\hline Parental support [std] & 0.037 & $(0.031)$ \\
\hline Harsh parenting [.33] & $0.025 *$ & $(0.014)$ \\
\hline Parent uni. aspirations [.51] & 0.011 & $(0.017)$ \\
\hline School environment [std] & -0.008 & $(0.037)$ \\
\hline Classroom hard to manage & -0.026 & $(0.050)$ \\
\hline Teacher engagement [std] & 0.022 & $(0.035)$ \\
\hline Teacher tired of teaching & -0.066 & $(0.048)$ \\
\hline
\end{tabular}

$\overline{\text { This table reports coefficient estimates of instrumental variable regressions of }}$ student's educational inputs in wave 2 on standardized average peer ability in wave 1 in our estimation sample containing 232 schools, 850 classrooms, and 11,744 students. Peer ability and its instrument are constructed using the "mixed IV" approach described in Section 3.6.2. All models include school fixed effects, and students' own ability and educational inputs in wave 1. Standard errors are clustered at the classroom level. *, ** and *** denote significance levels at the 10\%, 5\% and 1\%. 
Table C.13: Effects of Peer Ability Using Sojourner (2013) Correction for Incomplete Classroom Sampling

\begin{tabular}{|c|c|c|c|c|c|c|}
\hline \multirow{2}{*}{$\begin{array}{l}\text { Outcome: } \\
\text { Test scores [std] }\end{array}$} & \multicolumn{6}{|c|}{$\begin{array}{l}\text { Effect of peer test scores [std] with Sojourner (2013) } \\
\text { correction for peer test scores missing not at random }\end{array}$} \\
\hline & $\begin{array}{l}0.133 * * * \\
(0.039)\end{array}$ & $* \begin{array}{l}0.090 * * \\
(0.037)\end{array}$ & $\begin{array}{c}0.089 * * \\
(0.037)\end{array}$ & $\begin{array}{c}0.092 * * \\
(0.036)\end{array}$ & $\begin{array}{l}0.095 * * * \\
(0.035)\end{array}$ & $\begin{array}{c}0.098 * * \\
(0.035)\end{array}$ \\
\hline School effort [std] & $\begin{array}{l}-0.020 \\
(0.055)\end{array}$ & $\begin{array}{l}-0.028 \\
(0.051)\end{array}$ & $\begin{array}{c}-0.030 \\
(0.051)\end{array}$ & $\begin{array}{c}-0.038 \\
(0.051)\end{array}$ & $\begin{array}{r}-0.046 \\
(0.050)\end{array}$ & $\begin{array}{c}-0.042 \\
(0.049)\end{array}$ \\
\hline Initiative in class [std] & $\begin{array}{c}-0.064 \\
(0.062)\end{array}$ & $\begin{array}{c}-0.057 \\
(0.055)\end{array}$ & $\begin{array}{c}-0.057 \\
(0.055)\end{array}$ & $\begin{array}{c}-0.067 \\
(0.054)\end{array}$ & $\begin{array}{c}-0.074 \\
(0.053)\end{array}$ & $\begin{array}{c}-0.069 \\
(0.053)\end{array}$ \\
\hline Truancy [std] & & & $\begin{array}{c}0.004 \\
(0.050)\end{array}$ & $\begin{array}{c}0.006 \\
(0.052)\end{array}$ & $\begin{array}{c}0.005 \\
(0.051)\end{array}$ & $\begin{array}{c}0.004 \\
(0.050)\end{array}$ \\
\hline Cheated on exams [.48] & $\begin{array}{c}0.050 \\
(0.034)\end{array}$ & & $\begin{array}{c}0.032 \\
(0.028)\end{array}$ & & $\begin{array}{c}0.040 \\
(0.029)\end{array}$ & $\begin{array}{c}0.042 \\
(0.028)\end{array}$ \\
\hline Academic self-efficacy [std] & $\begin{array}{c}-0.026 \\
(0.054)\end{array}$ & $\begin{aligned}-0.0 \\
(0.0\end{aligned}$ & $\begin{array}{c}-0.014 \\
(0.046)\end{array}$ & -0.0 & 0.0 & $\begin{array}{c}0.006 \\
(0.045)\end{array}$ \\
\hline Mental health [std] & $\begin{array}{c}-0.036 \\
(0.050)\end{array}$ & $\begin{array}{r}-0.0 \\
(0.0\end{array}$ & $\begin{array}{c}-0.035 \\
(0.045)\end{array}$ & $\begin{array}{r}-0.0 \\
(0.0\end{array}$ & $\begin{array}{c}-0.025 \\
(0.044)\end{array}$ & $\begin{array}{c}-0.018 \\
(0.044)\end{array}$ \\
\hline University aspirations [.57] & $\begin{array}{l}0.050 * * \\
(0.024)\end{array}$ & & $\begin{array}{r}0.0 \\
(0.0\end{array}$ & & & $\begin{array}{r}0.035 * \\
(0.020)\end{array}$ \\
\hline University expectations [.44] & $\begin{array}{l}0.050 * * \\
(0.023)\end{array}$ & $\begin{array}{l}0.0 \\
(0.0\end{array}$ & 0.0 & & $\begin{array}{r}0.0 \\
(0.0\end{array}$ & $\begin{array}{c}0.036 * \\
(0.020)\end{array}$ \\
\hline Private tutoring [std] & $\begin{array}{c}-0.001 \\
(0.048)\end{array}$ & & $\begin{array}{r}-0.0 \\
(0.0\end{array}$ & & & $\begin{array}{c}0.023 \\
(0.041)\end{array}$ \\
\hline Time with parents [std] & $\begin{array}{c}0.098 * \\
(0.056)\end{array}$ & $\begin{array}{r}0.1 \\
(0.0\end{array}$ & $\begin{array}{l}0.1 \\
(0.0\end{array}$ & $\begin{array}{r}0.1 \\
0.0\end{array}$ & $\begin{array}{c}0.16 \\
0.04\end{array}$ & $\begin{array}{c}0.168 * * \\
(0.047)\end{array}$ \\
\hline Conflict with parents [.31] & $\begin{array}{c}0.004 \\
(0.025)\end{array}$ & $\begin{array}{r}-0.0 \\
(0.0\end{array}$ & $\begin{array}{r}-0.0 \\
(0.0\end{array}$ & & $\begin{array}{r}-0.0 \\
(0.0\end{array}$ & \\
\hline Parental strictness [std] & $\begin{array}{c}0.030 \\
(0.052)\end{array}$ & & $\begin{array}{c}0.060 \\
(0.045)\end{array}$ & & & $\begin{array}{c}0.058 \\
(0.043)\end{array}$ \\
\hline Parental support [std] & & & & & & \\
\hline Harsh parenting [.33] & $\begin{array}{c}0.016 \\
(0.023)\end{array}$ & & $\begin{array}{l}0.0 \\
0.0\end{array}$ & & $\begin{array}{r}0.0 \\
0.0\end{array}$ & $\begin{array}{r}0.0 \\
0.0\end{array}$ \\
\hline Parent uni. aspirations [.51] & $\begin{array}{c}0.022 \\
(0.027)\end{array}$ & $\begin{array}{c}0.003 \\
(0.022)\end{array}$ & $\begin{array}{c}0.003 \\
(0.022)\end{array}$ & $\begin{array}{c}-0.000 \\
(0.022)\end{array}$ & $\begin{array}{r}0.0 \\
0.0\end{array}$ & $\begin{array}{c}0.004 \\
(0.021)\end{array}$ \\
\hline School environment [std] & $\begin{array}{c}0.004 \\
(0.066)\end{array}$ & $\begin{array}{c}-0.049 \\
(0.057)\end{array}$ & $\begin{array}{c}-0.045 \\
(0.056)\end{array}$ & $\begin{array}{c}-0.044 \\
(0.056)\end{array}$ & $\begin{array}{c}-0.059 \\
(0.054)\end{array}$ & $\begin{array}{c}-0.050 \\
(0.054)\end{array}$ \\
\hline Classroom hard to manage [.33] & $\begin{array}{r}-0.153 * \\
(0.079)\end{array}$ & $\begin{array}{c}-0.113 \\
(0.073)\end{array}$ & $\begin{array}{c}-0.117 \\
(0.072)\end{array}$ & $\begin{array}{r}-0.132 * \\
(0.076)\end{array}$ & $\begin{array}{r}-0.124 * \\
(0.073)\end{array}$ & $\begin{array}{r}-0.124 * \\
(0.073)\end{array}$ \\
\hline Teacher engagement [std] & $\begin{array}{c}0.080 \\
(0.058)\end{array}$ & $\begin{array}{c}0.026 \\
(0.052)\end{array}$ & $\begin{array}{c}0.025 \\
(0.053)\end{array}$ & $\begin{array}{c}0.008 \\
(0.052)\end{array}$ & $\begin{array}{c}0.021 \\
(0.051)\end{array}$ & $\begin{array}{c}0.024 \\
(0.051)\end{array}$ \\
\hline Teacher tired of teaching [.49] & $\begin{array}{c}-0.166 * * \\
(0.082)\end{array}$ & $\begin{array}{c}-0.098 \\
(0.074)\end{array}$ & $\begin{array}{c}-0.091 \\
(0.074)\end{array}$ & $\begin{array}{c}-0.127 * \\
(0.076)\end{array}$ & $\begin{array}{c}-0.130 * \\
(0.073)\end{array}$ & $\begin{array}{c}-0.114 \\
(0.073)\end{array}$ \\
\hline $\begin{array}{l}\text { Share peers observed: } \\
\times \text { School FE } \\
\times \text { School K-cile FE }\end{array}$ & Y & 25 & 20 & 15 & 10 & 5 \\
\hline $\begin{array}{l}\text { 11,029 students. These est } \\
\text { ojourner (2013) and implem } \\
\text { clude school fixed effects, anc }\end{array}$ & D & * & 1010 & L $D t$ & . & . \\
\hline
\end{tabular}


Table C.14: Corrected P-values for the Effect of Peer Ability using Young (2019)'s Randomization Inference, and Romano and Wolf (2005b)'s Step-Down Familywise Error Rate Adjustment Procedures

Corrected p-values for the

effect of peer test scores [std] using:

\begin{tabular}{cc}
\hline Young (2019) & Romano and Wolf $(2005 \mathrm{~b})$ \\
Randomization-t inference & Step-down procedure
\end{tabular}

(1)

\section{Outcomes:}

Test scores

\subsection{8}

\subsection{6}

School effort

0.058

0.382

Initiative in class

0.589

0.948

Truancy

0.673

0.956

Cheated on exams

0.287

0.860

Academic self-efficacy

0.487

0.914

Mental health

0.176

0.774

University aspirations

0.122

0.680

University expectations

0.078

0.394

Private tutoring

0.928

0.982

Time with parents

0.000

$\mathbf{0 . 0 1 4}$

Conflict with parents

0.146

0.742

Parental strictness

0.136

0.670

Parental support

0.220

0.800

Harsh parenting

0.192

0.774

Parent uni. Aspirations

0.916

0.982

School environment

0.305

0.860

Classroom hard to manage

0.248

0.858

Teacher engagement

0.421

0.890

Teacher tired of teaching

0.216

This table corrected p-values for our main results using i) Young (2019)'s randomization-t inference procedure to account for high-leverage, finite sample properties of the model error term, and the complex sampling structure of our data (Col. (1) based on 499 permutations), and ii) Romano and Wolf (2005b)'s step-down procedure for controlling for familywise error rate in multiple hypotheses testing implemented using Clarke et al. (2019) rwolf Stata package (Col. (2), based on 499 replications). p-values smaller than 0.10 are shown in italics and smaller than 0.05 in bold. 
Table C.15: Heterogeneous and Mediated Effects of Peer Ability

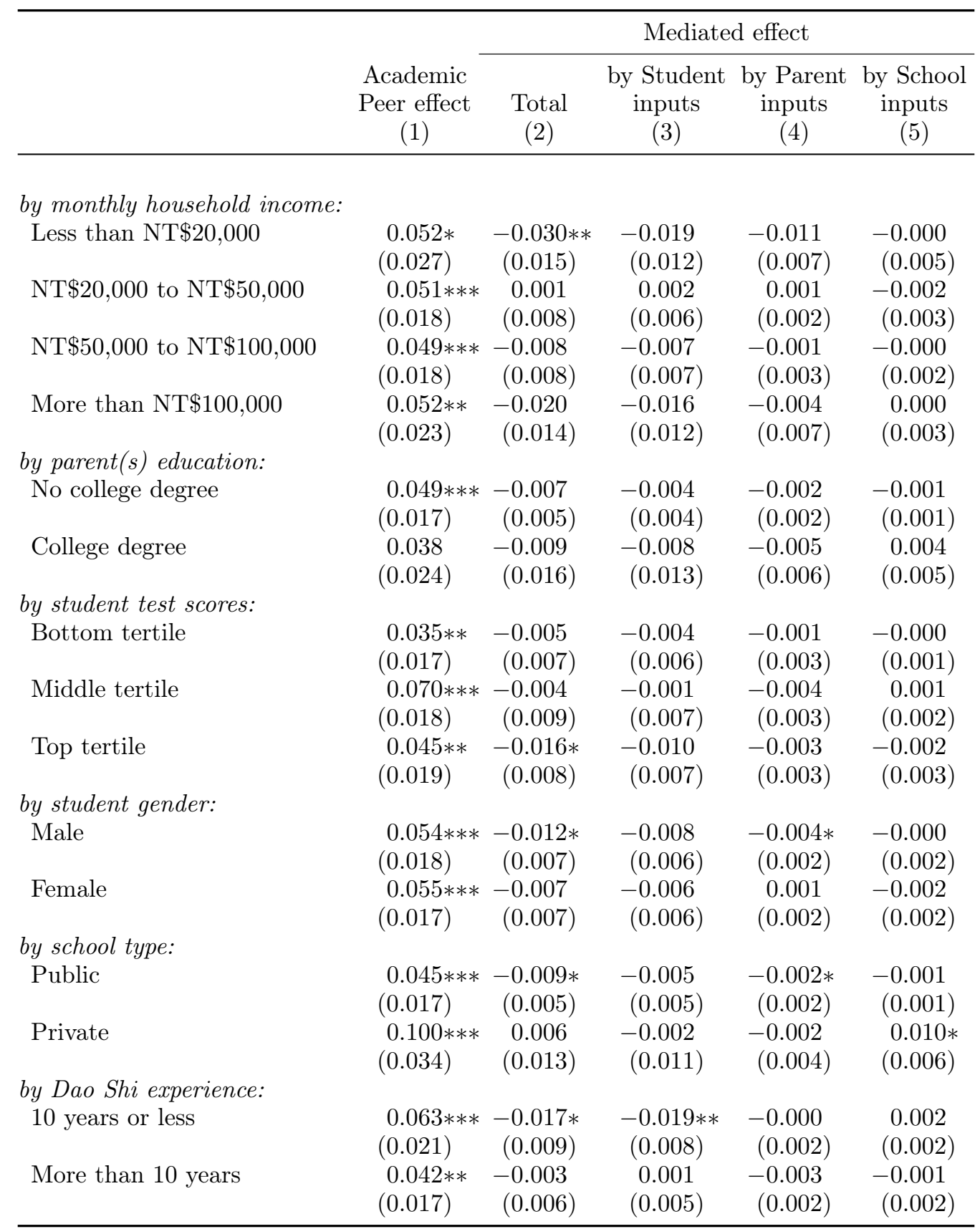

This table reports peer and mediated effects based on Gelbach (2016)'s decomposition using only within-school variation in our estimation sample containing 232 schools, 850 classrooms, and 11,029 students. These estimates are produced using a modified version of the b1x2 Stata package. Rows present the peer and mediated effects for different subgroups defined based on wave 1 variables. All models control for school fixed effects, student test scores, average peer test scores, and educational inputs in wave 1. Standard errors are clustered at the classroom level. *, ** and *** denote significance levels at the 10\%, 5\% and $1 \%$. 
Table C.16: Heterogeneous Value-Added

\begin{tabular}{|c|c|c|}
\hline \multirow[t]{2}{*}{ Outcome: } & \multicolumn{2}{|c|}{ Student test scores in Wave 2 [std] } \\
\hline & $\begin{array}{l}\text { Coef. Est. } \\
\text { (1) }\end{array}$ & $\begin{array}{l}\text { Std. err. } \\
\quad(2)\end{array}$ \\
\hline \multicolumn{3}{|l|}{$\begin{array}{l}\text { Value-added coef. interaction: } \\
\text { peer test scores [std] with: }\end{array}$} \\
\hline School effort [std] & $0.019 * *$ & $(0.008)$ \\
\hline Initiative in class [std] & $0.029 * * *$ & $(0.007)$ \\
\hline Truancy [std] & -0.007 & $(0.007)$ \\
\hline Cheated on exams [.48] & 0.011 & $(0.011)$ \\
\hline Academic self-efficacy [std] & -0.003 & $(0.006)$ \\
\hline Mental health [std] & -0.003 & $(0.006)$ \\
\hline University aspirations [.57] & 0.013 & $(0.015)$ \\
\hline University expectations [.44] & -0.012 & $(0.015)$ \\
\hline Private tutoring [std] & $-0.019 * * *$ & $(0.007)$ \\
\hline Time with parents [std] & $-0.013 * *$ & $(0.005)$ \\
\hline Conflict with parents [.31] & -0.008 & $(0.012)$ \\
\hline Parental strictness [std] & -0.004 & $(0.006)$ \\
\hline Parental support [std] & $-0.016 * *$ & $(0.006)$ \\
\hline Harsh parenting [.33] & -0.006 & $(0.012)$ \\
\hline Parent uni. aspirations [.51] & 0.013 & $(0.013)$ \\
\hline School environment [std] & 0.008 & $(0.006)$ \\
\hline Class hard to manage [.33] & $-0.045 * * *$ & $(0.016)$ \\
\hline Teacher engagement [std] & -0.001 & $(0.006)$ \\
\hline Teacher tired of teaching [.49] & -0.004 & $(0.015)$ \\
\hline $\mathrm{R} 2$ & \multicolumn{2}{|c|}{0.71} \\
\hline Schools & \multicolumn{2}{|c|}{232} \\
\hline Classes & \multicolumn{2}{|c|}{833} \\
\hline Students & \multicolumn{2}{|c|}{10,490} \\
\hline
\end{tabular}

This table reports coefficient estimates of regressing student test scores in wave 2 on educational inputs in wave 2, all interacted with peer test scores in wave 1, in our estimation sample containing 232 schools, 833 classes, and up to 10,490 students. Rows present coefficients of different regressors interacted with peer test scores. Unconditional means of each input are shown in square brackets and [std] marks inputs that have been standardized to have a mean of zero and a standard deviation of one. All models control for main effects of wave 2 inputs, school fixed effects, student test scores, average peer test scores, and educational inputs in wave 1. Standard errors clustered at the classroom level in parentheses. 
Table C.17: 35 Education Systems in Comparative Perspective in TIMSS 1999

\begin{tabular}{|c|c|c|c|c|c|c|c|c|c|c|}
\hline \multirow{2}{*}{\multicolumn{2}{|c|}{$\begin{array}{c}\text { Average } \\
\text { class } \\
\text { size } \\
(1) \\
\end{array}$}} & \multirow{2}{*}{$\begin{array}{l}\text { Student- } \\
\text { to-teacher } \\
\text { ratio } \\
\quad(2)\end{array}$} & \multirow{2}{*}{$\begin{array}{l}\text { School- } \\
\text { days } \\
\text { yearly } \\
(3)\end{array}$} & \multirow{2}{*}{$\begin{array}{l}\text { Study } \\
\text { hours } \\
\text { daily } \\
(4)\end{array}$} & \multicolumn{6}{|c|}{ Percentage of: } \\
\hline & & & & & $\begin{array}{l}\text { Math in } \\
\text { groups } \\
(5)\end{array}$ & $\begin{array}{l}\text { absent } \\
\text { daily } \\
(6)\end{array}$ & $\begin{array}{c}\text { school } \\
\text { dropout } \\
(7)\end{array}$ & $\begin{array}{c}\text { parents } \\
\text { monitoring } \\
(8)\end{array}$ & $\begin{array}{c}5+y \cdot \exp . \\
\text { teachers } \\
(9)\end{array}$ & $\begin{array}{l}\text { weekly }+ \\
\text { class disr. } \\
\quad(10)\end{array}$ \\
\hline \multicolumn{11}{|c|}{ Australasia and Pacific } \\
\hline Taiwan & 39 & 18 & 221 & 2.0 & 12.5 & 1.3 & 1.7 & 33.4 & 67.7 & 30.1 \\
\hline Japan & 36 & 20 & 223 & 1.7 & 12.5 & 3.0 & 0.3 & 4.0 & 32.3 & 4.5 \\
\hline South Korea & 42 & 24 & 225 & 1.6 & 18.5 & 0.5 & 0.8 & 9.2 & 20.6 & 43.0 \\
\hline Hong Kong & 39 & 20 & 176 & 1.6 & 6.9 & 1.4 & 1.9 & 6.9 & 64.7 & 36.2 \\
\hline Singapore & 37 & 20 & 180 & 3.5 & 32.5 & 1.6 & 1.0 & 1.8 & 49.1 & 32.2 \\
\hline Indonesia & 42 & 23 & 251 & 3.0 & 27.5 & 2.7 & 1.9 & 74.6 & 75.6 & 20.5 \\
\hline Malaysia & 38 & 19 & 198 & 3.8 & 38.2 & 3.8 & 3.9 & 28.5 & 42.4 & 25.6 \\
\hline Philippines & 51 & 35 & 204 & 3.3 & 53.0 & 5.1 & 8.5 & 28.0 & 65.0 & 27.4 \\
\hline Thailand & 39 & 31 & 202 & 2.9 & 51.1 & 3.7 & 3.1 & 57.0 & 64.7 & 12.6 \\
\hline New Zealand & 26 & 16 & 188 & 2.0 & 43.5 & 6.9 & 7.9 & 0.8 & 55.7 & 68.1 \\
\hline Australia & 26 & 16 & 196 & 2.0 & 27.8 & 7.6 & 6.0 & 0.1 & 57.7 & 73.1 \\
\hline \multicolumn{11}{|c|}{ Europe and Central Asia } \\
\hline Netherlands & 25 & 17 & 191 & 2.2 & 27.8 & 3.0 & 2.0 & 1.0 & 77.5 & 76.1 \\
\hline Belgium & 19 & 10 & 175 & 3.0 & 7.3 & 3.0 & 2.2 & 3.6 & 74.1 & 40.4 \\
\hline Italy & 20 & 13 & 210 & 3.6 & 27.7 & 6.3 & 1.5 & 20.7 & 63.7 & 46.6 \\
\hline Cyprus & 29 & 14 & 160 & 2.8 & 16.8 & 3.1 & 2.2 & 4.3 & 23.1 & 54.5 \\
\hline Finland & 20 & 12 & 186 & 1.8 & 18.5 & 5.2 & 1.4 & 1.5 & 70.7 & 50.0 \\
\hline Latvia & 22 & 13 & 176 & 3.0 & 11.3 & 5.2 & 2.0 & 17.1 & 71.4 & 37.5 \\
\hline Moldova & 26 & 17 & 205 & 3.3 & 31.2 & 4.5 & 2.7 & 34.8 & 78.0 & 29.1 \\
\hline Czech & 24 & 19 & 197 & 1.9 & 8.5 & 7.7 & 1.8 & 4.8 & 66.1 & 62.9 \\
\hline Hungary & 32 & 12 & 185 & 2.8 & 7.3 & 4.7 & 2.2 & 3.8 & 71.6 & 41.2 \\
\hline Bulgaria & 21 & 15 & 172 & 3.0 & 27.9 & 6.9 & 2.5 & 8.6 & 71.2 & 22.0 \\
\hline Romania & 24 & 19 & 159 & 3.4 & 23.9 & 3.5 & 1.9 & 12.3 & 65.9 & 17.0 \\
\hline Slovak & 25 & 18 & 194 & 2.3 & 15.9 & 7.0 & 1.7 & 3.1 & 66.8 & 59.8 \\
\hline Slovenia & 22 & 14 & 175 & 2.5 & 10.3 & 3.0 & 1.2 & 12.0 & 75.3 & 61.1 \\
\hline Macedonia & 27 & 21 & 176 & 3.5 & 41.8 & 1.9 & 1.5 & 15.5 & 78.7 & 13.1 \\
\hline Russia & 24 & 15 & 195 & 3.2 & 15.6 & 4.2 & 2.5 & 32.8 & 73.6 & 13.4 \\
\hline Turkey & 39 & 63 & 181 & 3.6 & 22.2 & 3.2 & 4.9 & 32.2 & 52.0 & 14.5 \\
\hline \multicolumn{11}{|c|}{ North America } \\
\hline Canada & 27 & 20 & 188 & 2.2 & 40.1 & 5.4 & 5.5 & 4.6 & 58.2 & 60.3 \\
\hline United States & 26 & 18 & 180 & 2.1 & 44.9 & 5.6 & 9.0 & 7.7 & 60.9 & 69.3 \\
\hline \multicolumn{11}{|c|}{ Central and Latin America } \\
\hline Chile & 34 & 37 & 193 & 2.4 & 57.6 & 6.4 & 5.1 & 2.5 & 67.1 & 45.9 \\
\hline \multicolumn{11}{|c|}{ Middle East and Africa } \\
\hline Iran & 32 & 27 & 209 & 4.1 & 38.6 & 2.4 & 2.3 & 27.5 & 26.7 & 21.4 \\
\hline Israel & 34 & 14 & 199 & 2.7 & 39.8 & 5.1 & 1.6 & 3.5 & 61.5 & 60.8 \\
\hline Jordan & 35 & 23 & 191 & 3.8 & 50.6 & 2.9 & 3.4 & 21.3 & 55.2 & 27.6 \\
\hline Morocco & 28 & 24 & 207 & 3.3 & 44.5 & 4.0 & 7.6 & 11.0 & 70.7 & 31.5 \\
\hline South Africa & 48 & 37 & 194 & 3.1 & 53.3 & 8.3 & 8.3 & 40.0 & 68.0 & 38.6 \\
\hline Tunisia & 34 & 23 & 205 & 3.7 & 24.3 & 2.4 & 2.3 & 61.1 & 26.2 & 54.0 \\
\hline
\end{tabular}




\section{C.3 The Fishing Algorithm}

In this Appendix, we explain the steps of our Fishing Algorithm introduced in Section 3.4.2 in detail. We illustrate its use in the TEPS data. We also provide simulation evidence of its performance in Appendix C.4.

\section{C.3.1 Sorting of students into classrooms within schools in TEPS}

As discussed in Section 3.3, Taiwan has an explicit mandate of random assignment of students to classrooms within schools. We first test whether the TEPS data is consistent with this mandate without imposing any sample restrictions and refer to this as our "initial sample". This initial sample includes a total of 20,055 students assigned to 1,244 classrooms across 333 schools in wave 1, for whom we have data from either students, parents, teachers or school administrators' questionnaires. Most students can be matched across questionnaires - we lose fewer than 1,000 observations due to questionnaire non-match - yet we estimate our initial tests on this unrestricted sample to limit the influence of selective questionnaire attrition.

We first run sorting tests on student wave 1 standardized test scores, as well as on each characteristic that we can unambiguously treat as pre-assignment; that is, variables capturing either fixed traits or events prior to entering junior high school.

Standardized test scores are not strictly measured pre-assignment; they were taken by students during the first weeks of the first junior high school academic year, shortly after assignment to classrooms. However, it is highly doubtful that only a few weeks' worth of exposure to peers could generate considerable peer effects already. Moreover, these test scores were never revealed to students, parents, teachers or school administrators so there is no chance of re-sorting of classrooms after initial assignment based on the results of these exams. However, finding sorting on standardized test scores would still be consistent with students being assigned to classrooms based on other ability or academic performance measures that are either known to the parents, teachers, or school administrators. In this spirit, we analyze standardized test scores in this paper.

To run sorting tests loosely follow the within-school equation:

$$
Y_{i c s 1}=\beta \bar{Y}_{i c s 1}^{-i}+\mu_{s}+\epsilon_{i c s 1}
$$


where $Y_{i c s 1}$ is the characteristic of student $i$ in class $c$ in school $s$ in wave 1, which is pre-determined at the time of assignment, $\bar{Y}_{i c s 1}^{-i}$ is the class leave-out mean of the same variable $Y$ at wave 1 (the classroom peer mean of characteristic ), $\mu_{s}$ is school-invariant unobserved heterogeneity which we account for using school fixed effects, and $\epsilon_{i c s 1}$ is a conditionally uncorrelated model error term.

The sorting statistic of interest is closely related to with the standard normal as a reference distribution in large samples. A positive over critical values in the distribution indicates positive sorting of students into classrooms based on the tested pre-determined characteristic. However, Guryan et al. (2009) observe that, under random assignment, $\hat{\beta}$ presents a small negative bias which seems to disappear when controlling for school-level leave-out-mean of the characteristic in sorting tests. Jochmans (2020) shows that Guryan et al. (2009)'s empirical correction results in low power for detecting sorting, derives analytical expressions for this bias in within-school estimators and proposes a bias-corrected that solves this power issue. In our sorting tests, we present $\hat{t}$ using the more commonly found Guryan et al. (2009) method and the very recent Jochmans (2020) improvement.

The second and third columns of Table C.18 show the sorting test statistics for all predetermined characteristics we consider. There is plenty of evidence suggesting that students are sorted into classrooms with similar peers in the initial sample - certainly for test scores, but also for family income and parental education, intellectual curiosity during primary school, private tutoring before entering junior high school, gifted academic and art class assignment, and on parents' efforts to influence the student's classroom assignment. Sorting on test scores in this sample is already reason enough for thinking that estimates of higher-ability peer effects might be biased. Yet further balancing tests on higher-ability peers - which regress $Y_{i c s 1}$ on $\overline{\text { TestScores }}_{i c s 1}$ - also show that higher-ability peers are also related to several pre-determined characteristics at baseline. These balancing test results are shown in the last two columns of Table C.18. ${ }^{1}$

\footnotetext{
${ }^{1}$ Note that, due to the large number of pre-treatment characteristics we test and the many students and classes in TEPS, we are more likely to find imbalances than many previous academic peer effect studies. The size of our detected imbalances is relatively small generally (very) small. In fact, simple back-of-the-envelope calculations suggest that in other datasets commonly used to estimate peer effects, such as the Project STAR data, imbalances of this size would have gone undetected.
} 
Table C.18: Balancing and Sorting Tests on the TEPS Initial Sample

\begin{tabular}{|c|c|c|c|c|c|}
\hline \multirow{3}{*}{ Treatment Variable: } & \multirow[b]{3}{*}{ Students } & \multicolumn{2}{|c|}{ Sorting tests (t-statistic) } & \multicolumn{2}{|c|}{ Balancing tests } \\
\hline & & \multicolumn{2}{|c|}{$\begin{array}{c}\text { Peer outcome } \\
\text { leave-out mean }\end{array}$} & \multicolumn{2}{|c|}{$\begin{array}{c}\text { Peer ability } \\
\text { leave-out-mean [std }\end{array}$} \\
\hline & & $\begin{array}{c}\text { s Guryan et al. } \\
(2009) \\
(2)\end{array}$ & $\begin{array}{c}\text { Jochmans } \\
(2020) \\
(3)\end{array}$ & $\begin{array}{l}\text { Coef. } \\
(4)\end{array}$ & $\begin{array}{c}\text { Std. err. } \\
\text { (5) }\end{array}$ \\
\hline \multicolumn{6}{|l|}{ Pre-assignment characteristics: } \\
\hline Female student & 19,957 & 2.2 & -0.9 & 0.012 & $(0.007)$ \\
\hline Student born before 1989 & 19,866 & -0.4 & 1.4 & $-0.011 * *$ & $(0.005)$ \\
\hline Household income $>\mathrm{NT} \$ 100 \mathrm{k} / \mathrm{mo}$ & 19,629 & 0.9 & 2.2 & $0.014 * * *$ & k $(0.004)$ \\
\hline College-educated parent(s) & 19,073 & 1.1 & 3.5 & $0.036 * * *$ & $k(0.005)$ \\
\hline Parent(s) work in government & 18,979 & 1.3 & 2.2 & $0.024 * * *$ & $k(0.004)$ \\
\hline Ethnic minority parent(s) & 19,070 & 1.5 & 1.9 & $-0.011 * * *$ & $(0.004)$ \\
\hline \multicolumn{6}{|l|}{ Since primary school: } \\
\hline Student always prioritized studies & 19,830 & -2.1 & 1.5 & -0.006 & $(0.005)$ \\
\hline Student always reviews lessons & 19,813 & 0.0 & 2.6 & -0.002 & $(0.004)$ \\
\hline Student likes new things & 19,771 & 1.0 & 2.9 & 0.005 & $(0.006)$ \\
\hline \multicolumn{6}{|l|}{ During primary school: } \\
\hline Was truant in primary school & 19,674 & 1.3 & 0.4 & $-0.022 * * *$ & $k(0.005)$ \\
\hline Student had mental health issues & 19,670 & 0.0 & 0.3 & 0.001 & $(0.006)$ \\
\hline Student quarreled with parents & 19,691 & -0.5 & -1.0 & -0.006 & $(0.006)$ \\
\hline \multicolumn{6}{|l|}{ Before junior high school: } \\
\hline Had private tutoring & 19,720 & 1.5 & 2.5 & $0.024 * * *$ & $k(0.006)$ \\
\hline Family help with homework & 18,976 & 1.3 & 1.2 & 0.006 & $(0.004)$ \\
\hline Student in gifted academic class & 19,779 & 2.3 & 4.3 & $0.074 * * *$ & $(0.009)$ \\
\hline Student in arts gifted class & 19,779 & 4.8 & 5.5 & $0.033 * * *$ & $k(0.010)$ \\
\hline $\begin{array}{l}\text { Parents made efforts to place child } \\
\text { in better class }\end{array}$ & 19,698 & 5.8 & 4.8 & $0.050 * * *$ & $k(0.006)$ \\
\hline
\end{tabular}

Note: Estimates in our trimmed sample of 333 schools and 1,257 classrooms. All estimators include school fixed effects. The reference distribution for the Guryan et al. (2009) and the Jochmans (2020) sorting statistics is the standard normal. t-statistics larger than critical values for a two-sided test are shown in italics for 95\% confidence and in bold for 99\% confidence. The last column reports cluster-robust standard errors at the classroom level. ***, ** and ${ }^{*}$ mark estimates statistically different from zero at the 90, 95 and 99 percent confidence level. 
Our next step is to characterize the deviations from random assignment in this initial sample in order to hopefully correct them. In Taiwan, class assignment is tasked to schools themselves, as opposed to being done at the regional or school district level. Because of this, we suspect that deviations from random assignment in our data could come directly from having (hopefully few) non-compliant schools, and direct our efforts towards finding these schools. All results in Table C.18 suggest that, in these sorter schools, students assigned to higher-ability peers are also higher ability themselves and are also generally more advantaged in other respects. These schools might have sorted students into classrooms directly based on academic ability/performance, perhaps by assignment them to "gifted" classrooms together, and perhaps also as a response of parental pressure on the school. All these are informative insights in the next steps of our Fishing Algorithm.

\section{C.3.2 The Fishing Algorithm}

The Fishing Algorithm is a data-driven method we developed to detect schools that are likely not compliant with Taiwan's national mandate to randomly assign students to classrooms. The algorithm combines permutation-based measures of the degree of sorting in the data with latent-class modeling techniques. Despite seeming complex, the intuition behind the procedure is simple and its implementation is fast. Its steps are described in Box 1.

In the first step, we identify whether there is evidence of sorting and/or imbalance in the data. Table C.18 describes the results of these tests for the TEPS initial sample. Since our study focuses on estimating the effect of higher-ability classroom peers, we identify student test scores as our key pre-assignment characteristic for the remaining steps.

In the second step, we construct our school-level measure of sorting of students into classrooms based on standardized test scores. We base our measure on the Herfindahl-Hirschman index, the most prominent measure of market concentration in economics. In school $s$ with classrooms $c=1 \ldots C$, we define our measure as:

$$
H_{s}=\sum_{c=1}^{C}\left(\frac{\overline{\text { TestScores }}_{c s}}{\sum_{c=1}^{C} \overline{\text { TestScores }}_{c s}}\right)^{2}
$$

Where $\overline{\text { TestScores }}_{c s}$ is the average standardized test score in classroom $c$ of school $s .^{2} H_{s}$ is a measure of the concentration (or sorting) of student test scores into 


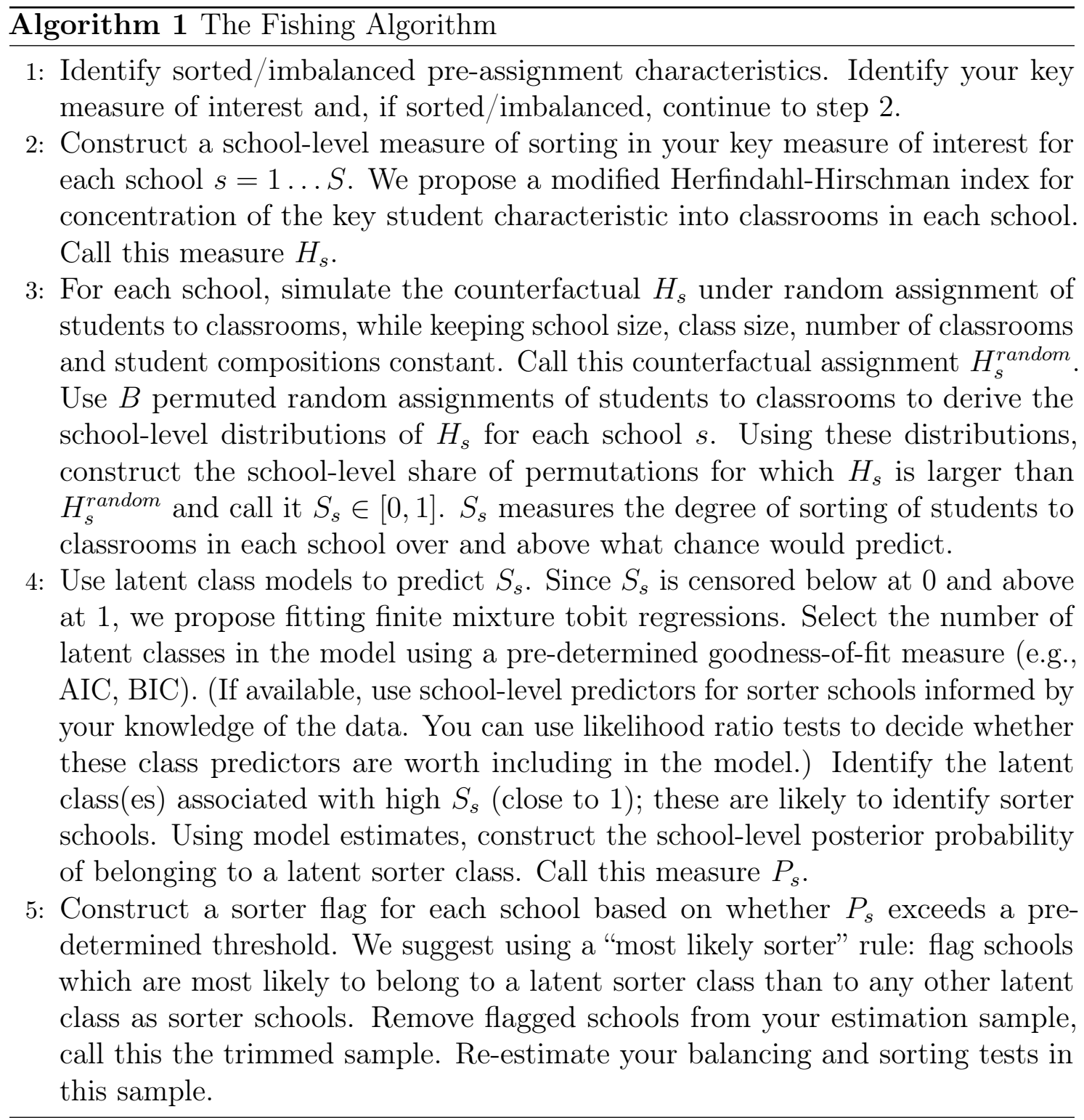


classrooms within each school, and it will range between $1 / C$ (if $\overline{\text { TestScores }}_{c s}$ is identical in all classrooms) to 1 (if all students with positive test scores are together in one classroom - which is ridiculous with test scores but more easy to think of when measuring sorting by e.g., race or gender). At this point our constructed $H_{s}$ includes test score sorting data for each of the 333 schools in TEPS. ${ }^{3}$

In the third step, we construct counterfactual distributions of $H_{s}$ for each of the 333 schools in TEPS that reflect random assignment of students to classrooms within schools. To do this, we construct $B=400$ permutations of random assignment of students to classroom within each school maintaining each schools' data structure; that is, maintaining the student number and composition in each school, and the exact number and size of classrooms in each school. Ensuring the data structure is maintained is crucial for computing randomization-based statistics (Young, 2019). For each permutation $b=1 \ldots B$ we thus end up with a measure $H_{s}^{\text {random }}$ that reflects one way that school sorting could have looked like if classrooms were randomly assigned within schools. Since we do this $B=400$ times, we end up with a distribution of this school concentration index based on 400 counterfactual classroom assignments for each school. We then construct $S_{s}$ for each school: the share of the 400 permutations for which the actual school concentration $H_{s}$ strictly exceeds the simulated concentration under random assignment $H_{s}^{\text {random }}$. For example, in a school where the actual score concentration was larger than 350 out of 400 simulated concentrations, $S_{s}$ would take the value of $350 / 400=0.875$.

At this point, it is important to highlight why $S_{s}$ is a superior measure of classroom sorting than $H_{s}$, especially to capture sorting on characteristics that are relatively rare. To do this, imagine trying to measure sorting based on race in a school with three classrooms and one racial minority student. Even if this school fully complies with random assignment, the measure $H_{s}$ will equal 1, implying full sorting. This is because, in any classroom configuration"all" minority students will be in the same classroom. The measure $S_{s}$, however, will equal 0 - implying perfect sorting because in no permutation will $H_{s}$ strictly exceed $H_{s}^{\text {random }}$. Generalizing based on this example, the key lesson is that $S_{s}$ naturally normalizes classroom concentration to reflect the rarity of the characteristic of interest at the school level, a very useful

\footnotetext{
${ }^{3}$ It is important to note that by standardized test scores we mean "scores from a standardized test" rather than "test scores that have been standardized to have a mean of zero and a standard deviation of one". Steps 3 through 5 of the Fishing Algorithm work much better if $H_{s}$ is constructed from test scores (or any other measure) that is weakly positive (i.e., with support in $[0 ; \infty)$ ).
} 
Figure C.1: The School-Level Concentration of Classrooms Based on Test Scores, With $B=400$ Classroom Assignment Permutations

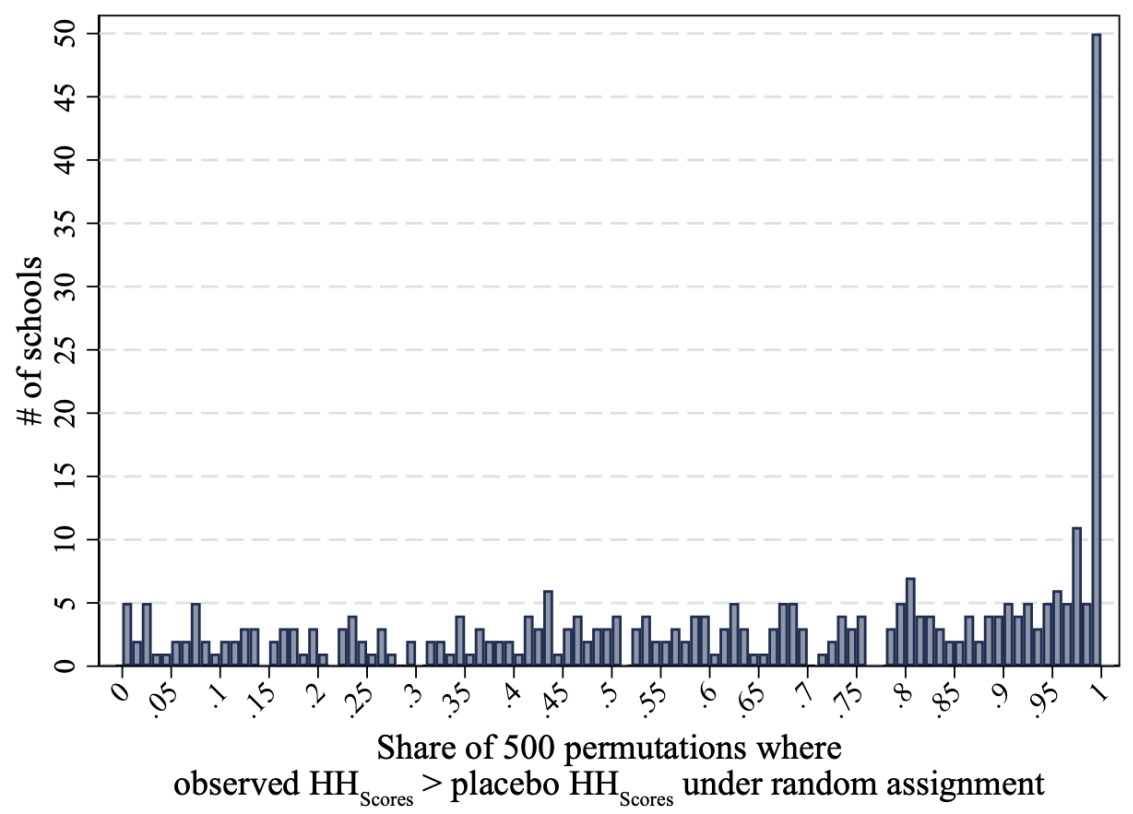

Note: This figure shows the school-level distribution of our measure for whether schools sort students into classrooms more strongly than chance would allow, given the school size, number and classroom size and student composition. The probability of being a sorter school is the posterior probability of being in a latent class classified as sorters by us and calculated based on a finite mixture model of school sorting using several school averages of parental characteristics as class predictors.

property. ${ }^{4}$

Figure C.1 shows the distribution of our school-level measure of classroom concentration based on test scores, $S_{s}$, for all 333 schools in the TEPS data. If all schools in TEPS would have perfectly complied with random assignment of students to classrooms, we would expect this to closely resemble a standardized uniform distribution. The figure suggests that most schools are likely complying with the random assignment mandate, yet a small but non-negligible share of schools show very high degree of sorting that is inconsistent with random assignment. With a quick graphical analysis of the distribution, one could conclude that schools in the rightmost part of the distribution - with $S_{s}>0.9$ which adds up to roughly 80 schools - are much more likely be defying the mandate of random assignment.

\footnotetext{
${ }^{4} \mathrm{~A}$ second, perhaps subtler, lesson is that we can only interpret as evidence of strong classroom non-sorting when the characteristic of interest is prevalent in the school (i.e., when the number of students with that characteristic exceed the number of classes in the school).
} 
At this stage just dropping these 80 schools from our data would be rather crude. Under random assignment, we should still expect that some schools, by chance, ended up grouping students with similar test scores. Blindly trimming these schools could therefore lead to "over-trimming": removing schools that have high sorting by chance. One problem with over-trimming is that it can lead to negative sorting tests in the trimmed sample. Another, perhaps more serious, problem is that it would remove legitimate variation from the estimation sample that could be crucial for identifying peer effect. These worst-case scenarios could result in a loss of power for identifying peer effects in the trimmed sample and, if peer effects are extremely non-linear, over-trimming could bias peer effect estimates downwards. In the fourth and key innovative step of our Fishing Algorithm, we try to disentangle schools that have strong sorting by chance from schools that are defying the mandate of random assignment using latent class models of $S_{s}$. Our preferred method is to fit a finite mixture model (FMM) of $S_{s}$ to recover a predicted probability of being a school defying the mandate of random assignment to classrooms for each school. ${ }^{5}$ One good reason for using FMM is that, based on its estimates, we can construct the posterior probability of belonging to each latent class modeled and. Once we have identified which class is likely to capture sorter schools, this gives us a direct estimate of school-level probability to be a sorter, which we then use to construct our likely sorter school flag.

For this step, there are four key choices to make: i) the correct model given the distribution of $S_{s}$, ii) the number of latent classes, iii) the class-level predictors (if any), and iv) the classification rule that flags a school as sorter. We discuss these choices and our approach to making them in turn:

1. For modelling the distribution of $S_{s}$, we opted for fitting a FMM tobit to account for the censoring of $S_{s}$ at 1 . For other characteristics or in other datasets where $S_{s}$ shows less censoring, one can always fit beta or linear regression FMMs instead. In the TEPS data all these alternatives yield similar results.

2. We chose the number of latent classes that minimizes the Bayesian Information Criterion. In the TEPS data this was a 3-class model. Of these three classes,

\footnotetext{
${ }^{5}$ We have also worked on procedures that detect sorter schools based on several $P_{s}$ indices to detect, for example, one type of sorter school that sorts students to classrooms based on test scores, and a second type that sorts students based on their history of truancy - using unsupervised machine learning techniques such as hierarchical cluster analysis.
} 
only one had a conspicuously large predicted mean for $S_{s}$, which was very close to 1 . We identified this as the latent class of sorter schools. The other two classes had much lower predicted means for $S_{s}$, both close to 0.5. Using the Akaike Information Criterion we would have chosen a 2-class model instead; a sorter latent class with a predicted very close to 1 and a non-sorter latent class with predicted close to 0.5. Both models would have classified schools near-identically. Models with more than 3 latent classes did not improve fit much but did increase optimization complexity and often had issues converging.

3. We chose school-level class predictors that were significantly related to $S_{s}$. In the TEPS data these are schools means for: children who report being in academically gifted classrooms, parents who push for their children to be assigned to particular classrooms, ethnic minority students, private tutoring lessons before joining junior high school, and two measures of baseline student effort. All these measures were positive predictors of belonging to the sorter latent class, most of the statistically significant at conventional levels. These predictors meaningfully improved the model performance and, since models with and without class predictors are nested, one can make the choice to include these in the final model specification based on a likelihood ratio test.

4. For flagging schools as sorters we constructed for each school the probability of belonging to the sorter latent class $P_{s}$ - the class with predicted $S_{s}$ close to 1 - based on the FMM estimates with class predictors. We then opted for classifying sorter schools as schools which were most likely to belong to the sorter latent class than to any other class. Different thresholds can of course be justified, but this is a reasonable one with a clear a priori justification. Our model results are not sensitive to other reasonable classification thresholds such as $P_{s}$ being larger than the sum of all other predicted class probabilities.

Table C.19 shows the results of the Finite Mixture Model estimation in the TEPS data. The latent class marginal means and variances suggest that the third latent class clearly identifies sorter schools, and the posterior latent class probability for this class suggests that 24.6 percent of schools might be sorters. The latent class predictor coefficients are also consistent with the third latent class identifying sorter schools, and most of these predictors are statistically significant. 
Table C.19: The Fishing Algorithm: Finite-Mixture Model Estimates

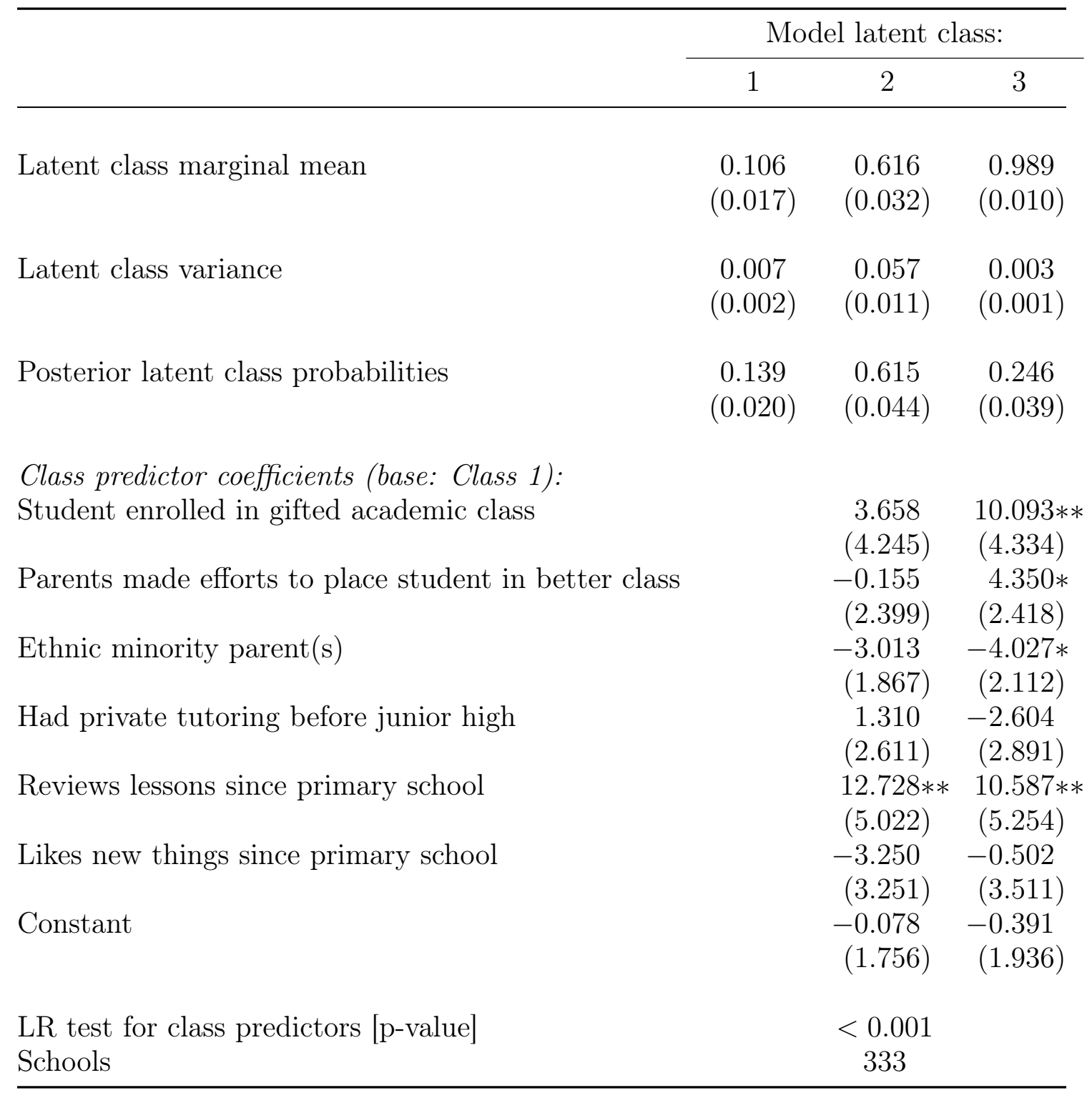

Note: Finite Mixture Model (FMM) estimates in the complete TEPS sample of 333 schools using school-level data. The dependent variable is modeled via a Tobit, and class posterior predicted probabilities of an FMM model without class covariates are used as initial values to improve model convergence. ***, ** and * mark estimates statistically different from zero at the 90\%, 95\% and 99\% confidence level. 
Figure 3.2 in Section 3.4.2 shows the schools eventually flagged as sorters by our Fishing Algorithm across the distribution of $S_{s}$. We overlay the probability of being a sorter school $P_{s}$ (on the right y-axis) in a scatterplot, with 0.5 as a dashed horizontal reference line. Our Fishing Algorithm flags 106 schools where $P_{s}$ is largest as sorters. As expected, most flagged schools have $S_{s}>0.9$, though a few schools with lower values of $S_{s}$ are also flagged. In the TEPS data, the algorithm failed to identify non-sorter schools with very high $S_{s}$. It is possible, of course, that all these schools with high $S_{s}$ are in fact sorters, yet it is more likely that the FMM class predictors are just not strong enough to discern the non-sorters among this group. As discussed above and in Section 3.4.2, this could lead to over-trimming and in fact we do see some evidence of this in Table 3.2, which shows sorting and balancing tests in our sample trimmed of the 106 schools flagged as sorters. Yet evidence of over-trimming is not strong enough to be concerning.

As a final point in this appendix, we show that out applying our Fishing Algorithm in the TEPS data does not introduce any evident selectivity in our estimation samples. Table C.20 shows that our initial sample including all the TEPS data remains very similar to our trimmed sample - which includes all information from schools not flagged as sorters by our Fishing Algorithm, and also remains similar to the our most restricted estimation sample - which includes only students for which we observe test scores, educational inputs and other key characteristics in both TEPS waves. The only difference is that the more restrictive samples have slightly larger schools, which can easily happen if smaller schools are more likely to systematically sort students into classrooms (because e.g., they are more likely to be special schools with exeptions to the national mandate, or have lower invigilance). 
Table C.20: Summary Statistics of Key Variables in TEPS Across Samples

\begin{tabular}{|c|c|c|c|}
\hline & \multicolumn{3}{|c|}{ Mean of characteristics in sample: } \\
\hline & $\begin{array}{c}\text { TEPS } \\
(1)\end{array}$ & $\begin{array}{c}\text { Trimmed } \\
(2)\end{array}$ & $\begin{array}{c}\text { Estimation } \\
(3)\end{array}$ \\
\hline \multicolumn{4}{|l|}{ Characteristics: } \\
\hline Student test scores (unstandardized) & 40.85 & 40.59 & 41.05 \\
\hline Female student & 0.50 & 0.50 & 0.48 \\
\hline Student year of birth & 1988.59 & 1988.59 & 1988.60 \\
\hline No. of siblings of student & 1.77 & 1.77 & 1.75 \\
\hline Responding parent is female & 0.64 & 0.64 & 0.63 \\
\hline Ethnic minority father & 0.05 & 0.05 & 0.04 \\
\hline Two-parent household & 0.86 & 0.86 & 0.87 \\
\hline Father's birth year & 1958.61 & 1958.67 & 1958.63 \\
\hline Father has post-secondary education & 0.12 & 0.12 & 0.12 \\
\hline Unemployed father & 0.11 & 0.11 & 0.10 \\
\hline \multicolumn{4}{|l|}{ Household monthly income is } \\
\hline NT $\$ 20,000$ or less & 0.11 & 0.11 & 0.10 \\
\hline NT $\$ 20,000-N T \$ 50,000$ & 0.41 & 0.41 & 0.41 \\
\hline NT $\$ 50,000-\mathrm{NT} \$ 100,000$ & 0.35 & 0.35 & 0.36 \\
\hline More than NT $\$ 100,000$ & 0.14 & 0.14 & 0.14 \\
\hline Classroom size & 35.88 & 36.41 & 36.51 \\
\hline Male-to-female students ratio & 0.52 & 0.52 & 0.52 \\
\hline Number of sampled students in school & 67.03 & 67.66 & 65.60 \\
\hline School size & 4,122 & 5,040 & 5,220 \\
\hline School sampling rate & 0.19 & 0.18 & 0.17 \\
\hline No. of students (approx.) & 20,055 & 14,044 & 11,029 \\
\hline
\end{tabular}




\section{C.4 Fishing Algorithm in Simulated Data}

In this appendix, we use simulated data to validate our fishing algorithm and investigate its performance. Ideally, we would want to provide evidence from MonteCarlo simulations of the performance of the algorithm in detecting schools that systematically sort students into classrooms. Unfortunately, we cannot provide Monte-Carlo evidence over many simulations - say, over 10,000 realizations of the same data generating process - since i) Steps 4 and 5 of the algorithm require making some decisions that cannot be automatized (see Box 1 in C.3) and ii) the finite mixture models in Step 4 often have convergence issues that demand making additional decisions, such as trying out different optimization procedures, grid search across different parameter values, or try out various initial latent class probabilities. Nevertheless, we provide as extensive evidence of the performance of our fishing algorithm as our setting allows, and highlight lessons learned along the way. These lessons will prove useful to researchers intending to implement our fishing algorithm in their data. In addition, we have coded flexible simulation programs in Stata which will be available with the published version of this paper.

\section{C.4.1 The Data Generating Process (DGP) for our Simulations}

We simulate data that closely follows our empirical setting in Taiwan: students are divided into schools and, within schools, assigned to classrooms. The only characteristic that varies across students is their ability. Classrooms are simple groupings of students within schools. Students in the same classroom can end up being similar or dissimilar to one another, depending partly on chance and partly on whether their school randomly assigns students to classrooms. Schools can differ in two dimensions: whether they actively sort students of similar ability into classrooms (sorter schools) or not (non-sorter schools), and-for sorter schools - the degree to which they sort students into classrooms. In addition, we also simulate a school-level variable that predicts whether the school is sorting or non-sorting. These three parameters - the number of sorting schools, the strength of sorting within sorting schools, and the strength of the sorting school predictor - are the key parameters we vary across our simulations. All other parameters, such as school size and classroom size, are kept constant across DGPs.

Specifically, for each DGP we simulate data from 300 schools. We stochastically vary the number of students across schools between 50 and 70 with an independent uniform distribution, $U[50,70]$, mostly as a legacy for implementing the Guryan et al. 
(2009) sorting test. Their method accounted for a small negative bias in classical sorting tests by controlling for school-level leave-out-mean of student ability, but this correction only works well when there is variation in school size in the data. For our exercises, however, we implement instead the solution proposed by Jochmans (2020), who derives analytical expressions for this negative bias and proposes a biascorrected test with better power and implementable without school-size variation. Once we have schools filled with students, we assign ability to students according to ability $\sim U[0,1]$.

At this point, we randomly determine which schools are the sorting schools that sort students into classrooms based on ability, and which schools are non-sorting schools. The number of sorting schools, $N_{\text {sorting }}$, is the first key parameter we vary across DGPs. Here we also generate predictor, the variable predicting whether a school is a sorter or a non-sorter, given by:

$$
\text { predictor }=1\{\text { sortingschool }\} \times p+U[0,1] \times(1-p)
$$

where $1\{$ sortingschool $\}$ is a dummy variable which flags sorting schools, $p \in[0,1]$ is a predictor strength parameter, and $U[0,1]$ is another independent random uniform. If $p$ equals 1 , predictor will be a perfect determinant of whether a school is systematically sorting students into classrooms; if $p$ equals zero, predictor will be completely uninformative for school type. The predictor strength $p$ is the second key parameter we vary across DGPs.

Within each school we then sort students based on the sortingstrength parameter in this school, and then sequentially assign them to similar-sized classrooms of roughly 15 students. sortingstrength is key for simulating student sorting into classrooms for some schools but not others, as is defined as:

$$
\text { sortingstrength }= \begin{cases}\theta \text { ability }+(1-\theta) U[0,1] & \text { if student is in a sorting school } \\ U[0,1] & \text { otherwise }\end{cases}
$$

where $\theta \in[0,1]$ is the parameter that governs the sorting strength in sorting schools and we vary it across DGPs. The way this parameter works is best explained with a few examples.

When $\theta$ is one, sortingstrength equals ability in sorting schools and a random 
uniform for non-sorting schools. This implies that in sorting schools, students will be assigned to classrooms based on their ability, with the first classroom having the top 15 students, the second classroom the top 15 among the remaining students, and so on. This simulates very strong sorting of students into classrooms in a scenario we refer to as "perfect stacking". In non-sorter schools, students will be randomly assigned to classrooms. If instead $\theta$ is zero, sortingstrength becomes a random uniform for all schools (sorting and non-sorting, resulting in random assignment of students to classrooms across the entire simulated data. Values of $\theta$ between zero and one will vary the strength of sorting, or stacking, in sorting schools while keeping random assignment in non-sorting schools. This $\theta$ is the second key parameter we vary across DGPs.

To make sure there is enough identifying variation in peer aggregates of ability, we ensure that no classroom has fewer than 10 students - which can happen because initial classroom size is set to 15 but variation in school size can occasionally lead to a classroom of fewer than 10 students. When this happens, we randomly redistribute students in these small classrooms to all other remaining classrooms, such that classrooms are always larger than 15 students.

We test the performance of our fishing algorithm using simulated data from three versions of our DGP that correspond to cases of particular interest for an econometrician interested in applying our method:

1. $N_{\text {sorting }}=50 ; \theta=0.8 ; p=0.8: 50$ strongly sorting schools with a good sorting predictor

2. $N_{\text {sorting }}=50 ; \theta=0.8 ; p=0.1: 50$ strong sorting schools with a weak sorting predictor

3. $N_{\text {sorting }}=300 ; \theta=0.15:$ all schools are weak sorters, with a good sorting predictor

The first is an ideal case where the researcher can detect the few schools that violate random assignment in the data, and has access to good enough predictors to detect whether a school is sorting systematically students. The second case showcases the limitations of our fishing algorithm when the researcher does not have access to reasonable predictors of sorter schools. The third case simulates the unfortunate situation where all schools sort students into classrooms, enough to invalidate random assignment in the data but with no hopes of being able to fish out sorter schools with our method-or any other for that matter. 


\section{C.4.2 Performance of the Fishing Algorithm}

After producing data using this DGP, we then i) test the degree of sorting in the simulated data, ii) run our fishing algorithm following the steps in Box 1, iii) evaluate the performance of our fishing algorithm in detecting sorter schools in the simulated data, and iv) estimate the degree of sorting in the data once the detected sorter schools are removed. These four sets of results are presented in Panels A, B, C and $\mathrm{D}$ in the tables below.

We simulate five different realizations of each DGP and present the results of our fishing algorithm for each. For each simulation, we present our results in columns (1) through (5) of the tables below. The downslide of this approach is that it produces less systematic evidence of the performance of our algorithm than would Monte Carlo simulations. The upside, apart from being feasible, is that we can demonstrate the several decisions required from the researcher to use our method, explain the reasoning behind them, and showcase results of situation when, by chance, our method does not perform well.

\section{Case 1: Few Strong Sorter Schools and a Strong Class Predictor}

Table C.21 shows the performance of the Fishing algorithm in five simulated datasets with 50 strongly sorting schools and access to a good predictor for whether schools are sorters. Panel A shows Jochmans' (2020) sorting test t-statistic estimated using the simulated student-level data. When positive and larger than critical values of the standard normal distribution, these t-statistics indicate positive sorting of students into classrooms based on ability. As expected, our simulated data shows strong evidence of sorting (first row) and this evidence is coming solely from the few sorter schools (second and third rows).

Panel B shows the steps to select the best Finite Mixture Models (FMM) to detect sorter schools. These FMMs are estimated using school-level data where the outcome is our measure of ability concentration in classrooms $\left(S_{s}\right.$, see C.3). We first estimate FMMs with 2, 3, and 4 potential latent classes. We select the best among these models based on goodness of fit, using the smallest Bayesian Information Criteria (BIC); the BIC of the preferred model is marked in bold in each column. 
Table C.21: Fishing Algorithm Performance in Five Simulated Datasets with 50 Strongly Sorting Schools ( $N_{\text {sorting }}=50, \theta=0.8$ ) and Access to a Good Predictor for Whether Schools Are Sorters $(p=0.8)$

Simulation number $=$

(3)

(4)

$(5)$

Panel A: Sorting t-statistic in student-level data if DGP were known

Jochmans (2020) sorting t-statistic:

for all schools

6.4

6.6

6.6

6.9

6.7

for non-sorter schools

$-1.2$

$-0.5$

$-0.4$

1.7

0.1

for sorter schools

6.6

6.7

6.8

6.7

6.8

Panel B: Finite Mixture Model selection on school-level data

Model BIC for:
2 latent classes
3 latent classes
4 latent classes

$\begin{array}{lllll}\mathbf{3 1 6 . 7} & \mathbf{3 3 6 . 7} & 326.6 & \mathbf{3 1 3 . 4} & 327.8 \\ 327.3 & 344.5 & \mathbf{3 2 2 . 4} & 320.6 & \mathbf{3 2 7 . 0} \\ 318.6 & 350.5 & 334.6 & 325.3 & 330.3\end{array}$

$0.000 \quad-\quad-\quad 0.000 \quad-$

Predicted sorting strength measure for:

$\begin{array}{llllll}\text { class } 1 & 0.48 & 0.13 & 0.09 & 0.53 & 0.11 \\ \text { class } 2 & \mathbf{1 . 0 2} & \mathbf{0 . 7 3} & 0.53 & \mathbf{1 . 0 1} & 0.55 \\ \text { class } 3 & - & - & \mathbf{1 . 0 3} & - & \mathbf{1 . 0 2} \\ \text { class } 4 & - & - & - & - & -\end{array}$

Panel C: Selected FMM model performance for defier classification

\begin{tabular}{llllll}
\hline Schools identified as defiers & 50 & 225 & 76 & 50 & 71 \\
Correctly classified schools (as \%) & 100.0 & 41.7 & 91.3 & 100.0 & 93.0 \\
Pr[Non-sorter school | Defier] & 0.0 & 77.8 & 34.2 & 0.0 & 29.6 \\
Pr[Sorter school | Complier] & 0.0 & 0.0 & 0.0 & 0.0 & 0.0
\end{tabular}

Panel D: Sorting t-statistics in student-level data in classified schools

\begin{tabular}{llllll}
\hline Jochmans (2020) sorting t-statistic: & & & & & \\
for classified complier schools & -1.2 & $\mathbf{- 6 . 7}$ & $\mathbf{- 4 . 2}$ & 1.7 & $\mathbf{- 3 . 9}$ \\
for classified defier schools & $\mathbf{6 . 6}$ & $\mathbf{7 . 0}$ & $\mathbf{7 . 1}$ & $\mathbf{6 . 7}$ & $\mathbf{7 . 1}$
\end{tabular}

In Panels $A$ and $D$, numbers in bold mark values larger than the $5 \%$ critical value in the reference a standard normal distribution. In Panel B, numbers in bold correspond to the smallest Bayesian Information Criterion (BIC) and the largest predicted outcome mean, used to select the preferred model, and numbers in italics correspond to models that did not comply with convergence criteria. A missing Likelihood Ratio (LR) test p-value is missing in Panel B, indicating that either the model using sorting predictors for the latent classes or the model without predictors did not converge (almost always the former). 
FMMs often have convergence issues - one of the reasons why we cannot produce complete Monte Carlo evidence in this Appendix. We mark models that failed to converge in italics. After choosing the preferred number of latent classes based on the BIC, we then choose whether the preferred model will include the variable predictor as a latent class predictor. For this, we estimate FMMs with and without this latent class predictor and use a Likelihood Ratio (LR) test to choose between these nested models. Rejecting the null that the models are equal leads us to choose the model that includes predictor as a latent class predictor. Here too, we have missing values for the p-value of this LR test when either model does not converge. Finally, we show the marginal means for each class - the average outcome predicted for schools in each latent class - in the preferred model. These correspond to the predicted level of classroom concentration in schools in each latent class. We interpret the latent class(es) with unusually high predicted means as those that identify sorter schools. These are also marked in bold.

There are three broad lessons from Panel B of Table C.21. First, models with two or three latent classes are generally preferred, and models with four latent classes often have convergence issues. This relatively simple latent class structure is partly a direct result of our DGPs - which have, in fact, two latent classes of sorter and non-sorter schools - yet it confirms that the FMMs do not tend to over-fit latent classes in the data. Second, models that use latent class predictors also suffer convergence issues. This is a potential shortcoming, since we later show that these predictors can meaningfully improve the performance of our fishing algorithm. Third, there is almost always a latent class with a clearly larger predicted sorting strength, and the closer this prediction is to 1 it is that this class identifies sorter schools.

Panel C summarizes the performance of the preferred FMM for classifying sorter schools - schools which, in violation of random assignment, systematically sort students into classrooms. We flag sorter school as those for which the posterior latent class probability for the sorter class is larger than the sum of all the other posterior latent class probabilities, as described in C.3. We report four standard indicators to describe the performance of our algorithm at detecting schools that systematically sort students into classrooms: i) the number of schools classified as sorters (out of 300), ii) the percentage of schools that are correctly classified as sorter schools by the fishing algorithm and are truly sorter schools, iii) the probability of being wrongly classified as a sorter school and actually being a non-sorter school (false positives), and iv) the probability of being classified as a non-sorter school and truly being a sorter school (false negative). Overall, the algorithm performs very well for this DGP: in 2 out of 5 simulations, the algorithm perfectly separates sorter 
and non-sorter schools (col. (1) and col. (4)), and in 2 additional simulations it identifies no false negatives and only a few false positives (col. (3) and col. (5)).

In column (2) the fishing algorithm somewhat fails: the algorithm indicates that the majority of schools as sorters, over $50 \%$ of which are actually non-sorter schools. This failure is not complete, however, in the sense that the algorithm only becomes too stringent, but does not misclassify sorter schools as compliant. The good news is that our exercise reveals why this failure occurred: the selected FMM model in this instance could not use as a latent class predictor to identify the latent class with sorter schools, and consequently the predicted sorting strength for this model is 0.73 , well below that of all other models. The lesson for researchers applying our method here is that having access to a good predictor of whether schools are sorting will meaningfully improve the performance of our fishing algorithm, even in settings with few strongly sorting schools. Panel D shows the sorting test performance from Jochmans (2020) back in the student-level simulated data in nonsorter schools - those classified as non-sorters by the fishing algorithm. For the two models with perfect performance (Columns (1) and (4)), we see that the t-statistics match the non-sorter t-statistics in Panel A. For the other three models, we see negative and significant t-statistics (Columns (3) and (5)); much more negative for the worst-performing model (Column (2)).

Negative and significant t-statistics of sorting tests become increasingly more frequent as the rate of false positives increases - that is, the probability of wrongly classifying non-sorting schools as sorter schools. In C.3, we call this situation "over-trimming", corresponding to situations when the fishing algorithm wrongly excludes schools that are actually compliant with random assignment. The issue with over-trimming is that it could lead to censuring the distribution of peer effects.

Importantly, our algorithm can be used as a diagnostic tool for over-trimming, since a clear sign of over-trimming is a "flipping" sign of Jochmans (2020)'s t-statistic: a positive and significant t-statistic in the untrimmed data (as in Panel A) and a negative and significant t-statistic in the trimmed data (as in panel D). When this occurs, we suggest going back to the FMM specification to improve the classification performance, either my changing the number of classes or by exploring additional and hopefully better class predictors. An important early sign that the algorithm is able to discern sorter from non-sorter schools is a high predicted sorting strength for at least one latent class, like in Column (1), and Columns (3) to (5) in Panel B. 


\section{Case 2: Few Strong Sorter Schools and a Weak Class Predictor}

Table C.22 shows the performance of our algorithm in a DGP where there are still 50 strongly sorting schools, but the researcher only has access to a much weaker predictor of whether schools are sorters. This reflects the situation of researchers with either limited data or limited institutional knowledge to construct such predictors.

Panel A confirms that our simulated data conform to the intended DGP. Panel B illustrates that i) in these data the FMMs generally choose simpler 2-class structures, that ii) even with a much weaker predictor the FMMs tend to prefer models with class predictors, but that iii) the predicted sorting strength for the high-sorting class is much weaker (between 0.73 and 0.78 ) than when a good class predictor is available (in Table C.21). As a direct result, Panel C shows much higher rates of misclassification, driven entirely by a higher rate of non-sorter schools identified as sorters; all sorter schools are always correctly classified. As explained above, this will lead to over-trimming, Panel D confirms the presence of over-trimming: we find strong evidence of negative sorting in classified non-sorter schools, and positive sorting in the classified sorter schools. In sum, Table C.22 corroborates the importance of having a strong sorting predictor for good performance of our fishing algorithm, but it also indicates two useful diagnostics that can tell the researcher whether the algorithm is likely to be performing poorly: a relatively low predicted sorting strength for the high-sorting latent class, and a strong flipping for the Jochmans (2020) sorting t-statistic for the classified non-sorters subsample. Compared to the findings of Table C.21, the findings of Table C.22 indicate that finding one or multiple strong class predictors is crucial for preventing the algorithm from over-trimming the sample. 
Table C.22: Fishing Algorithm Performance in Five Simulated Datasets with 50 Strongly Sorting Schools ( $\left.N_{\text {sorting }}=50, \theta=0.8\right)$ but Only a Weak Predictor for Whether Schools Are Sorters $(p=0.1)$

Simulation number $=$

(2)

(3)

(4)

(5)

Panel A: Sorting t-statistic in student-level data if DGP were known

Jochmans (2020) sorting t-statistic:

for all schools

$6.7 \quad 7.0$

6.5

6.7

6.6

for non-sorter schools

$0.6 \quad 1.6$

$-1.8$

$-0.4$

$-0.8$

for sorter schools

$6.7 \quad 6.8$

6.8

6.8

6.7

Panel B: Finite Mixture Model selection on school-level data

\begin{tabular}{llllll}
\hline Model BIC for: & & & & & \\
2 latent classes & $\mathbf{3 0 7 . 5}$ & $\mathbf{3 2 2 . 9}$ & $\mathbf{3 2 4 . 1}$ & $\mathbf{3 0 8 . 7}$ & $\mathbf{3 2 3 . 4}$ \\
3 latent classes & 317.2 & 325.4 & 331.5 & 313.3 & 331.4 \\
4 latent classes & 329.7 & 336.9 & 342.0 & 330.1 & 346.1 \\
& & & & & \\
LR for model with sorting predictor (p-value) & 0.000 & 0.000 & 0.000 & 0.000 & - \\
& & & & & \\
Predicted sorting strength measure for: & & & & & \\
class 1 & 0.18 & 0.17 & 0.19 & 0.20 & 0.22 \\
class 2 & $\mathbf{0 . 7 3}$ & $\mathbf{0 . 7 4}$ & $\mathbf{0 . 7 8}$ & $\mathbf{0 . 7 8}$ & $\mathbf{0 . 7 7}$ \\
class 3 & - & - & - & - & - \\
class 4 & - & - & - & - & -
\end{tabular}

Panel C: Selected FMM model performance for defier classification

\begin{tabular}{llllll}
\hline Schools identified as defiers & 228 & 233 & 192 & 199 & 207 \\
Correctly classified schools (as \%) & 40.7 & 39.0 & 52.7 & 50.3 & 47.7 \\
Pr[Non-sorter school | Defier] & 78.1 & 78.5 & 74.0 & 74.9 & 75.8 \\
Pr[Sorter school | Complier] & 0.0 & 0.0 & 0.0 & 0.0 & 0.0
\end{tabular}

Panel D: Sorting t-statistics in student-level data in classified schools

\begin{tabular}{llllll}
\hline Jochmans (2020) sorting t-statistic: & & & & & \\
for classified complier schools & $-\mathbf{5 . 9}$ & $\mathbf{- 5 . 2}$ & $\mathbf{- 8 . 8}$ & $\mathbf{- 7 . 8}$ & $\mathbf{- 6 . 8}$ \\
for classified defier schools & $\mathbf{7 . 1}$ & $\mathbf{7 . 2}$ & $\mathbf{7 . 1}$ & $\mathbf{7 . 2}$ & $\mathbf{7 . 0}$
\end{tabular}

$\overline{\text { In Panels } A \text { and } D \text {, numbers in bold mark values larger than the } 5 \% \text { critical value in the reference }}$ a standard normal distribution. In Panel B, numbers in bold correspond to the smallest Bayesian Information Criterion (BIC) and the largest predicted outcome mean, used to select the preferred model, and numbers in italics correspond to models that did not comply with convergence criteria. A missing Likelihood Ratio (LR) test p-value is missing in Panel B, indicating that either the model using sorting predictors for the latent classes or the model without predictors did not converge (almost always the former). 


\section{Case 3: Weak but Generalized Sorting}

Table C.23 shows the performance of our fishing algorithm in a DGP that simulates sorting in all schools, weaker relatively to the previous DGP but strong enough that it would be detected by Jochmans (2020) t-statistic. This corresponds to setting with generalized violations of random assignment, such that no natural experiment could be salvaged from the data using our algorithm.

Panel A confirms that our simulated data conforms to this setting, producing tstatistics that significant around the $1 \%$ level. Panel B shows that i) the FMMs in this setting tend to choose 3 - and 4-class structures, ii) the sorter school predictor is never statistically significant at conventional levels, which was to be expected since all schools are sorters, and iii) the predicted sorting strength in the high-sorting latent class is higher than in Table C.22 but lower than in Table C.23. This high predicted sorting strength results in relatively few schools identified as sorters, as show in Panel C. Because the FMMs classify as sorters the schools where the strongest sorting occurs, Panel D again shows strong flipping in the Jochmans (2020) t-statistic.

Overall, Table C.23 indicates that situations where all schools sort students into classrooms (generalized sorting) compared to clustered sorting (cases 1 and 2) are characterized by i) relatively complex latent class structures, ii) relatively low model fit yet iii) high predicted sorting strengths for the high-sorting latent class even in the absence of good sorting school predictors (Panel B), and iv) flipping of the Jochmans (2020) sorting t-statistic for identified non-sorter schools (Panel D). 
Table C.23: Fishing Algorithm Performance in Five Simulated Datasets with All Weakly Sorting Schools ( $N_{\text {sorting }}=300, \theta=0.15$ )

Simulation number $=$

(2)

(3)

(4)

(5)

Panel A: Sorting t-statistic in student-level data if DGP were known

Jochmans (2020) sorting t-statistic:

for all schools

3.6

for non-sorter schools

for sorter schools

$-$

3.6

$4.5 \quad 4.3$

4.6

3.0

4.5

4.3

4.6

3.0

Panel B: Finite Mixture Model selection on school-level data

\begin{tabular}{llllll}
\hline Model BIC for: & & & & & \\
2 latent classes & 105.0 & 103.7 & 85.4 & 104.9 & 99.4 \\
3 latent classes & $\mathbf{9 3 . 4}$ & 92.5 & $\mathbf{6 6 . 3}$ & $\mathbf{1 0 3 . 1}$ & 95.0 \\
4 latent classes & 95.1 & $\mathbf{8 4 . 9}$ & 70.4 & 88.7 & $\mathbf{9 1 . 9}$ \\
& & & & & \\
LR for model with sorting predictor (p-value) & 0.818 & 0.280 & 0.170 & 0.066 & 0.850 \\
& & & & & \\
Predicted sorting strength measure for: & & & & & \\
class 1 & 0.16 & 0.15 & 0.09 & 0.13 & 0.05 \\
class 2 & 0.56 & 0.41 & 0.52 & 0.52 & 0.38 \\
class 3 & $\mathbf{0 . 9 3}$ & 0.72 & $\mathbf{0 . 9 2}$ & $\mathbf{0 . 9 0}$ & 0.81 \\
class 4 & - & $\mathbf{0 . 9 5}$ & - & - & $\mathbf{0 . 9 6}$
\end{tabular}

Panel C: Selected FMM model performance for defier classification

\begin{tabular}{lllllll}
\hline Schools identified as defiers & 79 & 66 & 82 & 107 & 46
\end{tabular}

$\begin{array}{llllll}\text { Correctly classified schools (as \%) } & 26.3 & 22.0 & 27.3 & 35.7 & 15.3\end{array}$

$\begin{array}{llllll}\text { Correctly classified schools (as \%) } & 26.3 & 22.0 & 27.3 & 35.7 & 15.3\end{array}$

$\operatorname{Pr}[$ Non-sorter school | Defier]

Pr[Sorter school | Complier]

Panel D: Sorting t-statistics in student-level data in classified schools

Jochmans (2020) sorting t-statistic:

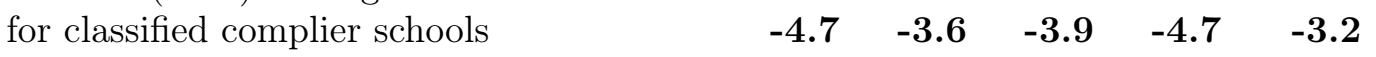

$\begin{array}{llllll}\text { for classified defier schools } & 6.7 & 6.9 & 7.5 & 7.5 & 5.6\end{array}$

$\overline{\text { In Panels } A \text { and } D \text {, numbers in bold mark values larger than the } 5 \% \text { critical value in the }}$ reference a standard normal distribution. In Panel B, numbers in bold correspond to the smallest Bayesian Information Criterion (BIC) and the largest predicted outcome mean, used to select the preferred model, and numbers in italics correspond to models that did not comply with convergence criteria. A missing Likelihood Ratio (LR) test p-value is missing in Panel $B$, indicating that either the model using sorting predictors for the latent classes or the model without predictors did not converge (almost always the former). 


\section{C.4.3 A Practitioner's Guide for Researchers Wanting to Use our Fishing Algorithm}

Our fishing algorithm combines several intuitive steps which are nonetheless somewhat technically complex. Drawing on the lessons illustrated in this section and on our own experience in developing this algorithm, we make the following suggestions to researchers intending to use our method:

1. Strive to find predictors of whether a school sorts students into classroom, even if these predictors are not perfect. Good predictors will meaningfully improve the performance of our method, even if it can still be applied without them. Place more trust in applications with institutionally sound sorting predictors that are also statistically and quantitatively strong inputs in your latent class model.

2. Your latent class that captures sorting schools will have a predicted sorting strength close to or exceeding 1. By the nature of our measure of sorting strength, sorting schools should have strengths very close to or greater than 1 . Latent classes with predicted sorting strengths much below 1 are therefore more likely to also capture non-sorting schools, increasing over-trimming problems. If your latent class model is not identifying classes with high enough predicted sorting strengths, this could be a sign that i) the class structure is not complex enough (solved by testing models with more latent classes), ii) your sorting school predictors are not good enough (solved by finding better predictors or a better structure for existing ones), or iii) sorting is too widespread in your data (only solved, sadly, by finding other data that reflects a better natural experiment).

3. Beware of sorting test flipping. Sorting test flipping - a large and positive sorting t-statistic in the whole data and a large and negative sorting t-statistic in the subsample of identified non-sorter schools - is a sign of either overtrimming or widespread sorting. 



\section{Appendix to Chapter 4}

\section{D.1 Data Preparation}

In this section, I explain how I construct my estimation sample, going from registry data to a dyadic sibling pair dataset. The estimation sample is a dyadic dataset, containing for every child, information about his closest older sibling. I first construct a dataset containing for each pupil her rank among her primary school classmates, along with information at the school-cohort level such as cohort size for each subject and for the complete standardized test. I start by appending yearly registries into a dataset of repeated cross-sections of pupils taking the standardized test. Few pupils repeat the test; I reshape the data into wide format and focus only on the first test, as I will later exclude test repeaters from my estimation sample. I keep these pupils for now, as I am interested in children's rank in their cohort, including classmates who are retaking the test. I set this dataset aside for later merging.

To construct a dataset of sibling which I later reshape into dyadic sibling pair format, I start from the national population registry, which records every person residing in the Netherlands. I keep only persons born since 1980, either born in the Netherlands or abroad; at this stage I have roughly 16.2 million persons. I merge in parental identifiers using family records, which yields 7,437,636 matches. I construct a mother identifier, to mark all children recorded with the same mother; only 32,636 children have no recorded mother.

I then merge education test taking registry information, and the dataset containing rank and school-cohort information. This match yields 1,670,714 matched observations; non-matched observations are mostly children residing in the Netherlands with no test taking information ( 5.75 million) and few are children with test taking information but not recorded as residents of the Netherlands (13,618 children). As records for the standardized test start in 2006, most non-matched children are simply born too early. 
At this stage, I make the following sample selection decisions that will later allow me to reshape the data into a dyadic sibling pair dataset: I drop all children in 1) families where no child ever took the test $(4,761,458$ children $), 2)$ families with at least once two children taking the test the same year (77,424 children), and 3) families with at least one test repeater child (12,424 children). I drop 4,852,653 unique child observations, and have a remaining sample of 2,643,323 children including 955,846 children with no test taking information.

I then construct sibling pair identifiers. I follow Karbownik and Özek (2019) and Black et al. (2017) and define a sibling as a child born to the same mother - and for $87 \%$ of children in the Netherlands, this also means living in the same residence. For every child, I assign a pair identifier that is only shared by the next oldest sibling who took the standardized test last, and who has not taken his first attempt on the standardized test at the same time as another sibling. Importantly, a child can be a younger child for one pair with pair identifier 1, and also be an older child for another pair with pair identifier 2 . To be able to reshape the data into dyadic format, I expand observations for children simultaneously belonging to two pairs. I then reshape the data to obtain one observation per sibling pair, in which the older sibling is always sibling 1 and the younger sibling is always sibling 2 . In this process, I am able to construct 531,780 unique pairs, for 975,256 unique children (1,063,560 observations after including expanded observations). These pairs constitute the baseline estimation sample.

Before reshaping the data into a dyadic format, I merge additional information in wide format for each child corresponding to the year immediately preceding the primary school exit test: 1) maternal and paternal employment status, working hours, earned income and taxable income, 2) maternal and paternal residence, 3) later life education records such as secondary school attendance, track and major, graduation, time to graduation, and post-secondary education attendance, and major. After merging this information to every single child in the sibling pairs dataset, I reshape the data into dyadic format and construct final variables which required information for both siblings of a pair to be constructed, such as age difference, same gender, and likelihood of having had the same primary school teacher. 


\section{D.2 Empirical Specification}

\section{Which Fixed Effects Should We Include?}

Table D.1 shows how the effect of older sibling rank on score varies across models including different types of fixed-effect. Columns (4) to (6) show our estimates of the effect of older sibling rank on scores across different types of fixed effect specifications, when I restrict the sample to siblings attending the same primary school. I find similar estimates when my model includes two-way fixed effects for the younger child's cohort and school (Column (4)) and when I add a fixed effect for the older sibling's cohort (Column (5)). I find a small estimate when I include a cohort-by-school fixed effect for the younger child's cohort and school, and a fixed effect for the older sibling's cohort. In this final specification, I find that a $1 \%$ increase in sibling's rank increases my standardized score by 1.4 point (corresponding roughly to $0.14 \mathrm{SD}$ ). Columns (1) to (3) show corresponding estimates when I don't restrict to siblings attending the same schools. Overall, estimates are similar, which is not surprising since $82 \%$ of siblings attend the same primary school.

Following Elsner and Isphording (2017), my preferred specification includes a cohortby-school fixed effect for the younger sibling, as this specification yields the most conservative estimates for the effect I am interested in estimating. Importantly, including one type of fixed effect over another has conceptual implications. A model with two-way fixed effect captures separately school-specific heterogeneity and cohort-specific heterogeneity. A model with cohort-by-school fixed-effect allows for nested heterogeneity. 
Table D.1: The Effects of Older Sibling Rank on Younger Sibling Test Scores Across Specifications

\begin{tabular}{|c|c|c|c|c|c|c|}
\hline Sample restrictions: & In same & e primary s & school & Likely & y same tear & acher \\
\hline Younger sibling outcome: & & & CITO $\mathrm{p}$ & points & & \\
\hline & $(1)$ & $(2)$ & $(3)$ & $(4)$ & (5) & (6) \\
\hline $\begin{array}{l}\text { Older sibling rank } \\
\text { in std. test score }\end{array}$ & $\begin{array}{l}2.533 * * * \\
(0.149)\end{array}$ & $\begin{array}{rl}* & 2.518 * * * \\
& (0.149)\end{array}$ & $\begin{array}{c}* 1.489 * * * \\
(0.173)\end{array}$ & $\begin{array}{l}* 2.463 * * * \\
(0.191)\end{array}$ & $\begin{array}{rl}* & 2.457 * * * \\
& (0.191)\end{array}$ & $\begin{array}{rl}* & 1.399 * * * \\
& (0.245)\end{array}$ \\
\hline Mean of dep.var & 536.02 & 536.02 & 536.02 & 535.75 & 535.75 & 535.75 \\
\hline SD of dep.var & 9.44 & 9.44 & 9.44 & 9.51 & 9.51 & 9.51 \\
\hline R-Squared & 0.26 & 0.26 & 0.37 & 0.27 & 0.27 & 0.43 \\
\hline Yg sib: Cohort FE & $\mathrm{Y}$ & $\mathrm{Y}$ & & $\mathrm{Y}$ & $\mathrm{Y}$ & \\
\hline Yg sib: School FE & $\mathrm{Y}$ & Y & & Y & $\mathrm{Y}$ & \\
\hline Old sib: Cohort FE & & $\mathrm{Y}$ & Y & & Y & Y \\
\hline Yg sib: school-by-cohort FE & & & $\mathrm{Y}$ & & & $\mathrm{Y}$ \\
\hline N. yg sib. schools & 6,144 & 6,144 & 6,144 & 5,950 & 5,950 & 5,950 \\
\hline Observations & 384,276 & 384,276 & 384,276 & 198,432 & 198,432 & 198,432 \\
\hline
\end{tabular}

Note: This table shows the results of dyadic regression analyses of the effect of older siblings' rank on younger siblings' standardized test score. Control variables: standardized test score of the older sibling (point dummies), number of parents with a migrant background (dummies), birth year of the younger sibling (dummies), household taxable income in the year preceding the younger sibling's test. Standard errors are clustered at the level of the younger sibling's school. *, **, and *** denote significance levels at the $10 \%, 5 \%$, and $1 \%$ respectively.

\section{Which Functional Form to Control for Sibling Absolute Ability?}

Table D.2 shows how our estimated effects vary depending on how we control for the older sibling's ability (in columns), across six different outcomes (in lines). This table shows results of regressions of the effect of older sibling rank on scores (line 1) and on teacher recommendations controlling linearly for the younger child's score (lines 2 to 6 ). For these regressions, I include school-by-cohort fixed effects for the younger sibling, older sibling cohort fixed effect, and restrict the sample to siblings likely to share the same primary school teacher. Column (1) shows our estimate when we control linearly for sibling standardized test score; Columns (2), (3) and (4) when I use respectively a quadratic, cubic and quartic polynomial; Column (5) when I use vintile dummies of raw score, and finally Column (6) when I use point dummies of the raw score.

Overall, we see that the effect size is relatively stable across controls of ability 
functional form for each outcome. When I study the effect of older sibling rank on standardized test scores, the estimate is smallest when controlling for sibling ability using a quadratic and cubic polynomial, but I prefer the specification with the point dummies because it is the most flexible specification. This specification also yields the most conservative estimates for the effect of older sibling rank on track recommendations, after controlling linearly for the younger child's score.

Based on my preferred specification, older sibling rank has a positive effect on standardized scores, but once I control for this score effect, there is no remaining effect on average on the track recommendations that younger siblings receive.

Table D.2: The Effects of Older Sibling Rank on Younger Sibling Test Scores Across Ability Functional Form

Control function:

Older sibling std. test score:

Linear Quadratic Cubic Quartic Vintile FE Point FE
$(2)$

(3)

(4)

(5)

(6)

Younger sibling Outcomes:

Std. test score

$$
\begin{array}{llllll}
2.821 * * * & 1.113 * * * & 1.113 * * * & 1.288 * * * & 1.761 * * * & 1.399 * * * \\
(0.217) & (0.242) & (0.242) & (0.244) & (0.242) & (0.245)
\end{array}
$$

Track recommendation:

\begin{tabular}{lcccccc} 
Academic & \multicolumn{2}{c}{$0.122 * * *-0.009$} & -0.009 & 0.004 & 0.009 & 0.009 \\
& $(0.007)$ & $(0.008)$ & $(0.008)$ & $(0.008)$ & $(0.008)$ & $(0.008)$ \\
General & $-0.036 * * *$ & $0.023 * *$ & $0.023 * *$ & 0.013 & 0.008 & 0.008 \\
& $(0.008)$ & $(0.009)$ & $(0.009)$ & $(0.009)$ & $(0.009)$ & $(0.009)$ \\
Vocational & $-0.031 * * *-0.018 *$ & $-0.018 *$ & -0.009 & -0.006 & -0.006 \\
& $(0.009)$ & $(0.010)$ & $(0.010)$ & $(0.010)$ & $(0.010)$ & $(0.010)$ \\
\hline Old sib: Cohort FE & $\mathrm{Y}$ & $\mathrm{Y}$ & $\mathrm{Y}$ & $\mathrm{Y}$ & $\mathrm{Y}$ & $\mathrm{Y}$ \\
Yg sib: school-by-cohort FE & $\mathrm{Y}$ & $\mathrm{Y}$ & $\mathrm{Y}$ & $\mathrm{Y}$ & $\mathrm{Y}$ & $\mathrm{Y}$ \\
N. young sib. schools & 5,950 & 5,950 & 5,950 & 5,950 & 5,950 & 5,950 \\
Observations & 198,432 & 198,432 & 198,432 & 198,432 & 198,432 & 198,432 \\
\hline
\end{tabular}

Note: This table shows the results of dyadic regression analyses of the effect of older siblings, rank on younger siblings' score and teacher subjective assessment, using various types of control function for older sibling overall test score. Each line and column represents one regression. Control variables: standardized test score of the older sibling, number of parents with a migrant background (dummies), birth year of the younger sibling (dummies), household taxable income in the year preceding the younger sibling's test. Regressions on younger sibling recommendations include an additional control variable for the standardized test score of the child (linear). Standard errors are clustered at the level of the younger sibling's school. Sample restriction: siblings likely to have the same primary school teacher. ${ }^{*},{ }^{*}$, and ${ }^{* * *}$ denote significance levels at the 10\%, 5\%, and 1\% respectively. 


\section{D.3 Additional Tables and Figures}

Table D.3: The Effects of Older Sibling Ranks on Younger Sibling Track Recommendation, Controlling for Older and Younger Sibling Overall Score

\begin{tabular}{lccc}
\hline Younger sibling outcome: & \multicolumn{3}{c}{ Track Recommendation: } \\
\cline { 2 - 4 } & $\begin{array}{c}\text { Academic } \\
(1)\end{array}$ & $\begin{array}{c}\text { General } \\
\text { Older sibling rank: }\end{array}$ & $\begin{array}{c}\text { Vocational } \\
(3)\end{array}$ \\
\cline { 2 - 4 } Overall rank & & & \\
& $(0.001$ & 0.008 & -0.006 \\
& & $(0.009)$ & $(0.010)$ \\
Dutch rank & $0.020 * *$ & 0.007 & 0.001 \\
& $(0.008)$ & $(0.009)$ & $(0.010)$ \\
& & & \\
Math rank & $0.022 * * *$ & 0.007 & -0.006 \\
& $(0.008)$ & $(0.009)$ & $(0.010)$ \\
\hline Mean of dep.var & 0.12 & 0.12 & 0.30 \\
SD of dep.var & 0.33 & 0.33 & 0.46 \\
Old sib: Cohort FE & $\mathrm{Y}$ & $\mathrm{Y}$ & $\mathrm{Y}$ \\
Young sib: school-by-cohort FE & $\mathrm{Y}$ & $\mathrm{Y}$ & $\mathrm{Y}$ \\
N. young sib. schools & 5,950 & 5,950 & 5,950 \\
Observations & 198,432 & 198,432 & 198,432 \\
\hline
\end{tabular}

Note: This table shows the results of dyadic regression analyses of the effect of older siblings' overall and subject-specific ranks on the track recommendations received by younger siblings, controlling for younger sibling overall score. Each cell represents one regression. Control variables: point dummies for older sibling overall score, number of parents with migrant background (categorical), year of birth of the younger sibling (categorical), taxable income of the household in the year preceding the younger sibling's standardized test. Sample restriction: siblings likely to have the same primary school teacher. Standard errors are clustered at the level of the younger child's school. *, ** and ${ }^{* * *}$ denote significance levels at the 10\%, 5\%, and $1 \%$ respectively. 
Table D.4: The Effect of Older Sibling Rank on Younger Sibling Track Recommendation, Controlling for Older Sibling Subject Scores and Younger Sibling Overall Score

Younger sibling outcome:

Track Recommendation:

Academic General Vocational

(1)

(2)

(3)

Older sibling rank:

Overall rank

$\begin{array}{lll}0.010 & 0.008 \quad-0.006\end{array}$

$(0.008) \quad(0.009) \quad(0.010)$

Dutch rank

$\begin{array}{rrr}0.015 * & 0.011 & -0.019 * \\ (0.008) & (0.009) & (0.010)\end{array}$

Math rank

$\begin{array}{lcc}0.018 * * & 0.012 & -0.024 * * \\ (0.008) & (0.009) & (0.010)\end{array}$

Mean of dep.var

0.12

0.12

0.30

SD of dep.var

Old sib: Cohort FE

Young sib: school-by-cohort FE

0.33

$\mathrm{Y}$

N. young sib. schools

Y

0.33

0.46

$\mathrm{Y}$

$\mathrm{Y}$

5,950

5,950

$\mathrm{Y}$

Observations

198,432

198,432

5,950

Note: This table shows the results of dyadic regression analyses of the effect of older siblings' overall rank on the track recommendations received by younger siblings, controlling for younger sibling overall score. Each cell represents one regression. Control variables: point dummies for older sibling subject-specific score (resp. overall score, Dutch language score and Math score), number of parents with migrant background (categorical), year of birth of the younger sibling (categorical), taxable income of the household in the year preceding the younger sibling's standardized test. Sample restriction: siblings likely to have the same primary school teacher. Standard errors are clustered at the level of the younger child's school. *, ** and ${ }^{* * *}$ denote significance levels at the 10\%, 5\%, and $1 \%$ respectively. 
Table D.5: Robustness of Sibling Rank Effects to Strategic Delay

Sample restrictions:

In school-age cohort:

Younger sibling outcomes: On time

Test Scores:

Any teacher

1)

Overall

Dutch

Math

assessment

(1)

(2)

(3)

(4)

(5)

Older sibling overall rank

$\begin{array}{cllll}0.032 * * & 1.407 * * * & 1.725 * * * & 1.169 * * * & 0.005 \\ (0.0132) & (0.282) & (0.421) & (0.370) & (0.004)\end{array}$

Mean of dep.var

0.83

536.31

80.28

47.59

0.75

SD of dep.var

0.37

9.14

16.91

13.37

0.43

R-Squared

0.31

0.43

0.62

0.55

0.92

Yg sib in school-age cohort

Old sib: Cohort FE

Yg sib: school-by-cohort FE

$\mathrm{Y}$

$\mathrm{Y}$

$\mathrm{Y}$

$\begin{array}{lll}\mathrm{Y} & \mathrm{Y} & \mathrm{Y} \\ \mathrm{Y} & \mathrm{Y} & \mathrm{Y}\end{array}$

Y

$\mathrm{Y}$

Y

5,872

5,892

5,892

5,892

$$
\mathrm{Y}
$$

N. yg sib. sch
Observations

154,780

160,537

160,537

160,537

5,950

Note: This table shows the results of dyadic regression analyses of the effect of older siblings' rank on younger siblings' probability to finish primary school on time (col. 1), on the scores of younger siblings who are on time (col. (2) to (4)), and on the probability of younger siblings to have a teacher subjective assessment. Each column represents one regression. Control variables: point dummies for older sibling score, number of parents with a migrant background (categorical), birth year of the younger sibling (categorical), the taxable income of the household in the year preceding the young child's test. Sample restriction: siblings likely to have the same primary school teacher, and from column (2) children who are in their "normal" testing year given their date of birth - that is, turning 12 within the year of their CITO first attempt. Standard errors are clustered at the level of the younger child's school. *, ** and *** denote significance levels at the 10\%, 5\%, and 1\% respectively. 


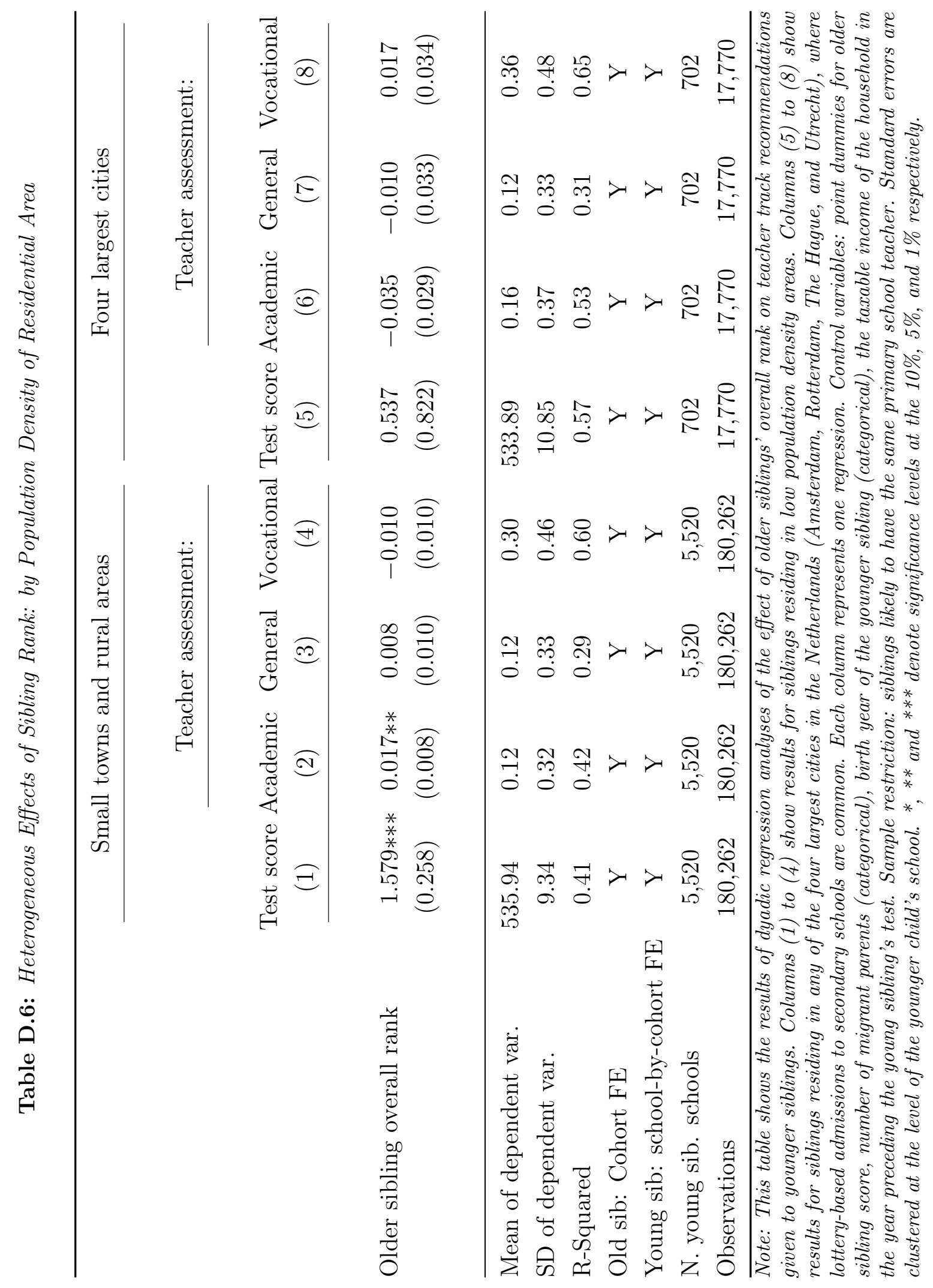




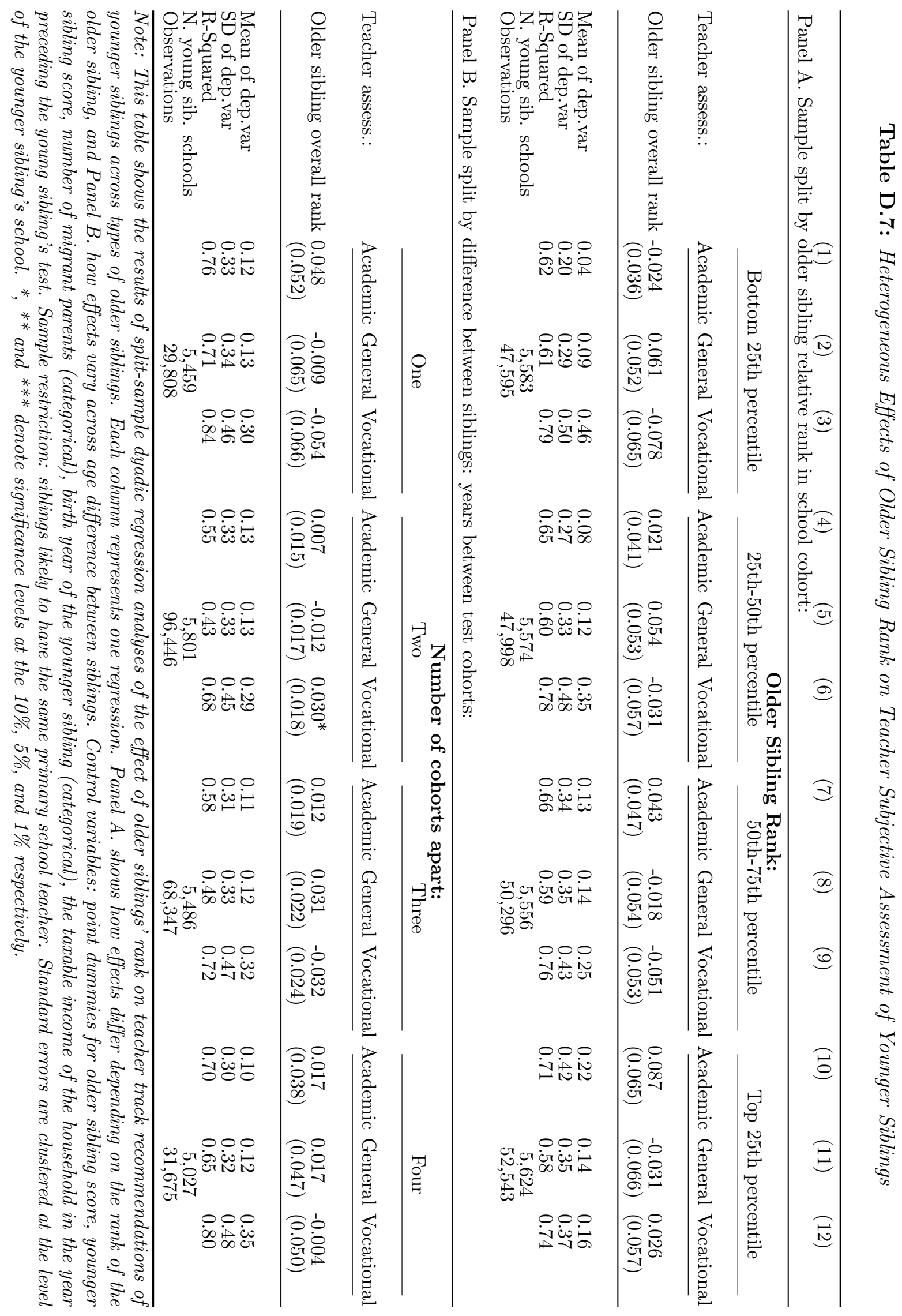




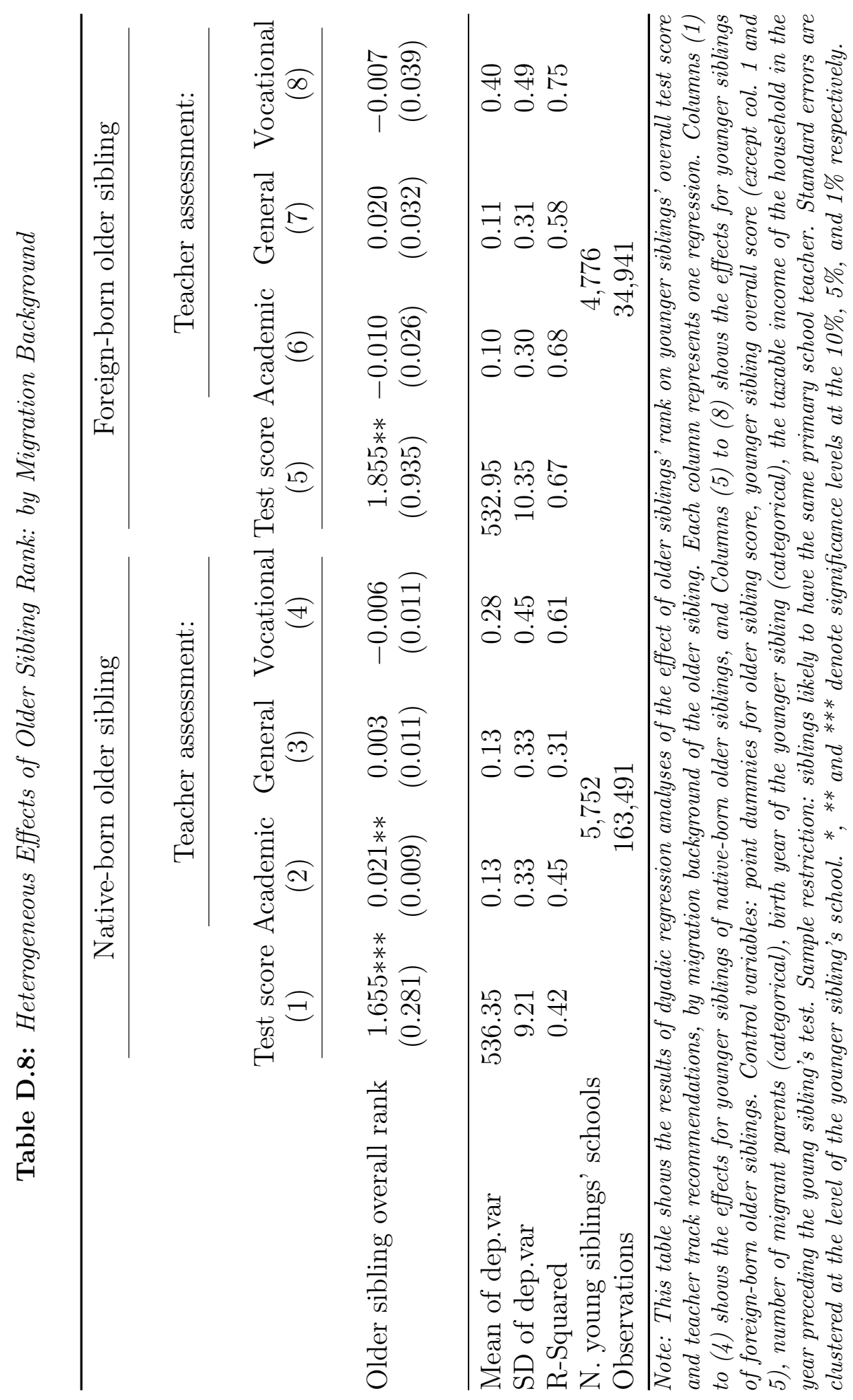


Table D.9: Heterogeneous Effects of Older Siblings' Rank: by Presence in the Household of Older Child Eligible to Means-Tested Higher Education Financial Aid

\begin{tabular}{|c|c|c|c|c|c|c|}
\hline Eligible Sibling: & & None & & & $t$ least ol & \\
\hline Test scores: & $\begin{array}{l}\text { Overall } \\
\text { (1) }\end{array}$ & $\begin{array}{l}\text { Dutch } \\
(2)\end{array}$ & $\begin{array}{c}\text { Math } \\
(3)\end{array}$ & $\begin{array}{l}\text { Overall } \\
(4)\end{array}$ & $\begin{array}{l}\text { Dutch } \\
(5)\end{array}$ & $\begin{array}{l}\text { Math } \\
(6)\end{array}$ \\
\hline $\begin{array}{l}\text { Interaction: At least one } \\
\# \text { older sibling rank }\end{array}$ & $\begin{array}{r}-0.119 \\
(0.534)\end{array}$ & $\begin{array}{r}-0.144 \\
(0.806)\end{array}$ & $\begin{array}{r}-0.276 \\
(0.713)\end{array}$ & $\begin{array}{c}0.435 \\
(3.885)\end{array}$ & $\begin{array}{c}0.791 \\
(5.967)\end{array}$ & $\begin{array}{c}1.654 \\
(5.157)\end{array}$ \\
\hline Mean of dep.var & 535.77 & 79.65 & 47.08 & 535.52 & 80.08 & 46.92 \\
\hline SD of dep.var & 9.52 & 17.35 & 13.72 & 9.31 & 17.09 & 13.67 \\
\hline R-Squared & 0.44 & 0.62 & 0.55 & 0.86 & 0.90 & 0.88 \\
\hline Old sib Cohort FE & $\mathrm{Y}$ & $\mathrm{Y}$ & $\mathrm{Y}$ & $\mathrm{Y}$ & $\mathrm{Y}$ & $\mathrm{Y}$ \\
\hline Yg. sib school-by-cohort FE & $\mathrm{Y}$ & $\mathrm{Y}$ & $\mathrm{Y}$ & $\mathrm{Y}$ & $\mathrm{Y}$ & $\mathrm{Y}$ \\
\hline N. yg siblings' schools & 5,939 & 5,939 & 5,939 & 4,194 & 4,194 & 4,194 \\
\hline Observations & 185,723 & 185,723 & 185,723 & 12,541 & 12,541 & 12,541 \\
\hline
\end{tabular}

Note: This table shows the results of dyadic regression analyses of the effect of older siblings' overall rank on younger siblings' scores, split by whether or not there was any older child eligible to means-tested financial aid for post-secondary education present in the household at the time when the younger sibling is taking his CITO test. Columns (1) to (3) show results for families where no other child was eligible to means-tested financial aid when the older sibling was taking the test, and while columns (4) to (6) show results for families where at least one other child was eligible at the time. Each column represents one regression. Control variables: point dummies for older sibling score, number of parents with a migrant background (categorical), birth year of the younger sibling (categorical), the taxable income of the household in the year preceding the young sibling's test. Sample restriction: siblings likely to have the same primary school teacher. Standard errors are clustered at the level of the younger child's school. ${ }^{*},{ }^{* *}$ and ${ }^{* * *}$ denote significance levels at the 10\%, 5\%, and $1 \%$ respectively. 
Table D.10: Heterogeneous Effects of Older Siblings' Rank: by Sibling Gender-Match

\section{Panel A. Older brothers}

Younger sibling outcomes:

Test Scores:

Teacher Assessment:

Overall Dutch Math Academic General Vocational

(2)

(3)

(4)

(5)

(6)

\begin{tabular}{|c|c|c|c|c|c|c|}
\hline \multirow{3}{*}{ Interaction: Male \# rank } & $(1)$ & \multicolumn{2}{|l|}{$(2)$} & $(4)$ & $(5)$ & $(6)$ \\
\hline & 0.325 & $1.840 * *$ & $k-1.091 * * *$ & 0.009 & 0.002 & -0.000 \\
\hline & $(0.268)$ & $(0.402)$ & $(0.347)$ & $(0.009)$ & $(0.010)$ & $(0.011)$ \\
\hline Mean of dep.var & 535.77 & 79.64 & 47.16 & 0.12 & 0.12 & 0.30 \\
\hline SD of dep.var & 9.47 & 17.29 & 13.67 & 0.33 & 0.33 & 0.46 \\
\hline R-Squared & 0.54 & 0.69 & 0.63 & 0.53 & 0.42 & 0.68 \\
\hline Old sib: Cohort FE & $\mathrm{Y}$ & Y & $\mathrm{Y}$ & $\mathrm{Y}$ & $\mathrm{Y}$ & $\mathrm{Y}$ \\
\hline Yg. sib: school-by-cohort FE & Y & $\mathrm{Y}$ & $\mathrm{Y}$ & $\mathrm{Y}$ & $\mathrm{Y}$ & $\mathrm{Y}$ \\
\hline N. young sib. schools & 5,807 & 5,807 & 5,807 & 5,807 & 5,807 & 5,807 \\
\hline Observations & 99,837 & 99,837 & 99,837 & 99,837 & 99,837 & 99,837 \\
\hline
\end{tabular}

\section{Panel B. Older sisters}

Younger sibling outcomes:

Test Scores:

Teacher Assessment:

Overall Dutch Math Academic General Vocational

Interaction: Female \# rank $\quad 0.500 * \quad-0.599 \quad 1.620 * * * \begin{array}{llll}0.016 * & -0.002 & -0.011\end{array}$

\begin{tabular}{lcccccc} 
& $(0.271)$ & $(0.406)$ & $(0.347)$ & $(0.009)$ & $(0.010)$ & $(0.011)$ \\
\hline Mean of dep.var & 535.73 & 79.71 & 46.97 & 0.12 & 0.12 & 0.30 \\
SD of dep.var & 9.55 & 17.38 & 13.76 & 0.33 & 0.33 & 0.46 \\
R-Squared & 0.55 & 0.69 & 0.64 & 0.53 & 0.42 & 0.68 \\
Old sib: Cohort FE & $\mathrm{Y}$ & $\mathrm{Y}$ & $\mathrm{Y}$ & $\mathrm{Y}$ & $\mathrm{Y}$ & $\mathrm{Y}$ \\
Yg. sib: school-by-cohort FE & $\mathrm{Y}$ & $\mathrm{Y}$ & $\mathrm{Y}$ & $\mathrm{Y}$ & $\mathrm{Y}$ & $\mathrm{Y}$ \\
N. young sib. schools & 5,784 & 5,784 & 5,784 & 5,784 & 5,784 & 5,784 \\
Observations & 98,595 & 98,595 & 98,595 & 98,595 & 98,595 & 98,595
\end{tabular}

Note: This table shows the results of dyadic regression analyses of the effect of older siblings' overall rank on their younger siblings' scores and subjective assessment of teachers, depending on gender composition of sibling pairs. Each column represents one regression. Control variables: point dummies for older sibling score, younger sibling score, number of migrant parents (categorical), birth year of the younger sibling (categorical), the taxable income of the household in the year preceding the young sibling's test. Sample restriction: siblings likely to have the same primary school teacher. Standard errors are clustered at the level of the younger child's school. *, ** and *** denote significance levels at the 10\%, 5\%, and $1 \%$ respectively. 
Table D.11: Sibling Rank When the Importance of Teacher Assessment Increases

\begin{tabular}{|c|c|c|c|}
\hline \multirow[t]{2}{*}{ Younger sibling outcome: } & \multicolumn{3}{|c|}{ Teacher Assessment: } \\
\hline & $\begin{array}{l}\text { Academic } \\
\text { (1) }\end{array}$ & $\begin{array}{c}\text { General } \\
(2)\end{array}$ & $\begin{array}{c}\text { Vocational } \\
(3)\end{array}$ \\
\hline Interaction: Post reform \# rank & $\begin{array}{l}0.067 * * * \\
(0.007)\end{array}$ & $\begin{array}{l}0.015 * * \\
(0.007)\end{array}$ & $\begin{array}{r}-0.011 \\
(0.009)\end{array}$ \\
\hline Mean of dep.var & 0.12 & 0.12 & 0.30 \\
\hline SD of dep.var & 0.32 & 0.33 & 0.46 \\
\hline R-Squared & 0.43 & 0.29 & 0.60 \\
\hline Old sib: Cohort FE & $\mathrm{Y}$ & $\mathrm{Y}$ & $\mathrm{Y}$ \\
\hline Yg. sib: school-by-cohort FE & $\mathrm{Y}$ & $\mathrm{Y}$ & Y \\
\hline N. young siblings' schools & 5,791 & 5,791 & 5,791 \\
\hline Observations & 193,243 & 193,243 & 193,243 \\
\hline
\end{tabular}

Note: This table shows the results of dyadic regression analyses of the effect of older siblings' rank on teacher subjective assessment of younger siblings, before and after a policy change in 2014. The 2014 reform decreases the weight of standardized scores compared to teacher recommendations for determining secondary schools and track admissions. Each column represents one regression. Control variables: point dummies for older sibling score, number of parents with migrant background (categorical), birth year of the younger sibling (categorical), the taxable income of the household in the year preceding the young sibling's test. Sample restriction: siblings likely to have the same primary school teacher. Standard errors are clustered at the level of the younger child's school. *, ** and ${ }^{* * *}$ denote significance levels at the 10\%, 5\%, and $1 \%$ respectively. 


\section{D.4 Long-Run Impacts of Own-Rank on Human Capital Accumulation}

I show in the main body of my study that older sibling rank has an effect on tracking recommendations of younger siblings. In this Appendix, I replicate results from the literature on rank in my setting for comparison.

Table D.12 reports estimates of the effect of own rank on later outcomes in the educational system; These results replicate some of the earlier findings in the literature on rank. I restrict the sample to children who are older siblings in pairs, and whose younger sibling is likely to have the same primary school teacher. I cluster standard errors at the school level.

Several articles on peer effects and rank effects effectively use school transitions from primary to secondary school the identification strategy (e.g. Kiss, 2013; Kramarz et al., 2015; Murphy and Weinhardt, Forthcoming; Denning et al., 2020). Secondary school peers are mostly new peers, because primary schools are typically chosen at the neighborhood level and are relatively small - roughly 7500 primary schools across the country, with on average 220 children registered in total -, compared to secondary schools which are chosen at a larger geographical area and are much larger institutions - roughly 650 schools across the country, with on average 1400 students registered in total.

Table D.12 reveals that own rank in primary school increases by 5.4 percentage point the probability to graduate from the general track, and decreases by 4.6 percentage point the probability to graduate from the vocational track. These results are in line with Elsner and Isphording (2017) who find that having a higher rank in middle school increases the probability of graduating from high school. I find that, however, rank in primary school has no significant effect on average on the quality of secondary school attended, as measured by the average score of pupils starting secondary school in the same cohort, and by the share of pupils with standardized scores above 545 . I interpret these results as evidence that rank contains an additional signaling value compared to standardized score alone, but mostly for pupils who would be candidates for the vocational or general track, but not really the top track.

The last part of Table D.12 shows that rank also has longer term effects on educational outcomes. My results are, however, in contrast with those found by Elsner and Isphording (2017) and Denning et al. (2020) on university enrolment. I find that 
at fixed ability, moving from the lowest to the best rank in one's primary school decreases on average by 2.3 percentage point the probability of ever being registered in post-secondary education. This negative effect is likely driven by institutional features of the Dutch educational system, in particular tracking. Rank shifts pupils from vocational education towards general education, and students graduating from general high school more likely to take a gap year before enrolling in university compared to vocational students who often work part-time in apprenticeships. Another interpretation is that put forward by Murphy and Weinhardt (Forthcoming) when they consider heterogeneity of the effect of class rank across SES. Lastly, I find that among students who do enroll in post-secondary education, moving from the worst to the best rank also decreases by 5 percentage point the probability of dropping out of one's university major, which is more in line with Elsner and Isphording (2017) and Murphy and Weinhardt (Forthcoming) who find that rank effects are mostly driven by students feeling more confident and becoming persistent in their later educational career. 


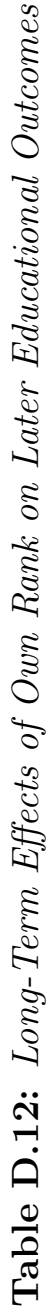

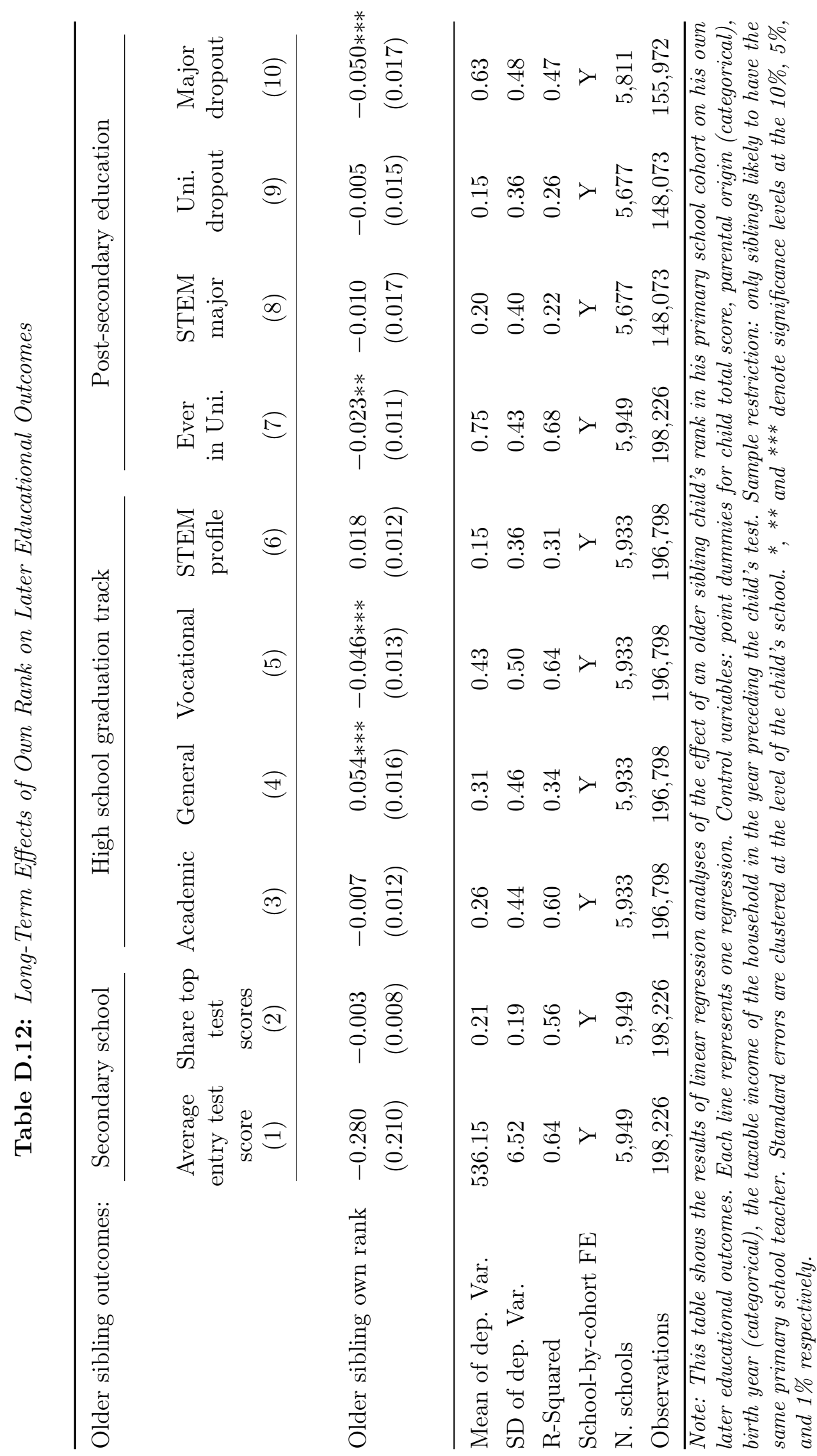





\section{Valorization Addedum}

This dissertation investigates the role of aspirations and beliefs in explaining children's educational outcomes, and explores how they can exacerbate socioeconomic disadvantage in education. Apart from the academic contribution of highlighting the importance of internal constraints in educational outcomes, the findings of this dissertation are also directly useful for policy making.

Chapter 1 proposes an easily-implementable methodology to estimate the potential size of aspirational poverty traps in a given population, and applies this methodology to the United States to show that 5.5 to $7 \%$ of the population could be located in aspirational poverty traps. This important result indicates that social interventions and welfare programs lifting aspirations could have a high potential for reducing achievement gap.

Chapter 2 shows that policy uncertainty about student finance can alone create or exacerbate achievement gaps as early as upon entering middle school. This finding highlights i) that access to higher education could be improved by reducing uncertainty in student finance, and ii) that uncertainty about future policies can harm vulnerable populations, exacerbating inequality of opportunity.

Chapter 3 indicates that high-achieving peers not only improve test scores, but also make children pay exert less school effort but increases their aspiration to go to university, and increase the time they spend with their parents. This study also finds that peer effects in test scores are not explained by intermediate effects on students' effort, aspirations and parental time investments. Together, these findings suggest that educational policies manipulating peer assignment - such as tracking, school admission policies, or classroom assignment policies - can in principle be leveraged to reduce achievement gaps, while increasing students' aspirations.

Chapter 4 shows that $i$ ) teachers rely on imperfect signals about children's ability, and form systematically biased expectations about their potential, ii) teacher bias can disadvantage migrant children, and iii) has long-lasting consequences on STEM major choice in high school. Suggestive findings also indicate that teacher bias can be limited through admissions policies in which teacher assessments have little weight. This study overall indicates that eliminating teacher bias should be a priority for education policies and explores the scope of two educational policies to this end. 


\section{Curriculum Vitae}

Alexandra de Gendre was born on 08 October 1990 in Longjumeau, near Paris, France. She received her French Baccalaureate degree (section Sciences and Mathematics, major Mathematics) in June 2008 from Lycee Saint-Charles in Athis-Mons near Paris. She then pursued a Classe Preparatoire aux Grandes Ecoles (section Humanities and Social Sciences - B/L) from September 2008 to June 2011, at Institut Sainte-Marie in Neuilly-sur-Seine, from which she graduated with honors and following which she was awarded a Bachelor of Arts in History and a Bachelor of Science in Economics from University Paris X. She then pursued a dual Master of Science in Statistics at Ensai (National School for Statistics and Information Analysis - Grande Ecole) and in Economics at Humboldt University in Berlin, Germany. She graduated from Ensai and Humboldt University in December 2014. During her university studies, Alexandra had several professional experiences, including as market analyst at PSA Peugeot Citroën in 2012, as research trainee at the OECD in 2013, and as consultant at Ekimetrics in Paris in 2014. During her PhD, Alexandra conducted research visits at the IZA Institute of Labour Economics in Germany, the Melbourne Institute of Applied Social and Economics Research in Australia, and the University of Zurich in Switzerland. She has presented her research at some of the leading international conferences in her field, including the European Society of Population Economics (ESPE), the Econometric Society (ES), the European Economic Association (EEA), the International Workshop of Applied Economics of Education (IWAEE), the IZA Summer School, and IZA and IZA/Briq workshops. Alexandra has refereed studies for Labour Economics and Health Economics. She has recently attracted seed funding from the Australian Research Council Centre of Excellence for Children and Families over the Life Course (Life Course Centre), and has been invited to join the prestigious IZA network. Alexandra is currently a Postdoctoral Research Fellow at the University of Sydney. 


\section{ROA Dissertation Series}

1. Lex Borghans (1993), Educational Choice and Labour Market Information, Maastricht, Research Centre for Education and the Labour Market.

2. Frank Cörvers (1999), The Impact of Human Capital on International Competitiveness and Trade Performance of Manufacturing Sectors, Maastricht, Research Centre for Education and the Labour Market.

3. Ben Kriechel (2003), Heterogeneity Among Displaced Workers, Maastricht, Research Centre for Education and the Labour Market.

4. Arnaud Dupuy (2004), Assignment and Substitution in the Labour Market, Maastricht, Research Centre for Education and the Labour Market.

5. Wendy Smits (2005), The Quality of Apprenticeship Training, Conflicting Interests of Firms and Apprentices, Maastricht, Research Centre for Education and the Labour Market.

6. Judith Semeijn (2005), Academic Competences and Labour Market Entry: Studies Among Dutch Graduates, Maastricht, Research Centre for Education and the Labour Market.

7. Jasper van Loo (2005), Training, Labor Market Outcomes and Self-Management, Maastricht, Research Centre for Education and the Labour Market.

8. Christoph Meng (2005), Discipline-Specific or Academic? Acquisition, Role and Value of Higher Education Competencies, Maastricht, Research Centre for Education and the Labour Market.

9. Andreas Ammermüller (2007), Institutional Effects in the Production of Education: Evidence from European Schooling Systems, Maastricht, Research Centre for Education and the Labour Market.

10. Bart Golsteyn (2007), The Ability to Invest in Human Capital, Maastricht, Research Centre for Education and the Labour Market.

11. Raymond Montizaan (2010), Pension Rights, human capital development and well-being, Maastricht, Research Centre for Education and the Labour Market.

12. Annemarie Nelen (2012), Part-Time Employment and Human Capital Devel- 
opment, Maastricht, Research Centre for Education and the Labour Market.

13. Jan Sauermann (2013), Human Capital, Incentives, and Performance Outcomes, Maastricht, Research Centre for Education and the Labour Market

14. Harald Ulrich Pfeifer (2013), Empirical Investigations of Costs and Benefits of Vocational Education and Training, Maastricht, Research Centre for Education and the Labour Market.

15. Charlotte Büchner (2013), Social Background, Educational Attainment and Labor Market Integration: An Exploration of Underlying Processes and Dynamics, Maastricht, Research Centre for Education and the Labour Market.

16. Martin Humburg (2014), Skills and the Employability of University Graduates, Maastricht, Research Centre for Education and the Labour Market.

17. Jan Feld (2014), Making the Invisible Visible, Essays on Overconfidence, Discrimination and Peer Effects, Maastricht, Research Centre for Education and the Labour Market.

18. Olga Skriabikova (2014), Preferences, Institutions, and Economic Outcomes: an Empirical Investigation, Maastricht, Research Centre for Education and the Labour Market.

19. Gabriele Marconi (2015), Higher Education in the National and Global Economy, Maastricht, Research Centre for Education and the Labour Market.

20. Nicolás Salamanca Acosta (2015), Economic Preferences and Financial RiskTaking, Maastricht, Research Centre for Education and the Labour Market.

21. Ahmed Elsayed Mohamed (2015), Essays on Working Hours, Maastricht, Research Centre for Education and the Labour Market.

22. Roxanne Amanda Korthals (2015), Tracking Students in Secondary Education, Consequences for Student Performance and Inequality, Maastricht, Research Centre for Education and the Labour Market.

23. Maria Zumbuehl (2015), Economic Preferences and Attitudes: Origins, Behavioral Impact, Stability and Measurement, Maastricht, Research Centre for Education and the Labour Market.

24. Anika Jansen (2016), Firms' incentives to provide apprenticeships - Studies 
on expected short- and long-term benefits, Maastricht, Research Centre for Education and the Labour Market.

25. Jos Maarten Arnold Frank Sanders (2016), Sustaining the employability of the low skilled worker: Development, mobility and work redesign, Maastricht, Research Centre for Education and the Labour Market.

26. Marion Collewet (2017), Working hours: preferences, well-being and productivity, Maastricht, Research Centre for Education and the Labour Market.

27. Tom Stolp (2018), Sorting in the Labor Market: The Role of Risk Preference and Stress, Maastricht, Research Centre for Education and the Labour Market.

28. Frauke Meyer (2019), Individual motives for (re-)distribution, Maastricht, Research Centre for Education and the Labour Market.

29. Maria Ferreira Sequeda (2019), Human Capital Development at School and Work, Maastricht, Research Centre for Education and the Labour Market.

30. Marie-Christine Martha Fregin (2019), Skill Matching and Outcomes: New Cross-Country Evidence, Maastricht, Research Centre for Education and the Labour Market.

31. Sanne Johanna Leontien van Wetten (2020), Human capital and employee entrepreneurship: The role of skills, personality characteristics and the work context, Maastricht, Research Centre for Education and the Labour Market.

32. Cécile Alice Jeanne Magnée (2020), Playing the hand you're dealt, The effects of family structure on children's personality and the effects of educational policy on educational outcomes of migrant children, Maastricht, Research Centre for Education and the Labour Market.

33. Merve Nezihe Özer (2020), Essays on drivers and long-term impact of migration, Maastricht, Research Centre for Education and the Labour Market.

34. Inge Ingeborg Henrica Maria Hooijen (2021), Place attractiveness, A study of the determinants playing a role in residential settlement behaviour, Maastricht, Research Centre for Education and the Labour Market.

35. Alexandra Marie Catherine de Gendre (2021), Behavioral Barriers to Success in Education, Maastricht, Research Centre for Education and the Labour Market. 